Prepared for the U.S. Department of Energy

under Contract DE-AC05-76RL01830

\title{
Revision of the APGEMS Dose Conversion Factor File Using Revised Factors from Federal Guidance Report 12 and 13
}

TR Hay

JP Rishel

September 2013

\section{Pacific Northwest}

NATIONAL LABORATORY

Proudly Operated by Battelle Since 1965 


\title{
DISCLAIMER
}

This report was prepared as an account of work sponsored by an agency of the United States Government. Neither the United States Government nor any agency thereof, nor Battelle Memorial Institute, nor any of their employees, makes any warranty, express or implied, or assumes any legal liability or responsibility for the accuracy, completeness, or usefulness of any information, apparatus, product, or process disclosed, or represents that its use would not infringe privately owned rights. Reference herein to any specific commercial product, process, or service by trade name, trademark, manufacturer, or otherwise does not necessarily constitute or imply its endorsement, recommendation, or favoring by the United States Government or any agency thereof, or Battelle Memorial Institute. The views and opinions of authors expressed herein do not necessarily state or reflect those of the United States Government or any agency thereof.

\author{
PACIFIC NORTHWEST NATIONAL LABORATORY \\ operated by \\ BATTELLE \\ for the \\ UNITED STATES DEPARTMENT OF ENERGY \\ under Contract DE-AC05-76RL01830
}

Printed in the United States of America
Available to DOE and DOE contractors from the Office of Scientific and Technical Information,
P.O. Box 62, Oak Ridge, TN 37831-0062;
ph: (865) 576-8401
fax: $(865) 576-5728$
email: reports@adonis.osti.gov
Available to the public from the National Technical Information Service
5301 Shawnee Rd., Alexandria, VA 22312 ph: (800) 553-NTIS (6847)
email: orders@ntis.gov $<$ http://www.ntis.gov/about/form.aspx $>$
Online ordering: http://www.ntis.gov

This document was printed on recycled paper. 


\title{
Revision of the APGEMS Dose Conversion Factor File Using Revised Factors from Federal Guidance Report
} 12 and 13.

\author{
TR Hay
}

JP Rishel

September 2013

Prepared for

the U.S. Department of Energy

under Contract DE-AC05-76RL01830

Pacific Northwest National Laboratory

Richland, Washington 99352 


\section{Summary}

The Air Pollutant Graphical Environmental Monitoring System (APGEMS) is used by the Hanford Emergency Operation Center (EOC) to provide refined plume modeling of releases involving radionuclides. The dose conversion factors (DCFs) used by APGEMS to convert air concentration to dose are stored in a file called HUDUFACT.dat; the DCFs are based primarily on ICRP 30 compiled in the late 1980's. This report updates the DCFs using more recent values reported in the Environmental Protection Agency's (EPAs) Federal Guidance Report (FGR) 12 and 13. FGR 12 provides external exposure (air submersion) DCFs for radionuclides in air; FGR 13 provides DCFs for radionuclides from inhalation. DCFs were updated for only those radionuclides listed in the original HUDUFACT.dat file. Since FGR 13 provides inhalation dose conversion factors as a function of age, revised DCF files were created for APGEMS for each age group. The "adult” DCF file is the most relevant to compare to the original DCF file being used in APGEMS; these DCF values are compared in this report. 



\section{Acronyms and Abbreviations}

$\begin{array}{ll}\text { APGEMS } & \text { Air Pollutant Graphical Environmental Monitoring System } \\ \text { DCF } & \text { Dose Conversion Factor } \\ \text { DOE } & \text { U.S. Department of Energy } \\ \text { EPA } & \text { U.S. Environmental Protection Agency } \\ \text { FGR } & \text { Federal Guidance Report } \\ \text { LET } & \text { Linear Energy Transfer }\end{array}$





\section{Contents}

Acronyms and Abbreviations
1.0 Introduction
2.0 Methodology 


\subsection{Introduction}

The Air Pollutant Graphical Environmental Monitoring System (APGEMS) is used by the Hanford Emergency Operation Center (EOC) to provide refined plume modeling of releases involving radionuclides. The dose conversion factors (DCFs) used by APGEMS to convert air concentration to dose are stored in a file called HUDUFACT.dat (dated 9/4/1990); the DCFs are based primarily on ICRP 30 compiled in the late 1980's.

This project involved revising the APGEMS HUDUFACT.dat file using more recent DCFs reported in the Environmental Protection Agency's (EPAs) Federal Guidance Report (FGR) 12 (Eckerman et al. 1993) and 13 (Eckerman et al. 1998). FGR 12 provides external exposure (air submersion) DCFs for radionuclides in air; FGR 13 provides DCFs for radionuclides from inhalation.

The update was performed by writing a Visual Basic (VB) script to extract the relevant factors from FGR 12/13 and write the corresponding HUDUFACT.dat file required by APGEMS. DCFs were updated for only those radionuclides listed in the existing HUDUFACT.dat file. Since FGR 13 provides inhalation dose conversion factors as a function of age, the script creates age-based dose conversion files, each in the HUDUFACT.dat file format that can be used in APGEMS. The "adult" (age 7300 days, and for some radionuclides, age 9125 days) dose conversion file are the most relevant DCFs to compare to the original values being used in APGEMS. 


\subsection{Methodology}

The original APGEMS dose conversion factors found in HUDUFACT.dat (dated 9/4/1990) are based on ICRP 30 and GENII outputs compiled in the late 1980's. Since then, the EPA has issued two guidance documents (FGR 12/13) for the purpose of providing federal and state agencies with technical information to assist in implementation of radiation protection programs. These guidance documents were used to update the file HUDUFACT.dat (dated 9/4/1990).

A VB script was written to extract the relevant dose conversion factors from FGR 12/13 and write the corresponding formatted dose conversion file (HUDUFACT.dat) required by APGEMS (the full script found in Appendix $\mathrm{C}$ of this report ). The script:

i. Reads in the existing HUDUFACT.dat file (dated 9/4/1990)

ii. For each radionuclide in HUDUFACT.dat, reads in the FGR 12/13 dose conversion data files and extract the relevant dose conversion factors

iii. Writes a revised HUDUFACT.dat file using the FGR 12/13 values (Note: since the inhalation dose conversion factors in FGR13 are now a function of age, age-based HUDUFACT.dat files were written for APGEMS; the "adult" file is most comparable to the original DCF file used by APGEMS)

iv. The revised, aged-base dose conversion factor files were validated by hand-checking the values against the factors listed in the FGR 12/13 reports

v. The revised dose conversion factors were compared against the existing values in HUDUFACT.dat file (dated 9/4/1990); these values were written to a separate file and are available for further review

\subsection{Units}

The units for the DCF factors in the original HUDUFACT.dat file are in the header of the file; the units are as follows:

\begin{tabular}{clllll}
\multicolumn{2}{c}{ AIR } & & & & \\
SUBMERSION & BONE & RED & & EFF DOSE \\
Sv/Yr per & LUNGS & SURFACE & MARROW & THYROID & EQUIV. \\
Bq/m3 & Sv/Bq & Sv/Bq & Sv/Bq & Sv/Bq & Sv/Bq
\end{tabular}

The FGR 12 and 13 DCFs units were converted, if necessary, to be consistent with the units in HUDUFACT.dat. 


\subsection{Results}

The VB script extracts data from FGR 12 for air submersion and FGR 13 for the inhalation dose conversion factors (DCFs). These data were arranged in a format consistent with that in the original DCF file HUDUFACT.dat. The original HUDUFACT.dat (dated 9/4/1990) file contains data for 1- and 50year committed dose. However, the 1-year DCFs are not available in FGR 12/13, nor are they used in the APGEMS; it was determined that the 1-year DCFs are in the file because, at one time, also used by the now obsolete HUDU program. Thus, to preserve the format in the revised HUDUFACT.dat DCF file, the 1 -year DCFs were made equivalent to the 50-year DCFs.

Finally, the original HUDUFACT.dat (dated 9/4/1990) file contains DCF data for adults. The revised inhalation DCFs in FGR 13 are available for different age groups (in days): 100, 365, 1825, 3650, 5475, and 7300/9125. Thus, separate files were created for each age group. The age group 7300/9125 days is the age group that corresponds more closely to the age group in the original file. Complete files, for each age group, are presented in appendix A and are available electronically.

\subsection{Conversion requirements}

In order to match the original HUDUFACT.dat (dated 9/4/1990) file format, a few conversions were needed. The lung transfer class from the FGR 13 is presented as Slow (S), Medium (M), and Fast (F) whereas, the original HUDUFACT.dat file is presented in Days (D), Weeks (W), and Years (Y). The assumed correspondence between the two systems is as follows:

$$
\begin{aligned}
& S=Y \\
& M=W \\
& F=D
\end{aligned}
$$

DCFs for the radionuclides that include high and low linear energy transfer (LET) are provided separately in FGR 13. Thus, to preserve the format in HUDUFACT.dat (dated 9/4/1990) the high and low LET were combined using the appropriate radiation weighting factors (20 for high LET, 1 for low LET) (ICRP 1990). The following equation is used:

$$
\text { Total DCF }=20 * \text { high LET DCF + Low LET DCF }
$$

There are a few radionuclides that are in HUDUFACT.dat (dated 9/4/1990) and do not have corresponding DCFs in FGR 13; these are primarily non-depositing radionuclides (e.g., noble gases) and include:
- $\operatorname{Ar}-39$
- $\operatorname{Ar}-41$
- $\mathrm{Kr}-83 \mathrm{~m}$
- $\mathrm{Kr}-85 \mathrm{~m}$
- $\mathrm{Kr}-85$
- $\mathrm{Kr}-87$
- $\mathrm{Kr}-88$

- $\mathrm{Kr}-89$

- Xe-137

- Nb-97m

- Xe-131m

- Xe-133m

- Xe-133

- Xe-135m

- $\mathrm{Xe-138}$

- $\operatorname{Pr}-144 m$

- Rn-222

- $\mathrm{Cm}-248$

- $\mathrm{Cf}-252$

- Xe-135 
For these radionuclides, the DCFs from the original HUDUFACT.dat (dated 9/4/1990) were used.

\subsection{Comparison of FRG12/13 to HUDUFACT.dat}

A VB script was created to compare the ratio of the revised DCFs from FRG12/13 to the original DCFs in HUDUFACT.dat (dated 9/4/1990). Figure 1 is a plot of the DCF ratios (FGR/original values) for effective dose equivalent. A value of one implies total agreement. Most radionuclides are within a factor of two and the largest difference is around a factor of 6 . The full results of the comparisons are presented in Appendix B. There are also two radionuclides in the original HUDUFACT.dat (dated 9/4/1990) file that had an air submersion value of zero, however, the revised DCF from FGR 12 is non-zero; these radionuclides are: Sn-121m and Sn-121.

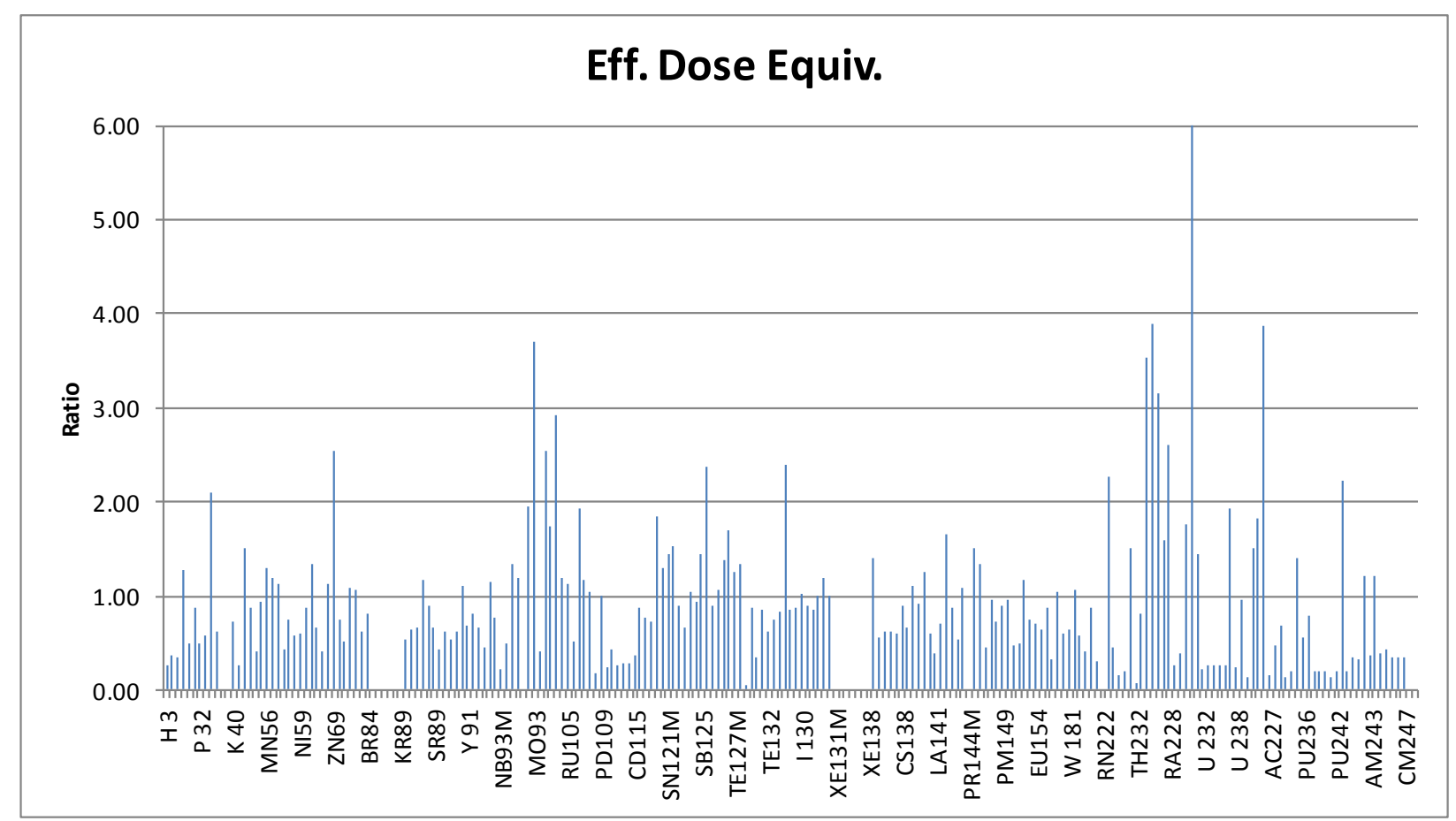

Figure 1. Ratio of the revised FGR13 effective dose equivalent DCFs to the original HUDUFACT.dat effective dose equivalent DCFs for the various radionuclides (Note: not all radionuclide labels are displayed).

\subsection{Quality Assurance}

The DCFs were hand-checked for several radionuclides to verify the values were correctly extracted by the VB script from the FGR 12/13 files and inserted into the revised (age-based) HUDUFACT.dat files. The VB script also generated a log file, which allowed the DCFs for each radionuclide to be easily compared and verified; Figure 1 and Appendix B provides ratios of the revised DCF's (from FGR12/13) to the original DCF's (from HUDUFACT.dat). Finally, the APGEMS program was tested with the revised HUDUFACT.dat file; it ran successfully with the revised DCFs.

To use a given DCF file, it simply has to be renamed as "HUDUFACT.DAT" and copied into the APGEMS StaticData folder. The 7300/9125 age group file should be used as the default 
HUDUFACT.DAT file. Appropriate quality control procedures should be employed by the APGEMS user or custodian to ensure that the default HUDUFACT file is always returned to active status after the termination of the current event or exercise. 


\subsection{References}

Eckerman, KF., Ryman, JC., 1993. External Exposure to Radionuclides in Air, Water, and Soil; Federal Guidance Report No. 12. EPA 402-R-93-081; http://ordose.ornl.gov/documents/fgr12.pdf, U.S. Environmental Protection Agency, Washington, DC.FGR 12.

Eckerman K F, Leggett R W, Nelson C B, Puskin J S and Richardson A C B 1998 Health risks from lowlevel environmental exposure to radionuclides Federal Guidance Report no 13, part I - interim version Environmental Protection Agency, Washington, DC.FGR 13.

ICRP 1990 Recommendations of the International Commission on Radiological Protection ICRP Publication 60. 


\section{Appendix A Dose Equiv. Factors for Acute Inhalation and Ext Air Submersion FGR12/13}

\begin{tabular}{|c|c|c|c|c|c|c|c|c|}
\hline Age & /9125 & & & & & & & \\
\hline se & to & & $\begin{array}{l}\text { or Acute I } \\
\text { AIR }\end{array}$ & $\begin{array}{r}\text { alation } \\
\text { Acute }\end{array}$ & $\begin{array}{l}\text { and Ext } \mathrm{Ai} \\
\text { Inhal. Con }\end{array}$ & $\begin{array}{l}\text { Submersio } \\
\text { itted Dose }\end{array}$ & $\begin{array}{r}\text { n FGR12 } \\
\text { Equival }\end{array}$ & ent \\
\hline & & & UBMERSION & & BONE & RED & & EFF DOSE \\
\hline & & & $\mathrm{v} / \mathrm{Yr}$ per & LUNGS & SURFACE & MARROW & THYROID & EQUIV. \\
\hline & & & $\mathrm{Bq} / \mathrm{m} 3$ & $\mathrm{~Sv} / \mathrm{Bq}$ & $\mathrm{Sv} / \mathrm{Bq}$ & $\mathrm{Sv} / \mathrm{Bq}$ & $\mathrm{Sv} / \mathrm{Bq}$ & $\mathrm{S} v / \mathrm{Bq}$ \\
\hline H 3 & Class: D & & $\odot . \odot \odot E \odot \odot$ & & & & & \\
\hline & 1 & Yr: & & $6.2 \mathrm{E}-12$ & $6.2 \mathrm{E}-12$ & $6.2 \mathrm{E}-12$ & $6.2 \mathrm{E}-12$ & $6.3 \mathrm{E}-12$ \\
\hline & 50 & Yr: & & $6.2 \mathrm{E}-12$ & $6.2 \mathrm{E}-12$ & $6.2 \mathrm{E}-12$ & $6.2 \mathrm{E}-12$ & $6.3 \mathrm{E}-12$ \\
\hline BE10 & Class: $Y$ & & $4.35 \mathrm{E}-09$ & & & & & \\
\hline & 1 & Yr: & & $2.8 \mathrm{E}-07$ & $6.2 \mathrm{E}-\odot 9$ & $2.1 \mathrm{E}-\odot 9$ & $7.1 \mathrm{E}-11$ & $3.5 E-08$ \\
\hline & 50 & Yr: & & $2.8 \mathrm{E}-07$ & $6.2 \mathrm{E}-09$ & $2.1 \mathrm{E}-09$ & 7.1E-11 & $3.5 \mathrm{E}-08$ \\
\hline C 14 & Class: D & & $8.20 \mathrm{E}-11$ & & & & & \\
\hline & 1 & Yr: & & 2. $\odot E-10$ & 1. $9 \mathrm{E}-10$ & $1.9 \mathrm{E}-10$ & 1. $9 \mathrm{E}-10$ & $2.0 \mathrm{E}-10$ \\
\hline & 50 & Yr: & & $2.0 \mathrm{E}-10$ & $1.9 \mathrm{E}-10$ & $1.9 \mathrm{E}-10$ & 1. $9 \mathrm{E}-10$ & $2.0 \mathrm{E}-10$ \\
\hline F 18 & Class: D & & 1. $44 \mathrm{E}-06$ & & & & & \\
\hline & 1 & Yr: & & $2.6 \mathrm{E}-11$ & $2.5 \mathrm{E}-11$ & $2.7 \mathrm{E}-11$ & 3. $4 \mathrm{E}-12$ & $2.8 \mathrm{E}-11$ \\
\hline & 50 & Yr: & & $2.6 \mathrm{E}-11$ & $2.5 \mathrm{E}-11$ & $2.7 \mathrm{E}-11$ & $3.4 \mathrm{E}-12$ & 2. $8 \mathrm{E}-11$ \\
\hline NA22 & Class: D & & $3.22 \mathrm{E}-06$ & & & & & \\
\hline & 1 & Yr: & & $9.2 \mathrm{E}-10$ & $2.2 \mathrm{E}-09$ & $1.6 \mathrm{E}-09$ & $9.6 \mathrm{E}-10$ & 1. $3 \mathrm{E}-09$ \\
\hline & 50 & Yr: & & $9.2 \mathrm{E}-10$ & $2.2 \mathrm{E}-\odot 9$ & $1.6 \mathrm{E}-\odot 9$ & $9.6 \mathrm{E}-10$ & 1. $3 \mathrm{E}-09$ \\
\hline NA24 & Class: D & & $6.56 \mathrm{E}-06$ & & & & & \\
\hline & 1 & Yr: & & 1. $4 \mathrm{E}-10$ & $2 . \odot E-10$ & $1.5 \mathrm{E}-10$ & 1. $2 \mathrm{E}-10$ & $2.8 \mathrm{E}-10$ \\
\hline & 50 & Yr: & & 1. $4 \mathrm{E}-10$ & $2 . \odot E-1 \odot$ & 1. $5 \mathrm{E}-10$ & 1. $2 \mathrm{E}-10$ & $2.8 \mathrm{E}-10$ \\
\hline P 32 & Class: D & & $1.69 \mathrm{E}-\odot 8$ & & & & & \\
\hline & 1 & Yr: & & $3.0 \mathrm{E}-10$ & $3.3 E-\odot 9$ & $3.3 E-\odot 9$ & $2.7 \mathrm{E}-10$ & $7.8 \mathrm{E}-10$ \\
\hline & 50 & Yr: & & $3.0 \mathrm{E}-10$ & $3.3 \mathrm{E}-09$ & $3.3 \mathrm{E}-09$ & $2.7 \mathrm{E}-10$ & $7.8 \mathrm{E}-10$ \\
\hline P 33 & Class: D & & $4.57 \mathrm{E}-10$ & & & & & \\
\hline & 1 & Yr: & & $5.3 E-11$ & $5.5 \mathrm{E}-10$ & $2.1 \mathrm{E}-10$ & $3.8 \mathrm{E}-11$ & $9.3 \mathrm{E}-11$ \\
\hline & 50 & Yr: & & $5.3 E-11$ & $5.5 E-10$ & $2.1 \mathrm{E}-10$ & $3.8 \mathrm{E}-11$ & $9.3 \mathrm{E}-11$ \\
\hline S 35 & Class: W & & $9.81 \mathrm{E}-11$ & & & & & \\
\hline & 1 & Yr: & & 1. $2 \mathrm{E}-\odot 8$ & $7.8 \mathrm{E}-12$ & $7.8 \mathrm{E}-12$ & $7.8 \mathrm{E}-12$ & 1. $4 \mathrm{E}-\odot 9$ \\
\hline & 50 & Yr: & & 1. $2 \mathrm{E}-\odot 8$ & $7.8 \mathrm{E}-12$ & $7.8 \mathrm{E}-12$ & 7. $8 \mathrm{E}-12$ & 1. $4 \mathrm{E}-09$ \\
\hline CL36 & Class: D & & $5.23 E-09$ & & & & & \\
\hline & 1 & Yr: & & 3. $0 \mathrm{E}-10$ & $2.7 \mathrm{E}-10$ & $2.7 \mathrm{E}-10$ & $2.7 \mathrm{E}-10$ & $3.3 E-10$ \\
\hline & $5 \odot$ & Yr: & & $3.0 \mathrm{E}-10$ & $2.7 \mathrm{E}-10$ & $2.7 \mathrm{E}-10$ & $2.7 \mathrm{E}-10$ & $3.3 \mathrm{E}-10$ \\
\hline AR39 & NobleGas & & $3.63 \mathrm{E}-\odot 9$ & & & & & \\
\hline & 1 & Yr: & & $\odot . \odot \mathrm{E}-\odot \odot$ & $\odot . \odot \mathrm{E}-\odot \odot$ & $\odot . \odot \mathrm{E}-\odot \odot$ & $\odot . \odot E-\odot \odot$ & $\odot . \odot \mathrm{E}-\odot \odot$ \\
\hline & 50 & Yr: & & $\odot . \odot \mathrm{E}-\odot \odot$ & $\odot . \odot \mathrm{E}-\odot \odot$ & $\odot . \odot \mathrm{E}-\odot \odot$ & $\odot . \odot \mathrm{E}-\odot \odot$ & $\odot . \odot \mathrm{E}-\odot \odot$ \\
\hline AR41 & NobleGas & & $1.94 \mathrm{E}-06$ & & & & & \\
\hline & 1 & Yr: & & $\odot . \odot \mathrm{E}-\odot \odot$ & $\odot . \odot \mathrm{E}-\odot \odot$ & $\odot . \odot \mathrm{E}-\odot \odot$ & $\odot . \odot \mathrm{E}-\odot \odot$ & $\odot . \odot \mathrm{E}-\odot \odot$ \\
\hline & 50 & Yr: & & $\odot . \odot \mathrm{E}-\odot \odot$ & $\odot . \odot \mathrm{E}-\odot \odot$ & $\odot . \odot \mathrm{E}-\odot \odot$ & $\odot . \odot \mathrm{E}-\odot \odot$ & $\odot . \odot \mathrm{E}-\odot \odot$ \\
\hline K 40 & Class: D & & $2.50 \mathrm{E}-07$ & & & & & \\
\hline & 1 & Yr: & & 1.7E- $\odot 9$ & $1.7 \mathrm{E}-\odot 9$ & $1.7 \mathrm{E}-\odot 9$ & 1. $7 \mathrm{E}-\odot 9$ & $2.1 \mathrm{E}-\odot 9$ \\
\hline & 50 & Yr: & & 1. . E- $\odot 9$ & 1. . E - $\odot 9$ & 1.7E-๑9 & 1. $7 \mathrm{E}-\odot 9$ & $2.1 \mathrm{E}-\odot 9$ \\
\hline CA41 & Class: W & & $\odot . \odot \odot \mathrm{E} \odot$ & & & & & \\
\hline & 1 & Yr: & & $1.6 \mathrm{E}-10$ & 1. $3 E-\odot 9$ & $4.9 \mathrm{E}-10$ & -13 & $9.5 \mathrm{E}-11$ \\
\hline & 50 & Yr: & & $1.6 \mathrm{E}-10$ & $1.3 \mathrm{E}-09$ & $4.9 \mathrm{E}-10$ & $9.5 \mathrm{E}-13$ & $9.5 \mathrm{E}-11$ \\
\hline CA45 & Class: W & & $4.83 E-10$ & & & & & \\
\hline & 1 & Yr: & & $2.1 \mathrm{E}-\odot 8$ & 1. $9 \mathrm{E}-\odot 9$ & $9.3 \mathrm{E}-10$ & 1. $2 \mathrm{E}-11$ & $2.7 \mathrm{E}-\odot 9$ \\
\hline & 50 & Yr: & & $2.1 \mathrm{E}-08$ & $1.9 \mathrm{E}-09$ & $9.3 \mathrm{E}-10$ & 1. $2 \mathrm{E}-11$ & 2. $7 \mathrm{E}-09$ \\
\hline SC46 & Class: Y & & $2.95 \mathrm{E}-06$ & & & & & \\
\hline & 1 & Yr: & & $4.5 E-08$ & 1. $2 \mathrm{E}-\odot 9$ & $E-\odot 9$ & 1. $2 \mathrm{E}-\odot 9$ & $6.8 \mathrm{E}-09$ \\
\hline & 50 & Yr: & & $4.5 \mathrm{E}-\odot 8$ & $1.2 \mathrm{E}-\odot 9$ & $1.6 \mathrm{E}-\odot 9$ & 1. $2 \mathrm{E}-09$ & $6.8 \mathrm{E}-09$ \\
\hline CR51 & Class: Y & & $4.35 E-\odot 8$ & & & & & \\
\hline & 1 & Yr: & & $1.8 \mathrm{E}-10$ & 1. $2 \mathrm{E}-11$ & 1. $3 E-11$ & $9.0 \mathrm{E}-12$ & $3.7 \mathrm{E}-11$ \\
\hline & 50 & Yr: & & $1.8 \mathrm{E}-10$ & 1. $2 \mathrm{E}-11$ & 1. $3 E-11$ & 9. $0 \mathrm{E}-12$ & $3.7 \mathrm{E}-11$ \\
\hline MN54 & Class: W & & $1.21 \mathrm{E}-06$ & & & & & \\
\hline & 1 & Yr: & & $6.5 \mathrm{E}-\odot 9$ & $1.2 \mathrm{E}-\odot 9$ & $1.2 \mathrm{E}-\odot 9$ & $6.7 \mathrm{E}-10$ & 1. $6 \mathrm{E}-09$ \\
\hline & $5 \odot$ & Yr: & & $6.5 \mathrm{E}-\odot 9$ & 1. $2 \mathrm{E}-\odot 9$ & 1. $2 \mathrm{E}-\odot 9$ & $6.7 \mathrm{E}-10$ & 1. $6 \mathrm{E}-09$ \\
\hline MN56 & Class: W & & $2.58 \mathrm{E}-06$ & & & & & \\
\hline & 1 & Yr: & & $3.8 \mathrm{E}-10$ & $7.7 \mathrm{E}-12$ & $9.9 \mathrm{E}-12$ & $6.4 \mathrm{E}-12$ & 1. $2 \mathrm{E}-10$ \\
\hline
\end{tabular}




\begin{tabular}{|c|c|c|c|c|c|c|c|c|}
\hline \multirow{3}{*}{ FE55 } & $5 \odot$ & Yr: & & $3.8 \mathrm{E}-10$ & $7.7 \mathrm{E}-12$ & $9.9 \mathrm{E}-12$ & $6.4 \mathrm{E}-12$ & 1. $2 \mathrm{E}-10$ \\
\hline & Class: W & & $\odot . \odot \odot Е \odot \odot ~$ & & & & & \\
\hline & $\begin{array}{r}1 \\
50\end{array}$ & Yr: & & 4. $0 \mathrm{E}-10$ & $6.7 \mathrm{E}-10$ & 1. $3 \mathrm{E}-09$ & $9.6 \mathrm{E}-11$ & 3. $9 \mathrm{E}-10$ \\
\hline \multirow[t]{3}{*}{ FE59 } & Class: W & & $1.77 \mathrm{E}-\odot 6$ & $4.0 E-10$ & $6.7 E-10$ & 1. 3E- - & & \\
\hline & 1 & Yr: & & $2.3 E-\odot 8$ & $9.8 \mathrm{E}-10$ & 1. $4 \mathrm{E}-\odot 9$ & $6.8 \mathrm{E}-10$ & $3.7 \mathrm{E}-09$ \\
\hline & 50 & Yr: & & 2. 3E- $\odot 8$ & $9.8 \mathrm{E}-10$ & 1. $4 \mathrm{E}-\odot 9$ & $6.8 \mathrm{E}-10$ & 3. $7 \mathrm{E}-09$ \\
\hline \multirow[t]{3}{*}{$\operatorname{co57}$} & Class: Y & & $1.57 \mathrm{E}-07$ & & & & & \\
\hline & 1 & Yr: & & $6.6 \mathrm{E}-\odot 9$ & $4.0 E-10$ & $2.4 \mathrm{E}-10$ & $1.9 \mathrm{E}-10$ & 1. $\odot E-\odot 9$ \\
\hline & 50 & Yr: & & $6.6 \mathrm{E}-\odot 9$ & 4. $0 \mathrm{E}-10$ & $2.4 \mathrm{E}-10$ & 1. $9 \mathrm{E}-10$ & 1. $\odot \mathrm{E}-\odot 9$ \\
\hline \multirow[t]{3}{*}{ C058 } & Class: Y & & $1.40 \mathrm{E}-06$ & & & & & \\
\hline & 1 & Yr: & & 1. 3E-०8 & $5.2 \mathrm{E}-10$ & $7.1 \mathrm{E}-10$ & $5.2 \mathrm{E}-10$ & $2.1 \mathrm{E}-09$ \\
\hline & 50 & Yr: & & 1. $3 E-08$ & $5.2 \mathrm{E}-10$ & $7.1 \mathrm{E}-10$ & $5.2 \mathrm{E}-10$ & $2.1 E-09$ \\
\hline \multirow[t]{3}{*}{$\mathrm{CO6 \odot}$} & Class: Y & & $3.75 \mathrm{E}-06$ & & & & & \\
\hline & 1 & Yr: & & $1.8 \mathrm{E}-07$ & $9.3 \mathrm{E}-\odot 9$ & $1.2 \mathrm{E}-08$ & $9.9 \mathrm{E}-\odot 9$ & $3.1 \mathrm{E}-08$ \\
\hline & 50 & Yr: & & $1.8 \mathrm{E}-07$ & $9.3 E-09$ & 1. $2 \mathrm{E}-08$ & $9.9 \mathrm{E}-09$ & $3.1 \mathrm{E}-08$ \\
\hline \multirow[t]{3}{*}{ NI59 } & Class: W & & $\odot . \odot \odot E \odot \odot ~$ & & & & & \\
\hline & 1 & Yr: & & $4.7 \mathrm{E}-10$ & $7.8 \mathrm{E}-11$ & $7.8 \mathrm{E}-11$ & $7.8 \mathrm{E}-11$ & 1. $3 \mathrm{E}-10$ \\
\hline & 50 & Yr: & & $4.7 \mathrm{E}-10$ & $7.8 \mathrm{E}-11$ & $7.8 \mathrm{E}-11$ & $7.8 \mathrm{E}-11$ & 1. $3 \mathrm{E}-10$ \\
\hline \multirow[t]{3}{*}{ NI63 } & Class: W & & $\odot . \odot \odot Е \odot \odot ~$ & & & & & \\
\hline & 1 & Yr: & & $2.5 \mathrm{E}-\odot 9$ & $1.8 \mathrm{E}-10$ & $1.8 \mathrm{E}-10$ & $1.8 \mathrm{E}-10$ & $4.7 \mathrm{E}-10$ \\
\hline & 50 & Yr: & & $2.5 E-\odot 9$ & $1.8 \mathrm{E}-10$ & $1.8 \mathrm{E}-10$ & $1.8 \mathrm{E}-10$ & $4.7 \mathrm{E}-10$ \\
\hline \multirow[t]{3}{*}{ NI65 } & Class: W & & $8.42 \mathrm{E}-07$ & & & & & \\
\hline & 1 & Yr: & & $3.2 \mathrm{E}-10$ & 2. $3 E-12$ & $3.0 \mathrm{E}-12$ & 2. $2 \mathrm{E}-12$ & $8.5 \mathrm{E}-11$ \\
\hline & 50 & Yr: & & $3.2 \mathrm{E}-10$ & 2. $3 \mathrm{E}-12$ & 3. $0 \mathrm{E}-12$ & 2. $2 \mathrm{E}-12$ & $8.5 \mathrm{E}-11$ \\
\hline \multirow[t]{3}{*}{ CU64 } & Class: D & & $2.69 \mathrm{E}-07$ & & & & & \\
\hline & 1 & Yr: & & $2.1 \mathrm{E}-11$ & $9.2 \mathrm{E}-12$ & $9.2 \mathrm{E}-12$ & $8.9 \mathrm{E}-12$ & $3.5 \mathrm{E}-11$ \\
\hline & 50 & Yr: & & $2.1 \mathrm{E}-11$ & $9.2 \mathrm{E}-12$ & $9.2 \mathrm{E}-12$ & $8.9 \mathrm{E}-12$ & $3.5 \mathrm{E}-11$ \\
\hline \multirow[t]{3}{*}{ ZN65 } & Class: Y & & $8.61 \mathrm{E}-07$ & & & & & \\
\hline & 1 & Yr: & & 1. $\odot E-\odot 8$ & $7.9 \mathrm{E}-10$ & 1. $\odot E-\odot 9$ & $7.9 \mathrm{E}-10$ & 2. $\odot \mathrm{E}-\odot 9$ \\
\hline & 50 & Yr: & & 1. $\odot E-\odot 8$ & $7.9 \mathrm{E}-10$ & 1. ๑E-๑9 & $7.9 \mathrm{E}-10$ & 2. $\odot \mathrm{E}-\odot 9$ \\
\hline ZN69M & Class: Y & & $5.83 E-07$ & & & & & \\
\hline & 1 & Yr: & & 1. 3E-०9 & 8. $\odot \mathrm{E}-12$ & 1. $2 \mathrm{E}-11$ & $5.7 \mathrm{E}-12$ & $2.7 \mathrm{E}-10$ \\
\hline & 50 & Yr: & & 1. $3 E-\odot 9$ & 8. $0 \mathrm{E}-12$ & 1. $2 \mathrm{E}-11$ & $5.7 \mathrm{E}-12$ & $2.7 \mathrm{E}-10$ \\
\hline ZN69 & Class: Y & & $6.31 \mathrm{E}-09$ & & & & & \\
\hline & 1 & Yr: & & 1. $2 \mathrm{E}-10$ & 2. $2 \mathrm{E}-15$ & $2.2 \mathrm{E}-15$ & 1.7E-15 & $2.8 \mathrm{E}-11$ \\
\hline & $5 \odot$ & Yr: & & 1. $2 \mathrm{E}-10$ & 2. $2 \mathrm{E}-15$ & $2.2 \mathrm{E}-15$ & 1.7E-15 & 2. $8 \mathrm{E}-11$ \\
\hline AS76 & Class: W & & $6.50 \mathrm{E}-\odot 7$ & & & & & \\
\hline & 1 & Yr: & & $3.4 \mathrm{E}-\odot 9$ & $3.4 \mathrm{E}-11$ & $3.8 \mathrm{E}-11$ & $3.2 \mathrm{E}-11$ & $7.4 \mathrm{E}-10$ \\
\hline & 50 & Yr: & & $3.4 \mathrm{E}-09$ & $3.4 \mathrm{E}-11$ & $3.8 \mathrm{E}-11$ & $3.2 \mathrm{E}-11$ & 7. $4 \mathrm{E}-10$ \\
\hline SE75 & Class: W & & $5.30 \mathrm{E}-07$ & & & & & \\
\hline & 1 & Yr: & & $5.3 E-09$ & $5.2 \mathrm{E}-10$ & $4.3 E-10$ & $3.2 \mathrm{E}-10$ & $1.1 \mathrm{E}-09$ \\
\hline & 50 & Yr: & & $5.3 E-09$ & $5.2 \mathrm{E}-10$ & 4. $3 E-10$ & $3.2 \mathrm{E}-10$ & 1. $1 \mathrm{E}-09$ \\
\hline SE79 & Class: W & & $1.24 \mathrm{E}-10$ & & & & & \\
\hline & 1 & Yr: & & 1. $9 \mathrm{E}-08$ & 1. $4 \mathrm{E}-10$ & 1. $4 \mathrm{E}-10$ & 1. $4 \mathrm{E}-10$ & $2.6 \mathrm{E}-\odot 9$ \\
\hline & 50 & Yr: & & 1. $9 \mathrm{E}-\odot 8$ & 1. $4 \mathrm{E}-10$ & $1.4 \mathrm{E}-10$ & $1.4 \mathrm{E}-10$ & 2. $6 \mathrm{E}-09$ \\
\hline BR82 & Class: D & & $3.85 E-06$ & & & & & \\
\hline & 1 & Yr: & & 1.7E-10 & $1.7 \mathrm{E}-10$ & $1.6 \mathrm{E}-10$ & 1. $7 \mathrm{E}-10$ & $3.5 \mathrm{E}-10$ \\
\hline & 50 & Yr: & & 1. $7 \mathrm{E}-10$ & 1. $7 \mathrm{E}-10$ & $1.6 \mathrm{E}-10$ & 1. $7 \mathrm{E}-10$ & $3.5 \mathrm{E}-10$ \\
\hline BR83 & Class: D & & $1.68 \mathrm{E}-08$ & & & & & \\
\hline & 1 & Yr: & & $2.5 \mathrm{E}-11$ & $2.9 \mathrm{E}-12$ & $2.9 \mathrm{E}-12$ & $2.9 \mathrm{E}-12$ & 1. $6 \mathrm{E}-11$ \\
\hline & 50 & Yr: & & $2.5 \mathrm{E}-11$ & $2.9 \mathrm{E}-12$ & $2.9 \mathrm{E}-12$ & $2.9 \mathrm{E}-12$ & 1. $6 \mathrm{E}-11$ \\
\hline BR84 & Class: D & & $2.84 \mathrm{E}-06$ & & & & & \\
\hline & 1 & Yr: & & 3. $0 \mathrm{E}-11$ & $3.4 \mathrm{E}-12$ & 3. $3 E-12$ & $3.5 E-12$ & 2. $2 \mathrm{E}-11$ \\
\hline & 50 & Yr: & & 3. $0 \mathrm{E}-11$ & 3. $4 \mathrm{E}-12$ & 3. $3 \mathrm{E}-12$ & $3.5 \mathrm{E}-12$ & 2. $2 \mathrm{E}-11$ \\
\hline KR83M & NobleGas & & $3.78 \mathrm{E}-11$ & & & & & \\
\hline & 1 & Yr: & & $\odot . \odot E-\odot \odot$ & $\odot . \odot E-\odot \odot$ & $\odot . \odot \mathrm{E}-\odot \odot$ & $\odot . \odot \mathrm{E}-\odot \odot$ & $\odot . \odot \mathrm{E}-\odot \odot$ \\
\hline & 50 & Yr: & & $\odot . \odot \mathrm{E}-\odot \odot$ & $\odot . \odot \mathrm{E}-\odot \odot$ & $\odot . \odot \mathrm{E}-\odot \odot$ & $\odot . \odot \mathrm{E}-\odot \odot$ & $\odot . \odot \mathrm{E}-\odot \odot$ \\
\hline KR85M & NobleGas & & $2.17 \mathrm{E}-\odot 7$ & & & & & \\
\hline & 1 & Yr: & & $\odot . \odot \mathrm{E}-\odot \odot$ & $\odot . \odot \mathrm{E}-\odot \odot$ & $E-\odot \odot$ & $\odot . \odot \mathrm{E}-\odot \odot$ & $\odot . \odot \mathrm{E}-\odot \odot$ \\
\hline & 50 & Yr: & & $\odot . \odot E-\odot \odot$ & $\odot . \odot E-\odot \odot$ & $\odot . \odot E-\odot \odot$ & $\odot . \odot \mathrm{E}-\odot \odot$ & $\odot . \odot \mathrm{E}-\odot \odot$ \\
\hline KR85 & NobleGas & & $7.57 \mathrm{E}-\odot 9$ & & & & & \\
\hline & 1 & Yr: & & $\odot . \odot \mathrm{E}-\odot \odot$ & $\odot . \odot \mathrm{E}-\odot \odot$ & $\odot . \odot \mathrm{E}-\odot \odot$ & $\odot . \odot \mathrm{E}-\odot \odot$ & $\odot . \odot \mathrm{E}-\odot \odot$ \\
\hline & 50 & Yr: & & $\odot . \odot \mathrm{E}-\odot \odot$ & $\odot . \odot E-\odot \odot$ & $\odot . \odot E-\odot \odot$ & $\odot . \odot \mathrm{E}-\odot \odot$ & $\odot . \odot \mathrm{E}-\odot \odot$ \\
\hline KR87 & NobleGas & & $1.26 \mathrm{E}-06$ & & & & & \\
\hline & 1 & Yr: & & $\odot . \odot \mathrm{E}-\odot \odot$ & $\odot . \odot \mathrm{E}-\odot \odot$ & $\odot . \odot \mathrm{E}-\odot \odot$ & $\odot . \odot \mathrm{E}-\odot \odot$ & $\odot . \odot \mathrm{E}-\odot \odot$ \\
\hline & $5 \odot$ & Yr: & & $\odot . \odot \mathrm{E}-\odot \odot$ & $\odot . \odot \mathrm{E}-\odot \odot$ & $\odot . \odot \mathrm{E}-\odot \odot$ & $\odot . \odot \mathrm{E}-\odot \odot$ & $\odot . \odot \mathrm{E}-\odot \odot$ \\
\hline KR88 & NobleGas & & $3.07 E-\odot 6$ & & & & & \\
\hline & 1 & Yr: & & $\odot . \odot \mathrm{E}-\odot \odot$ & $\odot . \odot \mathrm{E}-\odot \odot$ & $\odot . \odot \mathrm{E}-\odot \odot$ & $\odot . \odot \mathrm{E}-\odot \odot$ & $\odot . \odot \mathrm{E}-\odot \odot$ \\
\hline & 50 & Yr: & & $\odot . \odot \mathrm{E}-\odot \odot$ & $\odot . \odot \mathrm{E}-\odot \odot$ & $\odot . \odot \mathrm{E}-\odot \odot$ & $\odot . \odot \mathrm{E}-\odot \odot$ & $\odot . \odot E-\odot \odot$ \\
\hline KR89 & NobleGas & & $3.45 \mathrm{E}-06$ & & & & & \\
\hline
\end{tabular}




\begin{tabular}{|c|c|c|c|c|c|c|c|c|}
\hline & 1 & Yr: & & $\odot . \odot \mathrm{E}-\odot \odot$ & $\odot . \odot \mathrm{E}-\odot \odot$ & $\odot . \odot \mathrm{E}-\odot \odot$ & $\odot . \odot \mathrm{E}-\odot \odot$ & $\odot . \odot \mathrm{E}-\odot \odot$ \\
\hline & 50 & Yr: & & $\odot . \odot \mathrm{E}-\odot \odot$ & $\odot . \odot \mathrm{E}-\odot \odot$ & $\odot . \odot E-\odot \odot$ & $\odot . \odot E-\odot \odot$ & $\odot . \odot \mathrm{E}-\odot \odot$ \\
\hline \multirow[t]{3}{*}{ RB86 } & Class: D & & $1.56 \mathrm{E}-07$ & & & & & \\
\hline & 1 & Yr: & & $7.7 \mathrm{E}-10$ & $2.8 \mathrm{E}-\odot 9$ & 1. $4 \mathrm{E}-\odot 9$ & $7.4 \mathrm{E}-10$ & $9.3 \mathrm{E}-10$ \\
\hline & 50 & Yr: & & $7.7 \mathrm{E}-10$ & $2.8 \mathrm{E}-09$ & 1. $4 \mathrm{E}-\odot 9$ & $7.4 \mathrm{E}-10$ & $9.3 \mathrm{E}-10$ \\
\hline \multirow[t]{3}{*}{ RB87 } & Class: D & & $1 . \odot 4 E-\odot 9$ & & & & & \\
\hline & 1 & Yr: & & $4.1 \mathrm{E}-10$ & $1.6 \mathrm{E}-09$ & $7.8 \mathrm{E}-10$ & $3.9 \mathrm{E}-10$ & $5.0 \mathrm{E}-10$ \\
\hline & 50 & Yr: & & $4.1 \mathrm{E}-10$ & 1. $6 \mathrm{E}-\odot 9$ & $7.8 \mathrm{E}-10$ & $3.9 \mathrm{E}-10$ & $5.0 \mathrm{E}-10$ \\
\hline \multirow[t]{3}{*}{ RB88 } & Class: D & & $1.05 E-\odot 6$ & & & & & \\
\hline & 1 & Yr: & & $3.1 \mathrm{E}-11$ & 1. $9 \mathrm{E}-12$ & 1.7E-12 & 1.7E-12 & 1. $6 \mathrm{E}-11$ \\
\hline & 50 & Yr: & & $3.1 \mathrm{E}-11$ & 1. $9 \mathrm{E}-12$ & 1.7E-12 & 1.7E-12 & $1.6 \mathrm{E}-11$ \\
\hline \multirow[t]{3}{*}{ RB89 } & Class: D & & $3.19 \mathrm{E}-06$ & & & & & \\
\hline & 1 & Yr: & & $2.2 \mathrm{E}-11$ & $2.4 \mathrm{E}-12$ & 1. $9 \mathrm{E}-12$ & $1.8 \mathrm{E}-12$ & $1.4 \mathrm{E}-11$ \\
\hline & 50 & Yr: & & $2.2 \mathrm{E}-11$ & 2. $4 \mathrm{E}-12$ & 1. $9 \mathrm{E}-12$ & $1.8 \mathrm{E}-12$ & 1. $4 \mathrm{E}-11$ \\
\hline \multirow[t]{3}{*}{ SR85 } & Class: D & & $7.06 \mathrm{E}-07$ & & & & & \\
\hline & 1 & Yr: & & $2.2 \mathrm{E}-10$ & 7. $3 \mathrm{E}-10$ & $7.4 \mathrm{E}-10$ & $2.0 \mathrm{E}-10$ & $3.8 \mathrm{E}-10$ \\
\hline & 50 & Yr: & & $2.2 \mathrm{E}-10$ & 7. 3E-10 & $7.4 \mathrm{E}-10$ & $2.0 \mathrm{E}-10$ & $3.8 \mathrm{E}-10$ \\
\hline \multirow[t]{3}{*}{ SR89 } & Class: D & & 1. 38E-๑8 & & & & & \\
\hline & 1 & Yr: & & $2.0 \mathrm{E}-10$ & $5.4 \mathrm{E}-\odot 9$ & 4. 3E- - 9 & $1.8 \mathrm{E}-10$ & 1. $0 \mathrm{E}-\odot 9$ \\
\hline & 50 & Yr: & & $2.0 \mathrm{E}-10$ & $5.4 \mathrm{E}-09$ & 4. 3E- $\odot 9$ & $1.8 \mathrm{E}-10$ & 1. $0 \mathrm{E}-\odot 9$ \\
\hline \multirow[t]{3}{*}{ SR90 } & Class: D & & $3.10 \mathrm{E}-09$ & & & & & \\
\hline & 1 & Yr: & & $6.2 \mathrm{E}-10$ & $3.7 \mathrm{E}-07$ & $1.6 \mathrm{E}-07$ & 6. $0 \mathrm{E}-10$ & $2.4 \mathrm{E}-\odot 8$ \\
\hline & 50 & Yr: & & $6.2 \mathrm{E}-10$ & $3.7 \mathrm{E}-07$ & $1.6 \mathrm{E}-07$ & $6.0 \mathrm{E}-10$ & $2.4 \mathrm{E}-\odot 8$ \\
\hline \multirow[t]{3}{*}{ SR91 } & Class: D & & $1.03 \mathrm{E}-06$ & & & & & \\
\hline & 1 & Yr: & & $5.2 \mathrm{E}-11$ & $1.4 \mathrm{E}-10$ & 1. $3 \mathrm{E}-10$ & $2.6 \mathrm{E}-11$ & $1.6 \mathrm{E}-10$ \\
\hline & 50 & $\mathrm{Yr}:$ & & $5.2 \mathrm{E}-11$ & $1.4 \mathrm{E}-10$ & $1.3 E-10$ & $2.6 \mathrm{E}-11$ & $1.6 \mathrm{E}-10$ \\
\hline \multirow[t]{3}{*}{ SR92 } & Class: D & & $2.02 \mathrm{E}-06$ & & & & & \\
\hline & 1 & Yr: & & $3.7 \mathrm{E}-11$ & $8.9 \mathrm{E}-11$ & $6.1 \mathrm{E}-11$ & $1.6 \mathrm{E}-11$ & $9.8 \mathrm{E}-11$ \\
\hline & 50 & Yr: & & $3.7 \mathrm{E}-11$ & $8.9 \mathrm{E}-11$ & $6.1 \mathrm{E}-11$ & 1. $6 \mathrm{E}-11$ & $9.8 \mathrm{E}-11$ \\
\hline Y 90 & Class: $\mathrm{Y}$ & & $2.50 \mathrm{E}-08$ & & & & & \\
\hline & 1 & Yr: & & $7.8 \mathrm{E}-\odot 9$ & 1. $2 \mathrm{E}-12$ & 1. $2 \mathrm{E}-12$ & $4.2 \mathrm{E}-14$ & 1. $5 E-09$ \\
\hline & 50 & Yr: & & $7.8 \mathrm{E}-09$ & 1. $2 \mathrm{E}-12$ & 1. $2 \mathrm{E}-12$ & $4.2 \mathrm{E}-14$ & 1. $5 \mathrm{E}-\odot 9$ \\
\hline Y 91M & Class: $\mathrm{Y}$ & & $7.47 \mathrm{E}-07$ & & & & & \\
\hline & 1 & Yr: & & $5.0 \mathrm{E}-11$ & $7.2 \mathrm{E}-13$ & $9.4 \mathrm{E}-13$ & $6.8 \mathrm{E}-13$ & 1. $1 \mathrm{E}-11$ \\
\hline & 50 & Yr: & & $5.0 \mathrm{E}-11$ & $7.2 \mathrm{E}-13$ & $9.4 \mathrm{E}-13$ & $6.8 \mathrm{E}-13$ & $1.1 \mathrm{E}-11$ \\
\hline Y 91 & Class: Y & & 1.96E-๑8 & & & & & \\
\hline & 1 & Yr: & & 7. ०E-०8 & $6.4 \mathrm{E}-11$ & $6.4 \mathrm{E}-11$ & $2.8 \mathrm{E}-12$ & 8. $9 \mathrm{E}-\odot 9$ \\
\hline & 50 & Yr: & & $7 . \odot E-\odot 8$ & $6.4 \mathrm{E}-11$ & $6.4 \mathrm{E}-11$ & $2.8 \mathrm{E}-12$ & $8.9 \mathrm{E}-\odot 9$ \\
\hline Y 92 & Class: Y & & $4.16 E-07$ & & & & & \\
\hline & 1 & Yr: & & $7.0 \mathrm{E}-10$ & 1. 3E-12 & $1.8 \mathrm{E}-12$ & 1.1E-12 & $1.8 \mathrm{E}-10$ \\
\hline & 50 & Yr: & & 7. $0 \mathrm{E}-10$ & 1. $3 \mathrm{E}-12$ & $1.8 \mathrm{E}-12$ & $1.1 \mathrm{E}-12$ & $1.8 \mathrm{E}-10$ \\
\hline Y 93 & Class: $\mathrm{Y}$ & & $1.67 \mathrm{E}-07$ & & & & & \\
\hline & 1 & Yr: & & $1.6 \mathrm{E}-\odot 9$ & 1. $3 \mathrm{E}-12$ & $1.8 \mathrm{E}-12$ & $9.0 \mathrm{E}-13$ & $4.2 \mathrm{E}-10$ \\
\hline & 50 & Yr: & & $1.6 \mathrm{E}-\odot 9$ & 1. $3 \mathrm{E}-12$ & $1.8 \mathrm{E}-12$ & $9.0 \mathrm{E}-13$ & $4.2 \mathrm{E}-10$ \\
\hline ZR93 & Class: W & & $\odot . \odot \odot Е \odot \odot ~$ & & & & & \\
\hline & 1 & Yr: & & $2.8 \mathrm{E}-09$ & $5.0 \mathrm{E}-07$ & 4. ०E-๑8 & $3.5 \mathrm{E}-12$ & 1. $\mathrm{OE}-\odot 8$ \\
\hline & 50 & Yr: & & $2.8 E-\odot 9$ & $5.0 \mathrm{E}-07$ & 4. $\odot E-\odot 8$ & $3.5 \mathrm{E}-12$ & $1.0 \mathrm{E}-08$ \\
\hline ZR95 & Class: W & & $1.06 \mathrm{E}-06$ & & & & & \\
\hline & 1 & Yr: & & $3.1 \mathrm{E}-08$ & 1. 3E- - 88 & 2. 4E-०9 & $\mathrm{E}-10$ & $4.8 E-\odot 9$ \\
\hline & 50 & Yr: & & $3.1 \mathrm{E}-08$ & 1. $3 \mathrm{E}-08$ & $2.4 \mathrm{E}-\odot 9$ & $6.5 \mathrm{E}-10$ & $4.8 \mathrm{E}-09$ \\
\hline ZR97 & Class: W & & $2.81 E-07$ & & & & & \\
\hline & 1 & Yr: & & $3.4 \mathrm{E}-09$ & $6.4 \mathrm{E}-11$ & $7.8 \mathrm{E}-11$ & $2.8 \mathrm{E}-11$ & $9.2 \mathrm{E}-10$ \\
\hline & 50 & Yr: & & $3.4 \mathrm{E}-09$ & $6.4 \mathrm{E}-11$ & $7.8 \mathrm{E}-11$ & $2.8 \mathrm{E}-11$ & $9.2 \mathrm{E}-10$ \\
\hline NB93M & Class: Y & & $9.62 \mathrm{E}-11$ & & & & & \\
\hline & 1 & Yr: & & 1. 4E- $\odot 8$ & $5.4 \mathrm{E}-11$ & 2. $0 \mathrm{E}-11$ & 4. $7 \mathrm{E}-12$ & $1.8 \mathrm{E}-\odot 9$ \\
\hline & 50 & Yr: & & 1. $4 \mathrm{E}-08$ & $5.4 \mathrm{E}-11$ & 2. $0 \mathrm{E}-11$ & 4. $7 \mathrm{E}-12$ & $1.8 \mathrm{E}-09$ \\
\hline NB94 & Class: Y & & $2.27 \mathrm{E}-06$ & & & & & \\
\hline & 1 & Yr: & & $3.2 \mathrm{E}-07$ & 1. $2 \mathrm{E}-08$ & 1. $5 \mathrm{E}-08$ & 1. $2 \mathrm{E}-08$ & $4.9 E-\odot 8$ \\
\hline & 50 & Yr: & & $3.2 \mathrm{E}-07$ & 1. $2 \mathrm{E}-08$ & 1. $5 \mathrm{E}-\odot 8$ & 1. $2 \mathrm{E}-08$ & $4.9 \mathrm{E}-\odot 8$ \\
\hline NB95M & Class: Y & & $8.64 \mathrm{E}-08$ & & & & & \\
\hline & 1 & Yr: & & $6.2 \mathrm{E}-09$ & $3.4 \mathrm{E}-11$ & $3.8 \mathrm{E}-11$ & $2.6 \mathrm{E}-11$ & $8.8 \mathrm{E}-10$ \\
\hline & 50 & Yr: & & $6.2 \mathrm{E}-09$ & $3.4 \mathrm{E}-11$ & $3.8 \mathrm{E}-11$ & $2.6 \mathrm{E}-11$ & $8.8 \mathrm{E}-10$ \\
\hline NB95 & Class: Y & & $1.10 \mathrm{E}-06$ & & & & & \\
\hline & 1 & Yr: & & 1. $2 \mathrm{E}-08$ & $2.5 \mathrm{E}-10$ & $3.4 \mathrm{E}-10$ & $2.3 E-10$ & 1. $8 \mathrm{E}-\odot 9$ \\
\hline & 50 & Yr: & & $1.2 \mathrm{E}-08$ & $2.5 \mathrm{E}-10$ & $3.4 \mathrm{E}-10$ & $2.3 \mathrm{E}-10$ & $1.8 \mathrm{E}-09$ \\
\hline NB97M & Class: Y & & $1.04 \mathrm{E}-06$ & & & & & \\
\hline & 1 & Yr: & & 2. 4E-12 & $2.3 \mathrm{E}-14$ & $3.1 \mathrm{E}-14$ & $2.8 \mathrm{E}-14$ & $3.5 \mathrm{E}-13$ \\
\hline & 50 & Yr: & & 2. 4E-12 & $2.3 \mathrm{E}-14$ & $3.1 \mathrm{E}-14$ & $2.8 \mathrm{E}-14$ & $3.5 E-13$ \\
\hline NB97 & Class: $\mathrm{Y}$ & & $9.43 \mathrm{E}-07$ & & & & & \\
\hline & 1 & Yr: & & $1.7 \mathrm{E}-10$ & 1. $2 \mathrm{E}-12$ & $1.6 \mathrm{E}-12$ & $1.1 \mathrm{E}-12$ & $4.5 \mathrm{E}-11$ \\
\hline & 50 & Yr: & & $1.7 \mathrm{E}-10$ & 1. $2 \mathrm{E}-12$ & 1.6E-12 & 1.1E-12 & $4.5 \mathrm{E}-11$ \\
\hline
\end{tabular}




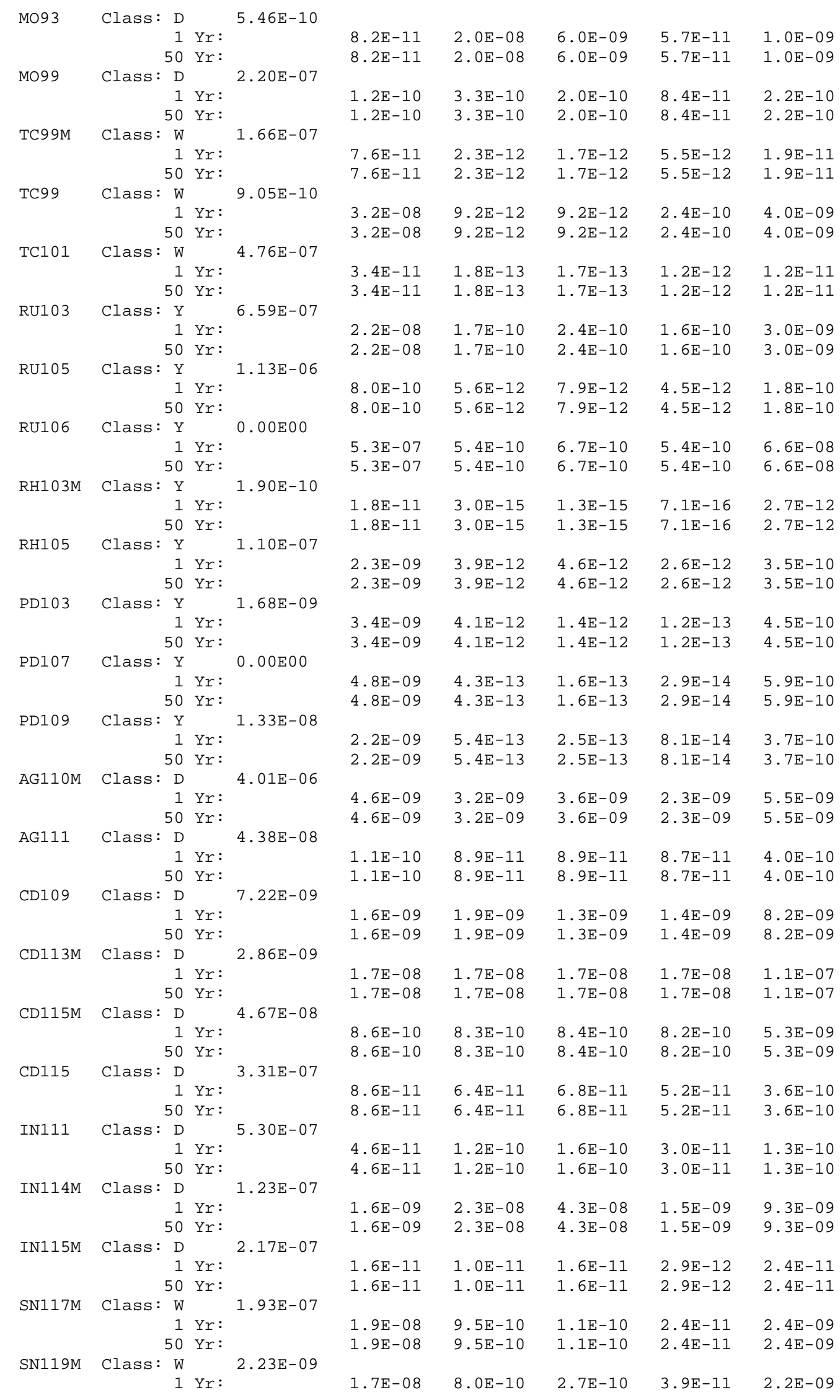




\begin{tabular}{|c|c|c|c|c|c|c|c|c|}
\hline & 50 & Yr: & & 1.7E-०8 & $8.0 \mathrm{E}-10$ & $2.7 \mathrm{E}-10$ & $3.9 \mathrm{E}-11$ & $2.2 \mathrm{E}-\odot 9$ \\
\hline \multirow[t]{3}{*}{ SN121M } & Class: W & & $1.66 \mathrm{E}-09$ & & & & & \\
\hline & 1 & Yr: & & $3.4 \mathrm{E}-08$ & $3.2 \mathrm{E}-\odot 9$ & 1.1E- 09 & $1.5 \mathrm{E}-10$ & $4.5 E-\odot 9$ \\
\hline & 50 & Yr: & & $3.4 \mathrm{E}-\odot 8$ & $3.2 \mathrm{E}-\odot 9$ & 1. $1 \mathrm{E}-\odot 9$ & $1.5 \mathrm{E}-10$ & $4.5 E-\odot 9$ \\
\hline \multirow[t]{3}{*}{ SN121 } & Class: W & & $1.23 \mathrm{E}-09$ & & & & & \\
\hline & 1 & Yr: & & 1. $5 \mathrm{E}-09$ & $4.2 \mathrm{E}-11$ & 3. $6 \mathrm{E}-12$ & $3.3 E-13$ & $2.3 \mathrm{E}-10$ \\
\hline & 50 & Yr: & & $1.5 \mathrm{E}-\odot 9$ & $4.2 \mathrm{E}-11$ & $3.6 \mathrm{E}-12$ & 3. $3 E-13$ & $2.3 \mathrm{E}-10$ \\
\hline \multirow[t]{3}{*}{ SN123 } & Class: W & & $2.20 \mathrm{E}-\odot 8$ & & & & & \\
\hline & 1 & Yr: & & $6.2 \mathrm{E}-08$ & 2. 3E- $\odot 9$ & $8.5 E-10$ & $1.1 \mathrm{E}-10$ & $8.1 \mathrm{E}-\odot 9$ \\
\hline & 50 & Yr: & & $6.2 \mathrm{E}-\odot 8$ & 2. $3 \mathrm{E}-\odot 9$ & $8.5 \mathrm{E}-10$ & $1.1 \mathrm{E}-10$ & $8.1 \mathrm{E}-09$ \\
\hline \multirow[t]{3}{*}{ SN125 } & Class: W & & $4.86 E-07$ & & & & & \\
\hline & 1 & Yr: & & $2 . \odot E-\odot 8$ & $5.8 \mathrm{E}-10$ & $3.5 \mathrm{E}-10$ & 5. $0 \mathrm{E}-11$ & $3.1 \mathrm{E}-09$ \\
\hline & 50 & Yr: & & $2.0 \mathrm{E}-08$ & $5.8 \mathrm{E}-10$ & $3.5 \mathrm{E}-10$ & $5.0 \mathrm{E}-11$ & $3.1 \mathrm{E}-09$ \\
\hline \multirow[t]{3}{*}{ SN126 } & Class: W & & $5.83 E-\odot 8$ & & & & & \\
\hline & 1 & Yr: & & $1.8 \mathrm{E}-07$ & $2.8 \mathrm{E}-\odot 8$ & $1.5 \mathrm{E}-08$ & 4. . $\mathrm{E}-\odot 9$ & $2.8 \mathrm{E}-\odot 8$ \\
\hline & 50 & Yr: & & $1.8 \mathrm{E}-07$ & $2.8 \mathrm{E}-08$ & $1.5 \mathrm{E}-\odot 8$ & 4. $7 \mathrm{E}-09$ & $2.8 \mathrm{E}-08$ \\
\hline \multirow{3}{*}{ SB124 } & Class: W & & $2.72 \mathrm{E}-\odot 6$ & & & & & \\
\hline & 1 & Yr: & & 4. 4E- -88 & 1. $9 \mathrm{E}-\odot 9$ & 1. $2 \mathrm{E}-\odot 9$ & $6.6 \mathrm{E}-10$ & $6.4 \mathrm{E}-\odot 9$ \\
\hline & 50 & Yr: & & $4.4 \mathrm{E}-08$ & 1. $9 \mathrm{E}-09$ & $1.2 \mathrm{E}-09$ & $6.6 \mathrm{E}-10$ & $6.4 \mathrm{E}-\odot 9$ \\
\hline \multirow[t]{3}{*}{ SB125 } & Class: W & & $5.9 \odot E-\odot 7$ & & & & & \\
\hline & 1 & Yr: & & $3.2 \mathrm{E}-08$ & $8.7 \mathrm{E}-09$ & 1.7E- 09 & $5.5 \mathrm{E}-10$ & $4.8 \mathrm{E}-\odot 9$ \\
\hline & 50 & Yr: & & $3.2 \mathrm{E}-08$ & 8. 7E-०9 & 1. $7 \mathrm{E}-\odot 9$ & $5.5 \mathrm{E}-10$ & $4.8 \mathrm{E}-09$ \\
\hline \multirow[t]{3}{*}{ SB126M } & Class: W & & $2.21 \mathrm{E}-06$ & & & & & \\
\hline & 1 & Yr: & & $4.7 \mathrm{E}-11$ & $9.6 \mathrm{E}-13$ & $1.1 \mathrm{E}-12$ & $9.6 \mathrm{E}-13$ & $1.9 \mathrm{E}-11$ \\
\hline & 50 & Yr: & & $4.7 \mathrm{E}-11$ & $9.6 \mathrm{E}-13$ & 1. $1 \mathrm{E}-12$ & $9.6 \mathrm{E}-13$ & $1.9 \mathrm{E}-11$ \\
\hline \multirow[t]{3}{*}{ SB126 } & Class: W & & $4.04 \mathrm{E}-\odot 6$ & & & & & \\
\hline & 1 & Yr: & & $1.7 \mathrm{E}-\odot 8$ & $6.5 \mathrm{E}-10$ & $6.5 \mathrm{E}-10$ & $3.6 \mathrm{E}-10$ & $2.9 \mathrm{E}-\odot 9$ \\
\hline & 50 & Yr: & & 1. 7E-०8 & $6.5 \mathrm{E}-10$ & $6.5 \mathrm{E}-10$ & $3.6 \mathrm{E}-10$ & $2.9 \mathrm{E}-09$ \\
\hline SB127 & Class: W & & $9.84 \mathrm{E}-07$ & & & & & \\
\hline & 1 & Yr: & & 1.1E- 08 & 1. $2 \mathrm{E}-10$ & 1. $2 \mathrm{E}-10$ & 4. $2 \mathrm{E}-11$ & 1. $7 \mathrm{E}-09$ \\
\hline & 50 & Yr: & & 1.1E- $\odot 8$ & 1. $2 \mathrm{E}-10$ & 1. $2 \mathrm{E}-10$ & $4.2 \mathrm{E}-11$ & 1. $7 \mathrm{E}-\odot 9$ \\
\hline TE123M & Class: W & & $1.84 \mathrm{E}-07$ & & & & & \\
\hline & 1 & Yr: & & $3 . \odot E-\odot 8$ & 1.1E- -08 & $1.1 \mathrm{E}-09$ & $4.1 \mathrm{E}-10$ & 4. $\odot E-\odot 9$ \\
\hline & 50 & $\mathrm{Yr}:$ & & $3.0 \mathrm{E}-08$ & 1. $1 \mathrm{E}-08$ & 1. 1E- 09 & $4.1 \mathrm{E}-10$ & $4.0 \mathrm{E}-09$ \\
\hline TE125M & Class: W & & 1.06E- 08 & & & & & \\
\hline & 1 & Yr: & & $2.6 \mathrm{E}-\odot 8$ & $4.8 \mathrm{E}-09$ & $4.2 \mathrm{E}-10$ & $2.6 \mathrm{E}-10$ & $3.4 \mathrm{E}-09$ \\
\hline & 50 & Yr: & & $2.6 \mathrm{E}-08$ & $4.8 E-09$ & $4.2 \mathrm{E}-10$ & $2.6 \mathrm{E}-10$ & $3.4 \mathrm{E}-09$ \\
\hline TE127M & Class: W & & $3.56 \mathrm{E}-\odot 9$ & & & & & \\
\hline & 1 & Yr: & & $5.6 \mathrm{E}-\odot 8$ & $9.0 \mathrm{E}-\odot 9$ & 2. $3 E-\odot 9$ & $8.6 \mathrm{E}-10$ & $7.4 \mathrm{E}-\odot 9$ \\
\hline & 50 & Yr: & & $5.6 \mathrm{E}-08$ & $9.0 \mathrm{E}-09$ & 2. $3 \mathrm{E}-09$ & $8.6 \mathrm{E}-10$ & $7.4 \mathrm{E}-09$ \\
\hline TE127 & Class: W & & $1.05 E-\odot 8$ & & & & & \\
\hline & 1 & Yr: & & $7.5 \mathrm{E}-10$ & 1. $7 \mathrm{E}-12$ & 1.7E-12 & $2.8 \mathrm{E}-12$ & $1.3 \mathrm{E}-10$ \\
\hline & 50 & Yr: & & $7.5 E-10$ & 1. $7 \mathrm{E}-12$ & 1. $7 \mathrm{E}-12$ & $2.8 \mathrm{E}-12$ & $1.3 \mathrm{E}-10$ \\
\hline TE129M & Class: W & & $4.92 \mathrm{E}-\odot 8$ & & & & & \\
\hline & 1 & Yr: & & $4.8 \mathrm{E}-08$ & $2.7 \mathrm{E}-\odot 9$ & 1. $2 \mathrm{E}-\odot 9$ & 1. $\odot E-\odot 9$ & $6.5 E-09$ \\
\hline & 50 & Yr: & & $4.8 E-08$ & 2. $7 \mathrm{E}-\odot 9$ & 1. $2 \mathrm{E}-\odot 9$ & 1. $\odot E-\odot 9$ & $6.5 E-09$ \\
\hline TE129 & Class: W & & $9.05 \mathrm{E}-08$ & & & & & \\
\hline & 1 & Yr: & & $1.5 \mathrm{E}-10$ & $3.6 \mathrm{E}-13$ & $3.5 \mathrm{E}-13$ & $3.5 E-13$ & $3.7 \mathrm{E}-11$ \\
\hline & 50 & Yr: & & $1.5 \mathrm{E}-10$ & $3.6 \mathrm{E}-13$ & $3.5 \mathrm{E}-13$ & $3.5 \mathrm{E}-13$ & $3.7 \mathrm{E}-11$ \\
\hline TE131M & Class: W & & $2.07 \mathrm{E}-06$ & & & & & \\
\hline & 1 & Yr: & & $4.6 \mathrm{E}-\odot 9$ & 1. $2 \mathrm{E}-10$ & $8.8 \mathrm{E}-11$ & $2.7 \mathrm{E}-\odot 9$ & 1.1E- -99 \\
\hline & 50 & Yr: & & $4.6 \mathrm{E}-09$ & $1.2 \mathrm{E}-10$ & $8.8 \mathrm{E}-11$ & $2.7 \mathrm{E}-09$ & $1.1 \mathrm{E}-09$ \\
\hline TE131 & Class: W & & $6.09 E-07$ & & & & & \\
\hline & 1 & Yr: & & $9.3 \mathrm{E}-11$ & $5.3 E-13$ & $5.2 \mathrm{E}-13$ & $4.8 \mathrm{E}-11$ & $2.9 \mathrm{E}-11$ \\
\hline & 50 & Yr: & & $9.3 \mathrm{E}-11$ & $5.3 \mathrm{E}-13$ & $5.2 \mathrm{E}-13$ & $4.8 \mathrm{E}-11$ & $2.9 \mathrm{E}-11$ \\
\hline TE132 & Class: W & & $2.95 \mathrm{E}-07$ & & & & & \\
\hline & 1 & Yr: & & 1. $0 \mathrm{E}-08$ & $2.9 \mathrm{E}-10$ & $2.2 \mathrm{E}-10$ & 4. 3E- $\odot 9$ & $2.1 \mathrm{E}-\odot 9$ \\
\hline & 50 & Yr: & & 1. $0 \mathrm{E}-08$ & $2.9 \mathrm{E}-10$ & $2.2 \mathrm{E}-10$ & 4. 3E- $\odot 9$ & $2.1 \mathrm{E}-09$ \\
\hline TE133M & Class: W & & $3.41 \mathrm{E}-06$ & & & & & \\
\hline & 1 & Yr: & & $2.6 \mathrm{E}-10$ & $4.4 \mathrm{E}-12$ & $5.5 \mathrm{E}-12$ & $1.6 \mathrm{E}-10$ & $8.9 \mathrm{E}-11$ \\
\hline & 50 & Yr: & & $2.6 \mathrm{E}-10$ & $4.4 \mathrm{E}-12$ & $5.5 \mathrm{E}-12$ & 1. $6 \mathrm{E}-10$ & $8.9 \mathrm{E}-11$ \\
\hline TE133 & Class: W & & $1.37 \mathrm{E}-06$ & & & & & \\
\hline & 1 & Yr: & & $5.3 \mathrm{E}-11$ & $5.1 \mathrm{E}-13$ & $5.8 \mathrm{E}-13$ & $3.6 \mathrm{E}-11$ & 2. $0 \mathrm{E}-11$ \\
\hline & 50 & Yr: & & $5.3 E-11$ & $5.1 \mathrm{E}-13$ & $5.8 \mathrm{E}-13$ & $3.6 \mathrm{E}-11$ & 2. $0 \mathrm{E}-11$ \\
\hline TE134 & Class: W & & $1.24 \mathrm{E}-06$ & & & & & \\
\hline & 1 & Yr: & & $2.1 \mathrm{E}-10$ & $4.1 \mathrm{E}-12$ & $5.1 \mathrm{E}-12$ & $2.6 \mathrm{E}-11$ & $6.7 \mathrm{E}-11$ \\
\hline & 50 & Yr: & & $2.1 \mathrm{E}-10$ & 4. $1 \mathrm{E}-12$ & $5.1 \mathrm{E}-12$ & $2.6 \mathrm{E}-11$ & $6.7 \mathrm{E}-11$ \\
\hline I 125 & Class: D & & $1.19 \mathrm{E}-\odot 8$ & & & & & \\
\hline & 1 & Yr: & & 1. $5 \mathrm{E}-11$ & $5.8 \mathrm{E}-11$ & $1.1 \mathrm{E}-11$ & 1. $\odot E-\odot 7$ & $5.2 \mathrm{E}-\odot 9$ \\
\hline & 50 & Yr: & & $1.5 \mathrm{E}-11$ & $5.8 \mathrm{E}-11$ & 1. $1 \mathrm{E}-11$ & 1. $0 \mathrm{E}-07$ & $5.2 \mathrm{E}-09$ \\
\hline I 129 & Class: D & & 8. 92E-०9 & & & & & \\
\hline
\end{tabular}




\begin{tabular}{|c|c|c|c|c|c|c|c|c|}
\hline & 1 & Yr: & & $6.2 \mathrm{E}-11$ & 1. $4 \mathrm{E}-10$ & $4.8 \mathrm{E}-11$ & $7.2 \mathrm{E}-07$ & $3.6 \mathrm{E}-08$ \\
\hline & 50 & Yr: & & $6.2 \mathrm{E}-11$ & $1.4 \mathrm{E}-10$ & $4.8 \mathrm{E}-11$ & $7.2 \mathrm{E}-07$ & $3.6 \mathrm{E}-08$ \\
\hline \multirow[t]{3}{*}{ I 130} & Class: D & & $3.05 E-06$ & & & & & \\
\hline & 1 & Yr: & & $5.6 \mathrm{E}-11$ & $3.6 \mathrm{E}-11$ & $3.3 E-11$ & 1. 3E - ๑8 & $6.8 \mathrm{E}-10$ \\
\hline & 50 & Yr: & & $5.6 \mathrm{E}-11$ & $3.6 \mathrm{E}-11$ & 3. $3 E-11$ & 1. $3 \mathrm{E}-08$ & $6.8 \mathrm{E}-10$ \\
\hline \multirow[t]{3}{*}{ I 131} & Class: D & & $5.33 E-\odot 7$ & & & & & \\
\hline & 1 & Yr: & & 6. ०E-11 & $4.9 \mathrm{E}-11$ & $3.7 \mathrm{E}-11$ & $1.5 E-07$ & 7. 4E- -99 \\
\hline & 50 & Yr: & & $6.0 \mathrm{E}-11$ & 4. $9 \mathrm{E}-11$ & $3.7 \mathrm{E}-11$ & $1.5 E-07$ & 7. 4E- -99 \\
\hline \multirow[t]{3}{*}{ I 132} & Class: D & & $3.31 \mathrm{E}-06$ & & & & & \\
\hline & 1 & Yr: & & $3.6 \mathrm{E}-11$ & 1. $2 \mathrm{E}-11$ & 1. $2 \mathrm{E}-11$ & 1. 4E- $\odot 9$ & $9.4 \mathrm{E}-11$ \\
\hline & 50 & Yr: & & $3.6 \mathrm{E}-11$ & 1. $2 \mathrm{E}-11$ & $1.2 \mathrm{E}-11$ & 1. 4E- $\odot 9$ & $9.4 \mathrm{E}-11$ \\
\hline \multirow[t]{3}{*}{ I 133} & Class: D & & $8.70 \mathrm{E}-07$ & & & & & \\
\hline & 1 & Yr: & & $4.2 \mathrm{E}-11$ & 2. $0 \mathrm{E}-11$ & $1.9 \mathrm{E}-11$ & $2.9 \mathrm{E}-\odot 8$ & $1.5 \mathrm{E}-09$ \\
\hline & 50 & Yr: & & $4.2 \mathrm{E}-11$ & 2. $0 \mathrm{E}-11$ & 1. $9 \mathrm{E}-11$ & $2.9 \mathrm{E}-08$ & 1. $5 \mathrm{E}-09$ \\
\hline \multirow[t]{3}{*}{ I 134} & Class: D & & $3.85 E-06$ & & & & & \\
\hline & 1 & Yr: & & $3.0 \mathrm{E}-11$ & $5.8 \mathrm{E}-12$ & $5.5 E-12$ & $2.6 \mathrm{E}-10$ & $4.5 \mathrm{E}-11$ \\
\hline & 50 & Yr: & & $3.0 \mathrm{E}-11$ & $5.8 \mathrm{E}-12$ & $5.5 \mathrm{E}-12$ & $2.6 \mathrm{E}-10$ & $4.5 \mathrm{E}-11$ \\
\hline \multirow[t]{3}{*}{ I 135} & Class: D & & $2.38 \mathrm{E}-06$ & & & & & \\
\hline & 1 & Yr: & & 4.0E-11 & $1.8 \mathrm{E}-11$ & 1.7E-11 & $5.8 E-09$ & $3.2 \mathrm{E}-10$ \\
\hline & 50 & Yr: & & 4. $0 \mathrm{E}-11$ & 1. $8 \mathrm{E}-11$ & 1. $7 \mathrm{E}-11$ & $5.8 \mathrm{E}-09$ & $3.2 \mathrm{E}-10$ \\
\hline \multirow[t]{3}{*}{ XE131M } & NobleGas & & $1.10 \mathrm{E}-08$ & & & & & \\
\hline & 1 & Yr: & & ๑.०Е-๑९ & $\odot . \odot \mathrm{E}-\odot \odot$ & $\odot . \odot \mathrm{E}-\odot \odot$ & $\odot . \odot-\odot \odot$ & $\odot . \odot \mathrm{E}-\odot \odot$ \\
\hline & 50 & Yr: & & $\odot . \odot \mathrm{E}-\odot \odot$ & $\odot . \odot \mathrm{E}-\odot \odot$ & $\odot . \odot \mathrm{E}-\odot \odot$ & $\odot . \odot \mathrm{E}-\odot \odot$ & $\odot . \odot \mathrm{E}-\odot \odot$ \\
\hline \multirow[t]{3}{*}{ XE133M } & NobleGas & & 4.07E-०8 & & & & & \\
\hline & 1 & Yr: & & $\odot . \odot \mathrm{E}-\odot \odot$ & $\odot . \odot \mathrm{E}-\odot \odot$ & $\odot . \odot \mathrm{E}-\odot \odot$ & $\odot . \odot \mathrm{E}-\odot \odot$ & $\odot . \odot \mathrm{E}-\odot \odot$ \\
\hline & 50 & Yr: & & $\odot . \odot \mathrm{E}-\odot \odot$ & $\odot . \odot \mathrm{E}-\odot \odot$ & $\odot . \odot \mathrm{E}-\odot \odot$ & $\odot . \odot \mathrm{E}-\odot \odot$ & $\odot . \odot \mathrm{E}-\odot \odot$ \\
\hline \multirow[t]{3}{*}{ XE133 } & NobleGas & & 4.23E- 08 & & & & & \\
\hline & 1 & Yr: & & $\odot . \odot \mathrm{E}-\odot \odot$ & $\odot . \odot \mathrm{E}-\odot \odot$ & $\odot . \odot \mathrm{E}-\odot \odot$ & $\odot . \odot \mathrm{E}-\odot \odot$ & $\odot . \odot \mathrm{E}-\odot \odot$ \\
\hline & 50 & Yr: & & $\odot . \odot \mathrm{E}-\odot \odot$ & $\odot . \odot \mathrm{E}-\odot \odot$ & $\odot . \odot \mathrm{E}-\odot \odot$ & $\odot . \odot \mathrm{E}-\odot \odot$ & $\odot . \odot \mathrm{E}-\odot \odot$ \\
\hline XE135M & NobleGas & & $5.99 E-07$ & & & & & \\
\hline & 1 & Yr: & & $\odot . \odot \mathrm{E}-\odot \odot$ & $\odot . \odot \mathrm{E}-\odot \odot$ & $\odot . \odot \mathrm{E}-\odot \odot$ & $\odot . \odot \mathrm{E}-\odot \odot$ & $\odot . \odot \mathrm{E}-\odot \odot$ \\
\hline & 50 & Yr: & & $\odot . \odot \mathrm{E}-\odot \odot$ & $\odot . \odot \mathrm{E}-\odot \odot$ & $\odot . \odot \mathrm{E}-\odot \odot$ & $\odot . \odot \mathrm{E}-\odot \odot$ & $\odot . \odot \mathrm{E}-\odot \odot$ \\
\hline XE135 & NobleGas & & $3.50 \mathrm{E}-07$ & & & & & \\
\hline & 1 & Yr: & & $\odot .0 \mathrm{E}-\odot \odot$ & $\odot . \odot \mathrm{E}-\odot \odot$ & $\odot . \odot E-\odot \odot$ & $\odot . \odot \mathrm{E}-\odot \odot$ & $\odot . \odot \mathrm{E}-\odot \odot$ \\
\hline & 50 & Yr: & & $\odot . \odot \mathrm{E}-\odot \odot$ & $\odot . \odot \mathrm{E}-\odot \odot$ & $\odot . \odot \mathrm{E}-\odot \odot$ & $\odot . \odot \mathrm{E}-\odot \odot$ & $\odot . \odot \mathrm{E}-\odot \odot$ \\
\hline XE137 & NobleGas & & $4.34 \mathrm{E}-07$ & & & & & \\
\hline & 1 & Yr: & & $\odot . \odot \mathrm{E}-\odot \odot$ & $\odot . \odot \mathrm{E}-\odot \odot$ & $\odot . \odot \mathrm{E}-\odot \odot$ & $\odot . \odot \mathrm{E}-\odot \odot$ & $\odot . \odot \mathrm{E}-\odot \odot$ \\
\hline & 50 & Yr: & & $\odot . \odot \mathrm{E}-\odot \odot$ & $\odot . \odot \mathrm{E}-\odot \odot$ & $\odot . \odot \mathrm{E}-\odot \odot$ & $\odot . \odot \mathrm{E}-\odot \odot$ & $\odot . \odot \mathrm{E}-\odot \odot$ \\
\hline XE138 & NobleGas & & $1.73 \mathrm{E}-06$ & & & & & \\
\hline & 1 & Yr: & & $\odot . \odot \mathrm{E}-\odot \odot$ & $\odot . \odot \mathrm{E}-\odot \odot$ & $\odot . \odot \mathrm{E}-\odot \odot$ & $\odot . \odot \mathrm{E}-\odot \odot$ & $\odot . \odot \mathrm{E}-\odot \odot$ \\
\hline & 50 & Yr: & & $\odot . \odot \mathrm{E}-\odot \odot$ & $\odot . \odot \mathrm{E}-\odot \odot$ & $\odot . \odot \mathrm{E}-\odot \odot$ & $\odot . \odot \mathrm{E}-\odot \odot$ & $\odot . \odot \mathrm{E}-\odot \odot$ \\
\hline CS134M & Class: D & & 2.51E- ๑8 & & & & & \\
\hline & 1 & Yr: & & $2.4 \mathrm{E}-11$ & $2.8 \mathrm{E}-12$ & $2.4 \mathrm{E}-12$ & 2. 4E-12 & $1.4 \mathrm{E}-11$ \\
\hline & 50 & Yr: & & $2.4 \mathrm{E}-11$ & $2.8 \mathrm{E}-12$ & $2.4 \mathrm{E}-12$ & 2. $4 \mathrm{E}-12$ & 1. $4 \mathrm{E}-11$ \\
\hline CS134 & Class: D & & $2.23 E-06$ & & & & & \\
\hline & 1 & Yr: & & 6. ०E-๑9 & $6.8 \mathrm{E}-09$ & $6.4 \mathrm{E}-09$ & $6.3 \mathrm{E}-09$ & $6.7 \mathrm{E}-09$ \\
\hline & 50 & Yr: & & 6. ๑E-๑9 & $6.8 \mathrm{E}-09$ & $6.4 \mathrm{E}-\odot 9$ & $6.3 E-\odot 9$ & $6.7 \mathrm{E}-09$ \\
\hline CS135 & Class: D & & $3.00 \mathrm{E}-10$ & & & & & \\
\hline & 1 & Yr: & & $6.7 \mathrm{E}-10$ & $6.5 \mathrm{E}-10$ & $6.5 \mathrm{E}-10$ & $6.5 \mathrm{E}-10$ & $6.9 \mathrm{E}-10$ \\
\hline & 50 & Yr: & & $6.7 \mathrm{E}-10$ & $6.5 \mathrm{E}-10$ & $6.5 \mathrm{E}-10$ & $6.5 \mathrm{E}-10$ & $6.9 \mathrm{E}-10$ \\
\hline CS136 & Class: D & & $3.13 E-06$ & & & & & \\
\hline & 1 & Yr: & & $9.7 \mathrm{E}-10$ & 1. 1E- $\odot 9$ & $9.9 \mathrm{E}-10$ & 1. $\odot E-\odot 9$ & 1. $2 \mathrm{E}-09$ \\
\hline & 50 & Yr: & & $9.7 \mathrm{E}-10$ & 1. 1E- $\odot 9$ & $9.9 \mathrm{E}-10$ & 1. $\odot E-\odot 9$ & 1. $2 \mathrm{E}-\odot 9$ \\
\hline CS137 & Class: D & & $2.93 E-09$ & & & & & \\
\hline & 1 & Yr: & & 4. 3E- -99 & 4. 7E- - 99 & $4.5 E-09$ & 4. 4E-०9 & 4.7E-०9 \\
\hline & 50 & Yr: & & 4. 3E-०9 & 4. . $\mathrm{E}-\odot 9$ & $4.5 E-09$ & 4. 4E-०9 & $4.7 \mathrm{E}-09$ \\
\hline CS138 & Class: D & & $3.63 \mathrm{E}-06$ & & & & & \\
\hline & 1 & Yr: & & $3.1 \mathrm{E}-11$ & $4.1 \mathrm{E}-12$ & 3. $9 \mathrm{E}-12$ & 4. $3 \mathrm{E}-12$ & $2.5 \mathrm{E}-11$ \\
\hline & 50 & Yr: & & $3.1 \mathrm{E}-11$ & $4.1 \mathrm{E}-12$ & $3.9 \mathrm{E}-12$ & 4. $3 E-12$ & $2.5 \mathrm{E}-11$ \\
\hline BA139 & Class: D & & $8.04 \mathrm{E}-08$ & & & & & \\
\hline & 1 & Yr: & & $2.9 \mathrm{E}-11$ & 1. $2 \mathrm{E}-11$ & 1. $2 \mathrm{E}-11$ & 1.7E-12 & $3.4 \mathrm{E}-11$ \\
\hline & 50 & Yr: & & $2.9 \mathrm{E}-11$ & 1. $2 \mathrm{E}-11$ & 1. $2 \mathrm{E}-11$ & 1. $7 \mathrm{E}-12$ & $3.4 \mathrm{E}-11$ \\
\hline BA140 & Class: D & & $2.55 \mathrm{E}-07$ & & & & & \\
\hline & 1 & Yr: & & $1.5 \mathrm{E}-10$ & $2.1 \mathrm{E}-09$ & $-\odot 9$ & 1. $2 \mathrm{E}-10$ & 1. $0 \mathrm{E}-\odot 9$ \\
\hline & 50 & Yr: & & $1.5 \mathrm{E}-10$ & $2.1 \mathrm{E}-\odot 9$ & $1.5 \mathrm{E}-\odot 9$ & 1. $2 \mathrm{E}-10$ & 1. $0 \mathrm{E}-09$ \\
\hline BA141 & Class: D & & $1.24 \mathrm{E}-06$ & & & & & \\
\hline & 1 & Yr: & & $2.4 \mathrm{E}-11$ & $6.0 \mathrm{E}-12$ & $5.6 \mathrm{E}-12$ & 1. $2 \mathrm{E}-12$ & $2.1 \mathrm{E}-11$ \\
\hline & 50 & Yr: & & $2.4 \mathrm{E}-11$ & $6.0 \mathrm{E}-12$ & $5.6 \mathrm{E}-12$ & 1. $2 \mathrm{E}-12$ & $2.1 \mathrm{E}-11$ \\
\hline BA142 & Class: D & & $1.53 \mathrm{E}-06$ & & & & & \\
\hline & 1 & Yr: & & $1.8 \mathrm{E}-11$ & $3.1 \mathrm{E}-12$ & $3.3 \mathrm{E}-12$ & 1. $3 E-12$ & 1. $5 \mathrm{E}-11$ \\
\hline & 50 & Yr: & & $1.8 \mathrm{E}-11$ & $3.1 \mathrm{E}-12$ & 3. $3 E-12$ & 1. $3 \mathrm{E}-12$ & $1.5 \mathrm{E}-11$ \\
\hline
\end{tabular}




\begin{tabular}{|c|c|c|c|c|c|c|c|c|}
\hline \multirow[t]{3}{*}{ LA140 } & Class: D & & $3.50 \mathrm{E}-06$ & & & & & \\
\hline & 1 & Yr: & & $1.8 \mathrm{E}-10$ & $2.7 \mathrm{E}-10$ & 3. $0 \mathrm{E}-10$ & 9. $0 \mathrm{E}-11$ & $5.7 \mathrm{E}-10$ \\
\hline & 50 & Yr: & & $1.8 \mathrm{E}-10$ & $2.7 \mathrm{E}-10$ & 3. $0 \mathrm{E}-10$ & 9. $0 \mathrm{E}-11$ & $5.7 \mathrm{E}-10$ \\
\hline \multirow[t]{3}{*}{ LA141 } & Class: D & & $9 . \odot 8 E-\odot 8$ & & & & & \\
\hline & 1 & Yr: & & $3.7 \mathrm{E}-11$ & $7.4 \mathrm{E}-11$ & 2. $3 \mathrm{E}-11$ & $8.1 \mathrm{E}-12$ & $6.3 \mathrm{E}-11$ \\
\hline & 50 & Yr: & & $3.7 \mathrm{E}-11$ & $7.4 \mathrm{E}-11$ & 2. $3 E-11$ & $8.1 \mathrm{E}-12$ & $6.3 \mathrm{E}-11$ \\
\hline \multirow[t]{3}{*}{ LA142 } & Class: D & & $4.32 \mathrm{E}-06$ & & & & & \\
\hline & 1 & Yr: & & $3.7 \mathrm{E}-11$ & 1. $2 \mathrm{E}-11$ & 1. $2 \mathrm{E}-11$ & $9.6 \mathrm{E}-12$ & $5.2 \mathrm{E}-11$ \\
\hline & $5 \odot$ & Yr: & & $3.7 \mathrm{E}-11$ & $1.2 \mathrm{E}-11$ & 1. $2 \mathrm{E}-11$ & $9.6 \mathrm{E}-12$ & $5.2 \mathrm{E}-11$ \\
\hline \multirow[t]{3}{*}{ CE141 } & Class: $Y$ & & $9.81 \mathrm{E}-\odot 8$ & & & & & \\
\hline & 1 & Yr: & & $3 . \odot E-\odot 8$ & $1.1 \mathrm{E}-10$ & $3.7 \mathrm{E}-11$ & 2. $3 \mathrm{E}-11$ & $3.8 \mathrm{E}-09$ \\
\hline & 50 & Yr: & & $3.0 E-\odot 8$ & 1. $1 \mathrm{E}-10$ & $3.7 \mathrm{E}-11$ & 2. $3 \mathrm{E}-11$ & $3.8 \mathrm{E}-09$ \\
\hline \multirow[t]{3}{*}{ CE143 } & Class: Y & & $3.82 E-07$ & & & & & \\
\hline & 1 & Yr: & & $5 . \odot E-\odot 9$ & 1. $4 \mathrm{E}-11$ & 1. $5 \mathrm{E}-11$ & $6.7 \mathrm{E}-12$ & $8.3 E-10$ \\
\hline & 50 & Yr: & & $5.0 \mathrm{E}-\odot 9$ & $1.4 \mathrm{E}-11$ & $1.5 \mathrm{E}-11$ & $6.7 \mathrm{E}-12$ & $8.3 \mathrm{E}-10$ \\
\hline \multirow[t]{3}{*}{ CE144 } & Class: $\mathrm{Y}$ & & $2.41 \mathrm{E}-08$ & & & & & \\
\hline & 1 & Yr: & & $4.2 \mathrm{E}-07$ & $2.1 \mathrm{E}-\odot 9$ & 1. $2 \mathrm{E}-09$ & 1. $4 \mathrm{E}-10$ & $5.3 E-08$ \\
\hline & 50 & Yr: & & $4.2 \mathrm{E}-07$ & $2.1 \mathrm{E}-09$ & 1. $2 \mathrm{E}-\odot 9$ & 1. $4 \mathrm{E}-10$ & $5.3 E-08$ \\
\hline \multirow[t]{3}{*}{ PR143 } & Class: $Y$ & & $6.12 \mathrm{E}-09$ & & & & & \\
\hline & 1 & Yr: & & 1. 8E-๑8 & $2.0 \mathrm{E}-12$ & 2. $\odot E-12$ & 1. $3 E-14$ & 2. $4 \mathrm{E}-\odot 9$ \\
\hline & 50 & Yr: & & $1.8 \mathrm{E}-08$ & 2. $\mathrm{OE}-12$ & 2. $0 \mathrm{E}-12$ & 1. $3 \mathrm{E}-14$ & 2. $4 \mathrm{E}-09$ \\
\hline \multirow[t]{3}{*}{ PR144M } & Class: $\mathrm{Y}$ & & $6.97 \mathrm{E}-\odot 9$ & & & & & \\
\hline & 1 & Yr: & & 4. $0 \mathrm{E}-11$ & $1.5 \mathrm{E}-14$ & $1.8 \mathrm{E}-14$ & 8.7E-15 & $5.1 \mathrm{E}-12$ \\
\hline & 50 & Yr: & & 4. $0 \mathrm{E}-11$ & 1. 5E-14 & $1.8 \mathrm{E}-14$ & 8. 7E-15 & $5.1 \mathrm{E}-12$ \\
\hline \multirow[t]{3}{*}{ PR144 } & Class: $\mathrm{Y}$ & & $8.36 \mathrm{E}-08$ & & & & & \\
\hline & 1 & Yr: & & $5.7 \mathrm{E}-11$ & 1. $4 \mathrm{E}-14$ & $1.6 \mathrm{E}-14$ & $1.6 \mathrm{E}-14$ & 1. $8 \mathrm{E}-11$ \\
\hline & 50 & Yr: & & $5.7 \mathrm{E}-11$ & 1. $4 \mathrm{E}-14$ & 1. $6 \mathrm{E}-14$ & 1. $6 \mathrm{E}-14$ & 1. $8 \mathrm{E}-11$ \\
\hline ND147 & Class: $Y$ & & $1.81 \mathrm{E}-07$ & & & & & \\
\hline & 1 & Yr: & & $1.8 \mathrm{E}-\odot 8$ & $9.9 \mathrm{E}-11$ & $3.4 \mathrm{E}-11$ & $1.6 \mathrm{E}-11$ & $2.4 \mathrm{E}-\odot 9$ \\
\hline & 50 & Yr: & & 1. $8 \mathrm{E}-08$ & $9.9 \mathrm{E}-11$ & 3. $4 \mathrm{E}-11$ & 1. $6 \mathrm{E}-11$ & 2. $4 \mathrm{E}-09$ \\
\hline PM147 & Class: Y & & $2.73 \mathrm{E}-10$ & & & & & \\
\hline & 1 & Yr: & & $3.9 \mathrm{E}-\odot 8$ & $4.9 \mathrm{E}-\odot 9$ & 4. $0 \mathrm{E}-10$ & 4. $0 \mathrm{E}-14$ & $4.9 \mathrm{E}-\odot 9$ \\
\hline & 50 & Yr: & & $3.9 \mathrm{E}-08$ & $4.9 \mathrm{E}-09$ & 4. $0 \mathrm{E}-10$ & 4. $0 \mathrm{E}-14$ & $4.9 \mathrm{E}-\odot 9$ \\
\hline PM148M & Class: Y & & $2.84 \mathrm{E}-06$ & & & & & \\
\hline & 1 & Yr: & & $3.9 \mathrm{E}-\odot 8$ & $8.3 E-10$ & 1. $\odot E-\odot 9$ & 7. $\odot E-10$ & $5.8 \mathrm{E}-09$ \\
\hline & 50 & Yr: & & $3.9 \mathrm{E}-08$ & $8.3 \mathrm{E}-10$ & 1. $\odot E-\odot 9$ & $7.0 \mathrm{E}-10$ & $5.8 \mathrm{E}-09$ \\
\hline PM148 & Class: $\mathrm{Y}$ & & $8.70 \mathrm{E}-\odot 7$ & & & & & \\
\hline & 1 & Yr: & & 1. $3 \mathrm{E}-08$ & $4.5 \mathrm{E}-11$ & $6.4 \mathrm{E}-11$ & $3.8 \mathrm{E}-11$ & 2. $2 \mathrm{E}-09$ \\
\hline & 50 & Yr: & & 1. $3 E-08$ & $4.5 E-11$ & $6.4 \mathrm{E}-11$ & $3.8 E-11$ & $2.2 E-09$ \\
\hline PM149 & Class: $\mathrm{Y}$ & & $2.24 \mathrm{E}-08$ & & & & & \\
\hline & 1 & Yr: & & 4. 3E- - 9 & 1. $0 \mathrm{E}-12$ & 1. $1 \mathrm{E}-12$ & $3.8 \mathrm{E}-13$ & 7. $3 \mathrm{E}-10$ \\
\hline & 50 & Yr: & & 4. 3E- $\odot 9$ & 1. $O E-12$ & 1. $1 \mathrm{E}-12$ & $3.8 \mathrm{E}-13$ & 7. $3 \mathrm{E}-10$ \\
\hline PM151 & Class: $\mathrm{Y}$ & & $4.42 \mathrm{E}-07$ & & & & & \\
\hline & 1 & Yr: & & $2.7 \mathrm{E}-\odot 9$ & $1.4 \mathrm{E}-11$ & $1.5 \mathrm{E}-11$ & $7.0 \mathrm{E}-12$ & $4.7 \mathrm{E}-10$ \\
\hline & 50 & Yr: & & 2. 7E-०9 & 1. $4 \mathrm{E}-11$ & $1.5 \mathrm{E}-11$ & $7.0 \mathrm{E}-12$ & $4.7 \mathrm{E}-10$ \\
\hline SM147 & Class: W & & ๑. О९Е๑० & & & & & \\
\hline & 1 & Yr: & & $2.4 \mathrm{E}-07$ & 1. $4 \mathrm{E}-05$ & $1.1 \mathrm{E}-06$ & 1. $6 \mathrm{E}-11$ & $9.6 \mathrm{E}-06$ \\
\hline & 50 & Yr: & & $2.4 \mathrm{E}-07$ & 1. $4 \mathrm{E}-05$ & $1.1 \mathrm{E}-06$ & 1. $6 \mathrm{E}-11$ & $9.6 \mathrm{E}-06$ \\
\hline SM151 & Class: W & & $7.79 \mathrm{E}-13$ & & & & & \\
\hline & 1 & Yr: & & $3.1 \mathrm{E}-\odot 9$ & 1. $1 \mathrm{E}-07$ & $8.7 \mathrm{E}-\odot 9$ & 1. $5 \mathrm{E}-13$ & 4. . $\mathrm{E}-\odot 9$ \\
\hline & 50 & Yr: & & $3.1 \mathrm{E}-09$ & $1.1 \mathrm{E}-07$ & $8.7 \mathrm{E}-09$ & $1.5 \mathrm{E}-13$ & 4. $\odot \mathrm{E}-\odot 9$ \\
\hline SM153 & Class: W & & $6.46 \mathrm{E}-\odot 8$ & & & & & \\
\hline & 1 & Yr: & & 4. .E- - 9 & $6.3 \mathrm{E}-11$ & $2.3 \mathrm{E}-11$ & 2. $2 \mathrm{E}-12$ & $6.3 \mathrm{E}-10$ \\
\hline & 50 & Yr: & & 4. $\odot E-\odot 9$ & $6.3 E-11$ & $2.3 E-11$ & 2. $2 \mathrm{E}-12$ & $6.3 \mathrm{E}-10$ \\
\hline EU152 & Class: W & & $1.67 \mathrm{E}-\odot 6$ & & & & & \\
\hline & 1 & Yr: & & $6.3 E-08$ & $2 . \odot E-\odot 7$ & $7.1 \mathrm{E}-\odot 8$ & 8. 3E- $\odot 9$ & $4.2 \mathrm{E}-08$ \\
\hline & 50 & Yr: & & $6.3 E-\odot 8$ & 2. $\odot E-\odot 7$ & E-๑8 & 8. 3E- $\odot 9$ & $4.2 \mathrm{E}-08$ \\
\hline EU154 & Class: W & & $1.82 \mathrm{E}-06$ & & & & & \\
\hline & 1 & Yr: & & 1. $\odot E-\odot 7$ & $4.1 \mathrm{E}-07$ & $9.2 \mathrm{E}-08$ & $7.5 \mathrm{E}-\odot 9$ & $5.3 E-08$ \\
\hline & 50 & Yr: & & $1.0 \mathrm{E}-07$ & $4.1 \mathrm{E}-07$ & $9.2 \mathrm{E}-\odot 8$ & $7.5 E-\odot 9$ & $5.3 E-08$ \\
\hline EU155 & Class: W & & $6.78 \mathrm{E}-08$ & & & & & \\
\hline & 1 & Yr: & & 1. $9 \mathrm{E}-\odot 8$ & 1. $2 \mathrm{E}-\odot 7$ & 1. ๑E-๑8 & $2.6 \mathrm{E}-10$ & 7. ๑E-๑9 \\
\hline & 50 & Yr: & & 1. $9 \mathrm{E}-\odot 8$ & 1. $2 \mathrm{E}-\odot 7$ & 1. $\odot E-\odot 8$ & $2.6 \mathrm{E}-10$ & 7. $\odot E-\odot 9$ \\
\hline EU156 & Class: W & & $2.02 \mathrm{E}-\odot 6$ & & & & & \\
\hline & 1 & Yr: & & $2.2 \mathrm{E}-\odot 8$ & 1. $3 E-\odot 9$ & $6.3 E-10$ & 1. $9 \mathrm{E}-10$ & $3.4 \mathrm{E}-\odot 9$ \\
\hline & 50 & Yr: & & $2.2 \mathrm{E}-\odot 8$ & 1. 3E-๑9 & $6.3 \mathrm{E}-10$ & 1. $9 \mathrm{E}-10$ & $3.4 \mathrm{E}-09$ \\
\hline GD153 & Class: D & & $9.84 \mathrm{E}-08$ & & & & & \\
\hline & 1 & Yr: & & $7.1 \mathrm{E}-10$ & & 4.8 & 1. $9 \mathrm{E}-10$ & $2.1 \mathrm{E}-09$ \\
\hline & 50 & Yr: & & $7.1 \mathrm{E}-10$ & $4.9 \mathrm{E}-\odot 8$ & $4.8 \mathrm{E}-09$ & $1.9 \mathrm{E}-10$ & $2.1 \mathrm{E}-09$ \\
\hline TB160 & Class: W & & $1.64 \mathrm{E}-06$ & & & & & \\
\hline & 1 & Yr: & & $4.6 \mathrm{E}-\odot 8$ & 1. $4 \mathrm{E}-\odot 8$ & $2.9 \mathrm{E}-\odot 9$ & $5.6 \mathrm{E}-10$ & 7. ०E-๑9 \\
\hline
\end{tabular}




\begin{tabular}{|c|c|c|c|c|c|c|c|c|}
\hline \multirow{3}{*}{ H0166M } & 50 & Yr: & & $4.6 \mathrm{E}-08$ & 1. 4E-०8 & $2.9 E-\odot 9$ & $5.6 \mathrm{E}-10$ & 7. $0 \mathrm{E}-\odot 9$ \\
\hline & Class: W & & $2.48 \mathrm{E}-06$ & & & & & \\
\hline & 1 & Yr: & & 1. $2 \mathrm{E}-\odot 7$ & $7.2 \mathrm{E}-07$ & $1.5 \mathrm{E}-07$ & $2.1 \mathrm{E}-\odot 8$ & 1. $2 \mathrm{E}-07$ \\
\hline \multirow{4}{*}{ W 181} & 50 & Yr: & & 1. $2 \mathrm{E}-07$ & $7.2 \mathrm{E}-07$ & $1.5 \mathrm{E}-07$ & $2.1 \mathrm{E}-\odot 8$ & 1. $2 \mathrm{E}-07$ \\
\hline & Class: D & & $3.69 \mathrm{E}-08$ & & & & & \\
\hline & 1 & Yr: & & 4. $3 E-12$ & $5.2 \mathrm{E}-11$ & 1.6E-11 & $2.8 \mathrm{E}-12$ & $2.7 \mathrm{E}-11$ \\
\hline & 50 & Yr: & & 4. $3 \mathrm{E}-12$ & $5.2 \mathrm{E}-11$ & 1. $6 \mathrm{E}-11$ & $2.8 \mathrm{E}-12$ & $2.7 \mathrm{E}-11$ \\
\hline \multirow[t]{3}{*}{ W 187} & Class: D & & $6.72 \mathrm{E}-07$ & & & & & \\
\hline & 1 & Yr: & & $3.8 \mathrm{E}-11$ & $5.5 \mathrm{E}-11$ & $2.5 \mathrm{E}-11$ & 1. $2 \mathrm{E}-11$ & $1.9 \mathrm{E}-10$ \\
\hline & 50 & Yr: & & $3.8 \mathrm{E}-11$ & $5.5 E-11$ & $2.5 \mathrm{E}-11$ & 1. $2 \mathrm{E}-11$ & 1. $9 \mathrm{E}-10$ \\
\hline \multirow[t]{3}{*}{ W 185} & Class: D & & $1.57 \mathrm{E}-\odot 9$ & & & & & \\
\hline & 1 & Yr: & & $2.1 \mathrm{E}-11$ & 1. $3 \mathrm{E}-10$ & $4.6 \mathrm{E}-11$ & $2.5 \mathrm{E}-12$ & 1. $2 \mathrm{E}-10$ \\
\hline & 50 & Yr: & & $2.1 \mathrm{E}-11$ & 1. $3 E-10$ & $4.6 \mathrm{E}-11$ & $2.5 \mathrm{E}-12$ & 1. $2 \mathrm{E}-10$ \\
\hline \multirow[t]{3}{*}{ RE187 } & Class: W & & ๑.००Е९९ & & & & & \\
\hline & 1 & Yr: & & $3.8 \mathrm{E}-11$ & 1. $5 \mathrm{E}-13$ & $1.5 \mathrm{E}-13$ & $3.8 \mathrm{E}-12$ & $6.3 \mathrm{E}-12$ \\
\hline & 50 & Yr: & & $3.8 E-11$ & $1.5 \mathrm{E}-13$ & $1.5 \mathrm{E}-13$ & $3.8 \mathrm{E}-12$ & $6.3 \mathrm{E}-12$ \\
\hline \multirow[t]{3}{*}{ IR192 } & Class: Y & & $1.14 \mathrm{E}-06$ & & & & & \\
\hline & 1 & Yr: & & $4.9 E-\odot 8$ & $5.7 \mathrm{E}-10$ & $6.6 \mathrm{E}-10$ & $4.7 \mathrm{E}-10$ & $6.6 \mathrm{E}-\odot 9$ \\
\hline & 50 & Yr: & & $4.9 \mathrm{E}-08$ & $5.7 \mathrm{E}-10$ & $6.6 \mathrm{E}-10$ & $4.7 \mathrm{E}-10$ & $6.6 \mathrm{E}-09$ \\
\hline \multirow[t]{3}{*}{ HG2O3 } & Class: D & & $3.28 \mathrm{E}-07$ & & & & & \\
\hline & 1 & Yr: & & $3.1 \mathrm{E}-10$ & $3.7 \mathrm{E}-10$ & $3.1 \mathrm{E}-10$ & $2.9 \mathrm{E}-10$ & $4.7 \mathrm{E}-10$ \\
\hline & 50 & Yr: & & $3.1 \mathrm{E}-10$ & $3.7 \mathrm{E}-10$ & $3.1 \mathrm{E}-10$ & $2.9 \mathrm{E}-10$ & $4.7 \mathrm{E}-10$ \\
\hline \multirow[t]{3}{*}{ RN222 } & NobleGas & & $5.61 \mathrm{E}-10$ & & & & & \\
\hline & 1 & Yr: & & $\odot . \odot \mathrm{E}-\odot \odot$ & $\odot . \odot \mathrm{E}-\odot \odot$ & $\odot . \odot \mathrm{E}-\odot \odot$ & $\odot . \odot \mathrm{Q}-\odot \odot$ & $\odot . \odot \mathrm{E}-\odot \odot$ \\
\hline & 50 & Yr: & & $\odot . \odot E-\odot \odot$ & $\odot . \odot \mathrm{E}-\odot \odot$ & $\odot . \odot \mathrm{E}-\odot \odot$ & $\odot . \odot \mathrm{E}-\odot \odot$ & $\odot . \odot \mathrm{E}-\odot \odot$ \\
\hline \multirow[t]{3}{*}{ TH227 } & Class: Y & & $1.40 \mathrm{E}-07$ & & & & & \\
\hline & 1 & Yr: & & $8.6 E-05$ & 7. 4E-०8 & $6.4 \mathrm{E}-09$ & $2.9 \mathrm{E}-10$ & 1. $0 \mathrm{E}-05$ \\
\hline & 50 & Yr: & & $8.6 E-05$ & $7.4 \mathrm{E}-\odot 8$ & $6.4 \mathrm{E}-\odot 9$ & $2.9 \mathrm{E}-10$ & 1. $0 \mathrm{E}-05$ \\
\hline TH228 & Class: Y & & $2.56 \mathrm{E}-09$ & & & & & \\
\hline & 1 & Yr: & & $3.3 E-04$ & $2.4 \mathrm{E}-05$ & 1. $9 \mathrm{E}-\odot 6$ & $6.9 \mathrm{E}-08$ & 4. $0 \mathrm{E}-05$ \\
\hline & 50 & Yr: & & $3.3 E-04$ & $2.4 \mathrm{E}-05$ & $1.9 \mathrm{E}-06$ & $6.9 \mathrm{E}-08$ & 4. $0 \mathrm{E}-05$ \\
\hline TH229 & Class: Y & & $1.06 \mathrm{E}-07$ & & & & & \\
\hline & 1 & Yr: & & $5.0 \mathrm{E}-\odot 4$ & $5.1 \mathrm{E}-\odot 4$ & $2.5 \mathrm{E}-05$ & 1. 7E- - 6 & $7.1 \mathrm{E}-05$ \\
\hline & 50 & Yr: & & $5 . \odot E-\odot 4$ & $5.1 \mathrm{E}-04$ & $2.5 \mathrm{E}-\odot 5$ & 1. $7 \mathrm{E}-\odot 6$ & $7.1 \mathrm{E}-05$ \\
\hline TH230 & Class: Y & & $4.70 \mathrm{E}-10$ & & & & & \\
\hline & 1 & Yr: & & 7.7E-०5 & $2.8 \mathrm{E}-04$ & 1. $\odot E-\odot 5$ & 3. $0 \mathrm{E}-07$ & 1. $4 \mathrm{E}-05$ \\
\hline & 50 & Yr: & & $7.7 \mathrm{E}-05$ & $2.8 E-\odot 4$ & 1. $\odot E-\odot 5$ & $3 . \odot E-\odot 7$ & 1. $4 \mathrm{E}-05$ \\
\hline TH231 & Class: Y & & 1. 45E- -88 & & & & & \\
\hline & 1 & Yr: & & $2.2 \mathrm{E}-\odot 9$ & $5.6 \mathrm{E}-11$ & $2.8 \mathrm{E}-12$ & $3.6 \mathrm{E}-13$ & $3.3 \mathrm{E}-10$ \\
\hline & 50 & Yr: & & 2. $2 \mathrm{E}-\odot 9$ & $5.6 \mathrm{E}-11$ & $2.8 \mathrm{E}-12$ & $3.6 \mathrm{E}-13$ & $3.3 \mathrm{E}-10$ \\
\hline TH232 & Class: Y & & $2.29 \mathrm{E}-10$ & & & & & \\
\hline & 1 & Yr: & & $1.6 \mathrm{E}-\odot 4$ & $2.9 E-\odot 4$ & 1. $2 \mathrm{E}-05$ & $8.2 \mathrm{E}-07$ & $2.5 \mathrm{E}-\odot 5$ \\
\hline & 50 & Yr: & & 1. $6 \mathrm{E}-04$ & $2.9 \mathrm{E}-04$ & $1.2 \mathrm{E}-\odot 5$ & $8.2 \mathrm{E}-07$ & $2.5 \mathrm{E}-05$ \\
\hline TH234 & Class: Y & & $9.30 \mathrm{E}-\odot 9$ & & & & & \\
\hline & 1 & Yr: & & $5.8 \mathrm{E}-08$ & $7.4 \mathrm{E}-11$ & $4.4 \mathrm{E}-11$ & $6.7 \mathrm{E}-12$ & 7. $7 \mathrm{E}-09$ \\
\hline & 50 & Yr: & & $5.8 \mathrm{E}-08$ & 7. $4 \mathrm{E}-11$ & $4.4 \mathrm{E}-11$ & $6.7 \mathrm{E}-12$ & 7. $7 \mathrm{E}-\odot 9$ \\
\hline RA223 & Class: W & & $1.73 \mathrm{E}-07$ & & & & & \\
\hline & 1 & Yr: & & $6.2 \mathrm{E}-05$ & $7.8 \mathrm{E}-07$ & $7.5 \mathrm{E}-\odot 8$ & $3.4 \mathrm{E}-\odot 9$ & 7. $4 \mathrm{E}-06$ \\
\hline & 50 & Yr: & & $6.2 E-05$ & $7.8 E-07$ & $7.5 E-\odot 8$ & 3. 4E- $\odot 9$ & $7.4 \mathrm{E}-06$ \\
\hline RA224 & Class: W & & 1. $36 \mathrm{E}-\odot 8$ & & & & & \\
\hline & 1 & Yr: & & $2.5 E-\odot 5$ & $4.1 \mathrm{E}-07$ & 4. . ०E-๑8 & 2. 3E-०9 & $3.0 E-\odot 6$ \\
\hline & 50 & Yr: & & $2.5 E-05$ & $4.1 \mathrm{E}-07$ & 4. ०E-๑8 & 2. 3E-०9 & $3.0 \mathrm{E}-06$ \\
\hline RA225 & Class: W & & $7.6 \odot E-\odot 9$ & & & & & \\
\hline & 1 & Yr: & & $5.2 \mathrm{E}-05$ & $2.2 \mathrm{E}-06$ & 2. $\odot \mathrm{E}-\odot 7$ & $6.4 \mathrm{E}-\odot 9$ & 6. 3E- 06 \\
\hline & 50 & Yr: & & $5.2 \mathrm{E}-05$ & $2.2 \mathrm{E}-06$ & 2. $\odot \mathrm{E}-\odot 7$ & $6.4 \mathrm{E}-\odot 9$ & $6.3 \mathrm{E}-06$ \\
\hline RA226 & Class: W & & 8. 96E-๑9 & & & & & \\
\hline & 1 & Yr: & & $2.8 \mathrm{E}-\odot 5$ & $7.4 \mathrm{E}-06$ & $5.2 \mathrm{E}-\odot 7$ & $2.4 \mathrm{E}-08$ & $3.5 \mathrm{E}-06$ \\
\hline & 50 & Yr: & & $2.8 E-05$ & $7.4 \mathrm{E}-06$ & $5.2 \mathrm{E}-07$ & 2. 4E- - 88 & $3.5 E-06$ \\
\hline RA228 & Class: W & & $\odot . \odot \odot Е \odot \odot ~$ & & & & & \\
\hline & 1 & Yr: & & $9.7 \mathrm{E}-06$ & $5.6 \mathrm{E}-05$ & 4.7E-०6 & $2.1 \mathrm{E}-07$ & $2.6 \mathrm{E}-06$ \\
\hline & 50 & Yr: & & $9.7 E-06$ & $5.6 \mathrm{E}-05$ & $4.7 \mathrm{E}-06$ & $2.1 \mathrm{E}-07$ & $2.6 \mathrm{E}-06$ \\
\hline PB210 & Class: D & & $1.42 \mathrm{E}-09$ & & & & & \\
\hline & 1 & Yr: & & 1. $2 \mathrm{E}-07$ & $2.9 \mathrm{E}-05$ & $3.2 \mathrm{E}-06$ & 1. $2 \mathrm{E}-07$ & $9.1 \mathrm{E}-07$ \\
\hline & 50 & Yr: & & 1. $2 \mathrm{E}-07$ & $2.9 \mathrm{E}-05$ & $3.2 \mathrm{E}-06$ & 1. $2 \mathrm{E}-07$ & $9.1 \mathrm{E}-07$ \\
\hline PB212 & Class: D & & $1.97 \mathrm{E}-07$ & & & & & \\
\hline & 1 & Yr: & & 8. 7E-๑9 & & 1. 1E-०8 & $3.4 \mathrm{E}-09$ & $1.8 \mathrm{E}-08$ \\
\hline & 50 & Yr: & & 8. 7E-०9 & $8.5 E-\odot 8$ & 1. 1E-๑8 & $3.4 \mathrm{E}-09$ & 1. $8 \mathrm{E}-08$ \\
\hline BI210 & Class: W & & $8.14 \mathrm{E}-\odot 9$ & & & & & \\
\hline & 1 & Yr: & & $7.7 \mathrm{E}-07$ & $4.7 \mathrm{E}-11$ & $4.7 \mathrm{E}-11$ & $4.7 \mathrm{E}-11$ & 9. 3E- - 8 \\
\hline & 50 & Yr: & & $7.7 \mathrm{E}-07$ & $4.7 \mathrm{E}-11$ & $4.7 \mathrm{E}-11$ & $4.7 \mathrm{E}-11$ & $9.3 \mathrm{E}-08$ \\
\hline BI212 & Class: W & & $2.83 E-07$ & & & & & \\
\hline
\end{tabular}




\begin{tabular}{|c|c|c|c|c|c|c|c|c|}
\hline & 1 & Yr: & & $2.0 \mathrm{E}-07$ & $2.1 \mathrm{E}-11$ & $2.1 \mathrm{E}-11$ & $2.1 \mathrm{E}-11$ & $3.1 \mathrm{E}-08$ \\
\hline & 50 & Yr: & & 2. $\odot \mathrm{E}-07$ & $2.1 \mathrm{E}-11$ & $2.1 \mathrm{E}-11$ & $2.1 \mathrm{E}-11$ & $3.1 \mathrm{E}-08$ \\
\hline \multirow[t]{3}{*}{ P0210 } & Class: W & & $1.23 \mathrm{E}-11$ & & & & & \\
\hline & 1 & Yr: & & $2.6 E-05$ & $2.8 \mathrm{E}-07$ & $4.6 E-07$ & 4. 9E- - & $3.3 E-06$ \\
\hline & 50 & Yr: & & $2.6 \mathrm{E}-05$ & $2.8 \mathrm{E}-07$ & $4.6 \mathrm{E}-07$ & 4. $9 \mathrm{E}-08$ & $3.3 E-06$ \\
\hline \multirow[t]{3}{*}{ U 232} & Class: $Y$ & & $3.72 \mathrm{E}-10$ & & & & & \\
\hline & 1 & Yr: & & $3.0 \mathrm{E}-04$ & 4. 1E- 05 & $3.3 E-06$ & 2. $\odot E-07$ & $3.7 \mathrm{E}-05$ \\
\hline & 50 & Yr: & & $3 . \odot E-\odot 4$ & $4.1 E-05$ & $3.3 E-\odot 6$ & 2. $\odot \mathrm{E}-07$ & $3.7 \mathrm{E}-05$ \\
\hline \multirow[t]{3}{*}{ U 233} & Class: $\mathrm{Y}$ & & $4.48 E-10$ & & & & & \\
\hline & 1 & Yr: & & 8.0E-05 & 8. ०E-๑7 & $6.8 \mathrm{E}-08$ & 1. 8E- - & $9.6 \mathrm{E}-06$ \\
\hline & 50 & Yr: & & 8. ०E- $\odot 5$ & $8.0 \mathrm{E}-07$ & $6.8 \mathrm{E}-\odot 8$ & 1. 8E- - & $9.6 \mathrm{E}-06$ \\
\hline \multirow[t]{3}{*}{ U 234} & Class: $Y$ & & $1.93 \mathrm{E}-10$ & & & & & \\
\hline & 1 & Yr: & & $7.8 E-05$ & $5.0 \mathrm{E}-\odot 7$ & $5.2 \mathrm{E}-\odot 8$ & 1. $6 \mathrm{E}-08$ & $9.4 \mathrm{E}-06$ \\
\hline & 50 & Yr: & & $7.8 E-05$ & $5.0 \mathrm{E}-\odot 7$ & $5.2 \mathrm{E}-\odot 8$ & 1. $6 \mathrm{E}-\odot 8$ & $9.4 \mathrm{E}-06$ \\
\hline \multirow[t]{3}{*}{ U 235} & Class: $\mathrm{Y}$ & & $2.04 \mathrm{E}-07$ & & & & & \\
\hline & 1 & Yr: & & $7.0 \mathrm{E}-05$ & $4.8 E-07$ & $5.0 \mathrm{E}-\odot 8$ & 1. $6 \mathrm{E}-08$ & $8.5 E-06$ \\
\hline & 50 & Yr: & & 7. ๑E-๑5 & $4.8 E-07$ & $5.0 \mathrm{E}-\odot 8$ & $1.6 \mathrm{E}-08$ & $8.5 E-06$ \\
\hline \multirow[t]{3}{*}{ U 236} & Class: $\mathrm{Y}$ & & $1.22 \mathrm{E}-10$ & & & & & \\
\hline & 1 & Yr: & & 7. 3E- - & $4.8 \mathrm{E}-07$ & 4. . 9E-०8 & 1. 5E-०8 & 8.7E- -06 \\
\hline & 50 & Yr: & & $7.3 E-05$ & $4.8 \mathrm{E}-07$ & 4. $9 \mathrm{E}-\odot 8$ & $1.5 \mathrm{E}-\odot 8$ & $8.7 \mathrm{E}-06$ \\
\hline \multirow[t]{3}{*}{ U 237} & Class: $Y$ & & $1.67 \mathrm{E}-07$ & & & & & \\
\hline & 1 & Yr: & & 1. 4E- - 88 & $3.4 \mathrm{E}-11$ & 1. $9 \mathrm{E}-11$ & $1.1 \mathrm{E}-11$ & 1. $9 \mathrm{E}-\odot 9$ \\
\hline & 50 & Yr: & & 1. 4E- -8 & $3.4 \mathrm{E}-11$ & 1. $9 \mathrm{E}-11$ & 1.1E-11 & 1. $9 \mathrm{E}-\odot 9$ \\
\hline \multirow[t]{3}{*}{ U 238} & Class: $Y$ & & $7.92 \mathrm{E}-11$ & & & & & \\
\hline & 1 & Yr: & & $6.7 \mathrm{E}-05$ & $4.6 E-07$ & 4. 9E- - & 1. $5 E-08$ & $8.0 E-06$ \\
\hline & 50 & Yr: & & $6.7 \mathrm{E}-05$ & $4.6 \mathrm{E}-07$ & 4. $9 \mathrm{E}-08$ & 1. $5 \mathrm{E}-08$ & $8.0 E-06$ \\
\hline \multirow[t]{3}{*}{ U 240} & Class: Y & & $1.85 E-09$ & & & & & \\
\hline & 1 & Yr: & & 2. $9 E-\odot 9$ & $5.1 \mathrm{E}-11$ & 1. 1E-11 & $4.4 \mathrm{E}-12$ & $5.8 \mathrm{E}-10$ \\
\hline & 50 & Yr: & & $2.9 \mathrm{E}-\odot 9$ & $5.1 \mathrm{E}-11$ & $1.1 \mathrm{E}-11$ & 4. $4 \mathrm{E}-12$ & $5.8 \mathrm{E}-10$ \\
\hline PA231 & Class: $\mathrm{Y}$ & & 4. .95E- - & & & & & \\
\hline & 1 & Yr: & & $1.5 \mathrm{E}-04$ & $6.0 \mathrm{E}-04$ & $2.4 \mathrm{E}-05$ & 1. $3 E-06$ & $2.9 \mathrm{E}-05$ \\
\hline & 50 & Yr: & & $1.5 E-04$ & $6 . \odot E-\odot 4$ & $2.4 \mathrm{E}-05$ & 1. $3 E-06$ & $2.9 \mathrm{E}-05$ \\
\hline PA233 & Class: $Y$ & & $2.7 \odot E-\odot 7$ & & & & & \\
\hline & 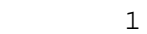 & Yr: & & 3.०E-०8 & $1.8 \mathrm{E}-10$ & 8. 3E-11 & $5.0 \mathrm{E}-11$ & $3.9 E-09$ \\
\hline & 50 & Yr: & & 3. ०E-०8 & 1. $8 \mathrm{E}-10$ & 8. $3 \mathrm{E}-11$ & $5.0 \mathrm{E}-11$ & $3.9 \mathrm{E}-\odot 9$ \\
\hline PA234 & Class: $Y$ & & $2.75 \mathrm{E}-06$ & & & & & \\
\hline & 1 & Yr: & & 1. 8E- - 9 & 1. $9 \mathrm{E}-11$ & $2.6 \mathrm{E}-11$ & 1. $4 \mathrm{E}-11$ & $4.2 \mathrm{E}-10$ \\
\hline & 50 & Yr: & & 1. 8E-०9 & 1. $9 \mathrm{E}-11$ & $2.6 \mathrm{E}-11$ & 1. $4 \mathrm{E}-11$ & $4.2 \mathrm{E}-10$ \\
\hline AC225 & Class: $\mathrm{Y}$ & & 2. .01E- -88 & & & & & \\
\hline & 1 & Yr: & & $7.1 \mathrm{E}-05$ & $3.8 \mathrm{E}-08$ & 4. 7E-๑9 & 2. $\odot E-\odot 9$ & $8.5 E-06$ \\
\hline & 50 & Yr: & & $7.1 \mathrm{E}-05$ & $3.8 \mathrm{E}-\odot 8$ & 4. 7E-०9 & 2. $\odot E-\odot 9$ & $8.5 E-06$ \\
\hline AC227 & Class: $Y$ & & $1.62 \mathrm{E}-10$ & & & & & \\
\hline & 1 & Yr: & & $4.1 \mathrm{E}-04$ & 1. $9 \mathrm{E}-\mathrm{-4}$ & 1. $0 \mathrm{E}-05$ & $9.5 \mathrm{E}-07$ & $5.5 E-05$ \\
\hline & 50 & Yr: & & $4.1 \mathrm{E}-04$ & 1. $9 \mathrm{E}-04$ & 1. $0 \mathrm{E}-05$ & $9.5 \mathrm{E}-07$ & $5.5 E-05$ \\
\hline AC228 & Class: Y & & $1.42 \mathrm{E}-06$ & & & & & \\
\hline & 1 & Yr: & & 1. $2 \mathrm{E}-07$ & 8. $6 \mathrm{E}-\odot 9$ & $6.8 \mathrm{E}-10$ & $3.2 \mathrm{E}-11$ & 1. $5 E-08$ \\
\hline & 50 & Yr: & & $1.2 \mathrm{E}-07$ & 8. 6E-๑9 & $6.8 \mathrm{E}-10$ & $3.2 \mathrm{E}-11$ & 1. $5 \mathrm{E}-\odot 8$ \\
\hline FR223 & Class: D & & $6.97 \mathrm{E}-\odot 8$ & & & & & \\
\hline & 1 & Yr: & & $8.6 \mathrm{E}-10$ & $8.0 \mathrm{E}-10$ & 8. $0 \mathrm{E}-10$ & 8. $0 \mathrm{E}-10$ & $9.0 \mathrm{E}-10$ \\
\hline & 50 & Yr: & & $8.6 \mathrm{E}-10$ & 8. $\odot E-1 \odot$ & 8. $\odot E-10$ & 8. $\odot E-1 \odot$ & $9.0 \mathrm{E}-10$ \\
\hline NP237 & Class: W & & $2.81 \mathrm{E}-\odot 8$ & & & & & \\
\hline & 1 & Yr: & & $2.9 E-05$ & 1. $0 \mathrm{E}-03$ & $3.9 E-05$ & 1. 3E- 06 & $2.3 E-05$ \\
\hline & 50 & Yr: & & $2.9 E-05$ & 1. $\odot E-\odot 3$ & $3.9 E-05$ & 1. 3E- 06 & 2. 3E- 05 \\
\hline NP238 & Class: W & & 8. $07 \mathrm{E}-\odot 7$ & & & & & \\
\hline & 1 & Yr: & & $5.5 E-09$ & $6.8 \mathrm{E}-08$ & $2.8 \mathrm{E}-\odot 9$ & 1. $0 \mathrm{E}-10$ & $2.1 \mathrm{E}-09$ \\
\hline & 50 & Yr: & & $5.5 \mathrm{E}-09$ & $6.8 \mathrm{E}-08$ & $2.8 \mathrm{E}-\odot 9$ & 1. $0 \mathrm{E}-10$ & $2.1 \mathrm{E}-\odot 9$ \\
\hline NP239 & Class: W & & $2.19 \mathrm{E}-07$ & & & & & \\
\hline & 1 & Yr: & & $6.3 E-09$ & $5.2 \mathrm{E}-10$ & $4.5 \mathrm{E}-11$ & 8. $2 \mathrm{E}-12$ & $9.3 \mathrm{E}-10$ \\
\hline & 50 & Yr: & & $6.3 E-09$ & $5.2 \mathrm{E}-10$ & $4.5 E-11$ & 8. $2 \mathrm{E}-12$ & $9.3 E-10$ \\
\hline PU236 & Class: $Y$ & & $1.48 \mathrm{E}-10$ & & & & & \\
\hline & 1 & Yr: & & 7. 4E- 05 & $5.1 \mathrm{E}-05$ & $2.8 \mathrm{E}-06$ & 8. 8E-๑8 & 1. $1 \mathrm{E}-05$ \\
\hline & 50 & Yr: & & $7.4 \mathrm{E}-05$ & $5.1 \mathrm{E}-05$ & $2.8 \mathrm{E}-06$ & 8. 8E- - & $1.1 \mathrm{E}-05$ \\
\hline PU237 & Class: $Y$ & & $5.58 \mathrm{E}-\odot 8$ & & & & & \\
\hline & 1 & Yr: & & 2. $9 \mathrm{E}-\odot 9$ & $7.4 \mathrm{E}-11$ & $2.8 \mathrm{E}-11$ & 1. $8 \mathrm{E}-11$ & $3.9 \mathrm{E}-10$ \\
\hline & 50 & Yr: & & $2.9 E-09$ & 7. $4 \mathrm{E}-11$ & $2.8 \mathrm{E}-11$ & 1. $8 \mathrm{E}-11$ & $3.9 \mathrm{E}-10$ \\
\hline PU238 & Class: $\mathrm{Y}$ & & $1.11 \mathrm{E}-10$ & & & & & \\
\hline & 1 & Yr: & & $9.3 \mathrm{E}-05$ & $1.6 \mathrm{E}-04$ & $8.3 E-06$ & $2.8 \mathrm{E}-07$ & $1.6 \mathrm{E}-05$ \\
\hline & 50 & Yr: & & $9.3 \mathrm{E}-05$ & 1. $6 \mathrm{E}-04$ & 8. 3E-๑6 & $2.8 E-07$ & $1.6 \mathrm{E}-05$ \\
\hline PU239 & Class: $Y$ & & $1.10 \mathrm{E}-10$ & & & & & \\
\hline & 1 & Yr: & & $8.7 E-05$ & $1.8 \mathrm{E}-04$ & $9.1 \mathrm{E}-06$ & 3. $2 \mathrm{E}-07$ & $1.6 \mathrm{E}-05$ \\
\hline & 50 & Yr: & & $8.7 \mathrm{E}-05$ & $1.8 \mathrm{E}-04$ & $9.1 \mathrm{E}-06$ & $3.2 \mathrm{E}-07$ & $1.6 \mathrm{E}-05$ \\
\hline
\end{tabular}




\begin{tabular}{|c|c|c|c|c|c|c|c|c|}
\hline \multirow[t]{3}{*}{ PU240 } & Class: Y & & $1.08 \mathrm{E}-10$ & & & & & \\
\hline & 1 & Yr: & & 8. 8E- 05 & $1.8 \mathrm{E}-\odot 4$ & $9.1 \mathrm{E}-06$ & $3.2 \mathrm{E}-07$ & 1. $6 \mathrm{E}-05$ \\
\hline & 50 & Yr: & & $8.8 \mathrm{E}-05$ & $1.8 \mathrm{E}-\odot 4$ & $9.1 \mathrm{E}-06$ & $3.2 \mathrm{E}-07$ & 1. $6 \mathrm{E}-05$ \\
\hline \multirow[t]{3}{*}{ PU241 } & Class: Y & & 2. $00 \mathrm{E}-12$ & & & & & \\
\hline & 1 & Yr: & & $4.6 \mathrm{E}-07$ & $4.1 \mathrm{E}-06$ & $1.8 \mathrm{E}-\odot 7$ & $7.1 \mathrm{E}-09$ & $1.8 \mathrm{E}-07$ \\
\hline & 50 & Yr: & & $4.6 \mathrm{E}-07$ & $4.1 E-06$ & $1.8 \mathrm{E}-\odot 7$ & $7.1 \mathrm{E}-09$ & 1. $8 \mathrm{E}-07$ \\
\hline \multirow[t]{3}{*}{ PU242 } & Class: Y & & $9.18 \mathrm{E}-11$ & & & & & \\
\hline & 1 & Yr: & & $8.1 E-05$ & $1.8 E-04$ & $8.7 E-\odot 6$ & 3. ०E-०7 & 1. $5 \mathrm{E}-05$ \\
\hline & 50 & Yr: & & $8.1 \mathrm{E}-05$ & $1.8 \mathrm{E}-\odot 4$ & 8. . $\mathrm{E}-06$ & 3. $\odot E-\odot 7$ & $1.5 \mathrm{E}-05$ \\
\hline \multirow[t]{3}{*}{ PU243 } & Class: Y & & $3.04 \mathrm{E}-\odot 8$ & & & & & \\
\hline & 1 & Yr: & & $4.7 \mathrm{E}-10$ & $1.5 \mathrm{E}-11$ & $9.9 E-13$ & 1. $6 \mathrm{E}-13$ & $8.7 \mathrm{E}-11$ \\
\hline & 50 & Yr: & & 4. $7 \mathrm{E}-10$ & $1.5 \mathrm{E}-11$ & $9.9 \mathrm{E}-13$ & 1. $6 \mathrm{E}-13$ & $8.7 \mathrm{E}-11$ \\
\hline \multirow[t]{3}{*}{ PU244 } & Class: Y & & $6.56 \mathrm{E}-11$ & & & & & \\
\hline & 1 & Yr: & & $7.5 E-05$ & 1. $6 \mathrm{E}-\odot 4$ & $8.2 \mathrm{E}-\odot 6$ & $2.9 E-07$ & 1. $4 \mathrm{E}-05$ \\
\hline & 50 & Yr: & & $7.5 E-05$ & $1.6 \mathrm{E}-04$ & $8.2 \mathrm{E}-\odot 6$ & $2.9 \mathrm{E}-07$ & 1. $4 \mathrm{E}-05$ \\
\hline \multirow{3}{*}{ AM241 } & Class: W & & $2.13 \mathrm{E}-08$ & & & & & \\
\hline & 1 & Yr: & & $3.7 \mathrm{E}-05$ & 1. $7 \mathrm{E}-\odot 3$ & $5.8 \mathrm{E}-\odot 5$ & $2.9 E-06$ & $4.2 E-05$ \\
\hline & 50 & Yr: & & $3.7 \mathrm{E}-05$ & 1. $7 \mathrm{E}-03$ & $5.8 \mathrm{E}-05$ & 2. $9 \mathrm{E}-06$ & $4.2 \mathrm{E}-05$ \\
\hline \multirow[t]{3}{*}{ AM242M } & Class: W & & $7.85 \mathrm{E}-10$ & & & & & \\
\hline & 1 & Yr: & & $8.8 \mathrm{E}-06$ & 1. $7 \mathrm{E}-\odot 3$ & $5.5 E-\odot 5$ & $2.9 E-06$ & $3.7 \mathrm{E}-05$ \\
\hline & 50 & Yr: & & $8.8 \mathrm{E}-06$ & 1. $7 \mathrm{E}-03$ & $5.5 E-05$ & 2. $9 \mathrm{E}-06$ & $3.7 \mathrm{E}-05$ \\
\hline \multirow[t]{3}{*}{ AM242 } & Class: W & & 1.93E-๑8 & & & & & \\
\hline & 1 & Yr: & & 1. 2E- - 7 & $9.2 \mathrm{E}-\odot 8$ & $6.3 E-\odot 9$ & 1. $2 \mathrm{E}-10$ & $1.7 \mathrm{E}-\odot 8$ \\
\hline & $5 \odot$ & Yr: & & 1. $2 E-07$ & $9.2 \mathrm{E}-08$ & $6.3 E-\odot 9$ & 1. $2 \mathrm{E}-10$ & 1. $7 \mathrm{E}-\odot 8$ \\
\hline \multirow[t]{3}{*}{ AM243 } & Class: W & & $5.87 \mathrm{E}-\odot 8$ & & & & & \\
\hline & 1 & Yr: & & $3.5 E-05$ & 1. $7 \mathrm{E}-\odot 3$ & $5.7 \mathrm{E}-\odot 5$ & $2.9 E-\odot 6$ & $4.1 \mathrm{E}-05$ \\
\hline & 50 & Yr: & & $3.5 E-05$ & 1.7E-०3 & $5.7 \mathrm{E}-\odot 5$ & $2.9 E-\odot 6$ & $4.1 \mathrm{E}-05$ \\
\hline \multirow[t]{3}{*}{ CM242 } & Class: W & & $1.27 \mathrm{E}-10$ & & & & & \\
\hline & 1 & Yr: & & $3.5 E-05$ & $2.7 \mathrm{E}-05$ & 1. $9 \mathrm{E}-\odot 6$ & $3.5 E-\odot 8$ & $5.2 \mathrm{E}-\odot 6$ \\
\hline & $5 \odot$ & Yr: & & $3.5 E-05$ & $2.7 \mathrm{E}-05$ & $1.9 \mathrm{E}-\odot 6$ & $3.5 \mathrm{E}-\odot 8$ & $5.2 \mathrm{E}-06$ \\
\hline \multirow[t]{3}{*}{ CM243 } & Class: W & & $1.67 \mathrm{E}-07$ & & & & & \\
\hline & 1 & Yr: & & 4. $0 \mathrm{E}-05$ & 1. $2 \mathrm{E}-03$ & $4.5 \mathrm{E}-\odot 5$ & 1. $7 \mathrm{E}-\odot 6$ & $3.2 \mathrm{E}-05$ \\
\hline & 50 & Yr: & & $4.0 \mathrm{E}-05$ & $1.2 \mathrm{E}-03$ & $4.5 \mathrm{E}-05$ & 1.7E- 06 & $3.2 \mathrm{E}-05$ \\
\hline \multirow[t]{3}{*}{ CM244 } & Class: W & & $1.08 \mathrm{E}-10$ & & & & & \\
\hline & 1 & Yr: & & $3.9 E-05$ & $9.2 \mathrm{E}-04$ & $3.9 E-\odot 5$ & 1. 3E-०6 & $2.7 \mathrm{E}-\odot 5$ \\
\hline & 50 & Yr: & & $3.9 \mathrm{E}-05$ & $9.2 \mathrm{E}-04$ & $3.9 \mathrm{E}-05$ & 1. $3 \mathrm{E}-06$ & $2.7 \mathrm{E}-05$ \\
\hline \multirow[t]{3}{*}{ CM245 } & Class: W & & $1.10 \mathrm{E}-07$ & & & & & \\
\hline & 1 & Yr: & & $3.6 \mathrm{E}-05$ & $1.8 \mathrm{E}-03$ & $5.9 E-05$ & 3. $0 \mathrm{E}-06$ & $4.2 \mathrm{E}-05$ \\
\hline & 50 & Yr: & & $3.6 \mathrm{E}-05$ & $1.8 \mathrm{E}-03$ & $5.9 E-05$ & $3.0 \mathrm{E}-06$ & $4.2 \mathrm{E}-05$ \\
\hline \multirow[t]{3}{*}{ CM246 } & Class: W & & $9.78 \mathrm{E}-11$ & & & & & \\
\hline & 1 & Yr: & & $3.6 \mathrm{E}-05$ & 1.7E-०3 & $5.8 \mathrm{E}-05$ & $2.9 \mathrm{E}-06$ & $4.2 \mathrm{E}-05$ \\
\hline & 50 & Yr: & & $3.6 \mathrm{E}-05$ & 1.7E-०3 & $5.8 \mathrm{E}-05$ & $2.9 \mathrm{E}-\odot 6$ & $4.2 \mathrm{E}-05$ \\
\hline \multirow[t]{3}{*}{ CM247 } & Class: W & & $4.38 \mathrm{E}-07$ & & & & & \\
\hline & 1 & Yr: & & $3.2 E-05$ & 1. $6 \mathrm{E}-\odot 3$ & $5.4 \mathrm{E}-\odot 5$ & $2.7 \mathrm{E}-06$ & $3.9 E-05$ \\
\hline & 50 & Yr: & & $3.2 \mathrm{E}-05$ & 1. $6 \mathrm{E}-03$ & $5.4 \mathrm{E}-\odot 5$ & $2.7 \mathrm{E}-\odot 6$ & $3.9 \mathrm{E}-05$ \\
\hline \multirow[t]{3}{*}{ CM248 } & Class: W & & $7.44 \mathrm{E}-11$ & & & & & \\
\hline & 1 & Yr: & & $6.3 E-05$ & $7.8 E-03$ & $6.2 \mathrm{E}-\odot 4$ & 2. $2 \mathrm{E}-\odot 8$ & $4.2 E-04$ \\
\hline & 50 & Yr: & & $6.3 E-05$ & $7.8 E-03$ & $6.2 \mathrm{E}-\odot 4$ & $2.2 E-08$ & $4.2 E-04$ \\
\hline \multirow[t]{3}{*}{ CF252 } & Class: W & & $1.15 \mathrm{E}-10$ & & & & & \\
\hline & 1 & Yr: & & $3.5 \mathrm{E}-05$ & $6.6 \mathrm{E}-04$ & $5.3 E-\odot 5$ & 1. 1E- -8 & $3.5 E-05$ \\
\hline & 50 & Yr: & & $3.5 \mathrm{E}-05$ & $6.6 \mathrm{E}-04$ & $5.3 \mathrm{E}-05$ & 1.1E- -8 & $3.5 \mathrm{E}-05$ \\
\hline
\end{tabular}




\section{Appendix A.2}

\section{Age 5475}

Dose Equiv. Factors for Acute Inhalation and Ext Air Submersion FGR12/13

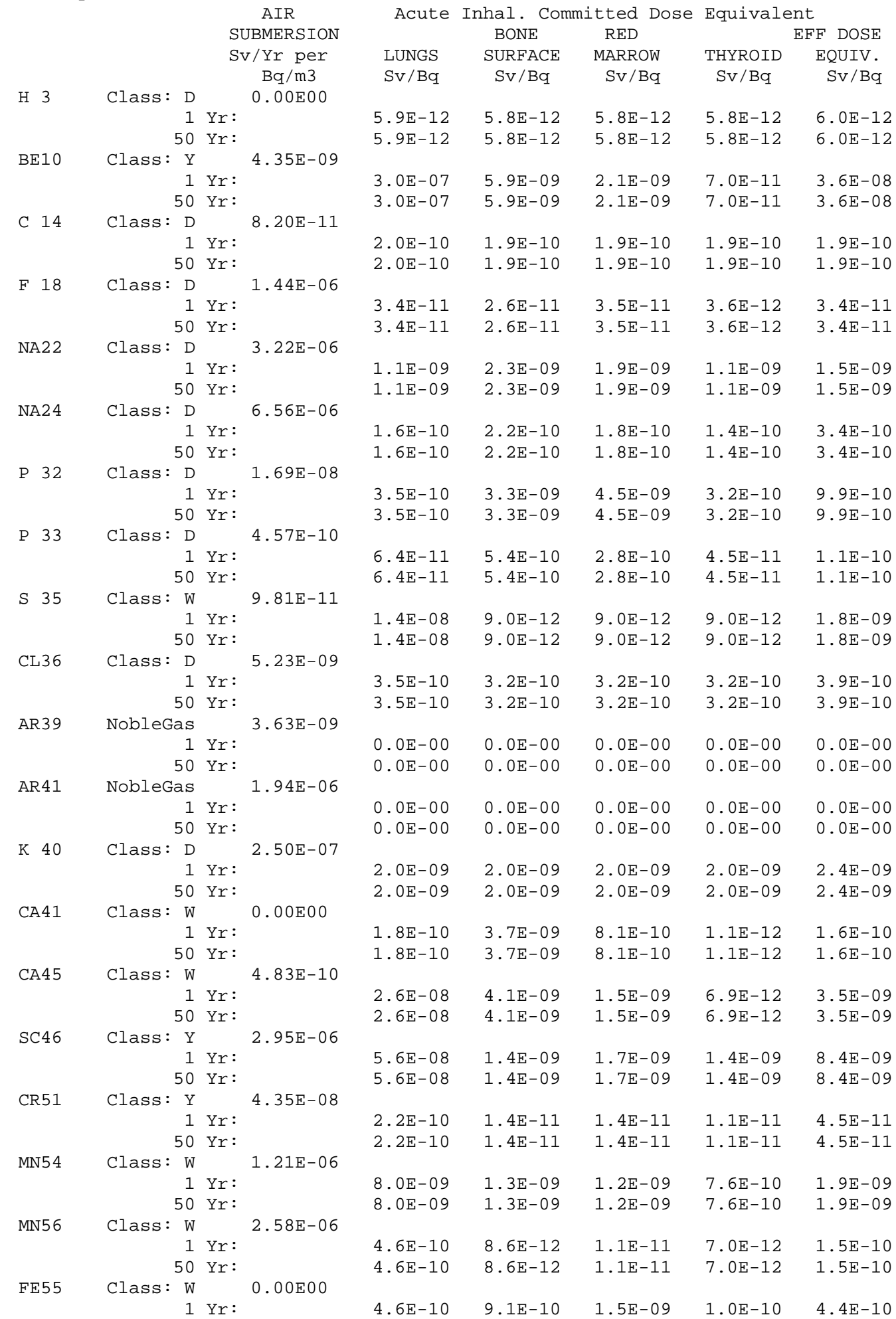




\begin{tabular}{|c|c|c|c|c|c|c|c|c|}
\hline & 50 & Yr: & & $4.6 \mathrm{E}-10$ & $9.1 \mathrm{E}-10$ & 1. $5 \mathrm{E}-\odot 9$ & 1. $0 \mathrm{E}-10$ & $4.4 \mathrm{E}-10$ \\
\hline \multirow[t]{3}{*}{ FE59 } & Class: W & & $1.77 \mathrm{E}-06$ & & & & & \\
\hline & 1 & Yr: & & $2.9 \mathrm{E}-\odot 8$ & 1. $3 \mathrm{E}-\odot 9$ & 1. $7 \mathrm{E}-\odot 9$ & 8. $0 \mathrm{E}-10$ & $4.6 \mathrm{E}-09$ \\
\hline & 50 & Yr: & & $2.9 \mathrm{E}-08$ & 1. 3E-०9 & 1. 7E- $\odot 9$ & $8.0 E-10$ & $4.6 E-\odot 9$ \\
\hline \multirow[t]{3}{*}{ C057 } & Class: Y & & $1.57 \mathrm{E}-07$ & & & & & \\
\hline & 1 & Yr: & & $7.9 E-\odot 9$ & $4.6 \mathrm{E}-10$ & $2.6 \mathrm{E}-10$ & $2.3 E-10$ & 1. $2 \mathrm{E}-09$ \\
\hline & 50 & Yr: & & $7.9 \mathrm{E}-09$ & $4.6 \mathrm{E}-10$ & $2.6 \mathrm{E}-10$ & $2.3 \mathrm{E}-10$ & 1. $2 \mathrm{E}-09$ \\
\hline \multirow[t]{3}{*}{ C058 } & Class: $Y$ & & $1.40 \mathrm{E}-06$ & & & & & \\
\hline & 1 & Yr: & & $1.6 \mathrm{E}-\odot 8$ & $6.1 \mathrm{E}-10$ & $7.6 \mathrm{E}-10$ & 6. $0 \mathrm{E}-10$ & $2.6 \mathrm{E}-\odot 9$ \\
\hline & 50 & Yr: & & $1.6 \mathrm{E}-08$ & $6.1 \mathrm{E}-10$ & $7.6 \mathrm{E}-10$ & $6.0 \mathrm{E}-10$ & $2.6 \mathrm{E}-\odot 9$ \\
\hline \multirow[t]{3}{*}{$\mathrm{C060}$} & Class: $\mathrm{Y}$ & & $3.75 E-06$ & & & & & \\
\hline & 1 & Yr: & & $2.1 \mathrm{E}-07$ & $9.9 \mathrm{E}-09$ & 1. $2 \mathrm{E}-08$ & 1. $0 \mathrm{E}-\odot 8$ & $3.4 \mathrm{E}-\odot 8$ \\
\hline & 50 & Yr: & & $2.1 \mathrm{E}-07$ & $9.9 \mathrm{E}-09$ & 1. $2 \mathrm{E}-\odot 8$ & 1. $\odot \mathrm{E}-\odot 8$ & $3.4 \mathrm{E}-\odot 8$ \\
\hline \multirow[t]{3}{*}{ NI59 } & Class: W & & 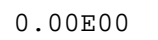 & & & & & \\
\hline & 1 & Yr: & & $5.4 \mathrm{E}-10$ & $7.9 \mathrm{E}-11$ & $7.9 \mathrm{E}-11$ & $7.9 \mathrm{E}-11$ & $1.4 \mathrm{E}-10$ \\
\hline & 50 & Yr: & & $5.4 \mathrm{E}-10$ & $7.9 \mathrm{E}-11$ & $7.9 \mathrm{E}-11$ & $7.9 \mathrm{E}-11$ & $1.4 \mathrm{E}-10$ \\
\hline \multirow[t]{3}{*}{ NI63 } & Class: W & & 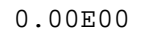 & & & & & \\
\hline & 1 & Yr: & & $2.8 \mathrm{E}-09$ & $1.9 \mathrm{E}-10$ & $1.9 \mathrm{E}-10$ & 1. $9 \mathrm{E}-10$ & $5.3 E-10$ \\
\hline & 50 & Yr: & & $2.8 \mathrm{E}-\odot 9$ & 1. $9 \mathrm{E}-10$ & 1. $9 \mathrm{E}-10$ & 1. $9 \mathrm{E}-10$ & $5.3 E-10$ \\
\hline \multirow[t]{3}{*}{ NI65 } & Class: W & & $8.42 \mathrm{E}-07$ & & & & & \\
\hline & 1 & Yr: & & $3.9 \mathrm{E}-10$ & $2.6 \mathrm{E}-12$ & $3.4 \mathrm{E}-12$ & $2.4 \mathrm{E}-12$ & 1. $0 \mathrm{E}-10$ \\
\hline & 50 & Yr: & & $3.9 \mathrm{E}-10$ & $2.6 \mathrm{E}-12$ & 3. $4 \mathrm{E}-12$ & 2. $4 \mathrm{E}-12$ & $1.0 \mathrm{E}-10$ \\
\hline \multirow[t]{3}{*}{ CU64 } & Class: D & & $2.69 \mathrm{E}-07$ & & & & & \\
\hline & 1 & Yr: & & $2.6 \mathrm{E}-11$ & 1.1E-11 & $1.1 \mathrm{E}-11$ & 1. $0 \mathrm{E}-11$ & $4.2 \mathrm{E}-11$ \\
\hline & 50 & Yr: & & $2.6 \mathrm{E}-11$ & $1.1 \mathrm{E}-11$ & $1.1 \mathrm{E}-11$ & $1.0 \mathrm{E}-11$ & $4.2 \mathrm{E}-11$ \\
\hline \multirow[t]{3}{*}{ ZN65 } & Class: $\mathrm{Y}$ & & $8.61 \mathrm{E}-07$ & & & & & \\
\hline & 1 & Yr: & & 1. 3E-๑8 & $8.8 \mathrm{E}-10$ & 1.1E-०9 & $9.0 \mathrm{E}-10$ & $2.4 \mathrm{E}-\odot 9$ \\
\hline & 50 & Yr: & & 1. $3 E-08$ & $8.8 \mathrm{E}-10$ & 1. 1E- $\odot 9$ & $9.0 \mathrm{E}-10$ & $2.4 \mathrm{E}-\odot 9$ \\
\hline ZN69M & Class: Y & & $5.83 E-07$ & & & & & \\
\hline & 1 & Yr: & & 1. $7 \mathrm{E}-09$ & $9.3 \mathrm{E}-12$ & 1. $3 \mathrm{E}-11$ & $6.2 \mathrm{E}-12$ & $3.3 \mathrm{E}-10$ \\
\hline & 50 & Yr: & & 1. 7E-०9 & $9.3 \mathrm{E}-12$ & 1. $3 \mathrm{E}-11$ & $6.2 \mathrm{E}-12$ & $3.3 E-10$ \\
\hline ZN69 & Class: Y & & $6.31 \mathrm{E}-09$ & & & & & \\
\hline & 1 & Yr: & & $1.6 \mathrm{E}-10$ & $2.5 \mathrm{E}-15$ & $2.7 \mathrm{E}-15$ & 2. $0 \mathrm{E}-15$ & $3.4 \mathrm{E}-11$ \\
\hline & 50 & Yr: & & 1. $6 \mathrm{E}-10$ & $2.5 \mathrm{E}-15$ & $2.7 \mathrm{E}-15$ & 2. $\odot E-15$ & 3. $4 \mathrm{E}-11$ \\
\hline AS76 & Class: W & & $6.50 E-07$ & & & & & \\
\hline & 1 & Yr: & & 4. ०E-०9 & $3.9 \mathrm{E}-11$ & $4.3 E-11$ & $3.7 \mathrm{E}-11$ & $8.8 \mathrm{E}-10$ \\
\hline & 50 & Yr: & & 4. ๑E-๑9 & $3.9 \mathrm{E}-11$ & 4. $3 E-11$ & 3. $7 \mathrm{E}-11$ & $8.8 \mathrm{E}-10$ \\
\hline SE75 & Class: W & & $5.30 E-07$ & & & & & \\
\hline & 1 & Yr: & & $6.6 \mathrm{E}-\odot 9$ & 6. $0 \mathrm{E}-10$ & $4.7 \mathrm{E}-10$ & $3.7 \mathrm{E}-10$ & 1. $3 E-09$ \\
\hline & 50 & Yr: & & $6.6 \mathrm{E}-09$ & $6.0 \mathrm{E}-10$ & 4. $7 \mathrm{E}-10$ & $3.7 \mathrm{E}-10$ & 1. $3 E-09$ \\
\hline SE79 & Class: W & & $1.24 \mathrm{E}-10$ & & & & & \\
\hline & 1 & Yr: & & $2.4 \mathrm{E}-08$ & $1.6 \mathrm{E}-10$ & $1.6 \mathrm{E}-10$ & $1.6 \mathrm{E}-10$ & $3.3 E-\odot 9$ \\
\hline & 50 & Yr: & & $2.4 \mathrm{E}-08$ & $1.6 \mathrm{E}-10$ & $1.6 \mathrm{E}-10$ & $1.6 \mathrm{E}-10$ & $3.3 E-\odot 9$ \\
\hline BR82 & Class: D & & $3.85 \mathrm{E}-06$ & & & & & \\
\hline & 1 & Yr: & & $2.0 \mathrm{E}-10$ & $1.9 \mathrm{E}-10$ & $1.8 \mathrm{E}-10$ & $1.9 \mathrm{E}-10$ & $4.2 \mathrm{E}-10$ \\
\hline & 50 & Yr: & & $2.0 \mathrm{E}-10$ & $1.9 \mathrm{E}-10$ & $1.8 \mathrm{E}-10$ & $1.9 \mathrm{E}-10$ & $4.2 \mathrm{E}-10$ \\
\hline BR83 & Class: D & & $1.68 \mathrm{E}-\odot 8$ & & & & & \\
\hline & 1 & Yr: & & $3.2 \mathrm{E}-11$ & 3. $4 \mathrm{E}-12$ & $3.4 \mathrm{E}-12$ & $3.4 \mathrm{E}-12$ & $1.9 \mathrm{E}-11$ \\
\hline & 50 & Yr: & & $3.2 \mathrm{E}-11$ & 3. $4 \mathrm{E}-12$ & $3.4 \mathrm{E}-12$ & $3.4 \mathrm{E}-12$ & $1.9 \mathrm{E}-11$ \\
\hline BR84 & Class: D & & $2.84 \mathrm{E}-06$ & & & & & \\
\hline & 1 & Yr: & & $3.7 \mathrm{E}-11$ & $3.9 \mathrm{E}-12$ & $3.8 \mathrm{E}-12$ & 4. $0 \mathrm{E}-12$ & $2.6 \mathrm{E}-11$ \\
\hline & 50 & Yr: & & $3.7 \mathrm{E}-11$ & $3.9 \mathrm{E}-12$ & $3.8 \mathrm{E}-12$ & 4. $0 \mathrm{E}-12$ & $2.6 \mathrm{E}-11$ \\
\hline KR83M & NobleGas & & $3.78 \mathrm{E}-11$ & & & & & \\
\hline & 1 & Yr: & & $\odot . \odot \mathrm{E}-\odot \odot$ & $\odot . \odot \mathrm{E}-\odot \odot$ & $\odot . \odot \mathrm{E}-\odot \odot$ & $\odot . \odot \mathrm{E}-\odot \odot$ & $\odot . \odot E-\odot \odot$ \\
\hline & 50 & Yr: & & $\odot . \odot \mathrm{E}-\odot \odot$ & $\odot . \odot \mathrm{E}-\odot \odot$ & $\odot . \odot \mathrm{E}-\odot \odot$ & $\odot . \odot \mathrm{E}-\odot \odot$ & $\odot . \odot \mathrm{E}-\odot \odot$ \\
\hline KR85M & NobleGas & & $2.17 \mathrm{E}-07$ & & & & & \\
\hline & 1 & Yr: & & $\odot . \odot \mathrm{E}-\odot \odot$ & $\odot . \odot \mathrm{E}-\odot \odot$ & $\odot . \odot \mathrm{E}-\odot \odot$ & $\odot . \odot \mathrm{E}-\odot \odot$ & $\odot . \odot \mathrm{E}-\odot \odot$ \\
\hline & 50 & Yr: & & $\odot . \odot \mathrm{E}-\odot \odot$ & $\odot . \odot \mathrm{E}-\odot \odot$ & $\odot . \odot \mathrm{E}-\odot \odot$ & $\odot . \odot \mathrm{E}-\odot \odot$ & $\odot . \odot \mathrm{E}-\odot \odot$ \\
\hline KR85 & NobleGas & & $7.57 \mathrm{E}-09$ & & & & & \\
\hline & 1 & Yr: & & $\odot . \odot \mathrm{E}-\odot \odot$ & $\odot . \odot \mathrm{E}-\odot \odot$ & $\odot . \odot \mathrm{E}-\odot \odot$ & $\odot . \odot \mathrm{E}-\odot \odot$ & $\odot . \odot \mathrm{E}-\odot \odot$ \\
\hline & 50 & Yr: & & $\odot . \odot \mathrm{E}-\odot \odot$ & $\odot . \odot \mathrm{E}-\odot \odot$ & $\odot . \odot \mathrm{E}-\odot \odot$ & $\odot . \odot \mathrm{E}-\odot \odot$ & $\odot . \odot \mathrm{E}-\odot \odot$ \\
\hline KR87 & NobleGas & & $1.26 \mathrm{E}-06$ & & & & & \\
\hline & 1 & Yr: & & $\odot . \odot \mathrm{E}-\odot \odot$ & $\odot . \odot \mathrm{E}-\odot \odot$ & $\odot . \odot \mathrm{E}-\odot \odot$ & $\odot . \odot \mathrm{E}-\odot \odot$ & $\odot . \odot \mathrm{E}-\odot \odot$ \\
\hline & 50 & Yr: & & $\odot . \odot \mathrm{E}-\odot \odot$ & $\odot . \odot \mathrm{E}-\odot \odot$ & $\odot . \odot \mathrm{E}-\odot \odot$ & $\odot . \odot \mathrm{E}-\odot \odot$ & $\odot . \odot \mathrm{E}-\odot \odot$ \\
\hline KR88 & NobleGas & & $3.07 E-06$ & & & & & \\
\hline & 1 & Yr: & & $\odot . \odot \mathrm{E}-\odot \odot$ & $\odot . \odot \mathrm{E}-\odot \odot$ & $\odot . \odot E-\odot \odot$ & $\odot . \odot \mathrm{E}-\odot \odot$ & $\odot . \odot \mathrm{E}-\odot \odot$ \\
\hline & 50 & Yr: & & $\odot . \odot \mathrm{E}-\odot \odot$ & $\odot . \odot E-\odot \odot$ & $\odot . \odot \mathrm{E}-\odot \odot$ & $\odot . \odot E-\odot \odot$ & $\odot . \odot \mathrm{E}-\odot \odot$ \\
\hline KR89 & NobleGas & & $3.45 E-06$ & & & & & \\
\hline & 1 & Yr: & & $\odot . \odot \mathrm{E}-\odot \odot$ & $\odot . \odot \mathrm{E}-\odot \odot$ & $\odot . \odot \mathrm{E}-\odot \odot$ & $\odot . \odot \mathrm{E}-\odot \odot$ & $\odot . \odot \mathrm{E}-\odot \odot$ \\
\hline & 50 & Yr: & & $\odot . \odot \mathrm{E}-\odot \odot$ & $\odot . \odot \mathrm{E}-\odot \odot$ & $\odot . \odot \mathrm{E}-\odot \odot$ & $\odot . \odot \mathrm{E}-\odot \odot$ & $\odot . \odot \mathrm{E}-\odot \odot$ \\
\hline RB86 & Class: D & & $1.56 E-07$ & & & & & \\
\hline
\end{tabular}




\begin{tabular}{|c|c|c|c|c|c|c|c|c|}
\hline & 1 & Yr: & & $9.1 \mathrm{E}-10$ & 2. 9E-๑9 & 1. 8E-๑9 & $8.8 \mathrm{E}-10$ & $1.1 \mathrm{E}-09$ \\
\hline & 50 & Yr: & & $9.1 \mathrm{E}-10$ & 2. . $9 \mathrm{E}-\odot 9$ & 1. 8E-๑9 & $8.8 \mathrm{E}-10$ & $1.1 \mathrm{E}-09$ \\
\hline \multirow[t]{3}{*}{ RB87 } & Class: D & & 1. $04 \mathrm{E}-\odot 9$ & & & & & \\
\hline & 1 & Yr: & & $4.9 E-10$ & 1. $6 \mathrm{E}-09$ & $9.9 \mathrm{E}-10$ & $4.6 \mathrm{E}-10$ & $5.9 \mathrm{E}-10$ \\
\hline & 50 & Yr: & & $4.9 \mathrm{E}-10$ & 1. $6 \mathrm{E}-\odot 9$ & $9.9 \mathrm{E}-10$ & $4.6 \mathrm{E}-10$ & $5.9 \mathrm{E}-10$ \\
\hline \multirow[t]{3}{*}{ RB88 } & Class: D & & $1.05 E-06$ & & & & & \\
\hline & 1 & Yr: & & $3.7 \mathrm{E}-11$ & $2.2 \mathrm{E}-12$ & $2.1 \mathrm{E}-12$ & 2. $0 \mathrm{E}-12$ & 1. $9 \mathrm{E}-11$ \\
\hline & 50 & Yr: & & $3.7 E-11$ & $2.2 \mathrm{E}-12$ & $2.1 \mathrm{E}-12$ & 2. $0 \mathrm{E}-12$ & 1. $9 \mathrm{E}-11$ \\
\hline \multirow[t]{3}{*}{ RB89 } & Class: D & & $3.19 E-06$ & & & & & \\
\hline & 1 & Yr: & & $2.7 \mathrm{E}-11$ & $2.6 \mathrm{E}-12$ & $2.2 \mathrm{E}-12$ & 2. $0 \mathrm{E}-12$ & $1.6 \mathrm{E}-11$ \\
\hline & 50 & Yr: & & $2.7 \mathrm{E}-11$ & $2.6 \mathrm{E}-12$ & $2.2 \mathrm{E}-12$ & 2. $\odot \mathrm{E}-12$ & $1.6 \mathrm{E}-11$ \\
\hline \multirow[t]{3}{*}{ SR85 } & Class: D & & $7.06 \mathrm{E}-07$ & & & & & \\
\hline & 1 & Yr: & & $5.8 \mathrm{E}-10$ & $2.6 \mathrm{E}-\odot 9$ & 2. $\odot E-\odot 9$ & $5.2 \mathrm{E}-10$ & $8.3 E-10$ \\
\hline & 50 & Yr: & & $5.8 \mathrm{E}-10$ & $2.6 \mathrm{E}-\odot 9$ & 2.०E-๑9 & $5.2 \mathrm{E}-10$ & $8.3 E-10$ \\
\hline \multirow[t]{3}{*}{ SR89 } & Class: D & & $1.38 \mathrm{E}-08$ & & & & & \\
\hline & 1 & Yr: & & $1.8 \mathrm{E}-10$ & 1. $5 E-\odot 8$ & 9. 7E-๑9 & $1.5 \mathrm{E}-10$ & 1. $7 \mathrm{E}-\odot 9$ \\
\hline & 50 & Yr: & & $1.8 \mathrm{E}-10$ & $1.5 E-08$ & 9. 7E-๑9 & $1.5 \mathrm{E}-10$ & 1. $.7 \mathrm{E}-09$ \\
\hline \multirow[t]{3}{*}{ SR90 } & Class: D & & $3.10 \mathrm{E}-\odot 9$ & & & & & \\
\hline & 1 & Yr: & & $7.9 \mathrm{E}-10$ & 1. 2E- - 06 & 3. 3E- - 7 & $7.6 E-10$ & $5.3 E-08$ \\
\hline & 50 & Yr: & & $7.9 \mathrm{E}-10$ & 1. $2 \mathrm{E}-06$ & $3.3 E-07$ & $7.6 \mathrm{E}-10$ & $5.3 E-08$ \\
\hline \multirow[t]{3}{*}{ SR91 } & Class: D & & 1. $03 \mathrm{E}-06$ & & & & & \\
\hline & 1 & Yr: & & $5.7 \mathrm{E}-11$ & $2.9 \mathrm{E}-10$ & $2.3 \mathrm{E}-10$ & $2.4 \mathrm{E}-11$ & 1. $7 \mathrm{E}-10$ \\
\hline & 50 & Yr: & & $5.7 \mathrm{E}-11$ & $2.9 \mathrm{E}-10$ & $2.3 \mathrm{E}-10$ & $2.4 \mathrm{E}-11$ & $1.7 \mathrm{E}-10$ \\
\hline \multirow[t]{3}{*}{ SR92 } & Class: D & & 2. $92 \mathrm{E}-06$ & & & & & \\
\hline & 1 & Yr: & & $4.2 \mathrm{E}-11$ & $2.0 E-10$ & $9.9 E-11$ & 1. $3 E-11$ & 1. $0 \mathrm{E}-10$ \\
\hline & 50 & Yr: & & $4.2 \mathrm{E}-11$ & $2.0 \mathrm{E}-1 \odot$ & $9.9 \mathrm{E}-11$ & 1. $3 \mathrm{E}-11$ & $1.0 \mathrm{E}-10$ \\
\hline \multirow[t]{3}{*}{ Y 90} & Class: Y & & $2.50 E-08$ & & & & & \\
\hline & 1 & Yr: & & $9.2 \mathrm{E}-\odot 9$ & 1. $2 \mathrm{E}-12$ & 1. 7E-12 & $5.0 \mathrm{E}-14$ & $1.8 \mathrm{E}-09$ \\
\hline & 50 & Yr: & & $9.2 \mathrm{E}-09$ & 1. $2 \mathrm{E}-12$ & 1. $7 \mathrm{E}-12$ & $5.0 \mathrm{E}-14$ & $1.8 \mathrm{E}-09$ \\
\hline Y 91M & Class: Y & & $7.47 E-07$ & & & & & \\
\hline & 1 & Yr: & & $5.9 \mathrm{E}-11$ & $8.1 \mathrm{E}-13$ & 1. $0 \mathrm{E}-12$ & $7.2 \mathrm{E}-13$ & 1. $4 \mathrm{E}-11$ \\
\hline & 50 & Yr: & & $5.9 E-11$ & $8.1 \mathrm{E}-13$ & 1. $0 \mathrm{E}-12$ & $7.2 \mathrm{E}-13$ & 1. $4 \mathrm{E}-11$ \\
\hline Y 91 & Class: Y & & 1. .96E-๑8 & & & & & \\
\hline & 1 & Yr: & & 8. ०E-०8 & $6.1 \mathrm{E}-11$ & $8.4 \mathrm{E}-11$ & $3.3 E-12$ & 1. $0 \mathrm{E}-08$ \\
\hline & 50 & Yr: & & 8. ०E-๑8 & $6.1 \mathrm{E}-11$ & $8.4 \mathrm{E}-11$ & 3. $3 E-12$ & 1. $0 \mathrm{E}-\odot 8$ \\
\hline Y 92 & Class: Y & & $4.16 \mathrm{E}-07$ & & & & & \\
\hline & 1 & Yr: & & $8.1 \mathrm{E}-10$ & 1. 4E-12 & 2. $0 \mathrm{E}-12$ & 1. $2 \mathrm{E}-12$ & $2.1 \mathrm{E}-10$ \\
\hline & $5 \odot$ & Yr: & & $8.1 E-10$ & 1. 4E-12 & $2.0 \mathrm{E}-12$ & 1. $2 \mathrm{E}-12$ & $2.1 \mathrm{E}-10$ \\
\hline Y 93 & Class: Y & & $1.67 \mathrm{E}-07$ & & & & & \\
\hline & 1 & Yr: & & 1. $9 \mathrm{E}-\odot 9$ & 1. $5 \mathrm{E}-12$ & 2. $0 \mathrm{E}-12$ & $9.8 \mathrm{E}-13$ & $5.0 \mathrm{E}-10$ \\
\hline & 50 & Yr: & & 1. $9 \mathrm{E}-\odot 9$ & 1. $5 \mathrm{E}-12$ & 2. $\mathrm{OE}-12$ & $9.8 \mathrm{E}-13$ & $5.0 \mathrm{E}-10$ \\
\hline ZR93 & Class: W & & ๑. ००Е७९ & & & & & \\
\hline & 1 & Yr: & & 3. 3E- 09 & $3.7 \mathrm{E}-07$ & 3. ०E-๑8 & $3.5 E-12$ & $7.8 \mathrm{E}-09$ \\
\hline & 50 & Yr: & & $3.3 E-09$ & $3.7 \mathrm{E}-07$ & 3. ०E-๑8 & $3.5 E-12$ & $7.8 \mathrm{E}-\odot 9$ \\
\hline ZR95 & Class: W & & $1.06 \mathrm{E}-06$ & & & & & \\
\hline & 1 & Yr: & & $3.9 \mathrm{E}-\odot 8$ & 1. $2 \mathrm{E}-\odot 8$ & $2.7 \mathrm{E}-\odot 9$ & $7.2 \mathrm{E}-10$ & $5.9 \mathrm{E}-\odot 9$ \\
\hline & 50 & Yr: & & $3.9 E-08$ & 1. $2 \mathrm{E}-08$ & $2.7 E-\odot 9$ & $7.2 \mathrm{E}-10$ & $5.9 E-\odot 9$ \\
\hline ZR97 & Class: W & & $2.81 E-07$ & & & & & \\
\hline & 1 & Yr: & & 4. 2E- 09 & $6.9 \mathrm{E}-11$ & $9.4 \mathrm{E}-11$ & $3.1 \mathrm{E}-11$ & $1.1 \mathrm{E}-09$ \\
\hline & 50 & Yr: & & 4. 2E-०9 & $6.9 \mathrm{E}-11$ & $9.4 \mathrm{E}-11$ & $3.1 \mathrm{E}-11$ & 1.1E- $\odot 9$ \\
\hline NB93M & Class: Y & & $9.62 \mathrm{E}-11$ & & & & & \\
\hline & 1 & Yr: & & $1.5 E-08$ & $5.2 \mathrm{E}-11$ & $2.2 \mathrm{E}-11$ & 4. $9 \mathrm{E}-12$ & 1. $9 \mathrm{E}-\odot 9$ \\
\hline & $5 \odot$ & Yr: & & 1. $5 E-\odot 8$ & $5.2 E-11$ & $2.2 E-11$ & $4.9 \mathrm{E}-12$ & 1. $9 \mathrm{E}-09$ \\
\hline NB94 & Class: Y & & $2.27 E-06$ & & & & & \\
\hline & 1 & Yr: & & $3.4 \mathrm{E}-07$ & 1. 2E- - 88 & 1. 5E- - & 1. $2 \mathrm{E}-08$ & $5.2 E-08$ \\
\hline & 50 & Yr: & & $3.4 \mathrm{E}-07$ & 1. $2 \mathrm{E}-08$ & 1. $5 \mathrm{E}-\odot 8$ & 1. $2 \mathrm{E}-08$ & $5.2 \mathrm{E}-08$ \\
\hline NB95M & Class: Y & & 8.64E- -8 & & & & & \\
\hline & 1 & Yr: & & $7.9 \mathrm{E}-09$ & $3.9 \mathrm{E}-11$ & $4.2 \mathrm{E}-11$ & $3.0 E-11$ & $1.1 \mathrm{E}-\odot 9$ \\
\hline & 50 & Yr: & & $7.9 \mathrm{E}-09$ & $3.9 \mathrm{E}-11$ & $4.2 \mathrm{E}-11$ & $3.0 E-11$ & 1. $1 \mathrm{E}-\odot 9$ \\
\hline NB95 & Class: Y & & $1.10 \mathrm{E}-06$ & & & & & \\
\hline & 1 & Yr: & & 1. 5E- - & $2.9 \mathrm{E}-10$ & $3.6 \mathrm{E}-10$ & $2.7 \mathrm{E}-10$ & $2.2 \mathrm{E}-\odot 9$ \\
\hline & 50 & Yr: & & $1.5 E-08$ & $2.9 \mathrm{E}-10$ & $3.6 \mathrm{E}-10$ & $2.7 \mathrm{E}-10$ & $2.2 \mathrm{E}-\odot 9$ \\
\hline NB97M & Class: Y & & 1. $\odot 4 \mathrm{E}-\odot 6$ & & & & & \\
\hline & 1 & Yr: & & 2. $4 \mathrm{E}-12$ & $2.3 E-14$ & $3.1 \mathrm{E}-14$ & $2.8 \mathrm{E}-14$ & $3.5 \mathrm{E}-13$ \\
\hline & 50 & Yr: & & 2. $4 \mathrm{E}-12$ & $2.3 \mathrm{E}-14$ & $3.1 \mathrm{E}-14$ & $2.8 \mathrm{E}-14$ & $3.5 \mathrm{E}-13$ \\
\hline NB97 & Class: Y & & $9.43 E-07$ & & & & & \\
\hline & 1 & Yr: & & $2.1 \mathrm{E}-10$ & 1. 4E-12 & 1. $8 \mathrm{E}-12$ & 1. $2 \mathrm{E}-12$ & $5.5 E-11$ \\
\hline & 50 & Yr: & & $2.1 \mathrm{E}-10$ & 1. $4 \mathrm{E}-12$ & $1.8 \mathrm{E}-12$ & 1. $2 \mathrm{E}-12$ & $5.5 E-11$ \\
\hline M093 & Class: D & & $5.46 \mathrm{E}-10$ & & & & & \\
\hline & 1 & Yr: & & $9.3 \mathrm{E}-11$ & $2.1 E-08$ & $6.2 \mathrm{E}-09$ & $6.5 \mathrm{E}-11$ & 1.1E- -99 \\
\hline & 50 & Yr: & & $9.3 \mathrm{E}-11$ & $2.1 \mathrm{E}-08$ & $6.2 \mathrm{E}-09$ & $6.5 \mathrm{E}-11$ & 1.1E- $\odot 9$ \\
\hline
\end{tabular}




\begin{tabular}{|c|c|c|c|c|c|c|c|c|}
\hline \multirow[t]{3}{*}{ M099 } & Class: D & & $2.20 \mathrm{E}-\odot 7$ & & & & & \\
\hline & 1 & Yr: & & 1. $4 \mathrm{E}-10$ & $3.4 \mathrm{E}-10$ & $2.6 \mathrm{E}-10$ & $9.9 \mathrm{E}-11$ & $2.7 \mathrm{E}-10$ \\
\hline & 50 & Yr: & & 1. $4 \mathrm{E}-10$ & $3.4 \mathrm{E}-10$ & $2.6 \mathrm{E}-10$ & $9.9 \mathrm{E}-11$ & $2.7 \mathrm{E}-10$ \\
\hline \multirow[t]{3}{*}{ TC99M } & Class: W & & $1.66 \mathrm{E}-\odot 7$ & & & & & \\
\hline & 1 & Yr: & & $9.8 \mathrm{E}-11$ & $2.7 \mathrm{E}-12$ & 1. $9 \mathrm{E}-12$ & $7.9 \mathrm{E}-12$ & $2.4 \mathrm{E}-11$ \\
\hline & 50 & Yr: & & $9.8 \mathrm{E}-11$ & 2. $7 \mathrm{E}-12$ & 1. $9 \mathrm{E}-12$ & $7.9 \mathrm{E}-12$ & 2. $4 \mathrm{E}-11$ \\
\hline \multirow[t]{3}{*}{ TC99 } & Class: W & & $9.05 \mathrm{E}-10$ & & & & & \\
\hline & 1 & Yr: & & 4. $0 E-\odot 8$ & $1.1 \mathrm{E}-11$ & 1.1E-11 & $3.6 \mathrm{E}-10$ & $5.0 \mathrm{E}-\odot 9$ \\
\hline & 50 & Yr: & & 4. ๑E-๑8 & 1. $1 \mathrm{E}-11$ & $1.1 \mathrm{E}-11$ & $3.6 \mathrm{E}-10$ & 5. $\odot \mathrm{E}-\odot 9$ \\
\hline \multirow[t]{3}{*}{ TC101 } & Class: W & & $4.76 \mathrm{E}-07$ & & & & & \\
\hline & 1 & Yr: & & 4. $3 E-11$ & $2.1 \mathrm{E}-13$ & 1. $9 \mathrm{E}-13$ & 1. $7 \mathrm{E}-12$ & 1. $4 \mathrm{E}-11$ \\
\hline & 50 & Yr: & & $4.3 E-11$ & $2.1 \mathrm{E}-13$ & 1. $9 \mathrm{E}-13$ & 1. $7 \mathrm{E}-12$ & 1. $4 \mathrm{E}-11$ \\
\hline \multirow[t]{3}{*}{ RU103 } & Class: Y & & $6.59 \mathrm{E}-07$ & & & & & \\
\hline & 1 & Yr: & & $2.7 \mathrm{E}-08$ & $2.0 \mathrm{E}-10$ & $2.5 \mathrm{E}-10$ & $1.9 \mathrm{E}-10$ & $3.6 \mathrm{E}-\odot 9$ \\
\hline & 50 & Yr: & & $2.7 \mathrm{E}-08$ & $2.0 \mathrm{E}-10$ & $2.5 \mathrm{E}-10$ & $1.9 \mathrm{E}-10$ & $3.6 \mathrm{E}-09$ \\
\hline \multirow[t]{3}{*}{ RU105 } & Class: $\mathrm{Y}$ & & $1.13 \mathrm{E}-06$ & & & & & \\
\hline & 1 & Yr: & & 1. $\odot E-\odot 9$ & $6.4 \mathrm{E}-12$ & $8.8 \mathrm{E}-12$ & $4.9 \mathrm{E}-12$ & 2. $2 \mathrm{E}-10$ \\
\hline & 50 & Yr: & & 1. $\odot E-\odot 9$ & $6.4 \mathrm{E}-12$ & $8.8 \mathrm{E}-12$ & $4.9 \mathrm{E}-12$ & 2. $2 \mathrm{E}-10$ \\
\hline \multirow[t]{3}{*}{ RU106 } & Class: Y & & $\odot . \odot \odot Е \odot \odot ~$ & & & & & \\
\hline & 1 & Yr: & & $5.7 \mathrm{E}-\odot 7$ & $6.1 \mathrm{E}-10$ & 7. $0 \mathrm{E}-10$ & 6. $\odot \mathrm{E}-10$ & 7. $0 \mathrm{E}-\odot 8$ \\
\hline & 50 & Yr: & & $5.7 \mathrm{E}-07$ & $6.1 \mathrm{E}-10$ & $7.0 \mathrm{E}-10$ & 6. $0 \mathrm{E}-10$ & $7.0 \mathrm{E}-\odot 8$ \\
\hline \multirow[t]{3}{*}{ RH103M } & Class: Y & & $1.90 \mathrm{E}-10$ & & & & & \\
\hline & 1 & Yr: & & 2. $\mathrm{OE}-11$ & $3.4 \mathrm{E}-15$ & $1.6 \mathrm{E}-15$ & $8.5 E-16$ & 3. $2 \mathrm{E}-12$ \\
\hline & 50 & Yr: & & $2.0 \mathrm{E}-11$ & $3.4 \mathrm{E}-15$ & $1.6 \mathrm{E}-15$ & $8.5 \mathrm{E}-16$ & 3. $2 \mathrm{E}-12$ \\
\hline \multirow[t]{3}{*}{ RH105 } & Class: Y & & $1.10 \mathrm{E}-07$ & & & & & \\
\hline & 1 & Yr: & & $2.9 \mathrm{E}-09$ & $4.5 \mathrm{E}-12$ & $5.3 E-12$ & 3. $0 \mathrm{E}-12$ & $4.4 \mathrm{E}-10$ \\
\hline & 50 & Yr: & & $2.9 \mathrm{E}-09$ & $4.5 \mathrm{E}-12$ & $5.3 \mathrm{E}-12$ & 3. $0 \mathrm{E}-12$ & $4.4 \mathrm{E}-10$ \\
\hline PD103 & Class: Y & & $1.68 \mathrm{E}-\odot 9$ & & & & & \\
\hline & 1 & Yr: & & $3.9 \mathrm{E}-09$ & $4.8 \mathrm{E}-12$ & $1.6 \mathrm{E}-12$ & $1.6 \mathrm{E}-13$ & $5.3 \mathrm{E}-10$ \\
\hline & 50 & Yr: & & $3.9 \mathrm{E}-09$ & $4.8 \mathrm{E}-12$ & $1.6 \mathrm{E}-12$ & 1. $6 \mathrm{E}-13$ & $5.3 E-10$ \\
\hline PD107 & Class: Y & & $\odot . \odot \odot Е \odot \odot$ & & & & & \\
\hline & 1 & Yr: & & $5 . \odot E-\odot 9$ & $4.1 \mathrm{E}-13$ & $1.8 \mathrm{E}-13$ & $3.1 \mathrm{E}-14$ & $6.1 \mathrm{E}-10$ \\
\hline & 50 & Yr: & & $5.0 \mathrm{E}-09$ & $4.1 \mathrm{E}-13$ & 1. $8 \mathrm{E}-13$ & $3.1 \mathrm{E}-14$ & $6.1 \mathrm{E}-10$ \\
\hline PD109 & Class: Y & & 1. $33 E-\odot 8$ & & & & & \\
\hline & 1 & $\mathrm{Yr}:$ & & $2.8 \mathrm{E}-\odot 9$ & $6.2 \mathrm{E}-13$ & $3.1 \mathrm{E}-13$ & $9.7 \mathrm{E}-14$ & $4.6 \mathrm{E}-10$ \\
\hline & 50 & Yr: & & $2.8 \mathrm{E}-\odot 9$ & $6.2 \mathrm{E}-13$ & $3.1 \mathrm{E}-13$ & $9.7 \mathrm{E}-14$ & $4.6 \mathrm{E}-10$ \\
\hline AG110M & Class: D & & $4.01 \mathrm{E}-06$ & & & & & \\
\hline & 1 & Yr: & & $5.6 \mathrm{E}-\odot 9$ & $3.7 \mathrm{E}-09$ & $3.9 \mathrm{E}-09$ & $2.7 \mathrm{E}-\odot 9$ & $6.4 \mathrm{E}-09$ \\
\hline & 50 & Yr: & & $5.6 \mathrm{E}-\odot 9$ & $3.7 \mathrm{E}-\odot 9$ & $3.9 \mathrm{E}-\odot 9$ & $2.7 \mathrm{E}-\odot 9$ & $6.4 \mathrm{E}-09$ \\
\hline AG111 & Class: D & & $4.38 \mathrm{E}-\odot 8$ & & & & & \\
\hline & 1 & Yr: & & 1. $4 \mathrm{E}-10$ & $1.1 \mathrm{E}-10$ & $1.1 \mathrm{E}-10$ & 1. $\odot \mathrm{E}-10$ & $4.8 \mathrm{E}-10$ \\
\hline & 50 & $Y r:$ & & $1.4 \mathrm{E}-10$ & 1. $1 \mathrm{E}-10$ & $1.1 \mathrm{E}-10$ & 1. $0 \mathrm{E}-10$ & $4.8 \mathrm{E}-10$ \\
\hline CD109 & Class: D & & $7.22 \mathrm{E}-\odot 9$ & & & & & \\
\hline & 1 & Yr: & & 1. $9 \mathrm{E}-\odot 9$ & $2.2 \mathrm{E}-09$ & 1. $4 \mathrm{E}-\odot 9$ & $1.5 \mathrm{E}-\odot 9$ & $9.2 \mathrm{E}-09$ \\
\hline & 50 & Yr: & & 1.9E-०9 & 2. $2 \mathrm{E}-\odot 9$ & 1. $4 \mathrm{E}-\odot 9$ & 1. $5 \mathrm{E}-\odot 9$ & $9.2 \mathrm{E}-09$ \\
\hline CD113M & Class: D & & $2.86 \mathrm{E}-\odot 9$ & & & & & \\
\hline & 1 & $\mathrm{Yr}:$ & & $1.8 \mathrm{E}-08$ & 1. $8 \mathrm{E}-08$ & $1.8 \mathrm{E}-08$ & 1. $8 \mathrm{E}-08$ & 1. $1 \mathrm{E}-07$ \\
\hline & 50 & Yr: & & 1. $8 \mathrm{E}-\odot 8$ & 1. $8 \mathrm{E}-08$ & 1. $8 \mathrm{E}-\odot 8$ & 1. $8 \mathrm{E}-\odot 8$ & 1. $1 \mathrm{E}-07$ \\
\hline CD115M & Class: D & & $4.67 \mathrm{E}-\odot 8$ & & & & & \\
\hline & 1 & Yr: & & 1. $\odot E-\odot 9$ & $9.8 \mathrm{E}-10$ & $9.8 \mathrm{E}-10$ & $9.7 \mathrm{E}-10$ & $6.4 \mathrm{E}-\odot 9$ \\
\hline & 50 & Yr: & & 1. $\odot E-\odot 9$ & $9.8 \mathrm{E}-10$ & $9.8 \mathrm{E}-10$ & $9.7 \mathrm{E}-10$ & $6.4 \mathrm{E}-\odot 9$ \\
\hline CD115 & Class: D & & $3.31 \mathrm{E}-07$ & & & & & \\
\hline & 1 & Yr: & & $1.1 \mathrm{E}-10$ & $7.5 \mathrm{E}-11$ & $7.9 \mathrm{E}-11$ & $6.1 \mathrm{E}-11$ & 4. $3 E-10$ \\
\hline & 50 & Yr: & & $1.1 \mathrm{E}-10$ & $7.5 \mathrm{E}-11$ & $7.9 \mathrm{E}-11$ & $6.1 \mathrm{E}-11$ & $4.3 \mathrm{E}-10$ \\
\hline IN111 & Class: D & & $5.30 \mathrm{E}-07$ & & & & & \\
\hline & 1 & Yr: & & $5.5 \mathrm{E}-11$ & $1.6 \mathrm{E}-10$ & 1. $9 \mathrm{E}-10$ & $3.4 \mathrm{E}-11$ & $1.6 \mathrm{E}-10$ \\
\hline & 50 & Yr: & & $5.5 \mathrm{E}-11$ & $1.6 \mathrm{E}-10$ & 1. $9 \mathrm{E}-10$ & 3. $4 \mathrm{E}-11$ & $1.6 \mathrm{E}-10$ \\
\hline IN114M & Class: D & & $1.23 \mathrm{E}-07$ & & & & & \\
\hline & 1 & Yr: & & 1. $9 \mathrm{E}-\odot 9$ & $3.9 \mathrm{E}-\odot 8$ & $5.9 \mathrm{E}-\odot 8$ & 1. $7 \mathrm{E}-\odot 9$ & 1.1E- -08 \\
\hline & 50 & Yr: & & 1. $9 \mathrm{E}-09$ & $3.9 E-08$ & $5.9 \mathrm{E}-08$ & 1. $7 \mathrm{E}-\odot 9$ & 1.1E- -88 \\
\hline IN115M & Class: D & & $2.17 \mathrm{E}-07$ & & & & & \\
\hline & 1 & Yr: & & $2.1 \mathrm{E}-11$ & 1. $6 \mathrm{E}-11$ & $2.2 \mathrm{E}-11$ & $3.3 \mathrm{E}-12$ & $2.8 \mathrm{E}-11$ \\
\hline & 50 & Yr: & & $2.1 \mathrm{E}-11$ & $1.6 \mathrm{E}-11$ & $2.2 \mathrm{E}-11$ & 3. $3 \mathrm{E}-12$ & 2. $8 \mathrm{E}-11$ \\
\hline SN117M & Class: W & & $1.93 E-07$ & & & & & \\
\hline & 1 & Yr: & & $2.4 \mathrm{E}-\odot 8$ & $9.3 \mathrm{E}-10$ & 1. $4 \mathrm{E}-10$ & $2.9 \mathrm{E}-11$ & $3.1 \mathrm{E}-09$ \\
\hline & 50 & Yr: & & $2.4 \mathrm{E}-08$ & $9.3 \mathrm{E}-10$ & $1.4 \mathrm{E}-10$ & 2. $9 \mathrm{E}-11$ & $3.1 \mathrm{E}-09$ \\
\hline SN119M & Class: W & & $2.23 \mathrm{E}-09$ & & & & & \\
\hline & 1 & Yr: & & 2. $\odot E-\odot 8$ & $\mathrm{E}-10$ & $3.4 \mathrm{E}$ & $4.4 \mathrm{E}-11$ & $2.6 \mathrm{E}-\odot 9$ \\
\hline & 50 & Yr: & & 2. ๑E-๑8 & 7. $9 \mathrm{E}-10$ & $3.4 \mathrm{E}-10$ & 4. $4 \mathrm{E}-11$ & $2.6 \mathrm{E}-09$ \\
\hline SN121M & Class: W & & $1.66 \mathrm{E}-\odot 9$ & & & & & \\
\hline & 1 & Yr: & & $4.2 \mathrm{E}-08$ & 3. $\odot E-\odot 9$ & 1. $4 \mathrm{E}-\odot 9$ & $1.6 \mathrm{E}-10$ & $5.5 E-09$ \\
\hline
\end{tabular}




\begin{tabular}{|c|c|c|c|c|c|c|c|c|}
\hline & 50 & Yr: & & $4.2 E-08$ & 3. $0 \mathrm{E}-\odot 9$ & 1. $4 \mathrm{E}-09$ & $1.6 \mathrm{E}-10$ & $5.5 E-\odot 9$ \\
\hline \multirow[t]{3}{*}{ SN121 } & Class: W & & $1.23 \mathrm{E}-09$ & & & & & \\
\hline & 1 & Yr: & & 1. $9 \mathrm{E}-09$ & 4. $0 \mathrm{E}-11$ & $5.0 \mathrm{E}-12$ & $3.9 \mathrm{E}-13$ & $2.9 \mathrm{E}-10$ \\
\hline & 50 & Yr: & & 1. $9 \mathrm{E}-\odot 9$ & $4.0 \mathrm{E}-11$ & 5. $\mathrm{OE}-12$ & $3.9 \mathrm{E}-13$ & $2.9 \mathrm{E}-10$ \\
\hline \multirow[t]{3}{*}{ SN123 } & Class: W & & $2.20 \mathrm{E}-08$ & & & & & \\
\hline & 1 & Yr: & & $7.2 \mathrm{E}-08$ & $2.2 \mathrm{E}-\odot 9$ & 1.1E- 09 & 1. $3 \mathrm{E}-10$ & $9.5 \mathrm{E}-\odot 9$ \\
\hline & 50 & Yr: & & 7. 2E- -88 & 2. $2 \mathrm{E}-\odot 9$ & 1.1E- 09 & 1. $3 \mathrm{E}-10$ & $9.5 \mathrm{E}-09$ \\
\hline \multirow[t]{3}{*}{ SN125 } & Class: W & & $4.86 E-07$ & & & & & \\
\hline & 1 & Yr: & & $2.3 E-08$ & $5.7 \mathrm{E}-10$ & $4.5 E-10$ & $5.8 \mathrm{E}-11$ & $3.6 \mathrm{E}-\odot 9$ \\
\hline & 50 & Yr: & & 2. 3E- $\odot 8$ & $5.7 \mathrm{E}-10$ & $4.5 \mathrm{E}-10$ & $5.8 \mathrm{E}-11$ & $3.6 \mathrm{E}-\odot 9$ \\
\hline \multirow[t]{3}{*}{ SN126 } & Class: W & & $5.83 E-08$ & & & & & \\
\hline & 1 & Yr: & & $2.1 \mathrm{E}-07$ & $2.8 \mathrm{E}-08$ & $1.6 \mathrm{E}-08$ & 5. ๑E-๑9 & $3.3 E-08$ \\
\hline & 50 & Yr: & & $2.1 \mathrm{E}-07$ & $2.8 \mathrm{E}-08$ & 1. $6 \mathrm{E}-\odot 8$ & 5. $\odot E-\odot 9$ & $3.3 E-08$ \\
\hline \multirow[t]{3}{*}{ SB124 } & Class: W & & $2.72 \mathrm{E}-06$ & & & & & \\
\hline & 1 & Yr: & & $5.3 E-08$ & $2.0 \mathrm{E}-\odot 9$ & 1. $4 \mathrm{E}-09$ & $7.4 \mathrm{E}-10$ & $7.7 \mathrm{E}-\odot 9$ \\
\hline & 50 & Yr: & & $5.3 E-08$ & 2. $\odot E-\odot 9$ & 1. $4 \mathrm{E}-\odot 9$ & $7.4 \mathrm{E}-10$ & $7.7 \mathrm{E}-09$ \\
\hline \multirow[t]{3}{*}{ SB125 } & Class: W & & $5.90 \mathrm{E}-07$ & & & & & \\
\hline & 1 & Yr: & & 4. $\odot E-\odot 8$ & $8.4 \mathrm{E}-\odot 9$ & 1. $9 \mathrm{E}-\odot 9$ & $6.1 \mathrm{E}-10$ & $5.8 \mathrm{E}-09$ \\
\hline & 50 & Yr: & & $4.0 \mathrm{E}-08$ & $8.4 \mathrm{E}-09$ & $1.9 \mathrm{E}-09$ & $6.1 \mathrm{E}-10$ & $5.8 \mathrm{E}-\odot 9$ \\
\hline \multirow[t]{3}{*}{ SB126M } & Class: W & & $2.21 \mathrm{E}-06$ & & & & & \\
\hline & 1 & Yr: & & $5.9 \mathrm{E}-11$ & 1. $1 \mathrm{E}-12$ & 1. $2 \mathrm{E}-12$ & 1. $0 \mathrm{E}-12$ & $2.3 \mathrm{E}-11$ \\
\hline & 50 & Yr: & & $5.9 \mathrm{E}-11$ & 1. $1 \mathrm{E}-12$ & 1. $2 \mathrm{E}-12$ & 1. $0 \mathrm{E}-12$ & 2. $3 E-11$ \\
\hline \multirow[t]{3}{*}{ SB126 } & Class: W & & 4.04E-๑6 & & & & & \\
\hline & 1 & Yr: & & $2.1 \mathrm{E}-08$ & $7.2 \mathrm{E}-10$ & $7.3 E-10$ & $4.1 \mathrm{E}-10$ & $3.5 E-09$ \\
\hline & 50 & Yr: & & 2.1E-08 & $7.2 \mathrm{E}-10$ & $7.3 \mathrm{E}-10$ & $4.1 \mathrm{E}-10$ & $3.5 \mathrm{E}-09$ \\
\hline \multirow[t]{3}{*}{ SB127 } & Class: W & & $9.84 \mathrm{E}-07$ & & & & & \\
\hline & 1 & Yr: & & $1.4 \mathrm{E}-\odot 8$ & 1. $3 \mathrm{E}-10$ & $1.5 \mathrm{E}-10$ & $4.7 \mathrm{E}-11$ & $2.1 \mathrm{E}-\odot 9$ \\
\hline & 50 & Yr: & & 1. $4 \mathrm{E}-08$ & 1. $3 E-10$ & $1.5 \mathrm{E}-10$ & 4. $7 \mathrm{E}-11$ & $2.1 \mathrm{E}-09$ \\
\hline TE123M & Class: W & & $1.84 \mathrm{E}-07$ & & & & & \\
\hline & 1 & Yr: & & $3.7 \mathrm{E}-\odot 8$ & 1. $\odot E-\odot 8$ & 1. $4 \mathrm{E}-\odot 9$ & $5.8 \mathrm{E}-10$ & 5. $\odot \mathrm{E}-\odot 9$ \\
\hline & 50 & Yr: & & $3.7 \mathrm{E}-08$ & 1. $\mathrm{OE}-\odot 8$ & 1. $4 \mathrm{E}-\odot 9$ & $5.8 \mathrm{E}-10$ & 5. $0 \mathrm{E}-\odot 9$ \\
\hline TE125M & Class: W & & $1.06 \mathrm{E}-08$ & & & & & \\
\hline & 1 & Yr: & & $3.3 E-\odot 8$ & 4. . $7 \mathrm{E}-09$ & $5.6 \mathrm{E}-10$ & $3.9 \mathrm{E}-10$ & 4. 3E- -09 \\
\hline & 50 & Yr: & & $3.3 E-08$ & 4. . $\mathrm{E}-\odot 9$ & $5.6 \mathrm{E}-10$ & $3.9 \mathrm{E}-10$ & $4.3 E-09$ \\
\hline TE127M & Class: W & & $3.56 \mathrm{E}-\odot 9$ & & & & & \\
\hline & 1 & Yr: & & 7. $\odot E-\odot 8$ & $8.6 \mathrm{E}-\odot 9$ & 3. $\odot E-\odot 9$ & 1. $3 \mathrm{E}-\odot 9$ & $9.2 \mathrm{E}-\odot 9$ \\
\hline & 50 & Yr: & & 7. ๑E-०8 & $8.6 \mathrm{E}-09$ & 3. ๑E-๑9 & 1. 3E- 09 & $9.2 \mathrm{E}-09$ \\
\hline TE127 & Class: W & & $1.05 \mathrm{E}-\odot 8$ & & & & & \\
\hline & 1 & Yr: & & $9.5 \mathrm{E}-10$ & 1. $9 \mathrm{E}-12$ & $2.2 \mathrm{E}-12$ & $4.1 \mathrm{E}-12$ & 1. $6 \mathrm{E}-10$ \\
\hline & 50 & Yr: & & $9.5 \mathrm{E}-10$ & $1.9 \mathrm{E}-12$ & 2. $2 \mathrm{E}-12$ & $4.1 \mathrm{E}-12$ & $1.6 \mathrm{E}-10$ \\
\hline TE129M & Class: W & & $4.92 \mathrm{E}-\odot 8$ & & & & & \\
\hline & 1 & Yr: & & $5.8 \mathrm{E}-08$ & $2.6 \mathrm{E}-09$ & $1.6 \mathrm{E}-09$ & $1.5 \mathrm{E}-09$ & 8. $0 \mathrm{E}-\odot 9$ \\
\hline & 50 & Yr: & & $5.8 E-08$ & $2.6 \mathrm{E}-09$ & 1.6E-०9 & 1. $5 \mathrm{E}-\odot 9$ & $8.0 \mathrm{E}-09$ \\
\hline TE129 & Class: W & & $9.05 E-\odot 8$ & & & & & \\
\hline & 1 & Yr: & & $1.9 \mathrm{E}-10$ & $4.1 \mathrm{E}-13$ & $4.1 \mathrm{E}-13$ & $4.4 \mathrm{E}-13$ & 4. $4 \mathrm{E}-11$ \\
\hline & 50 & Yr: & & 1. $9 \mathrm{E}-10$ & $4.1 \mathrm{E}-13$ & $4.1 \mathrm{E}-13$ & $4.4 \mathrm{E}-13$ & 4. $4 \mathrm{E}-11$ \\
\hline TE131M & Class: W & & $2.07 \mathrm{E}-06$ & & & & & \\
\hline & 1 & Yr: & & $5.8 \mathrm{E}-\odot 9$ & 1. $2 \mathrm{E}-10$ & 1. $\odot E-10$ & $4.1 \mathrm{E}-\odot 9$ & 1. $2 \mathrm{E}-09$ \\
\hline & $5 \odot$ & Yr: & & $5.8 \mathrm{E}-\odot 9$ & 1. $2 \mathrm{E}-10$ & 1. $0 \mathrm{E}-10$ & $4.1 \mathrm{E}-\odot 9$ & 1. $2 \mathrm{E}-\odot 9$ \\
\hline TE131 & Class: W & & $6.09 \mathrm{E}-07$ & & & & & \\
\hline & 1 & Yr: & & 1. $2 \mathrm{E}-10$ & $6.1 \mathrm{E}-13$ & $5.8 \mathrm{E}-13$ & 7. $3 E-11$ & $3.5 \mathrm{E}-11$ \\
\hline & 50 & Yr: & & $1.2 \mathrm{E}-10$ & $6.1 \mathrm{E}-13$ & $5.8 \mathrm{E}-13$ & 7. 3E-11 & $3.5 \mathrm{E}-11$ \\
\hline TE132 & Class: W & & $2.95 \mathrm{E}-07$ & & & & & \\
\hline & 1 & Yr: & & 1. $3 \mathrm{E}-\odot 8$ & $3.1 \mathrm{E}-10$ & $2.5 \mathrm{E}-10$ & $E-\odot 9$ & $2.6 \mathrm{E}-\odot 9$ \\
\hline & 50 & Yr: & & 1. $3 \mathrm{E}-08$ & $3.1 \mathrm{E}-10$ & $2.5 \mathrm{E}-10$ & $6.6 \mathrm{E}-09$ & $2.6 \mathrm{E}-\odot 9$ \\
\hline TE133M & Class: W & & $3.41 \mathrm{E}-06$ & & & & & \\
\hline & 1 & Yr: & & $3.2 \mathrm{E}-10$ & $5.0 \mathrm{E}-12$ & $6.1 \mathrm{E}-12$ & $2.5 \mathrm{E}-10$ & $1.1 \mathrm{E}-10$ \\
\hline & 50 & Yr: & & $3.2 \mathrm{E}-10$ & 5. $0 \mathrm{E}-12$ & $6.1 \mathrm{E}-12$ & $2.5 \mathrm{E}-10$ & $1.1 \mathrm{E}-10$ \\
\hline TE133 & Class: W & & $1.37 \mathrm{E}-06$ & & & & & \\
\hline & 1 & Yr: & & $6.6 \mathrm{E}-11$ & $5.7 \mathrm{E}-13$ & $6.5 \mathrm{E}-13$ & $E-11$ & $2.4 \mathrm{E}-11$ \\
\hline & 50 & Yr: & & $6.6 \mathrm{E}-11$ & $5.7 \mathrm{E}-13$ & $6.5 \mathrm{E}-13$ & $5.6 \mathrm{E}-11$ & $2.4 \mathrm{E}-11$ \\
\hline TE134 & Class: W & & $1.24 \mathrm{E}-\odot 6$ & & & & & \\
\hline & 1 & Yr: & & $2.6 \mathrm{E}-10$ & $4.6 \mathrm{E}-12$ & $5.7 \mathrm{E}-12$ & 3. $8 \mathrm{E}-11$ & $8.2 \mathrm{E}-11$ \\
\hline & 50 & Yr: & & $2.6 \mathrm{E}-10$ & $4.6 \mathrm{E}-12$ & $5.7 \mathrm{E}-12$ & $3.8 \mathrm{E}-11$ & $8.2 \mathrm{E}-11$ \\
\hline I 125 & Class: D & & $1.19 \mathrm{E}-\odot 8$ & & & & & \\
\hline & 1 & Yr: & & $1.5 \mathrm{E}-11$ & $5.5 \mathrm{E}-11$ & $1.1 \mathrm{E}-11$ & $5-07$ & 7. 3E- -99 \\
\hline & 50 & Yr: & & 1. $5 \mathrm{E}-11$ & $5.5 \mathrm{E}-11$ & 1. $1 \mathrm{E}-11$ & $1.5 \mathrm{E}-07$ & 7. $3 E-09$ \\
\hline I 129 & Class: D & & $8.92 \mathrm{E}-\odot 9$ & & & & & \\
\hline & 1 & Yr: & & $5.4 \mathrm{E}-11$ & $1.1 \mathrm{E}-10$ & $3.7 \mathrm{E}-11$ & $9.1 \mathrm{E}-07$ & $4.6 \mathrm{E}-\odot 8$ \\
\hline & 50 & Yr: & & $5.4 \mathrm{E}-11$ & 1. $1 \mathrm{E}-10$ & $3.7 \mathrm{E}-11$ & $9.1 \mathrm{E}-07$ & $4.6 \mathrm{E}-08$ \\
\hline I 130 & Class: D & & $3.05 \mathrm{E}-06$ & & & & & \\
\hline
\end{tabular}




\begin{tabular}{|c|c|c|c|c|c|c|c|c|}
\hline & 1 & Yr: & & $6.9 \mathrm{E}-11$ & $3.9 \mathrm{E}-11$ & $3.6 \mathrm{E}-11$ & 1. $9 \mathrm{E}-\odot 8$ & 1. $0 \mathrm{E}-\odot 9$ \\
\hline & 50 & Yr: & & $6.9 \mathrm{E}-11$ & $3.9 \mathrm{E}-11$ & $3.6 \mathrm{E}-11$ & 1. $9 \mathrm{E}-\odot 8$ & 1. $\odot E-\odot 9$ \\
\hline \multirow{3}{*}{ I 131} & Class: D & & $5.33 E-07$ & & & & & \\
\hline & 1 & Yr: & & $7.3 \mathrm{E}-11$ & $5.2 \mathrm{E}-11$ & $4.1 \mathrm{E}-11$ & $2.2 \mathrm{E}-07$ & $1.1 \mathrm{E}-08$ \\
\hline & 50 & Yr: & & 7. 3E-11 & $5.2 \mathrm{E}-11$ & $4.1 \mathrm{E}-11$ & $2.2 \mathrm{E}-\odot 7$ & $1.1 \mathrm{E}-08$ \\
\hline \multirow[t]{3}{*}{ I 132} & Class: D & & $3.31 \mathrm{E}-06$ & & & & & \\
\hline & 1 & Yr: & & $4.6 \mathrm{E}-11$ & 1. $4 \mathrm{E}-11$ & 1. $3 \mathrm{E}-11$ & $2.1 \mathrm{E}-09$ & 1. $4 \mathrm{E}-10$ \\
\hline & 50 & Yr: & & $4.6 \mathrm{E}-11$ & 1. $4 \mathrm{E}-11$ & 1. $3 \mathrm{E}-11$ & $2.1 \mathrm{E}-09$ & 1. $4 \mathrm{E}-10$ \\
\hline \multirow[t]{3}{*}{ I 133} & Class: D & & $8.70 \mathrm{E}-07$ & & & & & \\
\hline & 1 & Yr: & & $5.3 \mathrm{E}-11$ & $2.2 \mathrm{E}-11$ & $2.1 \mathrm{E}-11$ & 4. 4E- - 8 & 2. 3E-๑9 \\
\hline & 50 & Yr: & & $5.3 \mathrm{E}-11$ & $2.2 \mathrm{E}-11$ & $2.1 \mathrm{E}-11$ & 4. 4E- - 88 & 2. 3E- -99 \\
\hline \multirow[t]{3}{*}{ I 134} & Class: D & & $3.85 E-06$ & & & & & \\
\hline & 1 & Yr: & & $3.8 \mathrm{E}-11$ & $6.5 \mathrm{E}-12$ & $6.2 \mathrm{E}-12$ & $4.0 \mathrm{E}-10$ & $5.9 \mathrm{E}-11$ \\
\hline & 50 & Yr: & & $3.8 \mathrm{E}-11$ & $6.5 \mathrm{E}-12$ & $6.2 \mathrm{E}-12$ & $4.0 \mathrm{E}-10$ & $5.9 \mathrm{E}-11$ \\
\hline \multirow[t]{3}{*}{ I 135} & Class: D & & $2.38 \mathrm{E}-06$ & & & & & \\
\hline & 1 & Yr: & & $5.0 \mathrm{E}-11$ & $2.0 \mathrm{E}-11$ & $1.9 \mathrm{E}-11$ & $8.8 \mathrm{E}-09$ & $4.8 \mathrm{E}-10$ \\
\hline & 50 & Yr: & & $5.0 \mathrm{E}-11$ & 2. $0 \mathrm{E}-11$ & 1. $9 \mathrm{E}-11$ & $8.8 \mathrm{E}-\odot 9$ & $4.8 \mathrm{E}-10$ \\
\hline \multirow[t]{3}{*}{ XE131M } & NobleGas & & $1.10 \mathrm{E}-\odot 8$ & & & & & \\
\hline & 1 & Yr: & & $\odot . \odot \mathrm{E}-\odot \odot$ & $\odot . \odot \mathrm{E}-\odot \odot$ & $\odot . \odot \mathrm{E}-\odot \odot$ & $\odot . \odot \mathrm{E}-\odot \odot$ & $\odot . \odot E-\odot \odot$ \\
\hline & $5 \odot$ & Yr: & & $\odot . \odot \mathrm{E}-\odot \odot$ & $\odot . \odot \mathrm{E}-\odot \odot$ & $\odot . \odot \mathrm{E}-\odot \odot$ & $\odot . \odot \mathrm{E}-\odot \odot$ & $\odot . \odot \mathrm{E}-\odot \odot$ \\
\hline \multirow[t]{3}{*}{ XE133M } & NobleGas & & $4.07 \mathrm{E}-\odot 8$ & & & & & \\
\hline & 1 & Yr: & & $\odot . \odot \mathrm{E}-\odot \odot$ & $\odot . \odot \mathrm{E}-\odot \odot$ & $\odot . \odot \mathrm{E}-\odot \odot$ & $\odot . \odot \mathrm{E}-\odot \odot$ & $\odot . \odot \mathrm{E}-\odot \odot$ \\
\hline & 50 & Yr: & & $\odot . \odot \mathrm{E}-\odot \odot$ & $\odot . \odot \mathrm{E}-\odot \odot$ & $\odot . \odot \mathrm{E}-\odot \odot$ & $\odot . \odot \mathrm{E}-\odot \odot$ & $\odot . \odot \mathrm{E}-\odot \odot$ \\
\hline \multirow[t]{3}{*}{ XE133 } & NobleGas & & $4.23 E-08$ & & & & & \\
\hline & 1 & Yr: & & $\odot . \odot \mathrm{E}-\odot \odot$ & $\odot . \odot \mathrm{E}-\odot \odot$ & $\odot . \odot \mathrm{E}-\odot \odot$ & $\odot . \odot \mathrm{E}-\odot \odot$ & $\odot . \odot \mathrm{E}-\odot \odot$ \\
\hline & 50 & Yr: & & $\odot . \odot \mathrm{E}-\odot \odot$ & $\odot . \odot \mathrm{E}-\odot \odot$ & $\odot . \odot \mathrm{E}-\odot \odot$ & $\odot . \odot \mathrm{E}-\odot \odot$ & $\odot . \odot \mathrm{E}-\odot \odot$ \\
\hline \multirow[t]{3}{*}{ XE135M } & NobleGas & & $5.99 E-07$ & & & & & \\
\hline & 1 & Yr: & & $\odot . \odot \mathrm{E}-\odot \odot$ & $\odot . \odot \mathrm{E}-\odot \odot$ & $\odot . \odot \mathrm{E}-\odot \odot$ & $\odot . \odot \mathrm{E}-\odot \odot$ & $\odot . \odot \mathrm{E}-\odot \odot$ \\
\hline & 50 & Yr: & & $\odot . \odot \mathrm{E}-\odot \odot$ & $\odot . \odot E-\odot \odot$ & $\odot . \odot \mathrm{E}-\odot \odot$ & $\odot . \odot \mathrm{E}-\odot \odot$ & $\odot .0 \mathrm{E}-\odot \odot$ \\
\hline XE135 & NobleGas & & $3.50 E-07$ & & & & & \\
\hline & 1 & Yr: & & $\odot . \odot \mathrm{E}-\odot \odot$ & $\odot . \odot \mathrm{E}-\odot \odot$ & $\odot . \odot \mathrm{E}-\odot \odot$ & $\odot . \odot \mathrm{E}-\odot \odot$ & $\odot . \odot \mathrm{E}-\odot \odot$ \\
\hline & 50 & Yr: & & $\odot . \odot \mathrm{E}-\odot \odot$ & $\odot . \odot \mathrm{E}-\odot \odot$ & $\odot . \odot \mathrm{E}-\odot \odot$ & $\odot . \odot \mathrm{E}-\odot \odot$ & $\odot . \odot \mathrm{E}-\odot \odot$ \\
\hline XE137 & NobleGas & & $4.34 \mathrm{E}-07$ & & & & & \\
\hline & 1 & Yr: & & $\odot . \odot \mathrm{E}-\odot \odot$ & $\odot . \odot \mathrm{E}-\odot \odot$ & $\odot . \odot \mathrm{E}-\odot \odot$ & $\odot . \odot \mathrm{E}-\odot \odot$ & $\odot . \odot \mathrm{E}-\odot \odot$ \\
\hline & 50 & Yr: & & $\odot . \odot \mathrm{E}-\odot \odot$ & $\odot . \odot \mathrm{E}-\odot \odot$ & $\odot . \odot \mathrm{E}-\odot \odot$ & $\odot . \odot \mathrm{E}-\odot \odot$ & $\odot . \odot \mathrm{E}-\odot \odot$ \\
\hline XE138 & NobleGas & & $1.73 \mathrm{E}-06$ & & & & & \\
\hline & 1 & Yr: & & $\odot . \odot \mathrm{E}-\odot \odot$ & $\odot . \odot \mathrm{E}-\odot \odot$ & $\odot . \odot \mathrm{E}-\odot \odot$ & $\odot . \odot \mathrm{E}-\odot \odot$ & $\odot . \odot \mathrm{E}-\odot \odot$ \\
\hline & 50 & Yr: & & $\odot . \odot \mathrm{E}-\odot \odot$ & $\odot . \odot \mathrm{E}-\odot \odot$ & $\odot . \odot \mathrm{E}-\odot \odot$ & $\odot . \odot \mathrm{E}-\odot \odot$ & $\odot . \odot \mathrm{E}-\odot \odot$ \\
\hline CS134M & Class: D & & $2.51 E-08$ & & & & & \\
\hline & 1 & Yr: & & $3.2 \mathrm{E}-11$ & 3. $0 \mathrm{E}-12$ & $2.5 \mathrm{E}-12$ & $2.6 \mathrm{E}-12$ & $1.6 \mathrm{E}-11$ \\
\hline & 50 & Yr: & & $3.2 \mathrm{E}-11$ & 3. $0 \mathrm{E}-12$ & $2.5 \mathrm{E}-12$ & $2.6 \mathrm{E}-12$ & 1. $6 \mathrm{E}-11$ \\
\hline CS134 & Class: D & & $2.23 \mathrm{E}-06$ & & & & & \\
\hline & 1 & Yr: & & $5.9 \mathrm{E}-09$ & $6.4 \mathrm{E}-09$ & $6.0 \mathrm{E}-\odot 9$ & $6.1 \mathrm{E}-09$ & $6.4 \mathrm{E}-09$ \\
\hline & 50 & Yr: & & $5.9 \mathrm{E}-09$ & $6.4 \mathrm{E}-09$ & $6 . \odot E-\odot 9$ & $6.1 \mathrm{E}-09$ & $6.4 \mathrm{E}-09$ \\
\hline CS135 & Class: D & & $3.00 \mathrm{E}-10$ & & & & & \\
\hline & 1 & Yr: & & $6.5 \mathrm{E}-10$ & $6.3 \mathrm{E}-10$ & $6.3 \mathrm{E}-10$ & $6.3 \mathrm{E}-10$ & $6.8 \mathrm{E}-10$ \\
\hline & 50 & Yr: & & $6.5 \mathrm{E}-10$ & $6.3 \mathrm{E}-10$ & $6.3 \mathrm{E}-10$ & $6.3 \mathrm{E}-10$ & $6.8 \mathrm{E}-10$ \\
\hline CS136 & Class: D & & $3.13 E-06$ & & & & & \\
\hline & 1 & Yr: & & 1.1E-०9 & $1.2 \mathrm{E}-09$ & 1.1E- $\odot 9$ & 1.1E- -99 & 1. $4 \mathrm{E}-09$ \\
\hline & 50 & Yr: & & 1.1E- 09 & 1. $2 \mathrm{E}-09$ & $1.1 \mathrm{E}-09$ & 1.1E- $\odot 9$ & 1. $4 \mathrm{E}-09$ \\
\hline CS137 & Class: D & & $2.93 E-09$ & & & & & \\
\hline & 1 & Yr: & & $4.2 \mathrm{E}-09$ & 4. 4E- -99 & $4.2 \mathrm{E}-09$ & 4. 3E- - & $4.5 E-09$ \\
\hline & 50 & Yr: & & 4. $2 \mathrm{E}-\odot 9$ & 4. $4 \mathrm{E}-09$ & 4. 2E- $\odot 9$ & 4. 3E- $\odot 9$ & $4.5 E-\odot 9$ \\
\hline CS138 & Class: D & & $3.63 E-06$ & & & & & \\
\hline & 1 & Yr: & & $3.8 \mathrm{E}-11$ & $4.6 \mathrm{E}-12$ & $4.5 \mathrm{E}-12$ & $4.8 \mathrm{E}-12$ & $3.0 \mathrm{E}-11$ \\
\hline & 50 & Yr: & & $3.8 \mathrm{E}-11$ & $4.6 \mathrm{E}-12$ & $4.5 \mathrm{E}-12$ & $4.8 \mathrm{E}-12$ & 3. $0 \mathrm{E}-11$ \\
\hline BA139 & Class: D & & $8.04 \mathrm{E}-\odot 8$ & & & & & \\
\hline & 1 & Yr: & & $3.5 \mathrm{E}-11$ & $2.5 \mathrm{E}-11$ & $2.0 \mathrm{E}-11$ & 1. $2 \mathrm{E}-12$ & $3.1 \mathrm{E}-11$ \\
\hline & 50 & Yr: & & $3.5 \mathrm{E}-11$ & $2.5 \mathrm{E}-11$ & 2. $0 \mathrm{E}-11$ & 1. $2 \mathrm{E}-12$ & $3.1 \mathrm{E}-11$ \\
\hline BA140 & Class: D & & $2.55 E-\odot 7$ & & & & & \\
\hline & 1 & Yr: & & $4.7 \mathrm{E}-10$ & 8. 3E-०9 & 4.7E-०9 & $4.1 \mathrm{E}-10$ & 1. $6 \mathrm{E}-09$ \\
\hline & 50 & Yr: & & $4.7 \mathrm{E}-10$ & 8. उE- - 99 & $4.7 \mathrm{E}-\odot 9$ & $4.1 \mathrm{E}-10$ & $1.6 \mathrm{E}-09$ \\
\hline BA141 & Class: D & & $1.24 \mathrm{E}-06$ & & & & & \\
\hline & 1 & Yr: & & $2.9 \mathrm{E}-11$ & $=-11$ & $E-12$ & -12 & $2.1 \mathrm{E}-11$ \\
\hline & 50 & Yr: & & $2.9 \mathrm{E}-11$ & 1. $4 \mathrm{E}-11$ & $9.6 \mathrm{E}-12$ & $1.1 \mathrm{E}-12$ & $2.1 \mathrm{E}-11$ \\
\hline BA142 & Class: D & & $1.53 E-06$ & & & & & \\
\hline & 1 & Yr: & & $2.2 \mathrm{E}-11$ & $5.7 \mathrm{E}-12$ & 5. $0 \mathrm{E}-12$ & 1. $3 \mathrm{E}-12$ & 1. $6 \mathrm{E}-11$ \\
\hline & $5 \odot$ & Yr: & & $2.2 \mathrm{E}-11$ & $5.7 \mathrm{E}-12$ & $5.0 \mathrm{E}-12$ & 1. $3 \mathrm{E}-12$ & 1. $6 \mathrm{E}-11$ \\
\hline LA140 & Class: D & & $3.50 \mathrm{E}-06$ & & & & & \\
\hline & 1 & Yr: & & $2.2 \mathrm{E}-10$ & $2.9 \mathrm{E}-10$ & $3.6 \mathrm{E}-10$ & 1. $0 \mathrm{E}-10$ & $6.9 \mathrm{E}-10$ \\
\hline & 50 & Yr: & & 2. $2 \mathrm{E}-10$ & $2.9 \mathrm{E}-10$ & $3.6 \mathrm{E}-10$ & 1. $0 \mathrm{E}-10$ & $6.9 \mathrm{E}-10$ \\
\hline
\end{tabular}




\begin{tabular}{|c|c|c|c|c|c|c|c|c|}
\hline \multirow[t]{3}{*}{ LA141 } & Class: D & & $9.08 \mathrm{E}-\odot 8$ & & & & & \\
\hline & 1 & Yr: & & $4.5 \mathrm{E}-11$ & $7.3 E-11$ & $2.9 \mathrm{E}-11$ & $9.7 \mathrm{E}-12$ & $7.5 \mathrm{E}-11$ \\
\hline & 50 & Yr: & & $4.5 \mathrm{E}-11$ & $7.3 E-11$ & $2.9 \mathrm{E}-11$ & $9.7 \mathrm{E}-12$ & $7.5 \mathrm{E}-11$ \\
\hline \multirow[t]{3}{*}{ LA142 } & Class: D & & $4.32 E-06$ & & & & & \\
\hline & 1 & Yr: & & $4.6 \mathrm{E}-11$ & 1. $3 E-11$ & 1. $4 \mathrm{E}-11$ & $1.1 \mathrm{E}-11$ & $6.4 \mathrm{E}-11$ \\
\hline & 50 & Yr: & & $4.6 \mathrm{E}-11$ & 1. $3 E-11$ & 1. $4 \mathrm{E}-11$ & 1.1E-11 & $6.4 \mathrm{E}-11$ \\
\hline \multirow[t]{3}{*}{ CE141 } & Class: $\mathrm{Y}$ & & $9.81 \mathrm{E}-\odot 8$ & & & & & \\
\hline & 1 & Yr: & & $3.8 E-\odot 8$ & 1. $3 E-10$ & 4. $4 \mathrm{E}-11$ & 2. $8 \mathrm{E}-11$ & 4. $7 \mathrm{E}-\odot 9$ \\
\hline & $5 \odot$ & Yr: & & $3.8 \mathrm{E}-08$ & $1.3 E-10$ & $4.4 \mathrm{E}-11$ & $2.8 \mathrm{E}-11$ & $4.7 \mathrm{E}-09$ \\
\hline \multirow[t]{3}{*}{ CE143 } & Class: $Y$ & & $3.82 \mathrm{E}-07$ & & & & & \\
\hline & 1 & Yr: & & $6.2 \mathrm{E}-09$ & $1.6 \mathrm{E}-11$ & 1.7E-11 & $7.6 \mathrm{E}-12$ & 1. $0 \mathrm{E}-\odot 9$ \\
\hline & 50 & Yr: & & $6.2 \mathrm{E}-09$ & $1.6 \mathrm{E}-11$ & 1. $7 \mathrm{E}-11$ & $7.6 \mathrm{E}-12$ & 1. $0 \mathrm{E}-\odot 9$ \\
\hline \multirow[t]{3}{*}{ CE144 } & Class: Y & & $2.41 \mathrm{E}-\odot 8$ & & & & & \\
\hline & 1 & Yr: & & $4.6 \mathrm{E}-07$ & $2.5 \mathrm{E}-\odot 9$ & 1. $8 \mathrm{E}-\odot 9$ & $1.6 \mathrm{E}-10$ & $5.7 \mathrm{E}-\odot 8$ \\
\hline & 50 & Yr: & & $4.6 \mathrm{E}-07$ & $2.5 \mathrm{E}-\odot 9$ & $1.8 \mathrm{E}-09$ & $1.6 \mathrm{E}-10$ & $5.7 \mathrm{E}-08$ \\
\hline \multirow[t]{3}{*}{ PR143 } & Class: $\mathrm{Y}$ & & $6.12 \mathrm{E}-09$ & & & & & \\
\hline & 1 & Yr: & & $2.3 E-08$ & 1. $9 \mathrm{E}-12$ & $2.7 \mathrm{E}-12$ & 1. $5 \mathrm{E}-14$ & 3. $0 \mathrm{E}-\odot 9$ \\
\hline & 50 & Yr: & & $2.3 E-08$ & $1.9 \mathrm{E}-12$ & $2.7 \mathrm{E}-12$ & 1. $5 \mathrm{E}-14$ & $3.0 \mathrm{E}-09$ \\
\hline \multirow[t]{3}{*}{ PR144M } & Class: $Y$ & & $6.97 \mathrm{E}-\odot 9$ & & & & & \\
\hline & 1 & Yr: & & 4. $0 \mathrm{E}-11$ & 1. $5 \mathrm{E}-14$ & $1.8 \mathrm{E}-14$ & $8.7 \mathrm{E}-15$ & $5.1 \mathrm{E}-12$ \\
\hline & 50 & Yr: & & 4. $0 \mathrm{E}-11$ & $1.5 \mathrm{E}-14$ & $1.8 \mathrm{E}-14$ & $8.7 \mathrm{E}-15$ & $5.1 \mathrm{E}-12$ \\
\hline \multirow[t]{3}{*}{ PR144 } & Class: $\mathrm{Y}$ & & $8.36 \mathrm{E}-\odot 8$ & & & & & \\
\hline & 1 & Yr: & & $6.8 \mathrm{E}-11$ & $1.6 \mathrm{E}-14$ & 1. $7 \mathrm{E}-14$ & 1. $7 \mathrm{E}-14$ & $2.1 \mathrm{E}-11$ \\
\hline & 50 & Yr: & & $6.8 \mathrm{E}-11$ & $1.6 \mathrm{E}-14$ & 1.7E-14 & 1. $7 \mathrm{E}-14$ & $2.1 \mathrm{E}-11$ \\
\hline \multirow[t]{3}{*}{ ND147 } & Class: $\mathrm{Y}$ & & $1.81 \mathrm{E}-07$ & & & & & \\
\hline & 1 & Yr: & & $2.2 \mathrm{E}-\odot 8$ & 1. $\odot \mathrm{E}-1 \odot$ & $3.8 \mathrm{E}-11$ & 1. $9 \mathrm{E}-11$ & 3. $0 \mathrm{E}-\odot 9$ \\
\hline & 50 & Yr: & & 2. $2 \mathrm{E}-08$ & $1.0 \mathrm{E}-10$ & $3.8 \mathrm{E}-11$ & 1.9E-11 & $3.0 \mathrm{E}-09$ \\
\hline PM147 & Class: $\mathrm{Y}$ & & $2.73 \mathrm{E}-10$ & & & & & \\
\hline & 1 & Yr: & & $4.5 E-\odot 8$ & $4.6 \mathrm{E}-09$ & $4.2 \mathrm{E}-10$ & $4.4 \mathrm{E}-14$ & $5.7 \mathrm{E}-\odot 9$ \\
\hline & 50 & Yr: & & $4.5 \mathrm{E}-08$ & $4.6 \mathrm{E}-09$ & $4.2 \mathrm{E}-10$ & $4.4 \mathrm{E}-14$ & $5.7 \mathrm{E}-09$ \\
\hline PM148M & Class: Y & & $2.84 E-06$ & & & & & \\
\hline & 1 & Yr: & & 4. . $9 \mathrm{E}-\odot 8$ & $9.6 \mathrm{E}-10$ & 1.1E-๑9 & $8.1 \mathrm{E}-10$ & 7.1E- -99 \\
\hline & 50 & Yr: & & $4.9 \mathrm{E}-08$ & $9.6 \mathrm{E}-10$ & 1. $1 \mathrm{E}-\odot 9$ & $8.1 \mathrm{E}-10$ & $7.1 \mathrm{E}-09$ \\
\hline PM148 & Class: Y & & $8.70 \mathrm{E}-07$ & & & & & \\
\hline & 1 & Yr: & & 1. $6 \mathrm{E}-\odot 8$ & $5.1 \mathrm{E}-11$ & $7.2 \mathrm{E}-11$ & 4. $3 E-11$ & $2.6 \mathrm{E}-09$ \\
\hline & 50 & Yr: & & 1. $6 \mathrm{E}-08$ & $5.1 \mathrm{E}-11$ & $7.2 \mathrm{E}-11$ & $4.3 \mathrm{E}-11$ & $2.6 \mathrm{E}-09$ \\
\hline PM149 & Class: $\mathrm{Y}$ & & $2.24 \mathrm{E}-\odot 8$ & & & & & \\
\hline & 1 & Yr: & & $5.4 \mathrm{E}-\odot 9$ & 1.1E-12 & 1. $4 \mathrm{E}-12$ & 4. $3 E-13$ & $9.0 \mathrm{E}-10$ \\
\hline & 50 & Yr: & & $5.4 \mathrm{E}-09$ & $1.1 \mathrm{E}-12$ & 1. $4 \mathrm{E}-12$ & 4. $3 E-13$ & $9.0 \mathrm{E}-10$ \\
\hline PM151 & Class: $\mathrm{Y}$ & & $4.42 \mathrm{E}-07$ & & & & & \\
\hline & 1 & Yr: & & $3.4 \mathrm{E}-09$ & $1.6 \mathrm{E}-11$ & 1. $7 \mathrm{E}-11$ & $7.9 \mathrm{E}-12$ & $5.9 \mathrm{E}-10$ \\
\hline & 50 & Yr: & & $3.4 \mathrm{E}-09$ & $1.6 \mathrm{E}-11$ & $1.7 \mathrm{E}-11$ & $7.9 \mathrm{E}-12$ & $5.9 \mathrm{E}-10$ \\
\hline SM147 & Class: W & & 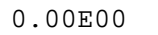 & & & & & \\
\hline & 1 & Yr: & & $2.8 \mathrm{E}-07$ & $1.3 \mathrm{E}-05$ & $1.1 \mathrm{E}-06$ & $1.8 \mathrm{E}-11$ & $9.6 \mathrm{E}-06$ \\
\hline & 50 & Yr: & & $2.8 \mathrm{E}-\odot 7$ & 1. 3E-๑5 & $1.1 \mathrm{E}-\odot 6$ & $1.8 \mathrm{E}-11$ & $9.6 \mathrm{E}-06$ \\
\hline SM151 & Class: W & & $7.79 \mathrm{E}-13$ & & & & & \\
\hline & 1 & Yr: & & $3.6 \mathrm{E}-09$ & 1. $\odot \mathrm{E}-\odot 7$ & $8.8 \mathrm{E}-\odot 9$ & 1.7E-13 & 4. $\odot E-\odot 9$ \\
\hline & 50 & Yr: & & $3.6 \mathrm{E}-09$ & 1. $\odot E-\odot 7$ & $8.8 \mathrm{E}-\odot 9$ & 1. $7 \mathrm{E}-13$ & 4. ०E- $\odot 9$ \\
\hline SM153 & Class: W & & $6.46 \mathrm{E}-\odot 8$ & & & & & \\
\hline & 1 & Yr: & & $5 . \odot E-\odot 9$ & $6.3 \mathrm{E}-11$ & $3.1 \mathrm{E}-11$ & $2.7 \mathrm{E}-12$ & $7.9 \mathrm{E}-10$ \\
\hline & 50 & Yr: & & $5.0 \mathrm{E}-09$ & $6.3 \mathrm{E}-11$ & 3. $1 \mathrm{E}-11$ & $2.7 \mathrm{E}-12$ & 7. $9 \mathrm{E}-10$ \\
\hline EU152 & Class: W & & $1.67 \mathrm{E}-06$ & & & & & \\
\hline & 1 & Yr: & & $7.2 \mathrm{E}-08$ & 1. $9 \mathrm{E}-\odot 7$ & $7.1 \mathrm{E}-\odot 8$ & 8. 3E- $\odot 9$ & 4. 3E- - 88 \\
\hline & 50 & Yr: & & $7.2 \mathrm{E}-08$ & 1. $9 \mathrm{E}-07$ & $7.1 \mathrm{E}-08$ & 8. 3E- 09 & $4.3 E-08$ \\
\hline EU154 & Class: W & & $1.82 \mathrm{E}-\odot 6$ & & & & & \\
\hline & 1 & Yr: & & 1. $2 \mathrm{E}-07$ & $3.9 \mathrm{E}-07$ & $9.4 \mathrm{E}-\odot 8$ & 7.6E- $\odot 9$ & $5.6 \mathrm{E}-08$ \\
\hline & 50 & Yr: & & 1. $2 \mathrm{E}-07$ & $3.9 E-07$ & $9.4 \mathrm{E}-\odot 8$ & 7. $6 \mathrm{E}-\odot 9$ & $5.6 \mathrm{E}-08$ \\
\hline EU155 & Class: W & & $6.78 \mathrm{E}-08$ & & & & & \\
\hline & 1 & Yr: & & $2.3 E-08$ & $1.1 \mathrm{E}-07$ & $1.1 \mathrm{E}-08$ & $2.7 \mathrm{E}-10$ & $7.6 \mathrm{E}-09$ \\
\hline & 50 & Yr: & & $2.3 E-08$ & $1.1 \mathrm{E}-07$ & 1. 1E- - 88 & $2.7 \mathrm{E}-10$ & $7.6 \mathrm{E}-09$ \\
\hline EU156 & Class: W & & $2.02 \mathrm{E}-06$ & & & & & \\
\hline & 1 & Yr: & & $2.7 \mathrm{E}-\odot 8$ & 1. $3 \mathrm{E}-\odot 9$ & $7.7 \mathrm{E}-10$ & $2.2 \mathrm{E}-10$ & 4. $2 \mathrm{E}-\odot 9$ \\
\hline & 50 & Yr: & & $2.7 \mathrm{E}-08$ & 1. $3 \mathrm{E}-09$ & $7.7 \mathrm{E}-10$ & 2. $2 \mathrm{E}-10$ & 4. $2 \mathrm{E}-09$ \\
\hline GD153 & Class: D & & $9.84 \mathrm{E}-08$ & & & & & \\
\hline & 1 & Yr: & & $8.6 \mathrm{E}-10$ & $4.8 E-\odot 8$ & $6.1 \mathrm{E}-09$ & $2.1 \mathrm{E}-10$ & 2. $5 \mathrm{E}-\odot 9$ \\
\hline & 50 & Yr: & & $8.6 \mathrm{E}-10$ & $4.8 E-\odot 8$ & $6.1 \mathrm{E}-\odot 9$ & $2.1 \mathrm{E}-10$ & $2.5 \mathrm{E}-\odot 9$ \\
\hline TB160 & Class: W & & $1.64 \mathrm{E}-06$ & & & & & \\
\hline & 1 & Yr: & & $5.7 \mathrm{E}-\odot 8$ & 1. 4E-๑8 & $E-\odot 9$ & $6.4 \mathrm{E}-10$ & $8.6 \mathrm{E}-09$ \\
\hline & 50 & Yr: & & $5.7 \mathrm{E}-08$ & 1. 4E-๑8 & $3.6 \mathrm{E}-09$ & $6.4 \mathrm{E}-10$ & $8.6 \mathrm{E}-\odot 9$ \\
\hline H0166M & Class: W & & $2.48 \mathrm{E}-06$ & & & & & \\
\hline & 1 & Yr: & & 1. $3 \mathrm{E}-07$ & $6.9 \mathrm{E}-\odot 7$ & 1. $5 \mathrm{E}-\odot 7$ & $2.1 \mathrm{E}-\odot 8$ & 1. $2 \mathrm{E}-07$ \\
\hline
\end{tabular}




\begin{tabular}{|c|c|c|c|c|c|c|c|c|}
\hline \multirow{4}{*}{ W 181} & 50 & Yr: & & 1. $3 \mathrm{E}-07$ & $6.9 \mathrm{E}-07$ & $1.5 \mathrm{E}-\odot 7$ & $2.1 \mathrm{E}-08$ & 1. $2 \mathrm{E}-07$ \\
\hline & Class: D & & $3.69 \mathrm{E}-08$ & & & & & \\
\hline & 1 & Yr: & & $5.4 \mathrm{E}-12$ & $5.6 \mathrm{E}-11$ & $2.0 \mathrm{E}-11$ & $3.1 \mathrm{E}-12$ & $3.3 E-11$ \\
\hline & 50 & Yr: & & $5.4 \mathrm{E}-12$ & $5.6 \mathrm{E}-11$ & 2. $\odot E-11$ & $3.1 \mathrm{E}-12$ & 3. $3 E-11$ \\
\hline \multirow{3}{*}{ W 187} & Class: D & & $6.72 \mathrm{E}-07$ & & & & & \\
\hline & 1 & Yr: & & $4.8 \mathrm{E}-11$ & $5.6 \mathrm{E}-11$ & 3. $0 \mathrm{E}-11$ & 1. $4 \mathrm{E}-11$ & $2.3 \mathrm{E}-10$ \\
\hline & 50 & Yr: & & $4.8 \mathrm{E}-11$ & $5.6 \mathrm{E}-11$ & $3.0 \mathrm{E}-11$ & 1. $4 \mathrm{E}-11$ & $2.3 \mathrm{E}-10$ \\
\hline \multirow[t]{3}{*}{ W 185} & Class: D & & $1.57 \mathrm{E}-09$ & & & & & \\
\hline & 1 & Yr: & & $2.7 \mathrm{E}-11$ & 1. $3 E-10$ & $6.1 \mathrm{E}-11$ & 3. $0 \mathrm{E}-12$ & 1. $4 \mathrm{E}-10$ \\
\hline & 50 & Yr: & & $2.7 \mathrm{E}-11$ & 1. $3 \mathrm{E}-10$ & $6.1 \mathrm{E}-11$ & 3. $0 \mathrm{E}-12$ & 1. $4 \mathrm{E}-10$ \\
\hline \multirow[t]{3}{*}{ RE187 } & Class: W & & $\odot . \odot \odot Е \odot \odot ~$ & & & & & \\
\hline & 1 & Yr: & & $4.4 \mathrm{E}-11$ & 1. $7 \mathrm{E}-13$ & $1.7 \mathrm{E}-13$ & $5.9 \mathrm{E}-12$ & 7. $5 \mathrm{E}-12$ \\
\hline & 50 & Yr: & & $4.4 \mathrm{E}-11$ & 1. $7 \mathrm{E}-13$ & 1.7E-13 & $5.9 \mathrm{E}-12$ & $7.5 \mathrm{E}-12$ \\
\hline \multirow[t]{3}{*}{ IR192 } & Class: Y & & $1.14 \mathrm{E}-06$ & & & & & \\
\hline & 1 & Yr: & & $6.1 \mathrm{E}-08$ & $6.7 \mathrm{E}-10$ & $7.1 \mathrm{E}-10$ & $5.5 \mathrm{E}-10$ & $8.2 \mathrm{E}-09$ \\
\hline & 50 & Yr: & & $6.1 \mathrm{E}-08$ & $6.7 \mathrm{E}-10$ & $7.1 \mathrm{E}-10$ & $5.5 \mathrm{E}-10$ & $8.2 \mathrm{E}-09$ \\
\hline \multirow[t]{3}{*}{$\mathrm{HG} 2 \mathrm{O} 3$} & Class: D & & $3.28 \mathrm{E}-07$ & & & & & \\
\hline & 1 & Yr: & & $3.6 \mathrm{E}-10$ & $4.1 \mathrm{E}-10$ & $3.6 \mathrm{E}-10$ & $3.4 \mathrm{E}-10$ & $5.5 \mathrm{E}-10$ \\
\hline & 50 & Yr: & & $3.6 \mathrm{E}-10$ & $4.1 \mathrm{E}-10$ & $3.6 \mathrm{E}-10$ & $3.4 \mathrm{E}-10$ & $5.5 \mathrm{E}-10$ \\
\hline \multirow[t]{3}{*}{ RN222 } & NobleGas & & $5.61 \mathrm{E}-10$ & & & & & \\
\hline & 1 & Yr: & & $\odot . \odot \mathrm{E}-\odot \odot$ & $\odot . \odot \mathrm{E}-\odot \odot$ & $\odot . \odot \mathrm{E}-\odot \odot$ & $\odot . \odot \mathrm{E}-\odot \odot$ & $\odot . \odot \mathrm{E}-\odot \odot$ \\
\hline & 50 & Yr: & & $\odot . \odot \mathrm{E}-\odot \odot$ & $\odot . \odot \mathrm{E}-\odot \odot$ & $\odot . \odot \mathrm{E}-\odot \odot$ & $\odot . \odot \mathrm{E}-\odot \odot$ & $\odot . \odot \mathrm{E}-\odot \odot$ \\
\hline \multirow[t]{3}{*}{ TH227 } & Class: Y & & $1.40 \mathrm{E}-07$ & & & & & \\
\hline & 1 & Yr: & & $1.1 \mathrm{E}-\odot 4$ & 1. $3 E-07$ & 1. $2 \mathrm{E}-\odot 8$ & $2.8 \mathrm{E}-10$ & 1. $3 E-05$ \\
\hline & 50 & Yr: & & $1.1 \mathrm{E}-04$ & 1. $3 E-07$ & 1. $2 \mathrm{E}-\odot 8$ & $2.8 \mathrm{E}-10$ & $1.3 E-05$ \\
\hline \multirow[t]{3}{*}{ TH228 } & Class: Y & & $2.56 \mathrm{E}-\odot 9$ & & & & & \\
\hline & 1 & Yr: & & $3.9 E-\odot 4$ & $2.6 \mathrm{E}-\mathrm{05}$ & $2.2 \mathrm{E}-\odot 6$ & $5.9 \mathrm{E}-\odot 8$ & $4.7 \mathrm{E}-05$ \\
\hline & 50 & Yr: & & $3.9 \mathrm{E}-04$ & $2.6 \mathrm{E}-05$ & $2.2 \mathrm{E}-\odot 6$ & $5.9 \mathrm{E}-08$ & $4.7 \mathrm{E}-05$ \\
\hline TH229 & Class: Y & & $1.06 \mathrm{E}-07$ & & & & & \\
\hline & 1 & Yr: & & $5.4 \mathrm{E}-\odot 4$ & $5.0 \mathrm{E}-04$ & $2.6 \mathrm{E}-05$ & 1. $7 \mathrm{E}-\odot 6$ & $7.6 \mathrm{E}-05$ \\
\hline & 50 & Yr: & & $5.4 \mathrm{E}-04$ & $5.0 \mathrm{E}-\odot 4$ & $2.6 \mathrm{E}-05$ & 1.7E-06 & $7.6 \mathrm{E}-05$ \\
\hline TH230 & Class: Y & & $4.70 \mathrm{E}-10$ & & & & & \\
\hline & 1 & Yr: & & $8.3 E-05$ & $2.6 \mathrm{E}-\mathrm{-} 4$ & 1. $\odot E-\odot 5$ & $3.1 \mathrm{E}-07$ & 1. $5 \mathrm{E}-05$ \\
\hline & 50 & Yr: & & 8. 3E- $\odot 5$ & $2.6 \mathrm{E}-\odot 4$ & 1. $\odot E-\odot 5$ & $3.1 \mathrm{E}-07$ & $1.5 \mathrm{E}-05$ \\
\hline TH231 & Class: Y & & $1.45 \mathrm{E}-\odot 8$ & & & & & \\
\hline & 1 & Yr: & & 2.7E-๑9 & $5.5 \mathrm{E}-11$ & 3. $0 \mathrm{E}-12$ & $4.1 \mathrm{E}-13$ & $4.1 \mathrm{E}-10$ \\
\hline & 50 & Yr: & & 2.7E-०9 & $5.5 \mathrm{E}-11$ & 3. $0 \mathrm{E}-12$ & $4.1 \mathrm{E}-13$ & $4.1 \mathrm{E}-10$ \\
\hline TH232 & Class: $\mathrm{Y}$ & & $2.29 \mathrm{E}-10$ & & & & & \\
\hline & 1 & Yr: & & $1.6 \mathrm{E}-04$ & $2.8 \mathrm{E}-\odot 4$ & 1. $3 \mathrm{E}-05$ & $8.8 \mathrm{E}-07$ & $2.5 \mathrm{E}-05$ \\
\hline & 50 & Yr: & & $1.6 \mathrm{E}-\odot 4$ & $2.8 \mathrm{E}-\odot 4$ & 1. $3 E-05$ & $8.8 \mathrm{E}-07$ & $2.5 \mathrm{E}-05$ \\
\hline TH234 & Class: Y & & $9.30 \mathrm{E}-\odot 9$ & & & & & \\
\hline & 1 & Yr: & & $6.8 \mathrm{E}-08$ & $8.2 \mathrm{E}-11$ & $6.4 \mathrm{E}-11$ & $7.8 \mathrm{E}-12$ & $9.1 \mathrm{E}-\odot 9$ \\
\hline & 50 & Yr: & & $6.8 \mathrm{E}-08$ & $8.2 \mathrm{E}-11$ & $6.4 \mathrm{E}-11$ & $7.8 \mathrm{E}-12$ & $9.1 \mathrm{E}-09$ \\
\hline RA223 & Class: W & & $1.73 E-07$ & & & & & \\
\hline & 1 & Yr: & & $7.7 \mathrm{E}-05$ & $3.1 \mathrm{E}-06$ & $2.1 \mathrm{E}-07$ & $3.2 E-09$ & $9.4 \mathrm{E}-06$ \\
\hline & 50 & Yr: & & 7.7E-05 & $3.1 \mathrm{E}-06$ & $2.1 \mathrm{E}-07$ & $3.2 \mathrm{E}-09$ & $9.4 \mathrm{E}-06$ \\
\hline RA224 & Class: W & & $1.36 \mathrm{E}-\odot 8$ & & & & & \\
\hline & 1 & Yr: & & $3.1 \mathrm{E}-05$ & $1.4 \mathrm{E}-06$ & $9.2 \mathrm{E}-\odot 8$ & $2.3 E-\odot 9$ & $3.7 \mathrm{E}-06$ \\
\hline & 50 & Yr: & & $3.1 \mathrm{E}-05$ & 1. $4 \mathrm{E}-06$ & $9.2 \mathrm{E}-\odot 8$ & 2. 3E- $\odot 9$ & $3.7 \mathrm{E}-06$ \\
\hline RA225 & Class: W & & $7.60 \mathrm{E}-\odot 9$ & & & & & \\
\hline & 1 & Yr: & & $6.5 \mathrm{E}-05$ & $5.2 \mathrm{E}-06$ & $4.4 \mathrm{E}-07$ & $5.3 E-09$ & $7.9 \mathrm{E}-06$ \\
\hline & 50 & Yr: & & $6.5 \mathrm{E}-05$ & $5.2 \mathrm{E}-06$ & $4.4 \mathrm{E}-07$ & $5.3 \mathrm{E}-09$ & $7.9 \mathrm{E}-06$ \\
\hline RA226 & Class: W & & $8.96 \mathrm{E}-09$ & & & & & \\
\hline & 1 & Yr: & & 3. 3E- 05 & $3.4 \mathrm{E}-05$ & 1. $5 \mathrm{E}-06$ & $2.8 \mathrm{E}-08$ & $4.5 E-06$ \\
\hline & 50 & Yr: & & $3.3 E-05$ & $3.4 \mathrm{E}-05$ & 1. $5 \mathrm{E}-\odot 6$ & $2.8 \mathrm{E}-\odot 8$ & $4.5 E-\odot 6$ \\
\hline RA228 & Class: W & & $\odot . \odot \odot Е \odot \odot ~$ & & & & & \\
\hline & 1 & Yr: & & 1. $2 \mathrm{E}-05$ & 1. $2 \mathrm{E}-\odot 4$ & 1. $0 \mathrm{E}-\odot 5$ & $2.7 \mathrm{E}-07$ & $4.3 E-06$ \\
\hline & 50 & Yr: & & 1. $2 \mathrm{E}-05$ & 1. $2 \mathrm{E}-04$ & 1. $0 \mathrm{E}-05$ & $2.7 \mathrm{E}-\odot 7$ & $4.3 E-06$ \\
\hline PB210 & Class: D & & $1.42 \mathrm{E}-09$ & & & & & \\
\hline & 1 & Yr: & & $1.8 \mathrm{E}-07$ & $4.6 \mathrm{E}-\odot 5$ & 4. $\odot E-\odot 6$ & $1.8 \mathrm{E}-07$ & 1. $3 E-06$ \\
\hline & 50 & Yr: & & $1.8 \mathrm{E}-07$ & $4.6 \mathrm{E}-05$ & 4. . E - 06 & $1.8 \mathrm{E}-07$ & 1. $3 E-06$ \\
\hline PB212 & Class: D & & $1.97 \mathrm{E}-07$ & & & & & \\
\hline & 1 & Yr: & & 1. $0 \mathrm{E}-08$ & $2.4 \mathrm{E}-07$ & $1.8 \mathrm{E}-08$ & $3.1 \mathrm{E}-09$ & $2.1 \mathrm{E}-08$ \\
\hline & 50 & Yr: & & 1. $\odot E-\odot 8$ & $2.4 \mathrm{E}-07$ & $1.8 \mathrm{E}-\odot 8$ & $3.1 \mathrm{E}-09$ & $2.1 \mathrm{E}-\odot 8$ \\
\hline BI210 & Class: W & & $8.14 \mathrm{E}-09$ & & & & & \\
\hline & 1 & Yr: & & $9.3 \mathrm{E}-07$ & $5.4 \mathrm{E}-11$ & $5.4 \mathrm{E}-11$ & $5.4 \mathrm{E}-11$ & 1.1E- -07 \\
\hline & 50 & Yr: & & $9.3 \mathrm{E}-07$ & $5.4 \mathrm{E}-11$ & $5.4 \mathrm{E}-11$ & $5.4 \mathrm{E}-11$ & 1. $1 \mathrm{E}-07$ \\
\hline BI212 & Class: W & & $2.83 E-07$ & & & & & \\
\hline & 1 & Yr: & & $2.5 \mathrm{E}-07$ & $2.5 \mathrm{E}-11$ & $2.5 \mathrm{E}-11$ & $2.4 \mathrm{E}-11$ & $3.8 \mathrm{E}-08$ \\
\hline & 50 & Yr: & & $2.5 \mathrm{E}-\odot 7$ & $2.5 \mathrm{E}-11$ & $2.5 \mathrm{E}-11$ & $2.4 \mathrm{E}-11$ & $3.8 \mathrm{E}-08$ \\
\hline P0210 & Class: W & & $1.23 \mathrm{E}-11$ & & & & & \\
\hline
\end{tabular}




\begin{tabular}{|c|c|c|c|c|c|c|c|c|}
\hline & 1 & Yr: & & $3.1 \mathrm{E}-05$ & $4.6 \mathrm{E}-07$ & $6.2 \mathrm{E}-07$ & $5.7 E-08$ & 4. ०E-०6 \\
\hline & 50 & Yr: & & $3.1 \mathrm{E}-05$ & $4.6 \mathrm{E}-07$ & $6.2 \mathrm{E}-07$ & 5.7E-๑8 & 4. . \\
\hline \multirow[t]{3}{*}{ U 232} & Class: Y & & $3.72 \mathrm{E}-10$ & & & & & \\
\hline & 1 & Yr: & & $3.1 \mathrm{E}-04$ & $4.6 \mathrm{E}-05$ & $3.5 E-06$ & 1. $9 \mathrm{E}-\odot 7$ & $3.8 \mathrm{E}-05$ \\
\hline & 50 & Yr: & & $3.1 \mathrm{E}-04$ & $4.6 \mathrm{E}-05$ & $3.5 E-06$ & 1. $9 \mathrm{E}-07$ & $3.8 \mathrm{E}-05$ \\
\hline \multirow[t]{3}{*}{ U 233} & Class: Y & & $4.48 \mathrm{E}-10$ & & & & & \\
\hline & 1 & Yr: & & 8.7E-05 & 1. $4 \mathrm{E}-06$ & 8. 4E- - & 1. $8 \mathrm{E}-08$ & $1.1 \mathrm{E}-05$ \\
\hline & 50 & Yr: & & 8. 7E- 05 & 1. $4 \mathrm{E}-06$ & 8. 4E- - ४ & 1. 8E- - & $1.1 \mathrm{E}-05$ \\
\hline \multirow[t]{3}{*}{ U 234} & Class: Y & & $1.93 \mathrm{E}-10$ & & & & & \\
\hline & 1 & Yr: & & $8.5 E-05$ & 1.1E-06 & 6. 6E- - & 1.7E-०8 & 1. $0 \mathrm{E}-05$ \\
\hline & 50 & Yr: & & $8.5 E-05$ & $1.1 \mathrm{E}-06$ & 6. 6E-๑8 & 1. 7E-๑8 & 1. $0 \mathrm{E}-05$ \\
\hline \multirow[t]{3}{*}{ U 235} & Class: Y & & $2.04 \mathrm{E}-07$ & & & & & \\
\hline & 1 & Yr: & & $7.6 \mathrm{E}-05$ & $9.9 \mathrm{E}-07$ & $6.4 \mathrm{E}-\odot 8$ & 1.7E-०8 & $9.2 \mathrm{E}-06$ \\
\hline & 50 & Yr: & & 7.6E- 05 & $9.9 \mathrm{E}-07$ & $6.4 \mathrm{E}-08$ & 1.7E-๑8 & $9.2 \mathrm{E}-06$ \\
\hline \multirow[t]{3}{*}{ U 236} & Class: Y & & $1.22 \mathrm{E}-10$ & & & & & \\
\hline & 1 & Yr: & & $7.9 \mathrm{E}-05$ & 1. $\odot E-06$ & $6.3 E-08$ & $1.6 \mathrm{E}-08$ & $9.5 \mathrm{E}-06$ \\
\hline & 50 & Yr: & & $7.9 \mathrm{E}-05$ & 1.0E-06 & $6.3 E-08$ & $1.6 \mathrm{E}-08$ & $9.5 \mathrm{E}-06$ \\
\hline \multirow[t]{3}{*}{ U 237} & Class: Y & & $1.67 \mathrm{E}-07$ & & & & & \\
\hline & 1 & Yr: & & 1. 8E- - & $4.6 \mathrm{E}-11$ & $2.2 \mathrm{E}-11$ & $1.4 \mathrm{E}-11$ & $2.4 \mathrm{E}-09$ \\
\hline & 50 & Yr: & & 1. $8 \mathrm{E}-08$ & $4.6 \mathrm{E}-11$ & 2. $2 \mathrm{E}-11$ & 1. $4 \mathrm{E}-11$ & $2.4 \mathrm{E}-09$ \\
\hline \multirow[t]{3}{*}{ U 238} & Class: Y & & 7.92E-11 & & & & & \\
\hline & 1 & Yr: & & $7.2 \mathrm{E}-05$ & $9.5 \mathrm{E}-07$ & $6.2 \mathrm{E}-08$ & 1. 5E- -8 & 8.7E-06 \\
\hline & 50 & Yr: & & $7.2 \mathrm{E}-05$ & $9.5 \mathrm{E}-07$ & $6.2 \mathrm{E}-\odot 8$ & 1. $5 \mathrm{E}-\odot 8$ & $8.7 \mathrm{E}-06$ \\
\hline \multirow[t]{3}{*}{ U 240} & Class: Y & & 1.85E- $\odot 9$ & & & & & \\
\hline & 1 & Yr: & & $3.6 \mathrm{E}-09$ & $5.0 \mathrm{E}-11$ & 1. $2 \mathrm{E}-11$ & 4. $9 \mathrm{E}-12$ & $7.1 \mathrm{E}-10$ \\
\hline & 50 & Yr: & & $3.6 \mathrm{E}-09$ & $5.0 E-11$ & 1. $2 \mathrm{E}-11$ & 4. $9 \mathrm{E}-12$ & $7.1 \mathrm{E}-10$ \\
\hline \multirow[t]{3}{*}{ PA231 } & Class: Y & & 4.95E- 08 & & & & & \\
\hline & 1 & Yr: & & $1.5 \mathrm{E}-04$ & $5.9 E-04$ & $2.4 \mathrm{E}-05$ & 1. 4E- 06 & 3. $0 \mathrm{E}-05$ \\
\hline & 50 & Yr: & & $1.5 E-\odot 4$ & $5.9 E-\odot 4$ & $2.4 \mathrm{E}-\odot 5$ & 1. 4E- 06 & $3.0 \mathrm{E}-05$ \\
\hline PA233 & Class: Y & & $2.7 \odot E-\odot 7$ & & & & & \\
\hline & 1 & Yr: & & $3.8 \mathrm{E}-08$ & $2.0 \mathrm{E}-10$ & $9.6 \mathrm{E}-11$ & $6.0 \mathrm{E}-11$ & 4. .9E- $\odot 9$ \\
\hline & 50 & Yr: & & $3.8 E-\odot 8$ & $2.0 \mathrm{E}-10$ & $9.6 \mathrm{E}-11$ & $6.0 \mathrm{E}-11$ & 4. $9 \mathrm{E}-09$ \\
\hline PA234 & Class: Y & & $2.75 E-\odot 6$ & & & & & \\
\hline & 1 & Yr: & & $2.3 E-09$ & $2.2 \mathrm{E}-11$ & $2.9 \mathrm{E}-11$ & $1.6 \mathrm{E}-11$ & $5.2 \mathrm{E}-10$ \\
\hline & 50 & Yr: & & 2. $3 E-\odot 9$ & $2.2 \mathrm{E}-11$ & $2.9 \mathrm{E}-11$ & $1.6 \mathrm{E}-11$ & $5.2 \mathrm{E}-10$ \\
\hline AC225 & Class: Y & & 2. .1E-๑8 & & & & & \\
\hline & 1 & Yr: & & 8. $9 \mathrm{E}-05$ & $5.9 \mathrm{E}-\odot 8$ & 8. 7E-๑9 & 2. 4E-๑9 & $1.1 \mathrm{E}-05$ \\
\hline & 50 & Yr: & & $8.9 E-05$ & $5.9 E-\odot 8$ & 8. 7E-๑9 & 2. 4E-๑9 & $1.1 \mathrm{E}-05$ \\
\hline AC227 & Class: Y & & $1.62 \mathrm{E}-10$ & & & & & \\
\hline & 1 & Yr: & & 4. 4E- - 4 & $1.8 \mathrm{E}-04$ & 1.1E-05 & $9.3 \mathrm{E}-07$ & $5.9 \mathrm{E}-05$ \\
\hline & 50 & Yr: & & 4. $4 \mathrm{E}-\odot 4$ & $1.8 \mathrm{E}-04$ & $1.1 \mathrm{E}-05$ & $9.3 \mathrm{E}-07$ & $5.9 \mathrm{E}-05$ \\
\hline AC228 & Class: Y & & $1.42 \mathrm{E}-06$ & & & & & \\
\hline & 1 & Yr: & & 1. $4 \mathrm{E}-07$ & $9.3 E-\odot 9$ & 8. ०E-10 & $2.9 \mathrm{E}-11$ & 1. $7 \mathrm{E}-08$ \\
\hline & 50 & Yr: & & 1. $4 \mathrm{E}-07$ & $9.3 E-\odot 9$ & 8. $0 \mathrm{E}-10$ & $2.9 \mathrm{E}-11$ & 1.7E-๑8 \\
\hline FR223 & Class: D & & 6.97E-๑8 & & & & & \\
\hline & 1 & Yr: & & 1. $0 E-\odot 9$ & $9.4 \mathrm{E}-10$ & $9.4 \mathrm{E}-10$ & $9.4 \mathrm{E}-10$ & 1.1E-09 \\
\hline & 50 & Yr: & & 1. $\odot E-\odot 9$ & $9.4 \mathrm{E}-10$ & $9.4 \mathrm{E}-10$ & $9.4 \mathrm{E}-10$ & 1. 1E-๑9 \\
\hline NP237 & Class: W & & 2.81E- 08 & & & & & \\
\hline & 1 & Yr: & & 3. 4E- 05 & $8.6 E-\odot 4$ & $3.7 \mathrm{E}-05$ & 1. 7E-๑6 & $2.2 \mathrm{E}-05$ \\
\hline & 50 & Yr: & & $3.4 \mathrm{E}-05$ & $8.6 \mathrm{E}-04$ & $3.7 \mathrm{E}-05$ & 1. 7E- 06 & $2.2 \mathrm{E}-05$ \\
\hline NP238 & Class: W & & 8. $07 \mathrm{E}-07$ & & & & & \\
\hline & 1 & Yr: & & $6.7 \mathrm{E}-09$ & $5.6 \mathrm{E}-\odot 8$ & $2.6 \mathrm{E}-\odot 9$ & 1. $3 \mathrm{E}-10$ & $2.2 \mathrm{E}-09$ \\
\hline & 50 & Yr: & & $6.7 \mathrm{E}-09$ & $5.6 \mathrm{E}-08$ & $2.6 \mathrm{E}-\odot 9$ & 1. $3 \mathrm{E}-10$ & 2. $2 \mathrm{E}-\odot 9$ \\
\hline NP239 & Class: W & & $2.19 E-07$ & & & & & \\
\hline & 1 & Yr: & & $8.1 E-09$ & $5.3 E-10$ & $5.7 \mathrm{E}-11$ & $9.7 \mathrm{E}-12$ & 1. $2 \mathrm{E}-09$ \\
\hline & 50 & Yr: & & $8.1 \mathrm{E}-09$ & $5.3 E-10$ & $5.7 \mathrm{E}-11$ & $9.7 \mathrm{E}-12$ & 1. $2 \mathrm{E}-09$ \\
\hline PU236 & Class: Y & & $1.48 \mathrm{E}-10$ & & & & & \\
\hline & 1 & Yr: & & $8.5 E-05$ & $4.5 E-05$ & $2.6 \mathrm{E}-06$ & $9.5 E-\odot 8$ & $1.2 \mathrm{E}-05$ \\
\hline & 50 & Yr: & & $8.5 E-05$ & $4.5 E-05$ & $2.6 \mathrm{E}-06$ & $9.5 E-\odot 8$ & 1. $2 \mathrm{E}-05$ \\
\hline PU237 & Class: Y & & $5.58 \mathrm{E}-\odot 8$ & & & & & \\
\hline & 1 & Yr: & & $3.6 \mathrm{E}-09$ & $8.4 \mathrm{E}-11$ & 3. $0 \mathrm{E}-11$ & $2.3 \mathrm{E}-11$ & $4.8 \mathrm{E}-10$ \\
\hline & 50 & Yr: & & $3.6 \mathrm{E}-09$ & $8.4 \mathrm{E}-11$ & $3.0 \mathrm{E}-11$ & $2.3 \mathrm{E}-11$ & $4.8 \mathrm{E}-10$ \\
\hline PU238 & Class: Y & & $1.11 \mathrm{E}-10$ & & & & & \\
\hline & 1 & Yr: & & 1. $\odot E-\odot 4$ & E-๑4 & $7.5 E-06$ & $3.0 \mathrm{E}-07$ & $1.7 \mathrm{E}-05$ \\
\hline & 50 & Yr: & & 1. $0 \mathrm{E}-\odot 4$ & 1. $5 \mathrm{E}-\odot 4$ & $7.5 \mathrm{E}-06$ & 3. $0 \mathrm{E}-07$ & 1.7E- 05 \\
\hline PU239 & Class: Y & & $1.10 \mathrm{E}-10$ & & & & & \\
\hline & 1 & Yr: & & $9.6 \mathrm{E}-05$ & 1. $7 \mathrm{E}-\odot 4$ & $8.5 E-06$ & $3.5 E-07$ & 1.7E-०5 \\
\hline & 50 & Yr: & & $9.6 \mathrm{E}-05$ & 1. . E - ๑4 & $8.5 E-06$ & $3.5 E-07$ & 1.7E-05 \\
\hline PU240 & Class: Y & & $1.08 \mathrm{E}-10$ & & & & & \\
\hline & 1 & Yr: & & $9.6 \mathrm{E}-05$ & $1.7 \mathrm{E}-04$ & $8.5 E-06$ & $3.5 E-07$ & 1.7E-05 \\
\hline & 50 & Yr: & & $9.6 \mathrm{E}-05$ & 1. $7 \mathrm{E}-\odot 4$ & $8.5 \mathrm{E}-06$ & $3.5 \mathrm{E}-07$ & $1.7 \mathrm{E}-05$ \\
\hline
\end{tabular}




\begin{tabular}{|c|c|c|c|c|c|c|c|c|}
\hline \multirow[t]{3}{*}{ PU241 } & Class: Y & & $2.00 \mathrm{E}-12$ & & & & & \\
\hline & 1 & Yr: & & $4.4 \mathrm{E}-07$ & 4. $\odot E-\odot 6$ & 1. $7 \mathrm{E}-\odot 7$ & 8. ०E-๑9 & 1. $7 \mathrm{E}-07$ \\
\hline & 50 & Yr: & & $4.4 \mathrm{E}-07$ & 4. $0 \mathrm{E}-06$ & 1. $7 \mathrm{E}-\odot 7$ & 8. ०E-๑9 & 1. $7 \mathrm{E}-07$ \\
\hline \multirow[t]{3}{*}{ PU242 } & Class: Y & & $9.18 \mathrm{E}-11$ & & & & & \\
\hline & 1 & Yr: & & $8.9 E-\odot 5$ & $1.6 \mathrm{E}-\odot 4$ & $8.1 \mathrm{E}-06$ & $3.3 E-07$ & $1.6 \mathrm{E}-05$ \\
\hline & 50 & Yr: & & $8.9 \mathrm{E}-05$ & $1.6 \mathrm{E}-\odot 4$ & $8.1 \mathrm{E}-\odot 6$ & $3.3 E-07$ & 1. $6 \mathrm{E}-05$ \\
\hline \multirow[t]{3}{*}{ PU243 } & Class: $Y$ & & $3.04 \mathrm{E}-\odot 8$ & & & & & \\
\hline & 1 & Yr: & & $6.0 \mathrm{E}-10$ & 1. $4 \mathrm{E}-11$ & $9.8 \mathrm{E}-13$ & 1. $8 \mathrm{E}-13$ & $9.2 \mathrm{E}-11$ \\
\hline & 50 & Yr: & & $6.0 \mathrm{E}-10$ & 1. $4 \mathrm{E}-11$ & $9.8 \mathrm{E}-13$ & $1.8 \mathrm{E}-13$ & $9.2 \mathrm{E}-11$ \\
\hline \multirow[t]{3}{*}{ PU244 } & Class: Y & & $6.56 \mathrm{E}-11$ & & & & & \\
\hline & 1 & Yr: & & $8.2 E-05$ & $1.5 E-04$ & $7.6 \mathrm{E}-06$ & $3.2 \mathrm{E}-07$ & 1. $5 \mathrm{E}-05$ \\
\hline & 50 & Yr: & & $8.2 \mathrm{E}-05$ & 1. $5 \mathrm{E}-\odot 4$ & $7.6 \mathrm{E}-06$ & $3.2 \mathrm{E}-\odot 7$ & $1.5 \mathrm{E}-05$ \\
\hline \multirow[t]{3}{*}{ AM241 } & Class: W & & $2.13 \mathrm{E}-08$ & & & & & \\
\hline & 1 & Yr: & & $4.4 \mathrm{E}-05$ & 1. $4 \mathrm{E}-03$ & $6.0 \mathrm{E}-05$ & $3.1 \mathrm{E}-06$ & 4. $0 \mathrm{E}-05$ \\
\hline & 50 & Yr: & & 4. $4 \mathrm{E}-05$ & 1. $4 \mathrm{E}-03$ & $6.0 \mathrm{E}-\odot 5$ & $3.1 \mathrm{E}-06$ & $4.0 \mathrm{E}-05$ \\
\hline \multirow[t]{3}{*}{ AM242M } & Class: W & & $7.85 \mathrm{E}-10$ & & & & & \\
\hline & 1 & Yr: & & 1. $0 \mathrm{E}-\odot 5$ & 1. $4 \mathrm{E}-\odot 3$ & $5.6 \mathrm{E}-05$ & $3.1 \mathrm{E}-06$ & $3.5 E-05$ \\
\hline & 50 & Yr: & & 1. $\odot E-\odot 5$ & 1. $4 \mathrm{E}-\odot 3$ & $5.6 \mathrm{E}-\odot 5$ & $3.1 \mathrm{E}-\odot 6$ & $3.5 E-05$ \\
\hline \multirow[t]{3}{*}{ AM242 } & Class: W & & 1.93E-๑8 & & & & & \\
\hline & 1 & Yr: & & 1. $4 \mathrm{E}-\odot 7$ & $1.1 \mathrm{E}-07$ & $1.1 \mathrm{E}-\odot 8$ & 1. $3 \mathrm{E}-10$ & $2.1 \mathrm{E}-\odot 8$ \\
\hline & 50 & Yr: & & $1.4 \mathrm{E}-07$ & $1.1 \mathrm{E}-07$ & 1.1E- - & 1. $3 E-10$ & $2.1 \mathrm{E}-08$ \\
\hline \multirow[t]{3}{*}{ AM243 } & Class: W & & $5.87 \mathrm{E}-\odot 8$ & & & & & \\
\hline & 1 & Yr: & & $4.2 E-05$ & 1. $4 \mathrm{E}-03$ & $6.0 \mathrm{E}-05$ & $3.1 \mathrm{E}-06$ & 4. $0 \mathrm{E}-05$ \\
\hline & 50 & Yr: & & $4.2 \mathrm{E}-05$ & 1. $4 \mathrm{E}-03$ & $6 . \odot E-\odot 5$ & $3.1 \mathrm{E}-06$ & $4.0 \mathrm{E}-05$ \\
\hline \multirow[t]{3}{*}{ CM242 } & Class: W & & $1.27 \mathrm{E}-10$ & & & & & \\
\hline & 1 & Yr: & & $4.3 E-05$ & $3.2 \mathrm{E}-05$ & $3.2 \mathrm{E}-06$ & $3.7 \mathrm{E}-\odot 8$ & $6.3 \mathrm{E}-06$ \\
\hline & 50 & Yr: & & 4. $3 \mathrm{E}-05$ & $3.2 \mathrm{E}-05$ & $3.2 \mathrm{E}-06$ & $3.7 \mathrm{E}-\odot 8$ & $6.3 \mathrm{E}-06$ \\
\hline \multirow[t]{3}{*}{ CM243 } & Class: W & & $1.67 \mathrm{E}-07$ & & & & & \\
\hline & 1 & Yr: & & $4.8 \mathrm{E}-05$ & $9.5 \mathrm{E}-\odot 4$ & 4.7E- - 5 & $1.8 \mathrm{E}-06$ & $3.0 \mathrm{E}-05$ \\
\hline & 50 & Yr: & & $4.8 E-05$ & $9.5 E-04$ & 4. 7E- $\odot 5$ & $1.8 \mathrm{E}-06$ & $3.0 \mathrm{E}-05$ \\
\hline \multirow[t]{3}{*}{ CM244 } & Class: W & & $1.08 \mathrm{E}-10$ & & & & & \\
\hline & 1 & Yr: & & $4.7 \mathrm{E}-05$ & $7.5 E-\odot 4$ & $4.1 \mathrm{E}-05$ & 1. $3 E-06$ & $2.6 \mathrm{E}-05$ \\
\hline & 50 & Yr: & & $4.7 \mathrm{E}-05$ & $7.5 E-\odot 4$ & $4.1 \mathrm{E}-05$ & 1. $3 E-06$ & $2.6 \mathrm{E}-05$ \\
\hline \multirow[t]{3}{*}{ CM245 } & Class: W & & $1.10 \mathrm{E}-07$ & & & & & \\
\hline & 1 & Yr: & & 4. $3 E-\odot 5$ & $1.5 \mathrm{E}-\odot 3$ & $6.1 \mathrm{E}-\odot 5$ & $3.3 E-\odot 6$ & $4.1 \mathrm{E}-05$ \\
\hline & 50 & Yr: & & 4. $3 E-05$ & $1.5 \mathrm{E}-\odot 3$ & $6.1 \mathrm{E}-05$ & $3.3 E-\odot 6$ & $4.1 \mathrm{E}-05$ \\
\hline \multirow[t]{3}{*}{ CM246 } & Class: W & & $9.78 \mathrm{E}-11$ & & & & & \\
\hline & 1 & Yr: & & 4. $3 E-05$ & $1.5 \mathrm{E}-\odot 3$ & $6.1 \mathrm{E}-05$ & $3.2 \mathrm{E}-06$ & $4.1 \mathrm{E}-05$ \\
\hline & 50 & Yr: & & 4. $3 E-05$ & $1.5 \mathrm{E}-\odot 3$ & $6.1 \mathrm{E}-05$ & $3.2 \mathrm{E}-06$ & $4.1 \mathrm{E}-05$ \\
\hline \multirow[t]{3}{*}{ CM247 } & Class: W & & $4.38 E-07$ & & & & & \\
\hline & 1 & Yr: & & $3.7 \mathrm{E}-05$ & 1. $4 \mathrm{E}-03$ & $5.6 \mathrm{E}-\odot 5$ & $3 . \odot E-\odot 6$ & $3.7 \mathrm{E}-05$ \\
\hline & 50 & Yr: & & $3.7 \mathrm{E}-05$ & 1. $4 \mathrm{E}-03$ & $5.6 \mathrm{E}-05$ & 3. $\odot E-\odot 6$ & $3.7 \mathrm{E}-05$ \\
\hline \multirow[t]{3}{*}{ CM248 } & Class: W & & $7.44 \mathrm{E}-11$ & & & & & \\
\hline & 1 & Yr: & & $6.3 \mathrm{E}-05$ & $7.8 \mathrm{E}-03$ & $6.2 \mathrm{E}-\odot 4$ & $2.2 \mathrm{E}-\odot 8$ & $4.2 E-04$ \\
\hline & 50 & Yr: & & $6.3 E-05$ & $7.8 \mathrm{E}-03$ & $6.2 \mathrm{E}-\odot 4$ & 2. $2 \mathrm{E}-08$ & $4.2 E-04$ \\
\hline \multirow[t]{3}{*}{ CF252 } & Class: W & & $1.15 \mathrm{E}-10$ & & & & & \\
\hline & 1 & Yr: & & $3.5 \mathrm{E}-05$ & $6.6 \mathrm{E}-\odot 4$ & $5.3 E-05$ & 1.1E- $\odot 8$ & $3.5 E-05$ \\
\hline & 50 & Yr: & & $3.5 E-05$ & $6.6 \mathrm{E}-\odot 4$ & $5.3 E-05$ & 1. $1 \mathrm{E}-\odot 8$ & $3.5 E-05$ \\
\hline
\end{tabular}




\section{Appendix A.3}

\section{Age 3650 days}

Dose Equiv. Factors for Acute Inhalation and Ext Air Submersion FGR12/13

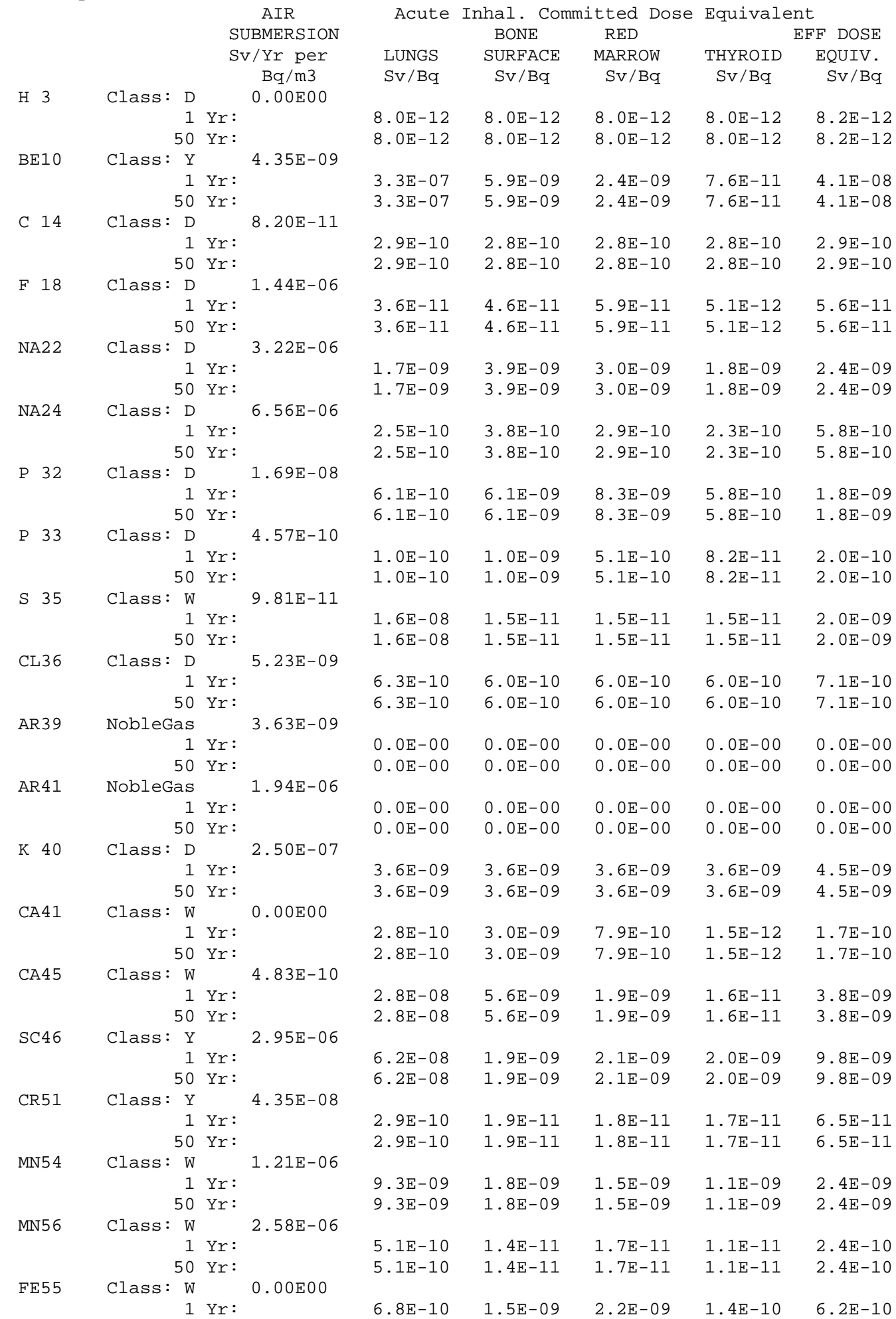




\begin{tabular}{|c|c|c|c|c|c|c|c|c|}
\hline & 50 & Yr: & & $6.8 \mathrm{E}-10$ & 1. $5 \mathrm{E}-\odot 9$ & 2. $2 \mathrm{E}-\odot 9$ & $1.4 \mathrm{E}-10$ & $6.2 \mathrm{E}-10$ \\
\hline \multirow[t]{3}{*}{ FE59 } & Class: W & & $1.77 \mathrm{E}-06$ & & & & & \\
\hline & 1 & Yr: & & $3.1 \mathrm{E}-08$ & 2. $\odot E-\odot 9$ & $2.5 \mathrm{E}-\odot 9$ & 1. $2 \mathrm{E}-\odot 9$ & $5.5 E-09$ \\
\hline & 50 & Yr: & & $3.1 \mathrm{E}-08$ & 2. $\odot E-\odot 9$ & $2.5 \mathrm{E}-\odot 9$ & 1. $2 \mathrm{E}-\odot 9$ & $5.5 E-\odot 9$ \\
\hline \multirow[t]{3}{*}{ C057 } & Class: Y & & $1.57 \mathrm{E}-07$ & & & & & \\
\hline & 1 & Yr: & & $9.7 \mathrm{E}-09$ & $5.7 \mathrm{E}-10$ & 3. $0 \mathrm{E}-10$ & $3.4 \mathrm{E}-10$ & $1.5 \mathrm{E}-\odot 9$ \\
\hline & 50 & Yr: & & $9.7 \mathrm{E}-09$ & $5.7 \mathrm{E}-10$ & $3.0 \mathrm{E}-10$ & $3.4 \mathrm{E}-10$ & 1. $5 \mathrm{E}-09$ \\
\hline \multirow[t]{3}{*}{ C058 } & Class: $Y$ & & $1.40 \mathrm{E}-06$ & & & & & \\
\hline & 1 & Yr: & & $1.8 \mathrm{E}-\odot 8$ & $8.1 \mathrm{E}-10$ & $9.1 \mathrm{E}-10$ & $8.8 \mathrm{E}-10$ & $3.2 \mathrm{E}-09$ \\
\hline & 50 & Yr: & & $1.8 \mathrm{E}-\odot 8$ & $8.1 \mathrm{E}-10$ & $9.1 \mathrm{E}-10$ & $8.8 \mathrm{E}-10$ & $3.2 \mathrm{E}-09$ \\
\hline \multirow[t]{3}{*}{$\mathrm{C060}$} & Class: Y & & $3.75 E-06$ & & & & & \\
\hline & 1 & Yr: & & $2.4 \mathrm{E}-07$ & 1. $2 \mathrm{E}-08$ & 1. $4 \mathrm{E}-08$ & 1. $4 \mathrm{E}-\odot 8$ & 4. $0 \mathrm{E}-\odot 8$ \\
\hline & 50 & Yr: & & $2.4 \mathrm{E}-\odot 7$ & 1. $2 \mathrm{E}-08$ & 1. $4 \mathrm{E}-\odot 8$ & 1. $4 \mathrm{E}-08$ & 4. $\odot \mathrm{E}-\odot 8$ \\
\hline \multirow[t]{3}{*}{ NI59 } & Class: W & & 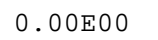 & & & & & \\
\hline & 1 & Yr: & & $8.1 \mathrm{E}-10$ & $1.0 \mathrm{E}-10$ & $1.0 \mathrm{E}-10$ & 1. $0 \mathrm{E}-10$ & $2.0 \mathrm{E}-10$ \\
\hline & 50 & Yr: & & $8.1 \mathrm{E}-10$ & 1. $0 \mathrm{E}-10$ & $1.0 \mathrm{E}-10$ & 1. $0 \mathrm{E}-10$ & $2.0 \mathrm{E}-10$ \\
\hline \multirow[t]{3}{*}{ NI63 } & Class: W & & 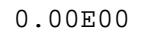 & & & & & \\
\hline & 1 & Yr: & & $3.7 \mathrm{E}-09$ & $2.5 \mathrm{E}-10$ & $2.5 \mathrm{E}-10$ & $2.5 \mathrm{E}-10$ & $7.0 \mathrm{E}-10$ \\
\hline & 50 & Yr: & & $3.7 \mathrm{E}-09$ & $2.5 \mathrm{E}-10$ & $2.5 \mathrm{E}-10$ & $2.5 \mathrm{E}-10$ & 7. $0 \mathrm{E}-10$ \\
\hline \multirow[t]{3}{*}{ NI65 } & Class: W & & $8.42 \mathrm{E}-07$ & & & & & \\
\hline & 1 & Yr: & & $4.3 \mathrm{E}-10$ & 4. 3E-12 & $5.1 \mathrm{E}-12$ & $3.8 \mathrm{E}-12$ & $1.6 \mathrm{E}-10$ \\
\hline & 50 & Yr: & & 4. $3 E-10$ & 4. 3E-12 & $5.1 \mathrm{E}-12$ & $3.8 \mathrm{E}-12$ & $1.6 \mathrm{E}-10$ \\
\hline \multirow[t]{3}{*}{ CU64 } & Class: D & & $2.69 \mathrm{E}-07$ & & & & & \\
\hline & 1 & Yr: & & $3.3 E-11$ & $1.8 \mathrm{E}-11$ & $1.8 \mathrm{E}-11$ & $1.8 \mathrm{E}-11$ & $7.6 \mathrm{E}-11$ \\
\hline & 50 & Yr: & & $3.3 \mathrm{E}-11$ & $1.8 \mathrm{E}-11$ & $1.8 \mathrm{E}-11$ & $1.8 \mathrm{E}-11$ & $7.6 \mathrm{E}-11$ \\
\hline \multirow[t]{3}{*}{ ZN65 } & Class: $\mathrm{Y}$ & & $8.61 \mathrm{E}-07$ & & & & & \\
\hline & 1 & Yr: & & $1.5 \mathrm{E}-\odot 8$ & $1.2 \mathrm{E}-\odot 9$ & 1. 3E-०9 & 1. 3E-०9 & $3.0 \mathrm{E}-\odot 9$ \\
\hline & 50 & Yr: & & $1.5 \mathrm{E}-08$ & 1. $2 \mathrm{E}-\odot 9$ & 1. 3E- $\odot 9$ & 1. 3E- $\odot 9$ & $3.0 \mathrm{E}-\odot 9$ \\
\hline ZN69M & Class: Y & & $5.83 E-07$ & & & & & \\
\hline & 1 & Yr: & & $1.8 \mathrm{E}-09$ & 1. $4 \mathrm{E}-11$ & 1. $9 \mathrm{E}-11$ & $9.5 \mathrm{E}-12$ & $5.4 \mathrm{E}-10$ \\
\hline & 50 & Yr: & & 1. 8E-०9 & 1. $4 \mathrm{E}-11$ & 1. $9 \mathrm{E}-11$ & $9.5 \mathrm{E}-12$ & $5.4 \mathrm{E}-10$ \\
\hline ZN69 & Class: Y & & $6.31 \mathrm{E}-09$ & & & & & \\
\hline & 1 & Yr: & & $1.6 \mathrm{E}-10$ & $4.9 \mathrm{E}-15$ & $5.3 \mathrm{E}-15$ & $3.9 \mathrm{E}-15$ & 4. $7 \mathrm{E}-11$ \\
\hline & 50 & Yr: & & 1. $6 \mathrm{E}-10$ & $4.9 \mathrm{E}-15$ & $5.3 E-15$ & $3.9 \mathrm{E}-15$ & 4. $7 \mathrm{E}-11$ \\
\hline AS76 & Class: W & & $6.50 E-07$ & & & & & \\
\hline & 1 & Yr: & & 4.7E-०9 & $7.2 \mathrm{E}-11$ & $7.7 \mathrm{E}-11$ & 7. $0 \mathrm{E}-11$ & 1. $4 \mathrm{E}-09$ \\
\hline & 50 & Yr: & & 4. 7E-०9 & $7.2 \mathrm{E}-11$ & $7.7 \mathrm{E}-11$ & $7.0 \mathrm{E}-11$ & 1. $4 \mathrm{E}-09$ \\
\hline SE75 & Class: W & & $5.30 E-07$ & & & & & \\
\hline & 1 & Yr: & & $7.5 E-09$ & $8.1 \mathrm{E}-10$ & $6.1 \mathrm{E}-10$ & $5.7 \mathrm{E}-10$ & $1.8 \mathrm{E}-09$ \\
\hline & 50 & Yr: & & $7.5 \mathrm{E}-09$ & $8.1 \mathrm{E}-10$ & $6.1 \mathrm{E}-10$ & $5.7 \mathrm{E}-10$ & $1.8 \mathrm{E}-09$ \\
\hline SE79 & Class: W & & $1.24 \mathrm{E}-10$ & & & & & \\
\hline & 1 & Yr: & & $2.6 \mathrm{E}-08$ & $2.6 \mathrm{E}-10$ & $2.6 \mathrm{E}-10$ & $2.6 \mathrm{E}-10$ & $4.8 \mathrm{E}-\odot 9$ \\
\hline & 50 & Yr: & & $2.6 \mathrm{E}-08$ & $2.6 \mathrm{E}-10$ & $2.6 \mathrm{E}-10$ & $2.6 \mathrm{E}-10$ & $4.8 \mathrm{E}-\odot 9$ \\
\hline BR82 & Class: D & & $3.85 \mathrm{E}-06$ & & & & & \\
\hline & 1 & Yr: & & $3.1 \mathrm{E}-10$ & $3.1 \mathrm{E}-10$ & $2.9 \mathrm{E}-10$ & $3.2 \mathrm{E}-10$ & $7.1 \mathrm{E}-10$ \\
\hline & 50 & Yr: & & $3.1 \mathrm{E}-10$ & $3.1 \mathrm{E}-10$ & $2.9 \mathrm{E}-10$ & $3.2 \mathrm{E}-10$ & $7.1 \mathrm{E}-10$ \\
\hline BR83 & Class: D & & $1.68 \mathrm{E}-\odot 8$ & & & & & \\
\hline & 1 & Yr: & & $3.5 \mathrm{E}-11$ & 6. $3 \mathrm{E}-12$ & 6. $3 \mathrm{E}-12$ & $6.3 \mathrm{E}-12$ & $3.1 \mathrm{E}-11$ \\
\hline & 50 & Yr: & & $3.5 E-11$ & $6.3 \mathrm{E}-12$ & $6.3 \mathrm{E}-12$ & $6.3 \mathrm{E}-12$ & $3.1 \mathrm{E}-11$ \\
\hline BR84 & Class: D & & $2.84 \mathrm{E}-06$ & & & & & \\
\hline & 1 & Yr: & & $4.4 \mathrm{E}-11$ & $6.7 \mathrm{E}-12$ & $6.4 \mathrm{E}-12$ & $6.9 \mathrm{E}-12$ & $4.4 \mathrm{E}-11$ \\
\hline & 50 & Yr: & & 4. $4 \mathrm{E}-11$ & $6.7 \mathrm{E}-12$ & $6.4 \mathrm{E}-12$ & $6.9 \mathrm{E}-12$ & $4.4 \mathrm{E}-11$ \\
\hline KR83M & NobleGas & & $3.78 \mathrm{E}-11$ & & & & & \\
\hline & 1 & Yr: & & $\odot . \odot \mathrm{E}-\odot \odot$ & $\odot . \odot \mathrm{E}-\odot \odot$ & $\odot . \odot \mathrm{E}-\odot \odot$ & $\odot . \odot \mathrm{E}-\odot \odot$ & $\odot . \odot E-\odot \odot$ \\
\hline & 50 & Yr: & & $\odot . \odot \mathrm{E}-\odot \odot$ & $\odot . \odot \mathrm{E}-\odot \odot$ & $\odot . \odot \mathrm{E}-\odot \odot$ & $\odot . \odot \mathrm{E}-\odot \odot$ & $\odot . \odot \mathrm{E}-\odot \odot$ \\
\hline KR85M & NobleGas & & $2.17 \mathrm{E}-07$ & & & & & \\
\hline & 1 & Yr: & & $\odot . \odot \mathrm{E}-\odot \odot$ & $\odot . \odot \mathrm{E}-\odot \odot$ & $\odot . \odot \mathrm{E}-\odot \odot$ & $\odot . \odot \mathrm{E}-\odot \odot$ & $\odot . \odot \mathrm{E}-\odot \odot$ \\
\hline & 50 & Yr: & & $\odot . \odot \mathrm{E}-\odot \odot$ & $\odot . \odot \mathrm{E}-\odot \odot$ & $\odot . \odot \mathrm{E}-\odot \odot$ & $\odot . \odot \mathrm{E}-\odot \odot$ & $\odot . \odot \mathrm{E}-\odot \odot$ \\
\hline KR85 & NobleGas & & $7.57 \mathrm{E}-09$ & & & & & \\
\hline & 1 & Yr: & & $\odot . \odot \mathrm{E}-\odot \odot$ & $\odot . \odot \mathrm{E}-\odot \odot$ & $\odot . \odot \mathrm{E}-\odot \odot$ & $\odot . \odot \mathrm{E}-\odot \odot$ & $\odot . \odot \mathrm{E}-\odot \odot$ \\
\hline & 50 & Yr: & & $\odot . \odot \mathrm{E}-\odot \odot$ & $\odot . \odot \mathrm{E}-\odot \odot$ & $\odot . \odot \mathrm{E}-\odot \odot$ & $\odot . \odot \mathrm{E}-\odot \odot$ & $\odot . \odot \mathrm{E}-\odot \odot$ \\
\hline KR87 & NobleGas & & $1.26 \mathrm{E}-06$ & & & & & \\
\hline & 1 & Yr: & & $\odot . \odot \mathrm{E}-\odot \odot$ & $\odot . \odot \mathrm{E}-\odot \odot$ & $\odot . \odot \mathrm{E}-\odot \odot$ & $\odot . \odot \mathrm{E}-\odot \odot$ & $\odot . \odot \mathrm{E}-\odot \odot$ \\
\hline & 50 & Yr: & & $\odot . \odot \mathrm{E}-\odot \odot$ & $\odot . \odot \mathrm{E}-\odot \odot$ & $\odot . \odot \mathrm{E}-\odot \odot$ & $\odot . \odot \mathrm{E}-\odot \odot$ & $\odot . \odot \mathrm{E}-\odot \odot$ \\
\hline KR88 & NobleGas & & $3.07 E-06$ & & & & & \\
\hline & 1 & Yr: & & $\odot . \odot \mathrm{E}-\odot \odot$ & $\odot . \odot \mathrm{E}-\odot \odot$ & $\odot . \odot E-\odot \odot$ & $\odot . \odot \mathrm{E}-\odot \odot$ & $\odot . \odot \mathrm{E}-\odot \odot$ \\
\hline & 50 & Yr: & & $\odot . \odot \mathrm{E}-\odot \odot$ & $\odot . \odot E-\odot \odot$ & $\odot . \odot \mathrm{E}-\odot \odot$ & $\odot . \odot E-\odot \odot$ & $\odot . \odot \mathrm{E}-\odot \odot$ \\
\hline KR89 & NobleGas & & $3.45 E-06$ & & & & & \\
\hline & 1 & Yr: & & $\odot . \odot \mathrm{E}-\odot \odot$ & $\odot . \odot \mathrm{E}-\odot \odot$ & $\odot . \odot \mathrm{E}-\odot \odot$ & $\odot . \odot \mathrm{E}-\odot \odot$ & $\odot . \odot \mathrm{E}-\odot \odot$ \\
\hline & 50 & Yr: & & $\odot . \odot \mathrm{E}-\odot \odot$ & $\odot . \odot \mathrm{E}-\odot \odot$ & $\odot . \odot \mathrm{E}-\odot \odot$ & $\odot . \odot \mathrm{E}-\odot \odot$ & $\odot . \odot \mathrm{E}-\odot \odot$ \\
\hline RB86 & Class: D & & $1.56 E-07$ & & & & & \\
\hline
\end{tabular}




\begin{tabular}{|c|c|c|c|c|c|c|c|c|}
\hline & 1 & Yr: & & 1. 6E-०9 & $5.4 \mathrm{E}-\odot 9$ & 3. 3E-०9 & 1. $6 \mathrm{E}-09$ & 2. $\odot E-\odot 9$ \\
\hline & 50 & Yr: & & 1. $6 \mathrm{E}-09$ & $5.4 \mathrm{E}-09$ & 3. $3 \mathrm{E}-\odot 9$ & $1.6 \mathrm{E}-09$ & 2. $\odot E-\odot 9$ \\
\hline \multirow[t]{3}{*}{ RB87 } & Class: D & & $1.04 \mathrm{E}-\odot 9$ & & & & & \\
\hline & 1 & Yr: & & $8.7 \mathrm{E}-10$ & 3. ०E-०9 & $1.8 \mathrm{E}-\odot 9$ & $8.4 \mathrm{E}-10$ & 1.1E- -99 \\
\hline & 50 & Yr: & & $8.7 \mathrm{E}-10$ & 3. ०E-०9 & 1. 8E-๑9 & $8.4 \mathrm{E}-10$ & 1. $1 \mathrm{E}-09$ \\
\hline \multirow[t]{3}{*}{ RB88 } & Class: D & & $1.05 E-06$ & & & & & \\
\hline & 1 & Yr: & & $4.4 \mathrm{E}-11$ & $3.8 \mathrm{E}-12$ & $3.6 \mathrm{E}-12$ & $3.6 \mathrm{E}-12$ & $3.2 \mathrm{E}-11$ \\
\hline & 50 & Yr: & & 4. $4 \mathrm{E}-11$ & $3.8 \mathrm{E}-12$ & $3.6 \mathrm{E}-12$ & $3.6 \mathrm{E}-12$ & 3. $2 \mathrm{E}-11$ \\
\hline \multirow[t]{3}{*}{ RB89 } & Class: D & & $3.19 \mathrm{E}-06$ & & & & & \\
\hline & 1 & Yr: & & 3. $0 \mathrm{E}-11$ & $4.6 \mathrm{E}-12$ & $3.7 \mathrm{E}-12$ & 3. 4E-12 & $2.7 \mathrm{E}-11$ \\
\hline & 50 & Yr: & & 3. $0 \mathrm{E}-11$ & $4.6 \mathrm{E}-12$ & $3.7 \mathrm{E}-12$ & $3.4 \mathrm{E}-12$ & $2.7 \mathrm{E}-11$ \\
\hline \multirow[t]{3}{*}{ SR85 } & Class: D & & $7.06 \mathrm{E}-07$ & & & & & \\
\hline & 1 & Yr: & & $6.1 \mathrm{E}-10$ & $2.8 \mathrm{E}-\odot 9$ & 1. $9 \mathrm{E}-\odot 9$ & $5.4 \mathrm{E}-10$ & $9.6 \mathrm{E}-10$ \\
\hline & 50 & Yr: & & $6.1 \mathrm{E}-10$ & $2.8 \mathrm{E}-\odot 9$ & 1. $9 \mathrm{E}-\odot 9$ & $5.4 \mathrm{E}-10$ & $9.6 \mathrm{E}-10$ \\
\hline \multirow[t]{3}{*}{ SR89 } & Class: D & & 1. 38E- - & & & & & \\
\hline & 1 & Yr: & & $3.5 \mathrm{E}-10$ & 1. 8E-०8 & 1. 1E-๑8 & $3.2 \mathrm{E}-10$ & $2.3 E-\odot 9$ \\
\hline & 50 & Yr: & & $3.5 \mathrm{E}-10$ & $1.8 \mathrm{E}-08$ & 1.1E-08 & $3.2 \mathrm{E}-10$ & $2.3 E-09$ \\
\hline \multirow[t]{3}{*}{ SR9๑ } & Class: D & & $3.10 \mathrm{E}-\odot 9$ & & & & & \\
\hline & 1 & Yr: & & 1. 3E-๑9 & $7.5 E-\odot 7$ & $2.6 \mathrm{E}-\odot 7$ & 1. 2E-०9 & $4.1 \mathrm{E}-08$ \\
\hline & 50 & Yr: & & 1. 3E-०9 & $7.5 \mathrm{E}-07$ & $2.6 \mathrm{E}-07$ & 1. $2 \mathrm{E}-09$ & $4.1 \mathrm{E}-\odot 8$ \\
\hline \multirow[t]{3}{*}{ SR91 } & Class: D & & 1. $03 \mathrm{E}-06$ & & & & & \\
\hline & 1 & Yr: & & 8. 0E-11 & 4. $0 \mathrm{E}-10$ & $2.9 E-10$ & 4. $3 \mathrm{E}-11$ & $3.2 \mathrm{E}-10$ \\
\hline & 50 & Yr: & & 8. $0 \mathrm{E}-11$ & $4.0 \mathrm{E}-10$ & $2.9 \mathrm{E}-10$ & 4. $3 \mathrm{E}-11$ & $3.2 \mathrm{E}-10$ \\
\hline \multirow[t]{3}{*}{ SR92 } & Class: D & & 2. $02 \mathrm{E}-06$ & & & & & \\
\hline & 1 & Yr: & & $5.4 \mathrm{E}-11$ & $2.8 \mathrm{E}-10$ & 1. 3E-10 & $2.4 \mathrm{E}-11$ & $2.0 \mathrm{E}-10$ \\
\hline & 50 & Yr: & & $5.4 \mathrm{E}-11$ & $2.8 \mathrm{E}-10$ & 1. $3 \mathrm{E}-10$ & 2. $4 \mathrm{E}-11$ & $2.0 \mathrm{E}-10$ \\
\hline \multirow[t]{3}{*}{ Y 90} & Class: Y & & $2.50 \mathrm{E}-\odot 8$ & & & & & \\
\hline & 1 & Yr: & & 1. 1E-๑8 & $2.1 \mathrm{E}-12$ & 2. $9 \mathrm{E}-12$ & 8. $6 \mathrm{E}-14$ & $2.7 \mathrm{E}-\odot 9$ \\
\hline & 50 & Yr: & & 1. 1E- 08 & $2.1 \mathrm{E}-12$ & 2. $9 \mathrm{E}-12$ & $8.6 \mathrm{E}-14$ & $2.7 \mathrm{E}-\odot 9$ \\
\hline Y 91M & Class: Y & & $7.47 \mathrm{E}-07$ & & & & & \\
\hline & 1 & Yr: & & 7. $0 \mathrm{E}-11$ & 1. $3 \mathrm{E}-12$ & 1. $5 \mathrm{E}-12$ & 1. $1 \mathrm{E}-12$ & 2. $0 \mathrm{E}-11$ \\
\hline & 50 & Yr: & & 7. $0 \mathrm{E}-11$ & 1. 3E-12 & $1.5 \mathrm{E}-12$ & 1.1E-12 & 2. $0 \mathrm{E}-11$ \\
\hline Y 91 & Class: Y & & 1. $96 \mathrm{E}-\odot 8$ & & & & & \\
\hline & 1 & Yr: & & 9. 6E-๑8 & $9.6 \mathrm{E}-11$ & 1. 3E-10 & 4.9E-12 & 1. $3 E-08$ \\
\hline & 50 & Yr: & & $9.6 \mathrm{E}-\odot 8$ & $9.6 \mathrm{E}-11$ & 1. $3 \mathrm{E}-10$ & $4.9 \mathrm{E}-12$ & 1. $3 E-\odot 8$ \\
\hline Y 92 & Class: Y & & $4.16 E-07$ & & & & & \\
\hline & 1 & Yr: & & $9.5 \mathrm{E}-10$ & $2.3 \mathrm{E}-12$ & 3. $0 \mathrm{E}-12$ & $1.8 \mathrm{E}-12$ & $3.5 \mathrm{E}-10$ \\
\hline & 50 & Yr: & & $9.5 \mathrm{E}-10$ & 2. $3 \mathrm{E}-12$ & 3. $0 \mathrm{E}-12$ & $1.8 \mathrm{E}-12$ & $3.5 \mathrm{E}-10$ \\
\hline Y 93 & Class: Y & & $1.67 \mathrm{E}-07$ & & & & & \\
\hline & 1 & Yr: & & 2. 2E- - 9 & $2.3 E-12$ & 3. OE-12 & $1.5 \mathrm{E}-12$ & $8.6 \mathrm{E}-10$ \\
\hline & 50 & Yr: & & $2.2 \mathrm{E}-09$ & 2. $3 \mathrm{E}-12$ & 3. $0 \mathrm{E}-12$ & 1. $5 \mathrm{E}-12$ & $8.6 \mathrm{E}-10$ \\
\hline ZR93 & Class: W & & ๑. ๑०Е๑९ & & & & & \\
\hline & 1 & Yr: & & $4.2 \mathrm{E}-09$ & 1. 7E-๑7 & 1. $6 \mathrm{E}-\odot 8$ & 4. 4E-12 & $4.2 \mathrm{E}-\odot 9$ \\
\hline & 50 & Yr: & & $4.2 \mathrm{E}-09$ & 1. $7 \mathrm{E}-07$ & 1. $6 \mathrm{E}-\odot 8$ & 4. $4 \mathrm{E}-12$ & $4.2 \mathrm{E}-\odot 9$ \\
\hline ZR95 & Class: W & & $1.06 \mathrm{E}-06$ & & & & & \\
\hline & 1 & Yr: & & $4.2 E-08$ & 1. 8E- - 8 & $3.7 \mathrm{E}-\odot 9$ & $9.9 \mathrm{E}-10$ & $6.8 \mathrm{E}-09$ \\
\hline & 50 & Yr: & & $4.2 \mathrm{E}-08$ & 1. 8E- - & $3.7 \mathrm{E}-09$ & $9.9 \mathrm{E}-10$ & $6.8 \mathrm{E}-09$ \\
\hline ZR97 & Class: W & & $2.81 E-07$ & & & & & \\
\hline & 1 & Yr: & & 4. 7E-๑9 & 1. 1E-10 & $1.5 \mathrm{E}-10$ & $4.8 \mathrm{E}-11$ & $1.8 \mathrm{E}-\odot 9$ \\
\hline & 50 & Yr: & & 4. 7E-०9 & $1.1 \mathrm{E}-10$ & 1. $5 \mathrm{E}-10$ & $4.8 \mathrm{E}-11$ & $1.8 \mathrm{E}-09$ \\
\hline NB93M & Class: Y & & $9.62 \mathrm{E}-11$ & & & & & \\
\hline & 1 & Yr: & & 2. $\odot E-\odot 8$ & 7. 3E-11 & $3.3 \mathrm{E}-11$ & 7. $0 \mathrm{E}-12$ & $2.5 E-\odot 9$ \\
\hline & 50 & Yr: & & 2. ०E-๑8 & 7. $3 \mathrm{E}-11$ & 3. $3 \mathrm{E}-11$ & 7. $0 \mathrm{E}-12$ & $2.5 \mathrm{E}-09$ \\
\hline NB94 & Class: Y & & $2.27 \mathrm{E}-06$ & & & & & \\
\hline & 1 & Yr: & & $3.8 E-07$ & 1. 4E-๑8 & 1. $6 \mathrm{E}-\odot 8$ & 1. 4E-०8 & $5.8 \mathrm{E}-08$ \\
\hline & 50 & Yr: & & $3.8 \mathrm{E}-07$ & 1. 4E- -88 & $1.6 \mathrm{E}-08$ & 1. 4E-०8 & $5.8 \mathrm{E}-\odot 8$ \\
\hline NB95M & Class: Y & & $8.64 \mathrm{E}-08$ & & & & & \\
\hline & 1 & Yr: & & $8.2 \mathrm{E}-09$ & $5.6 \mathrm{E}-11$ & $5.3 \mathrm{E}-11$ & $4.5 E-11$ & 1. $3 E-\odot 9$ \\
\hline & 50 & Yr: & & $8.2 \mathrm{E}-09$ & $5.6 \mathrm{E}-11$ & $5.3 \mathrm{E}-11$ & $4.5 \mathrm{E}-11$ & 1. $3 \mathrm{E}-09$ \\
\hline NB95 & Class: Y & & $1.10 \mathrm{E}-06$ & & & & & \\
\hline & 1 & Yr: & & 1. 6E-०8 & $3.9 \mathrm{E}-10$ & $4.4 \mathrm{E}-10$ & गE-10 & $2.6 \mathrm{E}-09$ \\
\hline & 50 & Yr: & & 1. $6 \mathrm{E}-08$ & $3.9 \mathrm{E}-10$ & $4.4 \mathrm{E}-10$ & 4. $0 \mathrm{E}-10$ & $2.6 \mathrm{E}-09$ \\
\hline NB97M & Class: Y & & 1. . $4 \mathrm{E}-06$ & & & & & \\
\hline & 1 & Yr: & & $2.4 \mathrm{E}-12$ & $2.3 \mathrm{E}-14$ & $3.1 \mathrm{E}-14$ & $2.8 \mathrm{E}-14$ & $3.5 E-13$ \\
\hline & 50 & Yr: & & $2.4 \mathrm{E}-12$ & $2.3 \mathrm{E}-14$ & $3.1 \mathrm{E}-14$ & $2.8 \mathrm{E}-14$ & $3.5 \mathrm{E}-13$ \\
\hline NB97 & Class: Y & & $9.43 E-07$ & & & & & \\
\hline & 1 & Yr: & & $2.2 \mathrm{E}-10$ & 2. $2 \mathrm{E}-12$ & $2.6 \mathrm{E}-12$ & 1. $9 \mathrm{E}-12$ & $8.1 \mathrm{E}-11$ \\
\hline & 50 & Yr: & & $2.2 \mathrm{E}-10$ & 2. $2 \mathrm{E}-12$ & $2.6 \mathrm{E}-12$ & 1. $9 \mathrm{E}-12$ & $8.1 \mathrm{E}-11$ \\
\hline M093 & Class: D & & $5.46 \mathrm{E}-10$ & & & & & \\
\hline & 1 & Yr: & & & & $7.6 \mathrm{E}-09$ & 1.1E-10 & $1.3 E-09$ \\
\hline & 50 & Yr: & & 1. $5 \mathrm{E}-10$ & $2.5 \mathrm{E}-\odot 8$ & $7.6 \mathrm{E}-\odot 9$ & $1.1 \mathrm{E}-10$ & 1. $3 \mathrm{E}-\odot 9$ \\
\hline
\end{tabular}




\begin{tabular}{|c|c|c|c|c|c|c|c|c|}
\hline \multirow[t]{3}{*}{ M099 } & Class: D & & $2.20 \mathrm{E}-\odot 7$ & & & & & \\
\hline & 1 & Yr: & & $2.2 \mathrm{E}-10$ & $6.3 \mathrm{E}-10$ & $4.6 \mathrm{E}-10$ & $1.8 \mathrm{E}-10$ & $4.8 \mathrm{E}-10$ \\
\hline & 50 & Yr: & & $2.2 \mathrm{E}-10$ & $6.3 \mathrm{E}-10$ & $4.6 \mathrm{E}-10$ & $1.8 \mathrm{E}-10$ & $4.8 \mathrm{E}-10$ \\
\hline \multirow[t]{3}{*}{ TC99M } & Class: W & & $1.66 \mathrm{E}-07$ & & & & & \\
\hline & 1 & Yr: & & $1.0 \mathrm{E}-10$ & $4.2 \mathrm{E}-12$ & $2.8 \mathrm{E}-12$ & 1. $3 \mathrm{E}-11$ & $3.4 \mathrm{E}-11$ \\
\hline & 50 & Yr: & & 1. $0 \mathrm{E}-10$ & $4.2 \mathrm{E}-12$ & $2.8 \mathrm{E}-12$ & 1. $3 \mathrm{E}-11$ & $3.4 \mathrm{E}-11$ \\
\hline \multirow[t]{3}{*}{ TC99 } & Class: W & & $9.05 \mathrm{E}-10$ & & & & & \\
\hline & 1 & Yr: & & 4. 4E-०8 & $1.8 \mathrm{E}-11$ & $1.8 \mathrm{E}-11$ & $5.5 \mathrm{E}-10$ & $5.6 \mathrm{E}-09$ \\
\hline & 50 & Yr: & & $4.4 \mathrm{E}-08$ & $1.8 \mathrm{E}-11$ & $1.8 \mathrm{E}-11$ & $5.5 \mathrm{E}-10$ & $5.6 \mathrm{E}-09$ \\
\hline \multirow[t]{3}{*}{ TC101 } & Class: W & & $4.76 E-07$ & & & & & \\
\hline & 1 & Yr: & & $4.5 \mathrm{E}-11$ & $3.2 \mathrm{E}-13$ & $2.8 \mathrm{E}-13$ & $2.8 \mathrm{E}-12$ & $2.1 \mathrm{E}-11$ \\
\hline & 50 & Yr: & & $4.5 E-11$ & $3.2 \mathrm{E}-13$ & $2.8 \mathrm{E}-13$ & $2.8 \mathrm{E}-12$ & $2.1 \mathrm{E}-11$ \\
\hline \multirow[t]{3}{*}{ RU103 } & Class: $\mathrm{Y}$ & & $6.59 \mathrm{E}-07$ & & & & & \\
\hline & 1 & Yr: & & 3. ०E- ๑8 & $2.7 \mathrm{E}-10$ & $3.0 \mathrm{E}-10$ & $2.8 \mathrm{E}-10$ & $4.2 \mathrm{E}-09$ \\
\hline & 50 & Yr: & & 3. ०E-०8 & $2.7 \mathrm{E}-10$ & $3.0 E-10$ & $2.8 \mathrm{E}-10$ & 4. $2 \mathrm{E}-09$ \\
\hline \multirow{3}{*}{ RU105 } & Class: $\mathrm{Y}$ & & $1.13 \mathrm{E}-06$ & & & & & \\
\hline & 1 & Yr: & & 1. 1E- 09 & $1.0 E-11$ & 1. $3 \mathrm{E}-11$ & $7.4 \mathrm{E}-12$ & $3.2 \mathrm{E}-10$ \\
\hline & 50 & Yr: & & 1.1E- $\odot 9$ & 1. $0 \mathrm{E}-11$ & 1. $3 \mathrm{E}-11$ & $7.4 \mathrm{E}-12$ & $3.2 \mathrm{E}-10$ \\
\hline \multirow[t]{3}{*}{ RU106 } & Class: Y & & ๑.๑०Е७९ & & & & & \\
\hline & 1 & Yr: & & $7.1 \mathrm{E}-07$ & $8.4 \mathrm{E}-10$ & $8.9 \mathrm{E}-10$ & $9.0 \mathrm{E}-10$ & 9. $0 \mathrm{E}-08$ \\
\hline & 50 & Yr: & & $7.1 \mathrm{E}-07$ & $8.4 \mathrm{E}-10$ & $8.9 \mathrm{E}-10$ & $9.0 \mathrm{E}-10$ & 9. ०E-०8 \\
\hline \multirow[t]{3}{*}{ RH103M } & Class: $\mathrm{Y}$ & & $1.90 \mathrm{E}-10$ & & & & & \\
\hline & 1 & Yr: & & $2.4 \mathrm{E}-11$ & $7.7 \mathrm{E}-15$ & $3.3 E-15$ & $1.8 \mathrm{E}-15$ & 4. $3 E-12$ \\
\hline & 50 & Yr: & & $2.4 \mathrm{E}-11$ & $7.7 \mathrm{E}-15$ & 3. $3 E-15$ & $1.8 \mathrm{E}-15$ & 4. $3 E-12$ \\
\hline \multirow[t]{3}{*}{ RH105 } & Class: $Y$ & & $1.10 \mathrm{E}-07$ & & & & & \\
\hline & 1 & Yr: & & 3. ०E-०9 & $7.1 \mathrm{E}-12$ & $7.9 \mathrm{E}-12$ & $5.0 \mathrm{E}-12$ & $5.6 \mathrm{E}-10$ \\
\hline & 50 & Yr: & & $3.0 E-\odot 9$ & $7.1 \mathrm{E}-12$ & $7.9 \mathrm{E}-12$ & 5. $0 \mathrm{E}-12$ & $5.6 \mathrm{E}-10$ \\
\hline PD103 & Class: Y & & $1.68 \mathrm{E}-09$ & & & & & \\
\hline & 1 & Yr: & & $4.7 \mathrm{E}-\odot 9$ & $1.2 \mathrm{E}-11$ & 3. $4 \mathrm{E}-12$ & $5.3 \mathrm{E}-13$ & $6.8 \mathrm{E}-10$ \\
\hline & 50 & Yr: & & 4. 7E-०9 & 1. $2 \mathrm{E}-11$ & $3.4 \mathrm{E}-12$ & $5.3 E-13$ & $6.8 \mathrm{E}-10$ \\
\hline PD107 & Class: $\mathrm{Y}$ & & $\odot . \odot \odot Е \odot \odot ~$ & & & & & \\
\hline & 1 & Yr: & & $6.2 \mathrm{E}-09$ & $5.1 \mathrm{E}-13$ & $2.4 \mathrm{E}-13$ & 4. $0 \mathrm{E}-14$ & $7.7 \mathrm{E}-10$ \\
\hline & 50 & Yr: & & $6.2 \mathrm{E}-\odot 9$ & $5.1 \mathrm{E}-13$ & $2.4 \mathrm{E}-13$ & 4. $0 \mathrm{E}-14$ & 7. $7 \mathrm{E}-10$ \\
\hline PD109 & Class: $\mathrm{Y}$ & & $1.33 E-08$ & & & & & \\
\hline & 1 & Yr: & & 2. .9E-๑9 & 1. $2 \mathrm{E}-12$ & $5.6 \mathrm{E}-13$ & $1.8 \mathrm{E}-13$ & $6.3 \mathrm{E}-10$ \\
\hline & 50 & Yr: & & 2. $9 \mathrm{E}-09$ & $1.2 \mathrm{E}-12$ & $5.6 \mathrm{E}-13$ & $1.8 \mathrm{E}-13$ & $6.3 \mathrm{E}-10$ \\
\hline AG110M & Class: D & & 4. . $1 \mathrm{E}-06$ & & & & & \\
\hline & 1 & Yr: & & $8.2 \mathrm{E}-09$ & $5.5 E-09$ & $5.7 \mathrm{E}-\odot 9$ & 4. 3E- $\odot 9$ & $9.8 \mathrm{E}-\odot 9$ \\
\hline & 50 & Yr: & & $8.2 \mathrm{E}-\odot 9$ & $5.5 E-\odot 9$ & $5.7 \mathrm{E}-\odot 9$ & 4. 3E- $\odot 9$ & $9.8 \mathrm{E}-09$ \\
\hline AG111 & Class: D & & $4.38 \mathrm{E}-\odot 8$ & & & & & \\
\hline & 1 & Yr: & & $2.2 \mathrm{E}-10$ & $1.8 \mathrm{E}-10$ & $1.8 \mathrm{E}-10$ & $1.8 \mathrm{E}-10$ & $8.8 \mathrm{E}-10$ \\
\hline & $5 \odot$ & Yr: & & $2.2 \mathrm{E}-10$ & $1.8 \mathrm{E}-10$ & $1.8 \mathrm{E}-10$ & $1.8 \mathrm{E}-10$ & $8.8 E-10$ \\
\hline CD109 & Class: D & & $7.22 \mathrm{E}-\odot 9$ & & & & & \\
\hline & 1 & Yr: & & $3.0 E-\odot 9$ & $3.6 \mathrm{E}-09$ & $2.3 E-\odot 9$ & $2.5 E-\odot 9$ & 1. 4E- - 88 \\
\hline & 50 & Yr: & & 3. $0 \mathrm{E}-\odot 9$ & $3.6 \mathrm{E}-09$ & 2. $3 E-\odot 9$ & $2.5 \mathrm{E}-\odot 9$ & 1. $4 \mathrm{E}-08$ \\
\hline CD113M & Class: D & & $2.86 E-\odot 9$ & & & & & \\
\hline & 1 & Yr: & & $2.2 \mathrm{E}-08$ & 2. $2 \mathrm{E}-\odot 8$ & $2.2 \mathrm{E}-\odot 8$ & $2.2 \mathrm{E}-08$ & 1. $4 \mathrm{E}-07$ \\
\hline & 50 & Yr: & & $2.2 \mathrm{E}-\odot 8$ & $2.2 \mathrm{E}-\odot 8$ & $2.2 \mathrm{E}-08$ & $2.2 \mathrm{E}-08$ & 1. $4 \mathrm{E}-07$ \\
\hline CD115M & Class: D & & $4.67 \mathrm{E}-\odot 8$ & & & & & \\
\hline & 1 & Yr: & & 1.7E-०9 & 1.7E- $\odot 9$ & 1.7E-०9 & 1. . E- $\odot 9$ & $9.9 \mathrm{E}-\odot 9$ \\
\hline & 50 & Yr: & & 1. 7E-०9 & 1. 7E-०9 & 1. 7E-०9 & 1. 7E-०9 & $9.9 \mathrm{E}-09$ \\
\hline CD115 & Class: D & & $3.31 \mathrm{E}-07$ & & & & & \\
\hline & 1 & Yr: & & $1.6 \mathrm{E}-10$ & $1.3 \mathrm{E}-10$ & 1. $3 \mathrm{E}-10$ & 1. $0 \mathrm{E}-10$ & $7.6 \mathrm{E}-10$ \\
\hline & 50 & Yr: & & $1.6 \mathrm{E}-10$ & $1.3 \mathrm{E}-10$ & 1. $3 \mathrm{E}-10$ & 1. $0 \mathrm{E}-10$ & $7.6 \mathrm{E}-10$ \\
\hline IN111 & Class: D & & $5.30 \mathrm{E}-07$ & & & & & \\
\hline & 1 & Yr: & & $8.1 \mathrm{E}-11$ & $2.7 \mathrm{E}-10$ & $3.1 \mathrm{E}-10$ & $5.3 E-11$ & $2.7 \mathrm{E}-10$ \\
\hline & 50 & Yr: & & $8.1 \mathrm{E}-11$ & $2.7 \mathrm{E}-10$ & $3.1 \mathrm{E}-10$ & $5.3 E-11$ & $2.7 \mathrm{E}-10$ \\
\hline IN114M & Class: D & & $1.23 \mathrm{E}-07$ & & & & & \\
\hline & 1 & Yr: & & $3.1 \mathrm{E}-09$ & $6.9 \mathrm{E}-08$ & E- 07 & E- $\odot 9$ & 1. $9 \mathrm{E}-08$ \\
\hline & 50 & Yr: & & $3.1 \mathrm{E}-09$ & $6.9 \mathrm{E}-\odot 8$ & 1. $\odot E-\odot 7$ & 3. $\odot E-\odot 9$ & 1. $9 \mathrm{E}-08$ \\
\hline IN115M & Class: D & & $2.17 \mathrm{E}-07$ & & & & & \\
\hline & 1 & Yr: & & $2.3 \mathrm{E}-11$ & $2.7 \mathrm{E}-11$ & $3.7 \mathrm{E}-11$ & $5.5 \mathrm{E}-12$ & $5.2 \mathrm{E}-11$ \\
\hline & 50 & Yr: & & $2.3 \mathrm{E}-11$ & $2.7 \mathrm{E}-11$ & $3.7 \mathrm{E}-11$ & $5.5 \mathrm{E}-12$ & $5.2 \mathrm{E}-11$ \\
\hline SN117M & Class: W & & 1. $93 \mathrm{E}-07$ & & & & & \\
\hline & 1 & Yr: & & $2.5 \mathrm{E}-\odot 8$ & 1. $6 \mathrm{E}-09$ & $2.3 \mathrm{E}-10$ & $4.6 \mathrm{E}-11$ & $3.4 \mathrm{E}-09$ \\
\hline & 50 & Yr: & & $2.5 E-\odot 8$ & $1.6 \mathrm{E}-\odot 9$ & $2.3 \mathrm{E}-10$ & $4.6 \mathrm{E}-11$ & $3.4 \mathrm{E}-\odot 9$ \\
\hline SN119M & Class: W & & $2.23 E-\odot 9$ & & & & & \\
\hline & 1 & Yr: & & $2.3 E-\odot 8$ & 1. $2 \mathrm{E}-09$ & $5.4 \mathrm{E}-10$ & $6.9 \mathrm{E}-11$ & $3.1 \mathrm{E}-09$ \\
\hline & 50 & Yr: & & $2.3 E-08$ & 1. $2 \mathrm{E}-\odot 9$ & $5.4 \mathrm{E}-10$ & $6.9 \mathrm{E}-11$ & $3.1 \mathrm{E}-09$ \\
\hline SN121M & Class: W & & $1.66 \mathrm{E}-09$ & & & & & \\
\hline & 1 & Yr: & & $4.7 \mathrm{E}-\odot 8$ & 4. 3E-०9 & $2.1 \mathrm{E}-\odot 9$ & $2.4 \mathrm{E}-10$ & $6.3 \mathrm{E}-09$ \\
\hline
\end{tabular}




\begin{tabular}{|c|c|c|c|c|c|c|c|c|}
\hline & 50 & Yr: & & 4.7E- - 88 & 4. 3E- -99 & $2.1 \mathrm{E}-09$ & $2.4 \mathrm{E}-10$ & $6.3 \mathrm{E}-\odot 9$ \\
\hline \multirow[t]{3}{*}{ SN121 } & Class: W & & $1.23 \mathrm{E}-09$ & & & & & \\
\hline & 1 & Yr: & & 2. $\odot E-\odot 9$ & $7.4 \mathrm{E}-11$ & $8.9 \mathrm{E}-12$ & $6.9 \mathrm{E}-13$ & $3.6 \mathrm{E}-10$ \\
\hline & 50 & Yr: & & 2. $\odot E-\odot 9$ & 7. $4 \mathrm{E}-11$ & $8.9 \mathrm{E}-12$ & $6.9 \mathrm{E}-13$ & $3.6 \mathrm{E}-10$ \\
\hline \multirow[t]{3}{*}{ SN123 } & Class: W & & $2.20 E-08$ & & & & & \\
\hline & 1 & Yr: & & $8.6 \mathrm{E}-08$ & $3.6 \mathrm{E}-\odot 9$ & 1. $8 \mathrm{E}-09$ & $2.0 \mathrm{E}-10$ & 1. $2 \mathrm{E}-\odot 8$ \\
\hline & 50 & Yr: & & $8.6 \mathrm{E}-\odot 8$ & $3.6 \mathrm{E}-09$ & $1.8 \mathrm{E}-\odot 9$ & 2. $\odot E-10$ & 1. $2 \mathrm{E}-08$ \\
\hline \multirow[t]{3}{*}{ SN125 } & Class: W & & $4.86 \mathrm{E}-07$ & & & & & \\
\hline & 1 & Yr: & & $2.7 \mathrm{E}-\odot 8$ & $9.2 \mathrm{E}-10$ & $7.5 \mathrm{E}-10$ & $8.8 \mathrm{E}-11$ & 5. ०E-๑9 \\
\hline & 50 & Yr: & & $2.7 \mathrm{E}-\odot 8$ & $9.2 \mathrm{E}-10$ & $7.5 \mathrm{E}-10$ & $8.8 \mathrm{E}-11$ & $5 . \odot E-\odot 9$ \\
\hline \multirow[t]{3}{*}{ SN126 } & Class: W & & $5.83 E-08$ & & & & & \\
\hline & 1 & Yr: & & $2.5 \mathrm{E}-\odot 7$ & $3.8 \mathrm{E}-08$ & $2.2 \mathrm{E}-\odot 8$ & $6.9 \mathrm{E}-09$ & $4.1 \mathrm{E}-\odot 8$ \\
\hline & 50 & Yr: & & $2.5 E-07$ & $3.8 E-08$ & $2.2 \mathrm{E}-\odot 8$ & $6.9 \mathrm{E}-\odot 9$ & $4.1 E-08$ \\
\hline \multirow[t]{3}{*}{ SB124 } & Class: W & & $2.72 \mathrm{E}-06$ & & & & & \\
\hline & 1 & Yr: & & $6.1 \mathrm{E}-08$ & $3.1 \mathrm{E}-09$ & 1. $9 \mathrm{E}-09$ & 1.1E- $\odot 9$ & $9.6 \mathrm{E}-\odot 9$ \\
\hline & 50 & Yr: & & $6.1 \mathrm{E}-08$ & $3.1 \mathrm{E}-09$ & 1. $9 \mathrm{E}-\odot 9$ & 1.1E-०9 & $9.6 \mathrm{E}-09$ \\
\hline \multirow[t]{3}{*}{ SB125 } & Class: W & & $5.90 \mathrm{E}-07$ & & & & & \\
\hline & 1 & Yr: & & $4.5 \mathrm{E}-\odot 8$ & 1. $1 \mathrm{E}-\odot 8$ & $2.5 \mathrm{E}-\odot 9$ & $8.6 \mathrm{E}-10$ & $6.9 \mathrm{E}-\odot 9$ \\
\hline & 50 & Yr: & & $4.5 \mathrm{E}-08$ & 1. $1 \mathrm{E}-08$ & $2.5 \mathrm{E}-09$ & $8.6 \mathrm{E}-10$ & $6.9 \mathrm{E}-\odot 9$ \\
\hline \multirow[t]{3}{*}{ SB126M } & Class: W & & $2.21 \mathrm{E}-06$ & & & & & \\
\hline & 1 & Yr: & & $6.3 \mathrm{E}-11$ & 1. $7 \mathrm{E}-12$ & 1. $7 \mathrm{E}-12$ & 1. $6 \mathrm{E}-12$ & $3.5 \mathrm{E}-11$ \\
\hline & 50 & Yr: & & $6.3 \mathrm{E}-11$ & 1. $7 \mathrm{E}-12$ & 1. $7 \mathrm{E}-12$ & 1. $6 \mathrm{E}-12$ & $3.5 \mathrm{E}-11$ \\
\hline \multirow[t]{3}{*}{ SB126 } & Class: W & & 4.04E-๑6 & & & & & \\
\hline & 1 & Yr: & & $2.3 E-08$ & 1.1E- 09 & $9.6 \mathrm{E}-10$ & $6.2 \mathrm{E}-10$ & $5.1 E-09$ \\
\hline & 50 & Yr: & & 2. 3E-०8 & 1. $1 \mathrm{E}-09$ & $9.6 \mathrm{E}-10$ & $6.2 \mathrm{E}-10$ & $5.1 \mathrm{E}-09$ \\
\hline \multirow[t]{3}{*}{ SB127 } & Class: W & & $9.84 \mathrm{E}-07$ & & & & & \\
\hline & 1 & Yr: & & $1.5 \mathrm{E}-\odot 8$ & $2.1 \mathrm{E}-10$ & $2.2 \mathrm{E}-10$ & $7.2 \mathrm{E}-11$ & $2.7 \mathrm{E}-\odot 9$ \\
\hline & 50 & Yr: & & $1.5 E-08$ & $2.1 \mathrm{E}-10$ & $2.2 \mathrm{E}-10$ & $7.2 \mathrm{E}-11$ & $2.7 \mathrm{E}-\odot 9$ \\
\hline TE123M & Class: W & & $1.84 \mathrm{E}-07$ & & & & & \\
\hline & 1 & Yr: & & 4. . E-๑8 & 1. $7 \mathrm{E}-08$ & 2. $2 \mathrm{E}-\odot 9$ & $9.0 \mathrm{E}-1 \odot$ & $5.7 \mathrm{E}-\odot 9$ \\
\hline & 50 & Yr: & & 4. $\odot E-\odot 8$ & 1. $7 \mathrm{E}-\odot 8$ & 2. $2 \mathrm{E}-\odot 9$ & $9.0 \mathrm{E}-10$ & $5.7 \mathrm{E}-09$ \\
\hline TE125M & Class: W & & $1.06 \mathrm{E}-08$ & & & & & \\
\hline & 1 & Yr: & & $3.5 E-\odot 8$ & $8.2 \mathrm{E}-09$ & $9.6 \mathrm{E}-10$ & $6.2 \mathrm{E}-10$ & $4.7 \mathrm{E}-09$ \\
\hline & 50 & Yr: & & $3.5 E-08$ & $8.2 E-09$ & $9.6 \mathrm{E}-10$ & $6.2 \mathrm{E}-10$ & $4.7 \mathrm{E}-09$ \\
\hline TE127M & Class: W & & $3.56 \mathrm{E}-\odot 9$ & & & & & \\
\hline & 1 & Yr: & & $7.6 \mathrm{E}-\odot 8$ & 1. $5 \mathrm{E}-08$ & $5.2 \mathrm{E}-\odot 9$ & $2.1 \mathrm{E}-09$ & 1.1E- -88 \\
\hline & 50 & Yr: & & $7.6 \mathrm{E}-08$ & $1.5 \mathrm{E}-\odot 8$ & $5.2 \mathrm{E}-\odot 9$ & 2.1E- 09 & $1.1 \mathrm{E}-08$ \\
\hline TE127 & Class: W & & $1.05 E-\odot 8$ & & & & & \\
\hline & 1 & Yr: & & $9.9 \mathrm{E}-10$ & $3.5 \mathrm{E}-12$ & $4.1 \mathrm{E}-12$ & 7. $2 \mathrm{E}-12$ & $2.4 \mathrm{E}-10$ \\
\hline & 50 & Yr: & & $9.9 \mathrm{E}-10$ & $3.5 \mathrm{E}-12$ & $4.1 \mathrm{E}-12$ & $7.2 \mathrm{E}-12$ & $2.4 \mathrm{E}-10$ \\
\hline TE129M & Class: W & & $4.92 \mathrm{E}-\odot 8$ & & & & & \\
\hline & 1 & Yr: & & $6.6 \mathrm{E}-08$ & 4. $7 \mathrm{E}-09$ & $2.8 \mathrm{E}-09$ & $2.5 E-\odot 9$ & $9.7 \mathrm{E}-\odot 9$ \\
\hline & 50 & Yr: & & $6.6 \mathrm{E}-08$ & 4. 7E- $\odot 9$ & $2.8 E-\odot 9$ & $2.5 \mathrm{E}-\odot 9$ & $9.7 \mathrm{E}-\odot 9$ \\
\hline TE129 & Class: W & & $9.05 E-\odot 8$ & & & & & \\
\hline & 1 & Yr: & & $2.0 \mathrm{E}-10$ & $7.2 \mathrm{E}-13$ & 7. $0 \mathrm{E}-13$ & $7.4 \mathrm{E}-13$ & $6.5 \mathrm{E}-11$ \\
\hline & 50 & Yr: & & 2. $\odot E-10$ & 7. $2 \mathrm{E}-13$ & 7. $0 \mathrm{E}-13$ & $7.4 \mathrm{E}-13$ & $6.5 \mathrm{E}-11$ \\
\hline TE131M & Class: W & & $2.07 \mathrm{E}-06$ & & & & & \\
\hline & 1 & Yr: & & $6.2 \mathrm{E}-\odot 9$ & $2.1 \mathrm{E}-10$ & 1. $5 \mathrm{E}-10$ & 6. $6 \mathrm{E}-\odot 9$ & 1. $9 \mathrm{E}-09$ \\
\hline & 50 & Yr: & & $6.2 \mathrm{E}-\odot 9$ & $2.1 \mathrm{E}-10$ & 1. $5 \mathrm{E}-10$ & $6.6 \mathrm{E}-\odot 9$ & 1. $9 \mathrm{E}-\odot 9$ \\
\hline TE131 & Class: W & & $6.09 \mathrm{E}-07$ & & & & & \\
\hline & 1 & Yr: & & 1. $2 \mathrm{E}-10$ & $9.5 \mathrm{E}-13$ & $8.6 \mathrm{E}-13$ & 1. $2 \mathrm{E}-10$ & $5.2 \mathrm{E}-11$ \\
\hline & 50 & Yr: & & $1.2 \mathrm{E}-10$ & $9.5 \mathrm{E}-13$ & $8.6 \mathrm{E}-13$ & $1.2 \mathrm{E}-10$ & $5.2 \mathrm{E}-11$ \\
\hline TE132 & Class: W & & $2.95 \mathrm{E}-07$ & & & & & \\
\hline & 1 & Yr: & & 1. $4 \mathrm{E}-\odot 8$ & $5.2 \mathrm{E}-10$ & $3.6 \mathrm{E}-10$ & 1. 1E- $\odot 8$ & $4.1 \mathrm{E}-09$ \\
\hline & 50 & Yr: & & 1. $4 \mathrm{E}-08$ & $5.2 \mathrm{E}-10$ & $3.6 \mathrm{E}-10$ & $1.1 \mathrm{E}-08$ & $4.1 \mathrm{E}-\odot 9$ \\
\hline TE133M & Class: W & & $3.41 \mathrm{E}-06$ & & & & & \\
\hline & 1 & Yr: & & $3.5 \mathrm{E}-10$ & $8.0 \mathrm{E}-12$ & $9.0 \mathrm{E}-12$ & $4.3 E-10$ & $1.7 \mathrm{E}-10$ \\
\hline & 50 & Yr: & & $3.5 \mathrm{E}-10$ & 8. $0 \mathrm{E}-12$ & $9.0 \mathrm{E}-12$ & $4.3 E-10$ & $1.7 \mathrm{E}-10$ \\
\hline TE133 & Class: W & & $1.37 \mathrm{E}-06$ & & & & & \\
\hline & 1 & Yr: & & $7.2 \mathrm{E}-11$ & $9.0 \mathrm{E}-13$ & $9.4 \mathrm{E}-13$ & $9.5 \mathrm{E}-11$ & $3.8 \mathrm{E}-11$ \\
\hline & 50 & Yr: & & $7.2 \mathrm{E}-11$ & $9.0 \mathrm{E}-13$ & $9.4 \mathrm{E}-13$ & $9.5 \mathrm{E}-11$ & $3.8 \mathrm{E}-11$ \\
\hline TE134 & Class: W & & $1.24 \mathrm{E}-\odot 6$ & & & & & \\
\hline & 1 & Yr: & & $2.7 \mathrm{E}-10$ & 7. $3 \mathrm{E}-12$ & $8.4 \mathrm{E}-12$ & $6.1 \mathrm{E}-11$ & 1. $2 \mathrm{E}-10$ \\
\hline & 50 & Yr: & & $2.7 \mathrm{E}-10$ & 7. $3 \mathrm{E}-12$ & $8.4 \mathrm{E}-12$ & $6.1 \mathrm{E}-11$ & 1. $2 \mathrm{E}-10$ \\
\hline I 125 & Class: D & & $1.19 \mathrm{E}-\odot 8$ & & & & & \\
\hline & 1 & Yr: & & $3.4 \mathrm{E}-11$ & $8.2 \mathrm{E}-11$ & 1. $8 \mathrm{E}-11$ & 2. 3E- -7 & $1.1 \mathrm{E}-08$ \\
\hline & 50 & Yr: & & $3.4 \mathrm{E}-11$ & 8. $2 \mathrm{E}-11$ & 1. $8 \mathrm{E}-11$ & 2. $3 \mathrm{E}-07$ & 1. $1 \mathrm{E}-08$ \\
\hline I 129 & Class: D & & $8.92 \mathrm{E}-\odot 9$ & & & & & \\
\hline & 1 & Yr: & & $9.1 \mathrm{E}-11$ & 1. $5 \mathrm{E}-10$ & $5.5 \mathrm{E}-11$ & 1. $3 E-06$ & $6.7 \mathrm{E}-08$ \\
\hline & 50 & Yr: & & $9.1 \mathrm{E}-11$ & $1.5 \mathrm{E}-10$ & $5.5 \mathrm{E}-11$ & 1. $3 E-06$ & $6.7 \mathrm{E}-08$ \\
\hline I 130 & Class: D & & $3.05 \mathrm{E}-06$ & & & & & \\
\hline
\end{tabular}




\begin{tabular}{|c|c|c|c|c|c|c|c|c|}
\hline & 1 & Yr: & & $9.9 \mathrm{E}-11$ & $6.3 \mathrm{E}-11$ & $5.7 \mathrm{E}-11$ & $3.1 \mathrm{E}-08$ & 1. $7 \mathrm{E}-\odot 9$ \\
\hline & 50 & Yr: & & $9.9 \mathrm{E}-11$ & $6.3 \mathrm{E}-11$ & $5.7 \mathrm{E}-11$ & $3.1 \mathrm{E}-08$ & 1.7E- -99 \\
\hline \multirow[t]{3}{*}{ I 131} & Class: D & & $5.33 E-07$ & & & & & \\
\hline & 1 & Yr: & & $1.1 \mathrm{E}-10$ & $7.8 \mathrm{E}-11$ & 6. ๑E-11 & $3.7 E-07$ & 1. $9 \mathrm{E}-\odot 8$ \\
\hline & 50 & Yr: & & $1.1 \mathrm{E}-10$ & $7.8 \mathrm{E}-11$ & $6.0 \mathrm{E}-11$ & $3.7 \mathrm{E}-07$ & 1. $9 \mathrm{E}-08$ \\
\hline \multirow[t]{3}{*}{ I 132} & Class: D & & $3.31 \mathrm{E}-06$ & & & & & \\
\hline & 1 & Yr: & & $5.7 \mathrm{E}-11$ & $2.3 E-11$ & $2.1 \mathrm{E}-11$ & $3.4 \mathrm{E}-09$ & $2.2 \mathrm{E}-10$ \\
\hline & 50 & Yr: & & $5.7 \mathrm{E}-11$ & 2. $3 E-11$ & $2.1 \mathrm{E}-11$ & 3. 4E- $\odot 9$ & $2.2 \mathrm{E}-10$ \\
\hline \multirow[t]{3}{*}{ I 133} & Class: D & & $8.70 \mathrm{E}-07$ & & & & & \\
\hline & 1 & Yr: & & $7.1 \mathrm{E}-11$ & $3.8 \mathrm{E}-11$ & $3.5 \mathrm{E}-11$ & 7. 4E-०8 & $3.8 \mathrm{E}-\odot 9$ \\
\hline & 50 & Yr: & & $7.1 \mathrm{E}-11$ & $3.8 \mathrm{E}-11$ & $3.5 \mathrm{E}-11$ & $7.4 \mathrm{E}-\odot 8$ & $3.8 \mathrm{E}-\odot 9$ \\
\hline \multirow[t]{3}{*}{ I 134} & Class: D & & $3.85 E-06$ & & & & & \\
\hline & 1 & Yr: & & $4.4 \mathrm{E}-11$ & $1.1 \mathrm{E}-11$ & 1. $0 \mathrm{E}-11$ & $6.5 \mathrm{E}-10$ & $9.8 \mathrm{E}-11$ \\
\hline & 50 & Yr: & & $4.4 \mathrm{E}-11$ & $1.1 \mathrm{E}-11$ & 1. $0 \mathrm{E}-11$ & $6.5 \mathrm{E}-10$ & $9.8 \mathrm{E}-11$ \\
\hline \multirow[t]{3}{*}{ I 135} & Class: D & & $2.38 \mathrm{E}-06$ & & & & & \\
\hline & 1 & Yr: & & $6.6 \mathrm{E}-11$ & $3.4 \mathrm{E}-11$ & $3.1 \mathrm{E}-11$ & 1.5E-08 & 8. $0 \mathrm{E}-10$ \\
\hline & 50 & Yr: & & $6.6 \mathrm{E}-11$ & $3.4 \mathrm{E}-11$ & $3.1 \mathrm{E}-11$ & $1.5 \mathrm{E}-08$ & 8. $0 \mathrm{E}-10$ \\
\hline \multirow[t]{3}{*}{ XE131M } & NobleGas & & 1.10E-๑8 & & & & & \\
\hline & 1 & Yr: & & $\odot . \odot \mathrm{E}-\odot \odot$ & $\odot . \odot-\odot \odot$ & $\odot . \odot \mathrm{E}-\odot \odot$ & $\odot . \odot E-\odot \odot$ & $\odot . \odot \mathrm{E}-\odot \odot$ \\
\hline & 50 & Yr: & & $\odot . \odot \mathrm{E}-\odot \odot$ & $\odot . \odot \mathrm{E}-\odot \odot$ & $\odot . \odot \mathrm{E}-\odot \odot$ & $\odot . \odot \mathrm{E}-\odot \odot$ & $\odot . \odot \mathrm{E}-\odot \odot$ \\
\hline \multirow[t]{3}{*}{ XE133M } & NobleGas & & 4. . & & & & & \\
\hline & 1 & Yr: & & $\odot . \odot \mathrm{E}-\odot \odot$ & $\odot . \odot \mathrm{E}-\odot \odot$ & $\odot . \odot \mathrm{E}-\odot \odot$ & $\odot . \odot \mathrm{E}-\odot \odot$ & $\odot . \odot \mathrm{E}-\odot \odot$ \\
\hline & 50 & Yr: & & $\odot . \odot \mathrm{E}-\odot \odot$ & $\odot . \odot \mathrm{E}-\odot \odot$ & $\odot . \odot \mathrm{E}-\odot \odot$ & $\odot . \odot \mathrm{E}-\odot \odot$ & $\odot . \odot \mathrm{E}-\odot \odot$ \\
\hline \multirow[t]{3}{*}{ XE133 } & NobleGas & & $4.23 E-\odot 8$ & & & & & \\
\hline & 1 & Yr: & & $\odot . \odot \mathrm{E}-\odot \odot$ & $\odot . \odot \mathrm{E}-\odot \odot$ & $\odot . \odot \mathrm{E}-\odot \odot$ & $\odot . \odot \mathrm{E}-\odot \odot$ & $\odot . \odot \mathrm{E}-\odot \odot$ \\
\hline & 50 & Yr: & & $\odot . \odot \mathrm{E}-\odot \odot$ & $\odot . \odot \mathrm{E}-\odot \odot$ & $\odot . \odot \mathrm{E}-\odot \odot$ & $\odot . \odot \mathrm{E}-\odot \odot$ & $\odot . \odot \mathrm{E}-\odot \odot$ \\
\hline \multirow[t]{3}{*}{ XE135M } & NobleGas & & $5.99 E-07$ & & & & & \\
\hline & 1 & Yr: & & $\odot . \odot \mathrm{E}-\odot \odot$ & $\odot . \odot \mathrm{E}-\odot \odot$ & $\odot . \odot \mathrm{E}-\odot \odot$ & $\odot . \odot \mathrm{E}-\odot \odot$ & $\odot . \odot \mathrm{E}-\odot \odot$ \\
\hline & 50 & Yr: & & $\odot . \odot \mathrm{E}-\odot \odot$ & $\odot . \odot \mathrm{E}-\odot \odot$ & $\odot . \odot \mathrm{E}-\odot \odot$ & $\odot . \odot \mathrm{E}-\odot \odot$ & $\odot . \odot \mathrm{E}-\odot \odot$ \\
\hline XE135 & NobleGas & & $3.5 \odot E-\odot 7$ & & & & & \\
\hline & 1 & Yr: & & $\odot . \odot \mathrm{E}-\odot \odot$ & $\odot . \odot E-\odot \odot$ & $\odot . \odot \mathrm{E}-\odot \odot$ & $\odot . \odot \mathrm{E}-\odot \odot$ & $\odot . \odot \mathrm{E}-\odot \odot$ \\
\hline & 50 & Yr: & & $\odot . \odot \mathrm{E}-\odot \odot$ & $\odot . \odot \mathrm{E}-\odot \odot$ & $\odot . \odot \mathrm{E}-\odot \odot$ & $\odot . \odot E-\odot \odot$ & $\odot .0 \mathrm{E}-\odot \odot$ \\
\hline XE137 & NobleGas & & $4.34 \mathrm{E}-07$ & & & & & \\
\hline & 1 & Yr: & & $\odot .0 \mathrm{E}-\odot \odot$ & $\odot . \odot E-\odot \odot$ & $\odot . \odot E-\odot \odot$ & $\odot . \odot E-\odot \odot$ & $\odot . \odot \mathrm{E}-\odot \odot$ \\
\hline & 50 & Yr: & & $\odot . \odot \mathrm{E}-\odot \odot$ & $\odot . \odot \mathrm{E}-\odot \odot$ & $\odot . \odot \mathrm{E}-\odot \odot$ & $\odot . \odot \mathrm{E}-\odot \odot$ & $\odot . \odot \mathrm{E}-\odot \odot$ \\
\hline XE138 & NobleGas & & $1.73 E-06$ & & & & & \\
\hline & 1 & Yr: & & $\odot . \odot \mathrm{E}-\odot \odot$ & $\odot . \odot \mathrm{E}-\odot \odot$ & $\odot . \odot \mathrm{E}-\odot \odot$ & $\odot . \odot \mathrm{E}-\odot \odot$ & $\odot . \odot \mathrm{E}-\odot \odot$ \\
\hline & 50 & Yr: & & $\odot . \odot \mathrm{E}-\odot \odot$ & $\odot . \odot \mathrm{E}-\odot \odot$ & $\odot . \odot \mathrm{E}-\odot \odot$ & $\odot . \odot \mathrm{E}-\odot \odot$ & $\odot . \odot \mathrm{E}-\odot \odot$ \\
\hline CS134M & Class: D & & $2.51 \mathrm{E}-08$ & & & & & \\
\hline & 1 & Yr: & & $3.2 \mathrm{E}-11$ & 4. 4E-12 & 3. $6 \mathrm{E}-12$ & $3.8 \mathrm{E}-12$ & $2.5 \mathrm{E}-11$ \\
\hline & 50 & Yr: & & $3.2 \mathrm{E}-11$ & 4. $4 \mathrm{E}-12$ & 3. $6 \mathrm{E}-12$ & $3.8 \mathrm{E}-12$ & $2.5 \mathrm{E}-11$ \\
\hline CS134 & Class: D & & $2.23 \mathrm{E}-06$ & & & & & \\
\hline & 1 & Yr: & & $4.6 \mathrm{E}-09$ & $5.1 \mathrm{E}-09$ & 4. . $\mathrm{E}-\odot 9$ & $5.1 \mathrm{E}-09$ & $5.3 \mathrm{E}-09$ \\
\hline & 50 & Yr: & & $4.6 \mathrm{E}-09$ & $5.1 \mathrm{E}-09$ & 4. . E - $\odot 9$ & $5.1 \mathrm{E}-09$ & $5.3 \mathrm{E}-09$ \\
\hline CS135 & Class: D & & $3.00 \mathrm{E}-10$ & & & & & \\
\hline & 1 & Yr: & & $5.5 E-10$ & $5.4 \mathrm{E}-10$ & $5.4 \mathrm{E}-10$ & $5.4 \mathrm{E}-10$ & $6.1 \mathrm{E}-10$ \\
\hline & 50 & Yr: & & $5.5 E-10$ & $5.4 \mathrm{E}-10$ & $5.4 \mathrm{E}-10$ & $5.4 \mathrm{E}-10$ & $6.1 \mathrm{E}-10$ \\
\hline CS136 & Class: D & & $3.13 E-06$ & & & & & \\
\hline & 1 & Yr: & & $1.5 E-09$ & 1. $6 \mathrm{E}-\odot 9$ & $1.5 \mathrm{E}-\odot 9$ & E- $\odot 9$ & $2.0 \mathrm{E}-\odot 9$ \\
\hline & 50 & Yr: & & 1. 5E-०9 & $1.6 \mathrm{E}-\odot 9$ & 1. 5E-०9 & 1. $6 \mathrm{E}-09$ & 2. $0 \mathrm{E}-\odot 9$ \\
\hline CS137 & Class: D & & $2.93 E-\odot 9$ & & & & & \\
\hline & 1 & Yr: & & 3. ЗE-๑9 & $3.5 E-\odot 9$ & $3.3 E-\odot 9$ & $3.5 E-09$ & $3.8 \mathrm{E}-09$ \\
\hline & 50 & Yr: & & 3. 3E- $\odot 9$ & $3.5 E-\odot 9$ & 3. ЗE-๑9 & $3.5 E-\odot 9$ & $3.8 \mathrm{E}-09$ \\
\hline CS138 & Class: D & & $3.63 E-06$ & & & & & \\
\hline & 1 & Yr: & & $4.5 \mathrm{E}-11$ & $7.9 E-12$ & $7.5 E-12$ & $8.1 \mathrm{E}-12$ & $5.0 \mathrm{E}-11$ \\
\hline & 50 & Yr: & & $4.5 \mathrm{E}-11$ & 7. $9 \mathrm{E}-12$ & $7.5 \mathrm{E}-12$ & $8.1 \mathrm{E}-12$ & $5.0 \mathrm{E}-11$ \\
\hline BA139 & Class: D & & ৪. .4E-๑8 & & & & & \\
\hline & 1 & Yr: & & $3.9 \mathrm{E}-11$ & $3.6 \mathrm{E}-11$ & $2.6 \mathrm{E}-11$ & $2.7 \mathrm{E}-12$ & $6.0 \mathrm{E}-11$ \\
\hline & 50 & Yr: & & $3.9 \mathrm{E}-11$ & $3.6 \mathrm{E}-11$ & $2.6 \mathrm{E}-11$ & 2. $7 \mathrm{E}-12$ & $6.0 \mathrm{E}-11$ \\
\hline BA140 & Class: D & & $2.55 E-07$ & & & & & \\
\hline & 1 & Yr: & & $5.0 \mathrm{E}-10$ & $9.5 E-09$ & $4.7 \mathrm{E}-\odot 9$ & 4. $0 \mathrm{E}-10$ & $2.4 \mathrm{E}-\odot 9$ \\
\hline & 50 & Yr: & & $5.0 \mathrm{E}-10$ & $9.5 E-09$ & 4. 7E- $\odot 9$ & 4. $0 \mathrm{E}-10$ & $2.4 \mathrm{E}-09$ \\
\hline BA141 & Class: D & & $1.24 \mathrm{E}-06$ & & & & & \\
\hline & 1 & Yr: & & $3.2 \mathrm{E}-11$ & 1. $9 \mathrm{E}-11$ & 1. $2 \mathrm{E}-11$ & $E-12$ & $3.8 \mathrm{E}-11$ \\
\hline & 50 & Yr: & & 3. $2 \mathrm{E}-11$ & 1. $9 \mathrm{E}-11$ & 1. $2 \mathrm{E}-11$ & 1. $9 \mathrm{E}-12$ & $3.8 \mathrm{E}-11$ \\
\hline BA142 & Class: D & & $1.53 \mathrm{E}-06$ & & & & & \\
\hline & 1 & Yr: & & $2.4 \mathrm{E}-11$ & $8.2 \mathrm{E}-12$ & $6.6 \mathrm{E}-12$ & $2.1 \mathrm{E}-12$ & $2.8 \mathrm{E}-11$ \\
\hline & 50 & Yr: & & $2.4 \mathrm{E}-11$ & 8. $2 \mathrm{E}-12$ & $6.6 \mathrm{E}-12$ & $2.1 \mathrm{E}-12$ & $2.8 \mathrm{E}-11$ \\
\hline LA140 & Class: D & & $3.50 \mathrm{E}-06$ & & & & & \\
\hline & 1 & Yr: & & $3.2 \mathrm{E}-10$ & $4.7 \mathrm{E}-10$ & $5.8 \mathrm{E}-10$ & $1.6 \mathrm{E}-10$ & 1. $2 \mathrm{E}-09$ \\
\hline & 50 & Yr: & & $3.2 \mathrm{E}-10$ & $4.7 \mathrm{E}-10$ & $5.8 \mathrm{E}-10$ & $1.6 \mathrm{E}-10$ & 1. $2 \mathrm{E}-09$ \\
\hline
\end{tabular}




\begin{tabular}{|c|c|c|c|c|c|c|c|c|}
\hline LA141 & Class: D & & $9.08 \mathrm{E}-\odot 8$ & & & & & \\
\hline & 1 & Yr: & & $5.6 \mathrm{E}-11$ & 1. $3 E-10$ & $5.1 \mathrm{E}-11$ & 1. $7 \mathrm{E}-11$ & $1.4 \mathrm{E}-10$ \\
\hline & 50 & Yr: & & $5.6 \mathrm{E}-11$ & 1. $3 \mathrm{E}-10$ & $5.1 \mathrm{E}-11$ & 1. $7 \mathrm{E}-11$ & 1. $4 \mathrm{E}-10$ \\
\hline LA142 & Class: D & & $4.32 E-\odot 6$ & & & & & \\
\hline & 1 & Yr: & & $5.7 \mathrm{E}-11$ & $2.1 \mathrm{E}-11$ & 2. $3 \mathrm{E}-11$ & 1.7E-11 & $1.1 \mathrm{E}-10$ \\
\hline & $5 \odot$ & Yr: & & $5.7 \mathrm{E}-11$ & $2.1 \mathrm{E}-11$ & 2. $3 \mathrm{E}-11$ & 1.7E-11 & $1.1 \mathrm{E}-10$ \\
\hline CE141 & Class: $\mathrm{Y}$ & & $9.81 \mathrm{E}-\odot 8$ & & & & & \\
\hline & 1 & Yr: & & 4. . $\mathrm{E}-\odot 8$ & 2. $0 \mathrm{E}-10$ & $5.8 \mathrm{E}-11$ & $4.4 \mathrm{E}-11$ & $5.2 \mathrm{E}-\odot 9$ \\
\hline & 50 & Yr: & & 4. $0 \mathrm{E}-\odot 8$ & $2.0 \mathrm{E}-10$ & $5.8 \mathrm{E}-11$ & 4. $4 \mathrm{E}-11$ & $5.2 \mathrm{E}-09$ \\
\hline CE143 & Class: Y & & $3.82 \mathrm{E}-07$ & & & & & \\
\hline & 1 & Yr: & & $6.7 \mathrm{E}-09$ & $2.6 \mathrm{E}-11$ & $2.4 \mathrm{E}-11$ & 1. $2 \mathrm{E}-11$ & 1. $4 \mathrm{E}-\odot 9$ \\
\hline & 50 & Yr: & & $6.7 \mathrm{E}-09$ & $2.6 \mathrm{E}-11$ & $2.4 \mathrm{E}-11$ & 1. $2 \mathrm{E}-11$ & 1. $4 \mathrm{E}-\odot 9$ \\
\hline CE144 & Class: $\mathrm{Y}$ & & $2.41 \mathrm{E}-08$ & & & & & \\
\hline & 1 & Yr: & & $5.7 \mathrm{E}-07$ & $3.5 \mathrm{E}-\odot 9$ & $2.7 \mathrm{E}-\odot 9$ & $2.4 \mathrm{E}-10$ & 7. $3 E-\odot 8$ \\
\hline & 50 & Yr: & & $5.7 \mathrm{E}-07$ & $3.5 \mathrm{E}-09$ & $2.7 \mathrm{E}-09$ & $2.4 \mathrm{E}-10$ & 7. $3 \mathrm{E}-08$ \\
\hline PR143 & Class: $\mathrm{Y}$ & & $6.12 \mathrm{E}-\odot 9$ & & & & & \\
\hline & 1 & Yr: & & $2.5 \mathrm{E}-08$ & 3. $4 \mathrm{E}-12$ & $4.7 \mathrm{E}-12$ & $2.6 \mathrm{E}-14$ & $3.6 \mathrm{E}-\odot 9$ \\
\hline & 50 & Yr: & & $2.5 \mathrm{E}-08$ & $3.4 \mathrm{E}-12$ & $4.7 \mathrm{E}-12$ & $2.6 \mathrm{E}-14$ & $3.6 \mathrm{E}-09$ \\
\hline PR144M & Class: $Y$ & & $6.97 \mathrm{E}-\odot 9$ & & & & & \\
\hline & 1 & Yr: & & 4. $0 \mathrm{E}-11$ & $1.5 \mathrm{E}-14$ & $1.8 \mathrm{E}-14$ & $8.7 \mathrm{E}-15$ & $5.1 \mathrm{E}-12$ \\
\hline & 50 & Yr: & & 4. $0 \mathrm{E}-11$ & 1. $5 \mathrm{E}-14$ & 1. $8 \mathrm{E}-14$ & $8.7 \mathrm{E}-15$ & $5.1 \mathrm{E}-12$ \\
\hline PR144 & Class: $\mathrm{Y}$ & & $8.36 \mathrm{E}-\odot 8$ & & & & & \\
\hline & 1 & Yr: & & $7.6 \mathrm{E}-11$ & $2.4 \mathrm{E}-14$ & $2.5 \mathrm{E}-14$ & $2.5 \mathrm{E}-14$ & 3. $3 \mathrm{E}-11$ \\
\hline & 50 & Yr: & & $7.6 \mathrm{E}-11$ & $2.4 \mathrm{E}-14$ & $2.5 \mathrm{E}-14$ & $2.5 \mathrm{E}-14$ & 3. $3 E-11$ \\
\hline ND147 & Class: $\mathrm{Y}$ & & $1.81 \mathrm{E}-07$ & & & & & \\
\hline & 1 & Yr: & & $2.4 \mathrm{E}-08$ & 1. $3 E-10$ & $5.1 \mathrm{E}-11$ & 3. $1 \mathrm{E}-11$ & $3.5 E-\odot 9$ \\
\hline & 50 & Yr: & & $2.4 \mathrm{E}-08$ & 1. $3 \mathrm{E}-10$ & $5.1 \mathrm{E}-11$ & $3.1 \mathrm{E}-11$ & $3.5 E-\odot 9$ \\
\hline PM147 & Class: Y & & $2.73 \mathrm{E}-10$ & & & & & \\
\hline & 1 & Yr: & & $5.3 E-08$ & $5.2 E-\odot 9$ & $5.6 \mathrm{E}-10$ & $6.4 \mathrm{E}-14$ & $6.7 \mathrm{E}-\odot 9$ \\
\hline & 50 & Yr: & & 5. 3E- - & $5.2 E-\odot 9$ & $5.6 \mathrm{E}-10$ & $6.4 \mathrm{E}-14$ & $6.7 \mathrm{E}-09$ \\
\hline PM148M & Class: $\mathrm{Y}$ & & $2.84 \mathrm{E}-\odot 6$ & & & & & \\
\hline & 1 & Yr: & & $5.4 \mathrm{E}-\odot 8$ & 1. $3 \mathrm{E}-\odot 9$ & 1. $3 \mathrm{E}-\odot 9$ & 1. $2 \mathrm{E}-09$ & $8.4 \mathrm{E}-\odot 9$ \\
\hline & 50 & Yr: & & 5. 4E- - & 1. 3E- $\odot 9$ & 1. $3 E-\odot 9$ & 1. $2 \mathrm{E}-\odot 9$ & $8.4 \mathrm{E}-09$ \\
\hline PM148 & Class: $\mathrm{Y}$ & & $8.70 \mathrm{E}-07$ & & & & & \\
\hline & 1 & Yr: & & 1. $8 \mathrm{E}-\odot 8$ & $7.5 \mathrm{E}-11$ & $9.6 \mathrm{E}-11$ & $6.4 \mathrm{E}-11$ & $3.7 \mathrm{E}-09$ \\
\hline & 50 & Yr: & & 1. $8 \mathrm{E}-08$ & $7.5 \mathrm{E}-11$ & $9.6 \mathrm{E}-11$ & $6.4 \mathrm{E}-11$ & $3.7 \mathrm{E}-09$ \\
\hline PM149 & Class: $\mathrm{Y}$ & & $2.24 \mathrm{E}-\odot 8$ & & & & & \\
\hline & 1 & Yr: & & $5.8 \mathrm{E}-09$ & 1. $8 \mathrm{E}-12$ & $2.2 \mathrm{E}-12$ & $6.6 \mathrm{E}-13$ & 1. $2 \mathrm{E}-09$ \\
\hline & 50 & Yr: & & $5.8 E-09$ & $1.8 \mathrm{E}-12$ & $2.2 \mathrm{E}-12$ & $6.6 \mathrm{E}-13$ & 1. $2 \mathrm{E}-09$ \\
\hline PM151 & Class: $\mathrm{Y}$ & & $4.42 \mathrm{E}-07$ & & & & & \\
\hline & 1 & Yr: & & $3.6 \mathrm{E}-09$ & $2.4 \mathrm{E}-11$ & $2.4 \mathrm{E}-11$ & 1. $2 \mathrm{E}-11$ & $8.1 \mathrm{E}-10$ \\
\hline & 50 & Yr: & & $3.6 \mathrm{E}-09$ & $2.4 \mathrm{E}-11$ & $2.4 \mathrm{E}-11$ & 1. $2 \mathrm{E}-11$ & $8.1 \mathrm{E}-10$ \\
\hline SM147 & Class: W & & 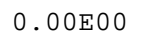 & & & & & \\
\hline & 1 & Yr: & & $3.8 \mathrm{E}-07$ & 1. $3 E-05$ & 1. $3 E-06$ & $2.8 \mathrm{E}-11$ & $1.1 \mathrm{E}-05$ \\
\hline & 50 & Yr: & & $3.8 \mathrm{E}-07$ & 1. $3 E-\odot 5$ & 1. $3 E-\odot 6$ & $2.8 \mathrm{E}-11$ & $1.1 \mathrm{E}-05$ \\
\hline SM151 & Class: W & & $7.79 \mathrm{E}-13$ & & & & & \\
\hline & 1 & Yr: & & 4. $6 \mathrm{E}-09$ & -07 & $E-\odot 8$ & $E-13$ & 4. $5 \mathrm{E}-09$ \\
\hline & 50 & Yr: & & $4.6 \mathrm{E}-09$ & $1.1 \mathrm{E}-07$ & 1. $0 \mathrm{E}-\odot 8$ & $2.6 \mathrm{E}-13$ & $4.5 E-\odot 9$ \\
\hline SM153 & Class: W & & $6.46 \mathrm{E}-08$ & & & & & \\
\hline & 1 & Yr: & & $=-09$ & -10 & $=-11$ & $E-12$ & $E-\odot 9$ \\
\hline & 50 & Yr: & & 5. 3E- $\odot 9$ & $1.1 \mathrm{E}-10$ & $E-11$ & $E-12$ & 1. $\odot E-\odot 9$ \\
\hline EU152 & Class: W & & $1.67 \mathrm{E}-\odot 6$ & & & & & \\
\hline & 1 & Yr: & & $8.2 \mathrm{E}-08$ & 2. $\odot E-07$ & $8.2 \mathrm{E}-08$ & $9.2 \mathrm{E}-09$ & $4.9 E-08$ \\
\hline & 50 & Yr: & & $8.2 \mathrm{E}-08$ & $2 . \odot E-\odot 7$ & $8.2 \mathrm{E}-\odot 8$ & $9.2 \mathrm{E}-09$ & $4.9 \mathrm{E}-08$ \\
\hline EU154 & Class: W & & $1.82 \mathrm{E}-06$ & & & & & \\
\hline & 1 & Yr: & & $E-07$ & -07 & -07 & & $=-08$ \\
\hline & 50 & Yr: & & 1. $3 \mathrm{E}-07$ & -07 & $-\odot 7$ & $\odot 9$ & E- ๑8 \\
\hline EU155 & Class: W & & $6.78 \mathrm{E}-\odot 8$ & & & & & \\
\hline & 1 & Yr: & & $2.6 \mathrm{E}-08$ & 1. $3 \mathrm{E}-07$ & $1.5 \mathrm{E}-\odot 8$ & $3.4 \mathrm{E}-10$ & $9.2 \mathrm{E}-09$ \\
\hline & 50 & Yr: & & $2.6 \mathrm{E}-\odot 8$ & 1. $3 \mathrm{E}-\odot 7$ & 1. $5 \mathrm{E}-\odot 8$ & $3.4 \mathrm{E}-10$ & $9.2 \mathrm{E}-\odot 9$ \\
\hline EU156 & Class: W & & $2.02 \mathrm{E}-06$ & & & & & \\
\hline & 1 & Yr: & & -08 & -09 & $=-\odot 9$ & -10 & BE-०9 \\
\hline & 50 & Yr: & & $3.1 \mathrm{E}-08$ & $2.1 \mathrm{E}-\odot 9$ & 1. $1 \mathrm{E}-\odot 9$ & $3.2 \mathrm{E}-10$ & $5.3 E-09$ \\
\hline GD153 & Class: D & & $9.84 \mathrm{E}-\odot 8$ & & & & & \\
\hline & 1 & Yr: & & 1. $2 \mathrm{E}-09$ & 7. .9E-०8 & $9.9 \mathrm{E}-\odot 9$ & $2.9 \mathrm{E}-10$ & $3.9 \mathrm{E}-09$ \\
\hline & 50 & Yr: & & 1. $2 \mathrm{E}-\odot 9$ & $7.9 \mathrm{E}-\odot 8$ & $9.9 \mathrm{E}-\odot 9$ & $2.9 \mathrm{E}-10$ & $3.9 \mathrm{E}-09$ \\
\hline TB160 & Class: W & & $1.64 \mathrm{E}-06$ & & & & & \\
\hline & 1 & Yr: & & $6.2 \mathrm{E}-\odot 8$ & & & & ๑E - ๑8 \\
\hline & 50 & Yr: & & $6.2 \mathrm{E}-08$ & 2. $2 \mathrm{E}-\odot 8$ & 5. $2 \mathrm{E}-\odot 9$ & $9.0 \mathrm{E}-1 \odot$ & 1. $\odot \mathrm{E}-\odot 8$ \\
\hline H0166M & Class: W & & $2.48 \mathrm{E}-06$ & & & & & \\
\hline & 1 & Yr: & & 1. $4 \mathrm{E}-07$ & $7.1 \mathrm{E}-07$ & $1.6 \mathrm{E}-07$ & $2.2 \mathrm{E}-\odot 8$ & 1. $3 \mathrm{E}-07$ \\
\hline
\end{tabular}




\begin{tabular}{|c|c|c|c|c|c|c|c|c|}
\hline \multirow{3}{*}{ W 181} & 50 & Yr: & & 1. $4 \mathrm{E}-\odot 7$ & $7.1 \mathrm{E}-07$ & $1.6 \mathrm{E}-\odot 7$ & $2.2 \mathrm{E}-\odot 8$ & 1. $3 \mathrm{E}-07$ \\
\hline & Class: D & & $3.69 \mathrm{E}-\odot 8$ & & & & & \\
\hline & 1 & Yr: & & $9.0 \mathrm{E}-12$ & $9.1 \mathrm{E}-11$ & $3.2 \mathrm{E}-11$ & 4. $7 \mathrm{E}-12$ & $5.9 \mathrm{E}-11$ \\
\hline & 50 & Yr: & & $9.0 \mathrm{E}-12$ & $9.1 \mathrm{E}-11$ & $3.2 \mathrm{E}-11$ & 4. 7E-12 & $5.9 \mathrm{E}-11$ \\
\hline \multirow[t]{3}{*}{ W 187} & Class: D & & $6.72 \mathrm{E}-07$ & & & & & \\
\hline & 1 & Yr: & & $5.9 \mathrm{E}-11$ & $9.9 \mathrm{E}-11$ & $4.8 \mathrm{E}-11$ & $2.2 \mathrm{E}-11$ & $4.3 \mathrm{E}-10$ \\
\hline & 50 & Yr: & & $5.9 \mathrm{E}-11$ & $9.9 \mathrm{E}-11$ & $4.8 \mathrm{E}-11$ & 2. $2 \mathrm{E}-11$ & $4.3 E-10$ \\
\hline \multirow[t]{3}{*}{ W 185} & Class: D & & $1.57 \mathrm{E}-09$ & & & & & \\
\hline & 1 & Yr: & & $2.9 \mathrm{E}-11$ & $2.3 \mathrm{E}-10$ & $1.1 \mathrm{E}-10$ & $5.3 \mathrm{E}-12$ & $2.7 \mathrm{E}-10$ \\
\hline & 50 & Yr: & & $2.9 \mathrm{E}-11$ & $2.3 \mathrm{E}-10$ & $1.1 \mathrm{E}-10$ & $5.3 \mathrm{E}-12$ & $2.7 \mathrm{E}-10$ \\
\hline \multirow{3}{*}{ RE187 } & Class: W & & $\odot . \odot \odot Е \odot \odot$ & & & & & \\
\hline & 1 & Yr: & & $6.7 \mathrm{E}-11$ & 3. $2 \mathrm{E}-13$ & $3.2 \mathrm{E}-13$ & $9.9 \mathrm{E}-12$ & 1. $2 \mathrm{E}-11$ \\
\hline & 50 & Yr: & & $6.7 \mathrm{E}-11$ & 3. $2 \mathrm{E}-13$ & $3.2 \mathrm{E}-13$ & $9.9 \mathrm{E}-12$ & 1. $2 \mathrm{E}-11$ \\
\hline \multirow[t]{3}{*}{ IR192 } & Class: Y & & $1.14 \mathrm{E}-06$ & & & & & \\
\hline & 1 & Yr: & & $6.8 \mathrm{E}-08$ & $8.7 \mathrm{E}-10$ & $8.4 \mathrm{E}-10$ & $8.3 E-10$ & $9.5 \mathrm{E}-\odot 9$ \\
\hline & 50 & Yr: & & $6.8 \mathrm{E}-08$ & $8.7 \mathrm{E}-10$ & $8.4 \mathrm{E}-10$ & $8.3 \mathrm{E}-10$ & $9.5 E-\odot 9$ \\
\hline \multirow[t]{3}{*}{ HG2O3 } & Class: D & & $3.28 \mathrm{E}-07$ & & & & & \\
\hline & 1 & Yr: & & $5.7 \mathrm{E}-10$ & $6.6 \mathrm{E}-10$ & $5.7 \mathrm{E}-10$ & $5.7 \mathrm{E}-10$ & $9.0 \mathrm{E}-10$ \\
\hline & 50 & Yr: & & $5.7 \mathrm{E}-10$ & $6.6 \mathrm{E}-10$ & $5.7 \mathrm{E}-10$ & $5.7 \mathrm{E}-10$ & $9.0 \mathrm{E}-10$ \\
\hline \multirow[t]{3}{*}{ RN222 } & NobleGas & & $5.61 \mathrm{E}-10$ & & & & & \\
\hline & 1 & Yr: & & $\odot . \odot \mathrm{E}-\odot \odot$ & $\odot . \odot \mathrm{E}-\odot \odot$ & $\odot . \odot-\odot \odot$ & $\odot . \odot \mathrm{E}-\odot \odot$ & $\odot . \odot \mathrm{E}-\odot \odot$ \\
\hline & 50 & Yr: & & $\odot . \odot \mathrm{E}-\odot \odot$ & $\odot . \odot \mathrm{E}-\odot \odot$ & $\odot . \odot \mathrm{E}-\odot \odot$ & $\odot . \odot \mathrm{E}-\odot \odot$ & $\odot . \odot \mathrm{E}-\odot \odot$ \\
\hline \multirow[t]{3}{*}{ TH227 } & Class: Y & & $1.40 \mathrm{E}-07$ & & & & & \\
\hline & 1 & Yr: & & 1. $2 \mathrm{E}-04$ & $1.8 \mathrm{E}-07$ & 1.7E-๑8 & $4.7 \mathrm{E}-10$ & 1. $4 \mathrm{E}-05$ \\
\hline & 50 & Yr: & & 1. $2 E-\odot 4$ & $1.8 E-07$ & 1. $7 \mathrm{E}-\odot 8$ & $4.7 \mathrm{E}-10$ & 1. $4 \mathrm{E}-05$ \\
\hline \multirow[t]{3}{*}{ TH228 } & Class: Y & & $2.56 \mathrm{E}-\odot 9$ & & & & & \\
\hline & 1 & Yr: & & $4.5 E-\odot 4$ & $3.0 \mathrm{E}-05$ & $3 . \odot E-\odot 6$ & 7. 3E- - 88 & $5.5 E-05$ \\
\hline & 50 & Yr: & & $4.5 E-04$ & $3.0 \mathrm{E}-05$ & $3 . \odot E-\odot 6$ & 7. 3E-๑8 & $5.5 E-\odot 5$ \\
\hline TH229 & Class: Y & & $1.06 \mathrm{E}-07$ & & & & & \\
\hline & 1 & Yr: & & $6.3 \mathrm{E}-04$ & $4.8 E-\odot 4$ & $2.7 \mathrm{E}-\odot 5$ & $1.8 \mathrm{E}-06$ & $8.7 \mathrm{E}-05$ \\
\hline & 50 & Yr: & & $6.3 E-04$ & $4.8 E-\odot 4$ & $2.7 \mathrm{E}-05$ & $1.8 \mathrm{E}-06$ & $8.7 E-05$ \\
\hline TH230 & Class: Y & & $4.70 \mathrm{E}-10$ & & & & & \\
\hline & 1 & Yr: & & $9.9 \mathrm{E}-05$ & $2.4 \mathrm{E}-\odot 4$ & $1.1 \mathrm{E}-05$ & $3.4 \mathrm{E}-07$ & 1.7E- 05 \\
\hline & 50 & Yr: & & $9.9 E-05$ & $2.4 \mathrm{E}-04$ & 1. 1E- 05 & $3.4 \mathrm{E}-07$ & 1.7E-05 \\
\hline TH231 & Class: Y & & 1. 45E- -88 & & & & & \\
\hline & 1 & Yr: & & $2.9 E-\odot 9$ & $5.4 \mathrm{E}-11$ & $3.7 \mathrm{E}-12$ & $6.2 \mathrm{E}-13$ & $5.3 E-10$ \\
\hline & 50 & Yr: & & $2.9 \mathrm{E}-\odot 9$ & $5.4 \mathrm{E}-11$ & $3.7 \mathrm{E}-12$ & $6.2 \mathrm{E}-13$ & $5.3 \mathrm{E}-10$ \\
\hline TH232 & Class: Y & & $2.29 \mathrm{E}-10$ & & & & & \\
\hline & 1 & Yr: & & 1. $7 \mathrm{E}-04$ & $2.6 \mathrm{E}-04$ & 1. 4E-०5 & $9.5 \mathrm{E}-07$ & $2.7 \mathrm{E}-05$ \\
\hline & 50 & Yr: & & 1. $7 \mathrm{E}-04$ & $2.6 E-04$ & $1.4 \mathrm{E}-05$ & $9.5 E-07$ & $2.7 \mathrm{E}-05$ \\
\hline TH234 & Class: Y & & $9.30 \mathrm{E}-\odot 9$ & & & & & \\
\hline & 1 & Yr: & & $7.9 \mathrm{E}-08$ & 1. $4 \mathrm{E}-10$ & 1. $0 \mathrm{E}-10$ & 1. $2 \mathrm{E}-11$ & $1.2 \mathrm{E}-\odot 8$ \\
\hline & 50 & Yr: & & $7.9 \mathrm{E}-\odot 8$ & 1. $4 \mathrm{E}-10$ & 1. $\odot \mathrm{E}-1 \odot$ & 1. $2 \mathrm{E}-11$ & 1. $2 \mathrm{E}-\odot 8$ \\
\hline RA223 & Class: W & & $1.73 \mathrm{E}-07$ & & & & & \\
\hline & 1 & Yr: & & $8.2 \mathrm{E}-05$ & $3.8 \mathrm{E}-06$ & $2.3 \mathrm{E}-\odot 7$ & $6.4 \mathrm{E}-09$ & $9.9 \mathrm{E}-06$ \\
\hline & 50 & Yr: & & $8.2 E-05$ & $3.8 \mathrm{E}-06$ & 2. $3 E-\odot 7$ & $6.4 \mathrm{E}-09$ & $9.9 \mathrm{E}-06$ \\
\hline RA224 & Class: W & & $1.36 \mathrm{E}-08$ & & & & & \\
\hline & 1 & Yr: & & $3.2 E-\odot 5$ & 1. $9 E-\odot 6$ & 1. 1E-๑7 & 4. $7 \mathrm{E}-\odot 9$ & $3.9 E-06$ \\
\hline & 50 & Yr: & & $3.2 E-05$ & 1. $9 \mathrm{E}-06$ & 1. 1E- - 7 & 4. 7E- - 9 & $3.9 E-06$ \\
\hline RA225 & Class: W & & $7.60 \mathrm{E}-\odot 9$ & & & & & \\
\hline & 1 & Yr: & & $6.9 \mathrm{E}-05$ & $6.8 \mathrm{E}-06$ & $5.5 \mathrm{E}-\odot 7$ & $9.7 \mathrm{E}-\odot 9$ & $8.4 \mathrm{E}-06$ \\
\hline & 50 & Yr: & & $6.9 \mathrm{E}-05$ & $6.8 E-06$ & $5.5 E-07$ & $9.7 \mathrm{E}-09$ & $8.4 \mathrm{E}-06$ \\
\hline RA226 & Class: W & & 8. . 96E-๑9 & & & & & \\
\hline & 1 & Yr: & & $3.8 \mathrm{E}-05$ & 1. $5 \mathrm{E}-\odot 5$ & $9.2 \mathrm{E}-\odot 7$ & $3.2 \mathrm{E}-\odot 8$ & $4.9 E-06$ \\
\hline & 50 & Yr: & & $3.8 E-05$ & $1.5 \mathrm{E}-05$ & $9.2 \mathrm{E}-07$ & $3.2 \mathrm{E}-08$ & $4.9 E-06$ \\
\hline RA228 & Class: W & & $\odot . \odot \odot Е \odot \odot$ & & & & & \\
\hline & 1 & Yr: & & 1. $4 \mathrm{E}-05$ & 1. $1 \mathrm{E}-04$ & 1. $0 \mathrm{E}-05$ & $2.9 \mathrm{E}-07$ & $4.6 \mathrm{E}-06$ \\
\hline & 50 & Yr: & & 1. $4 \mathrm{E}-05$ & 1. $1 \mathrm{E}-04$ & 1. $0 \mathrm{E}-05$ & $2.9 \mathrm{E}-07$ & $4.6 E-06$ \\
\hline PB210 & Class: D & & 1. 42E- 09 & & & & & \\
\hline & 1 & Yr: & & $2.7 \mathrm{E}-07$ & $3.1 \mathrm{E}-05$ & $4.6 \mathrm{E}-06$ & $2.7 \mathrm{E}-07$ & 1. $4 \mathrm{E}-06$ \\
\hline & 50 & Yr: & & $2.7 \mathrm{E}-07$ & $3.1 \mathrm{E}-05$ & $4.6 E-06$ & $2.7 \mathrm{E}-07$ & 1. $4 \mathrm{E}-\odot 6$ \\
\hline PB212 & Class: D & & $1.97 \mathrm{E}-07$ & & & & & \\
\hline & 1 & Yr: & & 1. 3E- - 8 & $3.4 \mathrm{E}-07$ & $2.5 E-\odot 8$ & $6.4 \mathrm{E}-\odot 9$ & $3.6 E-\odot 8$ \\
\hline & 50 & Yr: & & 1. 3E-०8 & $3.4 \mathrm{E}-07$ & $2.5 \mathrm{E}-\odot 8$ & $6.4 \mathrm{E}-09$ & $3.6 \mathrm{E}-08$ \\
\hline BI210 & Class: W & & $8.14 \mathrm{E}-09$ & & & & & \\
\hline & 1 & Yr: & & 1.1E-06 & $8.7 \mathrm{E}-11$ & $8.7 \mathrm{E}-11$ & $8.7 \mathrm{E}-11$ & 1. $3 E-07$ \\
\hline & 50 & Yr: & & 1.1E-06 & $8.7 \mathrm{E}-11$ & $8.7 \mathrm{E}-11$ & $8.7 \mathrm{E}-11$ & 1. $3 \mathrm{E}-07$ \\
\hline BI212 & Class: W & & $2.83 E-07$ & & & & & \\
\hline & 1 & Yr: & & $2.5 \mathrm{E}-07$ & $4.3 E-11$ & $4.4 \mathrm{E}-11$ & 4. $3 E-11$ & 4. 4E-๑8 \\
\hline & 50 & Yr: & & $2.5 E-07$ & 4. $3 \mathrm{E}-11$ & $4.4 \mathrm{E}-11$ & 4. $3 \mathrm{E}-11$ & $4.4 \mathrm{E}-08$ \\
\hline P0210 & Class: W & & $1.23 \mathrm{E}-11$ & & & & & \\
\hline
\end{tabular}




\begin{tabular}{|c|c|c|c|c|c|c|c|c|}
\hline & 1 & Yr: & & $3.5 \mathrm{E}-05$ & $7.9 \mathrm{E}-07$ & $1.1 \mathrm{E}-06$ & $9.6 \mathrm{E}-\odot 8$ & $4.6 \mathrm{E}-06$ \\
\hline & 50 & Yr: & & $3.5 E-05$ & $7.9 \mathrm{E}-07$ & $1.1 \mathrm{E}-06$ & $9.6 \mathrm{E}-\odot 8$ & $4.6 \mathrm{E}-06$ \\
\hline \multirow[t]{3}{*}{ U 232} & Class: $\mathrm{Y}$ & & $3.72 \mathrm{E}-10$ & & & & & \\
\hline & 1 & Yr: & & $3.5 E-04$ & $4.5 E-05$ & $3.9 E-06$ & 1. $9 \mathrm{E}-07$ & 4. $3 E-05$ \\
\hline & 50 & Yr: & & $3.5 E-\odot 4$ & $4.5 E-\odot 5$ & $3.9 E-\odot 6$ & 1. $9 \mathrm{E}-07$ & 4. $3 E-05$ \\
\hline \multirow[t]{3}{*}{ U 233} & Class: Y & & $4.48 \mathrm{E}-10$ & & & & & \\
\hline & 1 & Yr: & & 1. $0 \mathrm{E}-\odot 4$ & 1. 3E-०6 & $8.4 \mathrm{E}-08$ & 1.9E-๑8 & 1. $2 \mathrm{E}-05$ \\
\hline & 50 & Yr: & & 1. $0 \mathrm{E}-\odot 4$ & 1. $3 \mathrm{E}-06$ & $8.4 \mathrm{E}-08$ & 1. $9 \mathrm{E}-08$ & $1.2 \mathrm{E}-05$ \\
\hline \multirow[t]{3}{*}{ U 234} & Class: Y & & $1.93 \mathrm{E}-10$ & & & & & \\
\hline & 1 & Yr: & & 1. $\odot E-\odot 4$ & $9.2 \mathrm{E}-07$ & $6.6 \mathrm{E}-08$ & 1. $7 \mathrm{E}-\odot 8$ & 1. $2 \mathrm{E}-05$ \\
\hline & 50 & Yr: & & 1. $0 \mathrm{E}-\odot 4$ & $9.2 \mathrm{E}-07$ & $6.6 \mathrm{E}-08$ & 1.7E-๑8 & 1. $2 \mathrm{E}-05$ \\
\hline \multirow[t]{3}{*}{ U 235} & Class: Y & & $2.04 \mathrm{E}-07$ & & & & & \\
\hline & 1 & Yr: & & $9.1 \mathrm{E}-05$ & $8.6 \mathrm{E}-07$ & $6.4 \mathrm{E}-08$ & 1. $8 \mathrm{E}-\odot 8$ & $1.1 \mathrm{E}-05$ \\
\hline & 50 & Yr: & & $9.1 \mathrm{E}-05$ & $8.6 \mathrm{E}-07$ & $6.4 \mathrm{E}-08$ & $1.8 \mathrm{E}-08$ & 1. $1 \mathrm{E}-05$ \\
\hline \multirow[t]{3}{*}{ U 236} & Class: Y & & $1.22 \mathrm{E}-10$ & & & & & \\
\hline & 1 & Yr: & & $9.4 \mathrm{E}-05$ & $8.7 \mathrm{E}-07$ & $6.3 \mathrm{E}-08$ & $1.6 \mathrm{E}-08$ & 1.1E- 05 \\
\hline & 50 & Yr: & & $9.4 \mathrm{E}-05$ & $8.7 \mathrm{E}-07$ & $6.3 E-\odot 8$ & 1. $6 \mathrm{E}-08$ & 1.1E-05 \\
\hline \multirow[t]{3}{*}{ U 237} & Class: $\mathrm{Y}$ & & $1.67 \mathrm{E}-07$ & & & & & \\
\hline & 1 & Yr: & & 1. $9 \mathrm{E}-\odot 8$ & $6.6 \mathrm{E}-11$ & $3.0 \mathrm{E}-11$ & $2.1 \mathrm{E}-11$ & 2.7E-०9 \\
\hline & 50 & Yr: & & 1. $9 \mathrm{E}-\odot 8$ & $6.6 \mathrm{E}-11$ & 3. $0 \mathrm{E}-11$ & $2.1 \mathrm{E}-11$ & $2.7 \mathrm{E}-\odot 9$ \\
\hline \multirow[t]{3}{*}{ U 238} & Class: Y & & $7.92 \mathrm{E}-11$ & & & & & \\
\hline & 1 & Yr: & & $8.6 E-05$ & $8.2 \mathrm{E}-07$ & $6.2 \mathrm{E}-08$ & 1. $5 \mathrm{E}-\odot 8$ & 1. $0 \mathrm{E}-\odot 5$ \\
\hline & 50 & Yr: & & $8.6 \mathrm{E}-05$ & $8.2 \mathrm{E}-07$ & $6.2 \mathrm{E}-08$ & $1.5 \mathrm{E}-08$ & 1. $0 \mathrm{E}-\odot 5$ \\
\hline \multirow[t]{3}{*}{ U 240} & Class: $\mathrm{Y}$ & & $1.85 E-\odot 9$ & & & & & \\
\hline & 1 & Yr: & & $3.9 \mathrm{E}-09$ & 4. $9 \mathrm{E}-11$ & 1. $7 \mathrm{E}-11$ & $7.3 \mathrm{E}-12$ & $1.1 \mathrm{E}-09$ \\
\hline & 50 & Yr: & & $3.9 \mathrm{E}-\odot 9$ & $4.9 \mathrm{E}-11$ & 1. $7 \mathrm{E}-11$ & $7.3 E-12$ & 1. $1 \mathrm{E}-\odot 9$ \\
\hline \multirow[t]{3}{*}{ PA231 } & Class: Y & & 4.95E-०8 & & & & & \\
\hline & 1 & Yr: & & 1. $7 \mathrm{E}-\odot 4$ & $5.5 E-\odot 4$ & $2.5 E-\odot 5$ & $1.5 \mathrm{E}-\odot 6$ & $3.2 E-05$ \\
\hline & 50 & Yr: & & $1.7 \mathrm{E}-\odot 4$ & $5.5 E-\odot 4$ & $2.5 E-\odot 5$ & $1.5 \mathrm{E}-\odot 6$ & $3.2 \mathrm{E}-05$ \\
\hline PA233 & Class: $\mathrm{Y}$ & & $2.70 \mathrm{E}-\odot 7$ & & & & & \\
\hline & 1 & Yr: & & $4.1 \mathrm{E}-08$ & $3.0 \mathrm{E}-10$ & 1. $2 \mathrm{E}-10$ & $9.2 \mathrm{E}-11$ & $5.4 \mathrm{E}-\odot 9$ \\
\hline & 50 & Yr: & & $4.1 \mathrm{E}-08$ & $3.0 \mathrm{E}-10$ & $1.2 \mathrm{E}-10$ & $9.2 \mathrm{E}-11$ & $5.4 \mathrm{E}-09$ \\
\hline PA234 & Class: $\mathrm{Y}$ & & $2.75 \mathrm{E}-\odot 6$ & & & & & \\
\hline & 1 & Yr: & & $2.4 \mathrm{E}-\odot 9$ & $3.4 \mathrm{E}-11$ & $4.2 \mathrm{E}-11$ & $2.3 \mathrm{E}-11$ & $7.4 \mathrm{E}-10$ \\
\hline & 50 & Yr: & & $2.4 \mathrm{E}-09$ & $3.4 \mathrm{E}-11$ & $4.2 \mathrm{E}-11$ & $2.3 E-11$ & $7.4 \mathrm{E}-10$ \\
\hline AC225 & Class: Y & & 2. $\odot 1 E-\odot 8$ & & & & & \\
\hline & 1 & Yr: & & $9.3 \mathrm{E}-05$ & 1. $\odot E-\odot 7$ & $1.6 \mathrm{E}-08$ & 4. $9 \mathrm{E}-09$ & 1.1E-05 \\
\hline & 50 & Yr: & & $9.3 E-05$ & 1. $\odot E-\odot 7$ & $1.6 \mathrm{E}-08$ & 4. $9 \mathrm{E}-09$ & 1.1E-05 \\
\hline AC227 & Class: Y & & $1.62 \mathrm{E}-10$ & & & & & \\
\hline & 1 & Yr: & & $5.3 E-04$ & $1.6 \mathrm{E}-04$ & $1.1 \mathrm{E}-05$ & $9.5 \mathrm{E}-07$ & $6.9 \mathrm{E}-05$ \\
\hline & 50 & Yr: & & 5. $3 E-\odot 4$ & 1. $6 \mathrm{E}-\odot 4$ & $1.1 \mathrm{E}-05$ & $9.5 \mathrm{E}-07$ & $6.9 \mathrm{E}-05$ \\
\hline AC228 & Class: Y & & $1.42 \mathrm{E}-06$ & & & & & \\
\hline & 1 & Yr: & & 1. $7 \mathrm{E}-07$ & 1.1E-๑8 & $1.1 \mathrm{E}-\odot 9$ & $3.8 \mathrm{E}-11$ & 2. $\odot E-\odot 8$ \\
\hline & 50 & Yr: & & $1.7 \mathrm{E}-07$ & 1.1E- -8 & $1.1 \mathrm{E}-\odot 9$ & $3.8 \mathrm{E}-11$ & $2.0 \mathrm{E}-\odot 8$ \\
\hline FR223 & Class: D & & $6.97 \mathrm{E}-08$ & & & & & \\
\hline & 1 & Yr: & & $1.8 \mathrm{E}-09$ & 1.7E-०9 & 1. $7 \mathrm{E}-09$ & 1. $7 \mathrm{E}-\odot 9$ & 1. $9 \mathrm{E}-\odot 9$ \\
\hline & 50 & Yr: & & 1. $8 \mathrm{E}-09$ & 1. 7E-०9 & 1.7E-०9 & 1. 7E-०9 & 1. $9 \mathrm{E}-09$ \\
\hline NP237 & Class: W & & $2.81 \mathrm{E}-08$ & & & & & \\
\hline & 1 & Yr: & & 4. ๑E-๑5 & $6.8 \mathrm{E}-\odot 4$ & $4.2 \mathrm{E}-\odot 5$ & $2.0 E-06$ & $2.2 \mathrm{E}-\odot 5$ \\
\hline & 50 & Yr: & & $4.0 \mathrm{E}-05$ & $6.8 \mathrm{E}-\odot 4$ & $4.2 \mathrm{E}-05$ & 2. $0 \mathrm{E}-06$ & $2.2 \mathrm{E}-05$ \\
\hline NP238 & Class: W & & 8. $07 \mathrm{E}-07$ & & & & & \\
\hline & 1 & Yr: & & 7. 4E-०9 & 4. . $5-\odot 8$ & 3. ०E-๑9 & $=-10$ & $2.5 \mathrm{E}-09$ \\
\hline & 50 & Yr: & & $7.4 \mathrm{E}-\odot 9$ & 4. $5 \mathrm{E}-\odot 8$ & 3. $\odot E-\odot 9$ & $1.6 \mathrm{E}-10$ & 2. $5 \mathrm{E}-09$ \\
\hline NP239 & Class: W & & $2.19 E-07$ & & & & & \\
\hline & 1 & Yr: & & 8. 4E-०9 & $6.9 \mathrm{E}-10$ & $8.8 \mathrm{E}-11$ & $1.5 \mathrm{E}-11$ & 1. $4 \mathrm{E}-09$ \\
\hline & 50 & Yr: & & 8. 4E-०9 & $6.9 \mathrm{E}-10$ & 8. $8 \mathrm{E}-11$ & $1.5 \mathrm{E}-11$ & 1. $4 \mathrm{E}-\odot 9$ \\
\hline PU236 & Class: Y & & $1.48 \mathrm{E}-10$ & & & & & \\
\hline & 1 & Yr: & & 1. $\odot E-\odot 4$ & 4. $\odot E-\odot 5$ & $2.7 \mathrm{E}-\odot 6$ & 1. $1 \mathrm{E}-07$ & 1. $4 \mathrm{E}-05$ \\
\hline & 50 & Yr: & & 1. $0 \mathrm{E}-04$ & 4. $0 \mathrm{E}-05$ & $2.7 \mathrm{E}-06$ & $1.1 \mathrm{E}-07$ & 1. $4 \mathrm{E}-05$ \\
\hline PU237 & Class: Y & & $5.58 \mathrm{E}-08$ & & & & & \\
\hline & 1 & Yr: & & 4. $2 \mathrm{E}-\odot 9$ & $1.1 \mathrm{E}-10$ & $3.9 \mathrm{E}-11$ & $3.5 \mathrm{E}-11$ & $5.9 \mathrm{E}-10$ \\
\hline & 50 & Yr: & & $4.2 E-\odot 9$ & 1. $1 \mathrm{E}-10$ & $3.9 \mathrm{E}-11$ & $3.5 \mathrm{E}-11$ & $5.9 \mathrm{E}-10$ \\
\hline PU238 & Class: Y & & $1.11 \mathrm{E}-10$ & & & & & \\
\hline & 1 & Yr: & & 1. $2 \mathrm{E}-\odot 4$ & 1. $3 E-\odot 4$ & $7.3 E-\odot 6$ & $3.3 E-07$ & 1. $9 \mathrm{E}-05$ \\
\hline & 50 & Yr: & & 1. $2 \mathrm{E}-\odot 4$ & 1. $3 E-\odot 4$ & $7.3 E-06$ & $3.3 E-07$ & 1. $9 \mathrm{E}-05$ \\
\hline PU239 & Class: Y & & $1.10 \mathrm{E}-10$ & & & & & \\
\hline & 1 & Yr: & & $1.1 \mathrm{E}-04$ & $1.5 \mathrm{E}-\odot 4$ & $8.3 E-06$ & $3.8 \mathrm{E}-07$ & 1. $9 \mathrm{E}-05$ \\
\hline & 50 & Yr: & & $1.1 \mathrm{E}-04$ & 1. $5 E-\odot 4$ & $8.3 E-06$ & $3.8 \mathrm{E}-07$ & 1. $9 \mathrm{E}-05$ \\
\hline PU240 & Class: Y & & $1.08 \mathrm{E}-10$ & & & & & \\
\hline & 1 & Yr: & & 1.1E- $\odot 4$ & 1. $5 \mathrm{E}-\odot 4$ & $8.3 E-06$ & $3.8 \mathrm{E}-07$ & $1.9 \mathrm{E}-05$ \\
\hline & 50 & Yr: & & 1. $1 \mathrm{E}-04$ & 1. $5 E-04$ & 8. $3 E-06$ & $3.8 \mathrm{E}-07$ & 1. $9 \mathrm{E}-05$ \\
\hline
\end{tabular}




\begin{tabular}{|c|c|c|c|c|c|c|c|c|}
\hline \multirow[t]{3}{*}{ PU241 } & Class: Y & & $2.00 \mathrm{E}-12$ & & & & & \\
\hline & 1 & Yr: & & $4.6 \mathrm{E}-07$ & $3.5 E-\odot 6$ & 1. $7 \mathrm{E}-\odot 7$ & $8.7 \mathrm{E}-\odot 9$ & 1. $7 \mathrm{E}-07$ \\
\hline & 50 & Yr: & & $4.6 \mathrm{E}-07$ & $3.5 \mathrm{E}-06$ & 1. $7 \mathrm{E}-\odot 7$ & $8.7 \mathrm{E}-09$ & 1. $7 \mathrm{E}-07$ \\
\hline \multirow[t]{3}{*}{ PU242 } & Class: Y & & $9.18 \mathrm{E}-11$ & & & & & \\
\hline & 1 & Yr: & & $1.1 \mathrm{E}-\odot 4$ & 1. $4 \mathrm{E}-\odot 4$ & $7.9 \mathrm{E}-06$ & $3.6 \mathrm{E}-07$ & 1.7E-05 \\
\hline & 50 & Yr: & & 1. $1 \mathrm{E}-04$ & 1. $4 \mathrm{E}-\odot 4$ & $7.9 \mathrm{E}-\odot 6$ & $3.6 \mathrm{E}-07$ & 1. $7 \mathrm{E}-05$ \\
\hline \multirow[t]{3}{*}{ PU243 } & Class: $Y$ & & $3 . \odot 4 \mathrm{E}-\odot 8$ & & & & & \\
\hline & 1 & Yr: & & $6.2 \mathrm{E}-10$ & 1. 3E-11 & 1. $1 \mathrm{E}-12$ & $2.8 \mathrm{E}-13$ & 1. $4 \mathrm{E}-10$ \\
\hline & 50 & Yr: & & $6.2 \mathrm{E}-10$ & 1. 3E-11 & 1. $1 \mathrm{E}-12$ & $2.8 \mathrm{E}-13$ & 1. $4 \mathrm{E}-10$ \\
\hline \multirow[t]{3}{*}{ PU244 } & Class: Y & & $6.56 \mathrm{E}-11$ & & & & & \\
\hline & 1 & Yr: & & $9.7 \mathrm{E}-05$ & 1. $3 E-04$ & 7. $4 \mathrm{E}-06$ & $3.5 E-07$ & 1. $6 \mathrm{E}-05$ \\
\hline & 50 & Yr: & & $9.7 \mathrm{E}-05$ & 1. $3 E-\odot 4$ & $7.4 \mathrm{E}-06$ & $3.5 \mathrm{E}-\odot 7$ & $1.6 \mathrm{E}-05$ \\
\hline \multirow[t]{3}{*}{ AM241 } & Class: W & & $2.13 \mathrm{E}-\odot 8$ & & & & & \\
\hline & 1 & Yr: & & $5.1 \mathrm{E}-05$ & $1.2 \mathrm{E}-03$ & $6.9 \mathrm{E}-05$ & $3.6 \mathrm{E}-06$ & $4.1 \mathrm{E}-05$ \\
\hline & 50 & Yr: & & $5.1 \mathrm{E}-05$ & 1. $2 \mathrm{E}-03$ & $6.9 \mathrm{E}-05$ & $3.6 \mathrm{E}-06$ & $4.1 \mathrm{E}-05$ \\
\hline \multirow[t]{3}{*}{ AM242M } & Class: W & & $7.85 \mathrm{E}-10$ & & & & & \\
\hline & 1 & Yr: & & 1. $2 \mathrm{E}-05$ & 1. $2 \mathrm{E}-03$ & $6.3 E-05$ & $3.6 \mathrm{E}-06$ & $3.4 \mathrm{E}-05$ \\
\hline & 50 & Yr: & & 1. $2 \mathrm{E}-\odot 5$ & 1. $2 \mathrm{E}-\odot 3$ & $6.3 E-05$ & $3.6 \mathrm{E}-\odot 6$ & $3.4 \mathrm{E}-05$ \\
\hline \multirow[t]{3}{*}{ AM242 } & Class: W & & 1.93E-๑8 & & & & & \\
\hline & 1 & Yr: & & 1. $6 \mathrm{E}-\odot 7$ & 1. $4 \mathrm{E}-07$ & 1. $7 \mathrm{E}-\odot 8$ & $1.8 \mathrm{E}-10$ & $2.4 \mathrm{E}-\odot 8$ \\
\hline & 50 & Yr: & & $1.6 \mathrm{E}-07$ & 1. $4 \mathrm{E}-07$ & 1.7E- 08 & $1.8 \mathrm{E}-10$ & $2.4 \mathrm{E}-08$ \\
\hline \multirow[t]{3}{*}{ AM243 } & Class: W & & $5.87 \mathrm{E}-\odot 8$ & & & & & \\
\hline & 1 & Yr: & & $4.8 \mathrm{E}-05$ & $1.2 \mathrm{E}-03$ & $6.8 \mathrm{E}-05$ & $3.6 \mathrm{E}-06$ & 4. $0 \mathrm{E}-05$ \\
\hline & 50 & Yr: & & $4.8 E-05$ & $1.2 \mathrm{E}-03$ & $6.8 \mathrm{E}-05$ & $3.6 \mathrm{E}-06$ & $4.0 \mathrm{E}-05$ \\
\hline \multirow[t]{3}{*}{ CM242 } & Class: W & & $1.27 \mathrm{E}-10$ & & & & & \\
\hline & 1 & Yr: & & $4.8 E-05$ & $4.3 E-05$ & $5.0 \mathrm{E}-06$ & $5.3 E-08$ & 7. 3E- - 06 \\
\hline & 50 & Yr: & & $4.8 \mathrm{E}-05$ & $4.3 E-05$ & $5.0 \mathrm{E}-\odot 6$ & $5.3 E-\odot 8$ & 7. $3 \mathrm{E}-06$ \\
\hline \multirow[t]{3}{*}{ CM243 } & Class: W & & $1.67 \mathrm{E}-07$ & & & & & \\
\hline & 1 & Yr: & & $5.4 \mathrm{E}-05$ & $8.0 E-\odot 4$ & $5.7 \mathrm{E}-05$ & 2. $\odot E-\odot 6$ & $3.2 \mathrm{E}-05$ \\
\hline & 50 & Yr: & & $5.4 \mathrm{E}-05$ & $8.0 E-04$ & $5.7 E-\odot 5$ & 2. $\odot E-\odot 6$ & $3.2 \mathrm{E}-05$ \\
\hline \multirow[t]{3}{*}{ CM244 } & Class: W & & $1.08 \mathrm{E}-10$ & & & & & \\
\hline & 1 & Yr: & & $5.4 \mathrm{E}-05$ & $6.4 \mathrm{E}-04$ & $5.0 \mathrm{E}-05$ & $1.5 \mathrm{E}-06$ & $2.7 \mathrm{E}-05$ \\
\hline & 50 & Yr: & & $5.4 \mathrm{E}-05$ & $6.4 \mathrm{E}-04$ & $5.0 \mathrm{E}-\odot 5$ & 1. $5 \mathrm{E}-\odot 6$ & $2.7 \mathrm{E}-05$ \\
\hline \multirow[t]{3}{*}{ CM245 } & Class: W & & $1.10 \mathrm{E}-\odot 7$ & & & & & \\
\hline & 1 & Yr: & & 4. $9 \mathrm{E}-05$ & 1. $3 E-03$ & 7. $0 \mathrm{E}-05$ & $3.8 \mathrm{E}-\odot 6$ & $4.1 \mathrm{E}-05$ \\
\hline & 50 & Yr: & & 4. $9 \mathrm{E}-\odot 5$ & 1. $3 E-\odot 3$ & 7. $0 \mathrm{E}-\odot 5$ & $3.8 \mathrm{E}-\odot 6$ & $4.1 \mathrm{E}-05$ \\
\hline \multirow[t]{3}{*}{ CM246 } & Class: W & & $9.78 \mathrm{E}-11$ & & & & & \\
\hline & 1 & Yr: & & $4.9 E-05$ & $1.2 \mathrm{E}-\odot 3$ & $6.9 \mathrm{E}-05$ & $3.7 \mathrm{E}-06$ & $4.1 \mathrm{E}-05$ \\
\hline & 50 & Yr: & & $4.9 E-05$ & 1. $2 \mathrm{E}-03$ & $6.9 \mathrm{E}-05$ & $3.7 E-06$ & $4.1 \mathrm{E}-05$ \\
\hline \multirow[t]{3}{*}{ CM247 } & Class: W & & $4.38 E-07$ & & & & & \\
\hline & 1 & Yr: & & $4.3 E-05$ & $1.1 \mathrm{E}-03$ & $6.4 \mathrm{E}-05$ & $3.4 \mathrm{E}-06$ & $3.7 \mathrm{E}-05$ \\
\hline & 50 & Yr: & & 4. $3 E-05$ & $1.1 \mathrm{E}-03$ & $6.4 \mathrm{E}-05$ & $3.4 \mathrm{E}-06$ & $3.7 \mathrm{E}-05$ \\
\hline \multirow[t]{3}{*}{ CM248 } & Class: W & & $7.44 \mathrm{E}-11$ & & & & & \\
\hline & 1 & Yr: & & $6.3 \mathrm{E}-05$ & $7.8 \mathrm{E}-03$ & $6.2 \mathrm{E}-\odot 4$ & $2.2 \mathrm{E}-\odot 8$ & $4.2 E-04$ \\
\hline & 50 & Yr: & & $6.3 \mathrm{E}-05$ & $7.8 \mathrm{E}-03$ & $6.2 \mathrm{E}-\odot 4$ & $2.2 \mathrm{E}-\odot 8$ & $4.2 \mathrm{E}-\odot 4$ \\
\hline \multirow[t]{3}{*}{ CF252 } & Class: W & & $1.15 \mathrm{E}-10$ & & & & & \\
\hline & 1 & Yr: & & $3.5 \mathrm{E}-05$ & $6.6 \mathrm{E}-\odot 4$ & $5.3 E-05$ & 1.1E- $\odot 8$ & $3.5 E-05$ \\
\hline & 50 & Yr: & & $3.5 E-05$ & $6.6 \mathrm{E}-\odot 4$ & $5.3 E-05$ & 1. $1 \mathrm{E}-\odot 8$ & $3.5 E-05$ \\
\hline
\end{tabular}




\section{Appendix A.4}

Age 1825 days

Dose Equiv. Factors for Acute Inhalation and Ext Air Submersion FGR12/13

\begin{tabular}{|c|c|c|c|c|c|c|c|c|}
\hline & & & AIR & Acute & Inhal. Con & itted Dose & Equivale & \\
\hline & & & JBMERSION & & BONE & RED & & EFF DOSE \\
\hline & & & J/Yr per & LUNGS & SURFACE & MARROW & THYROID & EQUIV. \\
\hline & & & $\mathrm{Bq} / \mathrm{m} 3$ & $\mathrm{~Sv} / \mathrm{Bq}$ & $\mathrm{Sv} / \mathrm{Bq}$ & $\mathrm{Sv} / \mathrm{Bq}$ & $\mathrm{Sv} / \mathrm{Bq}$ & $\mathrm{S} v / \mathrm{Bq}$ \\
\hline H 3 & Class: D & & $\odot . \odot \odot Е \odot \odot ~$ & & & & & \\
\hline & 1 & Yr: & & 1.1E-11 & $1.1 \mathrm{E}-11$ & $1.1 \mathrm{E}-11$ & 1.1E-11 & 1. $1 \mathrm{E}-11$ \\
\hline & 50 & Yr: & & 1.1E-11 & 1. $1 \mathrm{E}-11$ & $1.1 \mathrm{E}-11$ & $1.1 \mathrm{E}-11$ & 1. $1 \mathrm{E}-11$ \\
\hline BE10 & Class: $Y$ & & $4.35 E-\odot 9$ & & & & & \\
\hline & 1 & Yr: & & $4.9 \mathrm{E}-07$ & 8. 4E-๑9 & $3.7 \mathrm{E}-\odot 9$ & $1.1 \mathrm{E}-10$ & $6.1 \mathrm{E}-08$ \\
\hline & 50 & Yr: & & $4.9 \mathrm{E}-07$ & $8.4 \mathrm{E}-\odot 9$ & $3.7 \mathrm{E}-\odot 9$ & $1.1 \mathrm{E}-10$ & $6.1 \mathrm{E}-08$ \\
\hline C 14 & Class: D & & $8.20 E-11$ & & & & & \\
\hline & 1 & Yr: & & $3.6 \mathrm{E}-10$ & $3.4 \mathrm{E}-10$ & $3.4 \mathrm{E}-10$ & $3.4 \mathrm{E}-10$ & $3.7 \mathrm{E}-10$ \\
\hline & 50 & Yr: & & $3.6 \mathrm{E}-10$ & $3.4 \mathrm{E}-10$ & $3.4 \mathrm{E}-10$ & $3.4 \mathrm{E}-10$ & $3.7 \mathrm{E}-10$ \\
\hline F 18 & Class: D & & $1.44 \mathrm{E}-06$ & & & & & \\
\hline & 1 & Yr: & & $4.8 \mathrm{E}-11$ & $7.9 \mathrm{E}-11$ & 1. $0 \mathrm{E}-10$ & $6.6 \mathrm{E}-12$ & $9.2 \mathrm{E}-11$ \\
\hline & 50 & Yr: & & $4.8 \mathrm{E}-11$ & $7.9 \mathrm{E}-11$ & 1. $0 \mathrm{E}-10$ & $6.6 \mathrm{E}-12$ & $9.2 \mathrm{E}-11$ \\
\hline NA22 & Class: D & & $3.22 \mathrm{E}-06$ & & & & & \\
\hline & 1 & Yr: & & $2.6 \mathrm{E}-\odot 9$ & $6.2 \mathrm{E}-\odot 9$ & $4.5 E-\odot 9$ & $2.8 \mathrm{E}-\odot 9$ & $3.8 \mathrm{E}-09$ \\
\hline & 50 & Yr: & & $2.6 \mathrm{E}-\odot 9$ & $6.2 \mathrm{E}-\odot 9$ & $4.5 E-\odot 9$ & $2.8 \mathrm{E}-\odot 9$ & $3.8 \mathrm{E}-09$ \\
\hline NA24 & Class: D & & $6.56 \mathrm{E}-06$ & & & & & \\
\hline & 1 & Yr: & & $3.8 \mathrm{E}-10$ & $6.0 \mathrm{E}-10$ & $4.4 \mathrm{E}-10$ & $3.4 \mathrm{E}-10$ & $9.6 \mathrm{E}-10$ \\
\hline & 50 & Yr: & & $3.8 \mathrm{E}-10$ & 6. $0 \mathrm{E}-10$ & $4.4 \mathrm{E}-10$ & $3.4 \mathrm{E}-10$ & $9.6 \mathrm{E}-10$ \\
\hline P 32 & Class: D & & $1.69 \mathrm{E}-08$ & & & & & \\
\hline & 1 & Yr: & & 1. ०E-०9 & $1.1 \mathrm{E}-08$ & $1.6 \mathrm{E}-\odot 8$ & $9.6 \mathrm{E}-10$ & $3.2 \mathrm{E}-\odot 9$ \\
\hline & 50 & Yr: & & 1. $0 \mathrm{E}-09$ & $1.1 \mathrm{E}-08$ & $1.6 \mathrm{E}-08$ & $9.6 \mathrm{E}-10$ & $3.2 \mathrm{E}-09$ \\
\hline P 33 & Class: D & & $4.57 \mathrm{E}-10$ & & & & & \\
\hline & 1 & Yr: & & $1.6 \mathrm{E}-10$ & $1.8 \mathrm{E}-09$ & $9.5 \mathrm{E}-10$ & $1.4 \mathrm{E}-10$ & $3.1 \mathrm{E}-10$ \\
\hline & 50 & Yr: & & 1. $6 \mathrm{E}-10$ & 1. $8 \mathrm{E}-\odot 9$ & $9.5 \mathrm{E}-10$ & 1. $4 \mathrm{E}-10$ & $3.1 \mathrm{E}-10$ \\
\hline S 35 & Class: W & & $9.81 \mathrm{E}-11$ & & & & & \\
\hline & 1 & Yr: & & $2.1 \mathrm{E}-08$ & $2.6 \mathrm{E}-11$ & $2.6 \mathrm{E}-11$ & $2.6 \mathrm{E}-11$ & $2.8 \mathrm{E}-\odot 9$ \\
\hline & 50 & Yr: & & $2.1 E-08$ & $2.6 \mathrm{E}-11$ & $2.6 \mathrm{E}-11$ & $2.6 \mathrm{E}-11$ & 2. $8 \mathrm{E}-09$ \\
\hline CL36 & Class: D & & $5.23 E-09$ & & & & & \\
\hline & 1 & Yr: & & 1. $0 \mathrm{E}-09$ & 1. $\odot E-\odot 9$ & 1. $\odot E-\odot 9$ & 1. $\odot E-\odot 9$ & 1. $2 \mathrm{E}-09$ \\
\hline & 50 & Yr: & & 1. $\odot E-\odot 9$ & 1. $\odot E-\odot 9$ & 1. $\odot E-\odot 9$ & 1. $\odot E-\odot 9$ & 1. $2 \mathrm{E}-\odot 9$ \\
\hline AR39 & NobleGas & & $3.63 E-09$ & & & & & \\
\hline & 1 & Yr: & & $\odot . \odot \mathrm{E}-\odot \odot$ & $\odot . \odot \mathrm{E}-\odot \odot$ & $\odot . \odot \mathrm{E}-\odot \odot$ & $\odot . \odot \mathrm{E}-\odot \odot$ & $\odot . \odot \mathrm{E}-\odot \odot$ \\
\hline & 50 & Yr: & & $\odot . \odot \mathrm{E}-\odot \odot$ & $\odot . \odot \mathrm{E}-\odot \odot$ & $\odot . \odot \mathrm{E}-\odot \odot$ & $\odot . \odot \mathrm{E}-\odot \odot$ & $\odot . \odot \mathrm{E}-\odot \odot$ \\
\hline AR41 & NobleGas & & $1.94 \mathrm{E}-06$ & & & & & \\
\hline & 1 & Yr: & & $\odot . \odot \mathrm{E}-\odot \odot$ & $\odot . \odot \mathrm{E}-\odot \odot$ & $\odot . \odot E-\odot \odot$ & $\odot . \odot \mathrm{E}-\odot \odot$ & $\odot . \odot \mathrm{E}-\odot \odot$ \\
\hline & 50 & Yr: & & $\odot . \odot \mathrm{E}-\odot \odot$ & $\odot . \odot \mathrm{E}-\odot \odot$ & $\odot . \odot \mathrm{E}-\odot \odot$ & $\odot . \odot \mathrm{E}-\odot \odot$ & $\odot . \odot \mathrm{E}-\odot \odot$ \\
\hline K 40 & Class: D & & $2.50 \mathrm{E}-07$ & & & & & \\
\hline & 1 & Yr: & & $6.0 \mathrm{E}-09$ & $6.0 \mathrm{E}-\odot 9$ & $6 . \odot \mathrm{E}-\odot 9$ & 6. ०E-०9 & 7. 4E- $\odot 9$ \\
\hline & 50 & Yr: & & $6.0 \mathrm{E}-09$ & $6.0 \mathrm{E}-\odot 9$ & $6 . \odot E-\odot 9$ & $6.0 \mathrm{E}-\odot 9$ & 7. 4E- $\odot 9$ \\
\hline CA41 & Class: W & & $\odot . \odot \odot Е \odot \odot ~$ & & & & & \\
\hline & 1 & Yr: & & $4.8 \mathrm{E}-10$ & 2. $\odot E-\odot 9$ & $6.7 \mathrm{E}-10$ & $2.7 \mathrm{E}-12$ & 1. $7 \mathrm{E}-10$ \\
\hline & 50 & Yr: & & $4.8 \mathrm{E}-10$ & 2. $\odot E-\odot 9$ & $6.7 \mathrm{E}-10$ & 2.7E-12 & $1.7 \mathrm{E}-10$ \\
\hline CA45 & Class: W & & $4.83 \mathrm{E}-10$ & & & & & \\
\hline & 1 & Yr: & & $3.8 \mathrm{E}-08$ & 7. $6 \mathrm{E}-09$ & $2.3 E-\odot 9$ & $E-11$ & $5.3 E-\odot 9$ \\
\hline & 50 & Yr: & & $3.8 \mathrm{E}-\odot 8$ & $7.6 \mathrm{E}-\odot 9$ & $2.3 E-\odot 9$ & $3.4 \mathrm{E}-11$ & $5.3 E-09$ \\
\hline SC46 & Class: $\mathrm{Y}$ & & $2.95 \mathrm{E}-06$ & & & & & \\
\hline & 1 & Yr: & & 8. 6E-๑8 & $3.1 \mathrm{E}-09$ & $2.9 \mathrm{E}-\odot 9$ & 3. 3E- -99 & 1. $4 \mathrm{E}-08$ \\
\hline & 50 & Yr: & & $8.6 \mathrm{E}-\odot 8$ & $3.1 \mathrm{E}-09$ & $2.9 \mathrm{E}-\odot 9$ & 3. 3E-०9 & 1. $4 \mathrm{E}-08$ \\
\hline CR51 & Class: $Y$ & & $4.35 E-08$ & & & & & \\
\hline & 1 & Yr: & & $4.5 \mathrm{E}-10$ & 3. $0 \mathrm{E}-11$ & $2.5 \mathrm{E}-11$ & $E-11$ & 1. $0 \mathrm{E}-10$ \\
\hline & 50 & Yr: & & $4.5 \mathrm{E}-10$ & $3.0 \mathrm{E}-11$ & $2.5 \mathrm{E}-11$ & $2.8 \mathrm{E}-11$ & 1. $0 \mathrm{E}-10$ \\
\hline MN54 & Class: W & & $1.21 \mathrm{E}-06$ & & & & & \\
\hline & 1 & Yr: & & 1. $4 \mathrm{E}-08$ & 3. ०E-๑9 & $2.1 \mathrm{E}-\odot 9$ & $1.8 \mathrm{E}-\odot 9$ & $3.9 \mathrm{E}-\odot 9$ \\
\hline & 50 & Yr: & & 1. 4E-०8 & 3. $\odot E-\odot 9$ & $2.1 \mathrm{E}-\odot 9$ & $1.8 \mathrm{E}-\odot 9$ & $3.9 \mathrm{E}-09$ \\
\hline MN56 & Class: W & & $2.58 \mathrm{E}-06$ & & & & & \\
\hline & 1 & Yr: & & $7.2 \mathrm{E}-10$ & $2.2 \mathrm{E}-11$ & $2.4 \mathrm{E}-11$ & 6 E-11 & $3.7 \mathrm{E}-10$ \\
\hline & 50 & Yr: & & $7.2 \mathrm{E}-10$ & $2.2 \mathrm{E}-11$ & $2.4 \mathrm{E}-11$ & 1. $6 \mathrm{E}-11$ & $3.7 \mathrm{E}-10$ \\
\hline FE55 & Class: W & & ๑. ๑९Е๑९ & & & & & \\
\hline & 1 & Yr: & & 1. $2 \mathrm{E}-09$ & 2. 3E- $\odot 9$ & $3.5 E-\odot 9$ & $2.4 \mathrm{E}-10$ & $9.9 \mathrm{E}-10$ \\
\hline & 50 & Yr: & & 1. $2 \mathrm{E}-\odot 9$ & 2. $3 \mathrm{E}-\odot 9$ & 3. $5 \mathrm{E}-09$ & 2. $4 \mathrm{E}-10$ & $9.9 \mathrm{E}-10$ \\
\hline FE59 & Class: W & & $1.77 \mathrm{E}-06$ & & & & & \\
\hline
\end{tabular}




\begin{tabular}{|c|c|c|c|c|c|c|c|c|}
\hline & 1 & Yr: & & $4.2 \mathrm{E}-\odot 8$ & $3.5 E-09$ & $4.1 \mathrm{E}-\odot 9$ & 1. $9 \mathrm{E}-09$ & 7. $9 \mathrm{E}-\odot 9$ \\
\hline & 50 & Yr: & & $4.2 \mathrm{E}-\odot 8$ & $3.5 E-\odot 9$ & $4.1 E-\odot 9$ & 1. $9 \mathrm{E}-\odot 9$ & 7. $9 \mathrm{E}-09$ \\
\hline \multirow[t]{3}{*}{ C057 } & Class: Y & & $1.57 \mathrm{E}-07$ & & & & & \\
\hline & 1 & Yr: & & 1. $5 \mathrm{E}-\odot 8$ & $8.8 \mathrm{E}-10$ & $4.1 \mathrm{E}-10$ & $5.8 \mathrm{E}-10$ & 2. $3 E-\odot 9$ \\
\hline & 50 & Yr: & & 1. $5 E-\odot 8$ & $8.8 \mathrm{E}-10$ & $4.1 \mathrm{E}-10$ & $5.8 \mathrm{E}-10$ & 2. $3 E-09$ \\
\hline \multirow[t]{3}{*}{ C058 } & Class: Y & & $1.40 \mathrm{E}-06$ & & & & & \\
\hline & 1 & Yr: & & $2.5 E-\odot 8$ & 1. $3 E-\odot 9$ & 1. $2 \mathrm{E}-\odot 9$ & 1. $5 \mathrm{E}-09$ & $4.6 \mathrm{E}-\odot 9$ \\
\hline & 50 & Yr: & & $2.5 E-\odot 8$ & 1. 3E-๑9 & 1. $2 \mathrm{E}-09$ & 1. $5 \mathrm{E}-\odot 9$ & $4.6 \mathrm{E}-\odot 9$ \\
\hline \multirow[t]{3}{*}{$\mathrm{C06 \odot}$} & Class: Y & & $3.75 \mathrm{E}-06$ & & & & & \\
\hline & 1 & Yr: & & $3.4 \mathrm{E}-07$ & 2. ๑E - ๑8 & 1. $9 \mathrm{E}-\odot 8$ & $2.3 E-\odot 8$ & $5.9 \mathrm{E}-08$ \\
\hline & 50 & Yr: & & $3.4 \mathrm{E}-07$ & 2. $\odot E-\odot 8$ & 1. $9 \mathrm{E}-08$ & 2. $3 E-08$ & $5.9 E-08$ \\
\hline \multirow[t]{3}{*}{ NI59 } & Class: W & & $\odot . \odot \odot Е \odot \odot$ & & & & & \\
\hline & 1 & Yr: & & 1. 4E- $\odot 9$ & $1.7 \mathrm{E}-10$ & 1.7E-10 & $1.7 \mathrm{E}-10$ & $3.4 \mathrm{E}-10$ \\
\hline & 50 & Yr: & & 1. 4E- -99 & $1.7 \mathrm{E}-10$ & $1.7 \mathrm{E}-10$ & $1.7 \mathrm{E}-10$ & $3.4 \mathrm{E}-10$ \\
\hline \multirow[t]{3}{*}{ NI63 } & Class: W & & ๑.๑०Е०९ & & & & & \\
\hline & 1 & Yr: & & $5.8 \mathrm{E}-09$ & $4.1 \mathrm{E}-10$ & $4.1 \mathrm{E}-10$ & $4.1 \mathrm{E}-10$ & 1.1E- -99 \\
\hline & 50 & Yr: & & $5.8 \mathrm{E}-\odot 9$ & $4.1 \mathrm{E}-10$ & $4.1 \mathrm{E}-10$ & $4.1 \mathrm{E}-10$ & 1. $1 \mathrm{E}-09$ \\
\hline \multirow[t]{3}{*}{ NI65 } & Class: W & & $8.42 \mathrm{E}-07$ & & & & & \\
\hline & 1 & Yr: & & $5.9 \mathrm{E}-10$ & $6.6 \mathrm{E}-12$ & $6.9 \mathrm{E}-12$ & $5.6 \mathrm{E}-12$ & $2.4 \mathrm{E}-10$ \\
\hline & 50 & Yr: & & $5.9 \mathrm{E}-10$ & $6.6 \mathrm{E}-12$ & $6.9 \mathrm{E}-12$ & $5.6 \mathrm{E}-12$ & $2.4 \mathrm{E}-10$ \\
\hline \multirow[t]{3}{*}{ CU64 } & Class: D & & $2.69 \mathrm{E}-07$ & & & & & \\
\hline & 1 & Yr: & & $4.9 \mathrm{E}-11$ & $2.9 \mathrm{E}-11$ & $2.8 \mathrm{E}-11$ & $2.8 \mathrm{E}-11$ & 1. $2 \mathrm{E}-10$ \\
\hline & 50 & Yr: & & $4.9 \mathrm{E}-11$ & $2.9 \mathrm{E}-11$ & $2.8 \mathrm{E}-11$ & $2.8 \mathrm{E}-11$ & 1. $2 \mathrm{E}-10$ \\
\hline \multirow[t]{3}{*}{ ZN65 } & Class: Y & & $8.61 \mathrm{E}-07$ & & & & & \\
\hline & 1 & Yr: & & $2.2 \mathrm{E}-\odot 8$ & 1. $9 \mathrm{E}-\odot 9$ & $1.8 \mathrm{E}-\odot 9$ & $2.1 \mathrm{E}-\odot 9$ & 4. $4 \mathrm{E}-\odot 9$ \\
\hline & 50 & Yr: & & $2.2 \mathrm{E}-08$ & 1. $9 \mathrm{E}-09$ & $1.8 E-09$ & $2.1 \mathrm{E}-09$ & 4. $4 \mathrm{E}-09$ \\
\hline \multirow[t]{3}{*}{ ZN69M } & Class: Y & & $5.83 E-07$ & & & & & \\
\hline & 1 & Yr: & & $2.4 \mathrm{E}-\odot 9$ & $2.2 \mathrm{E}-11$ & $2.4 \mathrm{E}-11$ & 1. $4 \mathrm{E}-11$ & $8.2 \mathrm{E}-10$ \\
\hline & 50 & Yr: & & $2.4 \mathrm{E}-\odot 9$ & $2.2 \mathrm{E}-11$ & $2.4 \mathrm{E}-11$ & 1. $4 \mathrm{E}-11$ & $8.2 \mathrm{E}-10$ \\
\hline ZN69 & Class: $\mathrm{Y}$ & & $6.31 E-09$ & & & & & \\
\hline & 1 & Yr: & & $2.1 \mathrm{E}-10$ & $8.2 \mathrm{E}-15$ & $9.1 \mathrm{E}-15$ & $6.4 \mathrm{E}-15$ & $6.9 \mathrm{E}-11$ \\
\hline & 50 & Yr: & & $2.1 \mathrm{E}-10$ & $8.2 \mathrm{E}-15$ & $9.1 \mathrm{E}-15$ & $6.4 \mathrm{E}-15$ & $6.9 \mathrm{E}-11$ \\
\hline AS76 & Class: W & & $6.50 \mathrm{E}-07$ & & & & & \\
\hline & 1 & Yr: & & $6.8 \mathrm{E}-\odot 9$ & $1.2 \mathrm{E}-10$ & 1. $2 \mathrm{E}-10$ & $1.1 \mathrm{E}-10$ & $2.2 \mathrm{E}-09$ \\
\hline & 50 & Yr: & & $6.8 \mathrm{E}-09$ & 1. $2 \mathrm{E}-10$ & 1. $2 \mathrm{E}-10$ & $1.1 \mathrm{E}-10$ & $2.2 \mathrm{E}-09$ \\
\hline SE75 & Class: W & & $5.30 \mathrm{E}-07$ & & & & & \\
\hline & 1 & Yr: & & $1.1 \mathrm{E}-08$ & $1.2 \mathrm{E}-09$ & $8.4 \mathrm{E}-10$ & $9.6 \mathrm{E}-10$ & $2.5 \mathrm{E}-\odot 9$ \\
\hline & 50 & Yr: & & 1.1E- - & 1. $2 \mathrm{E}-09$ & $8.4 \mathrm{E}-10$ & $9.6 \mathrm{E}-10$ & $2.5 \mathrm{E}-09$ \\
\hline SE79 & Class: W & & $1.24 \mathrm{E}-10$ & & & & & \\
\hline & 1 & Yr: & & $3.7 \mathrm{E}-\odot 8$ & $4.4 \mathrm{E}-10$ & $4.4 \mathrm{E}-10$ & $4.4 \mathrm{E}-10$ & $6.9 \mathrm{E}-\odot 9$ \\
\hline & 50 & Yr: & & $3.7 \mathrm{E}-08$ & 4. $4 \mathrm{E}-10$ & $4.4 \mathrm{E}-10$ & $4.4 \mathrm{E}-10$ & $6.9 \mathrm{E}-09$ \\
\hline BR82 & Class: D & & $3.85 E-06$ & & & & & \\
\hline & 1 & Yr: & & $4.7 \mathrm{E}-10$ & $4.6 \mathrm{E}-10$ & $4.1 \mathrm{E}-10$ & $4.8 \mathrm{E}-10$ & 1. $2 \mathrm{E}-\odot 9$ \\
\hline & 50 & Yr: & & $4.7 \mathrm{E}-10$ & $4.6 \mathrm{E}-10$ & $4.1 \mathrm{E}-10$ & $4.8 \mathrm{E}-10$ & 1. $2 \mathrm{E}-09$ \\
\hline BR83 & Class: D & & $1.68 \mathrm{E}-\odot 8$ & & & & & \\
\hline & 1 & Yr: & & $4.7 \mathrm{E}-11$ & 1.1E-11 & 1. $0 \mathrm{E}-11$ & 1. $1 \mathrm{E}-11$ & $5.0 \mathrm{E}-11$ \\
\hline & 50 & Yr: & & 4. $7 \mathrm{E}-11$ & $1.1 \mathrm{E}-11$ & 1. $0 \mathrm{E}-11$ & 1.1E-11 & $5.0 \mathrm{E}-11$ \\
\hline BR84 & Class: D & & $2.84 \mathrm{E}-\odot 6$ & & & & & \\
\hline & 1 & Yr: & & $6.2 \mathrm{E}-11$ & $1.1 \mathrm{E}-11$ & $9.9 \mathrm{E}-12$ & 1.1E-11 & $7.1 \mathrm{E}-11$ \\
\hline & 50 & Yr: & & $6.2 \mathrm{E}-11$ & $1.1 \mathrm{E}-11$ & $9.9 \mathrm{E}-12$ & 1.1E-11 & 7. $1 \mathrm{E}-11$ \\
\hline KR83M & NobleGas & & $3.78 \mathrm{E}-11$ & & & & & \\
\hline & 1 & Yr: & & $\odot . \odot \mathrm{E}-\odot \odot$ & $\odot . \odot \mathrm{E}-\odot \odot$ & $\odot . \odot \mathrm{E}-\odot \odot$ & $\odot . \odot \mathrm{E}-\odot \odot$ & $\odot . \odot \mathrm{E}-\odot \odot$ \\
\hline & 50 & Yr: & & $\odot . \odot E-\odot \odot$ & $\odot . \odot E-\odot \odot$ & $\odot . \odot E-\odot \odot$ & $\odot . \odot E-\odot \odot$ & $\odot . \odot \mathrm{E}-\odot \odot$ \\
\hline KR85M & NobleGas & & $2.17 \mathrm{E}-07$ & & & & & \\
\hline & 1 & Yr: & & $\odot . \odot \mathrm{E}-\odot \odot$ & $\odot . \odot \mathrm{E}-\odot \odot$ & $\odot . \odot \mathrm{E}-\odot \odot$ & $\odot . \odot \mathrm{E}-\odot \odot$ & $\odot . \odot \mathrm{E}-\odot \odot$ \\
\hline & 50 & Yr: & & $\odot . \odot \mathrm{E}-\odot \odot$ & $\odot . \odot \mathrm{E}-\odot \odot$ & $\odot . \odot \mathrm{E}-\odot \odot$ & $\odot . \odot \mathrm{E}-\odot \odot$ & $\odot . \odot \mathrm{E}-\odot \odot$ \\
\hline KR85 & NobleGas & & $7.57 \mathrm{E}-09$ & & & & & \\
\hline & 1 & Yr: & & $\odot . \odot \mathrm{E}-\odot \odot$ & $\odot . \odot \mathrm{E}-\odot \odot$ & $\odot . \odot \mathrm{E}-\odot \odot$ & $\odot . \odot \mathrm{E}-\odot \odot$ & $\odot . \odot \mathrm{E}-\odot \odot$ \\
\hline & 50 & Yr: & & $\odot . \odot \mathrm{E}-\odot \odot$ & $\odot . \odot \mathrm{E}-\odot \odot$ & $\odot . \odot \mathrm{E}-\odot \odot$ & $\odot . \odot \mathrm{E}-\odot \odot$ & $\odot . \odot \mathrm{E}-\odot \odot$ \\
\hline KR87 & NobleGas & & $1.26 \mathrm{E}-06$ & & & & & \\
\hline & 1 & Yr: & & $\odot . \odot \mathrm{E}-\odot \odot$ & $\odot . \odot \mathrm{E}-\odot \odot$ & $\odot . \odot \mathrm{E}-\odot \odot$ & $\odot . \odot \mathrm{E}-\odot \odot$ & $\odot . \odot \mathrm{E}-\odot \odot$ \\
\hline & 50 & Yr: & & $\odot . \odot \mathrm{E}-\odot \odot$ & $\odot . \odot E-\odot \odot$ & $\odot . \odot E-\odot \odot$ & $\odot . \odot \mathrm{E}-\odot \odot$ & $\odot . \odot \mathrm{E}-\odot \odot$ \\
\hline KR88 & NobleGas & & $3.07 \mathrm{E}-06$ & & & & & \\
\hline & 1 & Yr: & & $\odot . \odot \mathrm{E}-\odot \odot$ & $\odot . \odot \mathrm{E}-\odot \odot$ & $\odot . \odot \mathrm{E}-\odot \odot$ & $\odot . \odot \mathrm{E}-\odot \odot$ & $\odot . \odot \mathrm{E}-\odot \odot$ \\
\hline & 50 & Yr: & & $\odot . \odot \mathrm{E}-\odot \odot$ & $\odot . \odot \mathrm{E}-\odot \odot$ & $\odot . \odot \mathrm{E}-\odot \odot$ & $\odot . \odot E-\odot \odot$ & $\odot . \odot \mathrm{E}-\odot \odot$ \\
\hline KR89 & NobleGas & & $3.45 \mathrm{E}-06$ & & & & & \\
\hline & 1 & Yr: & & $\odot . \odot \mathrm{E}-\odot \odot$ & $\odot . \odot \mathrm{E}-\odot \odot$ & $\odot . \odot \mathrm{E}-\odot \odot$ & $\odot . \odot \mathrm{E}-\odot \odot$ & $\odot . \odot \mathrm{E}-\odot \odot$ \\
\hline & 50 & Yr: & & $\odot . \odot \mathrm{E}-\odot \odot$ & $\odot . \odot \mathrm{E}-\odot \odot$ & $\odot . \odot \mathrm{E}-\odot \odot$ & $\odot . \odot \mathrm{E}-\odot \odot$ & $\odot . \odot E-\odot \odot$ \\
\hline RB86 & Class: D & & $1.56 \mathrm{E}-\odot 7$ & & & & & \\
\hline & 1 & Yr: & & $2.7 \mathrm{E}-\odot 9$ & $9.6 \mathrm{E}-\odot 9$ & $5.9 \mathrm{E}-\odot 9$ & $2.7 \mathrm{E}-\odot 9$ & $3.4 \mathrm{E}-09$ \\
\hline & 50 & Yr: & & $2.7 \mathrm{E}-09$ & $9.6 \mathrm{E}-09$ & $5.9 \mathrm{E}-09$ & $2.7 E-09$ & $3.4 \mathrm{E}-\odot 9$ \\
\hline
\end{tabular}




\begin{tabular}{|c|c|c|c|c|c|c|c|c|}
\hline \multirow[t]{3}{*}{ RB87 } & Class: D & & 1. $\odot 4 \mathrm{E}-\odot 9$ & & & & & \\
\hline & 1 & Yr: & & 1. $4 \mathrm{E}-\odot 9$ & $5.3 E-09$ & $3.2 \mathrm{E}-\odot 9$ & 1. $4 \mathrm{E}-\odot 9$ & 1. $8 \mathrm{E}-\odot 9$ \\
\hline & 50 & Yr: & & 1. $4 \mathrm{E}-09$ & $5.3 E-09$ & $3.2 \mathrm{E}-09$ & 1. $4 \mathrm{E}-\odot 9$ & 1. $8 \mathrm{E}-\odot 9$ \\
\hline \multirow[t]{3}{*}{ RB88 } & Class: D & & $1.05 E-\odot 6$ & & & & & \\
\hline & 1 & Yr: & & $6.4 \mathrm{E}-11$ & $6.3 \mathrm{E}-12$ & $5.9 \mathrm{E}-12$ & $5.8 \mathrm{E}-12$ & $5.2 \mathrm{E}-11$ \\
\hline & 50 & Yr: & & $6.4 \mathrm{E}-11$ & $6.3 E-12$ & $5.9 \mathrm{E}-12$ & $5.8 \mathrm{E}-12$ & $5.2 \mathrm{E}-11$ \\
\hline \multirow[t]{3}{*}{ RB89 } & Class: D & & $3.19 \mathrm{E}-06$ & & & & & \\
\hline & 1 & Yr: & & 4. 3E-11 & $7.6 \mathrm{E}-12$ & $5.9 E-12$ & 5. $2 \mathrm{E}-12$ & $4.3 E-11$ \\
\hline & 50 & Yr: & & 4. 3E-11 & $7.6 \mathrm{E}-12$ & 5. $9 \mathrm{E}-12$ & 5. $2 \mathrm{E}-12$ & 4. $3 \mathrm{E}-11$ \\
\hline \multirow[t]{3}{*}{ SR85 } & Class: D & & $7.06 \mathrm{E}-07$ & & & & & \\
\hline & 1 & Yr: & & $6.8 \mathrm{E}-10$ & $2.9 \mathrm{E}-\odot 9$ & $1.8 \mathrm{E}-\odot 9$ & 6. $0 \mathrm{E}-10$ & 1.1E- -99 \\
\hline & 50 & Yr: & & $6.8 \mathrm{E}-10$ & $2.9 \mathrm{E}-\odot 9$ & $1.8 \mathrm{E}-\odot 9$ & 6. $0 \mathrm{E}-10$ & 1.1E- -99 \\
\hline \multirow[t]{3}{*}{ SR89 } & Class: D & & $1.38 \mathrm{E}-08$ & & & & & \\
\hline & 1 & Yr: & & $6.6 \mathrm{E}-10$ & $2.2 \mathrm{E}-\odot 8$ & 1. $2 \mathrm{E}-\odot 8$ & $6.1 \mathrm{E}-10$ & 3. $2 \mathrm{E}-\odot 9$ \\
\hline & 50 & Yr: & & $6.6 \mathrm{E}-10$ & $2.2 \mathrm{E}-08$ & 1. $2 \mathrm{E}-08$ & $6.1 \mathrm{E}-10$ & $3.2 \mathrm{E}-09$ \\
\hline \multirow[t]{3}{*}{ SR90 } & Class: D & & $3.10 \mathrm{E}-\odot 9$ & & & & & \\
\hline & 1 & Yr: & & $2.1 \mathrm{E}-\odot 9$ & $4.5 E-07$ & 1. $9 \mathrm{E}-\odot 7$ & 2. $\odot E-\odot 9$ & $3.1 \mathrm{E}-08$ \\
\hline & 50 & Yr: & & $2.1 \mathrm{E}-09$ & $4.5 \mathrm{E}-07$ & 1. $9 \mathrm{E}-07$ & 2. $\odot E-\odot 9$ & $3.1 \mathrm{E}-08$ \\
\hline \multirow[t]{3}{*}{ SR91 } & Class: D & & 1. $\odot 3 E-\odot 6$ & & & & & \\
\hline & 1 & Yr: & & 1. $3 E-10$ & $5.3 \mathrm{E}-10$ & $3.7 \mathrm{E}-10$ & $7.3 \mathrm{E}-11$ & $5.2 \mathrm{E}-10$ \\
\hline & 50 & Yr: & & 1. 3E-10 & $5.3 E-10$ & $3.7 \mathrm{E}-10$ & $7.3 E-11$ & $5.2 \mathrm{E}-10$ \\
\hline \multirow[t]{3}{*}{ SR92 } & Class: D & & $2 . \odot 2 E-\odot 6$ & & & & & \\
\hline & 1 & Yr: & & $8.3 \mathrm{E}-11$ & $3.9 \mathrm{E}-10$ & $1.8 \mathrm{E}-10$ & 4. $3 E-11$ & 3. $3 \mathrm{E}-10$ \\
\hline & 50 & Yr: & & $8.3 E-11$ & $3.9 \mathrm{E}-10$ & $1.8 \mathrm{E}-10$ & $4.3 E-11$ & 3. $3 \mathrm{E}-10$ \\
\hline \multirow[t]{3}{*}{ Y 90} & Class: $\mathrm{Y}$ & & $2.50 \mathrm{E}-\odot 8$ & & & & & \\
\hline & 1 & Yr: & & 1. $5 \mathrm{E}-08$ & 3. $9 \mathrm{E}-12$ & $5.5 \mathrm{E}-12$ & 1. $5 \mathrm{E}-13$ & 4. $2 \mathrm{E}-\odot 9$ \\
\hline & 50 & Yr: & & 1. 5E-०8 & 3. $9 \mathrm{E}-12$ & $5.5 \mathrm{E}-12$ & $1.5 \mathrm{E}-13$ & $4.2 E-09$ \\
\hline Y 91M & Class: Y & & $7.47 \mathrm{E}-07$ & & & & & \\
\hline & 1 & Yr: & & 1. $\odot \mathrm{E}-1 \odot$ & $2 . \odot \mathrm{E}-12$ & 2. $0 \mathrm{E}-12$ & $1.5 \mathrm{E}-12$ & $3.2 \mathrm{E}-11$ \\
\hline & 50 & Yr: & & 1. $0 \mathrm{E}-10$ & 2. $0 \mathrm{E}-12$ & 2. $0 \mathrm{E}-12$ & $1.5 \mathrm{E}-12$ & $3.2 \mathrm{E}-11$ \\
\hline Y 91 & Class: $Y$ & & $1.96 \mathrm{E}-\odot 8$ & & & & & \\
\hline & 1 & Yr: & & 1. $4 \mathrm{E}-07$ & $1.8 \mathrm{E}-10$ & $2.5 \mathrm{E}-10$ & $8.1 \mathrm{E}-12$ & 1. $9 \mathrm{E}-08$ \\
\hline & 50 & Yr: & & 1. $4 \mathrm{E}-07$ & $1.8 \mathrm{E}-10$ & $2.5 \mathrm{E}-10$ & $8 \cdot 1 \mathrm{E}-12$ & 1. $9 \mathrm{E}-08$ \\
\hline Y 92 & Class: Y & & $4.16 \mathrm{E}-07$ & & & & & \\
\hline & 1 & Yr: & & 1. $4 \mathrm{E}-\odot 9$ & $3.5 \mathrm{E}-12$ & $3.9 \mathrm{E}-12$ & $2.5 \mathrm{E}-12$ & $5.5 \mathrm{E}-10$ \\
\hline & 50 & Yr: & & 1. $4 \mathrm{E}-09$ & $3.5 \mathrm{E}-12$ & $3.9 \mathrm{E}-12$ & 2. $5 \mathrm{E}-12$ & $5.5 \mathrm{E}-10$ \\
\hline Y 93 & Class: Y & & $1.67 \mathrm{E}-07$ & & & & & \\
\hline & 1 & Yr: & & $3.2 \mathrm{E}-09$ & $3.6 \mathrm{E}-12$ & $4.1 \mathrm{E}-12$ & 2. $2 \mathrm{E}-12$ & 1. $4 \mathrm{E}-09$ \\
\hline & 50 & Yr: & & $3.2 \mathrm{E}-\odot 9$ & $3.6 \mathrm{E}-12$ & $4.1 \mathrm{E}-12$ & $2.2 \mathrm{E}-12$ & 1. $4 \mathrm{E}-09$ \\
\hline ZR93 & Class: W & & $\odot . \odot \odot Е \odot \odot$ & & & & & \\
\hline & 1 & Yr: & & $6.6 \mathrm{E}-09$ & 8. . E-๑8 & $9.2 \mathrm{E}-\odot 9$ & $6.9 \mathrm{E}-12$ & $2.9 \mathrm{E}-09$ \\
\hline & 50 & Yr: & & $6.6 \mathrm{E}-09$ & $8.7 \mathrm{E}-08$ & $9.2 \mathrm{E}-\odot 9$ & $6.9 \mathrm{E}-12$ & $2.9 \mathrm{E}-09$ \\
\hline ZR95 & Class: W & & $1.06 \mathrm{E}-06$ & & & & & \\
\hline & 1 & Yr: & & $5.7 \mathrm{E}-08$ & $3.2 \mathrm{E}-08$ & $5.8 \mathrm{E}-\odot 9$ & 1. $6 \mathrm{E}-09$ & $9.7 \mathrm{E}-\odot 9$ \\
\hline & 50 & Yr: & & $5.7 E-\odot 8$ & $3.2 \mathrm{E}-\odot 8$ & $5.8 \mathrm{E}-\odot 9$ & 1. $6 \mathrm{E}-\odot 9$ & $9.7 \mathrm{E}-09$ \\
\hline ZR97 & Class: W & & $2.81 \mathrm{E}-07$ & & & & & \\
\hline & 1 & Yr: & & $6.5 \mathrm{E}-09$ & $1.9 \mathrm{E}-10$ & $2.3 \mathrm{E}-10$ & $7.2 \mathrm{E}-11$ & $2.8 \mathrm{E}-09$ \\
\hline & 50 & Yr: & & $6.5 E-\odot 9$ & $1.9 \mathrm{E}-10$ & $2.3 \mathrm{E}-10$ & $7.2 \mathrm{E}-11$ & $2.8 \mathrm{E}-\odot 9$ \\
\hline NB93M & Class: Y & & $9.62 \mathrm{E}-11$ & & & & & \\
\hline & 1 & Yr: & & $3.2 E-08$ & 1. $5 \mathrm{E}-10$ & $6.6 \mathrm{E}-11$ & $1.1 \mathrm{E}-11$ & 4. $\odot E-\odot 9$ \\
\hline & 50 & Yr: & & $3.2 \mathrm{E}-08$ & $1.5 \mathrm{E}-10$ & $6.6 \mathrm{E}-11$ & $1.1 \mathrm{E}-11$ & $4.0 \mathrm{E}-09$ \\
\hline NB94 & Class: Y & & $2.27 \mathrm{E}-06$ & & & & & \\
\hline & 1 & Yr: & & $5.3 E-\odot 7$ & $2.1 \mathrm{E}-\odot 8$ & $2.1 \mathrm{E}-\odot 8$ & $2.2 \mathrm{E}-\odot 8$ & 8. 3E- - 08 \\
\hline & 50 & Yr: & & $5.3 E-07$ & $2.1 \mathrm{E}-08$ & $2.1 \mathrm{E}-08$ & $2.2 \mathrm{E}-08$ & 8. 3E- -88 \\
\hline NB95M & Class: Y & & $8.64 \mathrm{E}-\odot 8$ & & & & & \\
\hline & 1 & Yr: & & $1.1 \mathrm{E}-08$ & $9.8 \mathrm{E}-11$ & $7.7 \mathrm{E}-11$ & $7.4 \mathrm{E}-11$ & 1. $9 \mathrm{E}-\odot 9$ \\
\hline & 50 & Yr: & & 1.1E-08 & $9.8 \mathrm{E}-11$ & $7.7 \mathrm{E}-11$ & $7.4 \mathrm{E}-11$ & 1. $9 \mathrm{E}-\odot 9$ \\
\hline NB95 & Class: Y & & $1.10 \mathrm{E}-06$ & & & & & \\
\hline & 1 & Yr: & & $2.2 E-08$ & $6.4 \mathrm{E}-10$ & $6.0 \mathrm{E}-10$ & $6.7 \mathrm{E}-10$ & $3.6 \mathrm{E}-09$ \\
\hline & 50 & Yr: & & $2.2 \mathrm{E}-08$ & $6.4 \mathrm{E}-10$ & $6.0 \mathrm{E}-10$ & $6.7 \mathrm{E}-10$ & $3.6 \mathrm{E}-09$ \\
\hline NB97M & Class: $Y$ & & $1.04 \mathrm{E}-06$ & & & & & \\
\hline & 1 & Yr: & & $2.4 \mathrm{E}-12$ & $2.3 \mathrm{E}-14$ & $3.1 \mathrm{E}-14$ & $2.8 \mathrm{E}-14$ & $3.5 \mathrm{E}-13$ \\
\hline & 50 & Yr: & & 2. $4 \mathrm{E}-12$ & $2.3 E-14$ & $3.1 \mathrm{E}-14$ & $2.8 \mathrm{E}-14$ & $3.5 E-13$ \\
\hline NB97 & Class: Y & & $9.43 E-07$ & & & & & \\
\hline & 1 & Yr: & & $2.9 \mathrm{E}-10$ & $3.3 E-12$ & $E-12$ & $2.6 \mathrm{E}-12$ & 1. $2 \mathrm{E}-10$ \\
\hline & 50 & Yr: & & $2.9 \mathrm{E}-10$ & 3. $3 E-12$ & 3. $4 \mathrm{E}-12$ & $2.6 \mathrm{E}-12$ & 1. $2 \mathrm{E}-10$ \\
\hline M093 & Class: D & & $5.46 \mathrm{E}-10$ & & & & & \\
\hline & 1 & Yr: & & $2.6 \mathrm{E}-10$ & $2.9 \mathrm{E}-\odot 8$ & $9.2 \mathrm{E}-\odot 9$ & $1.8 \mathrm{E}-10$ & 1. $7 \mathrm{E}-\odot 9$ \\
\hline & 50 & Yr: & & $2.6 \mathrm{E}-10$ & $2.9 \mathrm{E}-\odot 8$ & $9.2 \mathrm{E}-\odot 9$ & $1.8 \mathrm{E}-10$ & 1. $7 \mathrm{E}-\odot 9$ \\
\hline M099 & Class: D & & $2.20 \mathrm{E}-07$ & & & & & \\
\hline & 1 & Yr: & & $3.5 \mathrm{E}-10$ & 1.1E- 09 & $8.2 \mathrm{E}-10$ & $2.9 \mathrm{E}-10$ & $7.7 \mathrm{E}-10$ \\
\hline
\end{tabular}




\begin{tabular}{|c|c|c|c|c|c|c|c|c|}
\hline & 50 & Yr: & & $3.5 \mathrm{E}-10$ & 1.1E-०9 & $8.2 \mathrm{E}-10$ & $2.9 \mathrm{E}-10$ & $7.7 \mathrm{E}-10$ \\
\hline \multirow[t]{3}{*}{ TC99M } & Class: W & & $1.66 \mathrm{E}-07$ & & & & & \\
\hline & 1 & Yr: & & 1. $3 \mathrm{E}-10$ & $6.1 \mathrm{E}-12$ & $3.6 \mathrm{E}-12$ & $2.7 \mathrm{E}-11$ & $5.1 \mathrm{E}-11$ \\
\hline & 50 & Yr: & & 1. $3 \mathrm{E}-10$ & $6.1 \mathrm{E}-12$ & $3.6 \mathrm{E}-12$ & $2.7 \mathrm{E}-11$ & $5.1 \mathrm{E}-11$ \\
\hline \multirow[t]{3}{*}{ TC99 } & Class: W & & $9.05 \mathrm{E}-10$ & & & & & \\
\hline & 1 & Yr: & & $6.1 \mathrm{E}-08$ & 3. $0 \mathrm{E}-11$ & 3. $0 \mathrm{E}-11$ & 1. 3E- 09 & $7.9 \mathrm{E}-09$ \\
\hline & 50 & Yr: & & $6.1 \mathrm{E}-08$ & 3. $0 \mathrm{E}-11$ & 3. $\odot \mathrm{E}-11$ & 1. 3E- $\odot 9$ & $7.9 \mathrm{E}-09$ \\
\hline \multirow[t]{3}{*}{ TC101 } & Class: W & & $4.76 \mathrm{E}-07$ & & & & & \\
\hline & 1 & Yr: & & $5.9 \mathrm{E}-11$ & $4.8 \mathrm{E}-13$ & $3.8 \mathrm{E}-13$ & $6.1 \mathrm{E}-12$ & $3.2 \mathrm{E}-11$ \\
\hline & 50 & Yr: & & $5.9 \mathrm{E}-11$ & $4.8 \mathrm{E}-13$ & $3.8 \mathrm{E}-13$ & $6.1 \mathrm{E}-12$ & 3. $2 \mathrm{E}-11$ \\
\hline \multirow[t]{3}{*}{ RU103 } & Class: Y & & $6.59 \mathrm{E}-07$ & & & & & \\
\hline & 1 & Yr: & & $4.2 \mathrm{E}-08$ & 4. $4 \mathrm{E}-10$ & $4.1 \mathrm{E}-10$ & $4.9 \mathrm{E}-10$ & $6 . \odot \mathrm{E}-\odot 9$ \\
\hline & 50 & Yr: & & $4.2 E-08$ & $4.4 \mathrm{E}-10$ & $4.1 \mathrm{E}-10$ & $4.9 \mathrm{E}-10$ & $6.0 \mathrm{E}-09$ \\
\hline \multirow[t]{3}{*}{ RU105 } & Class: Y & & $1.13 E-06$ & & & & & \\
\hline & 1 & Yr: & & 1. 4E-०9 & $1.5 \mathrm{E}-11$ & $1.6 \mathrm{E}-11$ & $1.1 \mathrm{E}-11$ & $4.9 \mathrm{E}-10$ \\
\hline & 50 & Yr: & & 1. 4E-०9 & $1.5 \mathrm{E}-11$ & 1. $6 \mathrm{E}-11$ & 1.1E-11 & $4.9 \mathrm{E}-10$ \\
\hline \multirow[t]{3}{*}{ RU106 } & Class: Y & & $\odot . \odot \odot Е \odot \odot ~$ & & & & & \\
\hline & 1 & Yr: & & 1.1E-०6 & 1. 4E-०9 & 1. 3E-०9 & 1. 6E-०9 & 1. $4 \mathrm{E}-07$ \\
\hline & 50 & Yr: & & $1.1 \mathrm{E}-06$ & 1. 4E- $\odot 9$ & 1. 3E- $\odot 9$ & 1. $6 \mathrm{E}-\odot 9$ & 1. $4 \mathrm{E}-07$ \\
\hline \multirow[t]{3}{*}{ RH103M } & Class: Y & & $1.90 \mathrm{E}-10$ & & & & & \\
\hline & 1 & Yr: & & $3.5 \mathrm{E}-11$ & 1. $5 \mathrm{E}-14$ & $5.5 E-15$ & $3.3 E-15$ & $6.7 \mathrm{E}-12$ \\
\hline & 50 & Yr: & & $3.5 \mathrm{E}-11$ & 1. $5 \mathrm{E}-14$ & $5.5 \mathrm{E}-15$ & 3. 3E-15 & $6.7 \mathrm{E}-12$ \\
\hline \multirow[t]{3}{*}{ RH105 } & Class: Y & & $1.10 \mathrm{E}-07$ & & & & & \\
\hline & 1 & Yr: & & 4. ०E-०9 & 1. $1 \mathrm{E}-11$ & 1. $0 \mathrm{E}-11$ & $7.9 \mathrm{E}-12$ & 8. $0 \mathrm{E}-10$ \\
\hline & 50 & Yr: & & 4. $\odot \mathrm{E}-\odot 9$ & 1. $1 \mathrm{E}-11$ & 1. $0 \mathrm{E}-11$ & 7. $9 \mathrm{E}-12$ & 8. $0 \mathrm{E}-10$ \\
\hline \multirow[t]{3}{*}{ PD103 } & Class: Y & & $1.68 \mathrm{E}-\odot 9$ & & & & & \\
\hline & 1 & Yr: & & $7.1 \mathrm{E}-09$ & $2.7 \mathrm{E}-11$ & $6.2 \mathrm{E}-12$ & 1. 8E-12 & 1. $0 \mathrm{E}-\odot 9$ \\
\hline & 50 & Yr: & & $7.1 E-09$ & $2.7 \mathrm{E}-11$ & $6.2 \mathrm{E}-12$ & $1.8 \mathrm{E}-12$ & 1. $0 \mathrm{E}-\odot 9$ \\
\hline PD107 & Class: Y & & $\odot . \odot \odot Е \odot \odot ~$ & & & & & \\
\hline & 1 & Yr: & & 1. ०E-०8 & $8.5 E-13$ & 4. $3 E-13$ & $6.4 \mathrm{E}-14$ & 1. $3 E-09$ \\
\hline & 50 & Yr: & & 1. $0 \mathrm{E}-08$ & $8.5 E-13$ & 4. $3 \mathrm{E}-13$ & $6.4 \mathrm{E}-14$ & 1. $3 \mathrm{E}-\odot 9$ \\
\hline PD109 & Class: Y & & $1.33 \mathrm{E}-08$ & & & & & \\
\hline & 1 & Yr: & & $3.9 \mathrm{E}-09$ & 2. $2 \mathrm{E}-12$ & $8.8 \mathrm{E}-13$ & $3.1 \mathrm{E}-13$ & $9.4 \mathrm{E}-10$ \\
\hline & 50 & Yr: & & $3.9 E-09$ & $2.2 \mathrm{E}-12$ & 8. 8E-13 & $3.1 \mathrm{E}-13$ & $9.4 \mathrm{E}-10$ \\
\hline AG110M & Class: D & & 4. $01 E-06$ & & & & & \\
\hline & 1 & Yr: & & 1. $2 \mathrm{E}-08$ & 8. ЗЕ-०9 & $7.9 E-\odot 9$ & 7. ०E-०९ & 1. $5 \mathrm{E}-08$ \\
\hline & 50 & Yr: & & 1. $2 \mathrm{E}-08$ & 8. 3E- $\odot 9$ & $7.9 E-\odot 9$ & $7 . \odot E-\odot 9$ & 1. $5 \mathrm{E}-08$ \\
\hline AG111 & Class: D & & 4. 38E- - 8 & & & & & \\
\hline & 1 & Yr: & & $3.5 \mathrm{E}-10$ & $3.1 \mathrm{E}-10$ & $3.1 \mathrm{E}-10$ & 3. $0 \mathrm{E}-10$ & 1. $4 \mathrm{E}-09$ \\
\hline & 50 & Yr: & & $3.5 \mathrm{E}-10$ & $3.1 \mathrm{E}-10$ & $3.1 \mathrm{E}-10$ & $3.0 \mathrm{E}-10$ & 1. $4 \mathrm{E}-\odot 9$ \\
\hline CD109 & Class: D & & $7.22 \mathrm{E}-\odot 9$ & & & & & \\
\hline & 1 & Yr: & & $5 . \odot E-\odot 9$ & $6.2 \mathrm{E}-\odot 9$ & $3.9 \mathrm{E}-\odot 9$ & $4.2 \mathrm{E}-\odot 9$ & $2.1 \mathrm{E}-08$ \\
\hline & 50 & Yr: & & 5. ०E-०9 & $6.2 E-09$ & $3.9 E-\odot 9$ & 4. $2 \mathrm{E}-09$ & $2.1 E-08$ \\
\hline CD113M & Class: D & & $2.86 \mathrm{E}-09$ & & & & & \\
\hline & 1 & Yr: & & $3.1 \mathrm{E}-08$ & $3.1 \mathrm{E}-08$ & $3.1 \mathrm{E}-08$ & $3.1 \mathrm{E}-\odot 8$ & 1. $8 \mathrm{E}-07$ \\
\hline & 50 & Yr: & & $3.1 \mathrm{E}-08$ & $3.1 \mathrm{E}-08$ & $3.1 \mathrm{E}-\odot 8$ & $3.1 \mathrm{E}-\odot 8$ & 1. $8 \mathrm{E}-07$ \\
\hline CD115M & Class: D & & $4.67 \mathrm{E}-08$ & & & & & \\
\hline & 1 & Yr: & & 2. $9 \mathrm{E}-\odot 9$ & 2. $9 \mathrm{E}-\odot 9$ & 2. $9 \mathrm{E}-\odot 9$ & $2.8 \mathrm{E}-\odot 9$ & 1. $5 \mathrm{E}-08$ \\
\hline & 50 & Yr: & & 2. $9 E-09$ & $2.9 E-\odot 9$ & 2. $9 \mathrm{E}-\odot 9$ & $2.8 E-\odot 9$ & 1. $5 \mathrm{E}-08$ \\
\hline CD115 & Class: D & & $3.31 \mathrm{E}-07$ & & & & & \\
\hline & 1 & Yr: & & $2.4 \mathrm{E}-10$ & 2. $0 \mathrm{E}-10$ & 2. $0 \mathrm{E}-10$ & 1. 7E-10 & 1. $2 \mathrm{E}-\odot 9$ \\
\hline & 50 & Yr: & & $2.4 \mathrm{E}-10$ & $2.0 E-1 \odot$ & 2. $0 \mathrm{E}-10$ & 1. $7 \mathrm{E}-10$ & 1. $2 \mathrm{E}-\odot 9$ \\
\hline IN111 & Class: D & & $5.30 \mathrm{E}-\Theta 7$ & & & & & \\
\hline & 1 & Yr: & & 1. $2 \mathrm{E}-10$ & $4.6 \mathrm{E}-10$ & $5.3 \mathrm{E}-10$ & $8.1 \mathrm{E}-11$ & $4.3 \mathrm{E}-10$ \\
\hline & 50 & Yr: & & 1. $2 \mathrm{E}-10$ & $4.6 \mathrm{E}-10$ & $5.3 \mathrm{E}-10$ & $8.1 \mathrm{E}-11$ & $4.3 \mathrm{E}-10$ \\
\hline IN114M & Class: D & & $1.23 E-07$ & & & & & \\
\hline & 1 & Yr: & & $5.2 \mathrm{E}-\odot 9$ & 1. 3E- - 7 & 1.9E-๑7 & $5.1 E-09$ & $3.4 \mathrm{E}-08$ \\
\hline & 50 & Yr: & & $5.2 \mathrm{E}-09$ & 1. $3 \mathrm{E}-07$ & 1. $9 \mathrm{E}-\odot 7$ & $5.1 \mathrm{E}-09$ & $3.4 \mathrm{E}-08$ \\
\hline IN115M & Class: D & & $2.17 \mathrm{E}-\odot 7$ & & & & & \\
\hline & 1 & Yr: & & $3.1 \mathrm{E}-11$ & $5.0 \mathrm{E}-11$ & $6.9 \mathrm{E}-11$ & 8. 9E-12 & $8.4 \mathrm{E}-11$ \\
\hline & 50 & Yr: & & $3.1 \mathrm{E}-11$ & $5.0 E-11$ & $6.9 \mathrm{E}-11$ & $8.9 E-12$ & $8.4 \mathrm{E}-11$ \\
\hline SN117M & Class: W & & $1.93 E-07$ & & & & & \\
\hline & 1 & Yr: & & $3.2 \mathrm{E}-08$ & 3. ०E-०9 & $4.1 \mathrm{E}-10$ & $7.6 \mathrm{E}-11$ & $4.6 \mathrm{E}-09$ \\
\hline & 50 & Yr: & & $3.2 E-08$ & 3. $\odot E-\odot 9$ & $4.1 \mathrm{E}-10$ & $7.6 \mathrm{E}-11$ & 4. $6 \mathrm{E}-\odot 9$ \\
\hline SN119M & Class: W & & $2.23 E-\odot 9$ & & & & & \\
\hline & 1 & Yr: & & 3. 4E- - 8 & $2.3 E-09$ & 1. $\odot \mathrm{E}-\odot 9$ & $1.2 \mathrm{E}-10$ & 4. . $\mathrm{E}-\odot 9$ \\
\hline & 50 & Yr: & & $3.4 \mathrm{E}-08$ & $2.3 E-\odot 9$ & 1. $\odot \mathrm{E}-\odot 9$ & 1. $2 \mathrm{E}-10$ & 4. $7 \mathrm{E}-09$ \\
\hline SN121M & Class: W & & $1.66 \mathrm{E}-\odot 9$ & & & & & \\
\hline & 1 & Yr: & & $6.5 \mathrm{E}-\odot 8$ & $7.9 E-\odot 9$ & $3.8 \mathrm{E}-09$ & $4.1 \mathrm{E}-10$ & $9.1 \mathrm{E}-09$ \\
\hline & 50 & Yr: & & $6.5 \mathrm{E}-08$ & $7.9 E-\odot 9$ & $3.8 \mathrm{E}-\odot 9$ & $4.1 \mathrm{E}-10$ & $9.1 \mathrm{E}-09$ \\
\hline SN121 & Class: W & & $1.23 E-\odot 9$ & & & & & \\
\hline
\end{tabular}




\begin{tabular}{|c|c|c|c|c|c|c|c|c|}
\hline & 1 & Yr: & & $2.6 \mathrm{E}-09$ & 1. $4 \mathrm{E}-10$ & 1. $7 \mathrm{E}-11$ & 1. $2 \mathrm{E}-12$ & $5.1 \mathrm{E}-10$ \\
\hline & 50 & Yr: & & $2.6 E-\odot 9$ & 1. $4 \mathrm{E}-10$ & 1.7E-11 & 1. $2 \mathrm{E}-12$ & $5.1 \mathrm{E}-10$ \\
\hline \multirow[t]{3}{*}{ SN123 } & Class: W & & $2.20 \mathrm{E}-\odot 8$ & & & & & \\
\hline & 1 & Yr: & & 1. 3E- -7 & $6.7 \mathrm{E}-09$ & $3.4 \mathrm{E}-\odot 9$ & $3.5 \mathrm{E}-10$ & 1. $8 \mathrm{E}-08$ \\
\hline & 50 & Yr: & & 1. $3 \mathrm{E}-07$ & $6.7 \mathrm{E}-\odot 9$ & $3.4 \mathrm{E}-\odot 9$ & $3.5 \mathrm{E}-10$ & 1. $8 \mathrm{E}-08$ \\
\hline \multirow[t]{3}{*}{ SN125 } & Class: W & & $4.86 E-07$ & & & & & \\
\hline & 1 & Yr: & & $3.9 \mathrm{E}-08$ & 1. $7 \mathrm{E}-09$ & 1. 4E- $\odot 9$ & 1. $4 \mathrm{E}-10$ & $7.6 \mathrm{E}-\odot 9$ \\
\hline & 50 & Yr: & & $3.9 E-08$ & 1. 7E-०9 & 1. 4E- 09 & 1. $4 \mathrm{E}-10$ & $7.6 E-09$ \\
\hline \multirow[t]{3}{*}{ SN126 } & Class: W & & $5.83 E-08$ & & & & & \\
\hline & 1 & Yr: & & $3.7 E-07$ & 6. 6E-०8 & $3.8 \mathrm{E}-08$ & 1.1E- 08 & $6.2 \mathrm{E}-08$ \\
\hline & 50 & Yr: & & $3.7 E-07$ & $6.6 \mathrm{E}-08$ & $3.8 \mathrm{E}-\odot 8$ & 1.1E- 08 & $6.2 \mathrm{E}-08$ \\
\hline \multirow[t]{3}{*}{ SB124 } & Class: W & & $2.72 \mathrm{E}-\odot 6$ & & & & & \\
\hline & 1 & Yr: & & $8.6 \mathrm{E}-08$ & $5.4 \mathrm{E}-\odot 9$ & 3. ०E-०9 & 1. $9 \mathrm{E}-\odot 9$ & 1. $4 \mathrm{E}-08$ \\
\hline & 50 & Yr: & & $8.6 \mathrm{E}-08$ & $5.4 \mathrm{E}-\odot 9$ & 3. ०E-๑9 & 1. $9 \mathrm{E}-\odot 9$ & 1. $4 \mathrm{E}-08$ \\
\hline \multirow[t]{3}{*}{ SB125 } & Class: W & & $5.90 \mathrm{E}-07$ & & & & & \\
\hline & 1 & Yr: & & $6.4 \mathrm{E}-08$ & 2. ๑E-๑8 & $4.2 \mathrm{E}-09$ & 1. $5 \mathrm{E}-\odot 9$ & 1. $0 \mathrm{E}-08$ \\
\hline & 50 & Yr: & & 6. 4E- - & 2. ๑E-๑8 & 4. 2E- $\odot 9$ & 1. $5 E-\odot 9$ & 1. $0 \mathrm{E}-08$ \\
\hline \multirow[t]{3}{*}{ SB126M } & Class: W & & $2.21 \mathrm{E}-06$ & & & & & \\
\hline & 1 & Yr: & & $8.6 \mathrm{E}-11$ & $2.6 \mathrm{E}-12$ & $2.3 E-12$ & $2.2 \mathrm{E}-12$ & $5.5 \mathrm{E}-11$ \\
\hline & 50 & Yr: & & $8.6 \mathrm{E}-11$ & $2.6 \mathrm{E}-12$ & 2. $3 \mathrm{E}-12$ & $2.2 \mathrm{E}-12$ & $5.5 \mathrm{E}-11$ \\
\hline \multirow[t]{3}{*}{ SB126 } & Class: W & & 4. . $4 \mathrm{E}-06$ & & & & & \\
\hline & 1 & Yr: & & $3.2 \mathrm{E}-08$ & 1. 8E-०9 & 1. 4E- $\odot 9$ & 1. ०E-०9 & 7. $5 \mathrm{E}-09$ \\
\hline & 50 & Yr: & & $3.2 \mathrm{E}-08$ & $1.8 \mathrm{E}-09$ & 1. 4E- $\odot 9$ & 1. $\odot E-\odot 9$ & 7. $5 \mathrm{E}-09$ \\
\hline \multirow[t]{3}{*}{ SB127 } & Class: W & & $9.84 \mathrm{E}-07$ & & & & & \\
\hline & 1 & Yr: & & 2. $\odot E-\odot 8$ & $3.6 \mathrm{E}-10$ & $3.6 \mathrm{E}-10$ & 1. $2 \mathrm{E}-10$ & $3.9 \mathrm{E}-09$ \\
\hline & 50 & Yr: & & 2. $\odot E-\odot 8$ & $3.6 \mathrm{E}-10$ & $3.6 \mathrm{E}-10$ & $1.2 \mathrm{E}-10$ & $3.9 \mathrm{E}-09$ \\
\hline \multirow[t]{3}{*}{ TE123M } & Class: W & & $1.84 \mathrm{E}-07$ & & & & & \\
\hline & 1 & Yr: & & $5.4 \mathrm{E}-08$ & $3.2 \mathrm{E}-08$ & 4. ०E-๑9 & 1. $9 \mathrm{E}-09$ & 8. $0 \mathrm{E}-\odot 9$ \\
\hline & 50 & Yr: & & $5.4 \mathrm{E}-08$ & $3.2 \mathrm{E}-\odot 8$ & 4. ๑E-๑9 & 1. $9 \mathrm{E}-\odot 9$ & 8. $0 \mathrm{E}-\odot 9$ \\
\hline TE125M & Class: W & & $1.06 \mathrm{E}-08$ & & & & & \\
\hline & 1 & Yr: & & $4.7 E-08$ & 1. $5 \mathrm{E}-\odot 8$ & $1.8 \mathrm{E}-\odot 9$ & 1. 4E- $\odot 9$ & $6.5 \mathrm{E}-09$ \\
\hline & 50 & Yr: & & $4.7 E-\odot 8$ & $1.5 \mathrm{E}-08$ & 1. 8E-๑9 & 1. 4E- $\odot 9$ & $6.5 \mathrm{E}-09$ \\
\hline TE127M & Class: W & & $3.56 \mathrm{E}-\odot 9$ & & & & & \\
\hline & 1 & Yr: & & $1.1 \mathrm{E}-07$ & $2.7 \mathrm{E}-\odot 8$ & $9.8 \mathrm{E}-09$ & 4. . $\mathrm{E}-\odot 9$ & 1. $5 \mathrm{E}-08$ \\
\hline & 50 & Yr: & & 1.1E-07 & 2. 7E- -08 & $9.8 \mathrm{E}-09$ & 4. . E- - 99 & 1. $5 \mathrm{E}-08$ \\
\hline TE127 & Class: W & & $1.05 E-\odot 8$ & & & & & \\
\hline & 1 & Yr: & & 1. 3E-๑9 & $6.1 \mathrm{E}-12$ & 7. 3E-12 & $1.5 \mathrm{E}-11$ & $3.6 \mathrm{E}-10$ \\
\hline & 50 & Yr: & & 1. 3E- $\odot 9$ & $6.1 \mathrm{E}-12$ & $7.3 E-12$ & 1. $5 \mathrm{E}-11$ & $3.6 \mathrm{E}-10$ \\
\hline TE129M & Class: W & & $4.92 \mathrm{E}-08$ & & & & & \\
\hline & 1 & Yr: & & $9.2 \mathrm{E}-08$ & $8.6 \mathrm{E}-09$ & $5.2 \mathrm{E}-\odot 9$ & $5.7 \mathrm{E}-09$ & 1. $4 \mathrm{E}-08$ \\
\hline & 50 & Yr: & & $9.2 \mathrm{E}-08$ & $8.6 E-09$ & $5.2 \mathrm{E}-09$ & 5. 7E- 09 & 1. $4 \mathrm{E}-08$ \\
\hline TE129 & Class: W & & $9.05 E-\odot 8$ & & & & & \\
\hline & 1 & Yr: & & $2.6 \mathrm{E}-10$ & 1. $2 \mathrm{E}-12$ & 1. $1 \mathrm{E}-12$ & 1. $3 \mathrm{E}-12$ & $9.9 \mathrm{E}-11$ \\
\hline & 50 & Yr: & & $2.6 \mathrm{E}-10$ & 1. $2 \mathrm{E}-12$ & 1. $1 \mathrm{E}-12$ & 1. $3 E-12$ & $9.9 \mathrm{E}-11$ \\
\hline TE131M & Class: W & & $2.07 E-06$ & & & & & \\
\hline & 1 & Yr: & & $8.4 \mathrm{E}-09$ & $3.6 \mathrm{E}-10$ & $2.1 \mathrm{E}-10$ & 1. $3 \mathrm{E}-\odot 8$ & $3.1 \mathrm{E}-09$ \\
\hline & 50 & Yr: & & 8. 4E- $\odot 9$ & $3.6 \mathrm{E}-10$ & $2.1 \mathrm{E}-10$ & 1. 3E- -8 & $3.1 \mathrm{E}-\odot 9$ \\
\hline TE131 & Class: W & & $6.09 E-07$ & & & & & \\
\hline & 1 & Yr: & & $1.7 \mathrm{E}-10$ & 1. $5 \mathrm{E}-12$ & 1. $2 \mathrm{E}-12$ & $2.4 \mathrm{E}-10$ & 8. $2 \mathrm{E}-11$ \\
\hline & $5 \odot$ & Yr: & & 1. $7 \mathrm{E}-10$ & 1. $5 \mathrm{E}-12$ & 1. $2 \mathrm{E}-12$ & $2.4 \mathrm{E}-10$ & 8. $2 \mathrm{E}-11$ \\
\hline TE132 & Class: W & & $2.95 \mathrm{E}-07$ & & & & & \\
\hline & 1 & Yr: & & 1. . $9 \mathrm{E}-\odot 8$ & $8.7 \mathrm{E}-10$ & $4.9 \mathrm{E}-10$ & $2.5 E-08$ & $6.5 \mathrm{E}-09$ \\
\hline & 50 & Yr: & & 1. $9 \mathrm{E}-\odot 8$ & $8.7 E-10$ & $4.9 E-10$ & $2.5 E-\odot 8$ & $6.5 E-\odot 9$ \\
\hline TE133M & Class: W & & $3.41 \mathrm{E}-06$ & & & & & \\
\hline & 1 & Yr: & & $4.8 \mathrm{E}-10$ & 1. $2 \mathrm{E}-11$ & 1. $2 \mathrm{E}-11$ & $9.4 \mathrm{E}-10$ & $2.8 \mathrm{E}-10$ \\
\hline & 50 & Yr: & & $4.8 \mathrm{E}-10$ & 1. $2 \mathrm{E}-11$ & 1. $2 \mathrm{E}-11$ & $9.4 \mathrm{E}-10$ & $2.8 \mathrm{E}-10$ \\
\hline TE133 & Class: W & & $1.37 \mathrm{E}-06$ & & & & & \\
\hline & 1 & Yr: & & $9.8 \mathrm{E}-11$ & 1. $4 \mathrm{E}-12$ & 1. $3 \mathrm{E}-12$ & $2.1 \mathrm{E}-10$ & $6.1 \mathrm{E}-11$ \\
\hline & 50 & Yr: & & $9.8 \mathrm{E}-11$ & 1. $4 \mathrm{E}-12$ & 1. $3 E-12$ & $2.1 \mathrm{E}-10$ & $6.1 \mathrm{E}-11$ \\
\hline TE134 & Class: W & & $1.24 \mathrm{E}-\odot 6$ & & & & & \\
\hline & 1 & Yr: & & $3.7 \mathrm{E}-10$ & $1.1 \mathrm{E}-11$ & 1.1E-11 & 1. $3 \mathrm{E}-10$ & $1.9 \mathrm{E}-10$ \\
\hline & 50 & Yr: & & $3.7 \mathrm{E}-10$ & $1.1 \mathrm{E}-11$ & $1.1 \mathrm{E}-11$ & $1.3 \mathrm{E}-10$ & $1.9 \mathrm{E}-10$ \\
\hline I 125 & Class: D & & $1.19 \mathrm{E}-08$ & & & & & \\
\hline & 1 & Yr: & & 4.7E-11 & $9.2 \mathrm{E}-11$ & 2. $0 \mathrm{E}-11$ & 3. $\odot E-\odot 7$ & 1. $5 E-\odot 8$ \\
\hline & 50 & Yr: & & 4. $7 \mathrm{E}-11$ & $9.2 \mathrm{E}-11$ & 2. $\mathrm{OE}-11$ & 3. $0 \mathrm{E}-07$ & 1. $5 \mathrm{E}-08$ \\
\hline I 129 & Class: D & & $8.92 \mathrm{E}-\odot 9$ & & & & & \\
\hline & 1 & Yr: & & $8.5 E-11$ & $1.1 \mathrm{E}-10$ & $4.2 \mathrm{E}-11$ & 1. $2 \mathrm{E}-06$ & $6.1 \mathrm{E}-08$ \\
\hline & 50 & Yr: & & $8.5 E-11$ & $1.1 \mathrm{E}-10$ & $4.2 \mathrm{E}-11$ & 1. $2 \mathrm{E}-06$ & $6.1 \mathrm{E}-08$ \\
\hline I 130 & Class: D & & $3.05 E-06$ & & & & & \\
\hline & 1 & Yr: & & $1.5 \mathrm{E}-10$ & $9.4 \mathrm{E}-11$ & 8.0E-11 & $6.8 \mathrm{E}-08$ & $3.5 E-09$ \\
\hline & 50 & Yr: & & $1.5 \mathrm{E}-10$ & $9.4 \mathrm{E}-11$ & 8. $0 \mathrm{E}-11$ & $6.8 \mathrm{E}-\odot 8$ & $3.5 E-09$ \\
\hline
\end{tabular}




\begin{tabular}{|c|c|c|c|c|c|c|c|c|}
\hline \multirow[t]{3}{*}{ I 131} & Class: D & & $5.33 E-07$ & & & & & \\
\hline & 1 & Yr: & & $1.6 \mathrm{E}-10$ & $1.1 \mathrm{E}-10$ & $8.4 \mathrm{E}-11$ & 7. 3E- -07 & $3.7 \mathrm{E}-08$ \\
\hline & 50 & Yr: & & $1.6 \mathrm{E}-10$ & $1.1 \mathrm{E}-10$ & $8.4 \mathrm{E}-11$ & 7. $3 E-\odot 7$ & $3.7 \mathrm{E}-08$ \\
\hline \multirow[t]{3}{*}{ I 132} & Class: D & & $3.31 \mathrm{E}-06$ & & & & & \\
\hline & 1 & Yr: & & $8.1 \mathrm{E}-11$ & $3.4 \mathrm{E}-11$ & $3.1 \mathrm{E}-11$ & 7.6E-๑9 & $4.6 \mathrm{E}-10$ \\
\hline & 50 & Yr: & & $8.1 \mathrm{E}-11$ & $3.4 \mathrm{E}-11$ & $3.1 \mathrm{E}-11$ & 7. $6 \mathrm{E}-\odot 9$ & $4.6 \mathrm{E}-10$ \\
\hline \multirow[t]{3}{*}{ I 133} & Class: D & & $8.7 \odot E-\odot 7$ & & & & & \\
\hline & 1 & Yr: & & 1.1E-10 & $5.9 \mathrm{E}-11$ & $5.3 \mathrm{E}-11$ & 1. $6 \mathrm{E}-07$ & $8.3 E-\odot 9$ \\
\hline & 50 & Yr: & & 1. $1 \mathrm{E}-10$ & $5.9 \mathrm{E}-11$ & $5.3 \mathrm{E}-11$ & $1.6 \mathrm{E}-07$ & $8.3 E-\odot 9$ \\
\hline \multirow[t]{3}{*}{ I 134} & Class: D & & $3.85 E-06$ & & & & & \\
\hline & 1 & Yr: & & $6.1 \mathrm{E}-11$ & $1.6 \mathrm{E}-11$ & $1.5 \mathrm{E}-11$ & 1. $5 \mathrm{E}-\odot 9$ & $1.8 \mathrm{E}-10$ \\
\hline & 50 & Yr: & & $6.1 \mathrm{E}-11$ & 1. $6 \mathrm{E}-11$ & 1. $5 \mathrm{E}-11$ & $1.5 \mathrm{E}-\odot 9$ & $1.8 \mathrm{E}-10$ \\
\hline \multirow[t]{3}{*}{ I 135} & Class: D & & $2.38 \mathrm{E}-06$ & & & & & \\
\hline & 1 & Yr: & & $9.6 \mathrm{E}-11$ & $5.1 \mathrm{E}-11$ & $4.5 \mathrm{E}-11$ & 3. 3E- - 88 & 1. $7 \mathrm{E}-09$ \\
\hline & 50 & Yr: & & $9.6 \mathrm{E}-11$ & $5.1 \mathrm{E}-11$ & $4.5 \mathrm{E}-11$ & 3. 3E- $\odot 8$ & 1. $7 \mathrm{E}-\odot 9$ \\
\hline \multirow{3}{*}{ XE131M } & NobleGas & & $1.10 \mathrm{E}-\odot 8$ & & & & & \\
\hline & 1 & Yr: & & $\odot . \odot \mathrm{E}-\odot \odot$ & $\odot . \odot \mathrm{E}-\odot \odot$ & $\odot . \odot \mathrm{E}-\odot \odot$ & $\odot . \odot \mathrm{E}-\odot \odot$ & $\odot . \odot \mathrm{E}-\odot \odot$ \\
\hline & 50 & Yr: & & $\odot . \odot \mathrm{E}-\odot \odot$ & $\odot . \odot \mathrm{E}-\odot \odot$ & $\odot . \odot \mathrm{E}-\odot \odot$ & $\odot . \odot \mathrm{E}-\odot \odot$ & $\odot . \odot \mathrm{E}-\odot \odot$ \\
\hline \multirow[t]{3}{*}{ XE133M } & NobleGas & & 4. . $7 \mathrm{E}-\odot 8$ & & & & & \\
\hline & 1 & Yr: & & $\odot . \odot \mathrm{E}-\odot \odot$ & $\odot . \odot \mathrm{E}-\odot \odot$ & $\odot . \odot \mathrm{E}-\odot \odot$ & $\odot . \odot \mathrm{E}-\odot \odot$ & $\odot . \odot \mathrm{E}-\odot \odot$ \\
\hline & 50 & Yr: & & $\odot . \odot \mathrm{E}-\odot \odot$ & $\odot . \odot \mathrm{E}-\odot \odot$ & $\odot . \odot \mathrm{E}-\odot \odot$ & $\odot . \odot \mathrm{E}-\odot \odot$ & $\odot . \odot \mathrm{E}-\odot \odot$ \\
\hline \multirow[t]{3}{*}{ XE133 } & NobleGas & & $4.23 E-08$ & & & & & \\
\hline & 1 & Yr: & & $\odot . \odot \mathrm{E}-\odot \odot$ & $\odot . \odot \mathrm{E}-\odot \odot$ & $\odot . \odot E-\odot \odot$ & $\odot . \odot \mathrm{E}-\odot \odot$ & $\odot . \odot E-\odot \odot$ \\
\hline & 50 & Yr: & & $\odot . \odot \mathrm{E}-\odot \odot$ & $\odot . \odot \mathrm{E}-\odot \odot$ & $\odot . \odot \mathrm{E}-\odot \odot$ & $\odot . \odot \mathrm{E}-\odot \odot$ & $\odot . \odot \mathrm{E}-\odot \odot$ \\
\hline \multirow[t]{3}{*}{ XE135M } & NobleGas & & $5.99 \mathrm{E}-07$ & & & & & \\
\hline & 1 & Yr: & & $\odot . \odot \mathrm{E}-\odot \odot$ & $\odot . \odot \mathrm{E}-\odot \odot$ & $\odot . \odot \mathrm{E}-\odot \odot$ & $\odot . \odot \mathrm{E}-\odot \odot$ & $\odot . \odot \mathrm{E}-\odot \odot$ \\
\hline & 50 & Yr: & & $\odot . \odot \mathrm{E}-\odot \odot$ & $\odot . \odot E-\odot \odot$ & $\odot . \odot \mathrm{E}-\odot \odot$ & $\odot . \odot \mathrm{E}-\odot \odot$ & $\odot . \odot \mathrm{E}-\odot \odot$ \\
\hline XE135 & NobleGas & & $3.50 \mathrm{E}-07$ & & & & & \\
\hline & 1 & Yr: & & $\odot . \odot E-\odot \odot$ & $\odot . \odot \mathrm{E}-\odot \odot$ & $\odot . \odot \mathrm{E}-\odot \odot$ & $\odot . \odot \mathrm{E}-\odot \odot$ & $\odot . \odot \mathrm{E}-\odot \odot$ \\
\hline & 50 & Yr: & & $\odot . \odot \mathrm{E}-\odot \odot$ & $\odot . \odot \mathrm{E}-\odot \odot$ & $\odot . \odot \mathrm{E}-\odot \odot$ & $\odot . \odot \mathrm{E}-\odot \odot$ & $\odot . \odot \mathrm{E}-\odot \odot$ \\
\hline XE137 & NobleGas & & $4.34 \mathrm{E}-07$ & & & & & \\
\hline & 1 & Yr: & & $\odot . \odot \mathrm{E}-\odot \odot$ & $\odot . \odot \mathrm{E}-\odot \odot$ & $\odot . \odot \mathrm{E}-\odot \odot$ & $\odot . \odot \mathrm{E}-\odot \odot$ & $\odot . \odot \mathrm{E}-\odot \odot$ \\
\hline & 50 & Yr: & & $\odot . \odot \mathrm{E}-\odot \odot$ & $\odot . \odot \mathrm{E}-\odot \odot$ & $\odot . \odot \mathrm{E}-\odot \odot$ & $\odot . \odot \mathrm{E}-\odot \odot$ & $\odot . \odot \mathrm{E}-\odot \odot$ \\
\hline XE138 & NobleGas & & $1.73 E-\odot 6$ & & & & & \\
\hline & 1 & Yr: & & $\odot . \odot \mathrm{E}-\odot \odot$ & $\odot . \odot E-\odot \odot$ & $\odot . \odot \mathrm{E}-\odot \odot$ & $\odot . \odot E-\odot \odot$ & $\odot . \odot \mathrm{E}-\odot \odot$ \\
\hline & 50 & Yr: & & $\odot . \odot \mathrm{E}-\odot \odot$ & $\odot . \odot \mathrm{E}-\odot \odot$ & $\odot . \odot \mathrm{E}-\odot \odot$ & $\odot . \odot \mathrm{E}-\odot \odot$ & $\odot .0 \mathrm{E}-\odot \odot$ \\
\hline CS134M & Class: D & & $2.51 E-08$ & & & & & \\
\hline & 1 & Yr: & & $4.1 \mathrm{E}-11$ & $6.6 \mathrm{E}-12$ & $5.4 \mathrm{E}-12$ & $5.7 \mathrm{E}-12$ & $3.8 \mathrm{E}-11$ \\
\hline & 50 & Yr: & & $4.1 \mathrm{E}-11$ & $6.6 \mathrm{E}-12$ & $5.4 \mathrm{E}-12$ & $5.7 \mathrm{E}-12$ & $3.8 \mathrm{E}-11$ \\
\hline CS134 & Class: D & & $2.23 E-06$ & & & & & \\
\hline & 1 & Yr: & & $4.1 \mathrm{E}-\odot 9$ & $4.5 E-\odot 9$ & $4.1 \mathrm{E}-09$ & 4. . $\mathrm{E}-\odot 9$ & $5.2 \mathrm{E}-\odot 9$ \\
\hline & 50 & Yr: & & 4. $1 \mathrm{E}-09$ & $4.5 E-\odot 9$ & $4.1 \mathrm{E}-09$ & 4. 7E-०9 & $5.2 E-09$ \\
\hline CS135 & Class: D & & $3.00 \mathrm{E}-10$ & & & & & \\
\hline & 1 & Yr: & & $5.3 E-10$ & $5.0 \mathrm{E}-10$ & $5.0 \mathrm{E}-10$ & $5.0 \mathrm{E}-10$ & $6.2 \mathrm{E}-10$ \\
\hline & 50 & Yr: & & $5.3 \mathrm{E}-10$ & $5.0 \mathrm{E}-10$ & $5.0 \mathrm{E}-10$ & $5.0 \mathrm{E}-10$ & $6.2 \mathrm{E}-10$ \\
\hline CS136 & Class: D & & $3.13 E-06$ & & & & & \\
\hline & 1 & Yr: & & 1. $9 \mathrm{E}-09$ & $2.2 \mathrm{E}-\odot 9$ & 1. $9 \mathrm{E}-\odot 9$ & $2.2 \mathrm{E}-\odot 9$ & $2.9 \mathrm{E}-\odot 9$ \\
\hline & 50 & Yr: & & 1. $9 \mathrm{E}-09$ & $2.2 \mathrm{E}-\odot 9$ & 1. $9 \mathrm{E}-\odot 9$ & $2.2 \mathrm{E}-\odot 9$ & $2.9 \mathrm{E}-\odot 9$ \\
\hline CS137 & Class: D & & $2.93 E-\odot 9$ & & & & & \\
\hline & 1 & Yr: & & 3. $\odot E-\odot 9$ & $3.1 \mathrm{E}-\odot 9$ & $2.9 E-\odot 9$ & $3.2 \mathrm{E}-\odot 9$ & $3.7 \mathrm{E}-09$ \\
\hline & 50 & Yr: & & 3. $\odot E-\odot 9$ & $3.1 \mathrm{E}-09$ & $2.9 \mathrm{E}-09$ & $3.2 \mathrm{E}-09$ & $3.7 \mathrm{E}-09$ \\
\hline CS138 & Class: D & & $3.63 E-06$ & & & & & \\
\hline & 1 & Yr: & & $6.4 \mathrm{E}-11$ & 1. $2 \mathrm{E}-11$ & $E-11$ & 1. 3E-11 & $8.2 \mathrm{E}-11$ \\
\hline & 50 & Yr: & & $6.4 \mathrm{E}-11$ & 1. $2 \mathrm{E}-11$ & $1.1 \mathrm{E}-11$ & 1. $3 \mathrm{E}-11$ & $8.2 \mathrm{E}-11$ \\
\hline BA139 & Class: D & & $8.04 \mathrm{E}-\odot 8$ & & & & & \\
\hline & 1 & Yr: & & $5.5 \mathrm{E}-11$ & 5. $0 \mathrm{E}-11$ & $3.5 \mathrm{E}-11$ & $5.2 \mathrm{E}-12$ & $1.1 \mathrm{E}-10$ \\
\hline & 50 & Yr: & & $5.5 \mathrm{E}-11$ & 5. $0 \mathrm{E}-11$ & $3.5 \mathrm{E}-11$ & $5.2 \mathrm{E}-12$ & 1. $1 \mathrm{E}-10$ \\
\hline BA140 & Class: D & & $2.55 \mathrm{E}-07$ & & & & & \\
\hline & 1 & Yr: & & $5.5 \mathrm{E}-10$ & $1.1 \mathrm{E}-08$ & $E-\odot 9$ & $\mathrm{E}-10$ & $3.6 \mathrm{E}-09$ \\
\hline & 50 & Yr: & & $5.5 \mathrm{E}-10$ & 1. $1 \mathrm{E}-\odot 8$ & $4.8 \mathrm{E}-\odot 9$ & $4.2 \mathrm{E}-10$ & $3.6 \mathrm{E}-\odot 9$ \\
\hline BA141 & Class: D & & $1.24 \mathrm{E}-06$ & & & & & \\
\hline & 1 & Yr: & & $4.4 \mathrm{E}-11$ & $2.5 \mathrm{E}-11$ & $1.6 \mathrm{E}-11$ & $3.3 E-12$ & $6.4 \mathrm{E}-11$ \\
\hline & 50 & Yr: & & 4. $4 \mathrm{E}-11$ & $2.5 \mathrm{E}-11$ & $1.6 \mathrm{E}-11$ & 3. $3 E-12$ & $6.4 \mathrm{E}-11$ \\
\hline BA142 & Class: D & & $1.53 \mathrm{E}-06$ & & & & & \\
\hline & 1 & Yr: & & $3.2 \mathrm{E}-11$ & 1.1E-11 & $8.5 E-12$ & $3.2 \mathrm{E}-12$ & $4.6 \mathrm{E}-11$ \\
\hline & 50 & Yr: & & $3.2 \mathrm{E}-11$ & 1.1E-11 & $8.5 \mathrm{E}-12$ & 3. $2 \mathrm{E}-12$ & $4.6 \mathrm{E}-11$ \\
\hline LA140 & Class: D & & $3.50 \mathrm{E}-06$ & & & & & \\
\hline & 1 & Yr: & & $4.8 \mathrm{E}-10$ & $7.9 \mathrm{E}-10$ & $9.6 \mathrm{E}-10$ & $2.5 \mathrm{E}-10$ & 2. $\odot E-\odot 9$ \\
\hline & 50 & Yr: & & $4.8 \mathrm{E}-10$ & $7.9 \mathrm{E}-10$ & $9.6 \mathrm{E}-10$ & $2.5 \mathrm{E}-10$ & 2. $\odot E-\odot 9$ \\
\hline LA141 & Class: D & & 9. ๑८E-๑৪ & & & & & \\
\hline & 1 & Yr: & & $8.2 \mathrm{E}-11$ & $2.4 \mathrm{E}-10$ & $9.3 \mathrm{E}-11$ & $2.8 \mathrm{E}-11$ & $2.3 \mathrm{E}-10$ \\
\hline
\end{tabular}




\begin{tabular}{|c|c|c|c|c|c|c|c|c|}
\hline & 50 & Yr: & & $8.2 \mathrm{E}-11$ & $2.4 \mathrm{E}-10$ & $9.3 \mathrm{E}-11$ & $2.8 \mathrm{E}-11$ & $2.3 \mathrm{E}-10$ \\
\hline \multirow[t]{2}{*}{ LA142 } & Class: D & Yr. & 4.32E-06 & $82 F_{-11}$ & $34 \mathrm{~F}-11$ & $35 E-11$ & $265-11$ & $18 \mathrm{~F}-10$ \\
\hline & $\begin{array}{r}1 \\
50\end{array}$ & Yr: & & $\begin{array}{l}8.2 \mathrm{E}-11 \\
8.2 \mathrm{E}-11\end{array}$ & 3. $4 \mathrm{E}-11$ & 3. $5 E-11$ & $\begin{array}{l}2.6 E-11 \\
2.6 E-11\end{array}$ & $\begin{array}{l}1.8 \mathrm{E}-10 \\
1.8 \mathrm{E}-10\end{array}$ \\
\hline \multirow[t]{3}{*}{ CE141 } & Class: Y & & $9.81 \mathrm{E}-08$ & & & & & \\
\hline & 1 & Yr: & & $5.3 E-08$ & $4.1 \mathrm{E}-10$ & $9.3 E-11$ & $7.4 \mathrm{E}-11$ & $7.1 \mathrm{E}-09$ \\
\hline & 50 & Yr: & & $5.3 E-08$ & $4.1 \mathrm{E}-10$ & $9.3 \mathrm{E}-11$ & $7.4 \mathrm{E}-11$ & $7.1 \mathrm{E}-09$ \\
\hline \multirow[t]{3}{*}{ CE143 } & Class: Y & & $3.82 \mathrm{E}-07$ & & & & & \\
\hline & 1 & Yr: & & $9.2 \mathrm{E}-09$ & $4.2 \mathrm{E}-11$ & 3. 3E-11 & 1.9E-11 & $2.1 \mathrm{E}-09$ \\
\hline & 50 & Yr: & & $9.2 \mathrm{E}-\odot 9$ & $4.2 \mathrm{E}-11$ & $3.3 E-11$ & 1. $9 \mathrm{E}-11$ & $2.1 \mathrm{E}-\odot 9$ \\
\hline \multirow[t]{3}{*}{ CE144 } & Class: Y & & $2.41 \mathrm{E}-08$ & & & & & \\
\hline & 1 & Yr: & & $8.7 E-07$ & $7.8 E-\odot 9$ & $6.1 \mathrm{E}-\odot 9$ & $4.1 \mathrm{E}-10$ & $1.1 \mathrm{E}-07$ \\
\hline & 50 & Yr: & & $8.7 \mathrm{E}-07$ & $7.8 \mathrm{E}-\odot 9$ & $6.1 \mathrm{E}-\odot 9$ & $4.1 \mathrm{E}-10$ & $1.1 \mathrm{E}-07$ \\
\hline \multirow[t]{3}{*}{ PR143 } & Class: Y & & $6.12 \mathrm{E}-09$ & & & & & \\
\hline & 1 & Yr: & & $3.4 \mathrm{E}-08$ & $6.3 E-12$ & $9.0 \mathrm{E}-12$ & $4.4 \mathrm{E}-14$ & $5.1 \mathrm{E}-\odot 9$ \\
\hline & 50 & Yr: & & $3.4 \mathrm{E}-08$ & $6.3 \mathrm{E}-12$ & $9.0 \mathrm{E}-12$ & $4.4 \mathrm{E}-14$ & $5.1 \mathrm{E}-09$ \\
\hline \multirow[t]{3}{*}{ PR144M } & Class: Y & & $6.97 \mathrm{E}-\odot 9$ & & & & & \\
\hline & 1 & Yr: & & 4. $0 \mathrm{E}-11$ & 1. 5E-14 & $1.8 \mathrm{E}-14$ & 8.7E-15 & $5.1 \mathrm{E}-12$ \\
\hline & 50 & Yr: & & 4. $0 \mathrm{E}-11$ & 1. $5 \mathrm{E}-14$ & 1. $8 \mathrm{E}-14$ & $8.7 \mathrm{E}-15$ & $5.1 \mathrm{E}-12$ \\
\hline \multirow[t]{3}{*}{ PR144 } & Class: Y & & 8.36E- -88 & & & & & \\
\hline & 1 & Yr: & & $1.1 \mathrm{E}-10$ & $3.7 \mathrm{E}-14$ & $3.4 \mathrm{E}-14$ & $3.5 \mathrm{E}-14$ & $5.2 \mathrm{E}-11$ \\
\hline & 50 & Yr: & & $1.1 \mathrm{E}-10$ & $3.7 \mathrm{E}-14$ & $3.4 \mathrm{E}-14$ & $3.5 \mathrm{E}-14$ & $5.2 \mathrm{E}-11$ \\
\hline \multirow[t]{3}{*}{ ND147 } & Class: Y & & $1.81 E-07$ & & & & & \\
\hline & 1 & Yr: & & $3.3 E-08$ & $2.3 \mathrm{E}-10$ & $7.5 E-11$ & $5.4 \mathrm{E}-11$ & $4.9 \mathrm{E}-09$ \\
\hline & 50 & Yr: & & $3.3 E-08$ & $2.3 \mathrm{E}-10$ & $7.5 \mathrm{E}-11$ & $5.4 \mathrm{E}-11$ & $4.9 \mathrm{E}-09$ \\
\hline \multirow[t]{3}{*}{ PM147 } & Class: Y & & $2.73 \mathrm{E}-10$ & & & & & \\
\hline & 1 & Yr: & & $7.8 E-08$ & 9. ०Е-०९ & 1. ๑E-๑9 & 1.1E-13 & 9. $9 \mathrm{E}-09$ \\
\hline & 50 & Yr: & & $7.8 \mathrm{E}-08$ & $9.0 \mathrm{E}-09$ & 1. $0 \mathrm{E}-09$ & $1.1 \mathrm{E}-13$ & $9.9 \mathrm{E}-09$ \\
\hline \multirow[t]{3}{*}{ PM148M } & Class: Y & & $2.84 \mathrm{E}-06$ & & & & & \\
\hline & 1 & Yr: & & 7. 5E- - & 2. 1E-०9 & 1. 8E- $\odot 9$ & $2.1 \mathrm{E}-09$ & 1. $2 \mathrm{E}-\odot 8$ \\
\hline & 50 & Yr: & & $7.5 E-08$ & $2.1 \mathrm{E}-09$ & $1.8 \mathrm{E}-09$ & $2.1 \mathrm{E}-09$ & 1. $2 \mathrm{E}-08$ \\
\hline PM148 & Class: Y & & $8.70 \mathrm{E}-07$ & & & & & \\
\hline & 1 & Yr: & & $2.6 \mathrm{E}-08$ & $1.2 \mathrm{E}-10$ & 1. $3 \mathrm{E}-10$ & 1. $0 \mathrm{E}-10$ & $5.5 E-09$ \\
\hline & 50 & Yr: & & $2.6 \mathrm{E}-08$ & 1. $2 \mathrm{E}-10$ & 1. $3 \mathrm{E}-10$ & 1. $0 \mathrm{E}-10$ & $5.5 E-09$ \\
\hline PM149 & Class: Y & & 2. 24E- - & & & & & \\
\hline & 1 & Yr: & & $7.9 \mathrm{E}-09$ & 2. $9 \mathrm{E}-12$ & 3. 4E-12 & 1.1E-12 & $1.8 \mathrm{E}-09$ \\
\hline & 50 & Yr: & & 7. 9E-๑9 & $2.9 \mathrm{E}-12$ & $3.4 \mathrm{E}-12$ & 1.1E-12 & $1.8 \mathrm{E}-09$ \\
\hline PM151 & Class: Y & & $4.42 \mathrm{E}-07$ & & & & & \\
\hline & 1 & Yr: & & $4.8 \mathrm{E}-09$ & $3.7 \mathrm{E}-11$ & $3.1 \mathrm{E}-11$ & 1. $9 \mathrm{E}-11$ & 1. 3E- 09 \\
\hline & 50 & Yr: & & $4.8 \mathrm{E}-\odot 9$ & $3.7 \mathrm{E}-11$ & $3.1 \mathrm{E}-11$ & 1. $9 \mathrm{E}-11$ & 1. $3 \mathrm{E}-09$ \\
\hline SM147 & Class: W & & ๑. ๑९Е๑९ & & & & & \\
\hline & 1 & Yr: & & $6.2 \mathrm{E}-07$ & 1.9E-05 & 2. ๑E-๑6 & 4. $9 \mathrm{E}-11$ & $1.6 \mathrm{E}-05$ \\
\hline & 50 & Yr: & & $6.2 \mathrm{E}-07$ & 1.9E-05 & $2.0 E-06$ & $4.9 \mathrm{E}-11$ & $1.6 \mathrm{E}-05$ \\
\hline SM151 & Class: W & & $7.79 \mathrm{E}-13$ & & & & & \\
\hline & 1 & Yr: & & $7.1 \mathrm{E}-09$ & $1.5 E-07$ & 1.6E-०8 & $4.4 \mathrm{E}-13$ & $6.6 \mathrm{E}-09$ \\
\hline & 50 & Yr: & & $7.1 \mathrm{E}-09$ & $1.5 \mathrm{E}-07$ & $1.6 \mathrm{E}-\odot 8$ & $4.4 \mathrm{E}-13$ & $6.6 \mathrm{E}-09$ \\
\hline SM153 & Class: W & & $6.46 \mathrm{E}-08$ & & & & & \\
\hline & 1 & Yr: & & $7.1 \mathrm{E}-09$ & $2.0 \mathrm{E}-10$ & $9.5 \mathrm{E}-11$ & $7.5 E-12$ & 1. $5 \mathrm{E}-09$ \\
\hline & 50 & Yr: & & $7.1 E-\odot 9$ & $2 . \odot E-1 \odot$ & $9.5 \mathrm{E}-11$ & $7.5 \mathrm{E}-12$ & 1. $5 \mathrm{E}-09$ \\
\hline EU152 & Class: W & & $1.67 \mathrm{E}-06$ & & & & & \\
\hline & 1 & Yr: & & 1. 2E- - 7 & $3.1 \mathrm{E}-07$ & 1. $2 \mathrm{E}-07$ & 1. 3E - ๑8 & $7.1 \mathrm{E}-\odot 8$ \\
\hline & 50 & Yr: & & $1.2 \mathrm{E}-07$ & $3.1 \mathrm{E}-07$ & 1. $2 \mathrm{E}-07$ & 1. 3E - 08 & $7.1 \mathrm{E}-08$ \\
\hline EU154 & Class: W & & 1. $82 \mathrm{E}-06$ & & & & & \\
\hline & 1 & Yr: & & 1. $9 \mathrm{E}-07$ & $6.9 \mathrm{E}-07$ & 1. $8 \mathrm{E}-07$ & E-०8 & $9.7 \mathrm{E}-08$ \\
\hline & 50 & Yr: & & 1. $9 \mathrm{E}-07$ & $6.9 \mathrm{E}-07$ & 1. $8 \mathrm{E}-07$ & 1. $2 \mathrm{E}-\odot 8$ & $9.7 \mathrm{E}-\odot 8$ \\
\hline EU155 & Class: W & & $6.78 \mathrm{E}-\odot 8$ & & & & & \\
\hline & 1 & Yr: & & $3.7 E-\odot 8$ & $2.2 \mathrm{E}-07$ & $2.6 \mathrm{E}-\odot 8$ & $5.3 E-10$ & 1. 4E- - 8 \\
\hline & 50 & Yr: & & $3.7 E-08$ & $2.2 \mathrm{E}-07$ & $2.6 E-08$ & $5.3 E-10$ & $1.4 \mathrm{E}-08$ \\
\hline EU156 & Class: W & & $2.02 \mathrm{E}-06$ & & & & & \\
\hline & 1 & Yr: & & 4. 3E- - 8 & $3.8 \mathrm{E}-09$ & $1.9 \mathrm{E}-\mathrm{O}$ & $5.1 \mathrm{E}-10$ & 7.7E- - 99 \\
\hline & 50 & Yr: & & 4. 3E- - 8 & $3.8 \mathrm{E}-09$ & 1. $9 \mathrm{E}-\odot 9$ & $5.1 \mathrm{E}-10$ & 7. $7 \mathrm{E}-09$ \\
\hline GD153 & Class: D & & 9. 84E- ๑8 & & & & & \\
\hline & 1 & Yr: & & 1. . & 1. 4E- - 7 & 1. 8E- -8 & $4.6 \mathrm{E}-10$ & $6.5 \mathrm{E}-\odot 9$ \\
\hline & 50 & Yr: & & 1. $9 \mathrm{E}-09$ & 1. 4E- - 07 & 1. $8 \mathrm{E}-08$ & $4.6 \mathrm{E}-10$ & $6.5 \mathrm{E}-09$ \\
\hline TB160 & Class: W & & $1.64 \mathrm{E}-06$ & & & & & \\
\hline & 1 & Yr: & & 8.6E-08 & 4. ०E- -8 & 9. ०E-०9 & 1. 4E-०9 & 1. $5 \mathrm{E}-\odot 8$ \\
\hline & 50 & Yr: & & $8.6 \mathrm{E}-08$ & 4. . & 9. ०E-๑9 & 1. $4 \mathrm{E}-09$ & 1. $5 \mathrm{E}-08$ \\
\hline H0166M & Class: W & & $2.48 \mathrm{E}-06$ & & & & & \\
\hline & 1 & Yr: & & 1.9E-07 & $9.9 \mathrm{E}-07$ & $2.2 \mathrm{E}-07$ & $2.9 E-08$ & $1.8 \mathrm{E}-07$ \\
\hline & 50 & Yr: & & 1. $9 \mathrm{E}-07$ & $9.9 \mathrm{E}-07$ & $2.2 \mathrm{E}-07$ & $2.9 \mathrm{E}-\odot 8$ & $1.8 \mathrm{E}-07$ \\
\hline W 181 & Class: D & & 3.69E-๑8 & & & & & \\
\hline
\end{tabular}




\begin{tabular}{|c|c|c|c|c|c|c|c|c|}
\hline & 1 & Yr: & & 1. $4 \mathrm{E}-11$ & $1.5 \mathrm{E}-10$ & $5.2 \mathrm{E}-11$ & 7. $3 \mathrm{E}-12$ & $9.5 \mathrm{E}-11$ \\
\hline & 50 & Yr: & & 1. $4 \mathrm{E}-11$ & $1.5 \mathrm{E}-10$ & $5.2 \mathrm{E}-11$ & 7. $3 E-12$ & $9.5 \mathrm{E}-11$ \\
\hline \multirow[t]{3}{*}{ W 187} & Class: D & & $6.72 \mathrm{E}-\odot 7$ & & & & & \\
\hline & 1 & Yr: & & $8.5 E-11$ & $1.7 \mathrm{E}-10$ & $7.4 \mathrm{E}-11$ & 3. $4 \mathrm{E}-11$ & $7.0 \mathrm{E}-10$ \\
\hline & 50 & Yr: & & $8.5 E-11$ & 1. $7 \mathrm{E}-10$ & $7.4 \mathrm{E}-11$ & $3.4 \mathrm{E}-11$ & 7. $0 \mathrm{E}-10$ \\
\hline \multirow[t]{3}{*}{ W 185} & Class: D & & $1.57 \mathrm{E}-\odot 9$ & & & & & \\
\hline & 1 & Yr: & & 4. $\odot \mathrm{E}-11$ & 4. 3E - 10 & $2.1 \mathrm{E}-10$ & $8.8 \mathrm{E}-12$ & $4.4 \mathrm{E}-10$ \\
\hline & 50 & Yr: & & 4. $0 \mathrm{E}-11$ & 4. $3 E-10$ & $2.1 \mathrm{E}-10$ & 8. $8 \mathrm{E}-12$ & $4.4 \mathrm{E}-10$ \\
\hline \multirow[t]{3}{*}{ RE187 } & Class: W & & $\odot . \odot \odot Е \odot \odot ~$ & & & & & \\
\hline & 1 & Yr: & & 1. $2 \mathrm{E}-10$ & $5.3 E-13$ & $5.3 E-13$ & $2.3 \mathrm{E}-11$ & 2. $0 \mathrm{E}-11$ \\
\hline & 50 & Yr: & & 1. $2 \mathrm{E}-10$ & 5. $3 E-13$ & 5. $3 E-13$ & 2. $3 E-11$ & $2.0 \mathrm{E}-11$ \\
\hline \multirow[t]{3}{*}{ IR192 } & Class: Y & & $1.14 \mathrm{E}-06$ & & & & & \\
\hline & 1 & Yr: & & $9.4 \mathrm{E}-\odot 8$ & 1. 4E-०9 & $1.1 \mathrm{E}-\odot 9$ & 1. 4E- -99 & 1. $4 \mathrm{E}-\odot 8$ \\
\hline & 50 & Yr: & & $9.4 \mathrm{E}-08$ & $1.4 \mathrm{E}-09$ & $1.1 \mathrm{E}-09$ & 1. $4 \mathrm{E}-\odot 9$ & 1. $4 \mathrm{E}-\odot 8$ \\
\hline \multirow{3}{*}{ HG2O3 } & Class: D & & $3.28 E-07$ & & & & & \\
\hline & 1 & Yr: & & $9.0 \mathrm{E}-10$ & 1. $\odot E-\odot 9$ & $8.9 \mathrm{E}-10$ & $9.3 \mathrm{E}-10$ & 1. $4 \mathrm{E}-09$ \\
\hline & 50 & Yr: & & $9.0 \mathrm{E}-1 \odot$ & 1. $\odot E-\odot 9$ & $8.9 \mathrm{E}-10$ & $9.3 \mathrm{E}-10$ & 1. $4 \mathrm{E}-09$ \\
\hline \multirow[t]{3}{*}{ RN222 } & NobleGas & & $5.61 \mathrm{E}-10$ & & & & & \\
\hline & 1 & Yr: & & $\odot . \odot \mathrm{E}-\odot \odot$ & $\odot . \odot \mathrm{E}-\odot \odot$ & $\odot . \odot \mathrm{E}-\odot \odot$ & $\odot . \odot \mathrm{E}-\odot \odot$ & $\odot . \odot \mathrm{E}-\odot \odot$ \\
\hline & 50 & Yr: & & $\odot . \odot \mathrm{E}-\odot \odot$ & $\odot . \odot \mathrm{E}-\odot \odot$ & $\odot . \odot \mathrm{E}-\odot \odot$ & $\odot . \odot \mathrm{E}-\odot \odot$ & $\odot . \odot \mathrm{E}-\odot \odot$ \\
\hline \multirow[t]{3}{*}{ TH227 } & Class: Y & & $1.4 \odot \mathrm{E}-\odot 7$ & & & & & \\
\hline & 1 & Yr: & & $1.6 E-\odot 4$ & $2.8 \mathrm{E}-\odot 7$ & $2.9 \mathrm{E}-\odot 8$ & $8.1 \mathrm{E}-10$ & $1.9 \mathrm{E}-05$ \\
\hline & 50 & Yr: & & $1.6 \mathrm{E}-04$ & $2.8 \mathrm{E}-07$ & $2.9 \mathrm{E}-08$ & $8.1 \mathrm{E}-10$ & $1.9 \mathrm{E}-05$ \\
\hline \multirow[t]{3}{*}{ TH228 } & Class: $\mathrm{Y}$ & & $2.56 \mathrm{E}-\odot 9$ & & & & & \\
\hline & 1 & Yr: & & $6.7 \mathrm{E}-04$ & $4.5 E-05$ & $5.1 \mathrm{E}-06$ & 1. $3 E-07$ & $8.2 \mathrm{E}-05$ \\
\hline & 50 & Yr: & & $6.7 E-04$ & $4.5 E-05$ & $5.1 \mathrm{E}-06$ & $1.3 E-07$ & $8.2 E-05$ \\
\hline \multirow[t]{3}{*}{ TH229 } & Class: Y & & $1.06 \mathrm{E}-07$ & & & & & \\
\hline & 1 & Yr: & & $9.6 \mathrm{E}-\odot 4$ & $5.3 E-\odot 4$ & $3.6 E-\odot 5$ & $2.4 \mathrm{E}-\odot 6$ & 1. $3 E-\odot 4$ \\
\hline & 50 & Yr: & & $9.6 \mathrm{E}-04$ & $5.3 E-\odot 4$ & $3.6 \mathrm{E}-05$ & $2.4 \mathrm{E}-06$ & 1. $3 E-04$ \\
\hline TH230 & Class: Y & & $4.70 \mathrm{E}-10$ & & & & & \\
\hline & 1 & Yr: & & 1. $5 \mathrm{E}-\odot 4$ & $2.5 \mathrm{E}-\odot 4$ & 1. $4 \mathrm{E}-05$ & $4.6 \mathrm{E}-07$ & $2.4 \mathrm{E}-05$ \\
\hline & 50 & Yr: & & 1. $5 \mathrm{E}-04$ & $2.5 E-04$ & 1. $4 \mathrm{E}-05$ & $4.6 \mathrm{E}-07$ & 2. $4 \mathrm{E}-05$ \\
\hline TH231 & Class: $\mathrm{Y}$ & & $1.45 \mathrm{E}-\odot 8$ & & & & & \\
\hline & 1 & Yr: & & 4. $0 \mathrm{E}-\odot 9$ & $6.2 \mathrm{E}-11$ & $5.1 \mathrm{E}-12$ & $9.9 \mathrm{E}-13$ & $7.7 \mathrm{E}-10$ \\
\hline & 50 & Yr: & & 4. ०E-०9 & $6.2 \mathrm{E}-11$ & $5.1 \mathrm{E}-12$ & $9.9 \mathrm{E}-13$ & $7.7 \mathrm{E}-10$ \\
\hline TH232 & Class: Y & & $2.29 \mathrm{E}-10$ & & & & & \\
\hline & 1 & Yr: & & $2.5 E-04$ & $2.9 \mathrm{E}-04$ & $1.8 \mathrm{E}-\odot 5$ & 1. $2 \mathrm{E}-\odot 6$ & $3.7 \mathrm{E}-05$ \\
\hline & 50 & Yr: & & $2.5 E-\odot 4$ & $2.9 \mathrm{E}-04$ & $1.8 \mathrm{E}-05$ & 1.2E-06 & $3.7 \mathrm{E}-05$ \\
\hline TH234 & Class: Y & & $9.30 \mathrm{E}-\odot 9$ & & & & & \\
\hline & 1 & Yr: & & $1.1 \mathrm{E}-07$ & $2.5 \mathrm{E}-10$ & $1.9 \mathrm{E}-10$ & 2. $0 \mathrm{E}-11$ & 1. $7 \mathrm{E}-\odot 8$ \\
\hline & 50 & Yr: & & $1.1 \mathrm{E}-07$ & $2.5 \mathrm{E}-10$ & $1.9 \mathrm{E}-10$ & 2. $0 \mathrm{E}-11$ & 1. $7 \mathrm{E}-\odot 8$ \\
\hline RA223 & Class: W & & $1.73 \mathrm{E}-07$ & & & & & \\
\hline & 1 & Yr: & & $1.1 \mathrm{E}-\odot 4$ & $4.5 E-06$ & $2.6 \mathrm{E}-\odot 7$ & 1. $2 \mathrm{E}-\odot 8$ & 1. $3 \mathrm{E}-05$ \\
\hline & 50 & Yr: & & $1.1 \mathrm{E}-04$ & $4.5 E-\odot 6$ & $2.6 \mathrm{E}-07$ & 1. $2 \mathrm{E}-\odot 8$ & 1. $3 E-05$ \\
\hline RA224 & Class: W & & $1.36 \mathrm{E}-\odot 8$ & & & & & \\
\hline & 1 & Yr: & & $4.4 \mathrm{E}-05$ & $2.3 E-06$ & 1. $3 E-\odot 7$ & $8.3 E-09$ & $5.3 E-06$ \\
\hline & 50 & Yr: & & 4. 4E- 05 & $2.3 E-06$ & 1. $3 E-07$ & 8. 3E-०9 & $5.3 E-06$ \\
\hline RA225 & Class: W & & $7.60 E-\odot 9$ & & & & & \\
\hline & 1 & Yr: & & $9.2 \mathrm{E}-\odot 5$ & $9.4 \mathrm{E}-\odot 6$ & 8. 3E- - 07 & 1. $8 \mathrm{E}-\odot 8$ & 1.1E- 05 \\
\hline & 50 & Yr: & & $9.2 \mathrm{E}-05$ & $9.4 \mathrm{E}-06$ & $8.3 E-07$ & $1.8 \mathrm{E}-08$ & $1.1 \mathrm{E}-05$ \\
\hline RA226 & Class: W & & $8.96 \mathrm{E}-09$ & & & & & \\
\hline & 1 & Yr: & & $5.7 \mathrm{E}-05$ & $8.6 \mathrm{E}-06$ & E- -97 & $4.8 \mathrm{E}-08$ & 7. $0 \mathrm{E}-06$ \\
\hline & 50 & Yr: & & $5.7 E-05$ & $8.6 \mathrm{E}-06$ & $6.7 \mathrm{E}-\odot 7$ & $4.8 \mathrm{E}-\odot 8$ & 7. $0 \mathrm{E}-06$ \\
\hline RA228 & Class: W & & 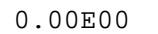 & & & & & \\
\hline & 1 & Yr: & & $2.2 \mathrm{E}-05$ & $1.2 \mathrm{E}-04$ & 1. $4 \mathrm{E}-05$ & 4. $4 \mathrm{E}-07$ & $6.3 \mathrm{E}-06$ \\
\hline & 50 & Yr: & & $2.2 E-05$ & 1. $2 \mathrm{E}-\odot 4$ & 1. $4 \mathrm{E}-05$ & 4. $4 \mathrm{E}-07$ & $6.3 E-06$ \\
\hline PB210 & Class: D & & $1.42 \mathrm{E}-\odot 9$ & & & & & \\
\hline & 1 & Yr: & & $3.7 E-07$ & 2. 3E- $\odot 5$ & $5.4 \mathrm{E}-\odot 6$ & $3.7 \mathrm{E}-07$ & $1.6 \mathrm{E}-06$ \\
\hline & 50 & Yr: & & $3.7 \mathrm{E}-07$ & $2.3 E-05$ & $5.4 \mathrm{E}-06$ & $3.7 \mathrm{E}-07$ & 1. $6 \mathrm{E}-06$ \\
\hline PB212 & Class: D & & $1.97 \mathrm{E}-07$ & & & & & \\
\hline & 1 & Yr: & & $2.1 \mathrm{E}-\odot 8$ & $4.6 \mathrm{E}-07$ & $3.5 E-\odot 8$ & 1. $2 \mathrm{E}-\odot 8$ & $5.5 \mathrm{E}-\odot 8$ \\
\hline & 50 & Yr: & & $2.1 \mathrm{E}-\odot 8$ & $4.6 \mathrm{E}-\odot 7$ & $3.5 \mathrm{E}-\odot 8$ & 1. $2 \mathrm{E}-\odot 8$ & $5.5 E-\odot 8$ \\
\hline BI210 & Class: W & & $8.14 \mathrm{E}-\odot 9$ & & & & & \\
\hline & 1 & Yr: & & $1.5 E-\odot 6$ & $1.5 \mathrm{E}-10$ & 1. $5 \mathrm{E}-10$ & $1.5 \mathrm{E}-10$ & 1. $9 \mathrm{E}-07$ \\
\hline & 50 & Yr: & & 1. $5 \mathrm{E}-06$ & $1.5 \mathrm{E}-10$ & 1. $5 E-10$ & $1.5 \mathrm{E}-10$ & 1. $9 \mathrm{E}-07$ \\
\hline BI212 & Class: W & & $2.83 E-07$ & & & & & \\
\hline & 1 & Yr: & & $3.3 E-07$ & $7.2 \mathrm{E}-11$ & $7.2 \mathrm{E}-11$ & $7.1 \mathrm{E}-11$ & $6.1 \mathrm{E}-08$ \\
\hline & 50 & Yr: & & 3. 3E- -07 & $7.2 \mathrm{E}-11$ & $7.2 \mathrm{E}-11$ & $7.1 \mathrm{E}-11$ & $6.1 \mathrm{E}-08$ \\
\hline P0210 & Class: W & & $1.23 \mathrm{E}-11$ & & & & & \\
\hline & 1 & Yr: & & $5.1 \mathrm{E}-05$ & $1.5 \mathrm{E}-06$ & 2. $\odot E-\odot 6$ & 1. $6 \mathrm{E}-07$ & $6.7 \mathrm{E}-06$ \\
\hline & 50 & Yr: & & $5.1 \mathrm{E}-05$ & $1.5 \mathrm{E}-06$ & 2. $. E-06$ & $1.6 \mathrm{E}-07$ & $6.7 \mathrm{E}-06$ \\
\hline
\end{tabular}




\begin{tabular}{|c|c|c|c|c|c|c|c|c|}
\hline \multirow[t]{3}{*}{ U 232} & Class: $Y$ & & $3.72 \mathrm{E}-10$ & & & & & \\
\hline & 1 & Yr: & & $5.3 E-04$ & $5.5 E-05$ & $5.5 E-06$ & $2.4 \mathrm{E}-\odot 7$ & $6.6 \mathrm{E}-05$ \\
\hline & 50 & Yr: & & $5.3 E-\odot 4$ & $5.5 \mathrm{E}-05$ & $5.5 \mathrm{E}-06$ & $2.4 \mathrm{E}-07$ & $6.6 \mathrm{E}-05$ \\
\hline \multirow[t]{3}{*}{ U 233} & Class: $Y$ & & $4.48 \mathrm{E}-10$ & & & & & \\
\hline & 1 & Yr: & & $1.6 \mathrm{E}-04$ & 1. $2 \mathrm{E}-06$ & $9.2 \mathrm{E}-\odot 8$ & $2.3 E-\odot 8$ & 1. $9 \mathrm{E}-05$ \\
\hline & 50 & Yr: & & 1. $6 \mathrm{E}-\odot 4$ & 1. $2 \mathrm{E}-06$ & $9.2 \mathrm{E}-\odot 8$ & $2.3 \mathrm{E}-08$ & 1. $9 \mathrm{E}-05$ \\
\hline \multirow[t]{3}{*}{ U 234} & Class: Y & & $1.93 \mathrm{E}-10$ & & & & & \\
\hline & 1 & Yr: & & $1.6 E-\odot 4$ & 8. ЗE - ०7 & $7.1 \mathrm{E}-\odot 8$ & $2.1 \mathrm{E}-08$ & 1. $9 \mathrm{E}-05$ \\
\hline & 50 & Yr: & & $1.6 \mathrm{E}-04$ & 8. उE- - 7 & $7.1 \mathrm{E}-08$ & 2.1E-०8 & $1.9 \mathrm{E}-05$ \\
\hline \multirow[t]{3}{*}{ U 235} & Class: $\mathrm{Y}$ & & $2.04 \mathrm{E}-07$ & & & & & \\
\hline & 1 & Yr: & & 1. $4 \mathrm{E}-04$ & $7.9 \mathrm{E}-07$ & $6.8 \mathrm{E}-\odot 8$ & $2.2 \mathrm{E}-\odot 8$ & 1. $7 \mathrm{E}-05$ \\
\hline & 50 & Yr: & & 1. $4 \mathrm{E}-04$ & $7.9 \mathrm{E}-07$ & $6.8 \mathrm{E}-\odot 8$ & 2. $2 \mathrm{E}-\odot 8$ & 1. $7 \mathrm{E}-05$ \\
\hline \multirow[t]{3}{*}{ U 236} & Class: Y & & $1.22 \mathrm{E}-10$ & & & & & \\
\hline & 1 & Yr: & & $1.5 E-\odot 4$ & $7.9 \mathrm{E}-07$ & $6.7 \mathrm{E}-08$ & 2. ๑E-๑8 & 1. $8 \mathrm{E}-05$ \\
\hline & 50 & Yr: & & 1. $5 \mathrm{E}-04$ & $7.9 \mathrm{E}-07$ & $6.7 \mathrm{E}-08$ & 2. $\odot E-\odot 8$ & $1.8 \mathrm{E}-05$ \\
\hline \multirow[t]{3}{*}{ U 237} & Class: $\mathrm{Y}$ & & $1.67 \mathrm{E}-07$ & & & & & \\
\hline & 1 & Yr: & & $2.5 E-\odot 8$ & $9.9 \mathrm{E}-11$ & $3.9 \mathrm{E}-11$ & $3.6 \mathrm{E}-11$ & $3.7 \mathrm{E}-09$ \\
\hline & 50 & Yr: & & $2.5 \mathrm{E}-08$ & $9.9 \mathrm{E}-11$ & $3.9 \mathrm{E}-11$ & $3.6 \mathrm{E}-11$ & $3.7 \mathrm{E}-09$ \\
\hline \multirow[t]{3}{*}{ U 238} & Class: $Y$ & & $7.92 \mathrm{E}-11$ & & & & & \\
\hline & 1 & Yr: & & 1. $3 E-\odot 4$ & $7.5 \mathrm{E}-07$ & $6.7 \mathrm{E}-08$ & 1. $9 \mathrm{E}-08$ & $1.6 \mathrm{E}-05$ \\
\hline & 50 & Yr: & & 1. 3E- - 4 & $7.5 \mathrm{E}-07$ & $6.7 \mathrm{E}-08$ & 1. $9 \mathrm{E}-08$ & $1.6 \mathrm{E}-05$ \\
\hline \multirow{3}{*}{ U 240} & Class: $Y$ & & $1.85 \mathrm{E}-\odot 9$ & & & & & \\
\hline & 1 & Yr: & & $5.4 \mathrm{E}-\odot 9$ & $5.7 \mathrm{E}-11$ & $2.1 \mathrm{E}-11$ & $1.1 \mathrm{E}-11$ & 1. $6 \mathrm{E}-\odot 9$ \\
\hline & 50 & Yr: & & $5.4 \mathrm{E}-\odot 9$ & $5.7 \mathrm{E}-11$ & $2.1 \mathrm{E}-11$ & 1. $1 \mathrm{E}-11$ & $1.6 \mathrm{E}-09$ \\
\hline \multirow[t]{3}{*}{ PA231 } & Class: $\mathrm{Y}$ & & $4.95 \mathrm{E}-\odot 8$ & & & & & \\
\hline & 1 & Yr: & & $2.5 \mathrm{E}-\odot 4$ & $5.9 \mathrm{E}-04$ & $3.2 \mathrm{E}-05$ & 2. $0 \mathrm{E}-06$ & 4. $3 E-05$ \\
\hline & 50 & Yr: & & $2.5 E-\odot 4$ & $5.9 E-\odot 4$ & $3.2 \mathrm{E}-\odot 5$ & 2. $\odot E-\odot 6$ & 4. $3 E-05$ \\
\hline PA233 & Class: $\mathrm{Y}$ & & $2.70 \mathrm{E}-07$ & & & & & \\
\hline & 1 & Yr: & & $5.5 E-\odot 8$ & $5.1 \mathrm{E}-10$ & $1.8 \mathrm{E}-10$ & $1.6 \mathrm{E}-10$ & 7. $5 \mathrm{E}-\odot 9$ \\
\hline & 50 & Yr: & & $5.5 E-08$ & $5.1 \mathrm{E}-10$ & $1.8 \mathrm{E}-10$ & $1.6 \mathrm{E}-10$ & $7.5 \mathrm{E}-09$ \\
\hline PA234 & Class: $Y$ & & $2.75 \mathrm{E}-\odot 6$ & & & & & \\
\hline & 1 & Yr: & & $3.2 E-09$ & $5.2 \mathrm{E}-11$ & $5.4 \mathrm{E}-11$ & $3.3 E-11$ & 1.1E- 09 \\
\hline & 50 & Yr: & & $3.2 \mathrm{E}-09$ & $5.2 \mathrm{E}-11$ & $5.4 \mathrm{E}-11$ & 3. 3E-11 & $1.1 \mathrm{E}-09$ \\
\hline AC225 & Class: $Y$ & & $2.01 \mathrm{E}-08$ & & & & & \\
\hline & 1 & Yr: & & 1. $2 \mathrm{E}-\odot 4$ & 1. $9 \mathrm{E}-\odot 7$ & $2.9 \mathrm{E}-\odot 8$ & 8. ๑E- $\odot 9$ & 1. $5 \mathrm{E}-05$ \\
\hline & 50 & Yr: & & $1.2 \mathrm{E}-04$ & 1. $9 \mathrm{E}-07$ & $2.9 \mathrm{E}-\odot 8$ & 8. $\odot \mathrm{E}-\odot 9$ & $1.5 \mathrm{E}-05$ \\
\hline AC227 & Class: $Y$ & & $1.62 \mathrm{E}-10$ & & & & & \\
\hline & 1 & Yr: & & $8.2 \mathrm{E}-\odot 4$ & $1.8 \mathrm{E}-\odot 4$ & $1.6 \mathrm{E}-\odot 5$ & 1. $2 \mathrm{E}-06$ & 1.1E- -04 \\
\hline & 50 & Yr: & & $8.2 \mathrm{E}-04$ & 1. $8 \mathrm{E}-04$ & $1.6 \mathrm{E}-05$ & 1. $2 \mathrm{E}-06$ & 1. $1 \mathrm{E}-04$ \\
\hline AC228 & Class: $Y$ & & $1.42 \mathrm{E}-\odot 6$ & & & & & \\
\hline & 1 & Yr: & & $2.5 \mathrm{E}-07$ & 1. $6 \mathrm{E}-08$ & 1. $9 \mathrm{E}-09$ & $6.4 \mathrm{E}-11$ & $3.0 \mathrm{E}-08$ \\
\hline & 50 & Yr: & & $2.5 E-07$ & $1.6 \mathrm{E}-08$ & 1. $9 \mathrm{E}-\odot 9$ & $6.4 \mathrm{E}-11$ & $3.0 \mathrm{E}-08$ \\
\hline FR223 & Class: D & & $6.97 \mathrm{E}-\odot 8$ & & & & & \\
\hline & 1 & Yr: & & $3.0 E-\odot 9$ & $2.9 \mathrm{E}-09$ & $2.9 \mathrm{E}-\odot 9$ & 2. . $\mathrm{E}-\odot 9$ & $3.2 \mathrm{E}-09$ \\
\hline & 50 & Yr: & & 3. $\odot E-\odot 9$ & $2.9 \mathrm{E}-\odot 9$ & $2.9 \mathrm{E}-\odot 9$ & $2.9 \mathrm{E}-\odot 9$ & 3. $2 \mathrm{E}-09$ \\
\hline NP237 & Class: W & & $2.81 \mathrm{E}-08$ & & & & & \\
\hline & 1 & Yr: & & $5.9 \mathrm{E}-05$ & $6.8 \mathrm{E}-04$ & $6.3 \mathrm{E}-05$ & $2.8 \mathrm{E}-06$ & $2.8 \mathrm{E}-05$ \\
\hline & 50 & Yr: & & $5.9 \mathrm{E}-05$ & $6.8 \mathrm{E}-04$ & $6.3 E-\odot 5$ & $2.8 \mathrm{E}-\odot 6$ & $2.8 \mathrm{E}-05$ \\
\hline NP238 & Class: W & & $8.07 \mathrm{E}-07$ & & & & & \\
\hline & 1 & Yr: & & 1. $\odot E-\odot 8$ & 4. $7 \mathrm{E}-08$ & $4.7 \mathrm{E}-\odot 9$ & 2. $2 \mathrm{E}-10$ & $3.5 \mathrm{E}-\odot 9$ \\
\hline & 50 & Yr: & & 1. ๑E-๑8 & 4. . E-०8 & 4.7E-09 & 2. $2 \mathrm{E}-10$ & $3.5 \mathrm{E}-09$ \\
\hline NP239 & Class: W & & $2.19 \mathrm{E}-07$ & & & & & \\
\hline & 1 & Yr: & & $1.1 \mathrm{E}-\odot 8$ & 1. $1 \mathrm{E}-\odot 9$ & 1. $5 \mathrm{E}-10$ & $2.4 \mathrm{E}-11$ & 2. $. \mathrm{E}-\odot 9$ \\
\hline & 50 & Yr: & & 1.1E-08 & 1. $1 \mathrm{E}-09$ & $1.5 \mathrm{E}-10$ & 2. $4 \mathrm{E}-11$ & $2.0 \mathrm{E}-09$ \\
\hline PU236 & Class: $\mathrm{Y}$ & & $1.48 \mathrm{E}-10$ & & & & & \\
\hline & 1 & Yr: & & 1. $5 \mathrm{E}-\odot 4$ & $4.4 \mathrm{E}-05$ & $3.7 \mathrm{E}-\odot 6$ & 1. $5 \mathrm{E}-\odot 7$ & $2.0 \mathrm{E}-\odot 5$ \\
\hline & 50 & Yr: & & 1. $5 \mathrm{E}-04$ & $4.4 \mathrm{E}-05$ & $3.7 \mathrm{E}-06$ & $1.5 \mathrm{E}-\odot 7$ & 2. $\odot E-05$ \\
\hline PU237 & Class: $Y$ & & $5.58 \mathrm{E}-\odot 8$ & & & & & \\
\hline & 1 & Yr: & & $6.2 \mathrm{E}-09$ & $1.8 \mathrm{E}-10$ & $E-11$ & $5.9 \mathrm{E}-11$ & $8.9 \mathrm{E}-10$ \\
\hline & 50 & Yr: & & $6.2 \mathrm{E}-\odot 9$ & 1. $8 \mathrm{E}-10$ & $5.5 \mathrm{E}-11$ & $5.9 \mathrm{E}-11$ & $8.9 \mathrm{E}-10$ \\
\hline PU238 & Class: $\mathrm{Y}$ & & $1.11 \mathrm{E}-10$ & & & & & \\
\hline & 1 & Yr: & & $1.8 \mathrm{E}-04$ & 1. $3 E-\odot 4$ & $9.3 \mathrm{E}-06$ & $4.5 \mathrm{E}-07$ & $2.7 \mathrm{E}-05$ \\
\hline & 50 & Yr: & & 1. $8 \mathrm{E}-04$ & 1. $3 E-\odot 4$ & $9.3 \mathrm{E}-\odot 6$ & $4.5 \mathrm{E}-\odot 7$ & $2.7 \mathrm{E}-05$ \\
\hline PU239 & Class: $Y$ & & $1.10 \mathrm{E}-10$ & & & & & \\
\hline & 1 & Yr: & & 1.7E-๑4 & $1.5 \mathrm{E}-\odot 4$ & $E-05$ & $5.2 \mathrm{E}-07$ & 2. $7 \mathrm{E}-05$ \\
\hline & 50 & Yr: & & 1. $7 \mathrm{E}-\odot 4$ & 1. $5 \mathrm{E}-\odot 4$ & 1. $\odot E-\odot 5$ & $5.2 \mathrm{E}-07$ & $2.7 \mathrm{E}-05$ \\
\hline PU240 & Class: $\mathrm{Y}$ & & $1.08 \mathrm{E}-10$ & & & & & \\
\hline & 1 & Yr: & & 1. $7 \mathrm{E}-\odot 4$ & 1. $5 \mathrm{E}-\odot 4$ & 1. $0 \mathrm{E}-\odot 5$ & $5.2 \mathrm{E}-07$ & $2.7 \mathrm{E}-05$ \\
\hline & 50 & Yr: & & 1. $7 \mathrm{E}-\odot 4$ & 1. $5 \mathrm{E}-\odot 4$ & 1. $0 \mathrm{E}-\odot 5$ & $5.2 \mathrm{E}-\odot 7$ & $2.7 \mathrm{E}-05$ \\
\hline PU241 & Class: Y & & $2.00 \mathrm{E}-12$ & & & & & \\
\hline & 1 & Yr: & & $6.4 \mathrm{E}-07$ & $3.6 \mathrm{E}-06$ & 1. $9 \mathrm{E}-07$ & 1. 1E- 08 & $2.0 \mathrm{E}-07$ \\
\hline
\end{tabular}




\begin{tabular}{|c|c|c|c|c|c|c|c|c|}
\hline & 50 & Yr: & & $6.4 \mathrm{E}-07$ & $3.6 \mathrm{E}-06$ & 1. $9 \mathrm{E}-07$ & 1. 1E- -8 & 2. $\odot E-\odot 7$ \\
\hline \multirow[t]{3}{*}{ PU242 } & Class: Y & & $9.18 \mathrm{E}-11$ & & & & & \\
\hline & 1 & Yr: & & 1. $6 \mathrm{E}-\odot 4$ & 1. 5E-๑4 & $9.8 \mathrm{E}-06$ & $4.9 \mathrm{E}-07$ & $2.5 \mathrm{E}-05$ \\
\hline & 50 & Yr: & & $1.6 \mathrm{E}-04$ & $1.5 \mathrm{E}-04$ & $9.8 \mathrm{E}-06$ & $4.9 \mathrm{E}-07$ & $2.5 \mathrm{E}-05$ \\
\hline \multirow[t]{3}{*}{ PU243 } & Class: Y & & 3. ०4E- - ४ & & & & & \\
\hline & 1 & Yr: & & $8.2 \mathrm{E}-10$ & 1. 4E-11 & 1. 4E-12 & $4.1 \mathrm{E}-13$ & $2.1 \mathrm{E}-10$ \\
\hline & 50 & Yr: & & $8.2 \mathrm{E}-10$ & 1. 4E-11 & 1. 4E-12 & $4.1 \mathrm{E}-13$ & $2.1 \mathrm{E}-10$ \\
\hline \multirow[t]{3}{*}{ PU244 } & Class: Y & & $6.56 \mathrm{E}-11$ & & & & & \\
\hline & 1 & Yr: & & 1. 5E-०4 & 1. 4E- - 4 & $9.3 \mathrm{E}-06$ & 4. 7E-07 & $2.3 E-05$ \\
\hline & 50 & Yr: & & $1.5 E-04$ & 1. 4E-०4 & $9.3 \mathrm{E}-06$ & $4.7 E-07$ & $2.3 E-05$ \\
\hline \multirow[t]{3}{*}{ AM241 } & Class: W & & $2.13 E-08$ & & & & & \\
\hline & 1 & Yr: & & $7.3 \mathrm{E}-05$ & 1. $2 \mathrm{E}-03$ & 1. $0 \mathrm{E}-\odot 4$ & $4.8 \mathrm{E}-06$ & $5.1 \mathrm{E}-05$ \\
\hline & 50 & Yr: & & $7.3 E-05$ & 1. $2 \mathrm{E}-\odot 3$ & 1. $\odot E-\odot 4$ & $4.8 \mathrm{E}-\odot 6$ & $5.1 \mathrm{E}-\odot 5$ \\
\hline \multirow[t]{3}{*}{ AM242M } & Class: W & & $7.85 \mathrm{E}-10$ & & & & & \\
\hline & 1 & Yr: & & $1.8 \mathrm{E}-05$ & 1. $2 \mathrm{E}-03$ & $9.1 \mathrm{E}-05$ & $4.8 \mathrm{E}-06$ & $4.1 \mathrm{E}-05$ \\
\hline & 50 & Yr: & & $1.8 \mathrm{E}-05$ & $1.2 \mathrm{E}-03$ & $9.1 \mathrm{E}-05$ & $4.8 \mathrm{E}-06$ & $4.1 \mathrm{E}-05$ \\
\hline \multirow[t]{3}{*}{ AM242 } & Class: W & & 1.93E-๑8 & & & & & \\
\hline & 1 & Yr: & & 2. 2E- - 7 & $2.4 \mathrm{E}-07$ & $3.2 E-\odot 8$ & 3. $0 \mathrm{E}-10$ & $3.6 \mathrm{E}-08$ \\
\hline & 50 & Yr: & & $2.2 \mathrm{E}-07$ & $2.4 \mathrm{E}-07$ & $3.2 \mathrm{E}-08$ & 3. $0 \mathrm{E}-10$ & $3.6 \mathrm{E}-08$ \\
\hline \multirow[t]{3}{*}{ AM243 } & Class: W & & $5.87 E-08$ & & & & & \\
\hline & 1 & Yr: & & $6.9 \mathrm{E}-05$ & 1. $2 \mathrm{E}-03$ & 1. $0 \mathrm{E}-\odot 4$ & $4.9 E-06$ & $5.0 E-05$ \\
\hline & 50 & Yr: & & $6.9 \mathrm{E}-05$ & 1. $2 \mathrm{E}-03$ & 1. $\odot \mathrm{E}-\odot 4$ & $4.9 \mathrm{E}-06$ & $5.0 \mathrm{E}-05$ \\
\hline \multirow[t]{3}{*}{ CM242 } & Class: W & & $1.27 \mathrm{E}-10$ & & & & & \\
\hline & 1 & Yr: & & $6.7 \mathrm{E}-05$ & 7. ०E-०5 & $9.4 \mathrm{E}-06$ & 8. 7E-०8 & $1.1 \mathrm{E}-05$ \\
\hline & 50 & Yr: & & $6.7 \mathrm{E}-05$ & 7. $0 \mathrm{E}-05$ & $9.4 \mathrm{E}-06$ & 8. 7E-०8 & $1.1 \mathrm{E}-05$ \\
\hline \multirow[t]{3}{*}{ CM243 } & Class: W & & $1.67 \mathrm{E}-07$ & & & & & \\
\hline & 1 & Yr: & & $7.8 E-\odot 5$ & 8. 8E- - 4 & 9. ๑E - ๑5 & $2.8 E-06$ & $4.2 \mathrm{E}-05$ \\
\hline & 50 & Yr: & & $7.8 E-05$ & 8. 8E-๑4 & 9. ๑E-๑5 & $2.8 \mathrm{E}-06$ & $4.2 \mathrm{E}-05$ \\
\hline \multirow[t]{3}{*}{ CM244 } & Class: W & & $1.08 \mathrm{E}-10$ & & & & & \\
\hline & 1 & Yr: & & $7.7 \mathrm{E}-05$ & 7. 4E-०4 & $8.2 \mathrm{E}-05$ & $2.1 \mathrm{E}-06$ & $3.8 \mathrm{E}-05$ \\
\hline & 50 & Yr: & & $7.7 E-05$ & $7.4 \mathrm{E}-04$ & $8.2 E-05$ & $2.1 \mathrm{E}-06$ & $3.8 \mathrm{E}-05$ \\
\hline \multirow[t]{3}{*}{ CM245 } & Class: W & & $1.10 \mathrm{E}-07$ & & & & & \\
\hline & 1 & Yr: & & $7.1 \mathrm{E}-05$ & 1. 3E- - 3 & 1. $0 \mathrm{E}-\odot 4$ & $5.1 \mathrm{E}-06$ & $5.2 \mathrm{E}-05$ \\
\hline & 50 & Yr: & & $7.1 \mathrm{E}-05$ & 1. $3 E-03$ & 1. ०E-०4 & $5.1 \mathrm{E}-06$ & $5.2 \mathrm{E}-05$ \\
\hline \multirow[t]{3}{*}{ CM246 } & Class: W & & $9.78 \mathrm{E}-11$ & & & & & \\
\hline & 1 & Yr: & & $7.1 \mathrm{E}-05$ & 1. 3E-03 & 1. ๑E - ०4 & 4. 9E-०6 & $5.1 \mathrm{E}-05$ \\
\hline & 50 & Yr: & & $7.1 \mathrm{E}-05$ & 1. 3E-03 & $1.0 \mathrm{E}-04$ & 4. 9E- 06 & $5.1 \mathrm{E}-05$ \\
\hline \multirow[t]{3}{*}{ CM247 } & Class: W & & $4.38 E-07$ & & & & & \\
\hline & 1 & Yr: & & $6.4 \mathrm{E}-05$ & 1. $2 \mathrm{E}-03$ & $9.5 \mathrm{E}-05$ & 4.6E-०6 & $4.7 \mathrm{E}-05$ \\
\hline & 50 & Yr: & & $6.4 \mathrm{E}-05$ & 1. $2 \mathrm{E}-03$ & $9.5 \mathrm{E}-\odot 5$ & $4.6 \mathrm{E}-06$ & 4. . $7 \mathrm{E}-05$ \\
\hline \multirow[t]{3}{*}{ CM248 } & Class: W & & $7.44 \mathrm{E}-11$ & & & & & \\
\hline & 1 & Yr: & & $6.3 E-05$ & $7.8 \mathrm{E}-03$ & $6.2 \mathrm{E}-\odot 4$ & 2. 2E-๑8 & $4.2 \mathrm{E}-04$ \\
\hline & 50 & Yr: & & $6.3 E-05$ & 7. 8E-०3 & $6.2 \mathrm{E}-04$ & 2. $2 \mathrm{E}-\odot 8$ & $4.2 \mathrm{E}-\odot 4$ \\
\hline \multirow[t]{3}{*}{ CF252 } & Class: W & & $1.15 \mathrm{E}-10$ & & & & & \\
\hline & 1 & Yr: & & 3.5E-05 & $6.6 \mathrm{E}-04$ & $5.3 E-05$ & 1.1E-๑8 & $3.5 \mathrm{E}-05$ \\
\hline & 50 & Yr: & & $3.5 E-05$ & $6.6 \mathrm{E}-04$ & $5.3 E-05$ & 1. 1E-๑8 & $3.5 \mathrm{E}-05$ \\
\hline
\end{tabular}




\section{Appendix A.5}

Age 365 days

Dose Equiv. Factors for Acute Inhalation and Ext Air Submersion FGR12/13

\begin{tabular}{|c|c|c|c|c|c|c|c|c|}
\hline & & & AIR & Acute & Inhal. Con & itted Dose & Equivale & \\
\hline & & & JBMERSION & & BONE & RED & & EFF DOSE \\
\hline & & & J/Yr per & LUNGS & SURFACE & MARROW & THYROID & EQUIV. \\
\hline & & & $\mathrm{Bq} / \mathrm{m} 3$ & $\mathrm{~Sv} / \mathrm{Bq}$ & $\mathrm{Sv} / \mathrm{Bq}$ & $\mathrm{Sv} / \mathrm{Bq}$ & $\mathrm{Sv} / \mathrm{Bq}$ & $\mathrm{S} v / \mathrm{Bq}$ \\
\hline H 3 & Class: D & & $\odot . \odot \odot \mathrm{E} \odot$ & & & & & \\
\hline & 1 & Yr: & & 1. $9 \mathrm{E}-11$ & 1. $9 \mathrm{E}-11$ & $1.9 \mathrm{E}-11$ & 1. $9 \mathrm{E}-11$ & 2. $0 \mathrm{E}-11$ \\
\hline & 50 & Yr: & & 1. $9 \mathrm{E}-11$ & 1. $9 \mathrm{E}-11$ & 1. $9 \mathrm{E}-11$ & 1. $9 \mathrm{E}-11$ & 2. $0 \mathrm{E}-11$ \\
\hline BE10 & Class: $Y$ & & $4.35 E-\odot 9$ & & & & & \\
\hline & 1 & Yr: & & $7.2 \mathrm{E}-07$ & 1. $2 \mathrm{E}-\odot 8$ & $5.9 \mathrm{E}-\odot 9$ & $1.6 \mathrm{E}-10$ & 9. ๑E-๑8 \\
\hline & 50 & Yr: & & $7.2 \mathrm{E}-07$ & 1. $2 \mathrm{E}-08$ & $5.9 \mathrm{E}-09$ & $1.6 \mathrm{E}-10$ & 9. $0 \mathrm{E}-\odot 8$ \\
\hline C 14 & Class: D & & $8.20 E-11$ & & & & & \\
\hline & 1 & Yr: & & $6.4 \mathrm{E}-10$ & $6.2 \mathrm{E}-10$ & $6.2 \mathrm{E}-10$ & $6.2 \mathrm{E}-10$ & $6.8 \mathrm{E}-10$ \\
\hline & 50 & Yr: & & $6.4 \mathrm{E}-10$ & $6.2 \mathrm{E}-10$ & $6.2 \mathrm{E}-10$ & $6.2 \mathrm{E}-10$ & $6.8 \mathrm{E}-10$ \\
\hline F 18 & Class: D & & $1.44 \mathrm{E}-06$ & & & & & \\
\hline & 1 & Yr: & & $7.8 \mathrm{E}-11$ & 1. $3 \mathrm{E}-10$ & $2.4 \mathrm{E}-10$ & $1.1 \mathrm{E}-11$ & $1.9 \mathrm{E}-10$ \\
\hline & 50 & Yr: & & $7.8 \mathrm{E}-11$ & 1. $3 \mathrm{E}-10$ & $2.4 \mathrm{E}-10$ & 1. $1 \mathrm{E}-11$ & 1. $9 \mathrm{E}-10$ \\
\hline NA22 & Class: D & & $3.22 \mathrm{E}-06$ & & & & & \\
\hline & 1 & Yr: & & $5.3 \mathrm{E}-09$ & $1.1 \mathrm{E}-08$ & $9.1 \mathrm{E}-\odot 9$ & $5.5 \mathrm{E}-\odot 9$ & 7. 3E- -99 \\
\hline & 50 & Yr: & & $5.3 E-\odot 9$ & 1. $1 \mathrm{E}-\odot 8$ & $9.1 \mathrm{E}-\odot 9$ & $5.5 \mathrm{E}-\odot 9$ & 7. $3 \mathrm{E}-09$ \\
\hline NA24 & Class: D & & $6.56 \mathrm{E}-06$ & & & & & \\
\hline & 1 & Yr: & & $7.7 \mathrm{E}-10$ & $1.1 \mathrm{E}-09$ & $9.2 \mathrm{E}-10$ & $6.8 \mathrm{E}-10$ & 1. $9 \mathrm{E}-09$ \\
\hline & 50 & Yr: & & $7.7 \mathrm{E}-10$ & 1.1E- 09 & $9.2 \mathrm{E}-10$ & $6.8 \mathrm{E}-10$ & 1. $9 \mathrm{E}-\odot 9$ \\
\hline P 32 & Class: D & & $1.69 \mathrm{E}-08$ & & & & & \\
\hline & 1 & Yr: & & $2.3 E-09$ & $1.8 \mathrm{E}-08$ & $3.7 \mathrm{E}-\odot 8$ & $2.2 \mathrm{E}-\odot 9$ & $7.5 \mathrm{E}-\odot 9$ \\
\hline & 50 & Yr: & & 2. $3 \mathrm{E}-09$ & 1. $8 \mathrm{E}-08$ & $3.7 \mathrm{E}-\odot 8$ & 2. $2 \mathrm{E}-\odot 9$ & $7.5 \mathrm{E}-\odot 9$ \\
\hline P 33 & Class: D & & $4.57 \mathrm{E}-10$ & & & & & \\
\hline & 1 & Yr: & & $3.5 \mathrm{E}-10$ & $3.0 E-\odot 9$ & 2. 3E- -99 & $3.1 \mathrm{E}-10$ & $7.8 \mathrm{E}-10$ \\
\hline & 50 & Yr: & & $3.5 \mathrm{E}-10$ & 3. $\odot E-\odot 9$ & $2.3 E-\odot 9$ & $3.1 \mathrm{E}-10$ & $7.8 \mathrm{E}-10$ \\
\hline S 35 & Class: W & & $9.81 \mathrm{E}-11$ & & & & & \\
\hline & 1 & Yr: & & $3.4 \mathrm{E}-\odot 8$ & $5.5 \mathrm{E}-11$ & $5.5 \mathrm{E}-11$ & $5.5 \mathrm{E}-11$ & $4.5 E-\odot 9$ \\
\hline & 50 & Yr: & & 3. $4 \mathrm{E}-08$ & $5.5 \mathrm{E}-11$ & $5.5 \mathrm{E}-11$ & $5.5 \mathrm{E}-11$ & $4.5 \mathrm{E}-09$ \\
\hline CL36 & Class: D & & $5.23 E-09$ & & & & & \\
\hline & 1 & Yr: & & $2.4 \mathrm{E}-09$ & 2. $3 \mathrm{E}-\odot 9$ & 2. $3 E-\odot 9$ & 2. $3 \mathrm{E}-09$ & $2.6 \mathrm{E}-09$ \\
\hline & 50 & Yr: & & $2.4 \mathrm{E}-09$ & 2. $3 E-\odot 9$ & $2.3 E-\odot 9$ & 2. $3 E-\odot 9$ & $2.6 \mathrm{E}-\odot 9$ \\
\hline AR39 & NobleGas & & $3.63 E-09$ & & & & & \\
\hline & 1 & Yr: & & $\odot . \odot \mathrm{E}-\odot \odot$ & $\odot . \odot \mathrm{E}-\odot \odot$ & $\odot . \odot \mathrm{E}-\odot \odot$ & $\odot . \odot \mathrm{E}-\odot \odot$ & $\odot . \odot \mathrm{E}-\odot \odot$ \\
\hline & 50 & Yr: & & $\odot . \odot \mathrm{E}-\odot \odot$ & $\odot . \odot \mathrm{E}-\odot \odot$ & $\odot . \odot \mathrm{E}-\odot \odot$ & $\odot . \odot \mathrm{E}-\odot \odot$ & $\odot . \odot \mathrm{E}-\odot \odot$ \\
\hline AR41 & NobleGas & & $1.94 \mathrm{E}-06$ & & & & & \\
\hline & 1 & Yr: & & $\odot . \odot \mathrm{E}-\odot \odot$ & $\odot . \odot \mathrm{E}-\odot \odot$ & $\odot . \odot E-\odot \odot$ & $\odot . \odot \mathrm{E}-\odot \odot$ & $\odot . \odot \mathrm{E}-\odot \odot$ \\
\hline & 50 & Yr: & & $\odot . \odot \mathrm{E}-\odot \odot$ & $\odot . \odot \mathrm{E}-\odot \odot$ & $\odot . \odot \mathrm{E}-\odot \odot$ & $\odot . \odot \mathrm{E}-\odot \odot$ & $\odot . \odot \mathrm{E}-\odot \odot$ \\
\hline K 40 & Class: D & & $2.50 \mathrm{E}-07$ & & & & & \\
\hline & 1 & Yr: & & 1. $4 \mathrm{E}-08$ & 1. $4 \mathrm{E}-\odot 8$ & 1. $3 E-\odot 8$ & 1. $4 \mathrm{E}-08$ & 1.7E-๑8 \\
\hline & 50 & Yr: & & 1. $4 \mathrm{E}-08$ & 1. $4 \mathrm{E}-08$ & 1. $3 E-\odot 8$ & 1. $4 \mathrm{E}-08$ & 1. $7 \mathrm{E}-\odot 8$ \\
\hline CA41 & Class: W & & $\odot . \odot \odot Е \odot \odot ~$ & & & & & \\
\hline & 1 & Yr: & & $8.9 \mathrm{E}-10$ & $2.1 \mathrm{E}-\odot 9$ & $8.7 \mathrm{E}-10$ & $5.3 \mathrm{E}-12$ & $2.6 \mathrm{E}-10$ \\
\hline & 50 & Yr: & & $8.9 \mathrm{E}-10$ & $2.1 \mathrm{E}-09$ & $8.7 \mathrm{E}-10$ & $5.3 \mathrm{E}-12$ & $2.6 \mathrm{E}-10$ \\
\hline CA45 & Class: W & & $4.83 E-10$ & & & & & \\
\hline & 1 & Yr: & & $6.1 \mathrm{E}-08$ & 1. $2 \mathrm{E}-08$ & $4.7 \mathrm{E}-\odot 9$ & $E-11$ & $8.7 \mathrm{E}-\odot 9$ \\
\hline & 50 & Yr: & & $6.1 \mathrm{E}-08$ & 1. $2 \mathrm{E}-\odot 8$ & $4.7 \mathrm{E}-\odot 9$ & $6.9 \mathrm{E}-11$ & 8. $7 \mathrm{E}-09$ \\
\hline SC46 & Class: $\mathrm{Y}$ & & $2.95 \mathrm{E}-06$ & & & & & \\
\hline & 1 & Yr: & & $1.4 \mathrm{E}-07$ & $5.7 \mathrm{E}-\odot 9$ & 4. 4E- $\odot 9$ & $5.6 \mathrm{E}-09$ & 2. 3E- - 88 \\
\hline & 50 & Yr: & & 1. $4 \mathrm{E}-07$ & $5.7 \mathrm{E}-\odot 9$ & 4. 4E- $\odot 9$ & $5.6 \mathrm{E}-\odot 9$ & 2. 3E-๑8 \\
\hline CR51 & Class: $Y$ & & $4.35 E-08$ & & & & & \\
\hline & 1 & Yr: & & $7.9 \mathrm{E}-10$ & $5.7 \mathrm{E}-11$ & $4.1 \mathrm{E}-11$ & -11 & $2.1 \mathrm{E}-10$ \\
\hline & 50 & Yr: & & $7.9 \mathrm{E}-10$ & $5.7 \mathrm{E}-11$ & $4.1 \mathrm{E}-11$ & 4. $9 \mathrm{E}-11$ & $2.1 \mathrm{E}-10$ \\
\hline MN54 & Class: W & & $1.21 \mathrm{E}-06$ & & & & & \\
\hline & 1 & Yr: & & $2.1 \mathrm{E}-08$ & $5.1 \mathrm{E}-\odot 9$ & $3.3 E-\odot 9$ & 3. $\odot E-\odot 9$ & $6.3 \mathrm{E}-09$ \\
\hline & 50 & Yr: & & $2.1 \mathrm{E}-08$ & $5.1 \mathrm{E}-\odot 9$ & $3.3 E-09$ & 3. $\odot E-\odot 9$ & $6.3 \mathrm{E}-09$ \\
\hline MN56 & Class: W & & $2.58 \mathrm{E}-06$ & & & & & \\
\hline & 1 & Yr: & & $1.2 \mathrm{E}-\odot 9$ & $4.4 \mathrm{E}-11$ & 4.6E-11 & $E-11$ & $7.8 \mathrm{E}-10$ \\
\hline & 50 & Yr: & & 1. $2 \mathrm{E}-09$ & $4.4 \mathrm{E}-11$ & $4.6 \mathrm{E}-11$ & $2.8 \mathrm{E}-11$ & $7.8 \mathrm{E}-10$ \\
\hline FE55 & Class: W & & ๑. ๑९Е๑९ & & & & & \\
\hline & 1 & Yr: & & $2.1 \mathrm{E}-09$ & 3. 3E- - 9 & 4. 3E-๑9 & 4. $0 \mathrm{E}-10$ & 1. 4E- -99 \\
\hline & 50 & Yr: & & $2.1 \mathrm{E}-09$ & $3.3 E-\odot 9$ & $4.3 E-09$ & 4. $0 \mathrm{E}-10$ & 1. $4 \mathrm{E}-\odot 9$ \\
\hline FE59 & Class: W & & $1.77 \mathrm{E}-06$ & & & & & \\
\hline
\end{tabular}




\begin{tabular}{|c|c|c|c|c|c|c|c|c|}
\hline & 1 & Yr: & & $6.7 \mathrm{E}-08$ & $7.2 \mathrm{E}-\odot 9$ & 7. 3E- $\odot 9$ & $3.7 \mathrm{E}-\odot 9$ & 1. $3 E-\odot 8$ \\
\hline & 50 & Yr: & & $6.7 \mathrm{E}-\odot 8$ & 7. $2 \mathrm{E}-\odot 9$ & 7. 3E-๑9 & $3.7 \mathrm{E}-\odot 9$ & 1. $3 E-\odot 8$ \\
\hline \multirow[t]{3}{*}{$\operatorname{co57}$} & Class: Y & & $1.57 \mathrm{E}-07$ & & & & & \\
\hline & 1 & Yr: & & $2.3 E-08$ & $1.5 \mathrm{E}-09$ & $6.0 \mathrm{E}-10$ & $9.1 \mathrm{E}-10$ & $3.7 \mathrm{E}-09$ \\
\hline & $5 \odot$ & Yr: & & $2.3 E-\odot 8$ & 1. $5 \mathrm{E}-\odot 9$ & 6. $0 \mathrm{E}-10$ & $9.1 \mathrm{E}-10$ & $3.7 \mathrm{E}-09$ \\
\hline \multirow[t]{3}{*}{ C058 } & Class: Y & & $1.40 \mathrm{E}-06$ & & & & & \\
\hline & 1 & Yr: & & $3.9 \mathrm{E}-08$ & $2.5 E-\odot 9$ & 1. $9 \mathrm{E}-\odot 9$ & 2. 4E-०9 & 7. $5 \mathrm{E}-09$ \\
\hline & 50 & Yr: & & $3.9 E-\odot 8$ & $2.5 E-\odot 9$ & 1. $9 \mathrm{E}-\odot 9$ & $2.4 \mathrm{E}-\odot 9$ & $7.5 \mathrm{E}-\odot 9$ \\
\hline \multirow[t]{3}{*}{ C06९ } & Class: Y & & $3.75 \mathrm{E}-06$ & & & & & \\
\hline & 1 & Yr: & & $4.9 \mathrm{E}-07$ & $3.2 \mathrm{E}-08$ & $2.6 \mathrm{E}-\odot 8$ & $3.5 \mathrm{E}-\odot 8$ & $8.6 \mathrm{E}-08$ \\
\hline & 50 & Yr: & & 4. $9 \mathrm{E}-07$ & $3.2 \mathrm{E}-08$ & $2.6 E-\odot 8$ & $3.5 E-08$ & 8. $6 \mathrm{E}-\odot 8$ \\
\hline \multirow[t]{3}{*}{ NI59 } & Class: W & & $\odot . \odot \odot Е \odot \odot ~$ & & & & & \\
\hline & 1 & Yr: & & $2.5 E-\odot 9$ & $2.8 \mathrm{E}-10$ & $2.8 \mathrm{E}-10$ & $2.8 \mathrm{E}-10$ & $6.1 \mathrm{E}-10$ \\
\hline & 50 & Yr: & & $2.5 \mathrm{E}-\odot 9$ & $2.8 \mathrm{E}-10$ & $2.8 \mathrm{E}-10$ & $2.8 \mathrm{E}-10$ & $6.1 \mathrm{E}-10$ \\
\hline \multirow[t]{3}{*}{ NI63 } & Class: W & & $\odot . \odot \odot Е \odot \odot ~$ & & & & & \\
\hline & 1 & Yr: & & $9.7 \mathrm{E}-09$ & $6.8 \mathrm{E}-10$ & $6.8 \mathrm{E}-10$ & $6.8 \mathrm{E}-10$ & 1. $9 \mathrm{E}-\odot 9$ \\
\hline & 50 & Yr: & & $9.7 \mathrm{E}-09$ & $6.8 \mathrm{E}-10$ & $6.8 \mathrm{E}-10$ & $6.8 \mathrm{E}-10$ & 1. $9 \mathrm{E}-09$ \\
\hline \multirow[t]{3}{*}{ NI65 } & Class: W & & $8.42 \mathrm{E}-07$ & & & & & \\
\hline & 1 & Yr: & & $9.5 \mathrm{E}-10$ & 1. $4 \mathrm{E}-11$ & 1. $2 \mathrm{E}-11$ & 1. $0 \mathrm{E}-11$ & $5.2 \mathrm{E}-10$ \\
\hline & 50 & Yr: & & $9.5 \mathrm{E}-10$ & 1. $4 \mathrm{E}-11$ & 1. $2 \mathrm{E}-11$ & 1. $0 \mathrm{E}-11$ & $5.2 \mathrm{E}-10$ \\
\hline \multirow[t]{3}{*}{ CU64 } & Class: D & & $2.69 \mathrm{E}-07$ & & & & & \\
\hline & 1 & Yr: & & $9.4 \mathrm{E}-11$ & $6.2 \mathrm{E}-11$ & $5.9 \mathrm{E}-11$ & $6.1 \mathrm{E}-11$ & $2.7 \mathrm{E}-10$ \\
\hline & 50 & Yr: & & $9.4 \mathrm{E}-11$ & $6.2 \mathrm{E}-11$ & $5.9 \mathrm{E}-11$ & $6.1 \mathrm{E}-11$ & $2.7 \mathrm{E}-10$ \\
\hline \multirow[t]{3}{*}{ ZN65 } & Class: Y & & $8.61 \mathrm{E}-07$ & & & & & \\
\hline & 1 & Yr: & & $3.2 \mathrm{E}-08$ & $3.4 \mathrm{E}-09$ & $2.6 \mathrm{E}-\odot 9$ & $3.5 E-09$ & $6.8 \mathrm{E}-09$ \\
\hline & 50 & Yr: & & $3.2 E-08$ & 3. 4E- -09 & $2.6 E-09$ & $3.5 \mathrm{E}-09$ & $6.8 \mathrm{E}-\odot 9$ \\
\hline \multirow[t]{3}{*}{ ZN69M } & Class: Y & & $5.83 E-07$ & & & & & \\
\hline & 1 & Yr: & & $3.9 \mathrm{E}-09$ & $4.5 E-11$ & $4.0 E-11$ & $2.4 \mathrm{E}-11$ & 1. $7 \mathrm{E}-\odot 9$ \\
\hline & 50 & Yr: & & $3.9 \mathrm{E}-\odot 9$ & $4.5 \mathrm{E}-11$ & 4. $0 \mathrm{E}-11$ & $2.4 \mathrm{E}-11$ & 1. $7 \mathrm{E}-\odot 9$ \\
\hline ZN69 & Class: Y & & $6.31 \mathrm{E}-09$ & & & & & \\
\hline & 1 & Yr: & & $3.2 \mathrm{E}-10$ & $1.8 \mathrm{E}-14$ & 2. $2 \mathrm{E}-14$ & 1. $5 \mathrm{E}-14$ & $1.5 \mathrm{E}-10$ \\
\hline & 50 & Yr: & & $3.2 \mathrm{E}-10$ & $1.8 \mathrm{E}-14$ & $2.2 \mathrm{E}-14$ & $1.5 \mathrm{E}-14$ & $1.5 \mathrm{E}-10$ \\
\hline AS76 & Class: W & & $6.50 \mathrm{E}-07$ & & & & & \\
\hline & 1 & Yr: & & 1.1E- 08 & $2.7 \mathrm{E}-10$ & $2.6 \mathrm{E}-10$ & $2.5 \mathrm{E}-10$ & $4.6 \mathrm{E}-09$ \\
\hline & 50 & Yr: & & 1. 1E-08 & $2.7 \mathrm{E}-10$ & $2.6 \mathrm{E}-10$ & $2.5 \mathrm{E}-10$ & $4.6 \mathrm{E}-09$ \\
\hline SE75 & Class: W & & $5.30 \mathrm{E}-\odot 7$ & & & & & \\
\hline & 1 & Yr: & & 1. 7E-๑8 & $2.3 E-09$ & 1. 4E- 09 & 1. 7E-०9 & $4.6 \mathrm{E}-\odot 9$ \\
\hline & 50 & Yr: & & 1. 7E-๑8 & 2. 3E- $\odot 9$ & 1. 4E-๑9 & 1.7E-०9 & 4. $6 \mathrm{E}-09$ \\
\hline SE79 & Class: W & & $1.24 \mathrm{E}-10$ & & & & & \\
\hline & 1 & Yr: & & $5.9 \mathrm{E}-\odot 8$ & $8.4 \mathrm{E}-10$ & $8.4 \mathrm{E}-10$ & $8.4 \mathrm{E}-10$ & $1.1 \mathrm{E}-08$ \\
\hline & 50 & Yr: & & $5.9 \mathrm{E}-\odot 8$ & $8.4 \mathrm{E}-10$ & $8.4 \mathrm{E}-10$ & $8.4 \mathrm{E}-10$ & $1.1 \mathrm{E}-08$ \\
\hline BR82 & Class: D & & $3.85 E-06$ & & & & & \\
\hline & 1 & Yr: & & $9.5 \mathrm{E}-10$ & $9.1 \mathrm{E}-10$ & $7.8 \mathrm{E}-10$ & $9.6 \mathrm{E}-10$ & $2.2 \mathrm{E}-\odot 9$ \\
\hline & 50 & Yr: & & $9.5 \mathrm{E}-10$ & $9.1 \mathrm{E}-10$ & $7.8 \mathrm{E}-10$ & $9.6 \mathrm{E}-10$ & 2. $2 \mathrm{E}-\odot 9$ \\
\hline BR83 & Class: D & & $1.68 \mathrm{E}-08$ & & & & & \\
\hline & 1 & Yr: & & $8.2 \mathrm{E}-11$ & $2.4 \mathrm{E}-11$ & $2.4 \mathrm{E}-11$ & $2.4 \mathrm{E}-11$ & $1.2 \mathrm{E}-10$ \\
\hline & 50 & Yr: & & $8.2 \mathrm{E}-11$ & $2.4 \mathrm{E}-11$ & $2.4 \mathrm{E}-11$ & 2. $4 \mathrm{E}-11$ & 1. $2 \mathrm{E}-10$ \\
\hline BR84 & Class: D & & $2.84 \mathrm{E}-\odot 6$ & & & & & \\
\hline & 1 & Yr: & & $1.1 \mathrm{E}-10$ & 2. $3 E-11$ & $2.1 \mathrm{E}-11$ & $2.2 \mathrm{E}-11$ & $1.6 \mathrm{E}-10$ \\
\hline & 50 & Yr: & & 1.1E-10 & $2.3 \mathrm{E}-11$ & $2.1 \mathrm{E}-11$ & 2. $2 \mathrm{E}-11$ & 1. $6 \mathrm{E}-10$ \\
\hline KR83M & NobleGas & & $3.78 \mathrm{E}-11$ & & & & & \\
\hline & 1 & Yr: & & $\odot . \odot \mathrm{E}-\odot \odot$ & $\odot . \odot \mathrm{E}-\odot \odot$ & $\odot . \odot \mathrm{E}-\odot \odot$ & $\odot . \odot \mathrm{E}-\odot \odot$ & $\odot . \odot \mathrm{E}-\odot \odot$ \\
\hline & 50 & Yr: & & $\odot . \odot \mathrm{E}-\odot \odot$ & $\odot . \odot \mathrm{E}-\odot \odot$ & $\odot . \odot \mathrm{E}-\odot \odot$ & $\odot . \odot \mathrm{E}-\odot \odot$ & $\odot . \odot \mathrm{E}-\odot \odot$ \\
\hline KR85M & NobleGas & & $2.17 \mathrm{E}-07$ & & & & & \\
\hline & 1 & Yr: & & $\odot . \odot \mathrm{E}-\odot \odot$ & $\odot . \odot \mathrm{E}-\odot \odot$ & $\odot . \odot \mathrm{E}-\odot \odot$ & $\odot . \odot \mathrm{E}-\odot \odot$ & $\odot . \odot \mathrm{E}-\odot \odot$ \\
\hline & 50 & Yr: & & $\odot . \odot \mathrm{E}-\odot \odot$ & $\odot . \odot \mathrm{E}-\odot \odot$ & $\odot . \odot \mathrm{E}-\odot \odot$ & $\odot . \odot \mathrm{E}-\odot \odot$ & $\odot . \odot \mathrm{E}-\odot \odot$ \\
\hline KR85 & NobleGas & & $7.57 \mathrm{E}-\odot 9$ & & & & & \\
\hline & 1 & Yr: & & $\odot . \odot \mathrm{E}-\odot \odot$ & $\odot . \odot \mathrm{E}-\odot \odot$ & $\odot . \odot \mathrm{E}-\odot \odot$ & $\odot . \odot \mathrm{E}-\odot \odot$ & $\odot . \odot \mathrm{E}-\odot \odot$ \\
\hline & 50 & Yr: & & $\odot . \odot \mathrm{E}-\odot \odot$ & $\odot . \odot \mathrm{E}-\odot \odot$ & $\odot . \odot \mathrm{E}-\odot \odot$ & $\odot . \odot E-\odot \odot$ & $\odot . \odot \mathrm{E}-\odot \odot$ \\
\hline KR87 & NobleGas & & $1.26 \mathrm{E}-06$ & & & & & \\
\hline & 1 & Yr: & & $\odot . \odot \mathrm{E}-\odot \odot$ & $\odot . \odot \mathrm{E}-\odot \odot$ & $\odot . \odot \mathrm{E}-\odot \odot$ & $\odot . \odot \mathrm{E}-\odot \odot$ & $\odot . \odot \mathrm{E}-\odot \odot$ \\
\hline & 50 & Yr: & & $\odot . \odot \mathrm{E}-\odot \odot$ & $\odot . \odot \mathrm{E}-\odot \odot$ & $\odot . \odot \mathrm{E}-\odot \odot$ & $\odot . \odot \mathrm{E}-\odot \odot$ & $\odot . \odot \mathrm{E}-\odot \odot$ \\
\hline KR88 & NobleGas & & $3.07 \mathrm{E}-06$ & & & & & \\
\hline & 1 & Yr: & & $\odot . \odot \mathrm{E}-\odot \odot$ & $\odot . \odot \mathrm{E}-\odot \odot$ & $\odot . \odot \mathrm{E}-\odot \odot$ & $\odot . \odot \mathrm{E}-\odot \odot$ & $\odot . \odot \mathrm{E}-\odot \odot$ \\
\hline & 50 & Yr: & & $\odot . \odot \mathrm{E}-\odot \odot$ & $\odot . \odot \mathrm{E}-\odot \odot$ & $\odot . \odot E-\odot \odot$ & $\odot . \odot \mathrm{E}-\odot \odot$ & $\odot . \odot \mathrm{E}-\odot \odot$ \\
\hline KR89 & NobleGas & & $3.45 \mathrm{E}-06$ & & & & & \\
\hline & 1 & Yr: & & $\odot . \odot \mathrm{E}-\odot \odot$ & $\odot . \odot \mathrm{E}-\odot \odot$ & $\odot . \odot \mathrm{E}-\odot \odot$ & $\odot . \odot \mathrm{E}-\odot \odot$ & $\odot . \odot \mathrm{E}-\odot \odot$ \\
\hline & 50 & Yr: & & $\odot . \odot \mathrm{E}-\odot \odot$ & $\odot . \odot \mathrm{E}-\odot \odot$ & $\odot . \odot \mathrm{E}-\odot \odot$ & $\odot . \odot \mathrm{E}-\odot \odot$ & $\odot . \odot \mathrm{E}-\odot \odot$ \\
\hline RB86 & Class: D & & $1.56 \mathrm{E}-07$ & & & & & \\
\hline & 1 & Yr: & & $6.1 \mathrm{E}-09$ & 1.7E- $\odot 8$ & 1. 4E- $\odot 8$ & 6. ๑E- $\odot 9$ & 7. . E- -99 \\
\hline & 50 & Yr: & & $6.1 \mathrm{E}-09$ & 1. 7E-०8 & 1. $4 \mathrm{E}-\odot 8$ & 6. ०E-๑9 & $7.7 \mathrm{E}-09$ \\
\hline
\end{tabular}




\begin{tabular}{|c|c|c|c|c|c|c|c|c|}
\hline \multirow[t]{3}{*}{ RB87 } & Class: D & & $1.04 \mathrm{E}-09$ & & & & & \\
\hline & 1 & Yr: & & $3.2 \mathrm{E}-09$ & $9.4 \mathrm{E}-09$ & $7.5 \mathrm{E}-\odot 9$ & 3. $2 \mathrm{E}-\odot 9$ & $4.1 \mathrm{E}-09$ \\
\hline & 50 & Yr: & & $3.2 \mathrm{E}-09$ & $9.4 \mathrm{E}-09$ & $7.5 \mathrm{E}-\odot 9$ & 3. $2 \mathrm{E}-\odot 9$ & 4.1E- -99 \\
\hline \multirow[t]{3}{*}{ RB88 } & Class: D & & $1.05 E-06$ & & & & & \\
\hline & 1 & Yr: & & $1.1 \mathrm{E}-10$ & 1. $4 \mathrm{E}-11$ & 1. $3 \mathrm{E}-11$ & 1. $3 \mathrm{E}-11$ & 1. $2 \mathrm{E}-10$ \\
\hline & 50 & Yr: & & 1. $1 \mathrm{E}-10$ & 1. $4 \mathrm{E}-11$ & 1. $3 \mathrm{E}-11$ & 1. $3 \mathrm{E}-11$ & 1. $2 \mathrm{E}-10$ \\
\hline \multirow[t]{3}{*}{ RB89 } & Class: D & & $3.19 \mathrm{E}-06$ & & & & & \\
\hline & 1 & Yr: & & $7.2 \mathrm{E}-11$ & 1. $5 \mathrm{E}-11$ & 1. $3 E-11$ & 1.1E-11 & $9.3 \mathrm{E}-11$ \\
\hline & 50 & Yr: & & $7.2 \mathrm{E}-11$ & 1. $5 \mathrm{E}-11$ & 1. $3 \mathrm{E}-11$ & 1.1E-11 & $9.3 \mathrm{E}-11$ \\
\hline \multirow[t]{3}{*}{ SR85 } & Class: D & & $7.06 \mathrm{E}-07$ & & & & & \\
\hline & 1 & Yr: & & $1.5 \mathrm{E}-\odot 9$ & $6.2 \mathrm{E}-09$ & $3.7 E-\odot 9$ & 1.1E- 09 & $2.3 E-\odot 9$ \\
\hline & 50 & Yr: & & 1. 5E-०9 & $6.2 \mathrm{E}-09$ & 3. 7E- $\odot 9$ & 1.1E- $\odot 9$ & 2. $3 E-\odot 9$ \\
\hline \multirow[t]{3}{*}{ SR89 } & Class: D & & $1.38 \mathrm{E}-\odot 8$ & & & & & \\
\hline & 1 & Yr: & & 1. $4 \mathrm{E}-09$ & $3.9 \mathrm{E}-\odot 8$ & $2.9 \mathrm{E}-\odot 8$ & 1. 3E-०9 & 7. $3 E-09$ \\
\hline & 50 & Yr: & & 1. $4 \mathrm{E}-09$ & $3.9 \mathrm{E}-08$ & $2.9 \mathrm{E}-\odot 8$ & 1. $3 \mathrm{E}-\odot 9$ & 7. 3E- -99 \\
\hline \multirow[t]{3}{*}{ SR90 } & Class: D & & $3.10 \mathrm{E}-09$ & & & & & \\
\hline & 1 & Yr: & & 4. 3E- -99 & $5.8 \mathrm{E}-07$ & 3. $3 \mathrm{E}-07$ & 4. 3E- $\odot 9$ & $5.2 \mathrm{E}-08$ \\
\hline & 50 & Yr: & & $4.3 E-\odot 9$ & $5.8 \mathrm{E}-07$ & $3.3 E-07$ & 4. 3E- $\odot 9$ & $5.2 \mathrm{E}-08$ \\
\hline \multirow[t]{3}{*}{ SR91 } & Class: D & & $1.03 \mathrm{E}-06$ & & & & & \\
\hline & 1 & Yr: & & $2.4 \mathrm{E}-10$ & $9.4 \mathrm{E}-10$ & 8. $3 E-10$ & 1. $4 \mathrm{E}-10$ & 1. $1 \mathrm{E}-09$ \\
\hline & 50 & Yr: & & $2.4 \mathrm{E}-10$ & $9.4 \mathrm{E}-10$ & 8. $3 E-10$ & 1. $4 \mathrm{E}-10$ & 1. $1 \mathrm{E}-09$ \\
\hline \multirow[t]{3}{*}{ SR92 } & Class: D & & $2.02 \mathrm{E}-\odot 6$ & & & & & \\
\hline & 1 & Yr: & & $1.6 \mathrm{E}-10$ & $6.9 \mathrm{E}-10$ & $4.0 \mathrm{E}-10$ & $8.6 \mathrm{E}-11$ & $7.1 \mathrm{E}-10$ \\
\hline & 50 & Yr: & & $1.6 \mathrm{E}-10$ & $6.9 \mathrm{E}-10$ & 4. $0 \mathrm{E}-10$ & $8.6 \mathrm{E}-11$ & $7.1 \mathrm{E}-10$ \\
\hline \multirow[t]{3}{*}{ Y 90} & Class: Y & & $2.50 \mathrm{E}-\odot 8$ & & & & & \\
\hline & 1 & Yr: & & $2.6 \mathrm{E}-08$ & $6.1 \mathrm{E}-12$ & 1. $3 E-11$ & 3. $2 \mathrm{E}-13$ & 8. $8 \mathrm{E}-\odot 9$ \\
\hline & 50 & Yr: & & $2.6 E-08$ & $6.1 \mathrm{E}-12$ & 1. $3 E-11$ & $3.2 \mathrm{E}-13$ & 8. 8E-०9 \\
\hline Y 91M & Class: $Y$ & & $7.47 \mathrm{E}-07$ & & & & & \\
\hline & 1 & Yr: & & 1. $7 \mathrm{E}-10$ & $3.9 \mathrm{E}-12$ & $3.5 \mathrm{E}-12$ & $2.5 \mathrm{E}-12$ & $5.9 \mathrm{E}-11$ \\
\hline & 50 & Yr: & & 1. $7 \mathrm{E}-10$ & 3. $9 \mathrm{E}-12$ & 3. $5 \mathrm{E}-12$ & $2.5 \mathrm{E}-12$ & $5.9 \mathrm{E}-11$ \\
\hline Y 91 & Class: $Y$ & & 1.96E-๑8 & & & & & \\
\hline & 1 & Yr: & & $2.4 \mathrm{E}-07$ & $2.6 \mathrm{E}-10$ & $5.2 \mathrm{E}-10$ & 1. $5 \mathrm{E}-11$ & $3.4 \mathrm{E}-08$ \\
\hline & 50 & Yr: & & $2.4 \mathrm{E}-07$ & $2.6 \mathrm{E}-10$ & $5.2 \mathrm{E}-10$ & 1. $5 \mathrm{E}-11$ & $3.4 \mathrm{E}-08$ \\
\hline Y 92 & Class: $Y$ & & $4.16 E-07$ & & & & & \\
\hline & 1 & Yr: & & $2.3 E-\odot 9$ & 7. $2 \mathrm{E}-12$ & $6.5 \mathrm{E}-12$ & 4. $3 E-12$ & 1. $2 \mathrm{E}-09$ \\
\hline & 50 & Yr: & & 2. 3E-०9 & $7.2 \mathrm{E}-12$ & $6.5 \mathrm{E}-12$ & 4. $3 \mathrm{E}-12$ & 1. $2 \mathrm{E}-09$ \\
\hline Y 93 & Class: $\mathrm{Y}$ & & $1.67 \mathrm{E}-07$ & & & & & \\
\hline & 1 & Yr: & & $5.3 E-\odot 9$ & 7. $2 \mathrm{E}-12$ & 7. $0 \mathrm{E}-12$ & $3.8 \mathrm{E}-12$ & 3. $0 \mathrm{E}-\odot 9$ \\
\hline & 50 & Yr: & & $5.3 E-\odot 9$ & 7. $2 \mathrm{E}-12$ & 7. $0 \mathrm{E}-12$ & $3.8 \mathrm{E}-12$ & 3. $\odot E-\odot 9$ \\
\hline ZR93 & Class: W & & $\odot . \odot \odot E \odot \odot ~$ & & & & & \\
\hline & 1 & Yr: & & $1.1 \mathrm{E}-08$ & $5.7 \mathrm{E}-08$ & $8.1 \mathrm{E}-\odot 9$ & 1. $4 \mathrm{E}-11$ & $3.1 \mathrm{E}-\odot 9$ \\
\hline & 50 & Yr: & & $1.1 \mathrm{E}-08$ & $5.7 \mathrm{E}-\odot 8$ & $8.1 \mathrm{E}-\odot 9$ & 1. $4 \mathrm{E}-11$ & $3.1 \mathrm{E}-09$ \\
\hline ZR95 & Class: W & & $1.06 \mathrm{E}-06$ & & & & & \\
\hline & 1 & Yr: & & $9.1 \mathrm{E}-\odot 8$ & 4. $3 E-08$ & 1. $\odot E-\odot 8$ & $2.5 E-\odot 9$ & 1. $6 \mathrm{E}-\odot 8$ \\
\hline & 50 & Yr: & & $9.1 \mathrm{E}-08$ & 4. $3 E-08$ & 1. $0 \mathrm{E}-\odot 8$ & $2.5 E-\odot 9$ & $1.6 \mathrm{E}-08$ \\
\hline ZR97 & Class: W & & $2.81 \mathrm{E}-07$ & & & & & \\
\hline & 1 & Yr: & & 1.1E- 08 & $3.5 \mathrm{E}-10$ & $4.7 \mathrm{E}-10$ & 1. $3 \mathrm{E}-10$ & $5.3 E-\odot 9$ \\
\hline & 50 & Yr: & & 1.1E- $\odot 8$ & $3.5 \mathrm{E}-10$ & $4.7 \mathrm{E}-10$ & 1. $3 \mathrm{E}-10$ & $5.3 E-\odot 9$ \\
\hline NB93M & Class: $\mathrm{Y}$ & & $9.62 \mathrm{E}-11$ & & & & & \\
\hline & 1 & Yr: & & $5.1 \mathrm{E}-\odot 8$ & $2.7 \mathrm{E}-10$ & 1. $3 \mathrm{E}-10$ & 2. $0 \mathrm{E}-11$ & $6.5 \mathrm{E}-\odot 9$ \\
\hline & 50 & Yr: & & $5.1 \mathrm{E}-08$ & $2.7 \mathrm{E}-10$ & $1.3 E-10$ & 2. $0 \mathrm{E}-11$ & $6.5 \mathrm{E}-09$ \\
\hline NB94 & Class: $Y$ & & $2.27 \mathrm{E}-06$ & & & & & \\
\hline & 1 & Yr: & & $7.6 \mathrm{E}-07$ & $3.2 \mathrm{E}-08$ & $2.7 \mathrm{E}-\odot 8$ & $3.2 \mathrm{E}-08$ & 1. $2 \mathrm{E}-07$ \\
\hline & 50 & Yr: & & $7.6 \mathrm{E}-07$ & $3.2 \mathrm{E}-08$ & $2.7 \mathrm{E}-\odot 8$ & $3.2 \mathrm{E}-08$ & 1. $2 \mathrm{E}-07$ \\
\hline NB95M & Class: $\mathrm{Y}$ & & $8.64 \mathrm{E}-08$ & & & & & \\
\hline & 1 & Yr: & & 1.7E-०8 & $1.8 \mathrm{E}-10$ & 1. $3 \mathrm{E}-10$ & 1. $2 \mathrm{E}-10$ & $3.4 \mathrm{E}-\odot 9$ \\
\hline & 50 & Yr: & & 1. 7E-०8 & 1. $8 \mathrm{E}-10$ & 1. $3 E-10$ & 1. $2 \mathrm{E}-10$ & $3.4 \mathrm{E}-\odot 9$ \\
\hline NB95 & Class: $Y$ & & $1.10 \mathrm{E}-06$ & & & & & \\
\hline & 1 & Yr: & & $3.5 E-\odot 8$ & 1. $2 \mathrm{E}-09$ & $E-10$ & $1.1 \mathrm{E}-\odot 9$ & $5.9 \mathrm{E}-\odot 9$ \\
\hline & 50 & Yr: & & $3.5 E-\odot 8$ & 1. $2 \mathrm{E}-\odot 9$ & $9.5 \mathrm{E}-10$ & 1. $1 \mathrm{E}-\odot 9$ & $5.9 \mathrm{E}-\odot 9$ \\
\hline NB97M & Class: $Y$ & & $1.04 \mathrm{E}-06$ & & & & & \\
\hline & 1 & Yr: & & $2.4 \mathrm{E}-12$ & $2.3 \mathrm{E}-14$ & $3.1 \mathrm{E}-14$ & $2.8 \mathrm{E}-14$ & $3.5 E-13$ \\
\hline & 50 & Yr: & & $2.4 \mathrm{E}-12$ & $2.3 E-14$ & $3.1 \mathrm{E}-14$ & $2.8 \mathrm{E}-14$ & $3.5 \mathrm{E}-13$ \\
\hline NB97 & Class: $Y$ & & $9.43 \mathrm{E}-07$ & & & & & \\
\hline & 1 & Yr: & & $4.7 \mathrm{E}-10$ & $6.7 \mathrm{E}-12$ & $5.7 \mathrm{E}-12$ & 4. $3 E-12$ & $2.6 \mathrm{E}-10$ \\
\hline & 50 & Yr: & & $4.7 \mathrm{E}-10$ & $6.7 \mathrm{E}-12$ & $5.7 \mathrm{E}-12$ & 4. $3 E-12$ & $2.6 \mathrm{E}-10$ \\
\hline M093 & Class: D & & $5.46 \mathrm{E}-10$ & & & & & \\
\hline & 1 & Yr: & & $5.7 \mathrm{E}-10$ & 4.1E- - 88 & 1. $3 E-\odot 8$ & $3.8 \mathrm{E}-10$ & $2.6 \mathrm{E}-\odot 9$ \\
\hline & 50 & Yr: & & $5.7 \mathrm{E}-10$ & 4. 1E- - 88 & 1. $3 E-\odot 8$ & $3.8 \mathrm{E}-10$ & $2.6 \mathrm{E}-\odot 9$ \\
\hline M099 & Class: D & & $2.20 \mathrm{E}-\odot 7$ & & & & & \\
\hline & 1 & Yr: & & $7.4 \mathrm{E}-10$ & 2. $\odot \mathrm{E}-\odot 9$ & 1. $9 \mathrm{E}-\odot 9$ & $6.4 \mathrm{E}-10$ & 1. $7 \mathrm{E}-\odot 9$ \\
\hline
\end{tabular}




\begin{tabular}{|c|c|c|c|c|c|c|c|c|}
\hline & 50 & Yr: & & $7.4 \mathrm{E}-10$ & 2. $0 \mathrm{E}-\odot 9$ & 1. $9 \mathrm{E}-\odot 9$ & $6.4 \mathrm{E}-10$ & 1. $7 \mathrm{E}-\odot 9$ \\
\hline \multirow[t]{3}{*}{ TC99M } & Class: W & & $1.66 \mathrm{E}-07$ & & & & & \\
\hline & 1 & Yr: & & $2.0 \mathrm{E}-10$ & 1. $2 \mathrm{E}-11$ & $5.9 \mathrm{E}-12$ & $5.7 \mathrm{E}-11$ & $9.9 \mathrm{E}-11$ \\
\hline & 50 & Yr: & & 2. $\odot E-10$ & 1. $2 \mathrm{E}-11$ & $5.9 \mathrm{E}-12$ & $5.7 \mathrm{E}-11$ & $9.9 \mathrm{E}-11$ \\
\hline \multirow[t]{3}{*}{ TC99 } & Class: W & & $9.05 \mathrm{E}-10$ & & & & & \\
\hline & 1 & Yr: & & $9.7 \mathrm{E}-08$ & $6.2 \mathrm{E}-11$ & $6.2 \mathrm{E}-11$ & $2.5 \mathrm{E}-\odot 9$ & 1. $3 E-08$ \\
\hline & 50 & Yr: & & $9.7 \mathrm{E}-08$ & $6.2 \mathrm{E}-11$ & $6.2 \mathrm{E}-11$ & $2.5 \mathrm{E}-\odot 9$ & 1. $3 \mathrm{E}-\odot 8$ \\
\hline \multirow[t]{3}{*}{ TC101 } & Class: W & & $4.76 \mathrm{E}-07$ & & & & & \\
\hline & 1 & Yr: & & $9.4 \mathrm{E}-11$ & $9.4 \mathrm{E}-13$ & 7. $\odot E-13$ & 1. 3E-11 & $7.2 \mathrm{E}-11$ \\
\hline & 50 & Yr: & & $9.4 \mathrm{E}-11$ & $9.4 \mathrm{E}-13$ & 7. $0 \mathrm{E}-13$ & 1. $3 \mathrm{E}-11$ & $7.2 \mathrm{E}-11$ \\
\hline \multirow[t]{3}{*}{ RU103 } & Class: Y & & $6.59 \mathrm{E}-07$ & & & & & \\
\hline & 1 & Yr: & & $6.7 \mathrm{E}-\odot 8$ & $8.5 \mathrm{E}-10$ & $6.6 \mathrm{E}-10$ & $7.6 \mathrm{E}-10$ & $9.9 \mathrm{E}-\odot 9$ \\
\hline & 50 & Yr: & & $6.7 \mathrm{E}-08$ & $8.5 \mathrm{E}-10$ & $6.6 \mathrm{E}-10$ & $7.6 \mathrm{E}-10$ & $9.9 \mathrm{E}-09$ \\
\hline \multirow[t]{3}{*}{ RU105 } & Class: Y & & $1.13 \mathrm{E}-06$ & & & & & \\
\hline & 1 & Yr: & & $2.3 E-\odot 9$ & $3.1 \mathrm{E}-11$ & $2.7 \mathrm{E}-11$ & $1.8 \mathrm{E}-11$ & $9.9 \mathrm{E}-10$ \\
\hline & 50 & Yr: & & $2.3 E-09$ & $3.1 \mathrm{E}-11$ & $2.7 \mathrm{E}-11$ & $1.8 \mathrm{E}-11$ & $9.9 \mathrm{E}-10$ \\
\hline \multirow[t]{3}{*}{ RU106 } & Class: Y & & 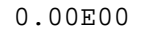 & & & & & \\
\hline & 1 & Yr: & & 1.7E-०6 & $2.5 \mathrm{E}-\odot 9$ & $2.2 \mathrm{E}-\odot 9$ & $2.5 E-\odot 9$ & $2.2 \mathrm{E}-07$ \\
\hline & 50 & Yr: & & 1. $7 \mathrm{E}-\odot 6$ & $2.5 \mathrm{E}-\odot 9$ & 2. $2 \mathrm{E}-\odot 9$ & $2.5 \mathrm{E}-\odot 9$ & 2. $2 \mathrm{E}-07$ \\
\hline \multirow[t]{3}{*}{ RH103M } & Class: $Y$ & & $1.90 \mathrm{E}-10$ & & & & & \\
\hline & 1 & Yr: & & $5.3 \mathrm{E}-11$ & $4.6 \mathrm{E}-14$ & 1. $3 \mathrm{E}-14$ & $8.7 \mathrm{E}-15$ & 1. $3 \mathrm{E}-11$ \\
\hline & 50 & Yr: & & $5.3 \mathrm{E}-11$ & 4. $6 \mathrm{E}-14$ & 1. $3 \mathrm{E}-14$ & 8.7E-15 & 1. $3 \mathrm{E}-11$ \\
\hline \multirow[t]{3}{*}{ RH105 } & Class: Y & & $1.10 \mathrm{E}-07$ & & & & & \\
\hline & 1 & Yr: & & $6.3 \mathrm{E}-09$ & $2.2 \mathrm{E}-11$ & $1.8 \mathrm{E}-11$ & 1. $5 \mathrm{E}-11$ & 1. $7 \mathrm{E}-\odot 9$ \\
\hline & 50 & Yr: & & $6.3 E-\odot 9$ & 2. $2 \mathrm{E}-11$ & $1.8 \mathrm{E}-11$ & 1. $5 \mathrm{E}-11$ & 1. $7 \mathrm{E}-\odot 9$ \\
\hline \multirow[t]{3}{*}{ PD103 } & Class: Y & & $1.68 \mathrm{E}-09$ & & & & & \\
\hline & 1 & Yr: & & $1.1 \mathrm{E}-08$ & $7.9 \mathrm{E}-11$ & $1.4 \mathrm{E}-11$ & $6.5 \mathrm{E}-12$ & $1.8 \mathrm{E}-\odot 9$ \\
\hline & 50 & Yr: & & 1.1E-08 & $7.9 \mathrm{E}-11$ & $1.4 \mathrm{E}-11$ & $6.5 \mathrm{E}-12$ & $1.8 \mathrm{E}-\odot 9$ \\
\hline PD107 & Class: $\mathrm{Y}$ & & ๑. ๑૦Е๑९ & & & & & \\
\hline & 1 & Yr: & & $1.6 \mathrm{E}-\odot 8$ & 1. $2 \mathrm{E}-12$ & $7.8 \mathrm{E}-13$ & $1.1 \mathrm{E}-13$ & 2. $\odot E-\odot 9$ \\
\hline & 50 & Yr: & & $1.6 \mathrm{E}-08$ & 1. $2 \mathrm{E}-12$ & $7.8 \mathrm{E}-13$ & 1. $1 \mathrm{E}-13$ & 2. $\odot E-\odot 9$ \\
\hline PD109 & Class: Y & & $1.33 \mathrm{E}-08$ & & & & & \\
\hline & 1 & Yr: & & $6.1 \mathrm{E}-09$ & $5.6 \mathrm{E}-12$ & $1.8 \mathrm{E}-12$ & $6.9 \mathrm{E}-13$ & 1. $9 \mathrm{E}-\odot 9$ \\
\hline & 50 & Yr: & & $6.1 \mathrm{E}-09$ & $5.6 \mathrm{E}-12$ & $1.8 \mathrm{E}-12$ & $6.9 \mathrm{E}-13$ & $1.9 \mathrm{E}-09$ \\
\hline AG110M & Class: D & & $4.01 \mathrm{E}-06$ & & & & & \\
\hline & 1 & Yr: & & 2. 3E- $\odot 8$ & 1. $6 \mathrm{E}-\odot 8$ & 1. $4 \mathrm{E}-\odot 8$ & 1. $3 E-\odot 8$ & $2.8 \mathrm{E}-\odot 8$ \\
\hline & 50 & Yr: & & $2.3 E-08$ & 1. $6 \mathrm{E}-08$ & 1. $4 \mathrm{E}-\odot 8$ & 1. $3 E-08$ & $2.8 \mathrm{E}-\odot 8$ \\
\hline AG111 & Class: D & & $4.38 \mathrm{E}-\odot 8$ & & & & & \\
\hline & 1 & Yr: & & $7.5 \mathrm{E}-10$ & $6.8 \mathrm{E}-10$ & $6.8 \mathrm{E}-10$ & $6.8 \mathrm{E}-10$ & $3.2 \mathrm{E}-09$ \\
\hline & 50 & Yr: & & $7.5 \mathrm{E}-10$ & $6.8 \mathrm{E}-10$ & $6.8 \mathrm{E}-10$ & $6.8 \mathrm{E}-10$ & $3.2 \mathrm{E}-09$ \\
\hline CD109 & Class: D & & $7.22 \mathrm{E}-09$ & & & & & \\
\hline & 1 & Yr: & & $9.7 \mathrm{E}-09$ & 1. $3 E-08$ & $7.5 \mathrm{E}-09$ & 8. ०E-०9 & $3.7 \mathrm{E}-\odot 8$ \\
\hline & 50 & Yr: & & $9.7 \mathrm{E}-09$ & 1. $3 E-08$ & $7.5 E-\odot 9$ & 8. $\odot E-\odot 9$ & $3.7 \mathrm{E}-08$ \\
\hline CD113M & Class: D & & $2.86 \mathrm{E}-\odot 9$ & & & & & \\
\hline & 1 & Yr: & & $5.1 \mathrm{E}-08$ & $5.1 \mathrm{E}-08$ & $5.1 \mathrm{E}-08$ & $5.1 \mathrm{E}-08$ & $2.7 \mathrm{E}-07$ \\
\hline & 50 & Yr: & & $5.1 \mathrm{E}-\odot 8$ & $5.1 \mathrm{E}-08$ & $5.1 \mathrm{E}-\odot 8$ & $5.1 \mathrm{E}-08$ & $2.7 \mathrm{E}-07$ \\
\hline CD115M & Class: D & & $4.67 \mathrm{E}-08$ & & & & & \\
\hline & 1 & Yr: & & $6.4 \mathrm{E}-09$ & $6.3 \mathrm{E}-09$ & $6.2 \mathrm{E}-\odot 9$ & $6.2 \mathrm{E}-\odot 9$ & $3.2 \mathrm{E}-\odot 8$ \\
\hline & 50 & Yr: & & $6.4 \mathrm{E}-09$ & $6.3 E-09$ & $6.2 \mathrm{E}-\odot 9$ & $6.2 \mathrm{E}-\odot 9$ & $3.2 \mathrm{E}-08$ \\
\hline CD115 & Class: D & & $3.31 \mathrm{E}-07$ & & & & & \\
\hline & 1 & Yr: & & $5.0 \mathrm{E}-10$ & $4.4 \mathrm{E}-10$ & 4. $0 \mathrm{E}-10$ & $3.7 \mathrm{E}-10$ & $2.6 \mathrm{E}-\odot 9$ \\
\hline & 50 & Yr: & & $5.0 \mathrm{E}-10$ & $4.4 \mathrm{E}-10$ & 4. $0 \mathrm{E}-10$ & $3.7 \mathrm{E}-10$ & $2.6 \mathrm{E}-09$ \\
\hline IN111 & Class: D & & $5.30 \mathrm{E}-07$ & & & & & \\
\hline & 1 & Yr: & & $2.3 E-10$ & 1. $2 \mathrm{E}-\odot 9$ & 1. $2 \mathrm{E}-\odot 9$ & $E-10$ & $8.7 \mathrm{E}-10$ \\
\hline & 50 & Yr: & & $2.3 \mathrm{E}-10$ & 1. $2 \mathrm{E}-09$ & 1. $2 \mathrm{E}-\odot 9$ & $1.6 \mathrm{E}-10$ & $8.7 \mathrm{E}-10$ \\
\hline IN114M & Class: D & & $1.23 \mathrm{E}-07$ & & & & & \\
\hline & 1 & Yr: & & 1.1E-๑8 & $3.7 \mathrm{E}-07$ & $4.4 \mathrm{E}-07$ & 1.1E- $\odot 8$ & $7.8 \mathrm{E}-\odot 8$ \\
\hline & 50 & Yr: & & $1.1 \mathrm{E}-08$ & $3.7 \mathrm{E}-07$ & 4. 4E- -07 & $1.1 \mathrm{E}-08$ & $7.8 E-08$ \\
\hline IN115M & Class: D & & $2.17 \mathrm{E}-07$ & & & & & \\
\hline & 1 & Yr: & & $5.5 \mathrm{E}-11$ & $=-10$ & $=-10$ & $=-11$ & $1.9 \mathrm{E}-10$ \\
\hline & 50 & Yr: & & $5.5 \mathrm{E}-11$ & 1. $4 \mathrm{E}-10$ & $1.6 \mathrm{E}-10$ & 1. $9 \mathrm{E}-11$ & 1. $9 \mathrm{E}-10$ \\
\hline SN117M & Class: W & & $1.93 E-07$ & & & & & \\
\hline & 1 & Yr: & & $5.0 \mathrm{E}-08$ & $4.6 \mathrm{E}-09$ & $8.9 \mathrm{E}-10$ & $1.4 \mathrm{E}-10$ & $7.7 \mathrm{E}-\odot 9$ \\
\hline & 50 & Yr: & & $5.0 \mathrm{E}-\odot 8$ & $4.6 \mathrm{E}-\odot 9$ & $8.9 \mathrm{E}-10$ & $1.4 \mathrm{E}-10$ & $7.7 \mathrm{E}-09$ \\
\hline SN119M & Class: W & & $2.23 E-09$ & & & & & \\
\hline & 1 & Yr: & & $5.5 E-\odot 8$ & $3.5 E-09$ & 2.๑E-๑9 & 2. $4 \mathrm{E}-10$ & $7.8 \mathrm{E}-09$ \\
\hline & 50 & Yr: & & $5.5 \mathrm{E}-08$ & $3.5 \mathrm{E}-\odot 9$ & 2. $\odot \mathrm{E}-\odot 9$ & $2.4 \mathrm{E}-10$ & 7. $8 \mathrm{E}-09$ \\
\hline SN121M & Class: W & & $1.66 \mathrm{E}-\odot 9$ & & & & & \\
\hline & 1 & Yr: & & $1.1 \mathrm{E}-07$ & 1. $2 \mathrm{E}-08$ & $7.1 \mathrm{E}-09$ & $7.1 \mathrm{E}-10$ & 1. $5 \mathrm{E}-08$ \\
\hline & 50 & Yr: & & $1.1 \mathrm{E}-07$ & 1. $2 \mathrm{E}-08$ & $7.1 \mathrm{E}-09$ & $7.1 \mathrm{E}-10$ & $1.5 \mathrm{E}-08$ \\
\hline SN121 & Class: W & & $1.23 \mathrm{E}-\odot 9$ & & & & & \\
\hline
\end{tabular}




\begin{tabular}{|c|c|c|c|c|c|c|c|c|}
\hline & 1 & Yr: & & $4.1 \mathrm{E}-09$ & $2.1 \mathrm{E}-10$ & $3.9 \mathrm{E}-11$ & $2.6 \mathrm{E}-12$ & 1.1E- -99 \\
\hline & 50 & Yr: & & $4.1 \mathrm{E}-09$ & $2.1 \mathrm{E}-10$ & $3.9 \mathrm{E}-11$ & $2.6 \mathrm{E}-12$ & 1. $1 \mathrm{E}-09$ \\
\hline \multirow[t]{3}{*}{ SN123 } & Class: W & & $2.20 \mathrm{E}-\odot 8$ & & & & & \\
\hline & 1 & Yr: & & $2.1 \mathrm{E}-07$ & 1. $0 \mathrm{E}-\odot 8$ & 7. ०E-๑9 & $6.9 \mathrm{E}-10$ & $3.1 \mathrm{E}-08$ \\
\hline & 50 & Yr: & & $2.1 \mathrm{E}-\odot 7$ & 1. $\odot E-\odot 8$ & 7. ๑E-๑9 & $6.9 \mathrm{E}-10$ & $3.1 \mathrm{E}-08$ \\
\hline \multirow[t]{3}{*}{ SN125 } & Class: W & & $4.86 E-07$ & & & & & \\
\hline & 1 & Yr: & & $6.5 E-\odot 8$ & $2.6 \mathrm{E}-\odot 9$ & $3.1 \mathrm{E}-\odot 9$ & $2.6 \mathrm{E}-10$ & $1.5 \mathrm{E}-08$ \\
\hline & 50 & Yr: & & $6.5 \mathrm{E}-\odot 8$ & $2.6 \mathrm{E}-\odot 9$ & $3.1 \mathrm{E}-09$ & $2.6 \mathrm{E}-10$ & 1. $5 \mathrm{E}-\odot 8$ \\
\hline \multirow[t]{3}{*}{ SN126 } & Class: W & & $5.83 E-08$ & & & & & \\
\hline & 1 & Yr: & & $6.0 \mathrm{E}-\odot 7$ & $9.8 \mathrm{E}-08$ & $6.5 \mathrm{E}-\odot 8$ & $1.8 \mathrm{E}-\odot 8$ & 1. $0 \mathrm{E}-\odot 7$ \\
\hline & 50 & Yr: & & $6.0 \mathrm{E}-07$ & $9.8 \mathrm{E}-08$ & $6.5 E-08$ & 1. $8 \mathrm{E}-08$ & 1. $0 \mathrm{E}-07$ \\
\hline \multirow[t]{3}{*}{ SB124 } & Class: W & & $2.72 \mathrm{E}-\odot 6$ & & & & & \\
\hline & 1 & Yr: & & 1. $4 \mathrm{E}-\odot 7$ & $8.8 \mathrm{E}-09$ & $5.5 E-\odot 9$ & $3.1 \mathrm{E}-09$ & $2.4 \mathrm{E}-08$ \\
\hline & 50 & Yr: & & 1. $4 \mathrm{E}-07$ & $8.8 \mathrm{E}-09$ & $5.5 \mathrm{E}-09$ & $3.1 \mathrm{E}-09$ & 2. $4 \mathrm{E}-08$ \\
\hline \multirow[t]{3}{*}{ SB125 } & Class: W & & $5.90 \mathrm{E}-07$ & & & & & \\
\hline & 1 & Yr: & & 1. $\odot E-07$ & $2.9 \mathrm{E}-08$ & $7.2 \mathrm{E}-\odot 9$ & $2.3 E-09$ & 1. $6 \mathrm{E}-08$ \\
\hline & 50 & Yr: & & 1. $\odot E-\odot 7$ & $2.9 \mathrm{E}-\odot 8$ & $7.2 \mathrm{E}-\odot 9$ & $2.3 E-09$ & 1. $6 \mathrm{E}-08$ \\
\hline \multirow[t]{3}{*}{ SB126M } & Class: W & & $2.21 \mathrm{E}-06$ & & & & & \\
\hline & 1 & Yr: & & 1. $4 \mathrm{E}-10$ & $5.0 \mathrm{E}-12$ & $4.1 \mathrm{E}-12$ & 3. $7 \mathrm{E}-12$ & 1. $2 \mathrm{E}-10$ \\
\hline & 50 & Yr: & & 1. $4 \mathrm{E}-10$ & 5. $0 \mathrm{E}-12$ & $4.1 \mathrm{E}-12$ & 3. $7 \mathrm{E}-12$ & 1. $2 \mathrm{E}-10$ \\
\hline \multirow[t]{3}{*}{ SB126 } & Class: W & & $4.04 \mathrm{E}-\odot 6$ & & & & & \\
\hline & 1 & Yr: & & $5.1 \mathrm{E}-08$ & $3.2 \mathrm{E}-\odot 9$ & 2. 4E- $\odot 9$ & 1.7E-०9 & 1. $4 \mathrm{E}-08$ \\
\hline & 50 & Yr: & & $5.1 \mathrm{E}-08$ & $3.2 \mathrm{E}-09$ & $2.4 \mathrm{E}-09$ & 1.7E-09 & 1. $4 \mathrm{E}-08$ \\
\hline \multirow[t]{3}{*}{ SB127 } & Class: W & & $9.84 \mathrm{E}-07$ & & & & & \\
\hline & 1 & Yr: & & $3.1 \mathrm{E}-\odot 8$ & $6.2 \mathrm{E}-10$ & $7.4 \mathrm{E}-10$ & 2. $\odot \mathrm{E}-10$ & 7. $4 \mathrm{E}-\odot 9$ \\
\hline & 50 & Yr: & & $3.1 \mathrm{E}-08$ & $6.2 \mathrm{E}-10$ & $7.4 \mathrm{E}-10$ & $2.0 \mathrm{E}-10$ & $7.4 \mathrm{E}-09$ \\
\hline \multirow[t]{3}{*}{ TE123M } & Class: W & & $1.84 \mathrm{E}-07$ & & & & & \\
\hline & 1 & Yr: & & $8.5 E-\odot 8$ & $4.8 E-08$ & $8.3 E-\odot 9$ & $3.9 E-\odot 9$ & 1. $3 E-08$ \\
\hline & 50 & Yr: & & $8.5 E-\odot 8$ & $4.8 \mathrm{E}-\odot 8$ & $8.3 E-\odot 9$ & $3.9 \mathrm{E}-\odot 9$ & 1. $3 E-08$ \\
\hline TE125M & Class: W & & $1.06 \mathrm{E}-\odot 8$ & & & & & \\
\hline & 1 & Yr: & & 7. 4E-०8 & $2.4 \mathrm{E}-\odot 8$ & 4. ०E-०9 & 3. ०E-०9 & 1.1E- -88 \\
\hline & 50 & Yr: & & $7.4 \mathrm{E}-08$ & $2.4 \mathrm{E}-08$ & 4. ०E-๑9 & 3. ०E-०9 & 1. $1 \mathrm{E}-08$ \\
\hline TE127M & Class: W & & $3.56 \mathrm{E}-\odot 9$ & & & & & \\
\hline & 1 & Yr: & & 1.7E- -7 & 4.1E-๑8 & $2.1 \mathrm{E}-08$ & $9.7 \mathrm{E}-09$ & $2.6 \mathrm{E}-08$ \\
\hline & 50 & Yr: & & 1.7E-07 & $4.1 \mathrm{E}-08$ & $2.1 \mathrm{E}-08$ & $9.7 \mathrm{E}-09$ & $2.6 \mathrm{E}-08$ \\
\hline TE127 & Class: W & & $1.05 E-\odot 8$ & & & & & \\
\hline & 1 & Yr: & & $2.1 \mathrm{E}-09$ & 1. $2 \mathrm{E}-11$ & 1. $7 \mathrm{E}-11$ & $3.4 \mathrm{E}-11$ & 7. $3 \mathrm{E}-10$ \\
\hline & 50 & Yr: & & $2.1 \mathrm{E}-\odot 9$ & 1. $2 \mathrm{E}-11$ & 1. $7 \mathrm{E}-11$ & $3.4 \mathrm{E}-11$ & 7. $3 \mathrm{E}-10$ \\
\hline TE129M & Class: W & & 4. 92E-๑8 & & & & & \\
\hline & 1 & Yr: & & $1.5 \mathrm{E}-07$ & 1. $4 \mathrm{E}-\odot 8$ & $1.2 \mathrm{E}-08$ & 1. $2 \mathrm{E}-08$ & $2.6 \mathrm{E}-08$ \\
\hline & 50 & Yr: & & $1.5 \mathrm{E}-07$ & 1. 4E-०8 & 1. $2 \mathrm{E}-08$ & 1. $2 \mathrm{E}-\odot 8$ & $2.6 \mathrm{E}-08$ \\
\hline TE129 & Class: W & & $9.05 \mathrm{E}-\odot 8$ & & & & & \\
\hline & 1 & Yr: & & $4.2 \mathrm{E}-10$ & $2.6 \mathrm{E}-12$ & $2.4 \mathrm{E}-12$ & $2.8 \mathrm{E}-12$ & 2. $2 \mathrm{E}-10$ \\
\hline & 50 & Yr: & & $4.2 \mathrm{E}-10$ & $2.6 \mathrm{E}-12$ & $2.4 \mathrm{E}-12$ & $2.8 \mathrm{E}-12$ & $2.2 \mathrm{E}-10$ \\
\hline TE131M & Class: W & & $2.07 \mathrm{E}-\odot 6$ & & & & & \\
\hline & 1 & Yr: & & 1. $3 E-08$ & $6.5 \mathrm{E}-10$ & $3.9 \mathrm{E}-10$ & $2.5 E-08$ & $5.9 \mathrm{E}-\odot 9$ \\
\hline & 50 & Yr: & & 1. $3 E-08$ & $6.5 \mathrm{E}-10$ & $3.9 \mathrm{E}-10$ & $2.5 E-08$ & $5.9 E-09$ \\
\hline TE131 & Class: W & & $6.09 E-07$ & & & & & \\
\hline & 1 & Yr: & & $2.7 \mathrm{E}-10$ & $2.9 \mathrm{E}-12$ & 2. $2 \mathrm{E}-12$ & $4.6 \mathrm{E}-10$ & 1. $7 \mathrm{E}-10$ \\
\hline & 50 & Yr: & & $2.7 \mathrm{E}-10$ & 2. $9 \mathrm{E}-12$ & $2.2 \mathrm{E}-12$ & $4.6 \mathrm{E}-10$ & $1.7 \mathrm{E}-10$ \\
\hline TE132 & Class: W & & $2.95 \mathrm{E}-07$ & & & & & \\
\hline & 1 & Yr: & & 3. $0 E-\odot 8$ & 1. $6 \mathrm{E}-\odot 9$ & $E-10$ & E-०8 & 1. $3 E-08$ \\
\hline & 50 & Yr: & & $3.0 E-\odot 8$ & 1. $6 \mathrm{E}-\odot 9$ & $8.6 \mathrm{E}-10$ & $5.3 E-\odot 8$ & 1. $3 \mathrm{E}-08$ \\
\hline TE133M & Class: W & & $3.41 \mathrm{E}-06$ & & & & & \\
\hline & 1 & Yr: & & $7.7 \mathrm{E}-10$ & $2.5 \mathrm{E}-11$ & $2.1 \mathrm{E}-11$ & 2. $\odot E-\odot 9$ & $5.9 \mathrm{E}-10$ \\
\hline & 50 & Yr: & & $7.7 \mathrm{E}-10$ & $2.5 \mathrm{E}-11$ & $2.1 \mathrm{E}-11$ & 2. $\odot E-\odot 9$ & $5.9 \mathrm{E}-10$ \\
\hline TE133 & Class: W & & $1.37 \mathrm{E}-06$ & & & & & \\
\hline & 1 & Yr: & & $1.6 \mathrm{E}-10$ & $2.7 \mathrm{E}-12$ & 2. $3 E-12$ & $4.5 E-10$ & 1. $3 \mathrm{E}-10$ \\
\hline & 50 & Yr: & & $1.6 \mathrm{E}-10$ & $2.7 \mathrm{E}-12$ & $2.3 \mathrm{E}-12$ & $4.5 \mathrm{E}-10$ & 1. $3 \mathrm{E}-10$ \\
\hline TE134 & Class: W & & $1.24 \mathrm{E}-06$ & & & & & \\
\hline & 1 & Yr: & & $5.9 \mathrm{E}-10$ & $2.3 \mathrm{E}-11$ & 1. $9 \mathrm{E}-11$ & $2.8 \mathrm{E}-10$ & 4. $0 \mathrm{E}-10$ \\
\hline & 50 & Yr: & & $5.9 \mathrm{E}-10$ & $2.3 E-11$ & 1. $9 \mathrm{E}-11$ & $2.8 \mathrm{E}-10$ & 4. $0 \mathrm{E}-10$ \\
\hline I 125 & Class: D & & $1.19 \mathrm{E}-\odot 8$ & & & & & \\
\hline & 1 & Yr: & & 1. $\odot E-10$ & $1.6 \mathrm{E}-10$ & $3.8 \mathrm{E}-11$ & $4.6 \mathrm{E}-07$ & 2. $3 E-08$ \\
\hline & 50 & Yr: & & 1. $0 \mathrm{E}-10$ & $1.6 \mathrm{E}-10$ & $3.8 \mathrm{E}-11$ & $4.6 \mathrm{E}-07$ & $2.3 E-08$ \\
\hline I 129 & Class: D & & $8.92 \mathrm{E}-\odot 9$ & & & & & \\
\hline & 1 & Yr: & & 1. $5 \mathrm{E}-10$ & $1.8 \mathrm{E}-10$ & $7.1 \mathrm{E}-11$ & 1. $7 \mathrm{E}-\odot 6$ & $8.6 \mathrm{E}-08$ \\
\hline & 50 & Yr: & & 1. $5 \mathrm{E}-10$ & $1.8 \mathrm{E}-10$ & $7.1 \mathrm{E}-11$ & 1.7E-06 & $8.6 \mathrm{E}-08$ \\
\hline I 130 & Class: D & & $3.05 E-\odot 6$ & & & & & \\
\hline & 1 & Yr: & & $2.8 \mathrm{E}-10$ & $1.8 \mathrm{E}-10$ & $1.6 \mathrm{E}-10$ & 1. $4 \mathrm{E}-\odot 7$ & 7. 4E- -99 \\
\hline & 50 & Yr: & & $2.8 \mathrm{E}-10$ & $1.8 \mathrm{E}-10$ & $1.6 \mathrm{E}-10$ & 1. $4 \mathrm{E}-\odot 7$ & 7. $4 \mathrm{E}-\odot 9$ \\
\hline
\end{tabular}




\begin{tabular}{|c|c|c|c|c|c|c|c|c|}
\hline \multirow[t]{3}{*}{ I 131} & Class: D & & $5.33 E-07$ & & & & & \\
\hline & 1 & Yr: & & 3. $0 \mathrm{E}-10$ & $2.0 \mathrm{E}-10$ & $1.6 \mathrm{E}-10$ & 1. $4 \mathrm{E}-06$ & 7. $2 \mathrm{E}-08$ \\
\hline & 50 & Yr: & & 3. $0 \mathrm{E}-10$ & 2. $\odot E-1 \odot$ & $1.6 \mathrm{E}-10$ & 1. $4 \mathrm{E}-\odot 6$ & 7. $2 \mathrm{E}-08$ \\
\hline \multirow[t]{3}{*}{ I 132} & Class: D & & $3.31 \mathrm{E}-06$ & & & & & \\
\hline & 1 & Yr: & & $1.5 \mathrm{E}-10$ & $6.9 \mathrm{E}-11$ & $6.1 \mathrm{E}-11$ & 1. $6 \mathrm{E}-08$ & $9.7 \mathrm{E}-10$ \\
\hline & 50 & Yr: & & 1. $5 \mathrm{E}-10$ & $6.9 \mathrm{E}-11$ & $6.1 \mathrm{E}-11$ & 1. $6 \mathrm{E}-\odot 8$ & $9.7 \mathrm{E}-10$ \\
\hline \multirow[t]{3}{*}{ I 133} & Class: D & & $8.7 \odot E-\odot 7$ & & & & & \\
\hline & 1 & Yr: & & 2. $0 \mathrm{E}-10$ & 1. $2 \mathrm{E}-10$ & 1.1E-10 & $3.5 E-07$ & $1.8 \mathrm{E}-\odot 8$ \\
\hline & 50 & Yr: & & $2.0 E-10$ & 1. $2 \mathrm{E}-10$ & $1.1 \mathrm{E}-10$ & $3.5 \mathrm{E}-07$ & $1.8 \mathrm{E}-08$ \\
\hline \multirow[t]{3}{*}{ I 134} & Class: D & & $3.85 E-06$ & & & & & \\
\hline & 1 & Yr: & & $1.1 \mathrm{E}-10$ & $3.3 E-11$ & $2.9 \mathrm{E}-11$ & $3.1 \mathrm{E}-09$ & $3.7 \mathrm{E}-10$ \\
\hline & 50 & Yr: & & $1.1 \mathrm{E}-10$ & 3. $3 E-11$ & $2.9 \mathrm{E}-11$ & $3.1 \mathrm{E}-09$ & $3.7 \mathrm{E}-10$ \\
\hline \multirow[t]{3}{*}{ I 135} & Class: D & & $2.38 \mathrm{E}-06$ & & & & & \\
\hline & 1 & Yr: & & $1.8 \mathrm{E}-10$ & $1.0 \mathrm{E}-10$ & $9.0 \mathrm{E}-11$ & 7. $0 \mathrm{E}-\odot 8$ & $3.7 \mathrm{E}-09$ \\
\hline & 50 & Yr: & & $1.8 \mathrm{E}-10$ & 1. $0 \mathrm{E}-10$ & $9.0 \mathrm{E}-11$ & 7. $0 \mathrm{E}-\odot 8$ & $3.7 \mathrm{E}-\odot 9$ \\
\hline \multirow{3}{*}{ XE131M } & NobleGas & & $1.10 \mathrm{E}-\odot 8$ & & & & & \\
\hline & 1 & Yr: & & $\odot . \odot \mathrm{E}-\odot \odot$ & $\odot . \odot \mathrm{E}-\odot \odot$ & $\odot . \odot \mathrm{E}-\odot \odot$ & $\odot . \odot \mathrm{E}-\odot \odot$ & $\odot . \odot \mathrm{E}-\odot \odot$ \\
\hline & 50 & Yr: & & $\odot . \odot \mathrm{E}-\odot \odot$ & $\odot . \odot \mathrm{E}-\odot \odot$ & $\odot . \odot \mathrm{E}-\odot \odot$ & $\odot . \odot \mathrm{E}-\odot \odot$ & $\odot . \odot \mathrm{E}-\odot \odot$ \\
\hline \multirow[t]{3}{*}{ XE133M } & NobleGas & & 4. . $7 \mathrm{E}-\odot 8$ & & & & & \\
\hline & 1 & Yr: & & $\odot . \odot \mathrm{E}-\odot \odot$ & $\odot . \odot \mathrm{E}-\odot \odot$ & $\odot . \odot \mathrm{E}-\odot \odot$ & $\odot . \odot \mathrm{E}-\odot \odot$ & $\odot . \odot \mathrm{E}-\odot \odot$ \\
\hline & 50 & Yr: & & $\odot . \odot \mathrm{E}-\odot \odot$ & $\odot . \odot \mathrm{E}-\odot \odot$ & $\odot . \odot \mathrm{E}-\odot \odot$ & $\odot . \odot \mathrm{E}-\odot \odot$ & $\odot . \odot \mathrm{E}-\odot \odot$ \\
\hline \multirow[t]{3}{*}{ XE133 } & NobleGas & & $4.23 E-08$ & & & & & \\
\hline & 1 & Yr: & & $\odot . \odot \mathrm{E}-\odot \odot$ & $\odot . \odot \mathrm{E}-\odot \odot$ & $\odot . \odot E-\odot \odot$ & $\odot . \odot \mathrm{E}-\odot \odot$ & $\odot . \odot E-\odot \odot$ \\
\hline & 50 & Yr: & & $\odot . \odot \mathrm{E}-\odot \odot$ & $\odot . \odot \mathrm{E}-\odot \odot$ & $\odot . \odot \mathrm{E}-\odot \odot$ & $\odot . \odot \mathrm{E}-\odot \odot$ & $\odot . \odot \mathrm{E}-\odot \odot$ \\
\hline \multirow[t]{3}{*}{ XE135M } & NobleGas & & $5.99 \mathrm{E}-07$ & & & & & \\
\hline & 1 & Yr: & & $\odot . \odot \mathrm{E}-\odot \odot$ & $\odot . \odot \mathrm{E}-\odot \odot$ & $\odot . \odot \mathrm{E}-\odot \odot$ & $\odot . \odot \mathrm{E}-\odot \odot$ & $\odot . \odot \mathrm{E}-\odot \odot$ \\
\hline & 50 & Yr: & & $\odot . \odot \mathrm{E}-\odot \odot$ & $\odot . \odot E-\odot \odot$ & $\odot . \odot \mathrm{E}-\odot \odot$ & $\odot . \odot \mathrm{E}-\odot \odot$ & $\odot . \odot \mathrm{E}-\odot \odot$ \\
\hline XE135 & NobleGas & & $3.50 \mathrm{E}-07$ & & & & & \\
\hline & 1 & Yr: & & $\odot . \odot E-\odot \odot$ & $\odot . \odot \mathrm{E}-\odot \odot$ & $\odot . \odot \mathrm{E}-\odot \odot$ & $\odot . \odot \mathrm{E}-\odot \odot$ & $\odot . \odot \mathrm{E}-\odot \odot$ \\
\hline & 50 & Yr: & & $\odot . \odot \mathrm{E}-\odot \odot$ & $\odot . \odot \mathrm{E}-\odot \odot$ & $\odot . \odot \mathrm{E}-\odot \odot$ & $\odot . \odot \mathrm{E}-\odot \odot$ & $\odot . \odot \mathrm{E}-\odot \odot$ \\
\hline XE137 & NobleGas & & $4.34 \mathrm{E}-07$ & & & & & \\
\hline & 1 & Yr: & & $\odot . \odot \mathrm{E}-\odot \odot$ & $\odot . \odot \mathrm{E}-\odot \odot$ & $\odot . \odot \mathrm{E}-\odot \odot$ & $\odot . \odot \mathrm{E}-\odot \odot$ & $\odot . \odot \mathrm{E}-\odot \odot$ \\
\hline & 50 & Yr: & & $\odot . \odot \mathrm{E}-\odot \odot$ & $\odot . \odot \mathrm{E}-\odot \odot$ & $\odot . \odot \mathrm{E}-\odot \odot$ & $\odot . \odot \mathrm{E}-\odot \odot$ & $\odot . \odot \mathrm{E}-\odot \odot$ \\
\hline XE138 & NobleGas & & $1.73 E-\odot 6$ & & & & & \\
\hline & 1 & Yr: & & $\odot . \odot \mathrm{E}-\odot \odot$ & $\odot . \odot E-\odot \odot$ & $\odot . \odot \mathrm{E}-\odot \odot$ & $\odot . \odot E-\odot \odot$ & $\odot . \odot \mathrm{E}-\odot \odot$ \\
\hline & 50 & Yr: & & $\odot . \odot \mathrm{E}-\odot \odot$ & $\odot . \odot \mathrm{E}-\odot \odot$ & $\odot . \odot \mathrm{E}-\odot \odot$ & $\odot . \odot \mathrm{E}-\odot \odot$ & $\odot .0 \mathrm{E}-\odot \odot$ \\
\hline CS134M & Class: D & & $2.51 E-08$ & & & & & \\
\hline & 1 & Yr: & & $6.7 \mathrm{E}-11$ & 1. $4 \mathrm{E}-11$ & 1. $2 \mathrm{E}-11$ & 1. $2 \mathrm{E}-11$ & $8.6 \mathrm{E}-11$ \\
\hline & 50 & Yr: & & $6.7 \mathrm{E}-11$ & 1. $4 \mathrm{E}-11$ & 1. $2 \mathrm{E}-11$ & 1. $2 \mathrm{E}-11$ & $8.6 \mathrm{E}-11$ \\
\hline CS134 & Class: D & & $2.23 E-06$ & & & & & \\
\hline & 1 & Yr: & & $5.6 \mathrm{E}-\odot 9$ & $5.9 \mathrm{E}-\odot 9$ & $5.2 \mathrm{E}-09$ & $6.3 \mathrm{E}-\odot 9$ & 7. 4E- $\odot 9$ \\
\hline & 50 & Yr: & & $5.6 \mathrm{E}-\odot 9$ & $5.9 E-\odot 9$ & $5.2 \mathrm{E}-09$ & $6.3 E-09$ & $7.4 \mathrm{E}-09$ \\
\hline CS135 & Class: D & & $3.00 \mathrm{E}-10$ & & & & & \\
\hline & 1 & Yr: & & $7.7 \mathrm{E}-10$ & $7.3 E-10$ & 7. $3 E-10$ & 7. $3 E-10$ & $9.9 \mathrm{E}-10$ \\
\hline & 50 & Yr: & & $7.7 \mathrm{E}-10$ & 7. $3 E-10$ & $7.3 E-10$ & 7. $3 E-10$ & $9.9 \mathrm{E}-10$ \\
\hline CS136 & Class: D & & $3.13 E-06$ & & & & & \\
\hline & 1 & Yr: & & $3.5 E-\odot 9$ & $3.8 E-09$ & $3.2 \mathrm{E}-\odot 9$ & 4. $\odot E-\odot 9$ & $5.3 E-\odot 9$ \\
\hline & 50 & Yr: & & $3.5 E-09$ & $3.8 \mathrm{E}-09$ & $3.2 \mathrm{E}-\odot 9$ & 4. $0 \mathrm{E}-\odot 9$ & $5.3 E-\odot 9$ \\
\hline CS137 & Class: D & & $2.93 E-\odot 9$ & & & & & \\
\hline & 1 & Yr: & & $4.2 \mathrm{E}-\odot 9$ & $4.3 E-09$ & 4. .E - ๑9 & $4.4 \mathrm{E}-09$ & $5.4 \mathrm{E}-\odot 9$ \\
\hline & 50 & Yr: & & $4.2 \mathrm{E}-09$ & $4.3 E-09$ & 4. ०E-०9 & 4. 4E- -09 & $5.4 \mathrm{E}-09$ \\
\hline CS138 & Class: D & & $3.63 E-06$ & & & & & \\
\hline & 1 & Yr: & & $1.1 \mathrm{E}-10$ & $2.6 \mathrm{E}-11$ & $2.4 \mathrm{E}-11$ & $2.6 \mathrm{E}-11$ & $1.8 \mathrm{E}-10$ \\
\hline & 50 & Yr: & & 1. $1 \mathrm{E}-10$ & $2.6 \mathrm{E}-11$ & $2.4 \mathrm{E}-11$ & $2.6 \mathrm{E}-11$ & $1.8 \mathrm{E}-10$ \\
\hline BA139 & Class: D & & $8.04 \mathrm{E}-\odot 8$ & & & & & \\
\hline & 1 & Yr: & & $9.2 \mathrm{E}-11$ & $8.8 \mathrm{E}-11$ & 8. $0 \mathrm{E}-11$ & 1. $1 \mathrm{E}-11$ & $2.4 \mathrm{E}-10$ \\
\hline & 50 & Yr: & & $9.2 \mathrm{E}-11$ & 8. $8 \mathrm{E}-11$ & 8. $0 \mathrm{E}-11$ & $1.1 \mathrm{E}-11$ & $2.4 \mathrm{E}-10$ \\
\hline BA140 & Class: D & & $2.55 \mathrm{E}-07$ & & & & & \\
\hline & 1 & Yr: & & 1. $3 \mathrm{E}-09$ & $2 . \odot E-\odot 8$ & E-๑8 & $E-10$ & $7.8 \mathrm{E}-09$ \\
\hline & 50 & Yr: & & 1. $3 \mathrm{E}-\odot 9$ & 2. ๑E-๑8 & 1.1E-๑8 & $8.4 \mathrm{E}-10$ & $7.8 \mathrm{E}-\odot 9$ \\
\hline BA141 & Class: D & & $1.24 \mathrm{E}-06$ & & & & & \\
\hline & 1 & Yr: & & $7.3 E-11$ & $4.5 \mathrm{E}-11$ & $3.7 \mathrm{E}-11$ & $6.6 \mathrm{E}-12$ & $1.4 \mathrm{E}-10$ \\
\hline & 50 & Yr: & & $7.3 E-11$ & $4.5 E-11$ & $3.7 \mathrm{E}-11$ & $6.6 \mathrm{E}-12$ & $1.4 \mathrm{E}-10$ \\
\hline BA142 & Class: D & & $1.53 \mathrm{E}-06$ & & & & & \\
\hline & 1 & Yr: & & $5.3 \mathrm{E}-11$ & $2.1 \mathrm{E}-11$ & $1.8 \mathrm{E}-11$ & $5.8 \mathrm{E}-12$ & $9.8 \mathrm{E}-11$ \\
\hline & 50 & Yr: & & $5.3 \mathrm{E}-11$ & $2.1 \mathrm{E}-11$ & $1.8 \mathrm{E}-11$ & $5.8 \mathrm{E}-12$ & $9.8 \mathrm{E}-11$ \\
\hline LA140 & Class: D & & $3.50 \mathrm{E}-06$ & & & & & \\
\hline & 1 & Yr: & & $9.5 \mathrm{E}-10$ & 1. 4E- $\odot 9$ & 2. ๑E-๑9 & $4.9 \mathrm{E}-10$ & 4. $2 \mathrm{E}-\odot 9$ \\
\hline & 50 & Yr: & & $9.5 \mathrm{E}-10$ & 1. $4 \mathrm{E}-\odot 9$ & 2. $\odot E-\odot 9$ & $4.9 \mathrm{E}-10$ & $4.2 \mathrm{E}-09$ \\
\hline LA141 & Class: D & & 9. ๑८E-๑৪ & & & & & \\
\hline & 1 & Yr: & & $1.5 \mathrm{E}-10$ & $3.9 \mathrm{E}-10$ & $2.1 \mathrm{E}-10$ & $6.2 \mathrm{E}-11$ & $5.5 \mathrm{E}-10$ \\
\hline
\end{tabular}




\begin{tabular}{|c|c|c|c|c|c|c|c|c|}
\hline \multirow{4}{*}{ LA142 } & 50 & Yr: & & $1.5 \mathrm{E}-10$ & $3.9 \mathrm{E}-10$ & $2.1 \mathrm{E}-10$ & $6.2 \mathrm{E}-11$ & $5.5 \mathrm{E}-10$ \\
\hline & Class: D & & $4.32 \mathrm{E}-06$ & & & & & \\
\hline & 1 & Yr: & & 1. $5 \mathrm{E}-10$ & $6.6 \mathrm{E}-11$ & 7. $0 \mathrm{E}-11$ & $5.1 \mathrm{E}-11$ & $3.8 \mathrm{E}-10$ \\
\hline & 50 & Yr: & & 1. $5 \mathrm{E}-10$ & $6.6 \mathrm{E}-11$ & 7. $0 \mathrm{E}-11$ & $5.1 \mathrm{E}-11$ & $3.8 \mathrm{E}-10$ \\
\hline \multirow[t]{3}{*}{ CE141 } & Class: Y & & $9.81 \mathrm{E}-08$ & & & & & \\
\hline & 1 & Yr: & & $8.5 E-\odot 8$ & $6.8 \mathrm{E}-10$ & 1. $7 \mathrm{E}-10$ & 1. $3 \mathrm{E}-10$ & 1. $2 \mathrm{E}-\odot 8$ \\
\hline & 50 & Yr: & & $8.5 \mathrm{E}-08$ & $6.8 \mathrm{E}-10$ & 1. $7 \mathrm{E}-10$ & 1. $3 \mathrm{E}-10$ & 1. $2 \mathrm{E}-08$ \\
\hline \multirow[t]{3}{*}{ CE143 } & Class: Y & & $3.82 \mathrm{E}-07$ & & & & & \\
\hline & 1 & Yr: & & 1. $5 \mathrm{E}-08$ & $9.1 \mathrm{E}-11$ & $5.5 \mathrm{E}-11$ & 3. $3 E-11$ & 4.1E- -99 \\
\hline & 50 & Yr: & & 1. $5 \mathrm{E}-08$ & $9.1 \mathrm{E}-11$ & $5.5 \mathrm{E}-11$ & 3. $3 \mathrm{E}-11$ & $4.1 \mathrm{E}-\odot 9$ \\
\hline \multirow[t]{3}{*}{ CE144 } & Class: Y & & $2.41 \mathrm{E}-\odot 8$ & & & & & \\
\hline & 1 & Yr: & & 1. $4 \mathrm{E}-06$ & 1.1E- -08 & 1.1E- $\odot 8$ & $6.8 \mathrm{E}-10$ & $1.8 \mathrm{E}-07$ \\
\hline & 50 & Yr: & & 1. $4 \mathrm{E}-\odot 6$ & 1. $1 \mathrm{E}-\odot 8$ & 1.1E- -88 & $6.8 \mathrm{E}-10$ & $1.8 \mathrm{E}-07$ \\
\hline \multirow[t]{3}{*}{ PR143 } & Class: Y & & $6.12 \mathrm{E}-\odot 9$ & & & & & \\
\hline & 1 & Yr: & & $5.5 \mathrm{E}-\odot 8$ & $9.6 \mathrm{E}-12$ & $2.1 \mathrm{E}-11$ & $9.6 \mathrm{E}-14$ & $9.2 \mathrm{E}-09$ \\
\hline & 50 & Yr: & & $5.5 E-08$ & $9.6 \mathrm{E}-12$ & $2.1 \mathrm{E}-11$ & $9.6 \mathrm{E}-14$ & $9.2 \mathrm{E}-09$ \\
\hline \multirow[t]{3}{*}{ PR144M } & Class: Y & & $6.97 \mathrm{E}-\odot 9$ & & & & & \\
\hline & 1 & Yr: & & 4. $0 \mathrm{E}-11$ & $1.5 \mathrm{E}-14$ & $1.8 \mathrm{E}-14$ & $8.7 \mathrm{E}-15$ & $5.1 \mathrm{E}-12$ \\
\hline & 50 & Yr: & & 4. $0 \mathrm{E}-11$ & 1. $5 \mathrm{E}-14$ & 1. $8 \mathrm{E}-14$ & $8.7 \mathrm{E}-15$ & $5.1 \mathrm{E}-12$ \\
\hline \multirow[t]{3}{*}{ PR144 } & Class: Y & & $8.36 \mathrm{E}-\odot 8$ & & & & & \\
\hline & 1 & Yr: & & $1.8 \mathrm{E}-10$ & $7.4 \mathrm{E}-14$ & $6.2 \mathrm{E}-14$ & $5.9 \mathrm{E}-14$ & 1. $2 \mathrm{E}-10$ \\
\hline & 50 & Yr: & & $1.8 \mathrm{E}-10$ & $7.4 \mathrm{E}-14$ & $6.2 \mathrm{E}-14$ & $5.9 \mathrm{E}-14$ & $1.2 \mathrm{E}-10$ \\
\hline \multirow[t]{3}{*}{ ND147 } & Class: $\mathrm{Y}$ & & $1.81 \mathrm{E}-07$ & & & & & \\
\hline & 1 & Yr: & & $5.2 \mathrm{E}-\odot 8$ & 4. $0 \mathrm{E}-10$ & 1. $3 \mathrm{E}-10$ & $9.0 \mathrm{E}-11$ & $8.7 \mathrm{E}-09$ \\
\hline & 50 & Yr: & & $5.2 \mathrm{E}-08$ & $4.0 \mathrm{E}-10$ & $1.3 \mathrm{E}-10$ & $9.0 \mathrm{E}-11$ & $8.7 \mathrm{E}-\odot 9$ \\
\hline \multirow[t]{3}{*}{ PM147 } & Class: $\mathrm{Y}$ & & $2.73 E-10$ & & & & & \\
\hline & 1 & Yr: & & 1. $2 \mathrm{E}-\odot 7$ & 1. 3E- - 88 & 1. $7 \mathrm{E}-09$ & 1.9E-13 & 1. $6 \mathrm{E}-\odot 8$ \\
\hline & 50 & Yr: & & $1.2 \mathrm{E}-07$ & 1. $3 E-\odot 8$ & 1. 7E- $\odot 9$ & 1. $9 \mathrm{E}-13$ & $1.6 \mathrm{E}-08$ \\
\hline PM148M & Class: $\mathrm{Y}$ & & $2.84 \mathrm{E}-\odot 6$ & & & & & \\
\hline & 1 & Yr: & & $1.2 \mathrm{E}-07$ & 4. . E - ०9 & $2.9 \mathrm{E}-\odot 9$ & 3. 3E- $\odot 9$ & $2.0 \mathrm{E}-\odot 8$ \\
\hline & 50 & Yr: & & $1.2 \mathrm{E}-07$ & 4. . E - ०9 & $2.9 \mathrm{E}-\odot 9$ & 3. 3E- $\odot 9$ & 2. $\odot E-\odot 8$ \\
\hline PM148 & Class: Y & & $8.70 \mathrm{E}-\odot 7$ & & & & & \\
\hline & 1 & Yr: & & $4.2 \mathrm{E}-08$ & $2.4 \mathrm{E}-10$ & $2.1 \mathrm{E}-10$ & $1.8 \mathrm{E}-10$ & 1.1E- -08 \\
\hline & 50 & Yr: & & $4.2 \mathrm{E}-08$ & $2.4 \mathrm{E}-10$ & $2.1 \mathrm{E}-10$ & $1.8 \mathrm{E}-10$ & 1.1E- -88 \\
\hline PM149 & Class: Y & & $2.24 \mathrm{E}-\odot 8$ & & & & & \\
\hline & 1 & Yr: & & 1. $3 \mathrm{E}-08$ & $5.3 \mathrm{E}-12$ & 7. $0 \mathrm{E}-12$ & $1.8 \mathrm{E}-12$ & $3.7 \mathrm{E}-\odot 9$ \\
\hline & 50 & Yr: & & 1. $3 E-08$ & 5. 3E-12 & $7.0 \mathrm{E}-12$ & 1. $8 \mathrm{E}-12$ & $3.7 \mathrm{E}-09$ \\
\hline PM151 & Class: Y & & $4.42 \mathrm{E}-07$ & & & & & \\
\hline & 1 & Yr: & & $7.6 \mathrm{E}-09$ & $7.6 \mathrm{E}-11$ & $4.9 \mathrm{E}-11$ & $3.2 \mathrm{E}-11$ & $2.7 \mathrm{E}-\odot 9$ \\
\hline & 50 & Yr: & & 7. $6 \mathrm{E}-\odot 9$ & $7.6 \mathrm{E}-11$ & $4.9 \mathrm{E}-11$ & $3.2 \mathrm{E}-11$ & $2.7 \mathrm{E}-\odot 9$ \\
\hline SM147 & Class: W & & ๑. О९Е๑९ & & & & & \\
\hline & 1 & Yr: & & $1.1 \mathrm{E}-06$ & $2.6 \mathrm{E}-05$ & $3.1 \mathrm{E}-06$ & $9.7 \mathrm{E}-11$ & $2.3 E-05$ \\
\hline & 50 & Yr: & & $1.1 \mathrm{E}-06$ & $2.6 \mathrm{E}-05$ & $3.1 \mathrm{E}-06$ & $9.7 \mathrm{E}-11$ & $2.3 E-05$ \\
\hline SM151 & Class: W & & $7.79 \mathrm{E}-13$ & & & & & \\
\hline & 1 & Yr: & & 1. $2 \mathrm{E}-\odot 8$ & $2.1 \mathrm{E}-07$ & $2.6 \mathrm{E}-\odot 8$ & $8.7 \mathrm{E}-13$ & 1. $0 \mathrm{E}-\odot 8$ \\
\hline & 50 & Yr: & & 1. $2 \mathrm{E}-08$ & $2.1 \mathrm{E}-07$ & $2.6 \mathrm{E}-\odot 8$ & $8.7 \mathrm{E}-13$ & 1. $0 \mathrm{E}-\odot 8$ \\
\hline SM153 & Class: W & & $6.46 \mathrm{E}-\odot 8$ & & & & & \\
\hline & 1 & Yr: & & 1.1E-०8 & $3.3 \mathrm{E}-10$ & $2.1 \mathrm{E}-10$ & $1.5 \mathrm{E}-11$ & $2.9 \mathrm{E}-\odot 9$ \\
\hline & $5 \odot$ & Yr: & & 1.1E- $\odot 8$ & $3.3 E-10$ & $2.1 \mathrm{E}-10$ & 1. $5 \mathrm{E}-11$ & $2.9 \mathrm{E}-\odot 9$ \\
\hline EU152 & Class: W & & $1.67 \mathrm{E}-06$ & & & & & \\
\hline & 1 & Yr: & & $1.8 \mathrm{E}-07$ & $4.4 \mathrm{E}-07$ & 1. $9 \mathrm{E}-07$ & $1.8 \mathrm{E}-08$ & 1. $\odot \mathrm{E}-\odot 7$ \\
\hline & 50 & Yr: & & $1.8 E-07$ & 4. $4 \mathrm{E}-07$ & $1.9 \mathrm{E}-07$ & $1.8 \mathrm{E}-08$ & $1.0 \mathrm{E}-07$ \\
\hline EU154 & Class: W & & $1.82 \mathrm{E}-06$ & & & & & \\
\hline & 1 & Yr: & & $3.0 \mathrm{E}-07$ & 1. $\odot \mathrm{E}-06$ & 2. $9 \mathrm{E}-07$ & $1.8 \mathrm{E}-08$ & $1.5 \mathrm{E}-07$ \\
\hline & 50 & Yr: & & 3. $\odot E-\odot 7$ & 1. $0 \mathrm{E}-06$ & $2.9 \mathrm{E}-07$ & 1. $8 \mathrm{E}-08$ & 1. $5 \mathrm{E}-07$ \\
\hline EU155 & Class: W & & $6.78 \mathrm{E}-\odot 8$ & & & & & \\
\hline & 1 & Yr: & & $5.9 \mathrm{E}-08$ & $3.3 \mathrm{E}-07$ & $4.5 \mathrm{E}-08$ & $8.2 \mathrm{E}-10$ & $2.3 E-\odot 8$ \\
\hline & 50 & Yr: & & $5.9 \mathrm{E}-08$ & $3.3 E-07$ & 4. 5E-๑8 & $8.2 \mathrm{E}-10$ & $2.3 E-\odot 8$ \\
\hline EU156 & Class: W & & $2.02 \mathrm{E}-06$ & & & & & \\
\hline & 1 & Yr: & & $6.9 \mathrm{E}-08$ & $6.1 \mathrm{E}-09$ & $3.9 \mathrm{E}-09$ & $8.8 \mathrm{E}-10$ & 1. $4 \mathrm{E}-\odot 8$ \\
\hline & 50 & Yr: & & $6.9 \mathrm{E}-08$ & $6.1 \mathrm{E}-09$ & $3.9 \mathrm{E}-09$ & $8.8 \mathrm{E}-10$ & 1. $4 \mathrm{E}-08$ \\
\hline GD153 & Class: D & & $9.84 \mathrm{E}-\odot 8$ & & & & & \\
\hline & 1 & Yr: & & $3.3 E-09$ & $2.2 \mathrm{E}-07$ & $3.7 \mathrm{E}-08$ & 8. $0 \mathrm{E}-10$ & 1. $2 \mathrm{E}-08$ \\
\hline & 50 & Yr: & & $3.3 E-09$ & 2. $2 \mathrm{E}-07$ & 3. $7 \mathrm{E}-\odot 8$ & 8. $0 \mathrm{E}-10$ & 1. $2 \mathrm{E}-\odot 8$ \\
\hline TB160 & Class: W & & $1.64 \mathrm{E}-06$ & & & & & \\
\hline & 1 & Yr: & & 1. $4 \mathrm{E}-07$ & $5.9 \mathrm{E}-\odot 8$ & 1. $8 \mathrm{E}-\odot 8$ & $2.4 \mathrm{E}-09$ & $2.5 \mathrm{E}-\odot 8$ \\
\hline & 50 & Yr: & & $1.4 \mathrm{E}-07$ & $5.9 \mathrm{E}-08$ & $1.8 \mathrm{E}-08$ & $2.4 \mathrm{E}-09$ & $2.5 \mathrm{E}-\odot 8$ \\
\hline H0166M & Class: W & & $2.48 \mathrm{E}-06$ & & & & & \\
\hline & 1 & Yr: & & $2.8 \mathrm{E}-07$ & 1. $4 \mathrm{E}-06$ & $3.0 \mathrm{E}-07$ & 4. ๑E-๑8 & $2.5 E-07$ \\
\hline & 50 & Yr: & & $2.8 \mathrm{E}-07$ & 1. $4 \mathrm{E}-06$ & $3.0 \mathrm{E}-07$ & 4. . $-\odot 8$ & $2.5 \mathrm{E}-07$ \\
\hline W 181 & Class: D & & $3.69 \mathrm{E}-\odot 8$ & & & & & \\
\hline
\end{tabular}




\begin{tabular}{|c|c|c|c|c|c|c|c|c|}
\hline & 1 & Yr: & & $3.0 E-11$ & $2.7 \mathrm{E}-10$ & $1.1 \mathrm{E}-10$ & 1. $4 \mathrm{E}-11$ & $2.0 \mathrm{E}-10$ \\
\hline & 50 & Yr: & & 3. $0 \mathrm{E}-11$ & $2.7 \mathrm{E}-10$ & $1.1 \mathrm{E}-10$ & 1. $4 \mathrm{E}-11$ & $2.0 \mathrm{E}-10$ \\
\hline \multirow[t]{3}{*}{ W 187} & Class: D & & $6.72 \mathrm{E}-\odot 7$ & & & & & \\
\hline & 1 & Yr: & & $1.6 \mathrm{E}-10$ & $3.0 \mathrm{E}-10$ & 1. $5 \mathrm{E}-10$ & 7. $0 \mathrm{E}-11$ & 1. $5 \mathrm{E}-\odot 9$ \\
\hline & 50 & Yr: & & $1.6 \mathrm{E}-10$ & $3 . \odot E-10$ & 1. $5 \mathrm{E}-10$ & 7. $\odot \mathrm{E}-11$ & 1. $5 \mathrm{E}-\odot 9$ \\
\hline \multirow[t]{3}{*}{ W 185} & Class: D & & $1.57 \mathrm{E}-\odot 9$ & & & & & \\
\hline & 1 & Yr: & & $6.7 \mathrm{E}-11$ & $6.7 \mathrm{E}-10$ & $4.7 \mathrm{E}-10$ & 2. $\odot \mathrm{E}-11$ & 1. $\odot E-\odot 9$ \\
\hline & 50 & Yr: & & $6.7 \mathrm{E}-11$ & $6.7 \mathrm{E}-10$ & 4. $7 \mathrm{E}-10$ & 2. $0 \mathrm{E}-11$ & 1. $\odot \mathrm{E}-\odot 9$ \\
\hline \multirow[t]{3}{*}{ RE187 } & Class: W & & $\odot . \odot \odot Е \odot \odot ~$ & & & & & \\
\hline & 1 & Yr: & & $2.1 \mathrm{E}-10$ & 1. $2 \mathrm{E}-12$ & 1. $2 \mathrm{E}-12$ & $4.9 \mathrm{E}-11$ & 4. $0 \mathrm{E}-11$ \\
\hline & 50 & Yr: & & $2.1 \mathrm{E}-10$ & 1. $2 \mathrm{E}-12$ & 1. $2 \mathrm{E}-12$ & 4. $9 \mathrm{E}-11$ & 4. $0 \mathrm{E}-11$ \\
\hline \multirow[t]{3}{*}{ IR192 } & Class: Y & & $1.14 \mathrm{E}-06$ & & & & & \\
\hline & 1 & Yr: & & $1.5 \mathrm{E}-07$ & $2.6 \mathrm{E}-\odot 9$ & $1.8 \mathrm{E}-\odot 9$ & 2. 3E- $\odot 9$ & $2.3 E-\odot 8$ \\
\hline & 50 & Yr: & & $1.5 \mathrm{E}-07$ & $2.6 \mathrm{E}-09$ & $1.8 \mathrm{E}-09$ & 2. $3 \mathrm{E}-\odot 9$ & 2. $3 \mathrm{E}-08$ \\
\hline \multirow{3}{*}{ HG2O3 } & Class: D & & $3.28 E-07$ & & & & & \\
\hline & 1 & Yr: & & 1. $9 \mathrm{E}-09$ & $2.1 \mathrm{E}-09$ & $1.8 \mathrm{E}-09$ & 1. $9 \mathrm{E}-\odot 9$ & $2.9 \mathrm{E}-09$ \\
\hline & 50 & Yr: & & 1. $9 \mathrm{E}-\odot 9$ & $2.1 \mathrm{E}-\odot 9$ & $1.8 \mathrm{E}-\odot 9$ & 1. $9 \mathrm{E}-\odot 9$ & $2.9 \mathrm{E}-\odot 9$ \\
\hline \multirow[t]{3}{*}{ RN222 } & NobleGas & & $5.61 \mathrm{E}-10$ & & & & & \\
\hline & 1 & Yr: & & $\odot . \odot \mathrm{E}-\odot \odot$ & $\odot . \odot \mathrm{E}-\odot \odot$ & $\odot . \odot \mathrm{E}-\odot \odot$ & $\odot . \odot \mathrm{E}-\odot \odot$ & $\odot . \odot \mathrm{E}-\odot \odot$ \\
\hline & 50 & Yr: & & $\odot . \odot \mathrm{E}-\odot \odot$ & $\odot . \odot \mathrm{E}-\odot \odot$ & $\odot . \odot \mathrm{E}-\odot \odot$ & $\odot . \odot \mathrm{E}-\odot \odot$ & $\odot . \odot \mathrm{E}-\odot \odot$ \\
\hline \multirow[t]{3}{*}{ TH227 } & Class: Y & & $1.4 \odot \mathrm{E}-\odot 7$ & & & & & \\
\hline & 1 & Yr: & & $2.5 E-\odot 4$ & $4.4 \mathrm{E}-\odot 7$ & $6.5 E-\odot 8$ & 1. $6 \mathrm{E}-\odot 9$ & 3. $0 E-05$ \\
\hline & 50 & Yr: & & $2.5 \mathrm{E}-04$ & $4.4 \mathrm{E}-07$ & $6.5 \mathrm{E}-08$ & 1.6E-09 & $3.0 E-\odot 5$ \\
\hline \multirow[t]{3}{*}{ TH228 } & Class: Y & & $2.56 \mathrm{E}-\odot 9$ & & & & & \\
\hline & 1 & Yr: & & $1.1 \mathrm{E}-03$ & $5.8 \mathrm{E}-05$ & $8.6 \mathrm{E}-06$ & $2.2 \mathrm{E}-07$ & 1. $3 E-04$ \\
\hline & 50 & Yr: & & $1.1 \mathrm{E}-03$ & $5.8 E-05$ & $8.6 \mathrm{E}-06$ & $2.2 \mathrm{E}-07$ & 1. $3 E-\odot 4$ \\
\hline \multirow[t]{3}{*}{ TH229 } & Class: Y & & $1.06 \mathrm{E}-07$ & & & & & \\
\hline & 1 & Yr: & & 1. $5 \mathrm{E}-\odot 3$ & $5.7 \mathrm{E}-\odot 4$ & $4.7 \mathrm{E}-\odot 5$ & $3 . \odot E-\odot 6$ & 1.9E-०4 \\
\hline & 50 & Yr: & & $1.5 \mathrm{E}-03$ & $5.7 \mathrm{E}-\odot 4$ & 4.7E- -55 & $3 . \odot E-\odot 6$ & 1. $9 \mathrm{E}-04$ \\
\hline TH230 & Class: $\mathrm{Y}$ & & $4.70 \mathrm{E}-10$ & & & & & \\
\hline & 1 & Yr: & & $2.4 \mathrm{E}-\odot 4$ & $2.6 \mathrm{E}-\odot 4$ & 2. $\odot E-\odot 5$ & $6.1 \mathrm{E}-07$ & $3.5 \mathrm{E}-05$ \\
\hline & 50 & Yr: & & $2.4 \mathrm{E}-04$ & $2.6 \mathrm{E}-04$ & 2. $0 E-05$ & $6.1 \mathrm{E}-07$ & $3.5 \mathrm{E}-05$ \\
\hline TH231 & Class: $\mathrm{Y}$ & & $1.45 \mathrm{E}-\odot 8$ & & & & & \\
\hline & 1 & Yr: & & $6.2 \mathrm{E}-09$ & $7.3 \mathrm{E}-11$ & $7.8 \mathrm{E}-12$ & 1. $8 \mathrm{E}-12$ & 1. $7 \mathrm{E}-09$ \\
\hline & 50 & Yr: & & $6.2 \mathrm{E}-09$ & $7.3 E-11$ & $7.8 \mathrm{E}-12$ & $1.8 \mathrm{E}-12$ & 1. $7 \mathrm{E}-09$ \\
\hline TH232 & Class: Y & & $2.29 \mathrm{E}-10$ & & & & & \\
\hline & 1 & Yr: & & $3.5 E-04$ & $3.0 \mathrm{E}-04$ & $2.3 E-05$ & $1.6 \mathrm{E}-06$ & $5.0 E-05$ \\
\hline & 50 & Yr: & & $3.5 E-04$ & $3.0 E-04$ & 2. 3E- -5 & $1.6 \mathrm{E}-06$ & $5.0 \mathrm{E}-05$ \\
\hline TH234 & Class: Y & & $9.30 \mathrm{E}-\odot 9$ & & & & & \\
\hline & 1 & Yr: & & 1. $9 \mathrm{E}-\odot 7$ & $4.1 \mathrm{E}-10$ & $4.3 E-10$ & $3.5 \mathrm{E}-11$ & $3.1 \mathrm{E}-08$ \\
\hline & 50 & Yr: & & 1. $9 \mathrm{E}-07$ & $4.1 \mathrm{E}-10$ & 4. $3 E-10$ & $3.5 \mathrm{E}-11$ & $3.1 \mathrm{E}-08$ \\
\hline RA223 & Class: W & & $1.73 \mathrm{E}-07$ & & & & & \\
\hline & 1 & Yr: & & 1. $7 \mathrm{E}-\odot 4$ & 8. 3E- - 6 & $6.3 \mathrm{E}-07$ & $2.6 \mathrm{E}-\odot 8$ & $2.1 \mathrm{E}-05$ \\
\hline & 50 & Yr: & & $1.7 \mathrm{E}-\odot 4$ & $8.3 E-\odot 6$ & $6.3 \mathrm{E}-07$ & $2.6 \mathrm{E}-\odot 8$ & $2.1 \mathrm{E}-05$ \\
\hline RA224 & Class: W & & $1.36 \mathrm{E}-\odot 8$ & & & & & \\
\hline & 1 & Yr: & & $6.7 \mathrm{E}-05$ & 4. 3E- - 6 & $3.2 \mathrm{E}-07$ & 1. $9 \mathrm{E}-08$ & $8.2 \mathrm{E}-06$ \\
\hline & 50 & Yr: & & $6.7 \mathrm{E}-05$ & $4.3 E-06$ & $3.2 \mathrm{E}-07$ & 1. $9 \mathrm{E}-\odot 8$ & $8.2 \mathrm{E}-06$ \\
\hline RA225 & Class: W & & $7.60 \mathrm{E}-\odot 9$ & & & & & \\
\hline & 1 & Yr: & & 1. $4 \mathrm{E}-04$ & $1.6 \mathrm{E}-05$ & 1. $9 \mathrm{E}-06$ & $3.7 \mathrm{E}-\odot 8$ & $1.8 \mathrm{E}-05$ \\
\hline & 50 & Yr: & & 1. $4 \mathrm{E}-04$ & $1.6 \mathrm{E}-05$ & $1.9 \mathrm{E}-06$ & $3.7 \mathrm{E}-08$ & $1.8 \mathrm{E}-05$ \\
\hline RA226 & Class: W & & $8.96 \mathrm{E}-\odot 9$ & & & & & \\
\hline & 1 & Yr: & & $9.0 \mathrm{E}-05$ & $1.1 \mathrm{E}-05$ & $1.1 \mathrm{E}-06$ & 8. ०E-๑8 & 1.1E-05 \\
\hline & 50 & Yr: & & $9.0 \mathrm{E}-05$ & 1. $1 \mathrm{E}-05$ & $1.1 \mathrm{E}-06$ & 8. ०E-๑8 & 1.1E- 05 \\
\hline RA228 & Class: W & & $\odot . \odot \odot E \odot \odot ~$ & & & & & \\
\hline & 1 & Yr: & & $3.6 \mathrm{E}-05$ & $1.6 \mathrm{E}-04$ & $2.4 \mathrm{E}-05$ & $7.5 \mathrm{E}-07$ & 1. $0 \mathrm{E}-05$ \\
\hline & 50 & Yr: & & $3.6 E-05$ & $1.6 E-\odot 4$ & $2.4 \mathrm{E}-05$ & $7.5 E-07$ & $1.0 \mathrm{E}-05$ \\
\hline PB210 & Class: D & & $1.42 \mathrm{E}-\odot 9$ & & & & & \\
\hline & 1 & Yr: & & $7.4 \mathrm{E}-07$ & $3 . \odot E-\odot 5$ & 1. $\odot E-\odot 5$ & 7. 4E- -07 & $2.9 \mathrm{E}-06$ \\
\hline & 50 & Yr: & & $7.4 \mathrm{E}-07$ & $3.0 \mathrm{E}-05$ & 1. $0 \mathrm{E}-05$ & $7.4 \mathrm{E}-07$ & 2.9E-๑6 \\
\hline PB212 & Class: D & & $1.97 \mathrm{E}-07$ & & & & & \\
\hline & 1 & Yr: & & 3. $9 \mathrm{E}-08$ & $8.2 \mathrm{E}-\odot 7$ & $8.1 \mathrm{E}-08$ & $2.6 \mathrm{E}-\odot 8$ & 1. $3 \mathrm{E}-07$ \\
\hline & 50 & Yr: & & $3.9 \mathrm{E}-08$ & $8.2 \mathrm{E}-07$ & $8.1 \mathrm{E}-08$ & $2.6 \mathrm{E}-\odot 8$ & 1. $3 \mathrm{E}-07$ \\
\hline BI210 & Class: W & & $8.14 \mathrm{E}-\odot 9$ & & & & & \\
\hline & 1 & Yr: & & $2.4 \mathrm{E}-06$ & $3.1 \mathrm{E}-10$ & $3.1 \mathrm{E}-10$ & $3.1 \mathrm{E}-10$ & 3. $\odot E-\odot 7$ \\
\hline & 50 & Yr: & & $2.4 \mathrm{E}-06$ & $3.1 \mathrm{E}-10$ & $3.1 \mathrm{E}-10$ & $3.1 \mathrm{E}-10$ & 3. $0 \mathrm{E}-07$ \\
\hline BI212 & Class: W & & $2.83 E-07$ & & & & & \\
\hline & 1 & Yr: & & $5 . \odot E-\odot 7$ & $1.6 \mathrm{E}-10$ & 1. $6 \mathrm{E}-10$ & $1.6 \mathrm{E}-10$ & $1.1 \mathrm{E}-07$ \\
\hline & 50 & Yr: & & $5.0 \mathrm{E}-07$ & $1.6 \mathrm{E}-10$ & 1. $6 \mathrm{E}-10$ & $1.6 \mathrm{E}-10$ & 1. $1 \mathrm{E}-07$ \\
\hline P0210 & Class: W & & $1.23 \mathrm{E}-11$ & & & & & \\
\hline & 1 & Yr: & & 8. $0 \mathrm{E}-05$ & $4.1 \mathrm{E}-06$ & $4.6 \mathrm{E}-06$ & $3.5 E-07$ & 1. $1 \mathrm{E}-05$ \\
\hline & 50 & Yr: & & $8.0 \mathrm{E}-05$ & $4.1 \mathrm{E}-06$ & $4.6 E-06$ & $3.5 \mathrm{E}-07$ & 1. $1 \mathrm{E}-05$ \\
\hline
\end{tabular}




\begin{tabular}{|c|c|c|c|c|c|c|c|c|}
\hline \multirow[t]{3}{*}{ U 232} & Class: $Y$ & & $3.72 \mathrm{E}-10$ & & & & & \\
\hline & 1 & Yr: & & $7.9 E-04$ & $6.7 \mathrm{E}-05$ & $7.8 \mathrm{E}-06$ & $3.2 \mathrm{E}-07$ & $9.7 \mathrm{E}-05$ \\
\hline & 50 & Yr: & & $7.9 \mathrm{E}-\odot 4$ & $6.7 \mathrm{E}-05$ & $7.8 E-06$ & $3.2 \mathrm{E}-07$ & $9.7 \mathrm{E}-05$ \\
\hline \multirow[t]{3}{*}{ U 233} & Class: $Y$ & & $4.48 \mathrm{E}-10$ & & & & & \\
\hline & 1 & Yr: & & $2.4 \mathrm{E}-04$ & 1. $3 E-06$ & $1.1 \mathrm{E}-07$ & $2.8 \mathrm{E}-\odot 8$ & $2.9 \mathrm{E}-05$ \\
\hline & 50 & Yr: & & $2.4 \mathrm{E}-\odot 4$ & 1. $3 E-06$ & $1.1 \mathrm{E}-07$ & $2.8 \mathrm{E}-08$ & $2.9 \mathrm{E}-05$ \\
\hline \multirow[t]{3}{*}{ U 234} & Class: $\mathrm{Y}$ & & $1.93 \mathrm{E}-10$ & & & & & \\
\hline & 1 & Yr: & & $2.4 \mathrm{E}-\odot 4$ & $8.9 E-\odot 7$ & ৪. 6E-๑8 & $2.6 \mathrm{E}-\odot 8$ & $2.9 \mathrm{E}-05$ \\
\hline & 50 & Yr: & & $2.4 \mathrm{E}-04$ & 8. $9 \mathrm{E}-07$ & $8.6 \mathrm{E}-\odot 8$ & $2.6 \mathrm{E}-08$ & $2.9 \mathrm{E}-05$ \\
\hline \multirow[t]{3}{*}{ U 235} & Class: $\mathrm{Y}$ & & $2.04 \mathrm{E}-07$ & & & & & \\
\hline & 1 & Yr: & & $2.2 \mathrm{E}-04$ & $8.4 \mathrm{E}-07$ & 8. 3E- - & $2.8 \mathrm{E}-\odot 8$ & $2.6 \mathrm{E}-05$ \\
\hline & 50 & Yr: & & $2.2 \mathrm{E}-\odot 4$ & $8.4 \mathrm{E}-07$ & 8. 3E- - & $2.8 \mathrm{E}-\odot 8$ & $2.6 \mathrm{E}-05$ \\
\hline \multirow[t]{3}{*}{ U 236} & Class: Y & & $1.22 \mathrm{E}-10$ & & & & & \\
\hline & 1 & Yr: & & $2.2 \mathrm{E}-\odot 4$ & $8.4 \mathrm{E}-07$ & $8.1 \mathrm{E}-08$ & $2.5 \mathrm{E}-\odot 8$ & $2.7 \mathrm{E}-\odot 5$ \\
\hline & 50 & Yr: & & $2.2 \mathrm{E}-04$ & $8.4 \mathrm{E}-07$ & $8.1 \mathrm{E}-08$ & $2.5 E-08$ & $2.7 \mathrm{E}-05$ \\
\hline \multirow[t]{3}{*}{ U 237} & Class: $\mathrm{Y}$ & & $1.67 \mathrm{E}-07$ & & & & & \\
\hline & 1 & Yr: & & $3.9 \mathrm{E}-\odot 8$ & $2.0 \mathrm{E}-10$ & $6.4 \mathrm{E}-11$ & $6.2 \mathrm{E}-11$ & $6.4 \mathrm{E}-09$ \\
\hline & 50 & Yr: & & $3.9 \mathrm{E}-08$ & $2.0 \mathrm{E}-10$ & $6.4 \mathrm{E}-11$ & $6.2 \mathrm{E}-11$ & $6.4 \mathrm{E}-09$ \\
\hline \multirow[t]{3}{*}{ U 238} & Class: $Y$ & & $7.92 \mathrm{E}-11$ & & & & & \\
\hline & 1 & Yr: & & $2.1 \mathrm{E}-\odot 4$ & $8.1 \mathrm{E}-07$ & $8.1 \mathrm{E}-08$ & $2.4 \mathrm{E}-08$ & $2.5 \mathrm{E}-05$ \\
\hline & 50 & Yr: & & $2.1 \mathrm{E}-04$ & $8.1 \mathrm{E}-07$ & $8.1 \mathrm{E}-08$ & $2.4 \mathrm{E}-08$ & $2.5 \mathrm{E}-05$ \\
\hline \multirow{3}{*}{ U 240} & Class: $Y$ & & $1.85 \mathrm{E}-\odot 9$ & & & & & \\
\hline & 1 & Yr: & & $8.6 \mathrm{E}-\odot 9$ & 7. $9 \mathrm{E}-11$ & $3.4 \mathrm{E}-11$ & $1.8 \mathrm{E}-11$ & $3.4 \mathrm{E}-\odot 9$ \\
\hline & 50 & Yr: & & $8.6 \mathrm{E}-\odot 9$ & $7.9 \mathrm{E}-11$ & $3.4 \mathrm{E}-11$ & $1.8 \mathrm{E}-11$ & $3.4 \mathrm{E}-09$ \\
\hline \multirow[t]{3}{*}{ PA231 } & Class: $\mathrm{Y}$ & & $4.95 \mathrm{E}-\odot 8$ & & & & & \\
\hline & 1 & Yr: & & $3.6 \mathrm{E}-04$ & $6.1 \mathrm{E}-04$ & $3.9 \mathrm{E}-05$ & $2.5 \mathrm{E}-06$ & $5.9 \mathrm{E}-05$ \\
\hline & 50 & Yr: & & $3.6 E-\odot 4$ & $6.1 \mathrm{E}-\odot 4$ & $3.9 \mathrm{E}-05$ & $2.5 \mathrm{E}-\odot 6$ & $5.9 \mathrm{E}-05$ \\
\hline PA233 & Class: $\mathrm{Y}$ & & $2.70 \mathrm{E}-07$ & & & & & \\
\hline & 1 & Yr: & & 8. 7E- - ४ & $8.4 \mathrm{E}-10$ & $3.2 \mathrm{E}-10$ & $2.6 \mathrm{E}-10$ & 1. $3 E-\odot 8$ \\
\hline & 50 & Yr: & & 8.7E-08 & $8.4 \mathrm{E}-10$ & $3.2 \mathrm{E}-10$ & 2. $6 \mathrm{E}-10$ & $1.3 E-08$ \\
\hline PA234 & Class: $\mathrm{Y}$ & & $2.75 \mathrm{E}-\odot 6$ & & & & & \\
\hline & 1 & Yr: & & $5.0 E-\odot 9$ & $1.1 \mathrm{E}-10$ & $8.7 \mathrm{E}-11$ & $5.7 \mathrm{E}-11$ & 2. $2 \mathrm{E}-09$ \\
\hline & 50 & Yr: & & $5.0 \mathrm{E}-\odot 9$ & $1.1 \mathrm{E}-10$ & 8. $7 \mathrm{E}-11$ & $5.7 \mathrm{E}-11$ & $2.2 \mathrm{E}-09$ \\
\hline AC225 & Class: $Y$ & & $2.01 \mathrm{E}-08$ & & & & & \\
\hline & 1 & Yr: & & 1. $9 \mathrm{E}-\odot 4$ & 4. $\odot \mathrm{E}-\odot 7$ & 8. . E- - & 1. $9 \mathrm{E}-\odot 8$ & 2. $3 E-05$ \\
\hline & 50 & Yr: & & 1. $9 \mathrm{E}-04$ & 4. $\odot E-\odot 7$ & 8. 7E-०8 & 1.9E-०8 & $2.3 E-05$ \\
\hline AC227 & Class: $Y$ & & $1.62 \mathrm{E}-10$ & & & & & \\
\hline & 1 & Yr: & & 1. $3 \mathrm{E}-03$ & $2 . \odot E-\odot 4$ & $2.3 E-\odot 5$ & $1.6 \mathrm{E}-06$ & 1. $6 \mathrm{E}-04$ \\
\hline & 50 & Yr: & & 1. $3 E-03$ & 2. $0 \mathrm{E}-04$ & 2. $3 E-05$ & $1.6 \mathrm{E}-06$ & $1.6 \mathrm{E}-04$ \\
\hline AC228 & Class: $Y$ & & $1.42 \mathrm{E}-\odot 6$ & & & & & \\
\hline & 1 & Yr: & & $3.9 \mathrm{E}-07$ & $2.1 \mathrm{E}-08$ & $3.2 \mathrm{E}-\odot 9$ & $1.1 \mathrm{E}-10$ & $4.9 \mathrm{E}-08$ \\
\hline & 50 & Yr: & & $3.9 E-07$ & $2.1 \mathrm{E}-08$ & $3.2 \mathrm{E}-\odot 9$ & 1. $1 \mathrm{E}-10$ & $4.9 \mathrm{E}-08$ \\
\hline FR223 & Class: D & & $6.97 \mathrm{E}-\odot 8$ & & & & & \\
\hline & 1 & Yr: & & $6.9 \mathrm{E}-09$ & $6.7 \mathrm{E}-09$ & $6.7 \mathrm{E}-\odot 9$ & 6. . E- $\odot 9$ & 7. $4 \mathrm{E}-\odot 9$ \\
\hline & 50 & Yr: & & $6.9 \mathrm{E}-\odot 9$ & $6.7 \mathrm{E}-09$ & $6.7 \mathrm{E}-\odot 9$ & $6.7 \mathrm{E}-\odot 9$ & 7. $4 \mathrm{E}-09$ \\
\hline NP237 & Class: W & & $2.81 \mathrm{E}-08$ & & & & & \\
\hline & 1 & Yr: & & $9.4 \mathrm{E}-05$ & 8. 3E- - 4 & 1. $\odot E-\odot 4$ & $4.2 \mathrm{E}-06$ & $4.1 \mathrm{E}-05$ \\
\hline & 50 & Yr: & & $9.4 \mathrm{E}-05$ & 8. 3E- - 4 & 1. $\odot E-\odot 4$ & $4.2 \mathrm{E}-\odot 6$ & $4.1 \mathrm{E}-05$ \\
\hline NP238 & Class: W & & $8.07 \mathrm{E}-07$ & & & & & \\
\hline & 1 & Yr: & & 1. $7 \mathrm{E}-\odot 8$ & $5.9 \mathrm{E}-08$ & $7.8 E-\odot 9$ & $3.6 \mathrm{E}-10$ & $5.8 \mathrm{E}-\odot 9$ \\
\hline & 50 & Yr: & & 1.7E-०8 & $5.9 \mathrm{E}-08$ & $7.8 E-09$ & $3.6 \mathrm{E}-10$ & $5.8 \mathrm{E}-09$ \\
\hline NP239 & Class: W & & $2.19 \mathrm{E}-07$ & & & & & \\
\hline & 1 & Yr: & & 1.7E-๑8 & 1. $9 \mathrm{E}-\odot 9$ & 3. $\odot E-10$ & 4. $3 E-11$ & $4.2 \mathrm{E}-09$ \\
\hline & 50 & Yr: & & 1. 7E-०8 & 1. $9 \mathrm{E}-09$ & 3. $0 \mathrm{E}-10$ & 4. 3E-11 & $4.2 E-09$ \\
\hline PU236 & Class: $\mathrm{Y}$ & & $1.48 \mathrm{E}-10$ & & & & & \\
\hline & 1 & Yr: & & $2.4 \mathrm{E}-\odot 4$ & $4.8 E-05$ & $5.7 \mathrm{E}-\odot 6$ & $2.2 \mathrm{E}-\odot 7$ & $3.1 \mathrm{E}-05$ \\
\hline & 50 & Yr: & & $2.4 \mathrm{E}-04$ & $4.8 E-05$ & $5.7 \mathrm{E}-06$ & 2. $2 \mathrm{E}-\odot 7$ & $3.1 \mathrm{E}-05$ \\
\hline PU237 & Class: $Y$ & & $5.58 \mathrm{E}-\odot 8$ & & & & & \\
\hline & 1 & Yr: & & 1. $0 E-08$ & $3.4 \mathrm{E}-10$ & $9.4 \mathrm{E}-11$ & $\mathrm{E}-11$ & 1. $5 E-\odot 9$ \\
\hline & 50 & Yr: & & 1. $\odot E-\odot 8$ & $3.4 \mathrm{E}-10$ & $9.4 \mathrm{E}-11$ & $9.9 \mathrm{E}-11$ & 1. $5 \mathrm{E}-09$ \\
\hline PU238 & Class: $\mathrm{Y}$ & & $1.11 \mathrm{E}-10$ & & & & & \\
\hline & 1 & Yr: & & $2.8 \mathrm{E}-\odot 4$ & 1. $3 E-\odot 4$ & 1. $3 E-05$ & $6.0 \mathrm{E}-07$ & 4. . \\
\hline & 50 & Yr: & & $2.8 E-04$ & 1. $3 E-\odot 4$ & 1. $3 E-05$ & $6 . \odot E-\odot 7$ & 4. . $\mathrm{E}-05$ \\
\hline PU239 & Class: $Y$ & & $1.10 \mathrm{E}-10$ & & & & & \\
\hline & 1 & Yr: & & $2.7 \mathrm{E}-\odot 4$ & $1.6 \mathrm{E}-\odot 4$ & 1. $4 \mathrm{E}-05$ & $E-07$ & $3.9 E-05$ \\
\hline & 50 & Yr: & & $2.7 \mathrm{E}-\odot 4$ & 1. $6 \mathrm{E}-\odot 4$ & 1. $4 \mathrm{E}-\odot 5$ & $6.9 \mathrm{E}-07$ & $3.9 \mathrm{E}-05$ \\
\hline PU240 & Class: $\mathrm{Y}$ & & $1.08 \mathrm{E}-10$ & & & & & \\
\hline & 1 & Yr: & & $2.7 \mathrm{E}-\odot 4$ & 1. $6 \mathrm{E}-\odot 4$ & 1. $4 \mathrm{E}-05$ & $6.9 \mathrm{E}-07$ & $3.9 \mathrm{E}-05$ \\
\hline & 50 & Yr: & & $2.7 \mathrm{E}-\odot 4$ & 1. $6 \mathrm{E}-\odot 4$ & 1. $4 \mathrm{E}-\odot 5$ & $6.9 \mathrm{E}-07$ & $3.9 \mathrm{E}-05$ \\
\hline PU241 & Class: $\mathrm{Y}$ & & 2. $0 \odot \mathrm{E}-12$ & & & & & \\
\hline & 1 & Yr: & & $8.6 E-07$ & $3.4 \mathrm{E}-06$ & $2 . \odot E-\odot 7$ & 1. 4E- $\odot 8$ & $2.4 \mathrm{E}-07$ \\
\hline
\end{tabular}




\begin{tabular}{|c|c|c|c|c|c|c|c|c|}
\hline & 50 & Yr: & & $8.6 \mathrm{E}-07$ & $3.4 \mathrm{E}-06$ & $2.0 \mathrm{E}-07$ & 1. $4 \mathrm{E}-08$ & $2.4 \mathrm{E}-07$ \\
\hline \multirow[t]{3}{*}{ PU242 } & Class: Y & & $9.18 \mathrm{E}-11$ & & & & & \\
\hline & 1 & Yr: & & $2.5 \mathrm{E}-\odot 4$ & 1. $5 \mathrm{E}-\odot 4$ & 1. $3 E-05$ & $6.6 \mathrm{E}-07$ & $3.6 \mathrm{E}-05$ \\
\hline & 50 & Yr: & & $2.5 \mathrm{E}-\odot 4$ & $1.5 \mathrm{E}-\odot 4$ & 1. $3 E-05$ & $6.6 \mathrm{E}-07$ & $3.6 \mathrm{E}-05$ \\
\hline \multirow[t]{3}{*}{ PU243 } & Class: $\mathrm{Y}$ & & $3.04 \mathrm{E}-08$ & & & & & \\
\hline & 1 & Yr: & & 1. $3 E-09$ & 1. $6 \mathrm{E}-11$ & $2.1 \mathrm{E}-12$ & $7.1 \mathrm{E}-13$ & $4.1 \mathrm{E}-10$ \\
\hline & 50 & Yr: & & 1. $3 E-\odot 9$ & $1.6 \mathrm{E}-11$ & $2.1 \mathrm{E}-12$ & $7.1 \mathrm{E}-13$ & $4.1 \mathrm{E}-10$ \\
\hline \multirow[t]{3}{*}{ PU244 } & Class: $\mathrm{Y}$ & & $6.56 \mathrm{E}-11$ & & & & & \\
\hline & 1 & Yr: & & $2.3 E-04$ & 1. $4 \mathrm{E}-\odot 4$ & $1.2 \mathrm{E}-\odot 5$ & $6.3 E-07$ & $3.4 \mathrm{E}-05$ \\
\hline & 50 & Yr: & & 2. $3 E-\odot 4$ & 1. $4 \mathrm{E}-\odot 4$ & 1. $2 \mathrm{E}-\odot 5$ & $6.3 \mathrm{E}-\odot 7$ & $3.4 \mathrm{E}-05$ \\
\hline \multirow[t]{3}{*}{ AM241 } & Class: W & & $2.13 E-08$ & & & & & \\
\hline & 1 & Yr: & & 1. $2 \mathrm{E}-\odot 4$ & 1. $4 \mathrm{E}-03$ & $1.8 \mathrm{E}-\odot 4$ & $6.4 \mathrm{E}-06$ & 7. $0 \mathrm{E}-\odot 5$ \\
\hline & 50 & Yr: & & 1. $2 \mathrm{E}-04$ & 1. $4 \mathrm{E}-03$ & 1. $8 \mathrm{E}-\odot 4$ & $6.4 \mathrm{E}-06$ & $7.0 \mathrm{E}-05$ \\
\hline \multirow[t]{3}{*}{ AM242M } & Class: W & & $7.85 \mathrm{E}-10$ & & & & & \\
\hline & 1 & Yr: & & $2.8 \mathrm{E}-05$ & 1. $3 E-03$ & $1.5 \mathrm{E}-04$ & $6.3 \mathrm{E}-06$ & $5.3 E-05$ \\
\hline & 50 & Yr: & & $2.8 \mathrm{E}-05$ & 1. $3 E-03$ & $1.5 \mathrm{E}-\odot 4$ & $6.3 \mathrm{E}-06$ & $5.3 E-05$ \\
\hline \multirow[t]{3}{*}{ AM242 } & Class: W & & 1. 93E-๑8 & & & & & \\
\hline & 1 & Yr: & & $3.5 E-07$ & $4.1 \mathrm{E}-07$ & $8.4 \mathrm{E}-\odot 8$ & $5.4 \mathrm{E}-10$ & $5.9 \mathrm{E}-08$ \\
\hline & 50 & Yr: & & $3.5 \mathrm{E}-07$ & $4.1 \mathrm{E}-07$ & $8.4 \mathrm{E}-08$ & $5.4 \mathrm{E}-10$ & $5.9 \mathrm{E}-08$ \\
\hline \multirow[t]{3}{*}{ AM243 } & Class: W & & $5.87 \mathrm{E}-\odot 8$ & & & & & \\
\hline & 1 & Yr: & & $1.1 \mathrm{E}-04$ & 1. $4 \mathrm{E}-03$ & $1.8 \mathrm{E}-\odot 4$ & $6.5 \mathrm{E}-06$ & $6.8 \mathrm{E}-05$ \\
\hline & 50 & Yr: & & $1.1 \mathrm{E}-04$ & $1.4 \mathrm{E}-03$ & 1. $8 \mathrm{E}-\odot 4$ & $6.5 \mathrm{E}-06$ & $6.8 \mathrm{E}-05$ \\
\hline \multirow[t]{3}{*}{ CM242 } & Class: W & & $1.27 \mathrm{E}-10$ & & & & & \\
\hline & 1 & Yr: & & $1.1 \mathrm{E}-04$ & 1. $2 \mathrm{E}-\odot 4$ & $2.5 \mathrm{E}-\odot 5$ & $1.6 \mathrm{E}-07$ & $1.8 \mathrm{E}-05$ \\
\hline & 50 & Yr: & & 1. $1 \mathrm{E}-04$ & 1. $2 \mathrm{E}-04$ & $2.5 \mathrm{E}-05$ & 1. $6 \mathrm{E}-\odot 7$ & $1.8 \mathrm{E}-05$ \\
\hline \multirow[t]{3}{*}{ CM243 } & Class: W & & $1.67 E-\odot 7$ & & & & & \\
\hline & 1 & Yr: & & 1. $2 \mathrm{E}-\odot 4$ & 1. $1 \mathrm{E}-03$ & 1.7E- -4 & $3.9 \mathrm{E}-\odot 6$ & $6.1 \mathrm{E}-05$ \\
\hline & 50 & Yr: & & 1. $2 \mathrm{E}-\odot 4$ & 1. $1 \mathrm{E}-03$ & 1. 7E-๑4 & $3.9 \mathrm{E}-\odot 6$ & $6.1 \mathrm{E}-05$ \\
\hline \multirow[t]{3}{*}{ CM244 } & Class: W & & $1.08 \mathrm{E}-10$ & & & & & \\
\hline & 1 & Yr: & & 1. $2 \mathrm{E}-\odot 4$ & $9.6 \mathrm{E}-04$ & $1.6 \mathrm{E}-\odot 4$ & 3. $\odot E-\odot 6$ & $5.6 \mathrm{E}-05$ \\
\hline & 50 & Yr: & & $1.2 \mathrm{E}-04$ & $9.6 \mathrm{E}-\odot 4$ & $1.6 \mathrm{E}-\odot 4$ & 3. $0 \mathrm{E}-06$ & $5.6 \mathrm{E}-05$ \\
\hline \multirow[t]{3}{*}{ CM245 } & Class: W & & $1.10 \mathrm{E}-07$ & & & & & \\
\hline & 1 & Yr: & & $1.1 \mathrm{E}-\odot 4$ & 1. $4 \mathrm{E}-03$ & 1. $8 \mathrm{E}-\odot 4$ & $6.7 \mathrm{E}-\odot 6$ & 7. $0 \mathrm{E}-\odot 5$ \\
\hline & 50 & Yr: & & $1.1 \mathrm{E}-04$ & 1. $4 \mathrm{E}-03$ & 1. $8 \mathrm{E}-04$ & $6.7 \mathrm{E}-06$ & 7. $0 \mathrm{E}-05$ \\
\hline \multirow[t]{3}{*}{ CM246 } & Class: W & & $9.78 \mathrm{E}-11$ & & & & & \\
\hline & 1 & Yr: & & 1.1E- -44 & 1. $4 \mathrm{E}-03$ & 1. $8 \mathrm{E}-\odot 4$ & $6.5 \mathrm{E}-06$ & $6.9 \mathrm{E}-05$ \\
\hline & 50 & Yr: & & $1.1 \mathrm{E}-04$ & 1. $4 \mathrm{E}-03$ & $1.8 \mathrm{E}-\odot 4$ & $6.5 \mathrm{E}-06$ & $6.9 \mathrm{E}-05$ \\
\hline \multirow[t]{3}{*}{ CM247 } & Class: W & & $4.38 \mathrm{E}-07$ & & & & & \\
\hline & 1 & Yr: & & 1. $0 \mathrm{E}-\odot 4$ & 1. $3 \mathrm{E}-\mathrm{-} 3$ & 1. $7 \mathrm{E}-\odot 4$ & $6.1 \mathrm{E}-06$ & $6.4 \mathrm{E}-05$ \\
\hline & 50 & Yr: & & 1. $0 \mathrm{E}-04$ & 1. $3 \mathrm{E}-03$ & $1.7 \mathrm{E}-04$ & $6.1 \mathrm{E}-06$ & $6.4 \mathrm{E}-05$ \\
\hline \multirow[t]{3}{*}{ CM248 } & Class: W & & $7.44 \mathrm{E}-11$ & & & & & \\
\hline & 1 & Yr: & & $6.3 E-05$ & $7.8 \mathrm{E}-03$ & $6.2 \mathrm{E}-\odot 4$ & $2.2 \mathrm{E}-\odot 8$ & $4.2 E-\odot 4$ \\
\hline & 50 & Yr: & & $6.3 E-05$ & $7.8 \mathrm{E}-03$ & $6.2 \mathrm{E}-\odot 4$ & 2. $2 \mathrm{E}-\odot 8$ & $4.2 \mathrm{E}-\odot 4$ \\
\hline \multirow[t]{3}{*}{ CF252 } & Class: W & & $1.15 \mathrm{E}-10$ & & & & & \\
\hline & 1 & Yr: & & $3.5 E-05$ & $6.6 \mathrm{E}-04$ & $5.3 \mathrm{E}-05$ & 1. 1E- -88 & $3.5 E-05$ \\
\hline & 50 & Yr: & & $3.5 E-05$ & $6.6 \mathrm{E}-\odot 4$ & $5.3 E-\odot 5$ & 1. $1 \mathrm{E}-08$ & $3.5 \mathrm{E}-05$ \\
\hline
\end{tabular}




\section{Appendix A.6}

Age 100 days

Dose Equiv. Factors for Acute Inhalation and Ext Air Submersion FGR12/13

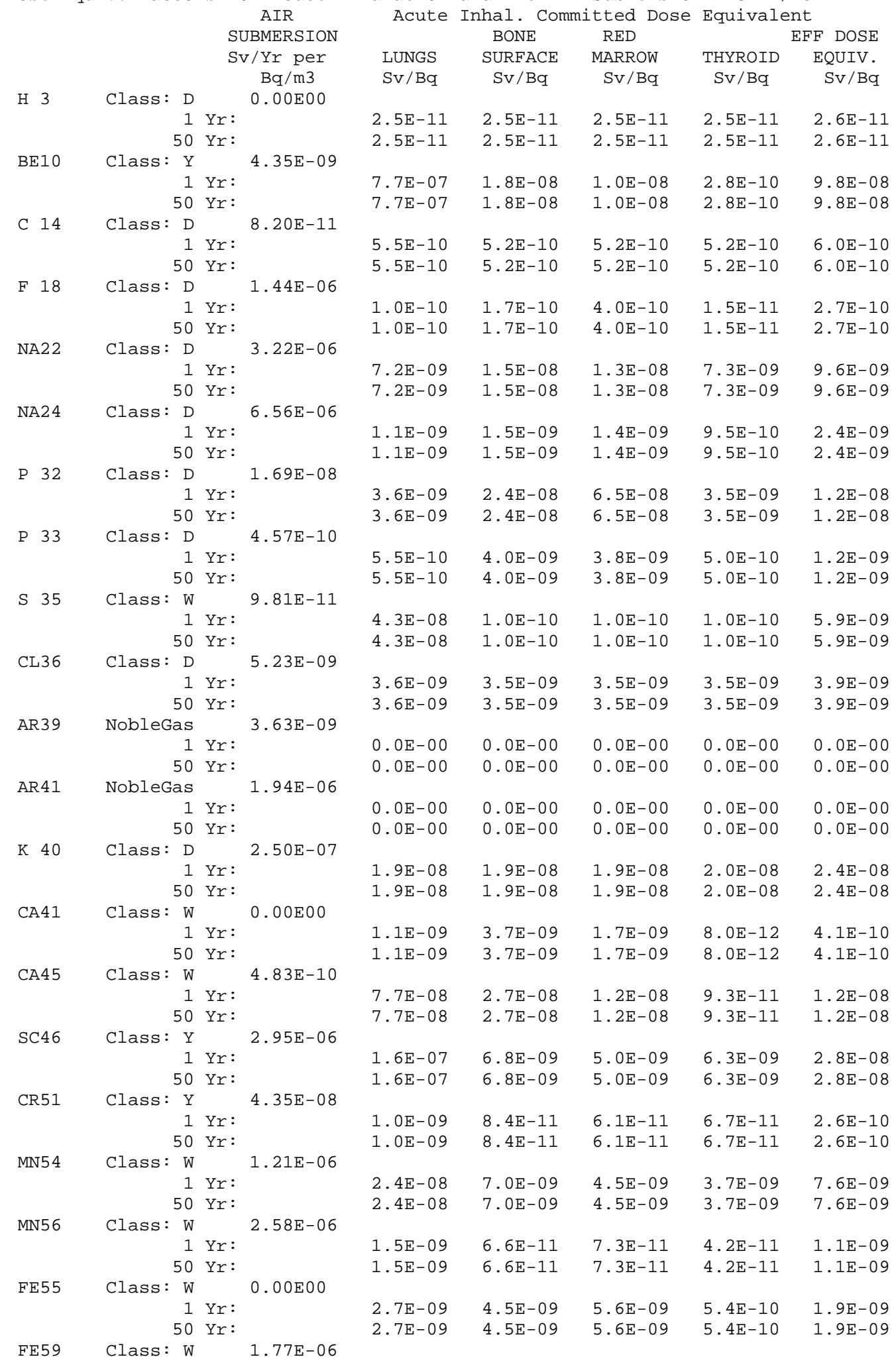




\begin{tabular}{|c|c|c|c|c|c|c|c|c|}
\hline & 1 & Yr: & & $8.5 \mathrm{E}-08$ & 1. $4 \mathrm{E}-\odot 8$ & 1. $4 \mathrm{E}-\odot 8$ & $5.5 \mathrm{E}-09$ & 1. $8 \mathrm{E}-\odot 8$ \\
\hline & 50 & Yr: & & $8.5 \mathrm{E}-\odot 8$ & 1. 4E-๑8 & 1. $4 \mathrm{E}-\odot 8$ & $5.5 E-\odot 9$ & 1. $8 \mathrm{E}-08$ \\
\hline \multirow[t]{3}{*}{ C057 } & Class: Y & & $1.57 \mathrm{E}-07$ & & & & & \\
\hline & 1 & Yr: & & $2.7 \mathrm{E}-\odot 8$ & 1.7E-०9 & $6.5 \mathrm{E}-10$ & $9.6 \mathrm{E}-10$ & 4. 3E- -99 \\
\hline & 50 & Yr: & & $2.7 \mathrm{E}-\odot 8$ & 1.7E-๑9 & $6.5 \mathrm{E}-10$ & $9.6 \mathrm{E}-10$ & 4. 3E- - 09 \\
\hline \multirow[t]{3}{*}{ C058 } & Class: Y & & $1.40 \mathrm{E}-06$ & & & & & \\
\hline & 1 & Yr: & & $4.7 \mathrm{E}-\odot 8$ & $3 . \odot E-\odot 9$ & $2.2 \mathrm{E}-\odot 9$ & $2.8 \mathrm{E}-\odot 9$ & 9. ०E-०९ \\
\hline & 50 & Yr: & & $4.7 \mathrm{E}-\odot 8$ & 3. $\odot E-\odot 9$ & $2.2 \mathrm{E}-09$ & $2.8 \mathrm{E}-\odot 9$ & 9. ०E-๑9 \\
\hline \multirow[t]{3}{*}{$\mathrm{C06 \odot}$} & Class: Y & & $3.75 \mathrm{E}-06$ & & & & & \\
\hline & 1 & Yr: & & $5.2 \mathrm{E}-07$ & $3.4 \mathrm{E}-08$ & $2.7 \mathrm{E}-\odot 8$ & $3.6 \mathrm{E}-\odot 8$ & $9.2 \mathrm{E}-08$ \\
\hline & 50 & Yr: & & $5.2 \mathrm{E}-07$ & $3.4 \mathrm{E}-08$ & $2.7 E-08$ & 3. $6 \mathrm{E}-08$ & $9.2 \mathrm{E}-08$ \\
\hline \multirow[t]{3}{*}{ NI59 } & Class: W & & $\odot . \odot \odot Е \odot \odot$ & & & & & \\
\hline & 1 & Yr: & & $3.1 \mathrm{E}-09$ & $3.6 \mathrm{E}-10$ & $3.6 \mathrm{E}-10$ & $3.6 \mathrm{E}-10$ & $7.8 \mathrm{E}-10$ \\
\hline & 50 & Yr: & & $3.1 \mathrm{E}-09$ & $3.6 \mathrm{E}-10$ & $3.6 \mathrm{E}-10$ & $3.6 \mathrm{E}-10$ & $7.8 \mathrm{E}-10$ \\
\hline \multirow[t]{3}{*}{ NI63 } & Class: W & & ๑.๑०Е०९ & & & & & \\
\hline & 1 & Yr: & & 1. $3 E-08$ & $8.6 \mathrm{E}-10$ & $8.6 \mathrm{E}-10$ & $8.6 \mathrm{E}-10$ & $2.5 E-\odot 9$ \\
\hline & 50 & Yr: & & 1. $3 E-\odot 8$ & $8.6 \mathrm{E}-10$ & $8.6 \mathrm{E}-10$ & $8.6 \mathrm{E}-10$ & $2.5 \mathrm{E}-09$ \\
\hline \multirow[t]{3}{*}{ NI65 } & Class: W & & $8.42 \mathrm{E}-07$ & & & & & \\
\hline & 1 & Yr: & & $1.2 \mathrm{E}-\odot 9$ & $2.0 \mathrm{E}-11$ & 1. $7 \mathrm{E}-11$ & $1.5 \mathrm{E}-11$ & $7.7 \mathrm{E}-10$ \\
\hline & 50 & Yr: & & 1. $2 \mathrm{E}-09$ & 2. $0 \mathrm{E}-11$ & 1. $7 \mathrm{E}-11$ & 1. $5 \mathrm{E}-11$ & $7.7 \mathrm{E}-10$ \\
\hline \multirow[t]{3}{*}{ CU64 } & Class: D & & $2.69 \mathrm{E}-07$ & & & & & \\
\hline & 1 & Yr: & & $1.5 \mathrm{E}-10$ & $1.1 \mathrm{E}-10$ & 1. $\odot \mathrm{E}-1 \odot$ & 1.1E-10 & $2.9 \mathrm{E}-10$ \\
\hline & 50 & Yr: & & 1. $5 \mathrm{E}-10$ & $1.1 \mathrm{E}-10$ & 1. $\odot E-10$ & $1.1 \mathrm{E}-10$ & $2.9 \mathrm{E}-10$ \\
\hline \multirow[t]{3}{*}{ ZN65 } & Class: Y & & $8.61 \mathrm{E}-07$ & & & & & \\
\hline & 1 & Yr: & & $3.6 \mathrm{E}-\odot 8$ & 4. $\odot E-\odot 9$ & $3 . \odot E-\odot 9$ & $3.9 E-\odot 9$ & 7. $7 \mathrm{E}-\odot 9$ \\
\hline & 50 & Yr: & & $3.6 E-08$ & 4. ०E-०9 & 3. ๑E-๑9 & $3.9 E-09$ & $7.7 \mathrm{E}-\odot 9$ \\
\hline \multirow[t]{3}{*}{ ZN69M } & Class: Y & & $5.83 E-07$ & & & & & \\
\hline & 1 & Yr: & & $5 . \odot E-\odot 9$ & $6.3 \mathrm{E}-11$ & $5.6 \mathrm{E}-11$ & $3.4 \mathrm{E}-11$ & $2.2 \mathrm{E}-\odot 9$ \\
\hline & 50 & Yr: & & $5 . \odot E-\odot 9$ & $6.3 \mathrm{E}-11$ & $5.6 \mathrm{E}-11$ & $3.4 \mathrm{E}-11$ & 2. $2 \mathrm{E}-\odot 9$ \\
\hline ZN69 & Class: Y & & $6.31 \mathrm{E}-09$ & & & & & \\
\hline & 1 & Yr: & & $4.2 \mathrm{E}-10$ & $4.4 \mathrm{E}-14$ & $5.7 \mathrm{E}-14$ & $3.8 \mathrm{E}-14$ & 2. $3 \mathrm{E}-10$ \\
\hline & 50 & Yr: & & $4.2 \mathrm{E}-10$ & $4.4 \mathrm{E}-14$ & $5.7 \mathrm{E}-14$ & $3.8 \mathrm{E}-14$ & 2. $3 \mathrm{E}-10$ \\
\hline AS76 & Class: W & & $6.5 \odot E-\odot 7$ & & & & & \\
\hline & 1 & Yr: & & $1.5 \mathrm{E}-\odot 8$ & $7.0 \mathrm{E}-10$ & $6.7 \mathrm{E}-10$ & $6.9 \mathrm{E}-10$ & $5.0 \mathrm{E}-\odot 9$ \\
\hline & 50 & Yr: & & 1. $5 \mathrm{E}-08$ & $7.0 \mathrm{E}-10$ & $6.7 \mathrm{E}-10$ & $6.9 \mathrm{E}-10$ & $5.0 \mathrm{E}-09$ \\
\hline SE75 & Class: W & & $5.30 \mathrm{E}-07$ & & & & & \\
\hline & 1 & Yr: & & $2.1 \mathrm{E}-08$ & $3.1 \mathrm{E}-09$ & 1. $9 \mathrm{E}-\odot 9$ & $2.2 \mathrm{E}-09$ & $6.0 \mathrm{E}-\odot 9$ \\
\hline & 50 & Yr: & & $2.1 \mathrm{E}-08$ & $3.1 \mathrm{E}-09$ & 1. $9 \mathrm{E}-09$ & 2. $2 \mathrm{E}-09$ & $6.0 \mathrm{E}-09$ \\
\hline SE79 & Class: W & & $1.24 \mathrm{E}-10$ & & & & & \\
\hline & 1 & Yr: & & 7. 4E-๑8 & 1. $3 E-\odot 9$ & 1. $3 E-09$ & 1. $3 E-09$ & 1. $4 \mathrm{E}-08$ \\
\hline & 50 & Yr: & & $7.4 \mathrm{E}-08$ & 1. 3E-०9 & 1. 3E-०9 & 1. 3E-०9 & 1. $4 \mathrm{E}-08$ \\
\hline BR82 & Class: D & & $3.85 \mathrm{E}-06$ & & & & & \\
\hline & 1 & Yr: & & 1. $3 E-\odot 9$ & 1. $3 E-\odot 9$ & $1.1 \mathrm{E}-\odot 9$ & 1. 3E-०9 & $2.8 \mathrm{E}-\odot 9$ \\
\hline & 50 & Yr: & & 1. 3E- $\odot 9$ & $1.3 \mathrm{E}-\odot 9$ & $1.1 \mathrm{E}-\odot 9$ & 1. 3E- $\odot 9$ & $2.8 \mathrm{E}-09$ \\
\hline BR83 & Class: D & & $1.68 \mathrm{E}-\odot 8$ & & & & & \\
\hline & 1 & Yr: & & $1.1 \mathrm{E}-10$ & $3.7 \mathrm{E}-11$ & $3.7 \mathrm{E}-11$ & $3.7 \mathrm{E}-11$ & $1.8 \mathrm{E}-10$ \\
\hline & 50 & Yr: & & $1.1 \mathrm{E}-10$ & $3.7 \mathrm{E}-11$ & $3.7 \mathrm{E}-11$ & 3. $7 \mathrm{E}-11$ & $1.8 \mathrm{E}-10$ \\
\hline BR84 & Class: D & & $2.84 \mathrm{E}-\odot 6$ & & & & & \\
\hline & 1 & Yr: & & 1. $4 \mathrm{E}-10$ & $3.3 E-11$ & $3.1 \mathrm{E}-11$ & $3.3 \mathrm{E}-11$ & $2.4 \mathrm{E}-10$ \\
\hline & 50 & Yr: & & 1. $4 \mathrm{E}-10$ & $3.3 \mathrm{E}-11$ & $3.1 \mathrm{E}-11$ & $3.3 E-11$ & $2.4 \mathrm{E}-10$ \\
\hline KR83M & NobleGas & & $3.78 \mathrm{E}-11$ & & & & & \\
\hline & 1 & Yr: & & $\odot . \odot \mathrm{E}-\odot \odot$ & $\odot . \odot \mathrm{E}-\odot \odot$ & $\odot . \odot \mathrm{E}-\odot \odot$ & $\odot . \odot E-\odot \odot$ & $\odot . \odot \mathrm{E}-\odot \odot$ \\
\hline & 50 & Yr: & & $\odot . \odot E-\odot \odot$ & $\odot . \odot E-\odot \odot$ & $\odot . \odot E-\odot \odot$ & $\odot . \odot E-\odot \odot$ & $\odot . \odot \mathrm{E}-\odot \odot$ \\
\hline KR85M & NobleGas & & $2.17 \mathrm{E}-\odot 7$ & & & & & \\
\hline & 1 & Yr: & & $\odot . \odot \mathrm{E}-\odot \odot$ & $\odot . \odot E-\odot \odot$ & $\odot . \odot E-\odot \odot$ & $\odot . \odot \mathrm{E}-\odot \odot$ & $\odot . \odot \mathrm{E}-\odot \odot$ \\
\hline & 50 & Yr: & & $\odot . \odot \mathrm{E}-\odot \odot$ & $\odot . \odot \mathrm{E}-\odot \odot$ & $\odot . \odot \mathrm{E}-\odot \odot$ & $\odot . \odot \mathrm{E}-\odot \odot$ & $\odot . \odot \mathrm{E}-\odot \odot$ \\
\hline KR85 & NobleGas & & $7.57 \mathrm{E}-09$ & & & & & \\
\hline & 1 & Yr: & & $\odot . \odot \mathrm{E}-\odot \odot$ & $\odot . \odot \mathrm{E}-\odot \odot$ & $\odot . \odot \mathrm{E}-\odot \odot$ & $\odot . \odot \mathrm{E}-\odot \odot$ & $\odot . \odot \mathrm{E}-\odot \odot$ \\
\hline & 50 & Yr: & & $\odot . \odot \mathrm{E}-\odot \odot$ & $\odot . \odot \mathrm{E}-\odot \odot$ & $\odot . \odot \mathrm{E}-\odot \odot$ & $\odot . \odot \mathrm{E}-\odot \odot$ & $\odot . \odot \mathrm{E}-\odot \odot$ \\
\hline KR87 & NobleGas & & $1.26 \mathrm{E}-06$ & & & & & \\
\hline & 1 & Yr: & & $\odot . \odot \mathrm{E}-\odot \odot$ & $\odot . \odot \mathrm{E}-\odot \odot$ & $\odot . \odot \mathrm{E}-\odot \odot$ & $\odot . \odot \mathrm{E}-\odot \odot$ & $\odot . \odot \mathrm{E}-\odot \odot$ \\
\hline & 50 & Yr: & & $\odot . \odot \mathrm{E}-\odot \odot$ & $\odot . \odot \mathrm{E}-\odot \odot$ & $\odot . \odot \mathrm{E}-\odot \odot$ & $\odot . \odot \mathrm{E}-\odot \odot$ & $\odot . \odot \mathrm{E}-\odot \odot$ \\
\hline KR88 & NobleGas & & $3.07 E-\odot 6$ & & & & & \\
\hline & 1 & Yr: & & $\odot . \odot \mathrm{E}-\odot \odot$ & $\odot . \odot \mathrm{E}-\odot \odot$ & $\odot . \odot \mathrm{E}-\odot \odot$ & $\odot . \odot \mathrm{E}-\odot \odot$ & $\odot . \odot \mathrm{E}-\odot \odot$ \\
\hline & 50 & Yr: & & $\odot . \odot \mathrm{E}-\odot \odot$ & $\odot . \odot \mathrm{E}-\odot \odot$ & $\odot . \odot \mathrm{E}-\odot \odot$ & $\odot . \odot E-\odot \odot$ & $\odot . \odot \mathrm{E}-\odot \odot$ \\
\hline KR89 & NobleGas & & $3.45 \mathrm{E}-06$ & & & & & \\
\hline & 1 & Yr: & & $\odot . \odot \mathrm{E}-\odot \odot$ & $\odot . \odot \mathrm{E}-\odot \odot$ & $\odot . \odot \mathrm{E}-\odot \odot$ & $\odot . \odot \mathrm{E}-\odot \odot$ & $\odot . \odot \mathrm{E}-\odot \odot$ \\
\hline & 50 & Yr: & & $\odot . \odot \mathrm{E}-\odot \odot$ & $\odot . \odot \mathrm{E}-\odot \odot$ & $\odot . \odot \mathrm{E}-\odot \odot$ & $\odot . \odot \mathrm{E}-\odot \odot$ & $\odot . \odot \mathrm{E}-\odot \odot$ \\
\hline RB86 & Class: D & & $1.56 \mathrm{E}-\odot 7$ & & & & & \\
\hline & 1 & Yr: & & $9.0 \mathrm{E}-\odot 9$ & $2.2 \mathrm{E}-\odot 8$ & $2.2 \mathrm{E}-08$ & 8. . E- $\odot 9$ & 1. $2 \mathrm{E}-08$ \\
\hline & 50 & Yr: & & $9.0 \mathrm{E}-\odot 9$ & $2.2 \mathrm{E}-08$ & $2.2 \mathrm{E}-08$ & $8.9 \mathrm{E}-09$ & 1. $2 \mathrm{E}-08$ \\
\hline
\end{tabular}




\begin{tabular}{|c|c|c|c|c|c|c|c|c|}
\hline \multirow[t]{3}{*}{ RB87 } & Class: D & & 1. $\odot 4 \mathrm{E}-\odot 9$ & & & & & \\
\hline & 1 & Yr: & & $4.5 E-09$ & $1.2 \mathrm{E}-08$ & 1.1E-๑8 & 4. $4 \mathrm{E}-\odot 9$ & 6. ०E-๑9 \\
\hline & 50 & Yr: & & $4.5 \mathrm{E}-\odot 9$ & 1. $2 \mathrm{E}-08$ & 1. $1 \mathrm{E}-\odot 8$ & 4. 4E- $\odot 9$ & 6. $\odot \mathrm{E}-\odot 9$ \\
\hline \multirow[t]{3}{*}{ RB88 } & Class: D & & $1.05 E-\odot 6$ & & & & & \\
\hline & 1 & Yr: & & 1. $5 \mathrm{E}-10$ & $2.1 \mathrm{E}-11$ & 2. $0 \mathrm{E}-11$ & 2. $0 \mathrm{E}-11$ & $2.0 \mathrm{E}-10$ \\
\hline & 50 & Yr: & & 1. $5 \mathrm{E}-10$ & $2.1 \mathrm{E}-11$ & 2. $0 \mathrm{E}-11$ & 2. $0 \mathrm{E}-11$ & 2. $0 \mathrm{E}-10$ \\
\hline \multirow[t]{3}{*}{ RB89 } & Class: D & & $3.19 \mathrm{E}-06$ & & & & & \\
\hline & 1 & Yr: & & $9.5 \mathrm{E}-11$ & $2.0 \mathrm{E}-11$ & 1. $9 \mathrm{E}-11$ & $1.5 \mathrm{E}-11$ & 1. $4 \mathrm{E}-10$ \\
\hline & 50 & Yr: & & $9.5 \mathrm{E}-11$ & 2. OE-11 & $1.9 \mathrm{E}-11$ & 1. $5 \mathrm{E}-11$ & 1. $4 \mathrm{E}-10$ \\
\hline \multirow[t]{3}{*}{ SR85 } & Class: D & & $7.06 \mathrm{E}-07$ & & & & & \\
\hline & 1 & Yr: & & $3.4 \mathrm{E}-\odot 9$ & $1.6 \mathrm{E}-\odot 8$ & $9.4 \mathrm{E}-\odot 9$ & 2. $2 \mathrm{E}-\odot 9$ & 4. 4E- -99 \\
\hline & 50 & Yr: & & $3.4 \mathrm{E}-\odot 9$ & 1. $6 \mathrm{E}-\odot 8$ & $9.4 \mathrm{E}-\odot 9$ & 2. $2 \mathrm{E}-\odot 9$ & 4. 4E- -99 \\
\hline \multirow[t]{3}{*}{ SR89 } & Class: D & & $1.38 \mathrm{E}-08$ & & & & & \\
\hline & 1 & Yr: & & 1. $7 \mathrm{E}-\odot 9$ & 9. ๑E - ๑४ & $8.9 \mathrm{E}-08$ & 1. $6 \mathrm{E}-\odot 9$ & 1. $5 \mathrm{E}-08$ \\
\hline & 50 & Yr: & & 1. $7 \mathrm{E}-09$ & $9.0 \mathrm{E}-08$ & 8. $9 \mathrm{E}-08$ & $1.6 \mathrm{E}-09$ & $1.5 \mathrm{E}-08$ \\
\hline \multirow[t]{3}{*}{ SR90 } & Class: D & & $3.10 \mathrm{E}-\odot 9$ & & & & & \\
\hline & 1 & Yr: & & $6.6 \mathrm{E}-09$ & 1. $3 E-\odot 6$ & $8.4 \mathrm{E}-07$ & $6.6 \mathrm{E}-\odot 9$ & 1. $2 \mathrm{E}-07$ \\
\hline & 50 & Yr: & & $6.6 \mathrm{E}-09$ & 1. $3 E-06$ & $8.4 \mathrm{E}-07$ & $6.6 \mathrm{E}-09$ & 1. $2 \mathrm{E}-07$ \\
\hline \multirow[t]{3}{*}{ SR91 } & Class: D & & 1. $\odot 3 E-\odot 6$ & & & & & \\
\hline & 1 & Yr: & & 3. $0 \mathrm{E}-10$ & 1. $9 \mathrm{E}-09$ & $2.2 \mathrm{E}-\odot 9$ & 1.7E-10 & 1. $5 \mathrm{E}-\odot 9$ \\
\hline & 50 & Yr: & & 3. $0 \mathrm{E}-10$ & 1. $9 \mathrm{E}-09$ & $2.2 \mathrm{E}-\odot 9$ & 1. $7 \mathrm{E}-10$ & $1.5 \mathrm{E}-09$ \\
\hline \multirow[t]{3}{*}{ SR92 } & Class: D & & $2 . \odot 2 E-\odot 6$ & & & & & \\
\hline & 1 & Yr: & & $1.9 \mathrm{E}-10$ & $1.4 \mathrm{E}-09$ & 1. $\odot E-\odot 9$ & $9.8 \mathrm{E}-11$ & $9.0 \mathrm{E}-10$ \\
\hline & 50 & Yr: & & 1. $9 \mathrm{E}-10$ & 1. $4 \mathrm{E}-\odot 9$ & 1. $\odot E-\odot 9$ & $9.8 \mathrm{E}-11$ & $9.0 \mathrm{E}-10$ \\
\hline \multirow[t]{3}{*}{ Y 90} & Class: $\mathrm{Y}$ & & $2.50 \mathrm{E}-\odot 8$ & & & & & \\
\hline & 1 & Yr: & & $3.3 E-08$ & 1. $3 \mathrm{E}-11$ & $3.8 \mathrm{E}-11$ & $8.6 \mathrm{E}-13$ & 1. $3 E-08$ \\
\hline & 50 & Yr: & & 3. 3E- -8 & 1. $3 \mathrm{E}-11$ & $3.8 \mathrm{E}-11$ & $8.6 \mathrm{E}-13$ & 1. $3 E-08$ \\
\hline Y 91M & Class: Y & & $7.47 \mathrm{E}-07$ & & & & & \\
\hline & 1 & Yr: & & $2.1 \mathrm{E}-10$ & $5.3 \mathrm{E}-12$ & $4.6 \mathrm{E}-12$ & $3.3 E-12$ & $7.5 \mathrm{E}-11$ \\
\hline & 50 & Yr: & & $2.1 \mathrm{E}-10$ & $5.3 \mathrm{E}-12$ & 4. $6 \mathrm{E}-12$ & 3. $3 E-12$ & $7.5 \mathrm{E}-11$ \\
\hline Y 91 & Class: $Y$ & & $1.96 \mathrm{E}-\odot 8$ & & & & & \\
\hline & 1 & Yr: & & $2.9 \mathrm{E}-07$ & $3.7 \mathrm{E}-10$ & $9.4 \mathrm{E}-10$ & $2.1 \mathrm{E}-11$ & 4. 3E- - 08 \\
\hline & 50 & Yr: & & $2.9 \mathrm{E}-07$ & $3.7 \mathrm{E}-10$ & $9.4 \mathrm{E}-10$ & $2.1 \mathrm{E}-11$ & $4.3 E-08$ \\
\hline Y 92 & Class: Y & & $4.16 \mathrm{E}-07$ & & & & & \\
\hline & 1 & Yr: & & $3.0 E-\odot 9$ & $9.9 \mathrm{E}-12$ & $8.7 \mathrm{E}-12$ & $5.8 \mathrm{E}-12$ & 1. $9 \mathrm{E}-\odot 9$ \\
\hline & 50 & Yr: & & 3. ०E-०9 & $9.9 \mathrm{E}-12$ & 8. $7 \mathrm{E}-12$ & $5.8 \mathrm{E}-12$ & $1.9 \mathrm{E}-09$ \\
\hline Y 93 & Class: Y & & $1.67 \mathrm{E}-07$ & & & & & \\
\hline & 1 & Yr: & & $6.9 \mathrm{E}-09$ & 1. $0 \mathrm{E}-11$ & $1.1 \mathrm{E}-11$ & $5.0 \mathrm{E}-12$ & $4.6 \mathrm{E}-09$ \\
\hline & 50 & Yr: & & $6.9 \mathrm{E}-09$ & 1. $0 \mathrm{E}-11$ & 1. $1 \mathrm{E}-11$ & $5.0 \mathrm{E}-12$ & $4.6 \mathrm{E}-09$ \\
\hline ZR93 & Class: W & & $\odot . \odot \odot Е \odot \odot$ & & & & & \\
\hline & 1 & Yr: & & 1. $4 \mathrm{E}-08$ & $4.2 E-\odot 8$ & $6.9 \mathrm{E}-09$ & 1. $9 \mathrm{E}-11$ & 3. 3E- -99 \\
\hline & 50 & Yr: & & 1. $4 \mathrm{E}-08$ & $4.2 \mathrm{E}-08$ & $6.9 \mathrm{E}-09$ & $1.9 \mathrm{E}-11$ & $3.3 E-09$ \\
\hline ZR95 & Class: W & & $1.06 \mathrm{E}-06$ & & & & & \\
\hline & 1 & Yr: & & 1.1E- -07 & $4.2 \mathrm{E}-\odot 8$ & 1. $2 \mathrm{E}-\odot 8$ & 2. . $\mathrm{E}-\odot 9$ & 2. $\odot E-\odot 8$ \\
\hline & 50 & Yr: & & 1. $1 \mathrm{E}-07$ & $4.2 \mathrm{E}-\odot 8$ & 1. $2 \mathrm{E}-\odot 8$ & $2.9 \mathrm{E}-\odot 9$ & 2. $\odot \mathrm{E}-\odot 8$ \\
\hline ZR97 & Class: W & & $2.81 \mathrm{E}-07$ & & & & & \\
\hline & 1 & Yr: & & 1. $4 \mathrm{E}-08$ & $4.9 \mathrm{E}-10$ & 8. $0 \mathrm{E}-10$ & $1.9 \mathrm{E}-10$ & $7.8 \mathrm{E}-09$ \\
\hline & 50 & Yr: & & 1. $4 \mathrm{E}-\odot 8$ & $4.9 \mathrm{E}-10$ & 8. $\odot E-10$ & 1. $9 \mathrm{E}-10$ & $7.8 \mathrm{E}-\odot 9$ \\
\hline NB93M & Class: Y & & $9.62 \mathrm{E}-11$ & & & & & \\
\hline & 1 & Yr: & & $5.7 \mathrm{E}-\odot 8$ & 4. $\odot E-10$ & $2.3 E-10$ & $2.7 \mathrm{E}-11$ & 7. $3 \mathrm{E}-\odot 9$ \\
\hline & 50 & Yr: & & $5.7 \mathrm{E}-08$ & 4. $0 \mathrm{E}-10$ & 2. $3 E-10$ & 2. $7 \mathrm{E}-11$ & $7.3 \mathrm{E}-09$ \\
\hline NB94 & Class: Y & & $2.27 \mathrm{E}-06$ & & & & & \\
\hline & 1 & Yr: & & $7.9 \mathrm{E}-\odot 7$ & $3.4 \mathrm{E}-\odot 8$ & $2.8 \mathrm{E}-\odot 8$ & $3.3 E-\odot 8$ & 1. $2 \mathrm{E}-07$ \\
\hline & 50 & Yr: & & $7.9 \mathrm{E}-07$ & $3.4 \mathrm{E}-\odot 8$ & $2.8 \mathrm{E}-\odot 8$ & 3. $3 E-\odot 8$ & 1. $2 \mathrm{E}-07$ \\
\hline NB95M & Class: Y & & $8.64 \mathrm{E}-\odot 8$ & & & & & \\
\hline & 1 & Yr: & & $2.2 E-\odot 8$ & $3.0 E-10$ & $2.4 \mathrm{E}-10$ & 1. $5 \mathrm{E}-10$ & $4.6 \mathrm{E}-\odot 9$ \\
\hline & $5 \odot$ & Yr: & & $2.2 \mathrm{E}-08$ & $3.0 \mathrm{E}-10$ & $2.4 \mathrm{E}-10$ & $1.5 \mathrm{E}-10$ & $4.6 \mathrm{E}-09$ \\
\hline NB95 & Class: Y & & $1.10 \mathrm{E}-06$ & & & & & \\
\hline & 1 & Yr: & & $4.4 \mathrm{E}-08$ & 1. $6 \mathrm{E}-09$ & $1.2 \mathrm{E}-09$ & 1. $3 \mathrm{E}-09$ & 7. $6 \mathrm{E}-\odot 9$ \\
\hline & 50 & Yr: & & $4.4 \mathrm{E}-08$ & $1.6 \mathrm{E}-\odot 9$ & 1. $2 \mathrm{E}-\odot 9$ & 1. $3 E-\odot 9$ & $7.6 \mathrm{E}-09$ \\
\hline NB97M & Class: $Y$ & & $1.04 \mathrm{E}-06$ & & & & & \\
\hline & 1 & Yr: & & $2.4 \mathrm{E}-12$ & $2.3 \mathrm{E}-14$ & $3.1 \mathrm{E}-14$ & $2.8 \mathrm{E}-14$ & $3.5 E-13$ \\
\hline & 50 & Yr: & & $2.4 \mathrm{E}-12$ & $2.3 E-14$ & $3.1 \mathrm{E}-14$ & $2.8 \mathrm{E}-14$ & $3.5 E-13$ \\
\hline NB97 & Class: Y & & $9.43 E-07$ & & & & & \\
\hline & 1 & Yr: & & $6.1 \mathrm{E}-10$ & $9.1 \mathrm{E}-12$ & 7. $4 \mathrm{E}-12$ & $5.8 \mathrm{E}-12$ & $3.9 \mathrm{E}-10$ \\
\hline & 50 & Yr: & & $6.1 \mathrm{E}-10$ & $9.1 \mathrm{E}-12$ & 7. $4 \mathrm{E}-12$ & $5.8 \mathrm{E}-12$ & $3.9 \mathrm{E}-10$ \\
\hline M093 & Class: D & & $5.46 \mathrm{E}-10$ & & & & & \\
\hline & 1 & Yr: & & $8.1 \mathrm{E}-10$ & $4.6 \mathrm{E}-\odot 8$ & 1. $5 \mathrm{E}-\odot 8$ & $5.7 \mathrm{E}-10$ & $3.1 \mathrm{E}-09$ \\
\hline & 50 & Yr: & & $8.1 \mathrm{E}-10$ & $4.6 \mathrm{E}-\odot 8$ & 1. $5 \mathrm{E}-\odot 8$ & $5.7 \mathrm{E}-10$ & $3.1 \mathrm{E}-09$ \\
\hline M099 & Class: D & & $2.20 \mathrm{E}-\odot 7$ & & & & & \\
\hline & 1 & Yr: & & 1. $2 \mathrm{E}-\odot 9$ & $2.8 \mathrm{E}-09$ & $3.3 E-\odot 9$ & 1. ०E-०9 & $2.3 E-\odot 9$ \\
\hline
\end{tabular}




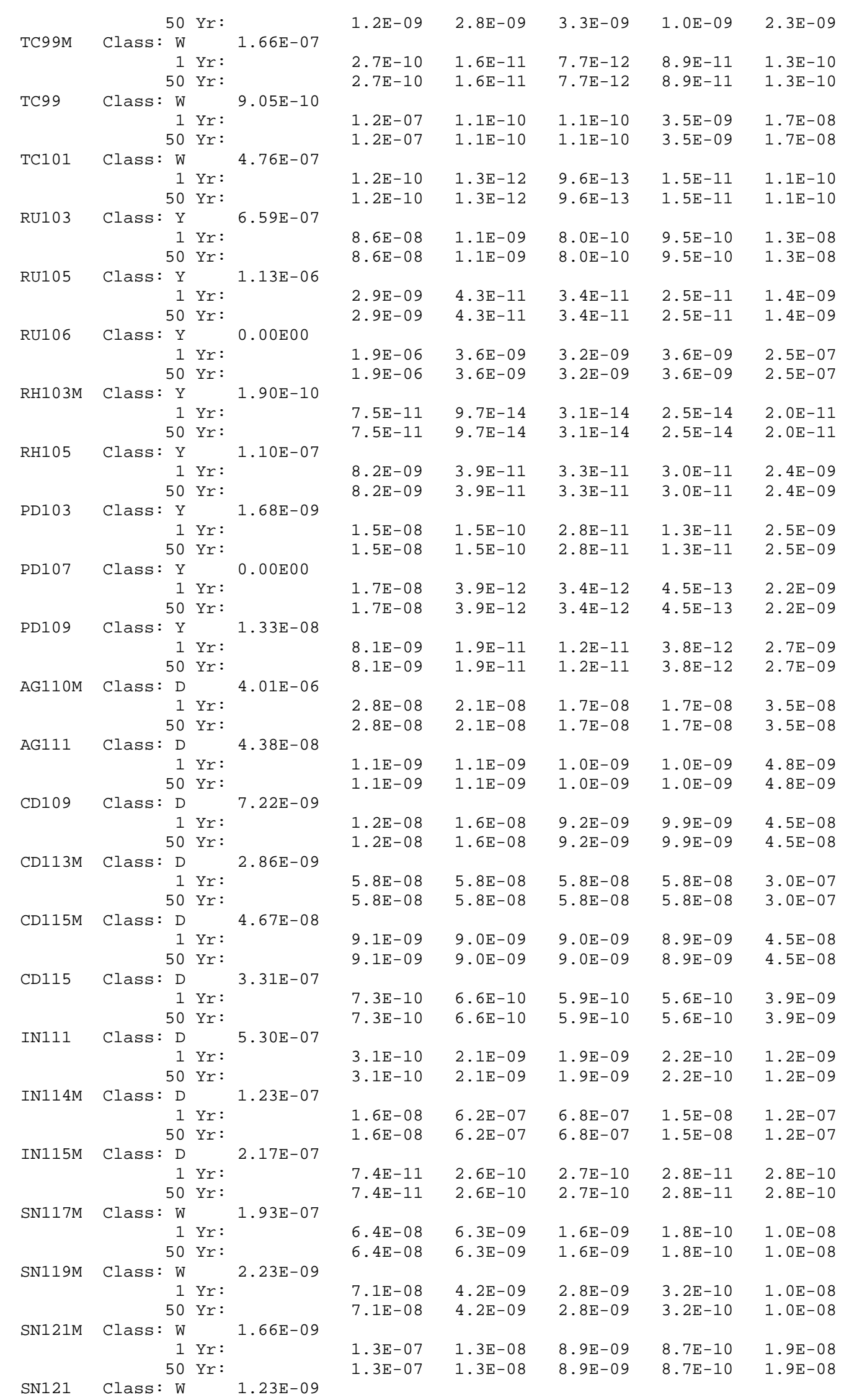




\begin{tabular}{|c|c|c|c|c|c|c|c|c|}
\hline & 1 & Yr: & & $5.3 E-\odot 9$ & $3.0 \mathrm{E}-10$ & $7.7 \mathrm{E}-11$ & $4.6 \mathrm{E}-12$ & 1.5E- 09 \\
\hline & $5 \odot$ & Yr: & & $5.3 E-\odot 9$ & $3.0 E-10$ & $7.7 \mathrm{E}-11$ & $4.6 \mathrm{E}-12$ & 1. $5 \mathrm{E}-09$ \\
\hline \multirow[t]{3}{*}{ SN123 } & Class: W & & 2. 20E-๑8 & & & & & \\
\hline & 1 & Yr: & & $2.5 \mathrm{E}-07$ & 1. $2 \mathrm{E}-\odot 8$ & 1. ०E-०8 & $9.5 \mathrm{E}-10$ & 4. . \\
\hline & 50 & Yr: & & $2.5 \mathrm{E}-07$ & 1. $2 \mathrm{E}-\odot 8$ & 1. $\odot E-\odot 8$ & $9.5 \mathrm{E}-10$ & 4. $\odot E-\odot 8$ \\
\hline \multirow[t]{3}{*}{ SN125 } & Class: W & & 4. 86E- -7 & & & & & \\
\hline & 1 & Yr: & & $8.2 \mathrm{E}-08$ & $3.4 \mathrm{E}-09$ & $5.5 E-09$ & $3.7 \mathrm{E}-10$ & $2.1 \mathrm{E}-08$ \\
\hline & 50 & Yr: & & $8.2 \mathrm{E}-\odot 8$ & 3. 4E- - 99 & $5.5 E-09$ & $3.7 \mathrm{E}-10$ & $2.1 \mathrm{E}-08$ \\
\hline \multirow[t]{3}{*}{ SN126 } & Class: W & & $5.83 E-\odot 8$ & & & & & \\
\hline & 1 & Yr: & & $7.1 \mathrm{E}-07$ & 1.1E- - & 7. .9E-०8 & 2. ๑E-๑8 & 1. $2 \mathrm{E}-07$ \\
\hline & 50 & Yr: & & $7.1 \mathrm{E}-07$ & $1.1 \mathrm{E}-07$ & 7. 9E-๑8 & 2. ๑E-๑8 & 1. $2 \mathrm{E}-07$ \\
\hline \multirow[t]{3}{*}{ SB124 } & Class: W & & $2.72 \mathrm{E}-06$ & & & & & \\
\hline & 1 & Yr: & & 1.7E- -7 & 1. 1E- 08 & $7.5 E-\odot 9$ & $3.7 \mathrm{E}-09$ & $3.1 \mathrm{E}-08$ \\
\hline & 50 & Yr: & & $1.7 \mathrm{E}-07$ & 1.1E- $\odot 8$ & $7.5 \mathrm{E}-\odot 9$ & $3.7 \mathrm{E}-\odot 9$ & $3.1 \mathrm{E}-08$ \\
\hline \multirow[t]{3}{*}{ SB125 } & Class: W & & $5.90 \mathrm{E}-07$ & & & & & \\
\hline & 1 & Yr: & & $1.2 \mathrm{E}-07$ & $3.1 \mathrm{E}-08$ & 8. $6 \mathrm{E}-\odot 9$ & $2.6 \mathrm{E}-09$ & $2.0 \mathrm{E}-\odot 8$ \\
\hline & 50 & Yr: & & 1. $2 \mathrm{E}-\odot 7$ & $3.1 E-\odot 8$ & 8. 6E-०9 & $2.6 \mathrm{E}-09$ & 2. $\odot E-\odot 8$ \\
\hline \multirow[t]{3}{*}{ SB126M } & Class: W & & $2.21 \mathrm{E}-06$ & & & & & \\
\hline & 1 & Yr: & & $1.8 \mathrm{E}-10$ & $6.8 \mathrm{E}-12$ & $5.5 E-12$ & $5.0 \mathrm{E}-12$ & 1.7E-10 \\
\hline & 50 & Yr: & & $1.8 \mathrm{E}-10$ & $6.8 \mathrm{E}-12$ & $5.5 \mathrm{E}-12$ & $5.0 \mathrm{E}-12$ & 1. $7 \mathrm{E}-10$ \\
\hline \multirow[t]{3}{*}{ SB126 } & Class: W & & 4. . $4 \mathrm{E}-06$ & & & & & \\
\hline & 1 & Yr: & & $6.5 \mathrm{E}-08$ & $4.2 \mathrm{E}-\odot 9$ & $3.3 E-09$ & $2.1 \mathrm{E}-09$ & $1.8 \mathrm{E}-08$ \\
\hline & 50 & Yr: & & $6.5 \mathrm{E}-08$ & $4.2 \mathrm{E}-\odot 9$ & $3.3 E-\odot 9$ & $2.1 \mathrm{E}-09$ & $1.8 \mathrm{E}-08$ \\
\hline \multirow[t]{3}{*}{ SB127 } & Class: W & & $9.84 \mathrm{E}-07$ & & & & & \\
\hline & 1 & Yr: & & 4. $0 \mathrm{E}-\odot 8$ & $8.2 \mathrm{E}-10$ & 1. $2 \mathrm{E}-09$ & $2.7 \mathrm{E}-10$ & 1. $0 \mathrm{E}-08$ \\
\hline & 50 & Yr: & & 4. . - - ๑8 & $8.2 \mathrm{E}-10$ & 1. $2 \mathrm{E}-\odot 9$ & $2.7 \mathrm{E}-10$ & 1. . E - ०8 \\
\hline \multirow[t]{3}{*}{ TE123M } & Class: W & & $1.84 \mathrm{E}-07$ & & & & & \\
\hline & 1 & Yr: & & $1.1 \mathrm{E}-07$ & $7.2 \mathrm{E}-08$ & $1.5 E-\odot 8$ & $5.5 E-\odot 9$ & $1.8 \mathrm{E}-08$ \\
\hline & 50 & Yr: & & $1.1 \mathrm{E}-07$ & 7. 2E- - & $1.5 \mathrm{E}-\odot 8$ & $5.5 E-\odot 9$ & 1. $8 \mathrm{E}-\odot 8$ \\
\hline TE125M & Class: W & & $1.06 \mathrm{E}-08$ & & & & & \\
\hline & 1 & Yr: & & $9.4 \mathrm{E}-08$ & $3.7 \mathrm{E}-08$ & $8.1 \mathrm{E}-09$ & 4. 4E- $\odot 9$ & 1. $4 \mathrm{E}-08$ \\
\hline & 50 & Yr: & & $9.4 \mathrm{E}-08$ & $3.7 \mathrm{E}-\odot 8$ & $8.1 \mathrm{E}-09$ & 4. $4 \mathrm{E}-09$ & 1. $4 \mathrm{E}-08$ \\
\hline TE127M & Class: W & & $3.56 \mathrm{E}-\odot 9$ & & & & & \\
\hline & 1 & Yr: & & $2.1 \mathrm{E}-07$ & $6.1 \mathrm{E}-08$ & $3.9 \mathrm{E}-\odot 8$ & 1. 4E- - & $3.5 \mathrm{E}-08$ \\
\hline & 50 & Yr: & & $2.1 \mathrm{E}-\odot 7$ & $6.1 \mathrm{E}-08$ & $3.9 \mathrm{E}-08$ & 1. $4 \mathrm{E}-\odot 8$ & $3.5 \mathrm{E}-08$ \\
\hline TE127 & Class: W & & 1. $05 E-\odot 8$ & & & & & \\
\hline & 1 & Yr: & & $2.7 \mathrm{E}-\odot 9$ & $2.4 \mathrm{E}-11$ & $4.1 \mathrm{E}-11$ & $5.9 \mathrm{E}-11$ & 1.1E- 09 \\
\hline & 50 & Yr: & & $2.7 \mathrm{E}-\odot 9$ & $2.4 \mathrm{E}-11$ & $4.1 \mathrm{E}-11$ & $5.9 \mathrm{E}-11$ & 1.1E- -99 \\
\hline TE129M & Class: W & & 4.92E- -8 & & & & & \\
\hline & 1 & Yr: & & 1. $9 \mathrm{E}-07$ & $2.3 E-\odot 8$ & $2.6 \mathrm{E}-08$ & 1. $9 \mathrm{E}-\odot 8$ & $3.5 \mathrm{E}-08$ \\
\hline & 50 & Yr: & & 1. $9 \mathrm{E}-07$ & 2. 3E-๑8 & $2.6 \mathrm{E}-\odot 8$ & 1. $9 \mathrm{E}-\odot 8$ & $3.5 \mathrm{E}-08$ \\
\hline TE129 & Class: W & & 9. ०5E- ๑8 & & & & & \\
\hline & 1 & Yr: & & $5.5 \mathrm{E}-10$ & $4.3 E-12$ & $4.1 \mathrm{E}-12$ & $4.5 \mathrm{E}-12$ & $3.4 \mathrm{E}-10$ \\
\hline & 50 & Yr: & & $5.5 \mathrm{E}-10$ & 4. $3 E-12$ & $4.1 \mathrm{E}-12$ & $4.5 E-12$ & $3.4 \mathrm{E}-10$ \\
\hline TE131M & Class: W & & $2.07 \mathrm{E}-06$ & & & & & \\
\hline & 1 & Yr: & & 1.7E-๑8 & $1.1 \mathrm{E}-\odot 9$ & $6.8 \mathrm{E}-10$ & $3.7 \mathrm{E}-08$ & 8. . E- $\odot 9$ \\
\hline & 50 & Yr: & & 1. 7E-०8 & 1. 1E- $\odot 9$ & $6.8 \mathrm{E}-10$ & $3.7 E-08$ & 8. $0 \mathrm{E}-09$ \\
\hline TE131 & Class: W & & $6.09 \mathrm{E}-07$ & & & & & \\
\hline & 1 & Yr: & & $3.4 \mathrm{E}-10$ & $4.1 \mathrm{E}-12$ & 3. $0 \mathrm{E}-12$ & $6.8 \mathrm{E}-10$ & $2.7 \mathrm{E}-10$ \\
\hline & 50 & Yr: & & $3.4 \mathrm{E}-10$ & $4.1 \mathrm{E}-12$ & 3. $0 \mathrm{E}-12$ & $6.8 \mathrm{E}-10$ & $2.7 \mathrm{E}-10$ \\
\hline TE132 & Class: W & & $2.95 \mathrm{E}-07$ & & & & & \\
\hline & 1 & Yr: & & 4. ๑E-๑8 & $2.6 \mathrm{E}-\odot 9$ & 1. 4E- $\odot 9$ & 8. 7E- ๑8 & 1. $6 \mathrm{E}-08$ \\
\hline & 50 & Yr: & & 4. . $-\odot 8$ & $2.6 \mathrm{E}-\odot 9$ & 1. $4 \mathrm{E}-09$ & 8. 7E-๑8 & 1. $6 \mathrm{E}-\odot 8$ \\
\hline TE133M & Class: W & & $3.41 \mathrm{E}-06$ & & & & & \\
\hline & 1 & Yr: & & 1. $0 \mathrm{E}-\odot 9$ & $3.5 E-11$ & $2.8 \mathrm{E}-11$ & 3. 3E- - 9 & $8.6 \mathrm{E}-10$ \\
\hline & 50 & Yr: & & 1. ०E-०9 & $3.5 \mathrm{E}-11$ & $2.8 \mathrm{E}-11$ & 3. $3 \mathrm{E}-\odot 9$ & $8.6 \mathrm{E}-10$ \\
\hline TE133 & Class: W & & 1. 37E- 06 & & & & & \\
\hline & 1 & Yr: & & $2.1 \mathrm{E}-10$ & $E-12$ & $E-12$ & $=-10$ & $2.0 \mathrm{E}-10$ \\
\hline & 50 & Yr: & & $2.1 \mathrm{E}-10$ & $3.8 \mathrm{E}-12$ & 3. $0 \mathrm{E}-12$ & $7.3 E-10$ & $2.0 E-10$ \\
\hline TE134 & Class: W & & $1.24 \mathrm{E}-06$ & & & & & \\
\hline & 1 & Yr: & & $7.6 \mathrm{E}-10$ & 3. $2 \mathrm{E}-11$ & $2.5 \mathrm{E}-11$ & $3.8 \mathrm{E}-10$ & $5.5 \mathrm{E}-10$ \\
\hline & 50 & Yr: & & $7.6 \mathrm{E}-10$ & $3.2 \mathrm{E}-11$ & $2.5 \mathrm{E}-11$ & $3.8 \mathrm{E}-10$ & $5.5 \mathrm{E}-10$ \\
\hline I 125 & Class: D & & 1.19E- 08 & & & & & \\
\hline & 1 & Yr: & & 1. $3 \mathrm{E}-10$ & $E-10$ & $5.2 \mathrm{E}-11$ & $E-07$ & $2.1 \mathrm{E}-08$ \\
\hline & 50 & Yr: & & 1. $3 E-10$ & 2. $3 \mathrm{E}-10$ & $5.2 \mathrm{E}-11$ & $4.1 \mathrm{E}-07$ & $2.1 \mathrm{E}-\odot 8$ \\
\hline I 129 & Class: D & & $8.92 \mathrm{E}-09$ & & & & & \\
\hline & 1 & Yr: & & $1.8 \mathrm{E}-10$ & $2.3 \mathrm{E}-10$ & $8.9 \mathrm{E}-11$ & 1. 4E- 06 & $7.2 \mathrm{E}-08$ \\
\hline & 50 & Yr: & & $1.8 \mathrm{E}-10$ & $2.3 E-10$ & 8. $9 \mathrm{E}-11$ & 1. $4 \mathrm{E}-06$ & $7.2 \mathrm{E}-08$ \\
\hline I 130 & Class: D & & $3.05 E-06$ & & & & & \\
\hline & 1 & Yr: & & $3.8 \mathrm{E}-10$ & $2.5 \mathrm{E}-10$ & $2.1 \mathrm{E}-10$ & $1.6 \mathrm{E}-07$ & $8.2 \mathrm{E}-09$ \\
\hline & 50 & Yr: & & $3.8 \mathrm{E}-10$ & $2.5 \mathrm{E}-10$ & $2.1 \mathrm{E}-10$ & $1.6 \mathrm{E}-07$ & $8.2 \mathrm{E}-09$ \\
\hline
\end{tabular}




\begin{tabular}{|c|c|c|c|c|c|c|c|c|}
\hline \multirow[t]{3}{*}{ I 131} & Class: D & & $5.33 E-07$ & & & & & \\
\hline & 1 & Yr: & & $3.9 \mathrm{E}-10$ & $2.6 \mathrm{E}-10$ & $2.2 \mathrm{E}-10$ & 1. $4 \mathrm{E}-06$ & 7. $2 \mathrm{E}-08$ \\
\hline & 50 & Yr: & & $3.9 \mathrm{E}-10$ & $2.6 \mathrm{E}-10$ & $2.2 \mathrm{E}-10$ & 1. $4 \mathrm{E}-06$ & $7.2 \mathrm{E}-\odot 8$ \\
\hline \multirow[t]{3}{*}{ I 132} & Class: D & & $3.31 \mathrm{E}-06$ & & & & & \\
\hline & 1 & Yr: & & 2. $0 \mathrm{E}-10$ & $9.8 \mathrm{E}-11$ & $8.6 \mathrm{E}-11$ & $1.8 \mathrm{E}-08$ & $1.1 \mathrm{E}-09$ \\
\hline & 50 & Yr: & & $2.0 E-10$ & $9.8 \mathrm{E}-11$ & $8.6 \mathrm{E}-11$ & 1. 8E- -8 & $1.1 \mathrm{E}-09$ \\
\hline \multirow[t]{3}{*}{ I 133} & Class: D & & $8.70 \mathrm{E}-07$ & & & & & \\
\hline & 1 & Yr: & & $2.8 \mathrm{E}-10$ & $1.8 \mathrm{E}-10$ & $1.6 \mathrm{E}-10$ & $3.8 \mathrm{E}-07$ & 1. $9 \mathrm{E}-08$ \\
\hline & 50 & Yr: & & $2.8 \mathrm{E}-10$ & $1.8 \mathrm{E}-10$ & $1.6 \mathrm{E}-10$ & $3.8 \mathrm{E}-07$ & $1.9 \mathrm{E}-08$ \\
\hline \multirow[t]{3}{*}{ I 134} & Class: D & & $3.85 E-06$ & & & & & \\
\hline & 1 & Yr: & & $1.4 \mathrm{E}-10$ & $4.7 \mathrm{E}-11$ & $4.1 \mathrm{E}-11$ & 3. 4E- $\odot 9$ & $4.6 \mathrm{E}-10$ \\
\hline & 50 & Yr: & & $1.4 \mathrm{E}-10$ & 4. $7 \mathrm{E}-11$ & $4.1 \mathrm{E}-11$ & $3.4 \mathrm{E}-\odot 9$ & $4.6 \mathrm{E}-10$ \\
\hline \multirow[t]{3}{*}{ I 135} & Class: D & & $2.38 \mathrm{E}-06$ & & & & & \\
\hline & 1 & Yr: & & $2.5 \mathrm{E}-10$ & $1.4 \mathrm{E}-10$ & 1. 3E-10 & 7.7E-०8 & $4.1 \mathrm{E}-09$ \\
\hline & 50 & Yr: & & $2.5 \mathrm{E}-10$ & $1.4 \mathrm{E}-10$ & $1.3 \mathrm{E}-10$ & $7.7 \mathrm{E}-08$ & $4.1 \mathrm{E}-09$ \\
\hline \multirow[t]{3}{*}{ XE131M } & NobleGas & & 1.10E- $९ 8$ & & & & & \\
\hline & 1 & Yr: & & $\odot . \odot \mathrm{E}-\odot \odot$ & $\odot . \odot \mathrm{E}-\odot \odot$ & $\odot . \odot \mathrm{E}-\odot \odot$ & $\odot . \odot \mathrm{E}-\odot \odot$ & $\odot . \odot \mathrm{E}-\odot \odot$ \\
\hline & 50 & Yr: & & $\odot . \odot \mathrm{E}-\odot \odot$ & $\odot . \odot \mathrm{E}-\odot \odot$ & $\odot . \odot \mathrm{E}-\odot \odot$ & $\odot . \odot \mathrm{E}-\odot \odot$ & $\odot . \odot \mathrm{E}-\odot \odot$ \\
\hline \multirow[t]{3}{*}{ XE133M } & NobleGas & & 4. . $7 \mathrm{E}-08$ & & & & & \\
\hline & 1 & Yr: & & $\odot . \odot \mathrm{E}-\odot \odot$ & $\odot . \odot \mathrm{E}-\odot \odot$ & $\odot . \odot \mathrm{E}-\odot \odot$ & $\odot . \odot \mathrm{E}-\odot \odot$ & $\odot . \odot \mathrm{E}-\odot \odot$ \\
\hline & 50 & Yr: & & $\odot . \odot \mathrm{E}-\odot \odot$ & $\odot . \odot \mathrm{E}-\odot \odot$ & $\odot . \odot \mathrm{E}-\odot \odot$ & $\odot . \odot \mathrm{E}-\odot \odot$ & $\odot . \odot \mathrm{E}-\odot \odot$ \\
\hline \multirow[t]{3}{*}{ XE133 } & NobleGas & & 4. 23E- -8 & & & & & \\
\hline & 1 & Yr: & & $\odot . \odot \mathrm{E}-\odot \odot$ & $\odot . \odot \mathrm{E}-\odot \odot$ & $\odot . \odot \mathrm{E}-\odot \odot$ & $\odot . \odot \mathrm{E}-\odot \odot$ & $\odot . \odot \mathrm{E}-\odot \odot$ \\
\hline & 50 & Yr: & & $\odot . \odot \mathrm{E}-\odot \odot$ & $\odot . \odot \mathrm{E}-\odot \odot$ & $\odot . \odot \mathrm{E}-\odot \odot$ & $\odot . \odot \mathrm{E}-\odot \odot$ & $\odot . \odot \mathrm{E}-\odot \odot$ \\
\hline \multirow[t]{3}{*}{ XE135M } & NobleGas & & $5.99 \mathrm{E}-\odot 7$ & & & & & \\
\hline & 1 & Yr: & & $\odot . \odot \mathrm{E}-\odot \odot$ & $\odot . \odot \mathrm{E}-\odot \odot$ & $\odot . \odot \mathrm{E}-\odot \odot$ & $\odot . \odot \mathrm{E}-\odot \odot$ & $\odot . \odot \mathrm{E}-\odot \odot$ \\
\hline & 50 & Yr: & & $\odot . \odot \mathrm{E}-\odot \odot$ & $\odot . \odot \mathrm{E}-\odot \odot$ & $\odot . \odot \mathrm{E}-\odot \odot$ & $\odot . \odot \mathrm{E}-\odot \odot$ & $\odot . \odot \mathrm{E}-\odot \odot$ \\
\hline XE135 & NobleGas & & $3.50 \mathrm{E}-07$ & & & & & \\
\hline & 1 & Yr: & & $\odot . \odot \mathrm{E}-\odot \odot$ & $\odot . \odot \mathrm{E}-\odot \odot$ & $\odot . \odot \mathrm{E}-\odot \odot$ & $\odot . \odot \mathrm{E}-\odot \odot$ & $\odot . \odot \mathrm{E}-\odot \odot$ \\
\hline & 50 & Yr: & & $\odot . \odot \mathrm{E}-\odot \odot$ & $\odot . \odot \mathrm{E}-\odot \odot$ & $\odot . \odot \mathrm{E}-\odot \odot$ & $\odot . \odot \mathrm{E}-\odot \odot$ & $\odot . \odot \mathrm{E}-\odot \odot$ \\
\hline XE137 & NobleGas & & $4.34 \mathrm{E}-07$ & & & & & \\
\hline & 1 & Yr: & & $\odot . \odot \mathrm{E}-\odot \odot$ & $\odot . \odot \mathrm{E}-\odot \odot$ & $\odot . \odot \mathrm{E}-\odot \odot$ & $\odot . \odot \mathrm{E}-\odot \odot$ & $\odot . \odot \mathrm{E}-\odot \odot$ \\
\hline & 50 & Yr: & & $\odot . \odot \mathrm{E}-\odot \odot$ & $\odot . \odot \mathrm{E}-\odot \odot$ & $\odot . \odot \mathrm{E}-\odot \odot$ & $\odot . \odot \mathrm{E}-\odot \odot$ & $\odot . \odot \mathrm{E}-\odot \odot$ \\
\hline XE138 & NobleGas & & $1.73 E-06$ & & & & & \\
\hline & 1 & Yr: & & $\odot . \odot \mathrm{E}-\odot \odot$ & $\odot . \odot \mathrm{E}-\odot \odot$ & $\odot . \odot \mathrm{E}-\odot \odot$ & $\odot . \odot E-\odot \odot$ & $\odot . \odot \mathrm{E}-\odot \odot$ \\
\hline & 50 & Yr: & & $\odot . \odot \mathrm{E}-\odot \odot$ & $\odot . \odot \mathrm{E}-\odot \odot$ & $\odot . \odot \mathrm{E}-\odot \odot$ & $\odot . \odot \mathrm{E}-\odot \odot$ & $\odot . \odot E-\odot \odot$ \\
\hline CS134M & Class: D & & 2.51E-๑8 & & & & & \\
\hline & 1 & Yr: & & $8.9 \mathrm{E}-11$ & $2.2 \mathrm{E}-11$ & $1.8 \mathrm{E}-11$ & 1. $9 \mathrm{E}-11$ & 1. $3 \mathrm{E}-10$ \\
\hline & 50 & Yr: & & $8.9 \mathrm{E}-11$ & 2. $2 \mathrm{E}-11$ & $1.8 \mathrm{E}-11$ & 1. $9 \mathrm{E}-11$ & 1. $3 \mathrm{E}-10$ \\
\hline CS134 & Class: D & & $2.23 E-06$ & & & & & \\
\hline & 1 & Yr: & & 9. ०E-๑9 & $9.5 \mathrm{E}-\odot 9$ & 8. 3E-०9 & 1. $\odot E-\odot 8$ & $1.1 \mathrm{E}-08$ \\
\hline & 50 & Yr: & & $9 . \odot E-\odot 9$ & $9.5 \mathrm{E}-09$ & 8. 3E-०9 & 1. $\odot \mathrm{E}-\odot 8$ & 1. $1 \mathrm{E}-08$ \\
\hline CS135 & Class: D & & $3.00 \mathrm{E}-10$ & & & & & \\
\hline & 1 & Yr: & & 1. $4 \mathrm{E}-09$ & 1. $3 \mathrm{E}-09$ & 1. 3E- $\odot 9$ & 1. 3E- $\odot 9$ & 1. $7 \mathrm{E}-09$ \\
\hline & 50 & Yr: & & 1. $4 \mathrm{E}-\odot 9$ & 1. $3 \mathrm{E}-\odot 9$ & 1. 3E-०9 & 1. $3 \mathrm{E}-\odot 9$ & 1. $7 \mathrm{E}-\odot 9$ \\
\hline CS136 & Class: D & & $3.13 E-06$ & & & & & \\
\hline & 1 & Yr: & & $5.2 \mathrm{E}-09$ & $5.7 \mathrm{E}-\odot 9$ & 4. . & $5.8 \mathrm{E}-\odot 9$ & 7. 3E- -99 \\
\hline & 50 & Yr: & & $5.2 \mathrm{E}-09$ & $5.7 \mathrm{E}-\odot 9$ & 4. . E- - & $5.8 \mathrm{E}-09$ & 7. 3E- $\odot 9$ \\
\hline CS137 & Class: D & & 2. $93 \mathrm{E}-\odot 9$ & & & & & \\
\hline & 1 & Yr: & & $7.1 \mathrm{E}-09$ & $7.3 E-09$ & $6.8 \mathrm{E}-09$ & 7. 4E-०9 & 8. 8E-०9 \\
\hline & 50 & Yr: & & $7.1 \mathrm{E}-09$ & 7. 3E- $\odot 9$ & $6.8 \mathrm{E}-\odot 9$ & 7. 4E- $\odot 9$ & $8.8 \mathrm{E}-09$ \\
\hline CS138 & Class: D & & $3.63 E-06$ & & & & & \\
\hline & 1 & Yr: & & $1.5 \mathrm{E}-10$ & $3.8 \mathrm{E}-11$ & $3.5 \mathrm{E}-11$ & $3.7 \mathrm{E}-11$ & $2.6 \mathrm{E}-10$ \\
\hline & $5 \odot$ & Yr: & & 1. $5 \mathrm{E}-10$ & $3.8 \mathrm{E}-11$ & $3.5 E-11$ & 3. $7 \mathrm{E}-11$ & $2.6 \mathrm{E}-10$ \\
\hline BA139 & Class: D & & 8. ๑4E-๑8 & & & & & \\
\hline & 1 & Yr: & & 1. $2 \mathrm{E}-10$ & 1.7E-10 & $2.1 \mathrm{E}-10$ & 1. $3 \mathrm{E}-11$ & $3.3 \mathrm{E}-10$ \\
\hline & 50 & Yr: & & 1. $2 \mathrm{E}-10$ & 1. $7 \mathrm{E}-10$ & $2.1 \mathrm{E}-10$ & 1. $3 E-11$ & 3. $3 E-10$ \\
\hline BA140 & Class: D & & $2.55 E-07$ & & & & & \\
\hline & 1 & Yr: & & $3.3 E-09$ & $5.8 \mathrm{E}-08$ & $E-\odot 8$ & LE - $\odot 9$ & 1. $3 \mathrm{E}-08$ \\
\hline & 50 & Yr: & & 3. 3E- $\odot 9$ & $5.8 \mathrm{E}-\odot 8$ & 4. 3E- - & $2.1 \mathrm{E}-\odot 9$ & 1. $3 E-08$ \\
\hline BA141 & Class: D & & $1.24 \mathrm{E}-06$ & & & & & \\
\hline & 1 & Yr: & & $9.4 \mathrm{E}-11$ & $9.5 \mathrm{E}-11$ & $9.8 \mathrm{E}-11$ & $8.2 \mathrm{E}-12$ & $1.9 \mathrm{E}-10$ \\
\hline & 50 & Yr: & & $9.4 \mathrm{E}-11$ & $9.5 \mathrm{E}-11$ & $9.8 \mathrm{E}-11$ & $8 \cdot 2 \mathrm{E}-12$ & 1. $9 \mathrm{E}-10$ \\
\hline BA142 & Class: D & & $1.53 \mathrm{E}-06$ & & & & & \\
\hline & 1 & Yr: & & $6.9 \mathrm{E}-11$ & $3.8 \mathrm{E}-11$ & $4.2 \mathrm{E}-11$ & $5 E-12$ & 1. $3 E-10$ \\
\hline & 50 & Yr: & & $6.9 \mathrm{E}-11$ & $3.8 \mathrm{E}-11$ & $4.2 \mathrm{E}-11$ & $7.5 \mathrm{E}-12$ & 1. $3 \mathrm{E}-10$ \\
\hline LA140 & Class: D & & $3.50 \mathrm{E}-06$ & & & & & \\
\hline & 1 & Yr: & & 1. 3E- $\odot 9$ & 1. $9 \mathrm{E}-\odot 9$ & 3. 2E- -9 & $6.8 \mathrm{E}-10$ & $5.8 \mathrm{E}-09$ \\
\hline & 50 & Yr: & & 1. $3 \mathrm{E}-09$ & 1. $9 \mathrm{E}-\odot 9$ & $3.2 \mathrm{E}-09$ & $6.8 \mathrm{E}-10$ & $5.8 \mathrm{E}-09$ \\
\hline LA141 & Class: D & & 9. ๑८E-๑৪ & & & & & \\
\hline & 1 & Yr: & & $2.1 \mathrm{E}-10$ & $4.7 \mathrm{E}-10$ & $3.4 \mathrm{E}-10$ & $9.5 \mathrm{E}-11$ & $8.6 \mathrm{E}-10$ \\
\hline
\end{tabular}




\begin{tabular}{|c|c|c|c|c|c|c|c|c|}
\hline \multirow{3}{*}{ LA142 } & 50 & Yr: & & $2.1 \mathrm{E}-10$ & 4. $7 \mathrm{E}-10$ & $3.4 \mathrm{E}-10$ & $9.5 \mathrm{E}-11$ & $8.6 \mathrm{E}-10$ \\
\hline & Class: D & & $4.32 \mathrm{E}-06$ & & & & & \\
\hline & 1 & Yr: & & $2.0 \mathrm{E}-1 \odot$ & $9.3 \mathrm{E}-11$ & 1. $\odot E-1 \odot$ & 7. 3E-11 & $5.3 \mathrm{E}-10$ \\
\hline & 50 & Yr: & & $2.0 \mathrm{E}-10$ & $9.3 \mathrm{E}-11$ & 1. $\odot \mathrm{E}-10$ & $7.3 \mathrm{E}-11$ & $5.3 \mathrm{E}-10$ \\
\hline \multirow[t]{3}{*}{ CE141 } & Class: Y & & $9.81 \mathrm{E}-08$ & & & & & \\
\hline & 1 & Yr: & & $1.1 \mathrm{E}-07$ & $2.2 \mathrm{E}-\odot 9$ & $5.4 \mathrm{E}-10$ & 1. $5 \mathrm{E}-10$ & 1. $6 \mathrm{E}-\odot 8$ \\
\hline & 50 & Yr: & & 1.1E- -7 & 2. $2 \mathrm{E}-09$ & $5.4 \mathrm{E}-10$ & $1.5 \mathrm{E}-10$ & $1.6 \mathrm{E}-08$ \\
\hline \multirow[t]{3}{*}{ CE143 } & Class: Y & & $3.82 \mathrm{E}-07$ & & & & & \\
\hline & 1 & Yr: & & 1.9E-०8 & $1.5 \mathrm{E}-10$ & 1. $2 \mathrm{E}-10$ & 4. $4 \mathrm{E}-11$ & $5.9 \mathrm{E}-09$ \\
\hline & 50 & Yr: & & 1. $9 \mathrm{E}-\odot 8$ & $1.5 \mathrm{E}-10$ & 1. $2 \mathrm{E}-10$ & $4.4 \mathrm{E}-11$ & $5.9 \mathrm{E}-09$ \\
\hline \multirow{3}{*}{ CE144 } & Class: Y & & $2.41 \mathrm{E}-\odot 8$ & & & & & \\
\hline & 1 & Yr: & & $1.6 \mathrm{E}-06$ & $2.4 \mathrm{E}-08$ & $2.8 \mathrm{E}-08$ & $9.8 \mathrm{E}-10$ & $2.1 \mathrm{E}-07$ \\
\hline & 50 & Yr: & & $1.6 \mathrm{E}-06$ & $2.4 \mathrm{E}-\odot 8$ & $2.8 \mathrm{E}-08$ & $9.8 \mathrm{E}-10$ & $2.1 \mathrm{E}-07$ \\
\hline \multirow[t]{3}{*}{ PR143 } & Class: $\mathrm{Y}$ & & $6.12 \mathrm{E}-09$ & & & & & \\
\hline & 1 & Yr: & & 7. ०E-๑8 & $3.8 \mathrm{E}-11$ & $1.1 \mathrm{E}-10$ & $4.9 \mathrm{E}-13$ & 1. $2 \mathrm{E}-08$ \\
\hline & 50 & Yr: & & $7.0 \mathrm{E}-08$ & $3.8 \mathrm{E}-11$ & $1.1 \mathrm{E}-10$ & 4. $9 \mathrm{E}-13$ & 1. $2 \mathrm{E}-08$ \\
\hline \multirow[t]{3}{*}{ PR144M } & Class: Y & & $6.97 \mathrm{E}-\odot 9$ & & & & & \\
\hline & 1 & Yr: & & 4. $\odot \mathrm{E}-11$ & 1. $5 \mathrm{E}-14$ & $1.8 \mathrm{E}-14$ & $8.7 \mathrm{E}-15$ & $5.1 \mathrm{E}-12$ \\
\hline & 50 & Yr: & & 4. $0 \mathrm{E}-11$ & $1.5 \mathrm{E}-14$ & $1.8 \mathrm{E}-14$ & $8.7 \mathrm{E}-15$ & $5.1 \mathrm{E}-12$ \\
\hline \multirow[t]{3}{*}{ PR144 } & Class: Y & & $8.36 \mathrm{E}-08$ & & & & & \\
\hline & 1 & Yr: & & $2.3 \mathrm{E}-10$ & 1. $\odot \mathrm{E}-13$ & $8.2 \mathrm{E}-14$ & 8. $0 \mathrm{E}-14$ & $1.9 \mathrm{E}-10$ \\
\hline & $5 \odot$ & Yr: & & $2.3 \mathrm{E}-10$ & 1. $0 \mathrm{E}-13$ & $8.2 \mathrm{E}-14$ & 8. $0 \mathrm{E}-14$ & $1.9 \mathrm{E}-10$ \\
\hline \multirow[t]{3}{*}{ ND147 } & Class: Y & & $1.81 \mathrm{E}-07$ & & & & & \\
\hline & 1 & Yr: & & $6.7 \mathrm{E}-\odot 8$ & $6.4 \mathrm{E}-10$ & $2.7 \mathrm{E}-10$ & $1.1 \mathrm{E}-10$ & 1. $2 \mathrm{E}-08$ \\
\hline & 50 & Yr: & & $6.7 \mathrm{E}-08$ & $6.4 \mathrm{E}-10$ & $2.7 \mathrm{E}-10$ & $1.1 \mathrm{E}-10$ & $1.2 \mathrm{E}-08$ \\
\hline \multirow[t]{3}{*}{ PM147 } & Class: Y & & $2.73 \mathrm{E}-10$ & & & & & \\
\hline & 1 & Yr: & & 1. $4 \mathrm{E}-07$ & $1.8 \mathrm{E}-08$ & $2.6 E-\odot 9$ & $2.9 \mathrm{E}-13$ & 1. $9 \mathrm{E}-\odot 8$ \\
\hline & 50 & Yr: & & $1.4 \mathrm{E}-07$ & $1.8 \mathrm{E}-08$ & $2.6 \mathrm{E}-09$ & $2.9 \mathrm{E}-13$ & $1.9 \mathrm{E}-08$ \\
\hline PM148M & Class: $\mathrm{Y}$ & & $2.84 \mathrm{E}-\odot 6$ & & & & & \\
\hline & 1 & Yr: & & $1.5 \mathrm{E}-07$ & $5.5 \mathrm{E}-\odot 9$ & $3.7 \mathrm{E}-09$ & $3.9 \mathrm{E}-\odot 9$ & $2.5 \mathrm{E}-08$ \\
\hline & 50 & Yr: & & $1.5 \mathrm{E}-07$ & $5.5 E-09$ & $3.7 \mathrm{E}-09$ & $3.9 \mathrm{E}-09$ & $2.5 \mathrm{E}-08$ \\
\hline PM148 & Class: Y & & $8.70 \mathrm{E}-\odot 7$ & & & & & \\
\hline & 1 & Yr: & & $5.4 \mathrm{E}-08$ & $3.5 \mathrm{E}-10$ & $4.0 E-10$ & $2.2 \mathrm{E}-10$ & 1. $5 \mathrm{E}-\odot 8$ \\
\hline & 50 & Yr: & & $5.4 \mathrm{E}-08$ & $3.5 \mathrm{E}-10$ & $4.0 E-10$ & $2.2 \mathrm{E}-10$ & 1. $5 \mathrm{E}-08$ \\
\hline PM149 & Class: Y & & $2.24 \mathrm{E}-\odot 8$ & & & & & \\
\hline & 1 & Yr: & & $1.7 \mathrm{E}-\odot 8$ & $1.5 \mathrm{E}-11$ & $3.4 \mathrm{E}-11$ & $2.6 \mathrm{E}-12$ & $5.3 E-09$ \\
\hline & 50 & Yr: & & 1. 7E-०8 & $1.5 \mathrm{E}-11$ & $3.4 \mathrm{E}-11$ & $2.6 \mathrm{E}-12$ & $5.3 E-09$ \\
\hline PM151 & Class: Y & & $4.42 \mathrm{E}-07$ & & & & & \\
\hline & 1 & Yr: & & $9.9 \mathrm{E}-\odot 9$ & $1.1 \mathrm{E}-10$ & $7.1 \mathrm{E}-11$ & 4. $2 \mathrm{E}-11$ & $3.5 E-\odot 9$ \\
\hline & 50 & Yr: & & $9.9 \mathrm{E}-\odot 9$ & $1.1 \mathrm{E}-10$ & $7.1 \mathrm{E}-11$ & $4.2 \mathrm{E}-11$ & $3.5 E-\odot 9$ \\
\hline SM147 & Class: W & & 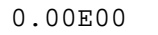 & & & & & \\
\hline & 1 & Yr: & & 1. $4 \mathrm{E}-06$ & $2.6 \mathrm{E}-05$ & $3.3 E-\odot 6$ & 1. $3 \mathrm{E}-10$ & $2.4 \mathrm{E}-05$ \\
\hline & 50 & Yr: & & 1. $4 \mathrm{E}-06$ & $2.6 E-05$ & $3.3 E-06$ & 1. $3 E-10$ & $2.4 \mathrm{E}-05$ \\
\hline SM151 & Class: W & & $7.79 \mathrm{E}-13$ & & & & & \\
\hline & 1 & Yr: & & 1. $5 \mathrm{E}-\odot 8$ & $2.2 \mathrm{E}-07$ & $2.8 \mathrm{E}-\odot 8$ & 1. $2 \mathrm{E}-12$ & 1. $1 \mathrm{E}-08$ \\
\hline & 50 & Yr: & & $1.5 \mathrm{E}-08$ & $2.2 \mathrm{E}-07$ & $2.8 \mathrm{E}-08$ & 1. $2 \mathrm{E}-12$ & $1.1 \mathrm{E}-08$ \\
\hline SM153 & Class: W & & $6.46 \mathrm{E}-\odot 8$ & & & & & \\
\hline & 1 & Yr: & & $1.5 \mathrm{E}-\odot 8$ & $4.3 E-10$ & $3.7 \mathrm{E}-10$ & 2. $\odot E-11$ & $4.2 E-09$ \\
\hline & 50 & Yr: & & $1.5 \mathrm{E}-\odot 8$ & $4.3 E-10$ & $3.7 \mathrm{E}-10$ & 2. $0 \mathrm{E}-11$ & $4.2 \mathrm{E}-09$ \\
\hline EU152 & Class: W & & $1.67 \mathrm{E}-06$ & & & & & \\
\hline & 1 & Yr: & & $2.0 \mathrm{E}-07$ & $4.5 E-07$ & 2. $\odot E-\odot 7$ & 1. $9 \mathrm{E}-\odot 8$ & 1. $1 \mathrm{E}-07$ \\
\hline & 50 & Yr: & & $2 . \odot E-\odot 7$ & $4.5 E-07$ & 2. $\odot E-\odot 7$ & 1. $9 \mathrm{E}-08$ & $1.1 \mathrm{E}-07$ \\
\hline EU154 & Class: W & & $1.82 \mathrm{E}-06$ & & & & & \\
\hline & 1 & Yr: & & $3.5 \mathrm{E}-07$ & $1.0 \mathrm{E}-06$ & $3.2 \mathrm{E}-07$ & 1. $9 \mathrm{E}-\odot 8$ & 1. $6 \mathrm{E}-07$ \\
\hline & 50 & Yr: & & $3.5 \mathrm{E}-07$ & 1. $\odot E-\odot 6$ & $3.2 \mathrm{E}-07$ & 1. $9 \mathrm{E}-08$ & 1. $6 \mathrm{E}-07$ \\
\hline EU155 & Class: W & & $6.78 \mathrm{E}-\odot 8$ & & & & & \\
\hline & 1 & Yr: & & $7.4 \mathrm{E}-08$ & $3.3 E-07$ & $5.0 \mathrm{E}-08$ & $8.7 \mathrm{E}-10$ & $2.6 \mathrm{E}-\odot 8$ \\
\hline & 50 & Yr: & & $7.4 \mathrm{E}-08$ & 3. $3 E-07$ & $5 . \odot E-\odot 8$ & $8.7 \mathrm{E}-10$ & $2.6 \mathrm{E}-08$ \\
\hline EU156 & Class: W & & $2.02 \mathrm{E}-\odot 6$ & & & & & \\
\hline & 1 & Yr: & & $8.8 \mathrm{E}-\odot 8$ & $7.5 E-\odot 9$ & $5.9 \mathrm{E}-\odot 9$ & $1.1 \mathrm{E}-09$ & 1. $9 \mathrm{E}-\odot 8$ \\
\hline & 50 & Yr: & & $8.8 \mathrm{E}-08$ & $7.5 \mathrm{E}-\odot 9$ & $5.9 \mathrm{E}-\odot 9$ & 1.1E- $\odot 9$ & $1.9 \mathrm{E}-08$ \\
\hline GD153 & Class: D & & $9.84 \mathrm{E}-\odot 8$ & & & & & \\
\hline & 1 & Yr: & & $3.9 \mathrm{E}-09$ & $2.5 E-07$ & $4.8 \mathrm{E}-\odot 8$ & $9.7 \mathrm{E}-10$ & 1. $5 \mathrm{E}-\odot 8$ \\
\hline & 50 & Yr: & & $3.9 E-09$ & $2.5 \mathrm{E}-07$ & $4.8 E-08$ & $9.7 \mathrm{E}-10$ & $1.5 \mathrm{E}-08$ \\
\hline TB160 & Class: W & & $1.64 \mathrm{E}-06$ & & & & & \\
\hline & 1 & Yr: & & $1.7 \mathrm{E}-07$ & $6.7 \mathrm{E}-08$ & $2.4 \mathrm{E}-\odot 8$ & $2.8 \mathrm{E}-\odot 9$ & $3.2 \mathrm{E}-\odot 8$ \\
\hline & 50 & Yr: & & $1.7 \mathrm{E}-07$ & $6.7 \mathrm{E}-08$ & $2.4 \mathrm{E}-08$ & $2.8 \mathrm{E}-09$ & $3.2 \mathrm{E}-08$ \\
\hline H0166M & Class: W & & $2.48 \mathrm{E}-06$ & & & & & \\
\hline & 1 & Yr: & & $3.2 \mathrm{E}-07$ & 1. $4 \mathrm{E}-06$ & $3.1 \mathrm{E}-07$ & 4.1E- $\odot 8$ & $2.6 \mathrm{E}-07$ \\
\hline & 50 & Yr: & & $3.2 \mathrm{E}-07$ & 1. $4 \mathrm{E}-\odot 6$ & $3.1 \mathrm{E}-07$ & 4. 1E- $\odot 8$ & $2.6 \mathrm{E}-07$ \\
\hline W 181 & Class: D & & $3.69 \mathrm{E}-08$ & & & & & \\
\hline
\end{tabular}




\begin{tabular}{|c|c|c|c|c|c|c|c|c|}
\hline & 1 & Yr: & & $4.2 \mathrm{E}-11$ & $3.7 \mathrm{E}-10$ & $1.6 \mathrm{E}-10$ & $2.1 \mathrm{E}-11$ & $2.6 \mathrm{E}-10$ \\
\hline & 50 & Yr: & & $4.2 \mathrm{E}-11$ & $3.7 \mathrm{E}-10$ & $1.6 \mathrm{E}-10$ & $2.1 \mathrm{E}-11$ & $2.6 \mathrm{E}-10$ \\
\hline \multirow[t]{3}{*}{ W 187} & Class: D & & $6.72 \mathrm{E}-\odot 7$ & & & & & \\
\hline & 1 & Yr: & & $2.2 \mathrm{E}-10$ & $4.3 \mathrm{E}-10$ & $2.5 \mathrm{E}-10$ & $1.1 \mathrm{E}-10$ & $2.1 \mathrm{E}-09$ \\
\hline & 50 & Yr: & & $2.2 \mathrm{E}-10$ & 4. $3 E-10$ & $2.5 \mathrm{E}-10$ & $1.1 \mathrm{E}-10$ & $2.1 \mathrm{E}-\odot 9$ \\
\hline \multirow[t]{3}{*}{ W 185} & Class: D & & $1.57 \mathrm{E}-\odot 9$ & & & & & \\
\hline & 1 & Yr: & & $9.6 \mathrm{E}-11$ & $8.9 \mathrm{E}-10$ & $7.9 \mathrm{E}-10$ & $3.5 \mathrm{E}-11$ & 1. $4 \mathrm{E}-09$ \\
\hline & 50 & Yr: & & $9.6 \mathrm{E}-11$ & $8.9 \mathrm{E}-10$ & $7.9 \mathrm{E}-10$ & $3.5 \mathrm{E}-11$ & 1. $4 \mathrm{E}-09$ \\
\hline \multirow[t]{3}{*}{ RE187 } & Class: W & & $\odot . \odot \odot Е \odot \odot ~$ & & & & & \\
\hline & 1 & Yr: & & $2.6 \mathrm{E}-10$ & $2.1 \mathrm{E}-12$ & $2.1 \mathrm{E}-12$ & $6.4 \mathrm{E}-11$ & $5.7 \mathrm{E}-11$ \\
\hline & 50 & Yr: & & $2.6 \mathrm{E}-10$ & $2.1 \mathrm{E}-12$ & $2.1 \mathrm{E}-12$ & $6.4 \mathrm{E}-11$ & $5.7 \mathrm{E}-11$ \\
\hline \multirow[t]{3}{*}{ IR192 } & Class: Y & & $1.14 \mathrm{E}-06$ & & & & & \\
\hline & 1 & Yr: & & 1. $9 \mathrm{E}-\odot 7$ & $3.1 \mathrm{E}-09$ & $2.1 \mathrm{E}-\odot 9$ & 2. 7E-๑9 & $2.8 \mathrm{E}-\odot 8$ \\
\hline & 50 & Yr: & & $1.9 \mathrm{E}-07$ & $3.1 \mathrm{E}-09$ & $2.1 \mathrm{E}-09$ & $2.7 \mathrm{E}-\odot 9$ & 2. $8 \mathrm{E}-\odot 8$ \\
\hline \multirow{3}{*}{ HG2O3 } & Class: D & & $3.28 E-07$ & & & & & \\
\hline & 1 & Yr: & & $2.6 \mathrm{E}-09$ & $2.9 \mathrm{E}-09$ & $2.5 E-\odot 9$ & $2.7 \mathrm{E}-\odot 9$ & $4.1 \mathrm{E}-09$ \\
\hline & 50 & Yr: & & $2.6 \mathrm{E}-\odot 9$ & $2.9 \mathrm{E}-\odot 9$ & $2.5 E-\odot 9$ & $2.7 \mathrm{E}-\odot 9$ & $4.1 \mathrm{E}-09$ \\
\hline \multirow[t]{3}{*}{ RN222 } & NobleGas & & $5.61 \mathrm{E}-10$ & & & & & \\
\hline & 1 & Yr: & & $\odot . \odot \mathrm{E}-\odot \odot$ & $\odot . \odot \mathrm{E}-\odot \odot$ & $\odot . \odot \mathrm{E}-\odot \odot$ & $\odot . \odot \mathrm{E}-\odot \odot$ & $\odot . \odot \mathrm{E}-\odot \odot$ \\
\hline & 50 & Yr: & & $\odot . \odot \mathrm{E}-\odot \odot$ & $\odot . \odot \mathrm{E}-\odot \odot$ & $\odot . \odot \mathrm{E}-\odot \odot$ & $\odot . \odot \mathrm{E}-\odot \odot$ & $\odot . \odot \mathrm{E}-\odot \odot$ \\
\hline \multirow[t]{3}{*}{ TH227 } & Class: Y & & $1.4 \odot \mathrm{E}-\odot 7$ & & & & & \\
\hline & 1 & Yr: & & $3.2 E-\odot 4$ & 1. $8 \mathrm{E}-\odot 6$ & $3.5 E-07$ & 5. 4E- $\odot 9$ & $3.9 E-\odot 5$ \\
\hline & 50 & Yr: & & $3.2 \mathrm{E}-04$ & $1.8 \mathrm{E}-06$ & $3.5 \mathrm{E}-07$ & $5.4 \mathrm{E}-09$ & $3.9 \mathrm{E}-05$ \\
\hline \multirow[t]{3}{*}{ TH228 } & Class: Y & & $2.56 \mathrm{E}-\odot 9$ & & & & & \\
\hline & 1 & Yr: & & 1. $3 \mathrm{E}-03$ & $8.0 E-05$ & 1. $4 \mathrm{E}-05$ & $3.5 \mathrm{E}-07$ & $1.6 \mathrm{E}-04$ \\
\hline & 50 & Yr: & & 1. 3E-०3 & $8.0 \mathrm{E}-05$ & $1.4 \mathrm{E}-05$ & $3.5 E-07$ & 1. $6 \mathrm{E}-\odot 4$ \\
\hline \multirow[t]{3}{*}{ TH229 } & Class: $\mathrm{Y}$ & & $1.06 \mathrm{E}-\odot 7$ & & & & & \\
\hline & 1 & Yr: & & $1.6 \mathrm{E}-03$ & $6.0 \mathrm{E}-\odot 4$ & $5.6 \mathrm{E}-\odot 5$ & $3.5 E-\odot 6$ & $2.1 E-04$ \\
\hline & 50 & Yr: & & $1.6 \mathrm{E}-03$ & $6.0 \mathrm{E}-\odot 4$ & $5.6 \mathrm{E}-05$ & $3.5 E-06$ & 2.1E- -4 \\
\hline TH230 & Class: Y & & $4.70 \mathrm{E}-10$ & & & & & \\
\hline & 1 & Yr: & & $2.7 \mathrm{E}-\odot 4$ & $2.7 \mathrm{E}-\odot 4$ & $2.4 \mathrm{E}-05$ & 7. 3E- - 7 & 4. $0 \mathrm{E}-05$ \\
\hline & 50 & Yr: & & $2.7 \mathrm{E}-04$ & $2.7 \mathrm{E}-04$ & $2.4 \mathrm{E}-05$ & 7. $3 \mathrm{E}-07$ & 4. $0 \mathrm{E}-05$ \\
\hline TH231 & Class: $\mathrm{Y}$ & & $1.45 \mathrm{E}-\odot 8$ & & & & & \\
\hline & 1 & Yr: & & $8.3 E-09$ & $1.1 \mathrm{E}-10$ & $1.6 \mathrm{E}-11$ & $2.8 \mathrm{E}-12$ & $2.4 \mathrm{E}-09$ \\
\hline & 50 & Yr: & & 8. 3E-०9 & $1.1 \mathrm{E}-10$ & $1.6 \mathrm{E}-11$ & $2.8 \mathrm{E}-12$ & $2.4 \mathrm{E}-09$ \\
\hline TH232 & Class: Y & & $2.29 \mathrm{E}-10$ & & & & & \\
\hline & 1 & Yr: & & $3.7 \mathrm{E}-04$ & $3.1 \mathrm{E}-04$ & $2.7 \mathrm{E}-\odot 5$ & $1.8 \mathrm{E}-06$ & $5.4 \mathrm{E}-05$ \\
\hline & 50 & Yr: & & $3.7 \mathrm{E}-04$ & $3.1 \mathrm{E}-04$ & $2.7 \mathrm{E}-05$ & $1.8 \mathrm{E}-06$ & $5.4 \mathrm{E}-05$ \\
\hline TH234 & Class: Y & & $9.30 \mathrm{E}-\odot 9$ & & & & & \\
\hline & 1 & Yr: & & $2.4 \mathrm{E}-\odot 7$ & $1.5 \mathrm{E}-\odot 9$ & 2. $\odot E-\odot 9$ & $5.6 \mathrm{E}-11$ & $4.1 \mathrm{E}-\odot 8$ \\
\hline & 50 & Yr: & & $2.4 \mathrm{E}-07$ & $1.5 E-09$ & 2. $\odot E-\odot 9$ & $5.6 \mathrm{E}-11$ & $4.1 \mathrm{E}-08$ \\
\hline RA223 & Class: W & & $1.73 \mathrm{E}-07$ & & & & & \\
\hline & 1 & Yr: & & $2.2 E-\odot 4$ & $3.1 \mathrm{E}-05$ & $3.2 \mathrm{E}-\odot 6$ & $4.8 \mathrm{E}-\odot 8$ & $2.8 \mathrm{E}-05$ \\
\hline & 50 & Yr: & & $2.2 \mathrm{E}-\odot 4$ & $3.1 \mathrm{E}-05$ & $3.2 \mathrm{E}-06$ & $4.8 \mathrm{E}-\odot 8$ & $2.8 \mathrm{E}-05$ \\
\hline RA224 & Class: W & & $1.36 \mathrm{E}-\odot 8$ & & & & & \\
\hline & 1 & Yr: & & $8.9 \mathrm{E}-05$ & 1. $4 \mathrm{E}-05$ & $1.5 \mathrm{E}-\odot 6$ & $3.7 \mathrm{E}-08$ & 1.1E- 05 \\
\hline & 50 & Yr: & & $8.9 E-05$ & 1. $4 \mathrm{E}-05$ & 1. $5 E-06$ & 3. 7E- - 8 & 1.1E-05 \\
\hline RA225 & Class: W & & $7.60 E-\odot 9$ & & & & & \\
\hline & 1 & Yr: & & 1. $9 \mathrm{E}-\odot 4$ & 4. 4E- $\odot 5$ & $6.3 E-\odot 6$ & $5.4 \mathrm{E}-\odot 8$ & $2.4 \mathrm{E}-05$ \\
\hline & 50 & Yr: & & 1. $9 \mathrm{E}-04$ & $4.4 \mathrm{E}-05$ & $6.3 E-06$ & $5.4 \mathrm{E}-08$ & $2.4 \mathrm{E}-05$ \\
\hline RA226 & Class: W & & $8.96 \mathrm{E}-\odot 9$ & & & & & \\
\hline & 1 & Yr: & & $1.2 \mathrm{E}-04$ & $3.2 \mathrm{E}-05$ & $3.8 \mathrm{E}-06$ & 1. $2 \mathrm{E}-07$ & 1. $5 \mathrm{E}-05$ \\
\hline & 50 & Yr: & & 1. $2 \mathrm{E}-04$ & $3.2 \mathrm{E}-05$ & $3.8 E-06$ & 1. $2 \mathrm{E}-07$ & $1.5 \mathrm{E}-05$ \\
\hline RA228 & Class: W & & $\odot . \odot \odot E \odot \odot ~$ & & & & & \\
\hline & 1 & Yr: & & $4.2 \mathrm{E}-05$ & $2.7 \mathrm{E}-04$ & $4.1 \mathrm{E}-05$ & 1. $2 \mathrm{E}-06$ & $1.5 \mathrm{E}-05$ \\
\hline & 50 & Yr: & & $4.2 E-05$ & $2.7 \mathrm{E}-\odot 4$ & $4.1 E-05$ & 1. $2 \mathrm{E}-06$ & 1. $5 \mathrm{E}-05$ \\
\hline PB210 & Class: D & & $1.42 \mathrm{E}-\odot 9$ & & & & & \\
\hline & 1 & Yr: & & 1. $2 \mathrm{E}-\odot 6$ & $4.8 E-\odot 5$ & $1.8 E-\odot 5$ & 1. $2 \mathrm{E}-06$ & $4.6 \mathrm{E}-06$ \\
\hline & 50 & Yr: & & 1. $2 \mathrm{E}-06$ & $4.8 \mathrm{E}-05$ & $1.8 \mathrm{E}-05$ & 1. $2 \mathrm{E}-\odot 6$ & $4.6 \mathrm{E}-06$ \\
\hline PB212 & Class: D & & $1.97 \mathrm{E}-07$ & & & & & \\
\hline & 1 & Yr: & & $5.3 E-08$ & 1.7E- -6 & 2. $\odot E-\odot 7$ & $3.5 \mathrm{E}-\odot 8$ & $2.0 \mathrm{E}-07$ \\
\hline & 50 & Yr: & & $5.3 E-08$ & 1. 7E- 06 & 2. $\odot \mathrm{E}-\odot 7$ & $3.5 E-08$ & $2 . \odot E-\odot 7$ \\
\hline BI210 & Class: W & & $8.14 \mathrm{E}-\odot 9$ & & & & & \\
\hline & 1 & Yr: & & $3.2 \mathrm{E}-\odot 6$ & $4.9 \mathrm{E}-10$ & $4.9 \mathrm{E}-10$ & $4.9 \mathrm{E}-10$ & $3.9 \mathrm{E}-07$ \\
\hline & 50 & Yr: & & $3.2 \mathrm{E}-06$ & $4.9 \mathrm{E}-10$ & $4.9 E-10$ & $4.9 \mathrm{E}-10$ & $3.9 E-07$ \\
\hline BI212 & Class: W & & $2.83 E-07$ & & & & & \\
\hline & 1 & Yr: & & $6.6 \mathrm{E}-07$ & $2.7 \mathrm{E}-10$ & $2.6 \mathrm{E}-10$ & $2.6 \mathrm{E}-10$ & $1.6 \mathrm{E}-07$ \\
\hline & 50 & Yr: & & $6.6 \mathrm{E}-07$ & $2.7 \mathrm{E}-10$ & $2.6 \mathrm{E}-10$ & $2.6 \mathrm{E}-10$ & 1. $6 \mathrm{E}-07$ \\
\hline P0210 & Class: W & & $1.23 \mathrm{E}-11$ & & & & & \\
\hline & 1 & Yr: & & $1.1 \mathrm{E}-04$ & $8.8 \mathrm{E}-06$ & $9.1 \mathrm{E}-06$ & $6.4 \mathrm{E}-07$ & $1.5 \mathrm{E}-05$ \\
\hline & 50 & Yr: & & 1. 1E- -4 & $8.8 E-06$ & $9.1 \mathrm{E}-06$ & $6.4 \mathrm{E}-07$ & 1. $5 \mathrm{E}-05$ \\
\hline
\end{tabular}




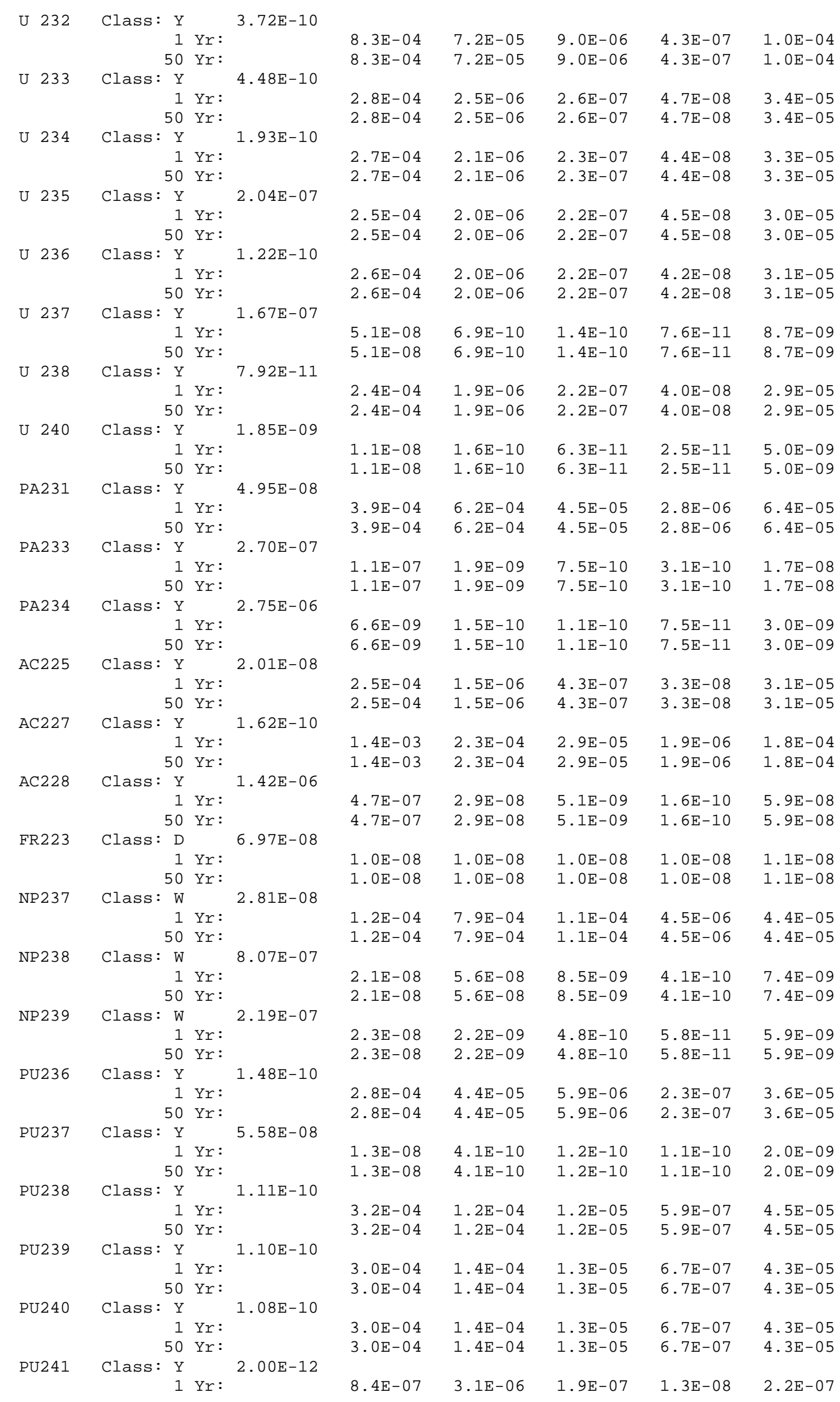




\begin{tabular}{|c|c|c|c|c|c|c|c|c|}
\hline \multirow{4}{*}{ PU242 } & 50 & Yr: & & $8.4 \mathrm{E}-07$ & $3.1 \mathrm{E}-06$ & 1.9E-०7 & 1. $3 E-\odot 8$ & $2.2 \mathrm{E}-07$ \\
\hline & Class: $\mathrm{Y}$ & & $9.18 \mathrm{E}-11$ & & & & & \\
\hline & 1 & Yr: & & $2.8 \mathrm{E}-\odot 4$ & $1.4 \mathrm{E}-04$ & 1. $3 \mathrm{E}-05$ & $6.4 \mathrm{E}-07$ & $4.0 \mathrm{E}-05$ \\
\hline & 50 & Yr: & & $2.8 \mathrm{E}-04$ & 1. $4 \mathrm{E}-\odot 4$ & 1. $3 \mathrm{E}-05$ & $6.4 \mathrm{E}-07$ & 4. . \\
\hline \multirow[t]{3}{*}{ PU243 } & Class: Y & & $3.04 \mathrm{E}-\odot 8$ & & & & & \\
\hline & 1 & Yr: & & 1.7E-०9 & $1.6 \mathrm{E}-11$ & $2.4 \mathrm{E}-12$ & $9.4 \mathrm{E}-13$ & $6.0 \mathrm{E}-10$ \\
\hline & 50 & Yr: & & 1.7E-०9 & 1. $6 \mathrm{E}-11$ & $2.4 \mathrm{E}-12$ & $9.4 \mathrm{E}-13$ & $6.0 \mathrm{E}-10$ \\
\hline \multirow[t]{3}{*}{ PU244 } & Class: Y & & $6.56 \mathrm{E}-11$ & & & & & \\
\hline & 1 & Yr: & & $2.6 \mathrm{E}-04$ & 1. 3E- -4 & $1.2 \mathrm{E}-05$ & $6.1 \mathrm{E}-07$ & $3.8 \mathrm{E}-05$ \\
\hline & 50 & Yr: & & $2.6 \mathrm{E}-04$ & 1. $3 E-04$ & 1. $2 \mathrm{E}-05$ & $6.1 \mathrm{E}-07$ & $3.8 \mathrm{E}-05$ \\
\hline \multirow[t]{3}{*}{ AM241 } & Class: W & & $2.13 \mathrm{E}-\odot 8$ & & & & & \\
\hline & 1 & Yr: & & $1.5 E-04$ & 1. $3 E-03$ & 2. $\odot E-\odot 4$ & $6.5 E-06$ & 7. 4E- -05 \\
\hline & 50 & Yr: & & $1.5 \mathrm{E}-\odot 4$ & 1. $3 E-03$ & $2 . \odot E-\odot 4$ & $6.5 \mathrm{E}-\odot 6$ & 7. $4 \mathrm{E}-05$ \\
\hline \multirow[t]{3}{*}{ AM242M } & Class: W & & $7.85 \mathrm{E}-10$ & & & & & \\
\hline & 1 & Yr: & & $3.2 \mathrm{E}-05$ & 1. $2 \mathrm{E}-03$ & $1.6 \mathrm{E}-\odot 4$ & $6.3 \mathrm{E}-06$ & $5.3 \mathrm{E}-05$ \\
\hline & 50 & Yr: & & $3.2 \mathrm{E}-05$ & 1. $2 \mathrm{E}-03$ & $1.6 \mathrm{E}-\odot 4$ & $6.3 \mathrm{E}-06$ & $5.3 \mathrm{E}-05$ \\
\hline \multirow[t]{3}{*}{ AM242 } & Class: W & & $1.93 E-08$ & & & & & \\
\hline & 1 & Yr: & & $4.5 \mathrm{E}-07$ & $4.1 \mathrm{E}-07$ & $1.1 \mathrm{E}-07$ & $7.0 \mathrm{E}-10$ & $7.5 E-08$ \\
\hline & 50 & Yr: & & $4.5 \mathrm{E}-07$ & $4.1 \mathrm{E}-07$ & $1.1 \mathrm{E}-07$ & 7. $0 \mathrm{E}-10$ & $7.5 \mathrm{E}-08$ \\
\hline \multirow[t]{3}{*}{ AM243 } & Class: W & & $5.87 \mathrm{E}-\odot 8$ & & & & & \\
\hline & 1 & Yr: & & 1. $4 \mathrm{E}-04$ & 1. 3E- 03 & 1.9E-๑4 & $6.5 \mathrm{E}-06$ & $7.2 \mathrm{E}-05$ \\
\hline & 50 & Yr: & & 1. $4 \mathrm{E}-04$ & 1. 3E-०3 & 1. $9 \mathrm{E}-\odot 4$ & $6.5 \mathrm{E}-06$ & $7.2 \mathrm{E}-05$ \\
\hline \multirow[t]{3}{*}{ CM242 } & Class: W & & $1.27 \mathrm{E}-10$ & & & & & \\
\hline & 1 & Yr: & & 1. $4 \mathrm{E}-\odot 4$ & 1. $2 \mathrm{E}-\odot 4$ & 3. $3 E-05$ & $2.1 \mathrm{E}-07$ & $2.2 \mathrm{E}-05$ \\
\hline & 50 & Yr: & & 1. $4 \mathrm{E}-04$ & 1. $2 \mathrm{E}-\odot 4$ & $3.3 E-05$ & $2.1 \mathrm{E}-07$ & $2.2 \mathrm{E}-05$ \\
\hline \multirow[t]{3}{*}{ CM243 } & Class: W & & $1.67 \mathrm{E}-07$ & & & & & \\
\hline & 1 & Yr: & & $1.6 \mathrm{E}-04$ & 1. $0 \mathrm{E}-\mathrm{O}$ & 1. $9 \mathrm{E}-\odot 4$ & 4. $\odot E-06$ & $6.7 \mathrm{E}-05$ \\
\hline & 50 & Yr: & & $1.6 \mathrm{E}-04$ & 1. $0 \mathrm{E}-03$ & 1. $9 \mathrm{E}-\odot 4$ & 4. . E - $\odot 6$ & $6.7 \mathrm{E}-05$ \\
\hline \multirow[t]{3}{*}{ CM244 } & Class: W & & $1.08 \mathrm{E}-10$ & & & & & \\
\hline & 1 & Yr: & & $1.6 \mathrm{E}-04$ & $9.2 \mathrm{E}-04$ & $1.8 \mathrm{E}-\odot 4$ & $3.2 \mathrm{E}-06$ & $6.2 \mathrm{E}-05$ \\
\hline & 50 & Yr: & & $1.6 \mathrm{E}-04$ & $9.2 \mathrm{E}-\odot 4$ & $1.8 \mathrm{E}-\odot 4$ & $3.2 \mathrm{E}-06$ & $6.2 \mathrm{E}-05$ \\
\hline \multirow[t]{3}{*}{ CM245 } & Class: W & & $1.10 \mathrm{E}-07$ & & & & & \\
\hline & 1 & Yr: & & 1. 4E-04 & 1. 3E- - 3 & $2 . \odot E-\odot 4$ & $6.8 \mathrm{E}-06$ & $7.4 \mathrm{E}-05$ \\
\hline & 50 & Yr: & & 1. $4 \mathrm{E}-04$ & 1. $3 E-03$ & $2.0 E-\odot 4$ & $6.8 \mathrm{E}-06$ & $7.4 \mathrm{E}-05$ \\
\hline \multirow[t]{3}{*}{ CM246 } & Class: W & & $9.78 \mathrm{E}-11$ & & & & & \\
\hline & 1 & Yr: & & 1. 4E- - 4 & 1. 3E- -3 & $2.0 \mathrm{E}-04$ & $6.6 \mathrm{E}-06$ & $7.3 \mathrm{E}-05$ \\
\hline & 50 & Yr: & & 1. $4 \mathrm{E}-04$ & 1. $3 E-03$ & 2. $\odot E-\odot 4$ & $6.6 \mathrm{E}-06$ & $7.3 \mathrm{E}-05$ \\
\hline \multirow[t]{3}{*}{ CM247 } & Class: W & & $4.38 \mathrm{E}-07$ & & & & & \\
\hline & 1 & Yr: & & 1. 3E-๑4 & $1.2 \mathrm{E}-\mathrm{-0}$ & $1.8 \mathrm{E}-\odot 4$ & $6.2 \mathrm{E}-06$ & $6.7 \mathrm{E}-05$ \\
\hline & 50 & Yr: & & 1. $3 \mathrm{E}-04$ & 1. $2 \mathrm{E}-03$ & $1.8 \mathrm{E}-04$ & $6.2 \mathrm{E}-06$ & $6.7 \mathrm{E}-05$ \\
\hline \multirow[t]{3}{*}{ CM248 } & Class: W & & $7.44 \mathrm{E}-11$ & & & & & \\
\hline & 1 & Yr: & & $6.3 E-05$ & $7.8 \mathrm{E}-03$ & $6.2 \mathrm{E}-04$ & $2.2 \mathrm{E}-08$ & $4.2 \mathrm{E}-\odot 4$ \\
\hline & 50 & Yr: & & $6.3 \mathrm{E}-05$ & $7.8 \mathrm{E}-03$ & $6.2 \mathrm{E}-04$ & $2.2 \mathrm{E}-08$ & $4.2 \mathrm{E}-\odot 4$ \\
\hline \multirow[t]{3}{*}{ CF252 } & Class: W & & $1.15 \mathrm{E}-10$ & & & & & \\
\hline & 1 & Yr: & & $3.5 E-05$ & $6.6 \mathrm{E}-04$ & $5.3 E-05$ & $1.1 \mathrm{E}-08$ & $3.5 E-05$ \\
\hline & 50 & Yr: & & $3.5 E-05$ & $6.6 \mathrm{E}-04$ & $5.3 \mathrm{E}-05$ & 1.1E-08 & $3.5 E-05$ \\
\hline
\end{tabular}




\section{Appendix B DCF Ratios}

\section{Appendix B.1}

The following figures (Figure B.1 - B.6) show the ratio of the revised Dose Conversion Factor (DCF) from FGR12/13 to the original HUDUFACT.dat DCF value. Only those radionuclides that had the largest differences (i.e., a ratio $<0.1$ or $>10$ ) are displayed. Some radionuclides show large changes from the original HUDUFACT.dat values to those found in FGR12/13, this is due to changes in dose conversion calculations and different dose modeling updates as new guidance has come available. Full listing of ratios are given in Appendix B.3.

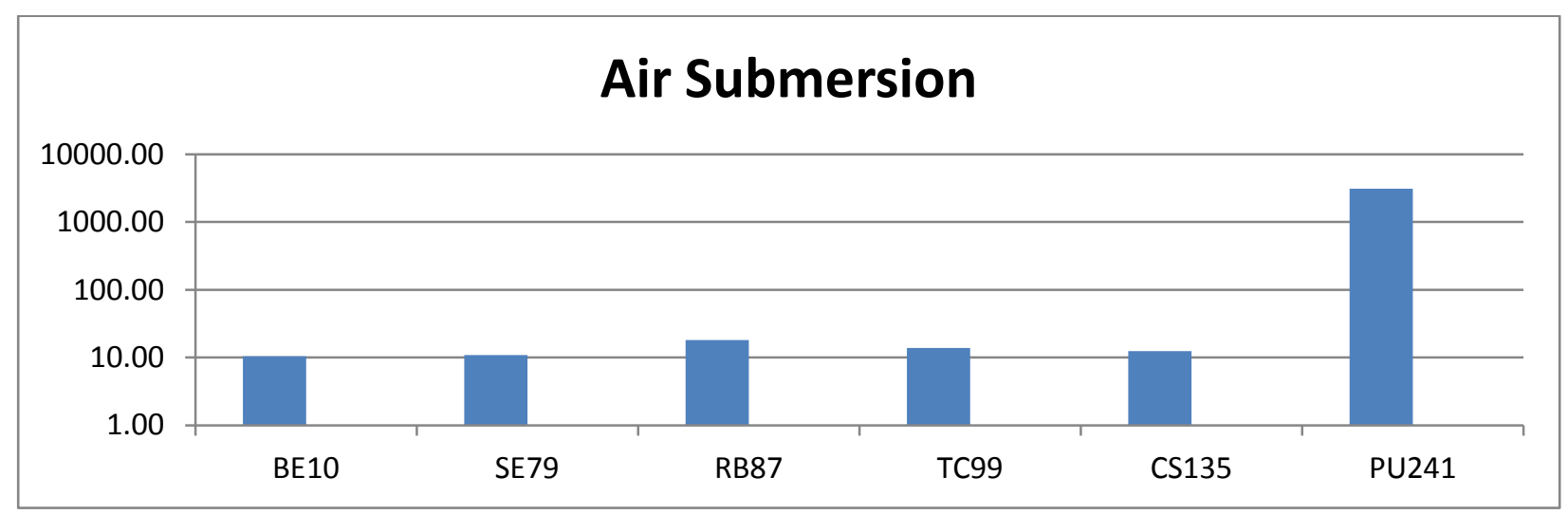

Figure B.1: Ratio of the revised FGR12 air submersion DCF to the original HUDUFACT.dat air submersion DCF for those radionuclides having the largest differences ( $\mathrm{y}$-axis uses log scale).

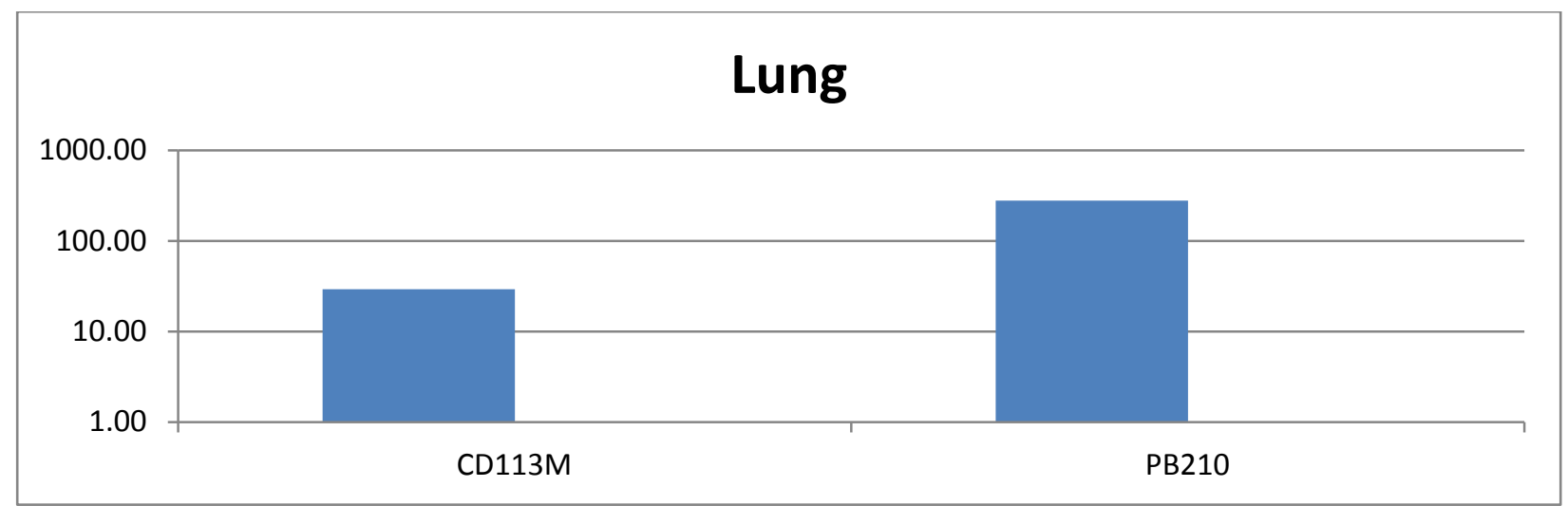

Figure B.2: Ratio of the revised FGR 13 lung DCF to the original HUDUFACT.dat lung DCF for those radionuclides having the largest differences (y-axis uses log scale). 


\section{Bone Surface}

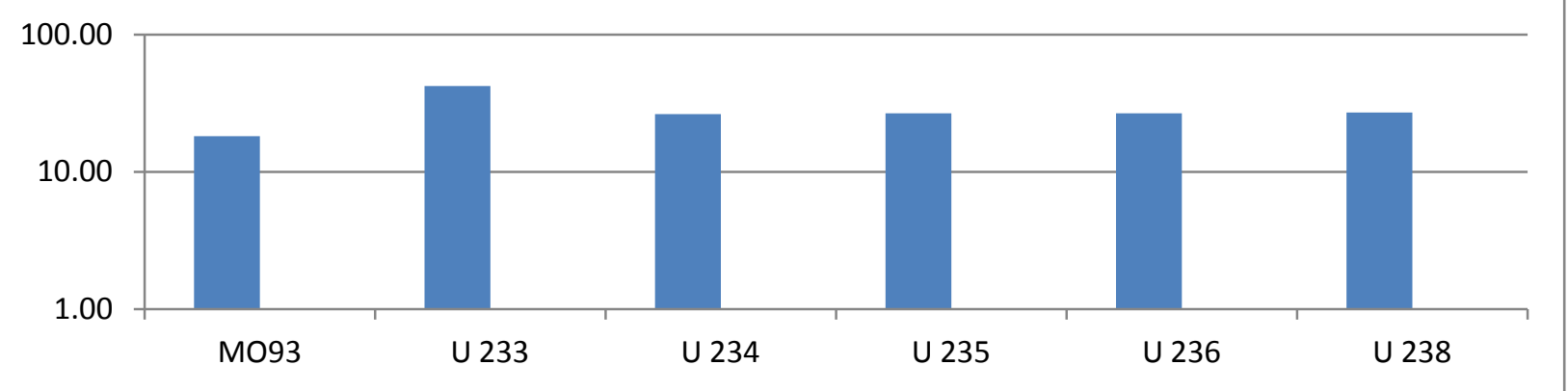

Figure B.3: Ratio of the revised FGR 13 bone surface DCF to the original HUDUFACT.dat bone surface DCF for those radionuclides having the largest differences (y-axis uses log scale).

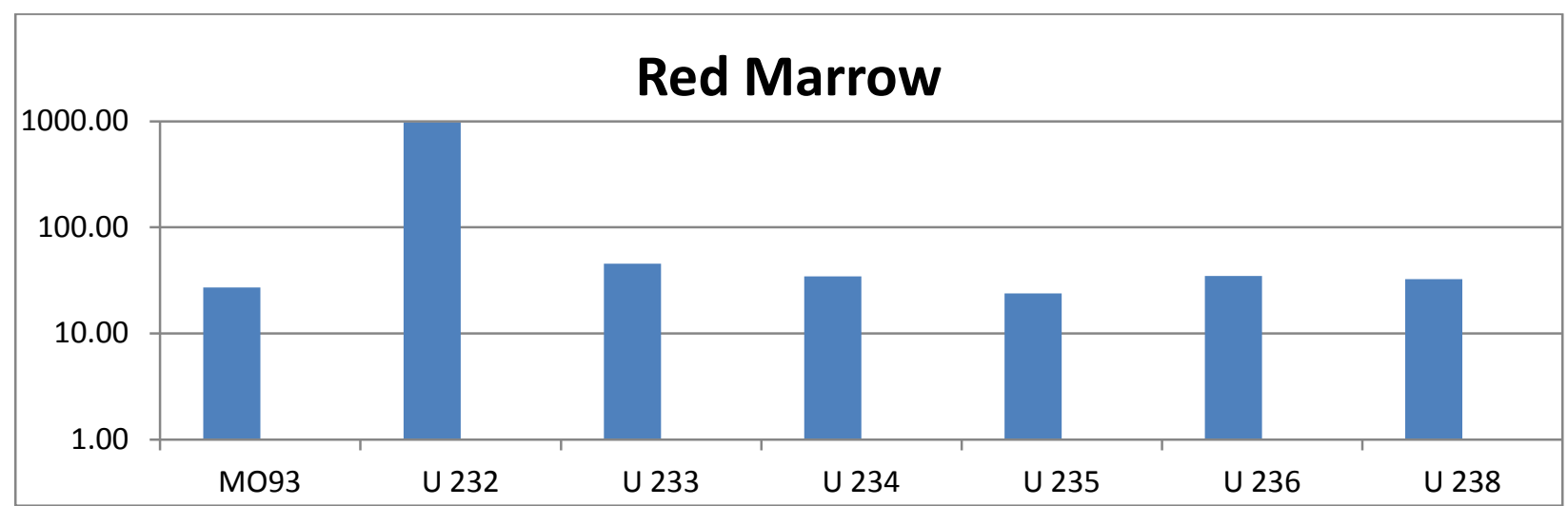

Figure B.4: Ratio of the revised FGR 13 red marrow DCF to the original HUDUFACT.dat red marrow DCF for those radionuclides having the largest differences (y-axis uses log scale).

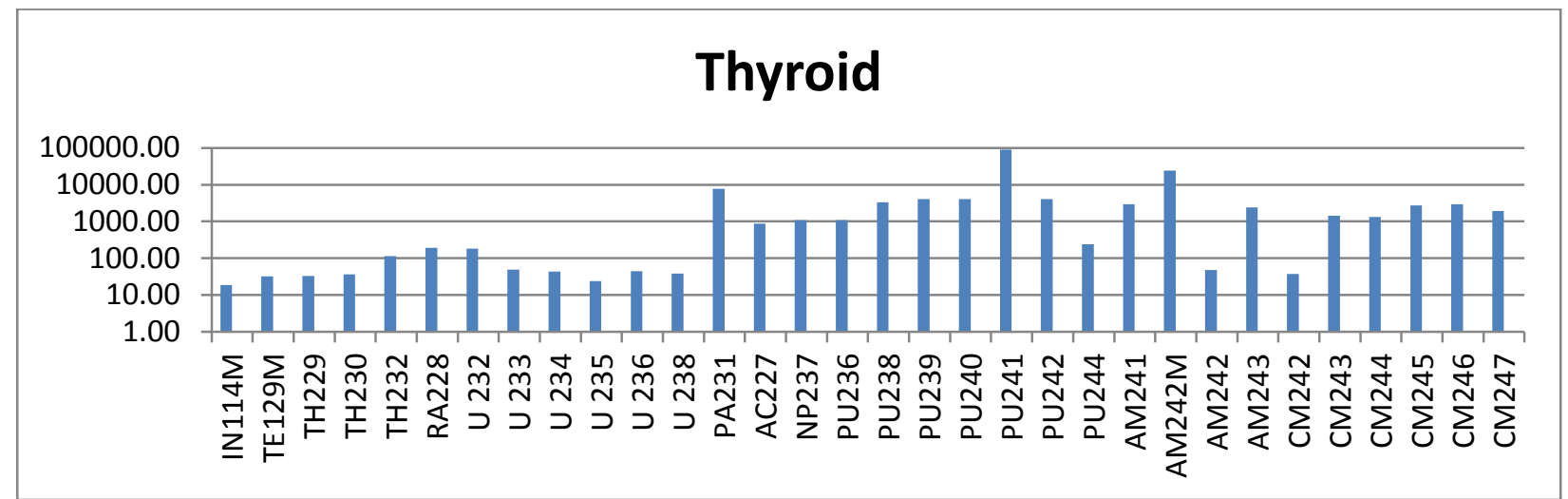

Figure B.5: Ratio of the revised FGR 13 thyroid DCF to the original HUDUFACT.dat thyroid DCF for those radionuclides having the largest differences (y-axis uses log scale). 


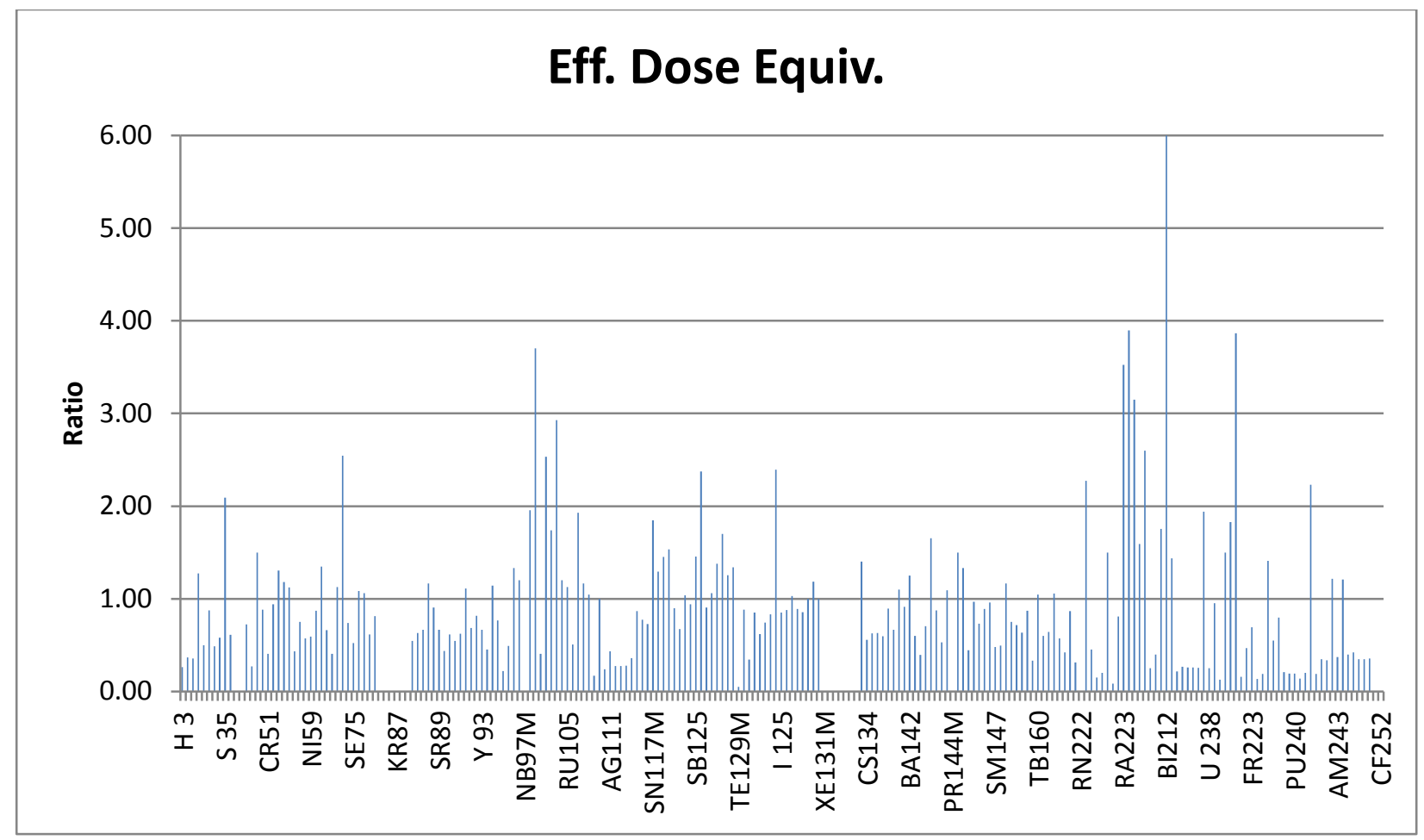

Figure B.6: Ratio of the revised FGR13 effective dose equivalent DCFs to the original HUDUFACT.dat effective dose equivalent DCFs for the various radionuclides (note: not all radionuclide labels are displayed). 


\section{Appendix B.2}

Listing of the revised FGR12/13 and Original HUDUFACT.dat DCFs (for adult) (Note: values with a * indicate where values were not in FGR12 and/or FGR 13; therefore original HUDUFACT.dat values were used where updated values were not found)

\begin{tabular}{|c|c|c|c|c|c|c|c|}
\hline source & $\mathrm{e}^{\text {nuclid }}$ & $\begin{array}{l}\text { air } \\
\text { submersion } \\
\text { Sv/Yr per } \\
\mathrm{Bq} / \mathrm{m3}\end{array}$ & $\begin{array}{l}\text { lung } \\
\text { Sv/Bq }\end{array}$ & $\begin{array}{l}\text { bone } \\
\text { surface } \\
\text { Sv/Bq }\end{array}$ & $\begin{array}{l}\text { red marrow } \\
\mathrm{Sv} / \mathrm{Bq}\end{array}$ & $\begin{array}{l}\text { thyroid } \\
\text { Sv/Bq }\end{array}$ & $\begin{array}{l}\text { eff dose } \\
\text { equiv } \\
\text { Sv/Bq }\end{array}$ \\
\hline FGR12/13 & H 3 & $\odot . \odot \odot \mathrm{E}+\odot \odot$ & $6.20 \mathrm{E}-12$ & $6.20 \mathrm{E}-12$ & $6.20 \mathrm{E}-12$ & $6.20 \mathrm{E}-12$ & $6.30 \mathrm{E}-12$ \\
\hline original & H 3 & $2.00 \mathrm{E}-16$ & $2.50 \mathrm{E}-11$ & $1.30 \mathrm{E}-11$ & $2.50 \mathrm{E}-11$ & $2.50 \mathrm{E}-11$ & $2.40 \mathrm{E}-11$ \\
\hline FGR12/13 & BE10 & 4. 40E-๑9 & $2.80 E-\odot 7$ & $6.20 E-\odot 9$ & $2.10 E-09$ & $7.10 \mathrm{E}-11$ & $3.50 E-08$ \\
\hline original & BE10 & $4.20 \mathrm{E}-10$ & $7.7 \odot \mathrm{E}-\odot 7$ & $2.20 \mathrm{E}-\odot 8$ & $6.70 \mathrm{E}-09$ & 1. $00 \mathrm{E}-11$ & $9.50 \mathrm{E}-\odot 8$ \\
\hline FGR12/13 & C 14 & $8.20 \mathrm{E}-11$ & $2.00 \mathrm{E}-10$ & $1.90 \mathrm{E}-10$ & $1.90 \mathrm{E}-10$ & $1.90 \mathrm{E}-10$ & 2. $00 \mathrm{E}-10$ \\
\hline original & C 14 & $1.40 \mathrm{E}-11$ & $5.80 \mathrm{E}-10$ & $3.00 \mathrm{E}-10$ & $5.80 \mathrm{E}-10$ & $5.80 \mathrm{E}-10$ & $5.60 \mathrm{E}-10$ \\
\hline FGR12/13 & F 18 & $1.40 \mathrm{E}-06$ & $2.60 \mathrm{E}-11$ & $2.50 \mathrm{E}-11$ & $2.70 \mathrm{E}-11$ & $3.40 \mathrm{E}-12$ & $2.80 E-11$ \\
\hline original & F 18 & $1.90 \mathrm{E}-\odot 6$ & $1.10 \mathrm{E}-10$ & $2.40 \mathrm{E}-11$ & $2.50 \mathrm{E}-11$ & $4.10 \mathrm{E}-12$ & $2.20 \mathrm{E}-11$ \\
\hline FGR12/13 & NA22 & $3.20 \mathrm{E}-\odot 6$ & $9.20 \mathrm{E}-10$ & $2.20 \mathrm{E}-\odot 9$ & $1.60 \mathrm{E}-\odot 9$ & $9.60 \mathrm{E}-10$ & 1. $30 \mathrm{E}-\odot 9$ \\
\hline original & NA22 & $3.90 \mathrm{E}-06$ & $2.4 \odot E-\odot 9$ & 4. $\odot \odot E-\odot 9$ & $3.30 \mathrm{E}-\odot 9$ & $2.10 E-\odot 9$ & $2.60 E-\odot 9$ \\
\hline FGR12/13 & NA24 & $6.60 \mathrm{E}-06$ & $1.40 \mathrm{E}-10$ & $2.00 \mathrm{E}-10$ & $1.50 \mathrm{E}-10$ & $1.20 \mathrm{E}-10$ & $2.80 \mathrm{E}-10$ \\
\hline original & NA24 & $8.10 \mathrm{E}-06$ & $1.20 \mathrm{E}-\odot 9$ & $2.80 \mathrm{E}-10$ & $2.20 \mathrm{E}-10$ & $1.60 \mathrm{E}-10$ & $3.20 \mathrm{E}-10$ \\
\hline FGR12/13 & P 32 & 1. $7 \odot \mathrm{E}-\odot 8$ & $3.00 \mathrm{E}-10$ & $3.30 E-\odot 9$ & $3.30 E-\odot 9$ & $2.70 \mathrm{E}-10$ & $7.80 \mathrm{E}-10$ \\
\hline original & P 32 & $6.30 \mathrm{E}-\odot 9$ & 2. $\odot \odot E-\odot 9$ & $5.90 \mathrm{E}-\odot 9$ & $6.20 \mathrm{E}-09$ & $5.10 \mathrm{E}-10$ & $1.60 E-09$ \\
\hline FGR12/13 & P 33 & $4.60 \mathrm{E}-10$ & $5.30 \mathrm{E}-11$ & $5.50 \mathrm{E}-10$ & $2.10 \mathrm{E}-10$ & $3.80 E-11$ & $9.30 \mathrm{E}-11$ \\
\hline original & P 33 & $4.90 \mathrm{E}-11$ & $2.30 \mathrm{E}-10$ & 1. $0 \odot E-\odot 9$ & $3.90 \mathrm{E}-10$ & $7.30 \mathrm{E}-11$ & $1.60 \mathrm{E}-10$ \\
\hline FGR12/13 & S 35 & $9.80 \mathrm{E}-11$ & $1.20 \mathrm{E}-\odot 8$ & $7.80 \mathrm{E}-12$ & $7.80 \mathrm{E}-12$ & $7.80 \mathrm{E}-12$ & $1.4 \odot E-\odot 9$ \\
\hline original & S 35 & $1.60 \mathrm{E}-11$ & $5.10 E-\odot 9$ & $2.20 \mathrm{E}-11$ & $4.60 \mathrm{E}-11$ & $4.60 \mathrm{E}-11$ & $6.70 \mathrm{E}-10$ \\
\hline FGR12/13 & CL36 & $5.20 \mathrm{E}-\odot 9$ & $3.00 \mathrm{E}-10$ & $2.70 \mathrm{E}-10$ & $2.70 \mathrm{E}-10$ & $2.70 \mathrm{E}-10$ & $3.30 \mathrm{E}-10$ \\
\hline original & CL36 & $7.50 \mathrm{E}-10$ & $8.20 \mathrm{E}-10$ & $2.60 \mathrm{E}-10$ & $5.10 \mathrm{E}-10$ & $5.30 \mathrm{E}-10$ & $5.40 \mathrm{E}-10$ \\
\hline *FGR12/13 & AR39 & $3.6 \odot E-\odot 9$ & $\odot . \odot \odot \mathrm{E}+\odot \odot$ & $\odot . \odot \odot E+\odot \odot$ & $\odot . \odot \odot E+\odot \odot$ & $\odot . \odot \odot E+\odot \odot$ & $\odot .00 \mathrm{E}+\odot \odot$ \\
\hline original & AR39 & $4.20 \mathrm{E}-10$ & $\odot . \odot \odot E+\odot \odot$ & $\odot . \odot \odot E+\odot \odot$ & $\odot . \odot \odot E+\odot \odot$ & $\odot . \odot \odot E+\odot \odot$ & $\odot . \odot \odot E+\odot \odot$ \\
\hline${ }^{*}$ FGR12/13 & AR41 & $1.90 \mathrm{E}-06$ & $\odot . \odot \odot \mathrm{E}+\odot \odot$ & $\odot . \odot \odot E+\odot \odot$ & $\odot . \odot \odot \mathrm{E}+\odot \odot$ & $\odot . \odot \odot E+\odot \odot$ & $\odot . \odot \odot E+\odot \odot$ \\
\hline original & AR41 & $2.10 \mathrm{E}-\odot 6$ & $\odot . \odot \odot E+\odot \odot$ & $\odot . \odot \odot E+\odot \odot$ & $\odot . \odot \odot E+\odot \odot$ & $\odot . \odot \odot E+\odot \odot$ & $\odot . \odot \odot E+\odot \odot$ \\
\hline FGR12/13 & K 40 & $2.50 E-\odot 7$ & 1. $70 \mathrm{E}-\odot 9$ & 1. $70 \mathrm{E}-\odot 9$ & 1. $7 \odot E-\odot 9$ & 1. $7 \odot E-\odot 9$ & $2.10 E-09$ \\
\hline
\end{tabular}




\begin{tabular}{|c|c|c|c|c|c|c|c|}
\hline original & K 40 & $2.90 \mathrm{E}-07$ & 1. $80 \mathrm{E}-\odot 9$ & $1.6 \odot \mathrm{E}-\odot 9$ & $3 . \odot \odot E-\odot 9$ & $3.10 \mathrm{E}-\odot 9$ & $2.90 E-\odot 9$ \\
\hline FGR12/13 & CA41 & $\odot . \odot \odot \mathrm{E}+\odot \odot$ & $1.60 \mathrm{E}-10$ & $1.30 \mathrm{E}-09$ & $4.90 \mathrm{E}-10$ & $9.50 \mathrm{E}-13$ & $9.50 \mathrm{E}-11$ \\
\hline original & CA41 & $2.70 \mathrm{E}-10$ & $4.40 \mathrm{E}-10$ & $3.60 \mathrm{E}-\odot 9$ & $1.5 \odot E-\odot 9$ & $9.10 \mathrm{E}-13$ & $3.50 \mathrm{E}-10$ \\
\hline FGR12/13 & CA45 & $4.80 \mathrm{E}-10$ & $2.10 \mathrm{E}-\odot 8$ & 1. $90 \mathrm{E}-\odot 9$ & $9.30 \mathrm{E}-10$ & $1.20 \mathrm{E}-11$ & $2.70 \mathrm{E}-\odot 9$ \\
\hline original & CA45 & $5.10 \mathrm{E}-11$ & $9.6 \odot \mathrm{E}-\odot 9$ & $4.30 E-\odot 9$ & $3 . \odot \odot E-\odot 9$ & $2.00 \mathrm{E}-11$ & 1. $80 \mathrm{E}-\odot 9$ \\
\hline FGR12/13 & SC46 & $3 . \odot \odot E-\odot 6$ & $4.50 E-\odot 8$ & $1.20 \mathrm{E}-\odot 9$ & $1.6 \odot \mathrm{E}-\odot 9$ & $1.20 \mathrm{E}-09$ & $6.80 E-\odot 9$ \\
\hline original & SC46 & $3.60 \mathrm{E}-06$ & $4.60 \mathrm{E}-\odot 8$ & $1.7 \odot E-\odot 9$ & $2.20 \mathrm{E}-\odot 9$ & $2.10 \mathrm{E}-09$ & $7.70 E-\odot 9$ \\
\hline FGR12/13 & CR51 & 4. 4०E-๑8 & $1.80 \mathrm{E}-10$ & $1.20 \mathrm{E}-11$ & $1.30 \mathrm{E}-11$ & $9.00 \mathrm{E}-12$ & $3.70 \mathrm{E}-11$ \\
\hline original & CR51 & $6.40 \mathrm{E}-08$ & $5.30 \mathrm{E}-10$ & $1.40 \mathrm{E}-11$ & $1.90 \mathrm{E}-11$ & $1.10 \mathrm{E}-11$ & $9.10 \mathrm{E}-11$ \\
\hline FGR12/13 & MN54 & $1.20 E-06$ & $6.50 \mathrm{E}-\odot 9$ & $1.20 \mathrm{E}-\odot 9$ & 1. $20 \mathrm{E}-\odot 9$ & $6.70 \mathrm{E}-10$ & 1. $60 \mathrm{E}-\odot 9$ \\
\hline original & MN54 & $1.50 \mathrm{E}-06$ & $6.70 \mathrm{E}-\odot 9$ & $1.20 \mathrm{E}-\odot 9$ & $1.10 \mathrm{E}-\odot 9$ & $7.40 \mathrm{E}-10$ & 1. $70 \mathrm{E}-09$ \\
\hline FGR12/13 & MN56 & $2.60 \mathrm{E}-06$ & $3.80 \mathrm{E}-10$ & $7.70 \mathrm{E}-12$ & $9.90 \mathrm{E}-12$ & $6.40 \mathrm{E}-12$ & $1.20 \mathrm{E}-10$ \\
\hline original & MN56 & $3.20 E-\odot 6$ & $5.50 \mathrm{E}-10$ & $8.60 \mathrm{E}-12$ & $1.10 \mathrm{E}-11$ & $6.50 \mathrm{E}-12$ & $9.20 \mathrm{E}-11$ \\
\hline FGR12/13 & FE55 & $\odot . \odot \odot \mathrm{E}+\odot \odot$ & 4. $\odot \odot \mathrm{E}-1 \odot$ & $6.70 \mathrm{E}-10$ & 1. $30 \mathrm{E}-\odot 9$ & $9.60 \mathrm{E}-11$ & $3.90 \mathrm{E}-10$ \\
\hline original & FE55 & $6.10 \mathrm{E}-10$ & $9.0 \odot \mathrm{E}-1 \odot$ & $7.50 \mathrm{E}-11$ & $1.60 \mathrm{E}-10$ & $1.90 \mathrm{E}-10$ & $3.30 \mathrm{E}-10$ \\
\hline FGR12/13 & FE59 & $1.80 E-06$ & $2.30 \mathrm{E}-\odot 8$ & $9.80 \mathrm{E}-10$ & 1. $4 \odot E-\odot 9$ & $6.80 \mathrm{E}-10$ & $3.70 E-\odot 9$ \\
\hline original & FE59 & $1.90 \mathrm{E}-\odot 6$ & 1. $40 \mathrm{E}-\odot 8$ & $9.30 \mathrm{E}-10$ & $1.2 \odot E-\odot 9$ & $1.20 \mathrm{E}-\odot 9$ & $3.30 E-09$ \\
\hline FGR12/13 & $\mathrm{C} 057$ & $1.60 \mathrm{E}-07$ & $6.60 \mathrm{E}-\odot 9$ & $4.0 \odot E-1 \odot$ & $2.40 \mathrm{E}-10$ & $1.90 \mathrm{E}-10$ & 1. $\odot \odot E-\odot 9$ \\
\hline original & C057 & $1.80 \mathrm{E}-07$ & 1. $70 \mathrm{E}-\odot 8$ & $4.40 \mathrm{E}-10$ & $5.70 \mathrm{E}-10$ & $2.70 \mathrm{E}-10$ & $2.30 \mathrm{E}-\odot 9$ \\
\hline FGR12/13 & $\mathrm{co58}$ & $1.40 \mathrm{E}-\odot 6$ & 1. $30 \mathrm{E}-\odot 8$ & $5.20 \mathrm{E}-10$ & $7.10 \mathrm{E}-10$ & $5.20 \mathrm{E}-10$ & $2.10 \mathrm{E}-\odot 9$ \\
\hline original & $\mathrm{C058}$ & $1.80 \mathrm{E}-06$ & $1.60 \mathrm{E}-\odot 8$ & $6.80 \mathrm{E}-10$ & $9.40 \mathrm{E}-10$ & $8.80 \mathrm{E}-10$ & $2.80 E-\odot 9$ \\
\hline FGR12/13 & $\mathrm{C060}$ & $3.80 E-06$ & $1.80 \mathrm{E}-\odot 7$ & $9.30 \mathrm{E}-\odot 9$ & $1.20 \mathrm{E}-\odot 8$ & $9.90 \mathrm{E}-\odot 9$ & $3.10 E-\odot 8$ \\
\hline original & $\mathrm{C060}$ & $4.30 \mathrm{E}-\odot 6$ & $3.50 \mathrm{E}-07$ & $1.30 \mathrm{E}-08$ & $1.70 \mathrm{E}-\odot 8$ & $1.60 \mathrm{E}-08$ & $5.40 \mathrm{E}-\odot 8$ \\
\hline FGR12/13 & NI59 & $\odot . \odot \odot \mathrm{E}+\odot \odot$ & $4.70 \mathrm{E}-10$ & $7.80 \mathrm{E}-11$ & $7.80 \mathrm{E}-11$ & $7.80 \mathrm{E}-11$ & $1.30 \mathrm{E}-10$ \\
\hline original & NI59 & $7.40 \mathrm{E}-10$ & 1. $10 \mathrm{E}-\odot 9$ & $4.30 \mathrm{E}-11$ & $8.70 \mathrm{E}-11$ & $1.10 \mathrm{E}-10$ & $2.20 \mathrm{E}-10$ \\
\hline FGR12/13 & NI63 & $\odot . \odot \odot E+\odot \odot$ & $2.5 \odot E-\odot 9$ & $1.80 \mathrm{E}-10$ & $1.80 \mathrm{E}-10$ & $1.80 \mathrm{E}-10$ & $4.70 \mathrm{E}-10$ \\
\hline original & NI63 & $6.50 \mathrm{E}-13$ & $2.8 \odot E-\odot 9$ & $1.20 \mathrm{E}-10$ & $2.40 \mathrm{E}-10$ & $2.40 \mathrm{E}-10$ & $5.40 \mathrm{E}-10$ \\
\hline FGR12/13 & NI65 & $8.4 \odot E-\odot 7$ & $3.20 \mathrm{E}-10$ & $2.30 \mathrm{E}-12$ & $3.0 \odot \mathrm{E}-12$ & $2.20 \mathrm{E}-12$ & $8.50 \mathrm{E}-11$ \\
\hline original & NI65 & 1. $\odot \odot E-\odot 6$ & 4. $\odot \odot E-1 \odot$ & $2.60 \mathrm{E}-12$ & $3.80 \mathrm{E}-12$ & $2.40 \mathrm{E}-12$ & $6.30 \mathrm{E}-11$ \\
\hline
\end{tabular}




\begin{tabular}{|c|c|c|c|c|c|c|c|}
\hline FGR12/13 & CU64 & $2.70 \mathrm{E}-07$ & $2.10 \mathrm{E}-11$ & $9.20 \mathrm{E}-12$ & $9.20 \mathrm{E}-12$ & $8.90 \mathrm{E}-12$ & $3.50 E-11$ \\
\hline original & CU64 & $3.60 \mathrm{E}-07$ & $2.00 \mathrm{E}-10$ & $2.30 \mathrm{E}-11$ & $1.40 \mathrm{E}-11$ & $1.20 \mathrm{E}-11$ & $5.30 \mathrm{E}-11$ \\
\hline FGR12/13 & ZN65 & $8.60 \mathrm{E}-07$ & 1. $.0 \mathrm{E}-\odot 8$ & $7.90 \mathrm{E}-10$ & 1. $\odot \odot E-\odot 9$ & $7.90 \mathrm{E}-10$ & 2. $\odot \odot E-\odot 9$ \\
\hline original & ZN65 & $1.10 \mathrm{E}-06$ & $2.10 \mathrm{E}-\odot 8$ & $3.30 E-\odot 9$ & $3.60 \mathrm{E}-\odot 9$ & $3.10 E-09$ & 4. . $90 E-09$ \\
\hline FGR12/13 & ZN69M & $5.8 \odot E-07$ & 1. $30 \mathrm{E}-\odot 9$ & 8. $00 \mathrm{E}-12$ & $1.20 \mathrm{E}-11$ & $5.70 \mathrm{E}-12$ & $2.70 \mathrm{E}-10$ \\
\hline original & ZN69M & $9.20 \mathrm{E}-07$ & 1. $\odot \odot E-\odot 9$ & $2.60 \mathrm{E}-11$ & $3.30 \mathrm{E}-11$ & 1. $40 \mathrm{E}-11$ & $2.40 \mathrm{E}-10$ \\
\hline FGR12/13 & ZN69 & $6.30 \mathrm{E}-\odot 9$ & 1. $20 \mathrm{E}-10$ & $2.20 \mathrm{E}-15$ & $2.20 \mathrm{E}-15$ & $1.70 \mathrm{E}-15$ & $2.80 \mathrm{E}-11$ \\
\hline original & ZN69 & $1.30 \mathrm{E}-\odot 9$ & $8.10 \mathrm{E}-11$ & $4.50 \mathrm{E}-14$ & $5.90 \mathrm{E}-14$ & $2.90 \mathrm{E}-14$ & $1.10 \mathrm{E}-11$ \\
\hline FGR12/13 & AS76 & $6.50 \mathrm{E}-07$ & $3.4 \odot E-\odot 9$ & $3.40 \mathrm{E}-11$ & $3.80 \mathrm{E}-11$ & $3.20 \mathrm{E}-11$ & $7.40 \mathrm{E}-10$ \\
\hline original & AS76 & $8.60 \mathrm{E}-07$ & $5.20 \mathrm{E}-\odot 9$ & $4.20 \mathrm{E}-11$ & $6.70 \mathrm{E}-11$ & $5.00 \mathrm{E}-11$ & 1. ๑०E-๑9 \\
\hline FGR12/13 & SE75 & $5.30 \mathrm{E}-07$ & $5.30 \mathrm{E}-\odot 9$ & $5.20 \mathrm{E}-10$ & $4.30 \mathrm{E}-10$ & $3.20 \mathrm{E}-10$ & $1.10 E-09$ \\
\hline original & SE75 & $6.10 \mathrm{E}-07$ & $5.30 \mathrm{E}-\odot 9$ & $1.10 \mathrm{E}-09$ & 1. $40 \mathrm{E}-\odot 9$ & $8.70 \mathrm{E}-10$ & $2.10 \mathrm{E}-09$ \\
\hline FGR12/13 & SE79 & $1.20 \mathrm{E}-10$ & 1. $90 \mathrm{E}-\odot 8$ & $1.40 \mathrm{E}-10$ & $1.40 \mathrm{E}-10$ & $1.40 \mathrm{E}-10$ & $2.60 \mathrm{E}-\odot 9$ \\
\hline original & SE79 & $1.10 \mathrm{E}-11$ & $9.30 \mathrm{E}-\odot 9$ & $3.10 \mathrm{E}-10$ & $6.10 \mathrm{E}-10$ & $6.10 \mathrm{E}-10$ & $2.40 E-09$ \\
\hline FGR12/13 & BR82 & $3.80 E-06$ & $1.70 \mathrm{E}-10$ & $1.70 \mathrm{E}-10$ & $1.60 \mathrm{E}-10$ & $1.70 \mathrm{E}-10$ & $3.50 \mathrm{E}-10$ \\
\hline original & BR82 & $4.80 E-06$ & $7.40 \mathrm{E}-10$ & $1.00 \mathrm{E}-10$ & $2.40 \mathrm{E}-10$ & $2.50 \mathrm{E}-10$ & $3.30 \mathrm{E}-10$ \\
\hline FGR12/13 & BR83 & $1.70 \mathrm{E}-\odot 8$ & $2.50 \mathrm{E}-11$ & $2.90 \mathrm{E}-12$ & $2.90 \mathrm{E}-12$ & $2.90 \mathrm{E}-12$ & $1.60 \mathrm{E}-11$ \\
\hline original & BR83 & $1.50 \mathrm{E}-\odot 8$ & $1.60 \mathrm{E}-10$ & 1.90E-12 & $3.70 \mathrm{E}-12$ & $3.80 \mathrm{E}-12$ & $2.60 \mathrm{E}-11$ \\
\hline FGR12/13 & BR84 & $2.80 \mathrm{E}-\odot 6$ & $3.00 \mathrm{E}-11$ & $3.40 \mathrm{E}-12$ & $3.30 \mathrm{E}-12$ & $3.50 \mathrm{E}-12$ & $2.20 \mathrm{E}-11$ \\
\hline original & BR84 & $3.30 \mathrm{E}-06$ & $1.60 \mathrm{E}-10$ & 1. 80E-12 & 3. $00 \mathrm{E}-12$ & $3.30 \mathrm{E}-12$ & $2.70 \mathrm{E}-11$ \\
\hline${ }^{*}$ FGR12/13 & KR83M & $3.80 \mathrm{E}-11$ & $\odot . \odot \odot E+\odot \odot$ & $\odot . \odot \odot E+\odot \odot$ & $\odot . \odot \odot E+\odot \odot$ & $\odot . \odot \odot E+\odot \odot$ & $\odot . \odot \odot E+\odot \odot$ \\
\hline original & KR83M & $5.20 \mathrm{E}-10$ & $\odot . \odot \odot \mathrm{E}+\odot \odot$ & $\odot . \odot \odot E+\odot \odot$ & $\odot . \odot \odot \mathrm{E}+\odot \odot$ & $\odot . \odot \odot E+\odot \odot$ & $\odot . \odot \odot E+\odot \odot$ \\
\hline *FGR12/13 & KR85M & $2.2 \odot E-\odot 7$ & $\odot . \odot \odot \mathrm{E}+\odot \odot$ & $\odot . \odot \odot E+\odot \odot$ & $\odot . \odot \odot \mathrm{E}+\odot \odot$ & $\odot . \odot \odot E+\odot \odot$ & $\odot . \odot \odot E+\odot \odot$ \\
\hline original & KR85M & $2.30 \mathrm{E}-07$ & $\odot . \odot \odot \mathrm{E}+\odot \odot$ & $\odot . \odot \odot \mathrm{E}+\odot \odot$ & $\odot . \odot \odot \mathrm{E}+\odot \odot$ & $\odot . \odot \odot E+\odot \odot$ & $\odot . \odot \odot E+\odot \odot$ \\
\hline${ }^{*}$ FGR12/13 & KR85 & $7.6 \odot E-\odot 9$ & $\odot . \odot \odot E+\odot \odot$ & $\odot . \odot \odot E+\odot \odot$ & $\odot . \odot \odot \mathrm{E}+\odot \odot$ & $\odot . \odot \odot E+\odot \odot$ & $\odot . \odot \odot E+\odot \odot$ \\
\hline original & KR85 & 4. $80 E-\odot 9$ & $\odot . \odot \odot \mathrm{E}+\odot \odot$ & $\odot . \odot \odot E+\odot \odot$ & $\odot . \odot \odot \mathrm{E}+\odot \odot$ & $\odot . \odot \odot E+\odot \odot$ & $\odot . \odot \odot E+\odot \odot$ \\
\hline${ }^{\star}$ FGR12/13 & KR87 & $1.30 \mathrm{E}-06$ & $\odot . \odot \odot \mathrm{E}+\odot \odot$ & $\odot . \odot \odot E+\odot \odot$ & $\odot . \odot \odot \mathrm{E}+\odot \odot$ & $\odot . \odot \odot E+\odot \odot$ & $\odot . \odot \odot E+\odot \odot$ \\
\hline original & KR87 & $1.70 \mathrm{E}-06$ & $\odot . \odot \odot \mathrm{E}+\odot \odot$ & $\odot . \odot \odot \mathrm{E}+\odot \odot$ & $\odot . \odot \odot \mathrm{E}+\odot \odot$ & $\odot . \odot \odot E+\odot \odot$ & $\odot . \odot ० \mathrm{E}+\odot \odot$ \\
\hline
\end{tabular}




\begin{tabular}{|c|c|c|c|c|c|c|c|}
\hline${ }^{*}$ FGR12/13 & KR88 & $3.10 \mathrm{E}-06$ & $\odot . \odot \odot E+\odot \odot$ & $\odot . \odot \odot E+\odot \odot$ & $\odot . \odot \odot E+\odot \odot$ & $\odot . \odot \odot E+\odot \odot$ & $\odot . \odot \odot \mathrm{E}+\odot \odot$ \\
\hline original & KR88 & $3.6 \odot E-\odot 6$ & $\odot . \odot \odot E+\odot \odot$ & $\odot . \odot \odot E+\odot \odot$ & $\odot . \odot \odot E+\odot \odot$ & $\odot . \odot \odot E+\odot \odot$ & $\odot . \odot \odot \mathrm{E}+\odot \odot$ \\
\hline${ }^{*}$ FGR12/13 & KR89 & $3.5 \odot E-06$ & $\odot . \odot \odot E+\odot \odot$ & $\odot . \odot \odot E+\odot \odot$ & $\odot . \odot \odot E+\odot \odot$ & $\odot . \odot \odot E+\odot \odot$ & $\odot . \odot \odot \mathrm{E}+\odot \odot$ \\
\hline original & KR89 & $3.50 E-06$ & $\odot . \odot \odot E+\odot \odot$ & $\odot . \odot \odot E+\odot \odot$ & $\odot . \odot \odot \mathrm{E}+\odot \odot$ & $\odot . \odot \odot E+\odot \odot$ & $\odot . \odot \odot \mathrm{E}+\odot \odot$ \\
\hline FGR12/13 & RB86 & $1.60 \mathrm{E}-07$ & $7.70 \mathrm{E}-10$ & $2.80 \mathrm{E}-\odot 9$ & $1.40 \mathrm{E}-\odot 9$ & $7.40 \mathrm{E}-10$ & $9.30 \mathrm{E}-10$ \\
\hline original & RB86 & $1.60 \mathrm{E}-07$ & 2. $\odot \odot E-\odot 9$ & $4.60 \mathrm{E}-\odot 9$ & $2.70 \mathrm{E}-\odot 9$ & 1. $40 \mathrm{E}-\odot 9$ & $1.70 \mathrm{E}-\odot 9$ \\
\hline FGR12/13 & RB87 & $1.00 \mathrm{E}-\odot 9$ & $4.10 \mathrm{E}-10$ & $1.60 \mathrm{E}-09$ & $7.80 \mathrm{E}-10$ & $3.90 \mathrm{E}-10$ & $5.0 \odot \mathrm{E}-10$ \\
\hline original & RB87 & $5.50 \mathrm{E}-11$ & $3.50 \mathrm{E}-10$ & $2.50 \mathrm{E}-09$ & $1.4 \odot \mathrm{E}-\odot 9$ & $7.30 \mathrm{E}-10$ & $7.90 \mathrm{E}-10$ \\
\hline FGR12/13 & RB88 & $1.10 \mathrm{E}-06$ & $3.10 \mathrm{E}-11$ & $1.90 \mathrm{E}-12$ & $1.70 \mathrm{E}-12$ & $1.70 \mathrm{E}-12$ & $1.60 \mathrm{E}-11$ \\
\hline original & RB88 & $1.30 \mathrm{E}-06$ & $1.50 \mathrm{E}-10$ & $1.80 \mathrm{E}-12$ & $1.80 \mathrm{E}-12$ & 1. $40 \mathrm{E}-12$ & $2.40 \mathrm{E}-11$ \\
\hline FGR12/13 & RB89 & $3.20 \mathrm{E}-06$ & $2.20 \mathrm{E}-11$ & $2.40 \mathrm{E}-12$ & $1.90 \mathrm{E}-12$ & $1.80 \mathrm{E}-12$ & $1.40 \mathrm{E}-11$ \\
\hline original & RB89 & $3.70 \mathrm{E}-06$ & $7.00 \mathrm{E}-11$ & $2.70 \mathrm{E}-12$ & $2.20 \mathrm{E}-12$ & $1.70 \mathrm{E}-12$ & $1.20 \mathrm{E}-11$ \\
\hline FGR12/13 & SR85 & $7.10 \mathrm{E}-07$ & $2.20 \mathrm{E}-10$ & $7.30 \mathrm{E}-10$ & $7.40 \mathrm{E}-10$ & $2.00 \mathrm{E}-10$ & $3.80 \mathrm{E}-10$ \\
\hline original & SR85 & $9.60 \mathrm{E}-07$ & $3.70 \mathrm{E}-10$ & $9.90 \mathrm{E}-10$ & $8.70 \mathrm{E}-10$ & $2.90 \mathrm{E}-10$ & $4.20 \mathrm{E}-10$ \\
\hline FGR12/13 & SR89 & $1.4 \odot E-\odot 8$ & $2.00 \mathrm{E}-10$ & $5.40 \mathrm{E}-\odot 9$ & $4.30 \mathrm{E}-09$ & $1.80 \mathrm{E}-10$ & 1. $00 \mathrm{E}-\odot 9$ \\
\hline original & SR89 & $4.80 E-\odot 9$ & $1.70 \mathrm{E}-09$ & 8. 3०E-०9 & $5.20 \mathrm{E}-09$ & $2.10 \mathrm{E}-10$ & $1.50 \mathrm{E}-09$ \\
\hline FGR12/13 & SR90 & $3.10 \mathrm{E}-09$ & $6.20 \mathrm{E}-10$ & $3.70 \mathrm{E}-07$ & $1.6 \odot \mathrm{E}-\odot 7$ & $6.00 \mathrm{E}-10$ & $2.40 \mathrm{E}-08$ \\
\hline original & SR90 & $3.50 \mathrm{E}-10$ & $1.10 \mathrm{E}-\odot 9$ & $6.50 \mathrm{E}-07$ & $2.90 \mathrm{E}-07$ & $3.40 \mathrm{E}-10$ & $5.50 \mathrm{E}-\odot 8$ \\
\hline FGR12/13 & SR91 & $1.00 \mathrm{E}-06$ & $5.20 \mathrm{E}-11$ & $1.40 \mathrm{E}-10$ & $1.30 \mathrm{E}-10$ & $2.60 \mathrm{E}-11$ & $1.60 \mathrm{E}-10$ \\
\hline original & SR91 & $1.20 \mathrm{E}-06$ & $9.00 \mathrm{E}-10$ & $1.10 \mathrm{E}-10$ & $1.10 \mathrm{E}-10$ & $3.60 \mathrm{E}-11$ & $2.60 \mathrm{E}-10$ \\
\hline FGR12/13 & SR92 & $2 . \odot \odot E-\odot 6$ & $3.70 \mathrm{E}-11$ & $8.90 \mathrm{E}-11$ & $6.10 \mathrm{E}-11$ & $1.60 \mathrm{E}-11$ & $9.80 \mathrm{E}-11$ \\
\hline original & SR92 & $2.60 \mathrm{E}-06$ & $7.10 \mathrm{E}-10$ & $3.10 \mathrm{E}-11$ & $3.00 \mathrm{E}-11$ & $2.00 \mathrm{E}-11$ & $1.80 \mathrm{E}-10$ \\
\hline FGR12/13 & Y 90 & $2.50 \mathrm{E}-08$ & $7.80 \mathrm{E}-\odot 9$ & $1.20 \mathrm{E}-12$ & $1.20 \mathrm{E}-12$ & $4.20 \mathrm{E}-14$ & $1.50 \mathrm{E}-\odot 9$ \\
\hline original & Y 90 & $1.30 \mathrm{E}-08$ & $9.70 \mathrm{E}-\odot 9$ & $1.60 \mathrm{E}-11$ & $1.60 \mathrm{E}-11$ & $5.50 \mathrm{E}-13$ & $2.40 \mathrm{E}-\odot 9$ \\
\hline FGR12/13 & Y 91M & $7.50 \mathrm{E}-07$ & $5.00 \mathrm{E}-11$ & $7.20 \mathrm{E}-13$ & $9.40 \mathrm{E}-13$ & $6.80 \mathrm{E}-13$ & $1.10 \mathrm{E}-11$ \\
\hline original & Y 91M & $1.10 \mathrm{E}-06$ & $7.10 \mathrm{E}-11$ & $6.30 \mathrm{E}-13$ & $8.00 \mathrm{E}-13$ & $5.10 \mathrm{E}-13$ & $9.90 \mathrm{E}-12$ \\
\hline FGR12/13 & Y 91 & $2 . \odot \odot E-๑ 8$ & 7. ๑०E-๑8 & $6.40 \mathrm{E}-11$ & $6.40 \mathrm{E}-11$ & $2.80 \mathrm{E}-12$ & $8.90 \mathrm{E}-\odot 9$ \\
\hline original & Y 91 & $1.10 \mathrm{E}-08$ & $9.90 \mathrm{E}-08$ & $3.20 \mathrm{E}-10$ & $3.10 \mathrm{E}-10$ & $8.50 \mathrm{E}-12$ & $1.30 \mathrm{E}-08$ \\
\hline FGR12/13 & Y 92 & $4.20 E-07$ & 7.00E-10 & $1.30 \mathrm{E}-12$ & $1.80 \mathrm{E}-12$ & $1.10 \mathrm{E}-12$ & $1.80 \mathrm{E}-10$ \\
\hline
\end{tabular}




\begin{tabular}{|c|c|c|c|c|c|c|c|}
\hline original & Y 92 & $5.10 \mathrm{E}-07$ & 1. $30 \mathrm{E}-\odot 9$ & $1.60 \mathrm{E}-12$ & $2.30 \mathrm{E}-12$ & $1.10 \mathrm{E}-12$ & $2.20 \mathrm{E}-10$ \\
\hline FGR12/13 & Y 93 & 1. $7 \odot \mathrm{E}-\odot 7$ & 1. $60 \mathrm{E}-\odot 9$ & 1. $30 \mathrm{E}-12$ & 1. $80 \mathrm{E}-12$ & $9.00 \mathrm{E}-13$ & $4.20 E-10$ \\
\hline original & Y 93 & $1.80 \mathrm{E}-07$ & $2.7 \odot E-\odot 9$ & $3.40 \mathrm{E}-12$ & $4.40 \mathrm{E}-12$ & $9.60 \mathrm{E}-13$ & $6.30 \mathrm{E}-10$ \\
\hline FGR12/13 & ZR93 & $\odot . \odot \odot \mathrm{E}+\odot \odot$ & $2.80 \mathrm{E}-\odot 9$ & $5 . \odot \odot E-\odot 7$ & 4. $\odot \odot E-\odot 8$ & $3.50 \mathrm{E}-12$ & 1. $\odot \odot E-\odot 8$ \\
\hline original & ZR93 & $4.80 E-13$ & 3. 30E- $\odot 9$ & $5.40 \mathrm{E}-\odot 7$ & 4. $4 \odot E-\odot 8$ & $2.80 \mathrm{E}-12$ & $2.20 E-08$ \\
\hline FGR12/13 & ZR95 & $1.10 \mathrm{E}-06$ & $3.10 \mathrm{E}-\odot 8$ & $1.30 \mathrm{E}-\odot 8$ & $2.4 \odot \mathrm{E}-\odot 9$ & $6.50 \mathrm{E}-10$ & $4.80 E-\odot 9$ \\
\hline original & ZR95 & $1.30 \mathrm{E}-06$ & 1. $80 \mathrm{E}-\odot 8$ & $2.30 \mathrm{E}-\odot 8$ & $3.30 E-09$ & $7.70 \mathrm{E}-10$ & $4.20 E-\odot 9$ \\
\hline FGR12/13 & ZR97 & $2.80 \mathrm{E}-\odot 7$ & $3.40 \mathrm{E}-\odot 9$ & $6.40 \mathrm{E}-11$ & $7.80 E-11$ & $2.80 \mathrm{E}-11$ & $9.20 \mathrm{E}-10$ \\
\hline original & ZR97 & $3.40 \mathrm{E}-07$ & 4. $\odot \odot E-\odot 9$ & $1.30 \mathrm{E}-10$ & $1.50 \mathrm{E}-10$ & $3.80 \mathrm{E}-11$ & $1.20 \mathrm{E}-\odot 9$ \\
\hline FGR12/13 & NB93M & $9.60 \mathrm{E}-11$ & 1. $4 \odot \mathrm{E}-\odot 8$ & $5.40 \mathrm{E}-11$ & $2.00 \mathrm{E}-11$ & 4. $70 \mathrm{E}-12$ & $1.80 \mathrm{E}-\odot 9$ \\
\hline original & NB93M & $2.90 \mathrm{E}-10$ & $6.70 \mathrm{E}-\odot 8$ & $2.20 \mathrm{E}-10$ & $2.4 \odot \mathrm{E}-11$ & $2.40 \mathrm{E}-12$ & $8.10 \mathrm{E}-\odot 9$ \\
\hline FGR12/13 & NB94 & $2.30 \mathrm{E}-06$ & $3.20 \mathrm{E}-\odot 7$ & $1.20 \mathrm{E}-\odot 8$ & $1.50 E-\odot 8$ & $1.20 \mathrm{E}-\odot 8$ & 4. $90 \mathrm{E}-\odot 8$ \\
\hline original & NB94 & $2.60 \mathrm{E}-06$ & $7.4 \odot \mathrm{E}-\odot 7$ & $1.60 \mathrm{E}-\odot 8$ & $1.80 \mathrm{E}-\odot 8$ & 1. $8 \odot E-\odot 8$ & 1. $\odot \odot E-\odot 7$ \\
\hline FGR12/13 & NB95M & $8.60 \mathrm{E}-08$ & $6.20 \mathrm{E}-09$ & $3.40 \mathrm{E}-11$ & $3.80 E-11$ & $2.60 \mathrm{E}-11$ & $8.80 \mathrm{E}-10$ \\
\hline original & NB95M & 1. $\odot \odot E-\odot 7$ & $3.10 \mathrm{E}-\odot 9$ & $6.40 \mathrm{E}-11$ & $5.70 \mathrm{E}-11$ & $3.70 \mathrm{E}-11$ & $6.60 \mathrm{E}-10$ \\
\hline FGR12/13 & NB95 & $1.10 \mathrm{E}-06$ & $1.20 \mathrm{E}-\odot 8$ & $2.50 \mathrm{E}-10$ & $3.40 \mathrm{E}-10$ & $2.30 \mathrm{E}-10$ & $1.80 \mathrm{E}-\odot 9$ \\
\hline original & NB95 & $1.50 \mathrm{E}-\odot 6$ & $8.4 \odot E-\odot 9$ & $5.20 \mathrm{E}-10$ & $4.50 \mathrm{E}-10$ & $3.60 \mathrm{E}-10$ & $1.5 \odot E-\odot 9$ \\
\hline${ }^{*} \mathrm{FGR} 12 / 13$ & NB97M & $1 . \odot \odot E-\odot 6$ & $9.90 \mathrm{E}-99$ & $9.90 \mathrm{E}-99$ & $9.90 \mathrm{E}-99$ & $9.90 \mathrm{E}-99$ & $9.90 \mathrm{E}-99$ \\
\hline original & NB97M & $1.10 \mathrm{E}-06$ & $2.40 \mathrm{E}-12$ & $2.30 \mathrm{E}-14$ & $3.10 \mathrm{E}-14$ & $2.80 \mathrm{E}-14$ & $3.50 E-13$ \\
\hline FGR12/13 & NB97 & $9.40 \mathrm{E}-07$ & $1.70 \mathrm{E}-10$ & $1.20 \mathrm{E}-12$ & $1.60 \mathrm{E}-12$ & $1.10 \mathrm{E}-12$ & $4.50 \mathrm{E}-11$ \\
\hline original & NB97 & $1.20 \mathrm{E}-06$ & $1.60 \mathrm{E}-10$ & $8.60 \mathrm{E}-13$ & $1.20 \mathrm{E}-12$ & $9.50 \mathrm{E}-13$ & $2.30 E-11$ \\
\hline FGR12/13 & M093 & $5.50 \mathrm{E}-10$ & $8.20 \mathrm{E}-11$ & $2.00 \mathrm{E}-\odot 8$ & $6.00 \mathrm{E}-\odot 9$ & $5.70 \mathrm{E}-11$ & 1. $\odot \odot E-\odot 9$ \\
\hline original & M093 & $1.40 \mathrm{E}-\odot 9$ & $6.40 \mathrm{E}-11$ & $1.10 \mathrm{E}-\odot 9$ & $2.20 \mathrm{E}-10$ & $7.30 \mathrm{E}-11$ & $2.70 \mathrm{E}-10$ \\
\hline FGR12/13 & M099 & $2.20 \mathrm{E}-07$ & $1.20 \mathrm{E}-10$ & $3.30 \mathrm{E}-10$ & $2.00 \mathrm{E}-10$ & $8.40 \mathrm{E}-11$ & $2.20 \mathrm{E}-10$ \\
\hline original & M099 & $2.50 \mathrm{E}-07$ & $1.10 \mathrm{E}-\odot 9$ & $6.60 \mathrm{E}-10$ & $4.10 \mathrm{E}-10$ & $1.20 \mathrm{E}-10$ & $5.40 \mathrm{E}-10$ \\
\hline FGR12/13 & TC99M & $1.7 \odot E-\odot 7$ & $7.60 \mathrm{E}-11$ & $2.30 \mathrm{E}-12$ & $1.70 \mathrm{E}-12$ & $5.50 \mathrm{E}-12$ & $1.90 \mathrm{E}-11$ \\
\hline original & TC99M & $1.6 \odot \mathrm{E}-\odot 7$ & $3.10 \mathrm{E}-11$ & $1.50 \mathrm{E}-12$ & $2.50 \mathrm{E}-12$ & $2.20 \mathrm{E}-11$ & $7.50 E-12$ \\
\hline FGR12/13 & TC99 & $9.10 \mathrm{E}-10$ & $3.20 \mathrm{E}-\odot 8$ & $9.20 \mathrm{E}-12$ & $9.20 \mathrm{E}-12$ & $2.40 \mathrm{E}-10$ & 4. $\odot \odot E-\odot 9$ \\
\hline original & TC99 & $6.60 \mathrm{E}-11$ & $1.60 \mathrm{E}-\odot 8$ & $2.10 \mathrm{E}-11$ & 4. $00 \mathrm{E}-11$ & 4. . $90 \mathrm{E}-\odot 9$ & $2.30 E-\odot 9$ \\
\hline
\end{tabular}




\begin{tabular}{|c|c|c|c|c|c|c|c|}
\hline FGR12/13 & TC101 & $4.80 E-07$ & $3.40 \mathrm{E}-11$ & $1.80 \mathrm{E}-13$ & $1.70 \mathrm{E}-13$ & $1.20 \mathrm{E}-12$ & 1. $20 \mathrm{E}-11$ \\
\hline original & TC101 & $7 . \odot \odot E-\odot 7$ & $3.10 \mathrm{E}-11$ & $1.40 \mathrm{E}-13$ & $1.90 \mathrm{E}-13$ & $2.50 \mathrm{E}-12$ & $4.10 \mathrm{E}-12$ \\
\hline FGR12/13 & RU103 & $6.60 \mathrm{E}-07$ & $2.20 \mathrm{E}-\odot 8$ & $1.70 \mathrm{E}-10$ & $2.40 \mathrm{E}-10$ & $1.60 \mathrm{E}-10$ & 3. ๑०E-๑9 \\
\hline original & RU103 & $9.4 \odot \mathrm{E}-07$ & 1. $60 \mathrm{E}-\odot 8$ & $2.20 \mathrm{E}-10$ & $3.10 \mathrm{E}-10$ & $2.50 \mathrm{E}-10$ & $2.50 E-\odot 9$ \\
\hline FGR12/13 & RU105 & $1.10 \mathrm{E}-06$ & $8.00 \mathrm{E}-10$ & $5.60 \mathrm{E}-12$ & $7.90 \mathrm{E}-12$ & $4.50 \mathrm{E}-12$ & $1.80 \mathrm{E}-10$ \\
\hline original & RU105 & $1.4 \odot E-\odot 6$ & $7.00 \mathrm{E}-10$ & $4.90 \mathrm{E}-12$ & $8.50 \mathrm{E}-12$ & $4.40 \mathrm{E}-12$ & $1.60 \mathrm{E}-10$ \\
\hline FGR12/13 & RU106 & $\odot . \odot \odot \mathrm{E}+\odot \odot$ & $5.30 \mathrm{E}-07$ & $5.40 \mathrm{E}-10$ & $6.70 \mathrm{E}-10$ & $5.40 \mathrm{E}-10$ & $6.60 \mathrm{E}-08$ \\
\hline original & RU106 & $4.20 \mathrm{E}-07$ & 1. $\odot \odot E-\odot 6$ & $6.40 \mathrm{E}-10$ & 1. $10 \mathrm{E}-\odot 9$ & $1 . \odot \odot E-\odot 9$ & 1. $30 \mathrm{E}-07$ \\
\hline FGR12/13 & RH103M & $1.90 \mathrm{E}-10$ & $1.80 \mathrm{E}-11$ & 3. $00 \mathrm{E}-15$ & $1.30 \mathrm{E}-15$ & $7.10 \mathrm{E}-16$ & $2.70 \mathrm{E}-12$ \\
\hline original & RH103M & 1. $30 \mathrm{E}-09$ & $9.90 \mathrm{E}-12$ & $2.60 \mathrm{E}-15$ & $3.80 \mathrm{E}-15$ & $1.60 \mathrm{E}-15$ & 1. $40 \mathrm{E}-12$ \\
\hline FGR12/13 & RH105 & $1.10 \mathrm{E}-07$ & $2.30 \mathrm{E}-\odot 9$ & $3.90 \mathrm{E}-12$ & $4.60 \mathrm{E}-12$ & $2.60 \mathrm{E}-12$ & $3.50 \mathrm{E}-10$ \\
\hline original & RH105 & $1.60 \mathrm{E}-07$ & 1. $\odot \odot E-\odot 9$ & $3.90 \mathrm{E}-12$ & $7.80 \mathrm{E}-12$ & $3.10 \mathrm{E}-12$ & $3.0 \odot \mathrm{E}-1 \odot$ \\
\hline FGR12/13 & PD103 & 1. $70 \mathrm{E}-\odot 9$ & $3.40 \mathrm{E}-\odot 9$ & $4.10 \mathrm{E}-12$ & 1. $40 \mathrm{E}-12$ & $1.20 \mathrm{E}-13$ & $4.50 \mathrm{E}-10$ \\
\hline original & PD103 & 1. $10 \mathrm{E}-\odot 8$ & $2.7 \odot E-\odot 9$ & $4.70 \mathrm{E}-12$ & $7.10 \mathrm{E}-12$ & $1.50 \mathrm{E}-13$ & $4.30 E-10$ \\
\hline FGR12/13 & PD107 & $\odot . \odot \odot E+\odot \odot$ & 4. $80 \mathrm{E}-\odot 9$ & $4.30 \mathrm{E}-13$ & $1.60 \mathrm{E}-13$ & $2.90 \mathrm{E}-14$ & $5.90 \mathrm{E}-10$ \\
\hline original & PD107 & $2.20 \mathrm{E}-14$ & $2.90 \mathrm{E}-\odot 8$ & $3.70 \mathrm{E}-13$ & $1.40 \mathrm{E}-13$ & $2.60 \mathrm{E}-14$ & $3.50 E-09$ \\
\hline FGR12/13 & PD109 & $1.30 \mathrm{E}-08$ & $2.20 \mathrm{E}-\odot 9$ & $5.40 \mathrm{E}-13$ & $2.50 \mathrm{E}-13$ & $8.10 \mathrm{E}-14$ & $3.70 \mathrm{E}-10$ \\
\hline original & PD109 & 1. $10 \mathrm{E}-08$ & $1.50 \mathrm{E}-\odot 9$ & 1. $0 \odot \mathrm{E}-12$ & $1.60 \mathrm{E}-12$ & $2.20 \mathrm{E}-13$ & $3.70 \mathrm{E}-10$ \\
\hline FGR12/13 & AG110M & $4 . \odot \odot E-\odot 6$ & $4.60 \mathrm{E}-\odot 9$ & $3.20 \mathrm{E}-\odot 9$ & $3.60 \mathrm{E}-\odot 9$ & $2.30 E-09$ & $5.50 E-\odot 9$ \\
\hline original & AG110M & $4.90 E-06$ & 1. $10 \mathrm{E}-08$ & $3.30 \mathrm{E}-\odot 9$ & 4. $90 \mathrm{E}-\odot 9$ & $3.10 \mathrm{E}-09$ & $2.30 \mathrm{E}-\odot 8$ \\
\hline FGR12/13 & AG111 & $4.4 \odot E-\odot 8$ & $1.10 \mathrm{E}-10$ & $8.90 \mathrm{E}-11$ & $8.90 \mathrm{E}-11$ & $8.70 \mathrm{E}-11$ & 4. $00 \mathrm{E}-10$ \\
\hline original & AG111 & $5.10 \mathrm{E}-08$ & 1. $\odot \odot E-\odot 9$ & $5.10 \mathrm{E}-11$ & $9.00 \mathrm{E}-11$ & $7.50 \mathrm{E}-11$ & $9.20 \mathrm{E}-10$ \\
\hline FGR12/13 & CD109 & $7.20 \mathrm{E}-\odot 9$ & 1. $6 \odot \mathrm{E}-\odot 9$ & 1. $90 \mathrm{E}-\odot 9$ & 1. $30 \mathrm{E}-\odot 9$ & $1.40 \mathrm{E}-\odot 9$ & $8.20 E-\odot 9$ \\
\hline original & CD109 & 1. $\odot \odot E-\odot 8$ & $7.80 \mathrm{E}-10$ & $1.20 \mathrm{E}-\odot 9$ & $2.7 \odot E-\odot 9$ & $2.50 E-\odot 9$ & $3 . \odot \odot E-\odot 8$ \\
\hline FGR12/13 & CD113M & $2.9 \odot E-\odot 9$ & 1. $70 \mathrm{E}-\odot 8$ & 1. $70 \mathrm{E}-\odot 8$ & 1. $70 \mathrm{E}-\odot 8$ & 1. $70 \mathrm{E}-08$ & $1.10 \mathrm{E}-07$ \\
\hline original & CD113M & $4.10 \mathrm{E}-10$ & $5.80 \mathrm{E}-10$ & $1.20 \mathrm{E}-\odot 8$ & $2.90 \mathrm{E}-\odot 8$ & 3. ๑०E-๑8 & 4. $\odot \odot E-\odot 7$ \\
\hline FGR12/13 & CD115M & $4.70 \mathrm{E}-\odot 8$ & $8.60 \mathrm{E}-10$ & $8.30 \mathrm{E}-10$ & $8.40 \mathrm{E}-10$ & $8.20 \mathrm{E}-10$ & $5.30 E-09$ \\
\hline original & CD115M & $4.50 E-08$ & 1. $90 \mathrm{E}-\odot 9$ & $8.20 \mathrm{E}-10$ & 1. $60 \mathrm{E}-\odot 9$ & $1.60 \mathrm{E}-09$ & 1. $90 \mathrm{E}-08$ \\
\hline
\end{tabular}




\begin{tabular}{|c|c|c|c|c|c|c|c|}
\hline FGR12/13 & CD115 & $3.30 \mathrm{E}-07$ & $8.60 \mathrm{E}-11$ & $6.40 \mathrm{E}-11$ & $6.80 \mathrm{E}-11$ & $5.20 \mathrm{E}-11$ & $3.60 \mathrm{E}-10$ \\
\hline original & CD115 & $3.80 \mathrm{E}-07$ & $1.10 \mathrm{E}-09$ & $9.00 \mathrm{E}-11$ & $1.20 \mathrm{E}-10$ & $8.70 \mathrm{E}-11$ & 1. $\odot \odot E-\odot 9$ \\
\hline FGR12/13 & IN111 & $5.30 \mathrm{E}-07$ & $4.60 \mathrm{E}-11$ & $1.20 \mathrm{E}-10$ & $1.60 \mathrm{E}-10$ & $3.00 \mathrm{E}-11$ & $1.30 \mathrm{E}-10$ \\
\hline original & IN111 & $5.5 \odot E-\odot 7$ & $1.90 \mathrm{E}-10$ & $1.10 \mathrm{E}-10$ & $9.80 \mathrm{E}-11$ & $1.90 \mathrm{E}-11$ & $1.50 \mathrm{E}-10$ \\
\hline FGR12/13 & IN114M & $1.20 \mathrm{E}-\odot 7$ & $1.60 \mathrm{E}-09$ & $2.30 \mathrm{E}-\odot 8$ & 4. $30 \mathrm{E}-\odot 8$ & $1.50 \mathrm{E}-09$ & $9.30 \mathrm{E}-09$ \\
\hline original & IN114M & $2.10 \mathrm{E}-07$ & $2.80 \mathrm{E}-\odot 9$ & 1. 80E- - 88 & $6.10 \mathrm{E}-09$ & $8.00 \mathrm{E}-11$ & $1.20 E-08$ \\
\hline FGR12/13 & IN115M & $2.20 \mathrm{E}-07$ & $1.60 \mathrm{E}-11$ & $1.00 \mathrm{E}-11$ & $1.60 \mathrm{E}-11$ & $2.90 \mathrm{E}-12$ & $2.40 \mathrm{E}-11$ \\
\hline original & IN115M & $3.10 \mathrm{E}-07$ & $1.30 \mathrm{E}-10$ & $6.50 \mathrm{E}-12$ & $5.10 \mathrm{E}-12$ & $2.50 \mathrm{E}-12$ & $3.30 \mathrm{E}-11$ \\
\hline FGR12/13 & SN117M & $1.90 \mathrm{E}-07$ & 1. $90 \mathrm{E}-\odot 8$ & $9.50 \mathrm{E}-10$ & $1.10 \mathrm{E}-10$ & $2.40 \mathrm{E}-11$ & $2.40 \mathrm{E}-\odot 9$ \\
\hline original & SN117M & $1.70 \mathrm{E}-07$ & $6.20 \mathrm{E}-09$ & $2.20 \mathrm{E}-\odot 9$ & $2.70 \mathrm{E}-10$ & $3.10 \mathrm{E}-11$ & $1.30 \mathrm{E}-09$ \\
\hline FGR12/13 & SN119M & $2.20 \mathrm{E}-\odot 9$ & $1.70 \mathrm{E}-\odot 8$ & $8.00 \mathrm{E}-10$ & $2.70 \mathrm{E}-10$ & $3.90 \mathrm{E}-11$ & $2.20 E-\odot 9$ \\
\hline original & SN119M & $7.20 \mathrm{E}-\odot 9$ & $1.10 \mathrm{E}-08$ & $1.10 \mathrm{E}-\odot 9$ & $4.70 \mathrm{E}-10$ & $4.20 \mathrm{E}-11$ & $1.70 \mathrm{E}-09$ \\
\hline FGR12/13 & SN121M & $1.70 \mathrm{E}-\odot 9$ & $3.40 \mathrm{E}-\odot 8$ & $3.20 \mathrm{E}-\odot 9$ & $1.10 \mathrm{E}-\odot 9$ & $1.50 \mathrm{E}-10$ & $4.50 E-09$ \\
\hline original & SN121M & $\odot . \odot \odot E+\odot \odot$ & $2.00 \mathrm{E}-\odot 8$ & $3.90 \mathrm{E}-\odot 9$ & $1.50 \mathrm{E}-\odot 9$ & $1.60 \mathrm{E}-10$ & $3.10 \mathrm{E}-09$ \\
\hline FGR12/13 & SN121 & $1.20 \mathrm{E}-\odot 9$ & $1.50 \mathrm{E}-\odot 9$ & $4.20 \mathrm{E}-11$ & $3.60 \mathrm{E}-12$ & $3.30 \mathrm{E}-13$ & $2.30 \mathrm{E}-10$ \\
\hline original & SN121 & $\odot . \odot \odot E+\odot \odot$ & $5.30 \mathrm{E}-10$ & $8.50 \mathrm{E}-12$ & $3.60 \mathrm{E}-12$ & $8.80 E-13$ & $1.50 \mathrm{E}-10$ \\
\hline FGR12/13 & SN123 & $2.20 \mathrm{E}-08$ & $6.20 \mathrm{E}-08$ & $2.30 \mathrm{E}-\odot 9$ & $8.50 \mathrm{E}-10$ & $1.10 \mathrm{E}-10$ & $8.10 E-09$ \\
\hline original & SN123 & $1.50 \mathrm{E}-08$ & $6.10 \mathrm{E}-\odot 8$ & $3.80 E-\odot 9$ & 1. $40 \mathrm{E}-\odot 9$ & $1.70 \mathrm{E}-10$ & $9.0 \odot \mathrm{E}-\odot 9$ \\
\hline FGR12/13 & SN125 & $4.90 \mathrm{E}-07$ & 2. $.00 \mathrm{E}-08$ & $5.80 \mathrm{E}-10$ & $3.50 \mathrm{E}-10$ & $5.00 \mathrm{E}-11$ & $3.10 E-09$ \\
\hline original & SN125 & $5.50 \mathrm{E}-07$ & $2.30 \mathrm{E}-\odot 8$ & $1.10 \mathrm{E}-09$ & $7.30 \mathrm{E}-10$ & $7.70 \mathrm{E}-11$ & $4.60 \mathrm{E}-09$ \\
\hline FGR12/13 & SN126 & $5.80 E-08$ & $1.80 \mathrm{E}-07$ & $2.80 E-08$ & $1.50 \mathrm{E}-08$ & $4.70 \mathrm{E}-09$ & $2.80 E-\odot 8$ \\
\hline original & SN126 & $4.60 \mathrm{E}-\odot 8$ & $1.50 \mathrm{E}-07$ & $3.30 \mathrm{E}-\odot 8$ & 1. $70 \mathrm{E}-\odot 8$ & $4.6 \odot E-\odot 9$ & $2.70 \mathrm{E}-\odot 8$ \\
\hline FGR12/13 & SB124 & $2.70 \mathrm{E}-\odot 6$ & $4.40 \mathrm{E}-\odot 8$ & 1. $90 \mathrm{E}-\odot 9$ & $1.20 \mathrm{E}-\odot 9$ & $6.60 \mathrm{E}-10$ & $6.40 \mathrm{E}-\odot 9$ \\
\hline original & SB124 & $3.60 \mathrm{E}-06$ & 4. 20E- - 8 & $1.30 \mathrm{E}-09$ & $1.10 \mathrm{E}-09$ & $6.80 \mathrm{E}-10$ & $6.80 E-09$ \\
\hline FGR12/13 & SB125 & $5.90 \mathrm{E}-07$ & $3.20 \mathrm{E}-\odot 8$ & 8. 70E-๑9 & $1.70 \mathrm{E}-\odot 9$ & $5.50 \mathrm{E}-10$ & $4.80 E-09$ \\
\hline original & SB125 & $8.40 \mathrm{E}-07$ & $2.20 \mathrm{E}-08$ & 1. $00 \mathrm{E}-09$ & $5.20 \mathrm{E}-10$ & $3.20 \mathrm{E}-10$ & $3.30 \mathrm{E}-09$ \\
\hline FGR12/13 & SB126M & $2.20 \mathrm{E}-\odot 6$ & $4.70 \mathrm{E}-11$ & $9.60 \mathrm{E}-13$ & $1.10 \mathrm{E}-12$ & $9.60 \mathrm{E}-13$ & $1.90 \mathrm{E}-11$ \\
\hline original & SB126M & $2.90 \mathrm{E}-06$ & $5.80 \mathrm{E}-11$ & $7.80 \mathrm{E}-13$ & $9.60 \mathrm{E}-13$ & $7.70 \mathrm{E}-13$ & $8.00 \mathrm{E}-12$ \\
\hline FGR12/13 & SB126 & 4. .०० - ๑6 & 1.7०E- -8 & $6.50 \mathrm{E}-10$ & $6.50 \mathrm{E}-10$ & $3.60 \mathrm{E}-10$ & 2. $90 E-\odot 9$ \\
\hline
\end{tabular}




\begin{tabular}{|c|c|c|c|c|c|c|c|}
\hline original & SB126 & $4.90 E-06$ & $1.4 \odot E-\odot 8$ & $7.00 \mathrm{E}-10$ & $8.00 \mathrm{E}-10$ & $4.80 E-10$ & $3.20 E-09$ \\
\hline FGR12/13 & SB127 & $9.80 \mathrm{E}-07$ & $1.10 \mathrm{E}-\odot 8$ & $1.20 \mathrm{E}-10$ & $1.20 \mathrm{E}-10$ & $4.20 \mathrm{E}-11$ & 1. $70 \mathrm{E}-\odot 9$ \\
\hline original & SB127 & $1.20 \mathrm{E}-06$ & $6.9 \odot E-\odot 9$ & $1.40 \mathrm{E}-10$ & $1.60 \mathrm{E}-10$ & $6.00 \mathrm{E}-11$ & 1. $60 \mathrm{E}-09$ \\
\hline FGR12/13 & TE123M & $1.80 \mathrm{E}-07$ & 3. ๑९E-๑8 & $1.10 \mathrm{E}-08$ & $1.10 \mathrm{E}-09$ & $4.10 \mathrm{E}-10$ & 4. $\odot \odot E-\odot 9$ \\
\hline original & TE123M & $1.60 \mathrm{E}-07$ & 1. $30 \mathrm{E}-\odot 8$ & $2.40 \mathrm{E}-\odot 8$ & $2.40 \mathrm{E}-\odot 9$ & $1.40 \mathrm{E}-10$ & $2.90 E-\odot 9$ \\
\hline FGR12/13 & TE125M & $1.10 \mathrm{E}-08$ & $2.60 \mathrm{E}-\odot 8$ & $4.80 E-\odot 9$ & $4.20 \mathrm{E}-10$ & $2.60 \mathrm{E}-10$ & $3.4 \odot E-\odot 9$ \\
\hline original & TE125M & $2.4 \odot E-\odot 8$ & 1. ๑๑E - ๑८ & $1.20 \mathrm{E}-\odot 8$ & $1.20 \mathrm{E}-\odot 9$ & $4.00 \mathrm{E}-11$ & 2. ๑๑E-๑९ \\
\hline FGR12/13 & TE127M & $3.60 \mathrm{E}-09$ & $5.60 \mathrm{E}-\odot 8$ & $9.0 \odot \mathrm{E}-\odot 9$ & $2.30 \mathrm{E}-\odot 9$ & $8.60 \mathrm{E}-10$ & $7.40 \mathrm{E}-\odot 9$ \\
\hline original & TE127M & $7.50 \mathrm{E}-\odot 9$ & 3. $30 \mathrm{E}-\odot 8$ & $2.10 \mathrm{E}-08$ & $5.40 \mathrm{E}-\odot 9$ & $1.00 \mathrm{E}-10$ & $5.90 \mathrm{E}-\odot 9$ \\
\hline FGR12/13 & TE127 & $1.10 \mathrm{E}-08$ & $7.50 \mathrm{E}-10$ & $1.70 \mathrm{E}-12$ & $1.70 \mathrm{E}-12$ & $2.80 \mathrm{E}-12$ & $1.30 \mathrm{E}-10$ \\
\hline original & TE127 & 1. $10 \mathrm{E}-08$ & $4.30 \mathrm{E}-10$ & $5.10 \mathrm{E}-12$ & $5.80 \mathrm{E}-12$ & $1.90 \mathrm{E}-12$ & $9.70 \mathrm{E}-11$ \\
\hline FGR12/13 & TE129M & $4.90 E-\odot 8$ & $4.80 E-\odot 8$ & $2.70 \mathrm{E}-\odot 9$ & $1.20 \mathrm{E}-09$ & 1. $00 \mathrm{E}-\odot 9$ & $6.50 \mathrm{E}-09$ \\
\hline original & TE129M & $5.90 \mathrm{E}-\odot 8$ & $3.90 \mathrm{E}-\odot 8$ & $7.10 \mathrm{E}-\odot 9$ & $3.10 \mathrm{E}-09$ & $3.10 \mathrm{E}-11$ & 1. $30 \mathrm{E}-07$ \\
\hline FGR12/13 & TE129 & $9.10 \mathrm{E}-08$ & $1.50 \mathrm{E}-10$ & $3.60 \mathrm{E}-13$ & $3.50 \mathrm{E}-13$ & $3.50 \mathrm{E}-13$ & 3. $70 \mathrm{E}-11$ \\
\hline original & TE129 & $1.10 \mathrm{E}-07$ & $1.60 \mathrm{E}-10$ & $9.80 \mathrm{E}-13$ & 1. $00 \mathrm{E}-12$ & $8.60 \mathrm{E}-14$ & 4. $20 \mathrm{E}-11$ \\
\hline FGR12/13 & TE131M & $2.10 \mathrm{E}-06$ & $4.60 \mathrm{E}-\odot 9$ & $1.20 \mathrm{E}-10$ & $8.80 E-11$ & $2.70 \mathrm{E}-\odot 9$ & 1. $10 \mathrm{E}-09$ \\
\hline original & TE131M & $2.60 \mathrm{E}-06$ & $2.20 \mathrm{E}-\odot 9$ & $2.50 \mathrm{E}-10$ & $1.40 \mathrm{E}-10$ & $3.20 E-08$ & $3.20 \mathrm{E}-\odot 9$ \\
\hline FGR12/13 & TE131 & $6.10 \mathrm{E}-07$ & $9.30 \mathrm{E}-11$ & $5.30 \mathrm{E}-13$ & $5.20 \mathrm{E}-13$ & $4.80 \mathrm{E}-11$ & $2.90 \mathrm{E}-11$ \\
\hline original & TE131 & $7.30 \mathrm{E}-07$ & $8.00 \mathrm{E}-11$ & $4.90 \mathrm{E}-13$ & $5.90 \mathrm{E}-13$ & $5.70 \mathrm{E}-10$ & $3.40 E-11$ \\
\hline FGR12/13 & TE132 & $2.90 \mathrm{E}-07$ & 1. $. \odot E-\odot 8$ & $2.90 \mathrm{E}-10$ & $2.20 \mathrm{E}-10$ & $4.30 E-09$ & $2.10 \mathrm{E}-\odot 9$ \\
\hline original & TE132 & $3.80 E-07$ & $1.4 \odot E-\odot 9$ & $4.40 \mathrm{E}-10$ & $8.60 \mathrm{E}-11$ & $4.30 E-08$ & $3.4 \odot E-\odot 9$ \\
\hline FGR12/13 & TE133M & $3.40 E-06$ & $2.60 \mathrm{E}-10$ & $4.40 \mathrm{E}-12$ & $5.50 \mathrm{E}-12$ & $1.60 \mathrm{E}-10$ & 8. $90 \mathrm{E}-11$ \\
\hline original & TE133M & $4.10 \mathrm{E}-06$ & $2.10 \mathrm{E}-10$ & $3.10 \mathrm{E}-12$ & 4. $00 \mathrm{E}-12$ & $2.20 E-\odot 9$ & $1.20 \mathrm{E}-10$ \\
\hline FGR12/13 & TE133 & $1.4 \odot E-\odot 6$ & $5.30 \mathrm{E}-11$ & $5.10 \mathrm{E}-13$ & $5.80 E-13$ & $3.60 \mathrm{E}-11$ & 2. $00 \mathrm{E}-11$ \\
\hline original & TE133 & $1.80 E-06$ & $4.80 E-11$ & $2.80 E-13$ & $3.70 \mathrm{E}-13$ & $5.00 \mathrm{E}-10$ & $2.40 \mathrm{E}-11$ \\
\hline FGR12/13 & TE134 & $1.20 E-06$ & $2.10 \mathrm{E}-10$ & $4.10 \mathrm{E}-12$ & $5.10 \mathrm{E}-12$ & $2.60 \mathrm{E}-11$ & $6.70 \mathrm{E}-11$ \\
\hline original & TE134 & $1.60 \mathrm{E}-06$ & $6.20 \mathrm{E}-11$ & 1. $20 \mathrm{E}-12$ & $1.20 \mathrm{E}-12$ & $3.60 \mathrm{E}-10$ & $2.80 \mathrm{E}-11$ \\
\hline FGR12/13 & I 125 & 1. $20 \mathrm{E}-\odot 8$ & $1.50 \mathrm{E}-11$ & $5.80 \mathrm{E}-11$ & $1.10 \mathrm{E}-11$ & $1.0 \odot \mathrm{E}-\odot 7$ & $5.20 E-09$ \\
\hline original & I 125 & $2.80 E-\odot 8$ & $1.10 \mathrm{E}-10$ & $1.60 \mathrm{E}-10$ & $1.20 \mathrm{E}-10$ & $2.00 \mathrm{E}-\odot 7$ & $6.10 \mathrm{E}-09$ \\
\hline
\end{tabular}




\begin{tabular}{|c|c|c|c|c|c|c|c|}
\hline FGR12/13 & I 129 & $8.90 \mathrm{E}-\odot 9$ & $6.20 \mathrm{E}-11$ & $1.40 \mathrm{E}-10$ & $4.80 E-11$ & $7.2 \odot E-\odot 7$ & $3.60 \mathrm{E}-\odot 8$ \\
\hline original & I 129 & $1.60 \mathrm{E}-08$ & $2.40 \mathrm{E}-10$ & $7.00 \mathrm{E}-10$ & $3.60 \mathrm{E}-10$ & $1.40 \mathrm{E}-06$ & $4.10 E-08$ \\
\hline FGR12/13 & I 130 & $3.10 \mathrm{E}-06$ & $5.60 \mathrm{E}-11$ & $3.60 \mathrm{E}-11$ & $3.30 \mathrm{E}-11$ & $1.30 \mathrm{E}-08$ & $6.80 \mathrm{E}-10$ \\
\hline original & I 130 & $3.70 \mathrm{E}-06$ & $6.20 \mathrm{E}-10$ & $5.40 \mathrm{E}-10$ & $1.90 \mathrm{E}-10$ & $1.60 \mathrm{E}-08$ & $6.60 \mathrm{E}-10$ \\
\hline FGR12/13 & I 131 & $5.30 \mathrm{E}-07$ & $6.00 \mathrm{E}-11$ & $4.90 \mathrm{E}-11$ & $3.70 \mathrm{E}-11$ & $1.50 \mathrm{E}-07$ & 7. $40 \mathrm{E}-\odot 9$ \\
\hline original & I 131 & $6.70 \mathrm{E}-07$ & $6.50 \mathrm{E}-10$ & $2.30 \mathrm{E}-\odot 9$ & $2.70 \mathrm{E}-10$ & $2.70 \mathrm{E}-07$ & $8.30 \mathrm{E}-\odot 9$ \\
\hline FGR12/13 & I 132 & $3.30 E-06$ & $3.60 \mathrm{E}-11$ & $1.20 \mathrm{E}-11$ & 1. $20 \mathrm{E}-11$ & $1.40 \mathrm{E}-09$ & $9.40 \mathrm{E}-11$ \\
\hline original & I 132 & $4.20 E-06$ & $2.80 \mathrm{E}-10$ & $6.90 \mathrm{E}-11$ & $7.00 \mathrm{E}-11$ & $1.30 \mathrm{E}-\odot 9$ & $1.10 \mathrm{E}-10$ \\
\hline FGR12/13 & I 133 & $8.70 E-07$ & $4.20 \mathrm{E}-11$ & $2.00 \mathrm{E}-11$ & $1.90 \mathrm{E}-11$ & $2.90 \mathrm{E}-\odot 8$ & $1.50 E-09$ \\
\hline original & I 133 & $1.10 \mathrm{E}-06$ & $8.30 \mathrm{E}-10$ & $2.90 \mathrm{E}-10$ & $2.20 \mathrm{E}-10$ & $4.30 E-08$ & $1.50 E-09$ \\
\hline FGR12/13 & I 134 & $3.80 E-06$ & $3.00 \mathrm{E}-11$ & $5.80 \mathrm{E}-12$ & $5.50 \mathrm{E}-12$ & $2.60 \mathrm{E}-10$ & $4.50 \mathrm{E}-11$ \\
\hline original & I 134 & $4.70 \mathrm{E}-06$ & $1.50 \mathrm{E}-10$ & $2.80 \mathrm{E}-11$ & $2.80 \mathrm{E}-11$ & $2.00 \mathrm{E}-10$ & $3.80 \mathrm{E}-11$ \\
\hline FGR12/13 & I 135 & $2.4 \odot E-\odot 6$ & 4. $00 \mathrm{E}-11$ & $1.80 \mathrm{E}-11$ & $1.70 \mathrm{E}-11$ & $5.80 E-09$ & $3.20 \mathrm{E}-10$ \\
\hline original & I 135 & $2.90 E-06$ & $4.60 \mathrm{E}-10$ & $1.80 \mathrm{E}-10$ & $1.30 \mathrm{E}-10$ & $6.80 \mathrm{E}-09$ & $3.20 \mathrm{E}-10$ \\
\hline${ }^{*}$ FGR12/13 & XE131M & $1.10 \mathrm{E}-08$ & $\odot . \odot \odot E+\odot \odot$ & $\odot . \odot \odot E+\odot \odot$ & $\odot . \odot \odot E+\odot \odot$ & $\odot . \odot \odot E+\odot \odot$ & $\odot . \odot \odot E+\odot \odot$ \\
\hline original & XE131M & $1.4 \odot E-\odot 8$ & $\odot . \odot \odot \mathrm{E}+\odot \odot$ & $\odot . \odot \odot \mathrm{E}+\odot \odot$ & $\odot . \odot \odot E+\odot \odot$ & $\odot . \odot \odot \mathrm{E}+\odot \odot$ & $\odot . \odot \odot E+\odot \odot$ \\
\hline *FGR12/13 & XE133M & 4.10E- -8 & $\odot . \odot \odot E+\odot \odot$ & $\odot . \odot \odot E+\odot \odot$ & $\odot . \odot \odot E+\odot \odot$ & $\odot . \odot \odot E+\odot \odot$ & $\odot . \odot \odot E+\odot \odot$ \\
\hline original & XE133M & $5.2 \odot E-\odot 8$ & $\odot . \odot \odot \mathrm{E}+\odot \odot$ & $\odot . \odot \odot E+\odot \odot$ & $\odot . \odot \odot E+\odot \odot$ & $\odot . \odot \odot E+\odot \odot$ & $\odot . \odot \odot E+\odot \odot$ \\
\hline *FGR12/13 & XE133 & $4.2 \odot E-\odot 8$ & $\odot . \odot \odot E+\odot \odot$ & $\odot . \odot \odot E+\odot \odot$ & $\odot . \odot \odot E+\odot \odot$ & $\odot . \odot \odot E+\odot \odot$ & $\odot . \odot \odot E+\odot \odot$ \\
\hline original & XE133 & 4. $30 \mathrm{E}-\odot 8$ & $\odot . \odot \odot \mathrm{E}+\odot \odot$ & $\odot . \odot \odot E+\odot \odot$ & $\odot . \odot \odot E+\odot \odot$ & $\odot . \odot \odot E+\odot \odot$ & $\odot . \odot \odot E+\odot \odot$ \\
\hline *FGR12/13 & XE135M & $6 . \odot \odot E-\odot 7$ & $\odot . \odot \odot E+\odot \odot$ & $\odot . \odot \odot E+\odot \odot$ & $\odot . \odot \odot E+\odot \odot$ & $\odot . \odot \odot E+\odot \odot$ & $\odot . \odot \odot E+\odot \odot$ \\
\hline original & XE135M & $7.90 \mathrm{E}-07$ & $\odot . \odot \odot \mathrm{E}+\odot \odot$ & $\odot . \odot \odot E+\odot \odot$ & $\odot . \odot \odot E+\odot \odot$ & $\odot . \odot \odot E+\odot \odot$ & $\odot . \odot \odot E+\odot \odot$ \\
\hline${ }^{*}$ FGR12/13 & XE135 & $3.50 \mathrm{E}-07$ & $\odot . \odot \odot \mathrm{E}+\odot \odot$ & $\odot . \odot \odot \mathrm{E}+\odot \odot$ & $\odot . \odot \odot E+\odot \odot$ & $\odot . \odot \odot \mathrm{E}+\odot \odot$ & $\odot . \odot \odot E+\odot \odot$ \\
\hline original & XE135 & 4. $\odot \odot E-\odot 7$ & $\odot . \odot \odot \mathrm{E}+\odot \odot$ & $\odot . \odot \odot E+\odot \odot$ & $\odot . \odot \odot E+\odot \odot$ & $\odot . \odot \odot E+\odot \odot$ & $\odot . \odot \odot E+\odot \odot$ \\
\hline${ }^{*}$ FGR12/13 & XE137 & $9.90 \mathrm{E}-99$ & $\odot . \odot \odot \mathrm{E}+\odot \odot$ & $\odot . \odot \odot E+\odot \odot$ & $\odot . \odot \odot E+\odot \odot$ & $\odot . \odot \odot E+\odot \odot$ & $\odot . \odot \odot E+\odot \odot$ \\
\hline original & XE137 & $4.30 \mathrm{E}-07$ & $\odot . \odot \odot \mathrm{E}+\odot \odot$ & $\odot . \odot \odot E+\odot \odot$ & $\odot . \odot \odot E+\odot \odot$ & $\odot . \odot \odot E+\odot \odot$ & $\odot . \odot \odot E+\odot \odot$ \\
\hline *FGR12/13 & XE138 & $1.70 \mathrm{E}-06$ & $\odot . \odot \odot E+\odot \odot$ & $\odot . \odot \odot E+\odot \odot$ & $\odot . \odot \odot E+\odot \odot$ & $\odot . \odot \odot E+\odot \odot$ & $\odot . \odot \odot E+\odot \odot$ \\
\hline original & XE138 & $2.20 \mathrm{E}-06$ & $\odot . \odot \odot \mathrm{E}+\odot \odot$ & $\odot . \odot \odot \mathrm{E}+\odot \odot$ & $\odot . \odot \odot E+\odot \odot$ & $\odot . \odot \odot E+\odot \odot$ & $\odot . \odot \odot E+\odot \odot$ \\
\hline
\end{tabular}




\begin{tabular}{|c|c|c|c|c|c|c|c|}
\hline FGR12/13 & CS134M & $2.50 \mathrm{E}-\odot 8$ & $2.40 \mathrm{E}-11$ & $2.80 \mathrm{E}-12$ & $2.40 \mathrm{E}-12$ & $2.40 \mathrm{E}-12$ & $1.40 \mathrm{E}-11$ \\
\hline original & $\mathrm{CS} 134 \mathrm{M}$ & $3.40 \mathrm{E}-\odot 8$ & $6.50 \mathrm{E}-11$ & $9.00 \mathrm{E}-13$ & $1.80 \mathrm{E}-12$ & 1. $70 \mathrm{E}-12$ & $1.0 \odot \mathrm{E}-11$ \\
\hline FGR12/13 & CS134 & $2.20 \mathrm{E}-\odot 6$ & $6 . \odot \odot E-\odot 9$ & $6.80 \mathrm{E}-\odot 9$ & $6.4 \odot E-\odot 9$ & $6.30 \mathrm{E}-\odot 9$ & $6.7 \odot E-\odot 9$ \\
\hline original & CS134 & $2.90 \mathrm{E}-06$ & $8.10 \mathrm{E}-\odot 9$ & $8.30 \mathrm{E}-\odot 9$ & 1. $10 \mathrm{E}-\odot 8$ & 1. $10 \mathrm{E}-\odot 8$ & $1.20 \mathrm{E}-\odot 8$ \\
\hline FGR12/13 & CS135 & $3.00 \mathrm{E}-10$ & $6.70 \mathrm{E}-10$ & $6.50 \mathrm{E}-10$ & $6.50 \mathrm{E}-10$ & $6.50 \mathrm{E}-10$ & $6.90 \mathrm{E}-10$ \\
\hline original & CS135 & $2.40 \mathrm{E}-11$ & $2.10 \mathrm{E}-10$ & $6.00 \mathrm{E}-10$ & $1.20 \mathrm{E}-\odot 9$ & 1. $20 \mathrm{E}-\odot 9$ & $1.10 \mathrm{E}-\odot 9$ \\
\hline FGR12/13 & CS136 & $3.10 \mathrm{E}-06$ & $9.70 \mathrm{E}-10$ & $1.10 \mathrm{E}-\odot 9$ & $9.90 \mathrm{E}-10$ & 1. $\odot \odot E-\odot 9$ & $1.20 \mathrm{E}-\odot 9$ \\
\hline original & CS136 & $3.70 \mathrm{E}-06$ & 1. $90 \mathrm{E}-\odot 9$ & $6.80 \mathrm{E}-10$ & $1.70 \mathrm{E}-\odot 9$ & 1. $90 \mathrm{E}-\odot 9$ & 1. $90 \mathrm{E}-\odot 9$ \\
\hline FGR12/13 & $\operatorname{CS} 137$ & $2.90 \mathrm{E}-\odot 9$ & $4.30 E-\odot 9$ & 4. $7 \odot E-\odot 9$ & $4.5 \odot E-\odot 9$ & 4. $40 \mathrm{E}-\odot 9$ & 4. $7 \odot E-\odot 9$ \\
\hline original & $\operatorname{CS} 137$ & $9.80 \mathrm{E}-07$ & 4. $\odot \odot E-\odot 9$ & $4.50 E-\odot 9$ & 7. ๑०E-๑9 & $8.10 E-\odot 9$ & 7. $90 \mathrm{E}-\odot 9$ \\
\hline FGR12/13 & $\mathrm{CS} 138$ & $3.60 \mathrm{E}-06$ & $3.10 \mathrm{E}-11$ & $4.10 \mathrm{E}-12$ & $3.90 \mathrm{E}-12$ & $4.30 \mathrm{E}-12$ & $2.50 \mathrm{E}-11$ \\
\hline original & $\mathrm{CS} 138$ & $4.50 E-06$ & $1.60 \mathrm{E}-10$ & $2.40 \mathrm{E}-12$ & $3.50 \mathrm{E}-12$ & $3.80 \mathrm{E}-12$ & $2.80 \mathrm{E}-11$ \\
\hline FGR12/13 & BA139 & ৪.๑०E-๑৪ & $2.90 \mathrm{E}-11$ & $1.20 \mathrm{E}-11$ & $1.20 \mathrm{E}-11$ & 1. $70 \mathrm{E}-12$ & $3.40 \mathrm{E}-11$ \\
\hline original & BA139 & $5.50 E-\odot 8$ & $2.60 \mathrm{E}-10$ & $2.60 \mathrm{E}-12$ & $2.90 \mathrm{E}-12$ & 1. $70 \mathrm{E}-12$ & $5.10 \mathrm{E}-11$ \\
\hline FGR12/13 & BA140 & $2.50 \mathrm{E}-07$ & $1.50 \mathrm{E}-10$ & $2.10 \mathrm{E}-\odot 9$ & $1.50 \mathrm{E}-\odot 9$ & $1.20 \mathrm{E}-10$ & $1.0 \odot E-\odot 9$ \\
\hline original & BA140 & $3.50 \mathrm{E}-07$ & $1.50 \mathrm{E}-\odot 9$ & $2.30 \mathrm{E}-\odot 9$ & $1.10 \mathrm{E}-\odot 9$ & $1.20 \mathrm{E}-10$ & $9.10 \mathrm{E}-10$ \\
\hline FGR12/13 & BA141 & $1.20 \mathrm{E}-06$ & $2.40 \mathrm{E}-11$ & 6. ๑०E-12 & $5.60 \mathrm{E}-12$ & $1.20 \mathrm{E}-12$ & $2.10 \mathrm{E}-11$ \\
\hline original & BA141 & $1.60 \mathrm{E}-06$ & $1.20 \mathrm{E}-10$ & $3.80 \mathrm{E}-12$ & $1.80 \mathrm{E}-12$ & 1. $00 \mathrm{E}-12$ & $2.30 E-11$ \\
\hline FGR12/13 & BA142 & $1.50 \mathrm{E}-\odot 6$ & $1.80 \mathrm{E}-11$ & $3.10 \mathrm{E}-12$ & 3. 30E-12 & $1.30 \mathrm{E}-12$ & $1.50 \mathrm{E}-11$ \\
\hline original & BA142 & $1.60 \mathrm{E}-06$ & $5.60 \mathrm{E}-11$ & $1.20 \mathrm{E}-12$ & $1.50 \mathrm{E}-12$ & 1. $00 \mathrm{E}-12$ & $1.20 \mathrm{E}-11$ \\
\hline FGR12/13 & LA140 & $3.50 \mathrm{E}-06$ & $1.80 \mathrm{E}-10$ & $2.70 \mathrm{E}-10$ & $3.00 \mathrm{E}-10$ & $9.00 \mathrm{E}-11$ & $5.70 \mathrm{E}-10$ \\
\hline original & LA140 & $4.10 \mathrm{E}-06$ & 1. $7 \odot \mathrm{E}-\odot 9$ & $4.30 \mathrm{E}-10$ & $4.80 \mathrm{E}-10$ & $1.30 \mathrm{E}-10$ & $9.50 \mathrm{E}-10$ \\
\hline FGR12/13 & LA141 & $9.10 \mathrm{E}-\odot 8$ & $3.70 \mathrm{E}-11$ & $7.40 \mathrm{E}-11$ & $2.30 \mathrm{E}-11$ & $8.10 \mathrm{E}-12$ & $6.30 E-11$ \\
\hline original & LA141 & $9.80 \mathrm{E}-\odot 8$ & $6.60 \mathrm{E}-10$ & $1.30 \mathrm{E}-10$ & $3.70 \mathrm{E}-11$ & 1. $00 \mathrm{E}-11$ & $1.60 \mathrm{E}-10$ \\
\hline FGR12/13 & LA142 & $4.30 \mathrm{E}-06$ & $3.70 \mathrm{E}-11$ & $1.20 \mathrm{E}-11$ & $1.20 \mathrm{E}-11$ & $9.60 \mathrm{E}-12$ & $5.20 \mathrm{E}-11$ \\
\hline original & LA142 & $5.20 E-06$ & $3.20 \mathrm{E}-10$ & 1. $30 \mathrm{E}-11$ & $1.60 \mathrm{E}-11$ & $9.30 \mathrm{E}-12$ & $7.40 \mathrm{E}-11$ \\
\hline FGR12/13 & CE141 & $9.80 \mathrm{E}-\odot 8$ & 3. ๑०E-๑8 & $1.10 \mathrm{E}-10$ & $3.70 \mathrm{E}-11$ & $2.30 \mathrm{E}-11$ & $3.80 E-09$ \\
\hline original & CE141 & $9.40 \mathrm{E}-\odot 8$ & $1.60 \mathrm{E}-\odot 8$ & $2.70 \mathrm{E}-10$ & $9.00 \mathrm{E}-11$ & $2.60 \mathrm{E}-11$ & $2.30 E-\odot 9$ \\
\hline FGR12/13 & CE143 & $3.80 E-07$ & 5. ๑९E-๑9 & 1. $40 \mathrm{E}-11$ & $1.50 \mathrm{E}-11$ & $6.70 \mathrm{E}-12$ & 8. $30 \mathrm{E}-10$ \\
\hline
\end{tabular}




\begin{tabular}{|c|c|c|c|c|c|c|c|}
\hline original & CE143 & $4.10 \mathrm{E}-07$ & 4. $\odot \odot E-\odot 9$ & $1.70 \mathrm{E}-11$ & $3.10 \mathrm{E}-11$ & $6.50 \mathrm{E}-12$ & $9.50 \mathrm{E}-10$ \\
\hline FGR12/13 & CE144 & $2.40 \mathrm{E}-\odot 8$ & $4.2 \odot E-\odot 7$ & $2.10 \mathrm{E}-\odot 9$ & 1. $20 \mathrm{E}-\odot 9$ & $1.40 \mathrm{E}-10$ & $5.30 E-08$ \\
\hline original & CE144 & $2.4 \odot E-\odot 8$ & $7.9 \odot E-\odot 7$ & $4.10 E-\odot 9$ & 2. ๑๑E-๑9 & $1.50 \mathrm{E}-10$ & 1. $\odot \odot E-\odot 7$ \\
\hline FGR12/13 & PR143 & $6.10 \mathrm{E}-09$ & 1. $80 \mathrm{E}-\odot 8$ & $2.0 \odot \mathrm{E}-12$ & 2. $\odot \odot \mathrm{E}-12$ & $1.30 \mathrm{E}-14$ & $2.4 \odot E-\odot 9$ \\
\hline original & PR143 & $1.30 \mathrm{E}-\odot 9$ & 1. $30 \mathrm{E}-\odot 8$ & $1.50 \mathrm{E}-11$ & $1.50 \mathrm{E}-11$ & $9.80 \mathrm{E}-14$ & $2.2 \odot E-\odot 9$ \\
\hline FGR12/13 & PR144M & 7.๑๑E-๑9 & $9.90 \mathrm{E}-99$ & $9.9 \odot \mathrm{E}-99$ & $9.9 \odot E-99$ & $9.90 \mathrm{E}-99$ & $9.90 \mathrm{E}-99$ \\
\hline original & PR144M & 1. ๑๑E-๑8 & 4. $00 \mathrm{E}-11$ & $1.50 \mathrm{E}-14$ & $1.80 \mathrm{E}-14$ & $8.70 E-15$ & $5.10 \mathrm{E}-12$ \\
\hline FGR12/13 & PR144 & $8.40 E-\odot 8$ & $5.70 \mathrm{E}-11$ & $1.40 \mathrm{E}-14$ & $1.60 \mathrm{E}-14$ & $1.60 \mathrm{E}-14$ & $1.80 \mathrm{E}-11$ \\
\hline original & PR144 & ८. ००E-๑8 & $9.70 \mathrm{E}-11$ & $2.10 \mathrm{E}-14$ & $2.70 \mathrm{E}-14$ & $1.80 \mathrm{E}-14$ & $1.20 \mathrm{E}-11$ \\
\hline FGR12/13 & ND147 & $1.8 \odot E-\odot 7$ & 1. $80 \mathrm{E}-\odot 8$ & $9.90 \mathrm{E}-11$ & $3.40 \mathrm{E}-11$ & $1.60 \mathrm{E}-11$ & $2.40 \mathrm{E}-\odot 9$ \\
\hline original & ND147 & $2.20 \mathrm{E}-07$ & 1. ๑०E-๑8 & $3.10 \mathrm{E}-10$ & $7.70 \mathrm{E}-11$ & $1.90 \mathrm{E}-11$ & $1.80 E-09$ \\
\hline FGR12/13 & PM147 & $2.70 \mathrm{E}-10$ & $3.90 \mathrm{E}-\odot 8$ & $4.9 \odot E-\odot 9$ & 4. $\odot \odot E-1 \odot$ & $4.00 \mathrm{E}-14$ & 4. $90 \mathrm{E}-\odot 9$ \\
\hline original & PM147 & $3.70 \mathrm{E}-11$ & $7.80 \mathrm{E}-\odot 8$ & $2 . \odot \odot E-\odot 8$ & $1.50 \mathrm{E}-\odot 9$ & $5.20 \mathrm{E}-14$ & $1.10 \mathrm{E}-08$ \\
\hline FGR12/13 & PM148M & $2.8 \odot E-\odot 6$ & $3.9 \odot \mathrm{E}-\odot 8$ & $8.30 \mathrm{E}-10$ & 1. ๑๑E-๑9 & $7.00 \mathrm{E}-10$ & $5.80 E-\odot 9$ \\
\hline original & PM148M & $3.80 E-06$ & $3.60 \mathrm{E}-\odot 8$ & $1.4 \odot E-\odot 9$ & 1. $40 \mathrm{E}-\odot 9$ & 1. $00 \mathrm{E}-\odot 9$ & $6.0 \odot E-\odot 9$ \\
\hline FGR12/13 & PM148 & $8.70 \mathrm{E}-07$ & 1. $30 \mathrm{E}-\odot 8$ & $4.50 E-11$ & $6.40 \mathrm{E}-11$ & $3.80 \mathrm{E}-11$ & $2.2 \odot E-\odot 9$ \\
\hline original & PM148 & $1.10 \mathrm{E}-06$ & 1. $40 \mathrm{E}-\odot 8$ & $7.40 \mathrm{E}-11$ & $1.10 \mathrm{E}-10$ & $3.90 \mathrm{E}-11$ & $3 . \odot \odot E-\odot 9$ \\
\hline FGR12/13 & PM149 & $2.20 \mathrm{E}-\odot 8$ & 4. $30 \mathrm{E}-\odot 9$ & $1.00 \mathrm{E}-12$ & $1.10 \mathrm{E}-12$ & $3.80 \mathrm{E}-13$ & $7.30 E-10$ \\
\hline original & PM149 & $1.90 \mathrm{E}-\odot 8$ & $3.20 \mathrm{E}-\odot 9$ & $5.30 \mathrm{E}-12$ & $5.80 \mathrm{E}-12$ & $4.40 E-13$ & $8.20 E-10$ \\
\hline FGR12/13 & PM151 & $4.4 \odot E-\odot 7$ & $2.7 \odot \mathrm{E}-\odot 9$ & $1.40 \mathrm{E}-11$ & $1.50 \mathrm{E}-11$ & $7.00 \mathrm{E}-12$ & $4.70 E-10$ \\
\hline original & PM151 & $5.80 \mathrm{E}-07$ & $1.70 \mathrm{E}-\odot 9$ & $1.70 \mathrm{E}-11$ & $2.80 \mathrm{E}-11$ & $6.60 \mathrm{E}-12$ & $4.90 \mathrm{E}-10$ \\
\hline FGR12/13 & SM147 & $\odot . \odot \odot \mathrm{E}+\odot \odot$ & $2.4 \odot E-\odot 7$ & $1.40 \mathrm{E}-\odot 5$ & $1.10 \mathrm{E}-\odot 6$ & $1.60 \mathrm{E}-11$ & $9.6 \odot \mathrm{E}-\odot 6$ \\
\hline original & SM147 & $\odot . \odot \odot E+\odot \odot$ & $7.60 \mathrm{E}-06$ & $3.40 E-\odot 4$ & $2.80 E-05$ & $4.00 \mathrm{E}-10$ & $2.00 E-05$ \\
\hline FGR12/13 & SM151 & $7.80 E-13$ & $3.10 \mathrm{E}-\odot 9$ & $1.10 \mathrm{E}-\odot 7$ & $8.7 \odot E-\odot 9$ & $1.50 \mathrm{E}-13$ & 4. $\odot \odot E-\odot 9$ \\
\hline original & SM151 & $8.0 \odot \mathrm{E}-12$ & $3.30 \mathrm{E}-\odot 9$ & $1.4 \odot \mathrm{E}-\odot 7$ & 1. $10 \mathrm{E}-\odot 8$ & $1.70 \mathrm{E}-13$ & $8.10 E-\odot 9$ \\
\hline FGR12/13 & SM153 & $6.50 \mathrm{E}-\odot 8$ & 4. ๑๑E-๑9 & $6.30 \mathrm{E}-11$ & $2.30 \mathrm{E}-11$ & $2.20 \mathrm{E}-12$ & $6.30 \mathrm{E}-10$ \\
\hline original & SM153 & $8.4 \odot E-\odot 8$ & $2.10 \mathrm{E}-\odot 9$ & $1.60 \mathrm{E}-10$ & $7.10 \mathrm{E}-11$ & $2.90 \mathrm{E}-12$ & $5.40 \mathrm{E}-10$ \\
\hline FGR12/13 & EU152 & $1.70 \mathrm{E}-06$ & $6.30 \mathrm{E}-\odot 8$ & $2 . \odot \odot E-\odot 7$ & $7.10 \mathrm{E}-08$ & $8.30 E-09$ & $4.20 E-\odot 8$ \\
\hline original & EU152 & $2.10 \mathrm{E}-06$ & $5.80 \mathrm{E}-\odot 8$ & $2.4 \odot E-\odot 7$ & 8. ๑๑E-๑8 & $8.00 \mathrm{E}-09$ & $5.60 \mathrm{E}-08$ \\
\hline
\end{tabular}




\begin{tabular}{|c|c|c|c|c|c|c|c|}
\hline FGR12/13 & EU154 & $1.80 \mathrm{E}-06$ & 1. $\odot \odot E-\odot 7$ & $4.10 \mathrm{E}-\odot 7$ & $9.2 \odot \mathrm{E}-\odot 8$ & $7.50 \mathrm{E}-09$ & $5.30 E-\odot 8$ \\
\hline original & EU154 & $2.10 \mathrm{E}-06$ & $7.9 \odot E-\odot 8$ & $5.2 \odot E-\odot 7$ & $1.10 \mathrm{E}-07$ & $6.70 \mathrm{E}-09$ & 7. 4०E- - \\
\hline FGR12/13 & EU155 & $6.80 \mathrm{E}-\odot 8$ & 1. $90 \mathrm{E}-\odot 8$ & $1.20 \mathrm{E}-07$ & 1. $.0 \mathrm{E}-\odot 8$ & $2.60 \mathrm{E}-10$ & 7. ๑०E-๑9 \\
\hline original & EU155 & $7.20 E-\odot 8$ & 1. $20 \mathrm{E}-\odot 8$ & $1.50 \mathrm{E}-07$ & 1. 40E-๑8 & 1. $30 \mathrm{E}-10$ & 1. $10 \mathrm{E}-08$ \\
\hline FGR12/13 & EU156 & $2 . \odot \odot E-\odot 6$ & $2.20 \mathrm{E}-\odot 8$ & $1.30 \mathrm{E}-\odot 9$ & $6.30 \mathrm{E}-10$ & $1.90 \mathrm{E}-10$ & 3. $40 \mathrm{E}-\odot 9$ \\
\hline original & EU156 & $2.50 E-06$ & 1. $90 \mathrm{E}-\odot 8$ & $2.80 E-\odot 9$ & 1. $20 \mathrm{E}-09$ & $2.30 \mathrm{E}-10$ & $3.90 \mathrm{E}-09$ \\
\hline FGR12/13 & GD153 & $9.80 E-\odot 8$ & $7.10 \mathrm{E}-10$ & 4. $90 \mathrm{E}-08$ & 4. 80E- -99 & $1.90 \mathrm{E}-10$ & $2.10 \mathrm{E}-\odot 9$ \\
\hline original & GD153 & $1.2 \odot E-\odot 7$ & 1. $30 \mathrm{E}-\odot 9$ & $9.20 \mathrm{E}-\odot 8$ & 1. $10 \mathrm{E}-\odot 8$ & $2.60 \mathrm{E}-10$ & $6.30 \mathrm{E}-09$ \\
\hline FGR12/13 & TB160 & $1.60 \mathrm{E}-06$ & $4.6 \odot E-\odot 8$ & $1.40 \mathrm{E}-08$ & $2.9 \odot E-\odot 9$ & $5.60 \mathrm{E}-10$ & 7. ๑०E-๑9 \\
\hline original & TB160 & $1.80 \mathrm{E}-06$ & 3. ๑९Е-๑8 & $2.50 \mathrm{E}-08$ & $4.50 \mathrm{E}-\odot 9$ & $6.50 \mathrm{E}-10$ & $6.70 \mathrm{E}-09$ \\
\hline FGR12/13 & H0166M & $2.50 \mathrm{E}-\odot 6$ & 1. $20 \mathrm{E}-07$ & $7.20 \mathrm{E}-07$ & $1.5 \odot \mathrm{E}-\odot 7$ & $2.10 \mathrm{E}-08$ & 1. $20 \mathrm{E}-07$ \\
\hline original & H0166М & $2.70 \mathrm{E}-06$ & $1.10 \mathrm{E}-07$ & $8.9 \odot E-\odot 7$ & $1.60 \mathrm{E}-07$ & $2.10 \mathrm{E}-08$ & 2. ๑९E-๑7 \\
\hline FGR12/13 & W 181 & $3.70 \mathrm{E}-\odot 8$ & 4. $30 \mathrm{E}-12$ & $5.20 \mathrm{E}-11$ & $1.60 \mathrm{E}-11$ & $2.80 \mathrm{E}-12$ & $2.70 \mathrm{E}-11$ \\
\hline original & W 181 & $3.30 E-\odot 8$ & $5.40 \mathrm{E}-11$ & 7. 30E-11 & $5.40 \mathrm{E}-11$ & $3.90 \mathrm{E}-12$ & $4.20 \mathrm{E}-11$ \\
\hline FGR12/13 & W 187 & $6.7 \odot \mathrm{E}-\odot 7$ & $3.80 E-11$ & $5.50 E-11$ & $2.50 \mathrm{E}-11$ & $1.20 \mathrm{E}-11$ & $1.90 \mathrm{E}-10$ \\
\hline original & W 187 & $8.40 \mathrm{E}-07$ & $6.20 \mathrm{E}-10$ & $1.10 \mathrm{E}-10$ & $3.70 \mathrm{E}-11$ & $1.60 \mathrm{E}-11$ & $1.80 \mathrm{E}-10$ \\
\hline FGR12/13 & W 185 & $1.6 \odot \mathrm{E}-\odot 9$ & $2.10 \mathrm{E}-11$ & $1.30 \mathrm{E}-10$ & $4.60 \mathrm{E}-11$ & $2.50 \mathrm{E}-12$ & $1.20 \mathrm{E}-10$ \\
\hline original & W 185 & $2.10 \mathrm{E}-10$ & $3.90 \mathrm{E}-10$ & $2.70 \mathrm{E}-10$ & $9.20 \mathrm{E}-11$ & $4.70 \mathrm{E}-12$ & $2.10 \mathrm{E}-10$ \\
\hline FGR12/13 & RE187 & $\odot . \odot \odot \mathrm{E}+\odot \odot$ & $3.80 \mathrm{E}-11$ & $1.50 \mathrm{E}-13$ & $1.50 \mathrm{E}-13$ & $3.80 \mathrm{E}-12$ & $6.30 \mathrm{E}-12$ \\
\hline original & RE187 & $\odot . \odot \odot \mathrm{E}+\odot \odot$ & $1.10 \mathrm{E}-10$ & 1. $30 \mathrm{E}-13$ & $2.70 \mathrm{E}-13$ & $6.90 \mathrm{E}-12$ & $1.50 \mathrm{E}-11$ \\
\hline FGR12/13 & IR192 & $1.10 \mathrm{E}-06$ & 4. $90 \mathrm{E}-\odot 8$ & $5.70 \mathrm{E}-10$ & $6.60 \mathrm{E}-10$ & $4.70 \mathrm{E}-10$ & $6.60 \mathrm{E}-09$ \\
\hline original & IR192 & $1.60 \mathrm{E}-\odot 6$ & $5.30 \mathrm{E}-\odot 8$ & $6.80 \mathrm{E}-10$ & $9.20 \mathrm{E}-10$ & $6.40 \mathrm{E}-10$ & $7.60 E-\odot 9$ \\
\hline FGR12/13 & HG2O3 & $3.30 \mathrm{E}-07$ & $3.10 \mathrm{E}-10$ & $3.70 \mathrm{E}-10$ & $3.10 \mathrm{E}-10$ & $2.90 \mathrm{E}-10$ & $4.70 E-10$ \\
\hline original & HG2O3 & $3.20 \mathrm{E}-07$ & $6.60 \mathrm{E}-10$ & $6.10 \mathrm{E}-10$ & $9.30 \mathrm{E}-10$ & $8.30 \mathrm{E}-10$ & $1.50 \mathrm{E}-\odot 9$ \\
\hline${ }^{*}$ FGR12/13 & RN222 & $5.60 \mathrm{E}-10$ & $\odot . \odot \odot E+\odot \odot$ & $\odot . \odot \odot E+\odot \odot$ & $\odot . \odot \odot E+\odot \odot$ & $\odot . \odot \odot E+\odot \odot$ & $\odot . \odot \odot E+\odot \odot$ \\
\hline original & RN222 & $3.30 E-06$ & $\odot . \odot \odot \mathrm{E}+\Theta \odot$ & $\odot . \odot \odot \mathrm{E}+\odot \odot$ & $\odot . \odot \odot \mathrm{E}+\odot \odot$ & $\odot . \odot \odot \mathrm{E}+\odot \odot$ & $\odot . \odot \odot \mathrm{E}+\odot \odot$ \\
\hline FGR12/13 & TH227 & $1.4 \odot E-\odot 7$ & $8.60 \mathrm{E}-05$ & 7. 40E-๑8 & $6.40 \mathrm{E}-09$ & $2.90 \mathrm{E}-10$ & 1. $\odot \odot E-\odot 5$ \\
\hline original & TH227 & $1.70 \mathrm{E}-\odot 7$ & $3.60 \mathrm{E}-05$ & $1.60 \mathrm{E}-06$ & 1. $30 \mathrm{E}-07$ & $3 . \odot \odot E-\odot 9$ & 4. 40E-०6 \\
\hline
\end{tabular}




\begin{tabular}{|c|c|c|c|c|c|c|c|}
\hline FGR12/13 & TH228 & $2.6 \odot \mathrm{E}-\odot 9$ & $3.30 E-\odot 4$ & $2.40 \mathrm{E}-05$ & $1.90 \mathrm{E}-\odot 6$ & $6.90 \mathrm{E}-\odot 8$ & 4. .००E- $\odot 5$ \\
\hline original & TH228 & $2.5 \odot E-\odot 9$ & $6.60 \mathrm{E}-\odot 4$ & $2.20 \mathrm{E}-\odot 4$ & $1.70 \mathrm{E}-05$ & $4.50 \mathrm{E}-08$ & $8.80 E-05$ \\
\hline FGR12/13 & TH229 & $1.10 \mathrm{E}-07$ & $5.0 \odot E-\odot 4$ & $5.10 \mathrm{E}-04$ & $2.50 \mathrm{E}-05$ & $1.70 \mathrm{E}-06$ & $7.10 \mathrm{E}-05$ \\
\hline original & TH229 & 1. $\odot \odot E-\odot 7$ & 2. .๑० - ๑3 & $5.70 E-03$ & $4.60 \mathrm{E}-04$ & $5.20 E-08$ & $4.70 E-04$ \\
\hline FGR12/13 & TH230 & $4.70 \mathrm{E}-10$ & $7.70 \mathrm{E}-\odot 5$ & $2.80 E-04$ & $1.00 \mathrm{E}-05$ & $3.00 \mathrm{E}-07$ & $1.40 \mathrm{E}-05$ \\
\hline original & TH230 & $5.10 \mathrm{E}-10$ & $3.00 \mathrm{E}-\odot 4$ & $8.50 \mathrm{E}-\odot 4$ & 7. .0०E- 05 & $8.20 \mathrm{E}-09$ & 7. $.0 \mathrm{E}-\odot 5$ \\
\hline FGR12/13 & TH231 & $1.40 \mathrm{E}-08$ & $2.20 \mathrm{E}-\odot 9$ & $5.60 \mathrm{E}-11$ & $2.80 \mathrm{E}-12$ & $3.60 \mathrm{E}-13$ & $3.30 \mathrm{E}-10$ \\
\hline original & TH231 & $1.40 \mathrm{E}-08$ & $8.10 \mathrm{E}-10$ & $2.90 \mathrm{E}-10$ & $3.70 \mathrm{E}-12$ & $2.80 \mathrm{E}-13$ & $2.20 \mathrm{E}-10$ \\
\hline FGR12/13 & TH232 & $2.30 \mathrm{E}-10$ & $1.60 \mathrm{E}-04$ & $2.90 \mathrm{E}-04$ & $1.20 \mathrm{E}-05$ & $8.20 \mathrm{E}-07$ & $2.50 E-05$ \\
\hline original & TH232 & $3.50 \mathrm{E}-10$ & $8.90 \mathrm{E}-\odot 4$ & $4.70 \mathrm{E}-03$ & $3.80 \mathrm{E}-\odot 4$ & 7. 20E-०9 & $3.00 E-04$ \\
\hline FGR12/13 & TH234 & $9.30 \mathrm{O}-\odot 9$ & $5.80 \mathrm{E}-\odot 8$ & $7.40 \mathrm{E}-11$ & $4.40 \mathrm{E}-11$ & $6.70 \mathrm{E}-12$ & 7. 7०E- $\odot 9$ \\
\hline original & TH234 & $3.90 \mathrm{E}-08$ & $6.40 \mathrm{E}-\odot 8$ & $4.10 \mathrm{E}-10$ & $2.40 \mathrm{E}-10$ & $1.20 \mathrm{E}-11$ & $9.50 \mathrm{E}-09$ \\
\hline FGR12/13 & RA223 & $1.70 \mathrm{E}-07$ & $6.20 \mathrm{E}-05$ & $7.80 \mathrm{E}-\odot 7$ & $7.50 \mathrm{E}-\odot 8$ & $3.40 \mathrm{E}-09$ & $7.40 E-06$ \\
\hline original & RA223 & $4.90 \mathrm{E}-07$ & $1.70 \mathrm{E}-05$ & $9 . \odot \odot E-\odot 7$ & $6.20 \mathrm{E}-\odot 8$ & $9.50 \mathrm{E}-\odot 9$ & $2.10 \mathrm{E}-06$ \\
\hline FGR12/13 & RA224 & $1.40 \mathrm{E}-08$ & $2.50 \mathrm{E}-05$ & 4.10E- -7 & 4. ๑०E-๑8 & $2.30 \mathrm{E}-\odot 9$ & $3.00 \mathrm{E}-\odot 6$ \\
\hline original & RA224 & $1.70 \mathrm{E}-\odot 8$ & $6.20 \mathrm{E}-06$ & $2.30 \mathrm{E}-\odot 7$ & $1.90 \mathrm{E}-\odot 8$ & $6.40 \mathrm{E}-09$ & $7.7 \odot \mathrm{E}-07$ \\
\hline FGR12/13 & RA225 & $7.6 \odot \mathrm{E}-\odot 9$ & $5.20 \mathrm{E}-05$ & $2.20 \mathrm{E}-06$ & 2. ๑०E-๑7 & 6. 40E-๑9 & $6.30 \mathrm{E}-06$ \\
\hline original & RA225 & $1.30 \mathrm{E}-08$ & $1.60 \mathrm{E}-05$ & $1.30 \mathrm{E}-\odot 6$ & $8.20 \mathrm{E}-\odot 8$ & $2.40 \mathrm{E}-\odot 9$ & $2.0 \odot E-\odot 6$ \\
\hline FGR12/13 & RA226 & 9. ๑०E-๑9 & $2.80 E-05$ & $7.40 \mathrm{E}-06$ & $5.20 \mathrm{E}-07$ & 2. 40E- -8 & $3.50 E-06$ \\
\hline original & RA226 & $6.60 \mathrm{E}-\odot 9$ & $1.60 \mathrm{E}-05$ & $7.00 \mathrm{E}-\odot 6$ & $3.90 \mathrm{E}-07$ & $2.80 E-\odot 9$ & $2.20 E-06$ \\
\hline FGR12/13 & RA228 & $\odot . \odot \odot E+\odot \odot$ & $9.70 \mathrm{E}-06$ & $5.60 \mathrm{E}-05$ & $4.70 \mathrm{E}-06$ & $2.10 \mathrm{E}-07$ & $2.60 \mathrm{E}-06$ \\
\hline original & RA228 & $5.40 \mathrm{E}-14$ & $6.70 \mathrm{E}-06$ & $5.70 \mathrm{E}-\odot 6$ & $5.50 \mathrm{E}-07$ & $1.10 \mathrm{E}-09$ & $1.00 \mathrm{E}-06$ \\
\hline FGR12/13 & PB210 & $1.40 \mathrm{E}-09$ & $1.20 \mathrm{E}-07$ & $2.90 \mathrm{E}-05$ & $3.20 \mathrm{E}-06$ & $1.20 \mathrm{E}-07$ & $9.10 \mathrm{E}-\odot 7$ \\
\hline original & PB210 & $2.20 \mathrm{E}-09$ & $4.30 \mathrm{E}-10$ & $5.40 \mathrm{E}-05$ & $3.80 \mathrm{E}-06$ & $3.20 \mathrm{E}-07$ & $3.60 E-06$ \\
\hline FGR12/13 & PB212 & $2 . \odot \odot E-\odot 7$ & 8. 7०E-๑9 & $8.50 \mathrm{E}-08$ & 1. 10E- 08 & $3.40 \mathrm{E}-09$ & $1.80 \mathrm{E}-08$ \\
\hline original & PB212 & $2.30 \mathrm{E}-07$ & $1.80 \mathrm{E}-07$ & $3.90 \mathrm{E}-07$ & $3.50 \mathrm{E}-08$ & 3. 40E-०9 & $4.50 E-08$ \\
\hline FGR12/13 & BI210 & $8.10 \mathrm{E}-\odot 9$ & $7.70 \mathrm{E}-\odot 7$ & $4.70 \mathrm{E}-11$ & $4.70 \mathrm{E}-11$ & $4.70 \mathrm{E}-11$ & $9.30 \mathrm{E}-08$ \\
\hline original & BI210 & $2.10 \mathrm{E}-09$ & 4.30E-07 & $3.80 \mathrm{E}-11$ & $6.70 \mathrm{E}-11$ & $6.60 \mathrm{E}-11$ & $5.30 \mathrm{E}-08$ \\
\hline FGR12/13 & BI212 & $2.8 \odot E-\odot 7$ & 2.00E-๑7 & $2.10 \mathrm{E}-11$ & $2.10 \mathrm{E}-11$ & $2.10 \mathrm{E}-11$ & $3.10 E-08$ \\
\hline
\end{tabular}




\begin{tabular}{|c|c|c|c|c|c|c|c|}
\hline original & BI212 & $2.80 E-06$ & 3. $80 \mathrm{E}-\odot 8$ & $2.40 \mathrm{E}-10$ & $6.70 \mathrm{E}-11$ & $5.00 \mathrm{E}-11$ & $5.10 E-09$ \\
\hline FGR12/13 & P0210 & $1.20 \mathrm{E}-11$ & $2.60 \mathrm{E}-05$ & $2.80 \mathrm{E}-07$ & $4.60 \mathrm{E}-07$ & $4.90 \mathrm{E}-\odot 8$ & $3.30 E-06$ \\
\hline original & P0210 & $1.60 \mathrm{E}-11$ & 1. $30 \mathrm{E}-05$ & $6.50 \mathrm{E}-\odot 8$ & $1.30 \mathrm{E}-07$ & $1.30 \mathrm{E}-07$ & $2.3 \odot E-\odot 6$ \\
\hline FGR12/13 & U 232 & $3.70 \mathrm{E}-10$ & $3.0 \odot E-\odot 4$ & $4.10 E-05$ & $3.30 E-06$ & $2 . \odot \odot E-\odot 7$ & $3.70 E-05$ \\
\hline original & U 232 & $4.90 \mathrm{E}-10$ & 1. $40 \mathrm{E}-\odot 3$ & $5.00 \mathrm{E}-\odot 6$ & $3.4 \odot E-\odot 9$ & $1.10 \mathrm{E}-09$ & $1.7 \odot E-\odot 4$ \\
\hline FGR12/13 & U 233 & $4.50 \mathrm{E}-10$ & 8. $\odot \odot E-๑ 5$ & $8.0 \odot E-\odot 7$ & $6.8 \odot E-\odot 8$ & 1. $80 \mathrm{E}-\odot 8$ & $9.60 \mathrm{E}-\odot 6$ \\
\hline original & U 233 & $4.20 \mathrm{E}-10$ & $3 . \odot \odot E-\odot 4$ & 1. $90 \mathrm{E}-\odot 8$ & $1.50 \mathrm{E}-\odot 9$ & $3.70 \mathrm{E}-10$ & $3.60 E-05$ \\
\hline FGR12/13 & U 234 & $1.90 \mathrm{E}-10$ & $7.80 \mathrm{E}-05$ & $5.00 \mathrm{E}-07$ & $5.20 \mathrm{E}-\odot 8$ & $1.60 \mathrm{E}-08$ & $9.4 \odot E-\odot 6$ \\
\hline original & U 234 & $3.60 \mathrm{E}-10$ & $3 . \odot \odot E-\odot 4$ & $1.90 \mathrm{E}-\odot 8$ & $1.50 \mathrm{E}-09$ & $3.70 \mathrm{E}-10$ & $3.60 E-05$ \\
\hline FGR12/13 & U 235 & $2 . \odot \odot E-\odot 7$ & $7.00 \mathrm{E}-\odot 5$ & $4.80 \mathrm{E}-07$ & $5 . \odot \odot E-\odot 8$ & $1.60 \mathrm{E}-08$ & $8.50 E-\odot 6$ \\
\hline original & U 235 & $1.70 \mathrm{E}-07$ & $2.80 \mathrm{E}-\odot 4$ & 1. 80E- - 8 & $2.10 \mathrm{E}-09$ & $6.70 \mathrm{E}-10$ & $3.30 E-05$ \\
\hline FGR12/13 & U 236 & $1.20 \mathrm{E}-10$ & $7.30 \mathrm{E}-05$ & $4.8 \odot E-\odot 7$ & 4. $90 \mathrm{E}-\odot 8$ & $1.50 \mathrm{E}-08$ & $8.70 E-\odot 6$ \\
\hline original & U 236 & $2.80 \mathrm{E}-10$ & $2.80 \mathrm{E}-\odot 4$ & $1.80 \mathrm{E}-08$ & 1. $4 \odot E-\odot 9$ & $3.40 \mathrm{E}-10$ & $3.4 \odot E-\odot 5$ \\
\hline FGR12/13 & U 237 & $1.7 \odot \mathrm{E}-\odot 7$ & 1. $40 \mathrm{E}-\odot 8$ & $3.40 \mathrm{E}-11$ & $1.90 \mathrm{E}-11$ & $1.10 \mathrm{E}-11$ & 1. $90 \mathrm{E}-\odot 9$ \\
\hline original & U 237 & $2.10 \mathrm{E}-07$ & $4.90 \mathrm{E}-\odot 9$ & $7.20 \mathrm{E}-11$ & $5.50 \mathrm{E}-11$ & $1.10 \mathrm{E}-11$ & $9.80 \mathrm{E}-10$ \\
\hline FGR12/13 & U 238 & $7.90 \mathrm{E}-11$ & $6.70 \mathrm{E}-05$ & $4.60 \mathrm{E}-07$ & $4.90 E-\odot 8$ & $1.50 E-08$ & ८. ๑๑E-๑6 \\
\hline original & U 238 & $2.40 \mathrm{E}-10$ & $2.7 \odot E-\odot 4$ & $1.70 \mathrm{E}-\odot 8$ & $1.50 \mathrm{E}-\odot 9$ & $3.90 \mathrm{E}-10$ & $3.20 E-05$ \\
\hline FGR12/13 & U 240 & $1.90 \mathrm{E}-\odot 9$ & $2.9 \odot E-\odot 9$ & $5.10 \mathrm{E}-11$ & $1.10 \mathrm{E}-11$ & $4.40 \mathrm{E}-12$ & $5.80 E-10$ \\
\hline original & U 240 & $6.50 \mathrm{E}-07$ & $2.30 \mathrm{E}-\odot 9$ & $9.80 \mathrm{E}-12$ & $1.20 \mathrm{E}-11$ & $4.00 \mathrm{E}-12$ & $6.10 \mathrm{E}-10$ \\
\hline FGR12/13 & PA231 & $5 . \odot \odot E-\odot 8$ & $1.50 \mathrm{E}-04$ & $6.0 \odot E-\odot 4$ & $2.40 \mathrm{E}-05$ & $1.30 E-06$ & $2.90 E-\odot 5$ \\
\hline original & PA231 & $5.50 \mathrm{E}-\odot 8$ & $7.50 \mathrm{E}-\odot 4$ & $3.60 \mathrm{E}-03$ & $2.90 \mathrm{E}-\odot 4$ & $1.70 \mathrm{E}-10$ & $2.30 E-\odot 4$ \\
\hline FGR12/13 & PA233 & $2.7 \odot E-\odot 7$ & 3. ๑९E-๑8 & $1.80 \mathrm{E}-10$ & $8.30 \mathrm{E}-11$ & $5.00 \mathrm{E}-11$ & $3.9 \odot E-\odot 9$ \\
\hline original & PA233 & $4 . \odot \odot E-\odot 7$ & 1. $70 \mathrm{E}-\odot 8$ & $4.60 \mathrm{E}-10$ & $1.60 \mathrm{E}-10$ & $5.70 \mathrm{E}-11$ & $2.60 E-\odot 9$ \\
\hline FGR12/13 & PA234 & $2.8 \odot E-\odot 6$ & 1. $8 \odot E-\odot 9$ & $1.90 \mathrm{E}-11$ & $2.60 \mathrm{E}-11$ & $1.40 \mathrm{E}-11$ & 4. $20 \mathrm{E}-10$ \\
\hline original & PA234 & $3.4 \odot E-\odot 6$ & $9.20 \mathrm{E}-10$ & $1.90 \mathrm{E}-11$ & $2.90 \mathrm{E}-11$ & $1.30 \mathrm{E}-11$ & $2.30 E-10$ \\
\hline FGR12/13 & AC225 & $2 . \odot \odot E-\odot 8$ & $7.10 \mathrm{E}-05$ & $3.80 E-\odot 8$ & $4.70 \mathrm{E}-\odot 9$ & $2 . \odot \odot E-\odot 9$ & $8.5 \odot E-\odot 6$ \\
\hline original & AC225 & $4.5 \odot E-07$ & 1. $80 \mathrm{E}-05$ & $4.7 \odot E-\odot 7$ & $3.8 \odot E-\odot 8$ & $2.10 \mathrm{E}-10$ & $2.20 E-\odot 6$ \\
\hline FGR12/13 & AC227 & $1.60 \mathrm{E}-10$ & $4.10 \mathrm{E}-\odot 4$ & $1.90 \mathrm{E}-\odot 4$ & 1. $00 \mathrm{E}-\odot 5$ & $9.50 \mathrm{E}-07$ & $5.50 E-05$ \\
\hline original & AC227 & $2.10 \mathrm{E}-10$ & $1.50 \mathrm{E}-03$ & $2.90 \mathrm{E}-03$ & $2.30 E-\odot 4$ & $1.10 \mathrm{E}-09$ & $3.50 E-\odot 4$ \\
\hline
\end{tabular}




\begin{tabular}{|c|c|c|c|c|c|c|c|}
\hline FGR12/13 & AC228 & $1.40 \mathrm{E}-06$ & $1.20 \mathrm{E}-\odot 7$ & $8.60 \mathrm{E}-\odot 9$ & $6.80 \mathrm{E}-10$ & 3. $20 \mathrm{E}-11$ & $1.50 E-08$ \\
\hline original & AC228 & $1.70 \mathrm{E}-\odot 6$ & $2.4 \odot \mathrm{E}-\odot 7$ & $5.60 E-08$ & 4.10E- $\odot 9$ & $6.50 \mathrm{E}-12$ & $3.20 E-08$ \\
\hline FGR12/13 & FR223 & $7.00 \mathrm{E}-\odot 8$ & $8.60 \mathrm{E}-10$ & $8.00 \mathrm{E}-10$ & $8.00 \mathrm{E}-10$ & $8.00 \mathrm{E}-10$ & $9.00 \mathrm{E}-10$ \\
\hline original & FR223 & $7 . \odot \odot E-\odot 8$ & 2. $\odot \odot E-\odot 9$ & $6.10 \mathrm{E}-10$ & 1. $20 \mathrm{E}-\odot 9$ & 1. $20 \mathrm{E}-09$ & 1. $30 \mathrm{E}-09$ \\
\hline FGR12/13 & NP237 & $2.80 E-\odot 8$ & 2. $90 \mathrm{E}-\odot 5$ & 1. $00 \mathrm{E}-\odot 3$ & $3.90 \mathrm{E}-05$ & $1.30 E-06$ & $2.30 E-05$ \\
\hline original & NP237 & $2.50 \mathrm{E}-\odot 8$ & $1.60 \mathrm{E}-05$ & $3.90 \mathrm{E}-03$ & $3.10 \mathrm{E}-04$ & $1.20 E-\odot 9$ & $1.70 E-\odot 4$ \\
\hline FGR12/13 & NP238 & $8.10 \mathrm{E}-07$ & $5.50 \mathrm{E}-\odot 9$ & $6.80 E-08$ & $2.80 E-\odot 9$ & $1.00 \mathrm{E}-10$ & $2.10 \mathrm{E}-\odot 9$ \\
\hline original & NP238 & $9.60 \mathrm{E}-07$ & 3. 30E- $\odot 9$ & $2.40 \mathrm{E}-07$ & 1. $90 \mathrm{E}-\odot 8$ & $2.40 \mathrm{E}-11$ & 1. $10 \mathrm{E}-\odot 8$ \\
\hline FGR12/13 & NP239 & $2.20 \mathrm{E}-\odot 7$ & $6.30 \mathrm{E}-09$ & $5.20 \mathrm{E}-10$ & $4.50 \mathrm{E}-11$ & $8.20 \mathrm{E}-12$ & $9.30 \mathrm{E}-10$ \\
\hline original & NP239 & $2.60 \mathrm{E}-07$ & $2.30 \mathrm{E}-\odot 9$ & $2.20 E-\odot 9$ & $1.40 \mathrm{E}-10$ & $7.50 \mathrm{E}-12$ & $6.60 \mathrm{E}-10$ \\
\hline FGR12/13 & PU236 & $1.50 \mathrm{E}-10$ & $7.40 \mathrm{E}-\odot 5$ & $5.10 E-05$ & $2.80 E-\odot 6$ & 8. ৪०E- - & $1.10 \mathrm{E}-05$ \\
\hline original & PU236 & $3.30 \mathrm{E}-10$ & $1.50 \mathrm{E}-\odot 4$ & $4.80 E-05$ & $3.60 \mathrm{E}-06$ & $8.00 \mathrm{E}-11$ & $2.00 E-05$ \\
\hline FGR12/13 & PU237 & $5.60 \mathrm{E}-\odot 8$ & $2.9 \odot E-\odot 9$ & $7.40 \mathrm{E}-11$ & $2.80 \mathrm{E}-11$ & $1.80 \mathrm{E}-11$ & $3.90 \mathrm{E}-10$ \\
\hline original & PU237 & $7 . \odot \odot E-\odot 8$ & $3.40 \mathrm{E}-\odot 9$ & $1.30 \mathrm{E}-10$ & $7.10 \mathrm{E}-11$ & $2.10 \mathrm{E}-11$ & $4.90 \mathrm{E}-10$ \\
\hline FGR12/13 & PU238 & $1.10 \mathrm{E}-10$ & $9.30 \mathrm{E}-05$ & $1.60 \mathrm{E}-04$ & $8.30 \mathrm{E}-06$ & $2.80 E-07$ & $1.60 \mathrm{E}-05$ \\
\hline original & PU238 & $2.70 \mathrm{E}-10$ & $3.20 E-\odot 4$ & $7.30 \mathrm{E}-\odot 4$ & $5.90 E-\odot 5$ & $8.50 \mathrm{E}-11$ & $7.70 E-05$ \\
\hline FGR12/13 & PU239 & $1.10 \mathrm{E}-10$ & $8.70 \mathrm{E}-05$ & $1.80 E-04$ & $9.10 \mathrm{E}-06$ & $3.20 E-07$ & $1.60 E-05$ \\
\hline original & PU239 & $1.80 \mathrm{E}-10$ & $3.10 \mathrm{E}-04$ & $8.50 E-\odot 4$ & $6.50 \mathrm{E}-05$ & $8.00 \mathrm{E}-11$ & $8.20 E-05$ \\
\hline FGR12/13 & PU240 & $1.10 \mathrm{E}-10$ & 8. 8०E- $९ 5$ & $1.80 E-04$ & $9.10 \mathrm{E}-06$ & $3.20 E-07$ & 1. $60 \mathrm{E}-\odot 5$ \\
\hline original & PU240 & $2.60 \mathrm{E}-10$ & $3.10 \mathrm{E}-04$ & $8.50 E-04$ & $6.50 \mathrm{E}-05$ & $8.00 \mathrm{E}-11$ & $8.20 E-05$ \\
\hline FGR12/13 & PU241 & $2.00 \mathrm{E}-12$ & $4.60 \mathrm{E}-07$ & $4.10 E-06$ & $1.80 \mathrm{E}-07$ & $7.10 \mathrm{E}-09$ & 1. $80 E-07$ \\
\hline original & PU241 & $6.50 \mathrm{E}-16$ & $3.10 \mathrm{E}-06$ & $1.80 \mathrm{E}-\odot 5$ & 1. $40 \mathrm{E}-06$ & 8. $.00 \mathrm{E}-14$ & 1. $30 \mathrm{E}-06$ \\
\hline FGR12/13 & PU242 & $9.20 \mathrm{E}-11$ & $8.10 \mathrm{E}-05$ & $1.80 \mathrm{E}-\odot 4$ & $8.70 \mathrm{E}-06$ & $3 . \odot \odot E-\odot 7$ & $1.50 E-05$ \\
\hline original & PU242 & $2.20 \mathrm{E}-10$ & $2.9 \odot E-\odot 4$ & $7.20 E-\odot 4$ & $6.00 \mathrm{E}-\odot 5$ & $7.40 \mathrm{E}-11$ & $7.40 \mathrm{E}-05$ \\
\hline FGR12/13 & PU243 & $3 . \odot \odot E-\odot 8$ & $4.70 \mathrm{E}-10$ & $1.50 \mathrm{E}-11$ & $9.90 \mathrm{E}-13$ & $1.60 \mathrm{E}-13$ & $8.70 \mathrm{E}-11$ \\
\hline original & PU243 & $2.40 \mathrm{E}-\odot 8$ & 2. $\odot \odot E-1 \odot$ & $5.80 \mathrm{E}-11$ & $6.30 \mathrm{E}-13$ & $1.10 \mathrm{E}-13$ & 3. $90 \mathrm{E}-11$ \\
\hline FGR12/13 & PU244 & $6.60 \mathrm{E}-11$ & $7.50 \mathrm{E}-05$ & $1.60 \mathrm{E}-\odot 4$ & $8.20 \mathrm{E}-06$ & $2.90 \mathrm{E}-07$ & $1.40 \mathrm{E}-05$ \\
\hline original & PU244 & $1.80 \mathrm{E}-10$ & $2.90 \mathrm{E}-\odot 4$ & $7.30 \mathrm{E}-04$ & $5.80 E-05$ & 1. $20 \mathrm{E}-\odot 9$ & $7.40 \mathrm{E}-05$ \\
\hline
\end{tabular}




\begin{tabular}{|c|c|c|c|c|c|c|c|}
\hline FGR12/13 & AM241 & $2.10 \mathrm{E}-08$ & 3. $70 \mathrm{E}-05$ & 1. $70 \mathrm{E}-03$ & $5.80 E-05$ & $2.9 \odot E-\odot 6$ & $4.20 E-05$ \\
\hline original & AM241 & 1. $90 \mathrm{E}-\odot 8$ & $1.80 \mathrm{E}-05$ & $2.20 \mathrm{E}-03$ & 1. $70 \mathrm{E}-\odot 4$ & 1. ๑०E-๑9 & $1.20 E-\odot 4$ \\
\hline FGR12/13 & AM242M & $7.90 \mathrm{E}-10$ & $8.80 E-\odot 6$ & 1. $70 \mathrm{E}-\odot 3$ & $5.50 \mathrm{E}-05$ & $2.90 \mathrm{E}-\odot 6$ & $3.70 E-05$ \\
\hline original & AM242M & 1. ๑०E-๑9 & $3.80 \mathrm{E}-06$ & 2. $.0 \mathrm{E}-\odot 3$ & 1. $7 \odot E-\odot 4$ & $1.20 \mathrm{E}-10$ & $1.10 \mathrm{E}-\odot 4$ \\
\hline FGR12/13 & AM242 & 1. $90 \mathrm{E}-\odot 8$ & 1. $20 \mathrm{E}-\odot 7$ & $9.20 \mathrm{E}-\odot 8$ & $6.30 \mathrm{E}-\odot 9$ & 1. $20 \mathrm{E}-10$ & 1. $70 \mathrm{E}-\odot 8$ \\
\hline original & AM242 & 2. $\odot \odot E-\odot 8$ & 4. $7 \odot E-\odot 8$ & $1.60 \mathrm{E}-07$ & $1.10 \mathrm{E}-08$ & $2.50 \mathrm{E}-12$ & 1. $40 \mathrm{E}-\odot 8$ \\
\hline FGR12/13 & AM243 & $5.90 \mathrm{E}-\odot 8$ & $3.50 \mathrm{E}-05$ & 1. $70 \mathrm{E}-\odot 3$ & $5.70 E-05$ & $2.90 \mathrm{E}-\odot 6$ & $4.10 E-05$ \\
\hline original & AM243 & 4. . $7 \odot E-\odot 8$ & 1. $70 \mathrm{E}-\odot 5$ & $2.10 \mathrm{E}-03$ & 1. $70 \mathrm{E}-\odot 4$ & 1. $20 \mathrm{E}-\odot 9$ & $1.10 \mathrm{E}-\odot 4$ \\
\hline FGR12/13 & CM242 & $1.30 \mathrm{E}-10$ & $3.50 \mathrm{E}-\odot 5$ & $2.70 \mathrm{E}-\odot 5$ & 1. $90 \mathrm{E}-\odot 6$ & $3.50 \mathrm{E}-\odot 8$ & $5.20 E-\odot 6$ \\
\hline original & CM242 & $2.70 \mathrm{E}-10$ & 1. $40 \mathrm{E}-\odot 5$ & $4.70 \mathrm{E}-05$ & $3.20 E-06$ & $9.40 \mathrm{E}-10$ & $4.30 E-06$ \\
\hline FGR12/13 & CM243 & $1.70 \mathrm{E}-07$ & 4. $\odot \odot E-\odot 5$ & $1.20 \mathrm{E}-03$ & $4.50 E-05$ & 1. $70 \mathrm{E}-\odot 6$ & $3.20 E-05$ \\
\hline original & CM243 & $1.90 \mathrm{E}-\odot 7$ & $1.80 \mathrm{E}-05$ & $1.40 \mathrm{E}-\odot 3$ & $1.20 \mathrm{E}-\odot 4$ & $1.20 \mathrm{E}-\odot 9$ & $8.00 E-\odot 5$ \\
\hline FGR12/13 & CM244 & $1.10 \mathrm{E}-10$ & $3.90 \mathrm{E}-05$ & $9.20 \mathrm{E}-\odot 4$ & $3.90 E-05$ & $1.30 \mathrm{E}-06$ & $2.70 E-\odot 5$ \\
\hline original & CM244 & $2.40 \mathrm{E}-10$ & 1. $80 \mathrm{E}-05$ & 1. $10 \mathrm{E}-03$ & $9.20 \mathrm{E}-05$ & $9.90 \mathrm{E}-10$ & $6.40 \mathrm{E}-05$ \\
\hline FGR12/13 & CM245 & $1.10 \mathrm{E}-07$ & $3.60 \mathrm{E}-05$ & 1. $80 \mathrm{E}-03$ & $5.90 E-05$ & 3. ๑९E-๑6 & $4.20 E-05$ \\
\hline original & CM245 & $9.60 \mathrm{E}-\odot 8$ & $1.70 \mathrm{E}-05$ & $2.10 \mathrm{E}-03$ & $1.7 \odot E-\odot 4$ & 1. $10 \mathrm{E}-\odot 9$ & $1.20 E-04$ \\
\hline FGR12/13 & CM246 & $9.80 \mathrm{E}-11$ & $3.60 \mathrm{E}-05$ & 1. $70 \mathrm{E}-\odot 3$ & $5.80 E-05$ & $2.90 \mathrm{E}-\odot 6$ & $4.20 E-05$ \\
\hline original & CM246 & $2.10 \mathrm{E}-10$ & $1.70 \mathrm{E}-05$ & $2.20 \mathrm{E}-\odot 3$ & 1. $7 \odot \mathrm{E}-\odot 4$ & $9.90 \mathrm{E}-10$ & 1. $20 \mathrm{E}-\odot 4$ \\
\hline FGR12/13 & CM247 & $4.40 \mathrm{E}-07$ & $3.20 \mathrm{E}-05$ & 1. $60 \mathrm{E}-03$ & $5.4 \odot E-05$ & $2.70 \mathrm{E}-\odot 6$ & $3.90 E-05$ \\
\hline original & CM247 & $7.40 \mathrm{E}-07$ & $1.50 \mathrm{E}-05$ & 2. $\odot \odot E-\odot 3$ & 1. $6 \odot E-\odot 4$ & 1. $40 \mathrm{E}-\odot 9$ & $1.10 \mathrm{E}-\odot 4$ \\
\hline *FGR12/13 & CM248 & $7.40 \mathrm{E}-11$ & $6.30 \mathrm{E}-05$ & $7.80 E-\odot 3$ & $6.20 E-\odot 4$ & $2.20 \mathrm{E}-\odot 8$ & $4.20 E-\odot 4$ \\
\hline original & CM248 & $1.70 \mathrm{E}-10$ & $6.30 \mathrm{E}-05$ & $7.80 \mathrm{E}-03$ & $6.20 E-\odot 4$ & $2.20 \mathrm{E}-\odot 8$ & $4.20 E-04$ \\
\hline *FGR12/13 & CF252 & $1.10 \mathrm{E}-10$ & $3.50 \mathrm{E}-\odot 5$ & $6.6 \odot E-\odot 4$ & $5.30 E-05$ & $1.10 \mathrm{E}-08$ & $3.50 E-05$ \\
\hline original & CF252 & $1.70 \mathrm{E}-10$ & $3.50 \mathrm{E}-05$ & $6.60 \mathrm{E}-\odot 4$ & $5.30 \mathrm{E}-05$ & $1.10 \mathrm{E}-\odot 8$ & $3.50 E-05$ \\
\hline
\end{tabular}




\section{Appendix B.3}

Ratio of the revised FGR12/13 DCFs to the original HUDUFACT.dat DCFs (for adult only) (NAN is given where both values were zero)

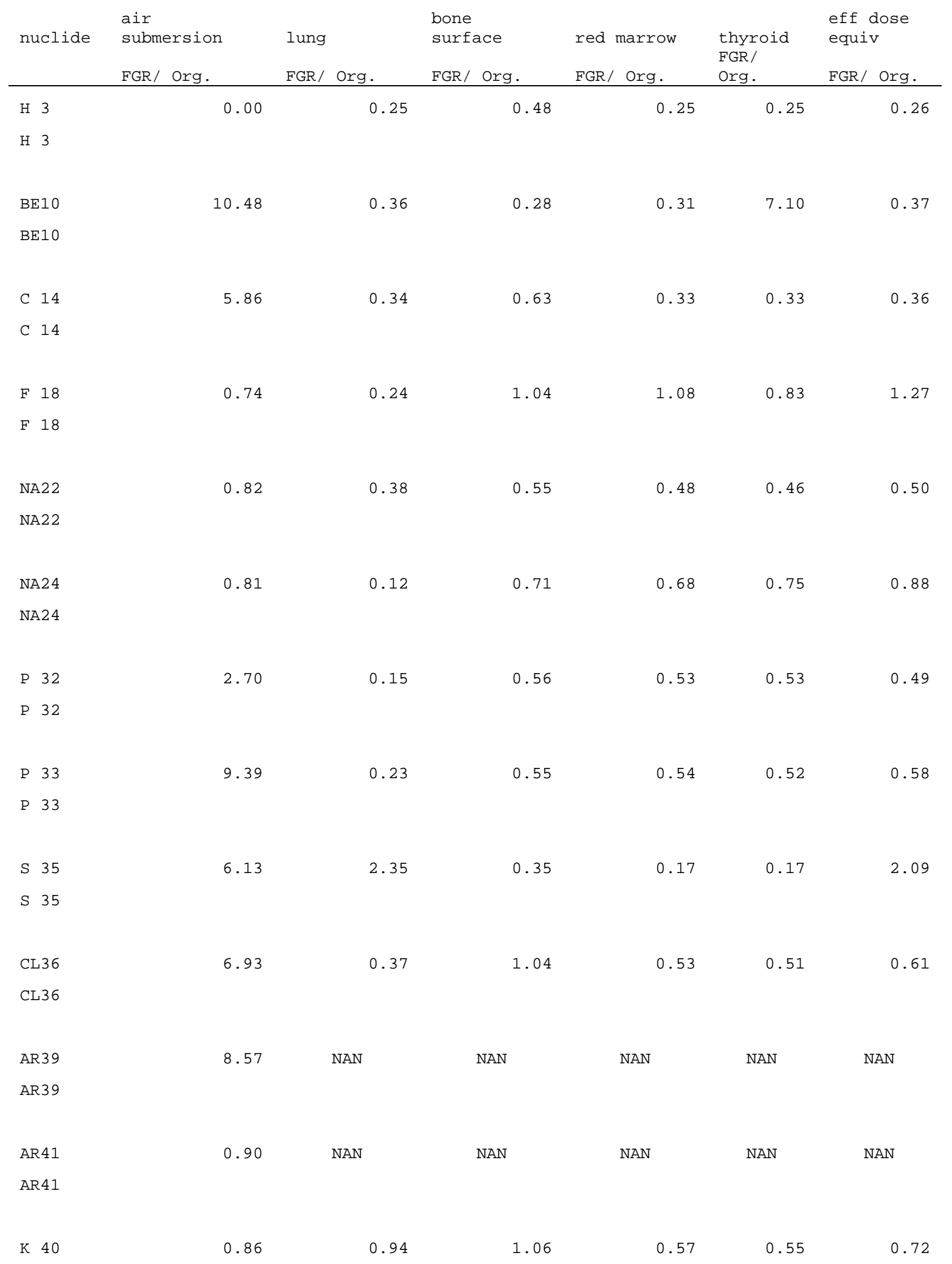




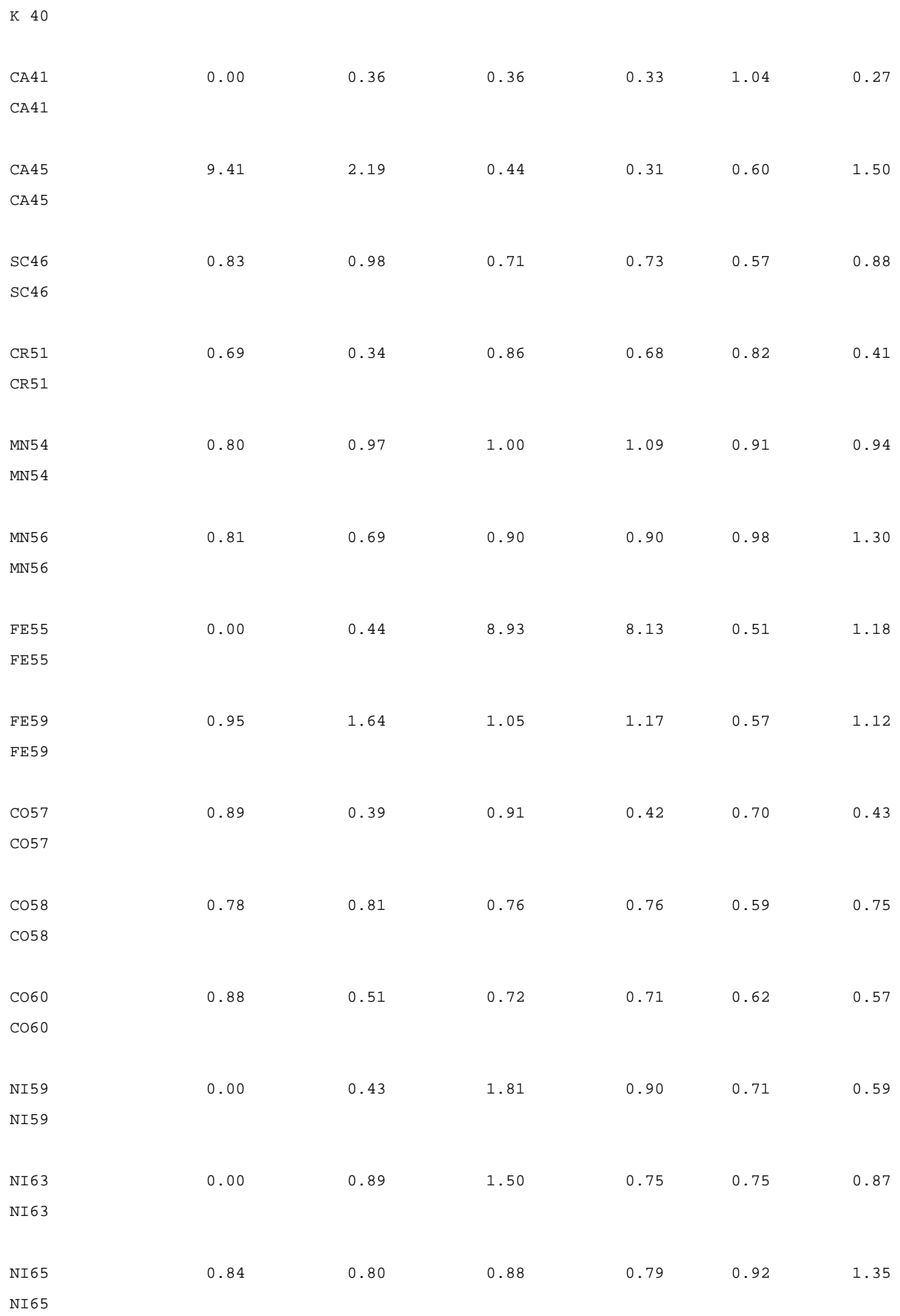




\begin{tabular}{|c|c|c|c|c|c|c|}
\hline CU64 & 0.75 & 0.11 & 0.40 & 0.66 & 0.74 & 0.66 \\
\hline \multicolumn{7}{|l|}{ CU64 } \\
\hline ZN65 & 0.78 & 0.48 & 0.24 & 0.28 & 0.25 & 0.41 \\
\hline \multicolumn{7}{|l|}{ ZN65 } \\
\hline ZN69M & 0.63 & 1.30 & 0.31 & 0.36 & 0.41 & 1.13 \\
\hline \multicolumn{7}{|l|}{ ZN69M } \\
\hline ZN69 & 4.85 & 1.48 & 0.05 & $\odot .04$ & 0.06 & 2.55 \\
\hline \multicolumn{7}{|l|}{ ZN69 } \\
\hline AS76 & 0.76 & 0.65 & 0.81 & 0.57 & 0.64 & 0.74 \\
\hline \multicolumn{7}{|l|}{ AS76 } \\
\hline SE75 & 0.87 & 1.00 & 0.47 & 0.31 & 0.37 & 0.52 \\
\hline \multicolumn{7}{|l|}{ SE75 } \\
\hline SE79 & 10.91 & 2.04 & 0.45 & 0.23 & 0.23 & 1.08 \\
\hline \multicolumn{7}{|l|}{ SE79 } \\
\hline BR82 & 0.79 & 0.23 & 1.70 & 0.67 & 0.68 & 1.06 \\
\hline \multicolumn{7}{|l|}{ BR82 } \\
\hline BR83 & 1.13 & 0.16 & 1.53 & 0.78 & 0.76 & 0.62 \\
\hline \multicolumn{7}{|l|}{ BR83 } \\
\hline BR84 & 0.85 & 0.19 & 1.89 & 1.10 & 1.06 & 0.81 \\
\hline \multicolumn{7}{|l|}{ BR84 } \\
\hline KR83M & 0.07 & NAN & NAN & NAN & NAN & NAN \\
\hline \multicolumn{7}{|l|}{ KR83M } \\
\hline KR85M & 0.96 & NAN & NAN & NAN & NAN & NAN \\
\hline \multicolumn{7}{|l|}{ KR85M } \\
\hline KR85 & 1.58 & NAN & NAN & NAN & NAN & NAN \\
\hline \multicolumn{7}{|l|}{ KR85 } \\
\hline KR87 & 0.76 & NAN & NAN & NAN & NAN & NAN \\
\hline KR87 & & & & & & \\
\hline
\end{tabular}




\begin{tabular}{|c|c|c|c|c|c|c|}
\hline KR88 & 0.86 & NAN & NAN & NAN & NAN & NAN \\
\hline \multicolumn{7}{|l|}{ KR88 } \\
\hline KR89 & 1.00 & NAN & NAN & NAN & NAN & NAN \\
\hline \multicolumn{7}{|l|}{ KR89 } \\
\hline RB86 & 1.00 & 0.39 & 0.61 & $\odot .52$ & 0.53 & 0.55 \\
\hline \multicolumn{7}{|l|}{ RB86 } \\
\hline RB87 & 18.18 & 1.17 & 0.64 & $\odot .56$ & 0.53 & 0.63 \\
\hline \multicolumn{7}{|l|}{ RB87 } \\
\hline RB88 & 0.85 & 0.21 & 1.06 & 0.94 & 1.21 & 0.67 \\
\hline \multicolumn{7}{|l|}{ RB88 } \\
\hline RB89 & 0.86 & 0.31 & $\odot .89$ & 0.86 & 1.06 & 1.17 \\
\hline \multicolumn{7}{|l|}{ RB89 } \\
\hline SR85 & 0.74 & 0.59 & 0.74 & 0.85 & 0.69 & 0.90 \\
\hline \multicolumn{7}{|l|}{ SR85 } \\
\hline SR89 & 2.92 & 0.12 & 0.65 & 0.83 & 0.86 & 0.67 \\
\hline \multicolumn{7}{|l|}{ SR89 } \\
\hline SR90 & 8.86 & 0.56 & 0.57 & 0.55 & 1.76 & 0.44 \\
\hline \multicolumn{7}{|l|}{ SR90 } \\
\hline SR91 & 0.83 & 0.06 & 1.27 & 1.18 & 0.72 & 0.62 \\
\hline \multicolumn{7}{|l|}{ SR91 } \\
\hline SR92 & 0.77 & 0.05 & 2.87 & 2.03 & 0.80 & 0.54 \\
\hline \multicolumn{7}{|l|}{ SR92 } \\
\hline Y 90 & 1.92 & 0.80 & $\odot . \odot 8$ & $\odot . \odot 8$ & 0.08 & 0.63 \\
\hline \multicolumn{7}{|l|}{ Y 90} \\
\hline Y 91M & 0.68 & 0.70 & 1.14 & 1.18 & 1.33 & 1.11 \\
\hline \multicolumn{7}{|l|}{ Y 91M } \\
\hline Y 91 & 1.82 & 0.71 & 0.20 & 0.21 & 0.33 & 0.68 \\
\hline \multicolumn{7}{|l|}{ Y 91} \\
\hline Y 92 & 0.82 & 0.54 & 0.81 & 0.78 & 1.00 & 0.82 \\
\hline
\end{tabular}




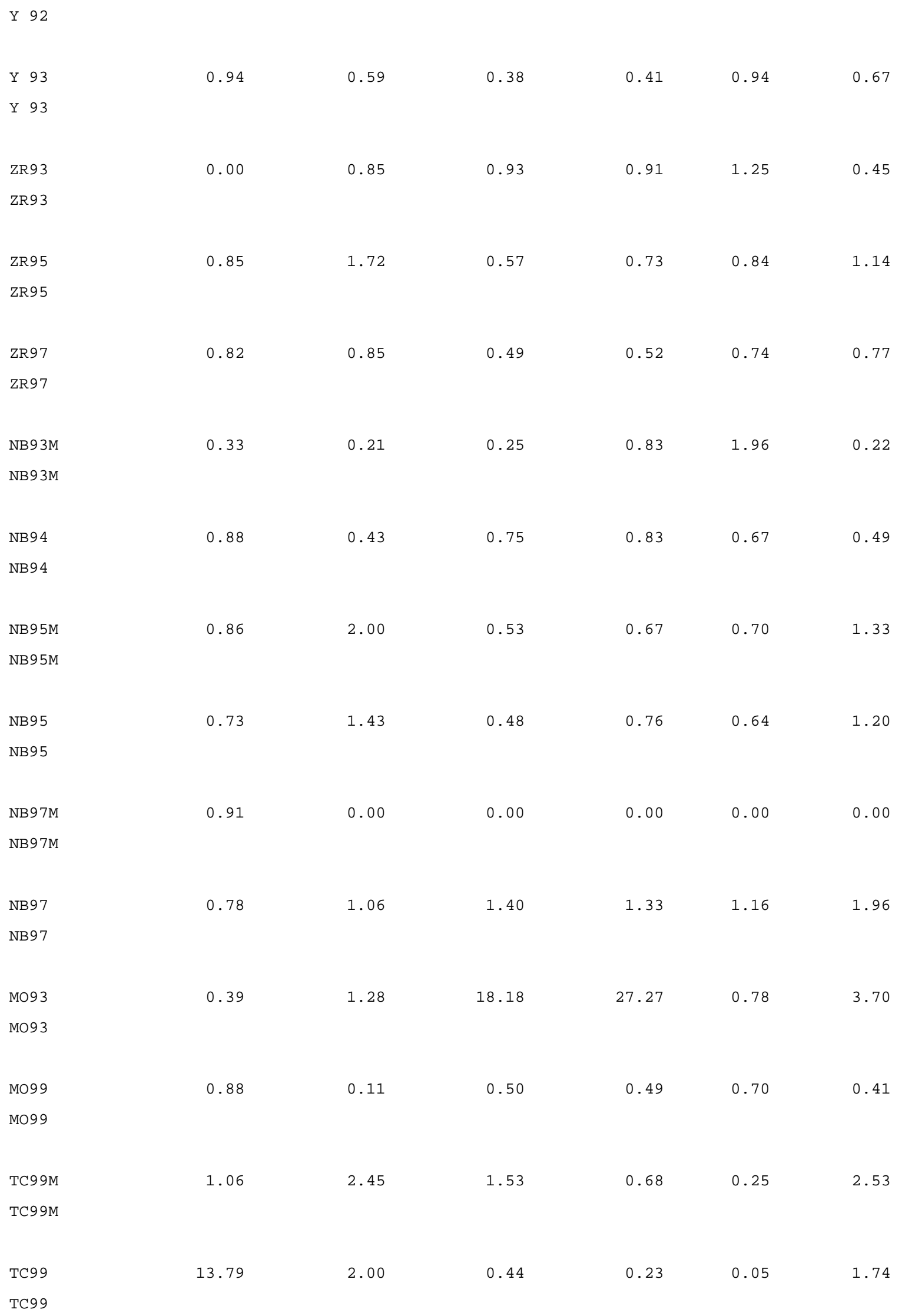




\begin{tabular}{|c|c|c|c|c|c|c|}
\hline TC101 & 0.69 & 1.10 & 1.29 & 0.89 & 0.48 & 2.93 \\
\hline \multicolumn{7}{|l|}{ TC101 } \\
\hline RU103 & 0.70 & 1.38 & 0.77 & 0.77 & 0.64 & 1.20 \\
\hline \multicolumn{7}{|l|}{ RU103 } \\
\hline RU105 & 0.79 & 1.14 & 1.14 & 0.93 & 1.02 & 1.13 \\
\hline \multicolumn{7}{|l|}{ RU105 } \\
\hline RU106 & $\odot . \odot \odot$ & 0.53 & 0.84 & 0.61 & 0.54 & 0.51 \\
\hline \multicolumn{7}{|l|}{ RU106 } \\
\hline RH103M & 0.15 & 1.82 & 1.15 & 0.34 & 0.44 & 1.93 \\
\hline \multicolumn{7}{|l|}{ RH103M } \\
\hline RH105 & 0.69 & 2.30 & 1.00 & $\odot .59$ & 0.84 & 1.17 \\
\hline \multicolumn{7}{|l|}{ RH105 } \\
\hline PD103 & 0.15 & 1.26 & 0.87 & 0.20 & 0.80 & 1.05 \\
\hline \multicolumn{7}{|l|}{ PD103 } \\
\hline PD107 & $\odot . \odot \odot$ & 0.17 & 1.16 & 1.14 & 1.12 & 0.17 \\
\hline \multicolumn{7}{|l|}{ PD107 } \\
\hline PD109 & 1.18 & 1.47 & 0.54 & 0.16 & 0.37 & 1.00 \\
\hline \multicolumn{7}{|l|}{ PD109 } \\
\hline AG110M & 0.82 & 0.42 & $\odot .97$ & 0.73 & 0.74 & 0.24 \\
\hline \multicolumn{7}{|l|}{ AG110M } \\
\hline AG111 & 0.86 & 0.11 & 1.75 & 0.99 & 1.16 & 0.43 \\
\hline \multicolumn{7}{|l|}{ AG111 } \\
\hline CD109 & 0.72 & 2.05 & 1.58 & 0.48 & 0.56 & 0.27 \\
\hline \multicolumn{7}{|l|}{ CD109 } \\
\hline CD113M & 7.07 & 29.31 & 1.42 & 0.59 & 0.57 & 0.28 \\
\hline \multicolumn{7}{|l|}{ CD113M } \\
\hline CD115M & 1.04 & 0.45 & 1.01 & 0.53 & 0.51 & 0.28 \\
\hline CD115M & & & & & & \\
\hline
\end{tabular}




\begin{tabular}{|c|c|c|c|c|c|c|}
\hline CD115 & 0.87 & $\odot .08$ & 0.71 & 0.57 & 0.60 & 0.36 \\
\hline \multicolumn{7}{|l|}{ CD115 } \\
\hline IN111 & 0.96 & 0.24 & 1.09 & 1.63 & 1.58 & 0.87 \\
\hline \multicolumn{7}{|l|}{ IN111 } \\
\hline IN114M & $\odot .57$ & 0.57 & 1.28 & 7.05 & 18.75 & 0.78 \\
\hline \multicolumn{7}{|l|}{ IN114M } \\
\hline IN115M & 0.71 & 0.12 & 1.54 & 3.14 & 1.16 & 0.73 \\
\hline \multicolumn{7}{|l|}{ IN115M } \\
\hline SN117M & 1.12 & 3.06 & 0.43 & 0.41 & 0.77 & 1.85 \\
\hline \multicolumn{7}{|l|}{ SN117M } \\
\hline SN119M & 0.31 & 1.55 & 0.73 & 0.57 & 0.93 & 1.29 \\
\hline \multicolumn{7}{|l|}{ SN119M } \\
\hline SN121M & NAN & 1.70 & 0.82 & 0.73 & 0.94 & 1.45 \\
\hline \multicolumn{7}{|l|}{ SN121M } \\
\hline SN121 & NAN & 2.83 & 4.94 & 1.00 & 0.38 & 1.53 \\
\hline \multicolumn{7}{|l|}{ SN121 } \\
\hline SN123 & 1.47 & 1.02 & 0.61 & 0.61 & 0.65 & 0.90 \\
\hline \multicolumn{7}{|l|}{ SN123 } \\
\hline SN125 & 0.89 & 0.87 & 0.53 & 0.48 & 0.65 & 0.67 \\
\hline \multicolumn{7}{|l|}{ SN125 } \\
\hline SN126 & 1.26 & 1.20 & 0.85 & 0.88 & 1.02 & 1.04 \\
\hline \multicolumn{7}{|l|}{ SN126 } \\
\hline SB124 & 0.75 & 1.05 & 1.46 & 1.09 & 0.97 & 0.94 \\
\hline \multicolumn{7}{|l|}{ SB124 } \\
\hline SB125 & 0.70 & 1.45 & 8.70 & 3.27 & 1.72 & 1.45 \\
\hline \multicolumn{7}{|l|}{ SB125 } \\
\hline SB126M & 0.76 & 0.81 & 1.23 & 1.15 & 1.25 & 2.38 \\
\hline \multicolumn{7}{|l|}{ SB126M } \\
\hline SB126 & $\odot .82$ & 1.21 & 0.93 & 0.81 & 0.75 & 0.91 \\
\hline
\end{tabular}




\begin{tabular}{|c|c|c|c|c|c|c|}
\hline SB127 & $\odot .82$ & 1.59 & 0.86 & 0.75 & 0.70 & 1.06 \\
\hline \multicolumn{7}{|l|}{ SB127 } \\
\hline TE123M & 1.13 & 2.31 & 0.46 & 0.46 & 2.93 & 1.38 \\
\hline \multicolumn{7}{|l|}{ TE123M } \\
\hline TE125M & 0.46 & 2.60 & 0.40 & 0.35 & 6.50 & 1.70 \\
\hline \multicolumn{7}{|l|}{ TE125M } \\
\hline TE127M & 0.48 & 1.70 & 0.43 & 0.43 & 8.60 & 1.25 \\
\hline \multicolumn{7}{|l|}{ TE127M } \\
\hline TE127 & 1.00 & 1.74 & 0.33 & 0.29 & 1.47 & 1.34 \\
\hline \multicolumn{7}{|l|}{ TE127 } \\
\hline TE129M & 0.83 & 1.23 & 0.38 & 0.39 & 32.26 & 0.05 \\
\hline \multicolumn{7}{|l|}{ TE129M } \\
\hline TE129 & 0.83 & 0.94 & 0.37 & 0.35 & 4.07 & 0.88 \\
\hline \multicolumn{7}{|l|}{ TE129 } \\
\hline TE131M & 0.81 & 2.09 & 0.48 & 0.63 & 0.08 & 0.34 \\
\hline \multicolumn{7}{|l|}{ TE131M } \\
\hline TE131 & 0.84 & 1.16 & 1.08 & 0.88 & $\odot . \odot 8$ & 0.85 \\
\hline \multicolumn{7}{|l|}{ TE131 } \\
\hline TE132 & 0.76 & 7.14 & 0.66 & 2.56 & 0.10 & 0.62 \\
\hline \multicolumn{7}{|l|}{ TE132 } \\
\hline TE133M & 0.83 & 1.24 & 1.42 & 1.38 & 0.07 & 0.74 \\
\hline \multicolumn{7}{|l|}{ TE133M } \\
\hline TE133 & 0.78 & 1.10 & 1.82 & 1.57 & 0.07 & 0.83 \\
\hline \multicolumn{7}{|l|}{ TE133 } \\
\hline TE134 & 0.75 & 3.39 & 3.42 & 4.25 & 0.07 & 2.39 \\
\hline \multicolumn{7}{|l|}{ TE134 } \\
\hline I 125 & 0.43 & 0.14 & 0.36 & $\odot . \odot 9$ & 0.50 & 0.85 \\
\hline I 125 & & & & & & \\
\hline
\end{tabular}




\begin{tabular}{|c|c|c|c|c|c|c|}
\hline I 129 & $\odot .56$ & 0.26 & 0.20 & 0.13 & 0.51 & 0.88 \\
\hline \multicolumn{7}{|l|}{ I 129} \\
\hline I 130 & 0.84 & $\odot .09$ & 0.07 & 0.17 & 0.81 & 1.03 \\
\hline \multicolumn{7}{|l|}{ I 130} \\
\hline I 131 & $\odot .79$ & $\odot .09$ & 0.02 & 0.14 & 0.56 & 0.89 \\
\hline \multicolumn{7}{|l|}{ I 131} \\
\hline I 132 & $\odot .79$ & 0.13 & 0.17 & 0.17 & 1.08 & 0.85 \\
\hline \multicolumn{7}{|l|}{ I 132} \\
\hline I 133 & $\odot .79$ & 0.05 & $\odot .07$ & 0.09 & 0.67 & 1.00 \\
\hline \multicolumn{7}{|l|}{ I 133} \\
\hline I 134 & $\odot .81$ & 0.20 & 0.21 & 0.20 & 1.30 & 1.18 \\
\hline \multicolumn{7}{|l|}{ I 134} \\
\hline I 135 & 0.83 & 0.09 & 0.10 & 0.13 & $\odot .85$ & $1.0 \odot$ \\
\hline \multicolumn{7}{|l|}{ I 135} \\
\hline XE131M & $\odot .79$ & NAN & NAN & NAN & NAN & NAN \\
\hline \multicolumn{7}{|l|}{ XE131M } \\
\hline XE133M & $\odot .79$ & NAN & NAN & NAN & NAN & NAN \\
\hline \multicolumn{7}{|l|}{ XE133M } \\
\hline XE133 & $\odot .98$ & NAN & NAN & NAN & NAN & NAN \\
\hline \multicolumn{7}{|l|}{ XE133 } \\
\hline XE135M & 0.76 & NAN & NAN & NAN & NAN & NAN \\
\hline \multicolumn{7}{|l|}{ XE135M } \\
\hline XE135 & 0.88 & NAN & NAN & NAN & NAN & NAN \\
\hline \multicolumn{7}{|l|}{ XE135 } \\
\hline XE137 & $\odot . \odot \odot$ & NAN & NAN & NAN & NAN & NAN \\
\hline \multicolumn{7}{|l|}{ XE137 } \\
\hline XE138 & 0.77 & NAN & NAN & NAN & NAN & NAN \\
\hline XE138 & & & & & & \\
\hline
\end{tabular}




\begin{tabular}{|c|c|c|c|c|c|c|}
\hline CS134M & 0.74 & 0.37 & 3.11 & 1.33 & 1.41 & 1.40 \\
\hline \multicolumn{7}{|l|}{ CS134M } \\
\hline CS134 & 0.76 & 0.74 & 0.82 & 0.58 & 0.57 & 0.56 \\
\hline \multicolumn{7}{|l|}{ CS134 } \\
\hline CS135 & 12.50 & 3.19 & 1.08 & 0.54 & 0.54 & 0.63 \\
\hline \multicolumn{7}{|l|}{ CS135 } \\
\hline CS136 & 0.84 & 0.51 & 1.62 & 0.58 & 0.53 & 0.63 \\
\hline \multicolumn{7}{|l|}{ CS136 } \\
\hline CS137 & $\odot . \odot \odot$ & 1.08 & 1.04 & 0.64 & 0.54 & 0.59 \\
\hline \multicolumn{7}{|l|}{ CS137 } \\
\hline CS138 & 0.80 & 0.19 & 1.71 & 1.11 & 1.13 & 0.89 \\
\hline \multicolumn{7}{|l|}{ CS138 } \\
\hline BA139 & 1.45 & 0.11 & 4.62 & 4.14 & 1.00 & 0.67 \\
\hline \multicolumn{7}{|l|}{ BA139 } \\
\hline BA140 & 0.71 & 0.10 & 0.91 & 1.36 & 1.00 & 1.10 \\
\hline \multicolumn{7}{|l|}{ BA140 } \\
\hline BA141 & 0.75 & 0.20 & 1.58 & 3.11 & 1.20 & 0.91 \\
\hline \multicolumn{7}{|l|}{ BA141 } \\
\hline BA142 & 0.94 & 0.32 & 2.58 & 2.20 & 1.30 & 1.25 \\
\hline \multicolumn{7}{|l|}{ BA142 } \\
\hline LA140 & 0.85 & 0.11 & 0.63 & 0.63 & 0.69 & 0.60 \\
\hline \multicolumn{7}{|l|}{ LA140 } \\
\hline LA141 & 0.93 & $\odot .06$ & 0.57 & 0.62 & 0.81 & 0.39 \\
\hline \multicolumn{7}{|l|}{ LA141 } \\
\hline LA142 & 0.83 & 0.12 & 0.92 & 0.75 & 1.03 & 0.70 \\
\hline \multicolumn{7}{|l|}{ LA142 } \\
\hline CE141 & 1.04 & 1.88 & 0.41 & $\odot .41$ & 0.88 & 1.65 \\
\hline \multicolumn{7}{|l|}{ CE141 } \\
\hline CE143 & 0.93 & 1.25 & 0.82 & 0.48 & 1.03 & 0.87 \\
\hline
\end{tabular}




\begin{tabular}{|c|c|c|c|c|c|c|}
\hline CE144 & $1.0 \odot$ & 0.53 & 0.51 & 0.60 & 0.93 & 0.53 \\
\hline \multicolumn{7}{|l|}{ CE144 } \\
\hline PR143 & 4.69 & 1.38 & 0.13 & 0.13 & 0.13 & 1.09 \\
\hline \multicolumn{7}{|l|}{ PR143 } \\
\hline PR144M & 0.70 & $\odot .0 \odot$ & 0.00 & $\odot . \odot \odot$ & $0.0 \odot$ & $\odot .0 \odot$ \\
\hline \multicolumn{7}{|l|}{ PR144M } \\
\hline PR144 & 1.05 & 0.59 & 0.67 & 0.59 & 0.89 & 1.50 \\
\hline \multicolumn{7}{|l|}{ PR144 } \\
\hline ND147 & 0.82 & 1.80 & 0.32 & 0.44 & 0.84 & 1.33 \\
\hline \multicolumn{7}{|l|}{ ND147 } \\
\hline PM147 & 7.30 & 0.50 & 0.25 & 0.27 & 0.77 & 0.45 \\
\hline \multicolumn{7}{|l|}{ PM147 } \\
\hline PM148M & 0.74 & 1.08 & 0.59 & 0.71 & 0.70 & 0.97 \\
\hline \multicolumn{7}{|l|}{ PM148M } \\
\hline PM148 & 0.79 & 0.93 & 0.61 & 0.58 & 0.97 & 0.73 \\
\hline \multicolumn{7}{|l|}{ PM148 } \\
\hline PM149 & 1.16 & 1.34 & 0.19 & 0.19 & 0.86 & 0.89 \\
\hline \multicolumn{7}{|l|}{ PM149 } \\
\hline PM151 & 0.76 & 1.59 & 0.82 & 0.54 & 1.06 & 0.96 \\
\hline \multicolumn{7}{|l|}{ PM151 } \\
\hline SM147 & NAN & 0.03 & 0.04 & $\odot . \odot 4$ & $\odot .04$ & 0.48 \\
\hline \multicolumn{7}{|l|}{ SM147 } \\
\hline SM151 & 0.10 & $\odot .94$ & 0.79 & $\odot .79$ & 0.88 & 0.49 \\
\hline \multicolumn{7}{|l|}{ SM151 } \\
\hline SM153 & 0.77 & 1.90 & 0.39 & 0.32 & 0.76 & 1.17 \\
\hline \multicolumn{7}{|l|}{ SM153 } \\
\hline EU152 & 0.81 & 1.09 & 0.83 & 0.89 & 1.04 & 0.75 \\
\hline EU152 & & & & & & \\
\hline
\end{tabular}




\begin{tabular}{|c|c|c|c|c|c|c|}
\hline EU154 & 0.86 & 1.27 & 0.79 & 0.84 & 1.12 & 0.72 \\
\hline \multicolumn{7}{|l|}{ EU154 } \\
\hline EU155 & 0.94 & 1.58 & 0.80 & 0.71 & 2.00 & 0.64 \\
\hline \multicolumn{7}{|l|}{ EU155 } \\
\hline EU156 & 0.80 & 1.16 & 0.46 & 0.53 & 0.83 & 0.87 \\
\hline \multicolumn{7}{|l|}{ EU156 } \\
\hline GD153 & 0.82 & 0.55 & 0.53 & 0.44 & 0.73 & 0.33 \\
\hline \multicolumn{7}{|l|}{ GD153 } \\
\hline TB160 & 0.89 & 1.53 & 0.56 & 0.64 & 0.86 & 1.04 \\
\hline \multicolumn{7}{|l|}{ TB160 } \\
\hline H0166M & 0.93 & 1.09 & 0.81 & 0.94 & 1.00 & 0.60 \\
\hline \multicolumn{7}{|l|}{ H0166M } \\
\hline W 181 & 1.12 & 0.08 & 0.71 & 0.30 & 0.72 & 0.64 \\
\hline \multicolumn{7}{|l|}{ W 181} \\
\hline W 187 & 0.80 & 0.06 & 0.50 & 0.68 & 0.75 & 1.06 \\
\hline \multicolumn{7}{|l|}{ W 187} \\
\hline W 185 & 7.62 & 0.05 & 0.48 & 0.50 & 0.53 & 0.57 \\
\hline \multicolumn{7}{|l|}{ W 185} \\
\hline RE187 & NAN & 0.35 & 1.15 & 0.56 & 0.55 & 0.42 \\
\hline \multicolumn{7}{|l|}{ RE187 } \\
\hline IR192 & 0.69 & 0.92 & 0.84 & 0.72 & 0.73 & 0.87 \\
\hline \multicolumn{7}{|l|}{ IR192 } \\
\hline HG2O3 & 1.03 & 0.47 & 0.61 & 0.33 & 0.35 & 0.31 \\
\hline \multicolumn{7}{|l|}{ HG2O3 } \\
\hline RN222 & $0.0 \odot$ & NAN & NAN & NAN & NAN & NAN \\
\hline \multicolumn{7}{|l|}{ RN222 } \\
\hline TH227 & 0.82 & 2.39 & 0.05 & 0.05 & 0.10 & 2.27 \\
\hline TH227 & & & & & & \\
\hline
\end{tabular}




\begin{tabular}{|c|c|c|c|c|c|c|}
\hline TH228 & 1.04 & 0.50 & 0.11 & 0.11 & 1.53 & 0.45 \\
\hline \multicolumn{7}{|l|}{ TH228 } \\
\hline TH229 & 1.10 & 0.25 & 0.09 & 0.05 & 32.69 & 0.15 \\
\hline \multicolumn{7}{|l|}{ TH229 } \\
\hline TH230 & 0.92 & 0.26 & 0.33 & 0.14 & 36.59 & 0.20 \\
\hline \multicolumn{7}{|l|}{ TH230 } \\
\hline TH231 & 1.00 & 2.72 & 0.19 & 0.76 & 1.29 & 1.50 \\
\hline \multicolumn{7}{|l|}{ TH231 } \\
\hline TH232 & 0.66 & 0.18 & 0.06 & 0.03 & 113.89 & 0.08 \\
\hline \multicolumn{7}{|l|}{ TH232 } \\
\hline TH234 & 0.24 & 0.91 & 0.18 & 0.18 & 0.56 & 0.81 \\
\hline \multicolumn{7}{|l|}{ TH234 } \\
\hline RA223 & 0.35 & 3.65 & 0.87 & 1.21 & 0.36 & 3.52 \\
\hline \multicolumn{7}{|l|}{ RA223 } \\
\hline RA224 & 0.82 & 4.03 & 1.78 & 2.11 & 0.36 & 3.90 \\
\hline \multicolumn{7}{|l|}{ RA224 } \\
\hline RA225 & 0.58 & 3.25 & 1.69 & 2.44 & 2.67 & 3.15 \\
\hline \multicolumn{7}{|l|}{ RA225 } \\
\hline RA226 & 1.36 & 1.75 & 1.06 & 1.33 & 8.57 & 1.59 \\
\hline \multicolumn{7}{|l|}{ RA226 } \\
\hline RA228 & $\odot . \odot \odot$ & 1.45 & 9.82 & 8.55 & 190.91 & 2.60 \\
\hline \multicolumn{7}{|l|}{ RA228 } \\
\hline PB210 & 0.64 & 279.07 & 0.54 & 0.84 & 0.38 & 0.25 \\
\hline \multicolumn{7}{|l|}{ PB210 } \\
\hline PB212 & 0.87 & 0.05 & 0.22 & 0.31 & $1.0 \odot$ & $0.4 \odot$ \\
\hline \multicolumn{7}{|l|}{ PB212 } \\
\hline BI210 & 3.86 & 1.79 & 1.24 & 0.70 & $\odot .71$ & 1.75 \\
\hline \multicolumn{7}{|l|}{ BI210 } \\
\hline BI212 & 0.10 & 5.26 & 0.09 & 0.31 & 0.42 & 6.08 \\
\hline
\end{tabular}


BI212

$\mathrm{P} 0210$

0.75

2.00

4.31

3.54

0.38

1.43

P0210

U 232

$\bullet .76$

$\odot .21$

8.20

970.59

181.82

0.22

U 232

U 233

1.07

0.27

42.11

45.33

48.65

0.27

U 233

U 234

0.53

0.26

26.32

34.67

43.24

0.26

U 234

U 235

1.18

0.25

26.67

23.81

23.88

0.26

U 235

U 236

0.43

$\odot .26$

26.67

$35.0 \odot$

44.12

0.26

U 236

U 237

๑. 81

2.86

0.47

0.35

1.00

1.94

U 237

บ 238

0.33

$\odot .25$

27.06

32.67

38.46

0.25

U 238

U 240

0.00

1.26

5.20

0.92

1.10

0.95

U 240

PA231

$\odot .91$

0.20

0.17

$\odot .08 \quad 7647.06$

0.13

PA231

PA233

0.68

1.76

$\odot .39$

0.52

0.88

1.50

PA233

PA234

0.82

1.96

1.00

0.90

1.08

1.83

PA234

AC225

$\odot .04$

3.94

$\odot . \odot 8$

0.12

9.52

3.86

AC225

AC227

0.76

$\odot .27$

0.07

$0.04 \quad 863.64$

$\odot .16$

AC227 


\begin{tabular}{|c|c|c|c|c|c|c|}
\hline AC228 & 0.82 & 0.50 & 0.15 & 0.17 & 4.92 & 0.47 \\
\hline \multicolumn{7}{|l|}{ AC228 } \\
\hline FR223 & 1.00 & 0.43 & 1.31 & 0.67 & 0.67 & 0.69 \\
\hline \multicolumn{7}{|l|}{ FR223 } \\
\hline NP237 & 1.12 & 1.81 & 0.26 & 0.13 & 1083.33 & 0.14 \\
\hline \multicolumn{7}{|l|}{ NP237 } \\
\hline NP238 & 0.84 & 1.67 & 0.28 & 0.15 & 4.17 & 0.19 \\
\hline \multicolumn{7}{|l|}{ NP238 } \\
\hline NP239 & 0.85 & 2.74 & 0.24 & 0.32 & 1.09 & 1.41 \\
\hline \multicolumn{7}{|l|}{ NP239 } \\
\hline PU236 & 0.45 & 0.49 & 1.06 & 0.78 & 1100.00 & 0.55 \\
\hline \multicolumn{7}{|l|}{ PU236 } \\
\hline PU237 & 0.80 & 0.85 & 0.57 & 0.39 & 0.86 & 0.80 \\
\hline \multicolumn{7}{|l|}{ PU237 } \\
\hline PU238 & 0.41 & 0.29 & 0.22 & 0.14 & 3294.12 & 0.21 \\
\hline \multicolumn{7}{|l|}{ PU238 } \\
\hline PU239 & 0.61 & 0.28 & 0.21 & 0.14 & 4000.00 & 0.20 \\
\hline \multicolumn{7}{|l|}{ PU239 } \\
\hline PU240 & 0.42 & 0.28 & 0.21 & 0.14 & $4000 . \odot \odot$ & 0.20 \\
\hline \multicolumn{7}{|l|}{ PU240 } \\
\hline PU241 & 3076.92 & 0.15 & 0.23 & 0.13 & 88750.00 & 0.14 \\
\hline \multicolumn{7}{|l|}{ PU241 } \\
\hline PU242 & 0.42 & 0.28 & 0.25 & 0.15 & 4054.05 & 0.20 \\
\hline \multicolumn{7}{|l|}{ PU242 } \\
\hline PU243 & 1.25 & 2.35 & 0.26 & 1.57 & 1.45 & 2.23 \\
\hline \multicolumn{7}{|l|}{ PU243 } \\
\hline PU244 & 0.37 & 0.26 & 0.22 & 0.14 & 241.67 & 0.19 \\
\hline PU244 & & & & & & \\
\hline
\end{tabular}




\begin{tabular}{|c|c|c|c|c|c|c|}
\hline AM241 & 1.11 & 2.06 & 0.77 & 0.34 & 2900.00 & 0.35 \\
\hline \multicolumn{7}{|l|}{ AM241 } \\
\hline AM242M & 0.79 & 2.32 & 0.85 & 0.32 & 24166.67 & 0.34 \\
\hline \multicolumn{7}{|l|}{ AM242M } \\
\hline AM242 & 0.95 & 2.55 & 0.58 & 0.57 & 48.00 & 1.21 \\
\hline \multicolumn{7}{|l|}{ AM242 } \\
\hline AM243 & 1.26 & 2.06 & 0.81 & 0.34 & 2416.67 & 0.37 \\
\hline \multicolumn{7}{|l|}{ AM243 } \\
\hline CM242 & 0.48 & 2.50 & 0.57 & 0.59 & 37.23 & 1.21 \\
\hline \multicolumn{7}{|l|}{ CM242 } \\
\hline CM243 & 0.89 & 2.22 & 0.86 & 0.38 & 1416.67 & $\odot .40$ \\
\hline \multicolumn{7}{|l|}{ CM243 } \\
\hline CM244 & 0.46 & 2.17 & 0.84 & 0.42 & 1313.13 & 0.42 \\
\hline \multicolumn{7}{|l|}{ CM244 } \\
\hline CM245 & 1.15 & 2.12 & $\odot .86$ & 0.35 & 2727.27 & $\odot .35$ \\
\hline \multicolumn{7}{|l|}{ CM245 } \\
\hline CM246 & 0.47 & 2.12 & 0.77 & 0.34 & 2929.29 & 0.35 \\
\hline \multicolumn{7}{|l|}{ CM246 } \\
\hline CM247 & $\odot .59$ & 2.13 & $\odot .80$ & 0.34 & 1928.57 & 0.35 \\
\hline \multicolumn{7}{|l|}{ CM247 } \\
\hline CM248 & 0.44 & 1.00 & 1.00 & 1.00 & 1.00 & 1.00 \\
\hline \multicolumn{7}{|l|}{ CM248 } \\
\hline CF252 & 0.65 & $1.0 \odot$ & 1.00 & 1.00 & 1.00 & 1.00 \\
\hline CF252 & & & & & & \\
\hline
\end{tabular}




\section{Appendix C Scripts}

\section{Appendix C.1}

\section{Script to create updated HUDUFACT file from FGR12/13}

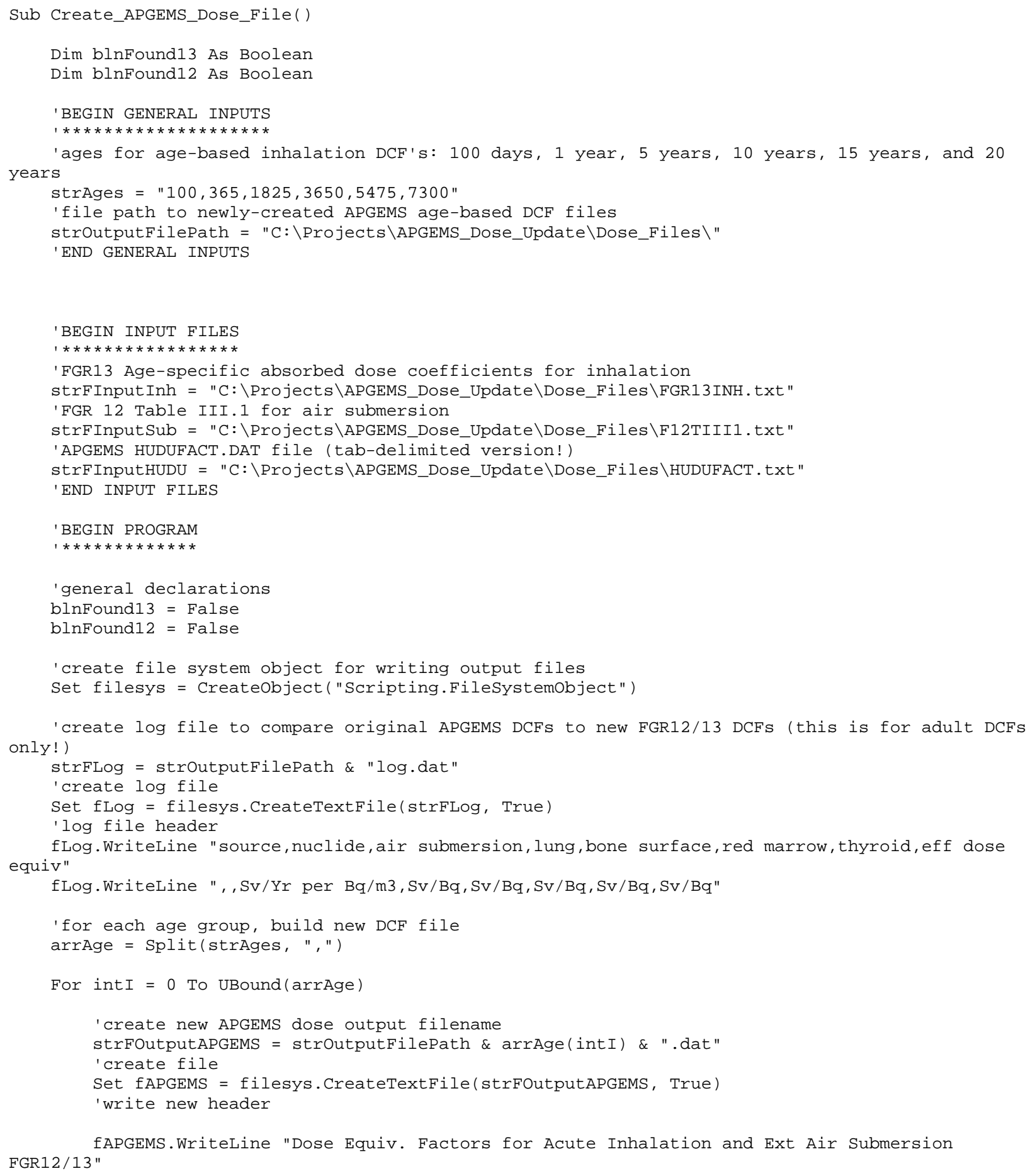




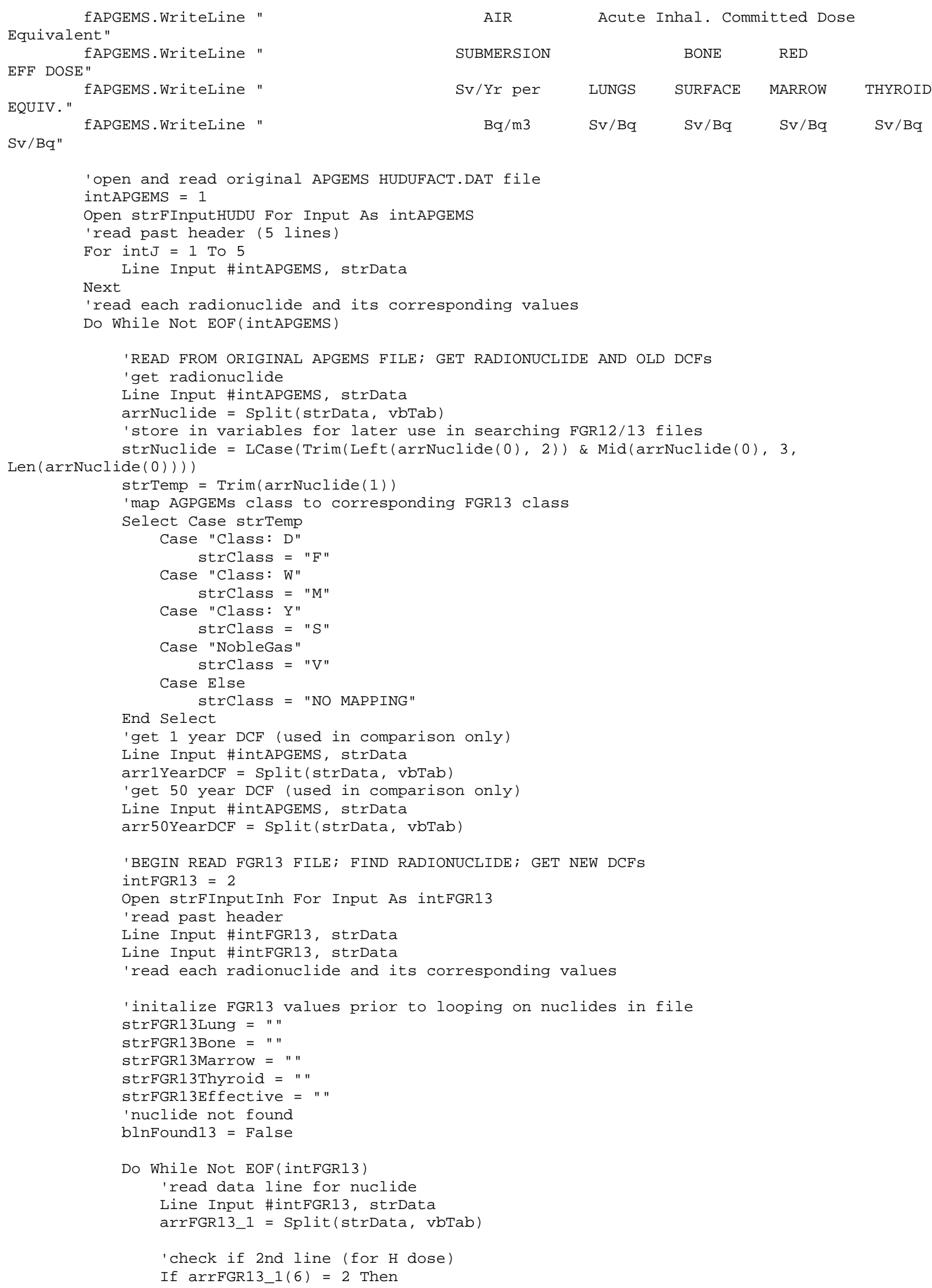




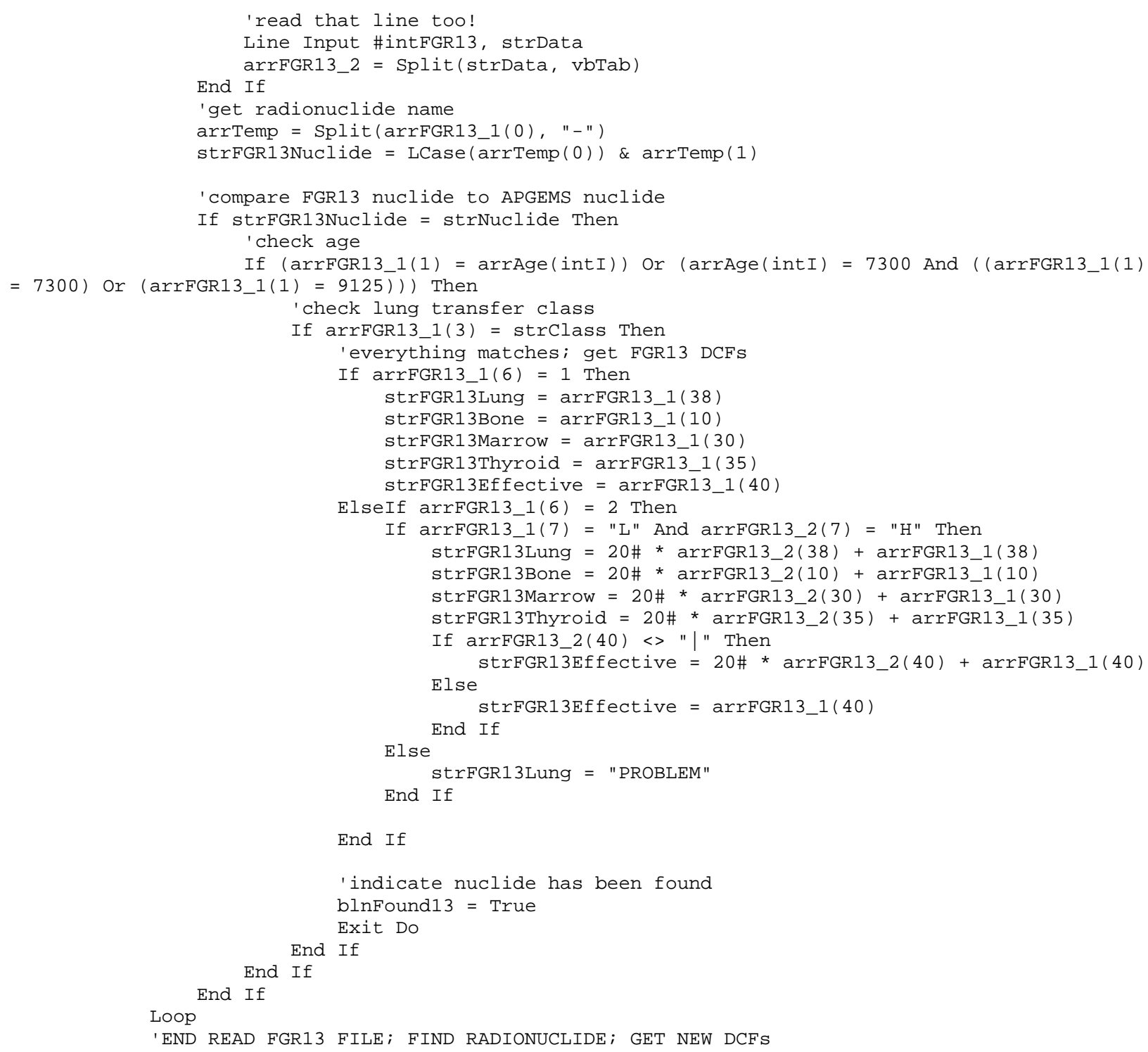


$\mathrm{Sv} / \mathrm{s}$ per $\mathrm{Bq} / \mathrm{m} 3$

'compare FGR12 nuclide to APGEMS nuclide

If strFGR12Nuclide = strNuclide Then

'column 27 ( $\odot$-based array) contains effective submersion dose in units of

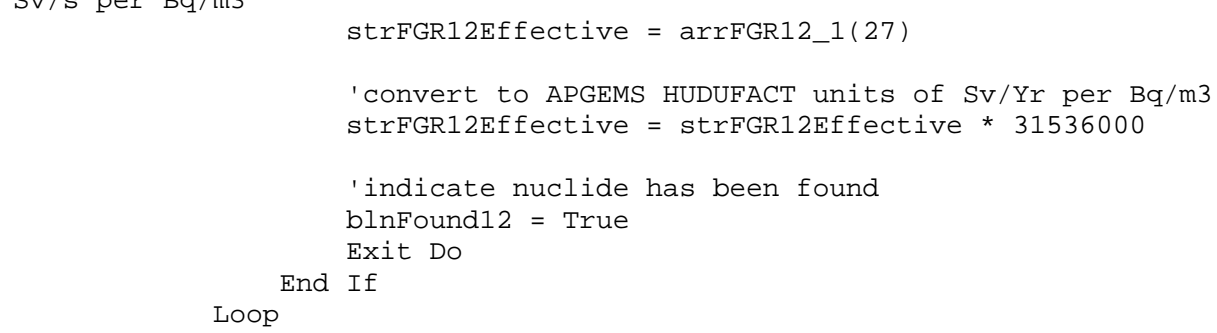

'END READ FGR12 FILE; FIND RADIONUCLIDE; GET NEW DCFS

'BEGIN WRITE OUTPUT FILE

'deal with missing values

If blnFound13 = False Then

'set values to missing

'strFGR13Lung $=9.9 \mathrm{E}-99$

'strFGR13Bone $=9.9 \mathrm{E}-99$

'strFGR13Marrow $=9.9 \mathrm{E}-99$

'strFGR13Thyroid $=9.9 \mathrm{E}-99$

'strFGR13Effective $=9.9 \mathrm{E}-99$

'set to HUDUFACT originals

strFGR13Lung $=\operatorname{arr50YearDCF}(4)$

strFGR13Bone $=\operatorname{arr50YearDCF}(5)$

strFGR13Marrow $=\operatorname{arr50YearDCF}(6)$

strFGR13Thyroid = $\operatorname{arr50YearDCF}(7)$

End If

strFGR13Effective $=\operatorname{arr50YearDCF}(8)$

If blnFound12 = False Then

'set values to missing

'strFGR12Effective $=9.9 \mathrm{E}-99$

'set to HUDUFACT originals

End If

strFGR12Effective = arrNuclide(3)

'write new APGEMS DCF values

fAPGEMS.WriteLine " " \& Format(arrNuclide(0), "!@@@@@@@@") \& arrNuclide(1) \& " "

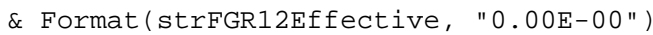

fAPGEMS.Writeline " 1 Yr: " \& Format(strFGR13Lung, "๑.

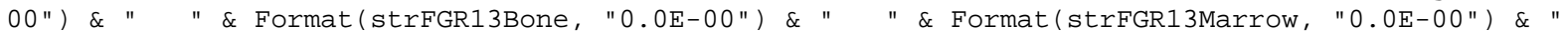

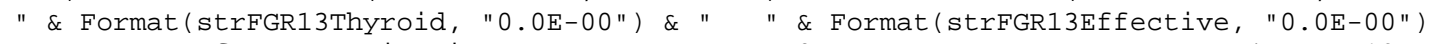

fAPGEMS. Writeline " $50 \mathrm{Yr}$ : " \& Format (strFGR13Lung, "O.OE-

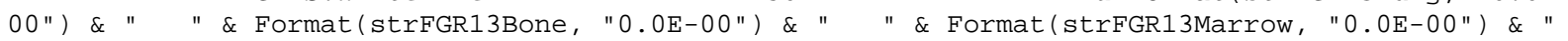

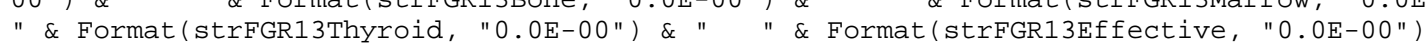

' $\log$ file

If $\operatorname{arrAge}($ int $I)=7300$ Then

'adult

' new FGR12/13 values

If $($ blnFound12 $=$ False) or (blnFound13 = False) Then

$1 *$ to indicate old HUDUFACT values are being used

fLog.Writeline "*FGR12/13," \& arrNuclide(๑) \& "," \& Format(strFGR12Effective,

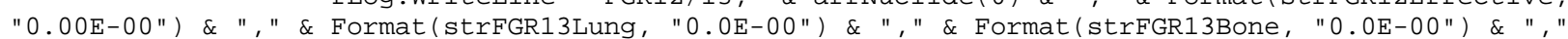

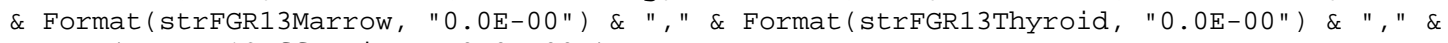

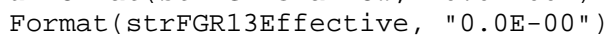

Else

'new FGR12/13 values being used

fLog.Writeline "FGR12/13," \& arrNuclide(๑) \& "," \& Format(strFGR12Effective,

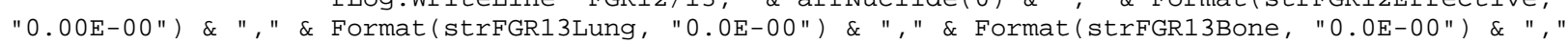

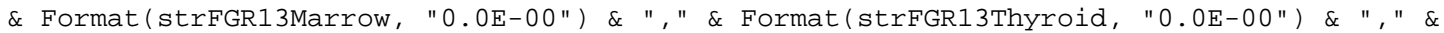

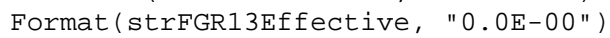

End If

'orignal APGEMS HUDUFACT values

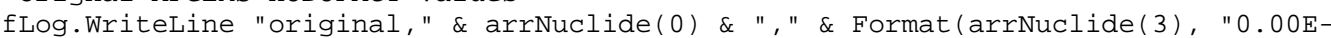

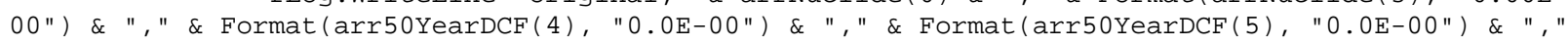




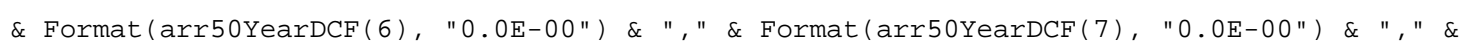

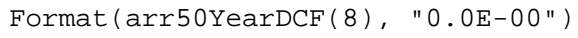

\section{End If} fLog.WriteLine ""

Close (intFGR13)

Close (intFGR12)

'END WRITE OUTPUT FILE

Loop

'close original APGEMS file close (intAPGEMS)

'close new APGEMS DCF File

Next

fAPGEMS. Close

Close (intAPGEMS)

'close new APGEMS DCF file

fAPGEMS. Close

'close log file

fLog.Close

End Sub 


\section{Appendix C.2}

\section{Script to create ratios and plot points} Sub APGEMS_Ratio()

' APGEMS_Ratio Macro

,

'this does the ratio of FGR12-13/APGEMS

strJ = ""

strB = ""

For intI $=3$ To 675 step 3

strJ = "J" \& intI

stro = "0" \& intI

strJo = strJ \& ":" \& stro

Range(str J). Select

ActiveCell. FormulaR1C1 $="=\operatorname{RC}[-7] / \mathrm{R}[1] \mathrm{C}[-7] "$

Range(str J). Select

ActiveWindow. ScrollColumn $=2$

Activewindow. Scrollcolumn $=3$

Activewindow. Smallscroll ToRight: $=1$

Range(strjo). Select

Next

Selection. FillRight

'this charts the ratio of FGR12-13/APGEMS

'Activesheet. Shapes. AddChart . Select

'ActiveChart. SetSourceData Source:=Range("' $\log$ '!\$J\$1:\$0\$3")

'Activechart. ChartType $=$ xlColumnclustered

Activechart.PlotArea.Select

For int J $=1$ To 6

Select Case int J

Case 1

strAlpha $=" \mathrm{~J} "$

Case 2

strAlpha $=" \mathrm{~K} "$

Case 3

strAlpha = "L"

Case 4

strAlpha = "M"

Case 5

$$
\text { strAlpha }=" N "
$$

Case 6

$$
\text { strAlpha }=" 0 "
$$

End Select

stry = ""

strX = ""

ActiveSheet. Shapes. AddChart. Select

ActiveChart. ChartType $=$ xlColumnclustered

Activechart.PlotArea. Select

For intI $=3$ To 675 step 3

If intI $=3$ Then

strY = strY \& "="

End If

strX $=\operatorname{strX\& ~} \&="$

strCompare = strAlpha \& intI

If IsNumeric(Range(strcompare).Value) Then

If Range(strCompare).Value > 10\# Then

stry = stry \& "'log'!\$" \& strAlpha \& "\$" \& intI

strX $=\operatorname{str} X \&$ \&' $\log '$ ! \$B\$" \& intI

If intI $<675$ Then

stry $=\operatorname{str} Y \& ", "$

$\operatorname{str} X=\operatorname{strX} \&$ \&," 
End If

End If

Next

If Right (strY, 1) = ", " Then

strY $=\operatorname{Left}($ strY, Len $(\operatorname{str} Y)-1)$

End If

$\operatorname{str} X=\operatorname{Left}(\operatorname{str} X, \operatorname{Len}(\operatorname{str} X)-1)$

Activechart.Seriescollection. NewSeries

Activechart.SeriesCollection(1). Values = stry

Next

Activechart.Seriescollection(1).XValues = strX

End Sub 



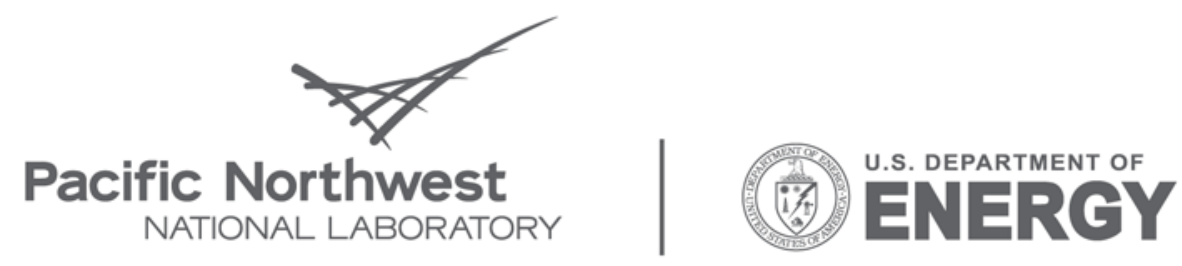

Proudly Operated by Battelle Since 1965

902 Battelle Boulevard

P.O. Box 999

Richland, WA 99352

1-888-375-PNNL (7665)

www.pnl.gov 